\title{
EEG-Kohärenzanalysen zu \\ kognitiven Prozessen im Arbeitsgedächtnis
}

\author{
Dissertation \\ zur Erlangung des Doktorgrades \\ der Mathematisch-Naturwissenschaftlichen Fakultäten \\ der Georg-August-Universität zu Göttingen
}

vorgelegt von

Nuria Vath

aus Hamburg 
D7

Referent: Prof. Dr. Gerd Lüer

Korreferent: Prof. Dr. Uta Lass

Tag der mündlichen Prüfung: 02.05.01 


\section{Vorwort}

Die vorliegende Arbeit wäre ohne Unterstützung und Mitwirkung von verschiedenen Seiten nicht zustande gekommen. An erster Stelle gilt mein Dank meinem verehrten Lehrer, Prof. Dr. Gerd Lüer, für die Anregung und Übertragung der Arbeit sowie Prof. Dr. Uta Lass für die umfassende und kompetente Betreuung. Dr. Dietrich Becker danke ich herzlich für die hilfreichen Diskussionen und für die äußerst kritische Textlektüre.

Die vorgestellte Untersuchung profitiert von einer Versuchsanordnung, die nach umfangreichen Vorarbeiten entstand und über viele Jahre hinweg von Prof. Dr. Lüer, Prof. Dr. Lass und Dr. Becker im Rahmen deutsch-chinesischer Forschungsprojekte optimiert wurde. Die Software zur Versuchssteuerung hat Herr Michael Felsmann erstellt, dem an dieser Stelle auch für die durch die EEG-Ableitung notwendig gewordenen programmtechnischen Änderungen bzw. Ergänzungen gedankt sei.

Bei Prof. Dr. Werner Krause möchte ich mich für die freundliche Aufnahme, die Bereitstellung von EEG-Geräten und Institutsräumlichkeiten sowie für wertvolle Ratschläge bezüglich der EEG-Ableitung bedanken. Prof. Dr. Bärbel Schack und Dr. Eva Möller danke ich für die kooperative Zusammenarbeit, die von der Einführung in die Problematik der EEG-Analyse über die Bereitstellung des EEG-Auswertungsprogramms bis zur Durchführung aufwendiger Ergebnisanalysen reichte. Schließlich sei noch Frau Christine Schleußner gedankt, die mir als EEG-Assistentin bei den Elektrodenpositionierungen hilfreich zur Seite stand.

Diese Arbeit widme ich meinem Mann als Dank für die besondere Geduld, Unterstützung und Ermunterung, die er mir in allen Phasen ihrer Entstehung hat zukommen lassen. 


\section{Inhaltsverzeichnis}

Theoretischer Teil

$1 \quad$ Einleitung

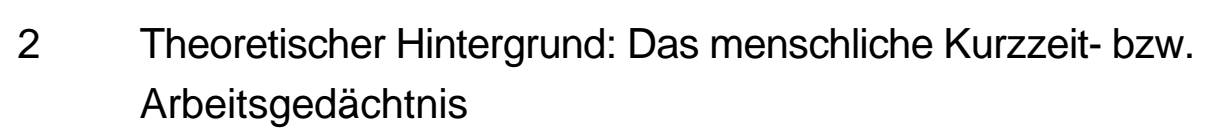

$2.1 \quad$ Das psychologische Konzept des Kurzzeitgedächtnisses 3

2.1.1 Das Kurzzeitgedächtnis als Arbeitsgedächtnis 3

2.1.2 Abruf aus dem Kurzzeitgedächtnis 10

2.2 Das Kurzzeitgedächtnis als Gegenstand neurowissenschaftlicher

Forschung

15

2.2.1 Neurophysiologische und anatomische Grundlagen des Gedächtnisses 16

2.2.2 Verfahren zur Untersuchung des Gedächtnisses 24

Bildgebende Verfahren: CAT, rCBF, PET und fMRT 25

\begin{tabular}{ll}
\hline MEG und EEG & 27
\end{tabular}

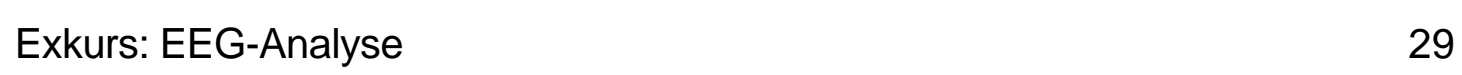

$3 \quad$ Neuropsychologische Untersuchungen zum Arbeitsgedächtnis

$3.1 \quad$ Klinisch-anatomische Studien 32

3.1.1 Beeinträchtigung des phonologischen Speichers 33

3.1.2 Beeinträchtigung des visuell-räumlichen Speichers 39

3.1.3 Beeinträchtigung der zentralen Exekutive $\quad 44$

$3.2 \quad$ Untersuchungen an gesunden Personen 49

3.2.1 Topographische Aspekte 49

\begin{tabular}{l|l|}
3.2 .2 & Dynamik der Verarbeitungsprozesse
\end{tabular}

$\begin{array}{lll}3.2 .3 & 68\end{array}$

3.3 Kritik der neuropsychologischen Untersuchungen zum Arbeitsgedächtnis 70 
$4 \quad$ Ableitung und Konkretisierung der eigenen Fragestellungen 74

\begin{tabular}{lll}
\hline 5 & 78
\end{tabular}

\begin{tabular}{lll}
5.1 & 78 \\
\hline
\end{tabular}

$\begin{array}{lll}5.2 & 78\end{array}$

$\begin{array}{lll}5.3 & 79\end{array}$

5.4 Versuchsmaterial 80

5.5 Übungsaufgabe und Versuchsaufgabe 81

5.6 Versuchsdurchführung 83

$\begin{array}{lll}5.7 & \text { Versuchsablauf } & 84\end{array}$

5.8 Statistische Auswertung 86

$6 \quad$ Ergebnisse 90

6.1 Antwortlatenzen 90

6.1.1 Falschantworten und Ausreißer 90

6.1.2 Einfluß von Reizart, Testitem und Sitzung 92

$\begin{array}{ll}6.1 .3 \text { Setgröße und Antwortlatenz } & 97\end{array}$

6.2 Synchronisationsprozesse elektrophysiologischer Signale 100

6.2.1 Aufbereitung der EEG-Daten 100

6.2.2 Sensitivität kohärenzanalytischer Kennwerte 100

6.2.3 Setgröße, mittlere Kohärenz und mittlere maximale Kohärenz 106

6.3 Zeitverlauf der Synchronisationsprozesse 112

6.3.1 Verlauf der gemittelten Bandkohärenzen 112

6.3.2 Setgröße und Zeitpunkt maximaler Kohärenz 117

6.3.3 Antwortlatenz und Zeitpunkt maximaler Kohärenz 121

7 Diskussion 126

$8 \quad$ Zusammenfassung 142

9 Anmerkungen 145

10 Literatur 149

Anhang 


\section{$1 \quad$ Einleitung}

Die Frage nach den neuronalen Grundlagen des Kurzzeitgedächtnisses stellt einen der aktuellsten Forschungsschwerpunkte der Neurowissenschaften dar. Der Zusammenschluß verschiedener naturwissenschaftlicher Fachdisziplinen hat seit einigen Jahren durch die Kombination unterschiedlicher methodischer Ansätze zu entscheidenden Fortschritten bei der Erforschung der menschlichen Großhirnrinde geführt.

Von der Möglichkeit, einen unmittelbaren Bezug zwischen bio-physiologischer und psychologischer Analyseebene herzustellen, profitiert insbesonders die kognitive Psychologie. Denn die im Rahmen der medizinischen Forschung entwickelten und verfeinerten bildgebenden Verfahren ermöglichen durch ihr hohes zeitliches und räumliches Auflösungsvermögen eine Untersuchung der neuronalen Grundlagen selbst schnell ablaufender psychischer Prozesse. Damit kann unter anderem die Gültigkeit psychologischer Modelle des Kurzzeitgedächtnisses auf der physiologischen Ebene geprüft werden.

Heute wird davon ausgegangen, daß es sich bei dem Kurzzeitgedächtnis um ein aus mehreren Subkomponenten zusammengesetztes "Arbeitsgedächtnis" handelt, das dynamisch Informationen verwaltet und verarbeitet und so eine Schlüsselposition bei der täglichen Bewältigung kognitiver Aufgaben einnimmt (Baddeley, 1998). Mit dieser Konzeption des Arbeitsgedächtnisses ist die Repräsentation und Verarbeitung begrifflicher versus bildhaft-anschaulicher Information im Gedächtnis wieder stärker in den Mittelpunkt der Forschung gerückt. In diesem Zusammenhang interessiert man sich $u$. a. für die psychologischen und neuronalen Grundlagen derjenigen Instanz, die die Aufmerksamkeit und den Informationsfluß von und zu den verbalen und bildhaft-räumlichen Kurzzeitspeichern im Arbeitsgedächtnis kontrolliert und koordiniert (sogenannte "zentrale Exekutive"). Die neuropsychologische Untersuchung dieser zentralen Instanz macht jedoch angesichts der Geschwindigkeit, mit der zahlreiche kognitive Prozesse beim Menschen ablaufen, nicht nur Verfahren mit hohem räumlichen, sondern vor allem mit hohem zeitlichen Auflösungsvermögen erforderlich (Birbaumer \& Schmidt, 1996). Ein Verfahren, das ein angemessen hohes zeitliches Auflösungsvermögen aufweist, ist die Ableitung hirnelektrischer Aktivität bzw. das Elektroenzephalogramm (EEG).

Die vorliegende Arbeit stellt einen Beitrag zur Untersuchung von Entscheidungsprozessen im Arbeitsgedächtnis mittels EEG dar. Im Mittelpunkt steht die Frage nach den neuronalen Grundlagen der verschiedenen Arbeitsgedächtniskomponenten beim Abruf von verbaler versus bildhafter Information. Darüber hinaus wird untersucht, welche statisti- 
schen Kennwerte sich beim Einsatz einer zeitlich hochauflösenden Meßmethode besonders für die Beschreibung schnell ablaufender kognitiver Prozesse eignen.

In Kapitel 2.1 wird zunächst das psychologische Konzept des Kurzzeitgedächtnisses erörtert. Es umfaßt die Darstellung von Eigenschaften und Theorien desselben sowie die Problematik des Abrufs aus dem Kurzzeitgedächtnis. In Kapitel 2.2 wird auf neurowissenschaftliche Aspekte des Kurzzeitgedächtnisses einschließlich der physiologischen und anatomischen Grundlagen und neuropsychologischen Untersuchungsverfahren eingegangen.

Kapitel 3 gibt den Forschungsstand zur theoretischen Konzeption des Arbeitsgedächtnisses wieder. Die Forschungsergebnisse werden grob nach den Subkomponenten des Arbeitsgedächtnismodells von Baddeley unterteilt. Im Anschluß an die zusammenfassende Bewertung der empirischen Studien (Kap. 3.3) werden im Kapitel 4 die eigenen Fragestellungen abgeleitet und konkretisiert. Die Darstellung und Diskussion der eigenen experimentellen Untersuchung erfolgt in den Kapiteln 5 bis 7. 


\section{Theoretischer Hintergrund: Das menschliche Kurzzeit- bzw. Arbeitsgedächtnis}

\subsection{Das psychologische Konzept des Kurzzeitgedächtnisses}

Die Dreiteilung des Gedächtnisses in sensorisches, Kurz- und Langzeitgedächtnis ist ein in der Gedächtnispsychologie übliches Gliederungsprinzip. Es wurde durch das "Modale Gedächtnismodell" von Atkinson und Shiffrin (1968) populär und entstand aufgrund der Beobachtung, daß Lernmaterial je nach Darbietungsart und -dauer unterschiedlich lange und in verschiedenem Umfang behalten und auf verschiedene Weise vergessen werden kann. In diesem Sinne wird das sensorische Gedächtnis als dasjenige mit der kürzesten Speicherdauer von nur einem Bruchteil einer Sekunde verstanden. Es ist noch Teil des peripheren sensorischen Systems und kann so als integraler Bestandteil des Wahrnehmungsprozesses betrachtet werden (Baddeley, 1998). Das Kurzzeitgedächtnis ermöglicht hingegen die Speicherung von Informationen für einige Sekunden. Es enthält nur die Information, die gerade (bewußt) genutzt wird, und der Umfang der Information, die im Kurzzeitgedächtnis gespeichert werden kann, ist eine grundlegende Begrenzung unserer geistigen Kapazität (Anderson, 1988). Im Langzeitgedächtnis werden sowohl spezifische, persönliche Erfahrungen (episodisches Gedächtnis) als auch allgemeines Wissen (deklaratives Gedächtnis) für lange Zeit gespeichert. Seine unbegrenzte Kapazität und die hohe Speicherdauer stellen den Hauptunterschied zum Kurzzeitgedächtnis dar, das mit seinem Übergangscharakter zwischen sensorischem und Langzeitgedächtnis eine Schlüsselposition einnimmt.

Entsprechend dieser Dreiteilung unterscheiden sich nun die Fragestellungen und experimentellen Verfahren, die in den damit entstandenen Forschungsgebieten zur Anwendung kommen. Obwohl diese Dreiteilung zweifelsfrei zur Steigerung der Übersichtlichkeit in der experimentellen Gedächtnispsychologie beiträgt, sollte sie nicht zur Annahme dreier separater, voneinander unabhängiger Gedächtnissysteme verleiten. Stattdessen sollte das Gedächtnis stets als ein Verarbeitungskontinuum begriffen werden, "das sich von der anfänglichen wahrnehmungsmäßigen Verarbeitung und der Verfügbarkeit im Bewußtsein bis zur Integration der Gedächtnisspur in die Wissensstruktur einer Person erstreckt" (Kintsch, 1982, S. 142). Nur so werden die Ergebnisse von Untersuchungen zu den einzelnen psychologischen Konzepten des Gedächtnisses interpretierbar.

\subsubsection{Das Kurzzeitgedächtnis als Arbeitsgedächtnis}

Die Konzipierung des unmittelbaren Gedächtnisses als Kurzzeitspeicher mit Arbeitsgedächtnisfunktion zeichnete das - wenngleich noch recht statische - modale Modell von Atkinson und Shiffrin aus. Viele Vorstellungen, die in späteren Gedächtnismodellen 
auftauchten, sind von Atkinson und Shiffrin vorweg genommen worden (z. B. der sogenannte "trade-off" zwischen Speicherung und Verarbeitung im Arbeitsgedächtnis und ein Kodierungsprozeß, der Formen höherer Verarbeitung entspricht). Schon kurz nach dessen Entwicklung ergaben sich jedoch experimentelle und konzeptuelle Probleme, die die Gültigkeit der Mehrspeichermodelle und damit die grundsätzliche strukturelle Teilung in primäres und sekundäres Gedächtnis wieder in Frage stellten. So konnte z. B. nachgewiesen werden, daß das längere Halten von Informationen im Kurzzeitgedächtnis nicht wie angenommen zu einem automatischen Transfer ins Langzeitgedächtnis führte (Tulving, 1966; Morton, 1967; Craik \& Watkins, 1973; Nickerson \& Adams, 1979; Bekerian \& Baddeley, 1980). Außerdem zeigten mehrere Untersuchungen, daß die sich aus dem modalen Modell ergebende Gleichsetzung von Rezenz-Effekten mit der alleinigen Reproduktion aus dem primären Gedächtnis zweifelhaft war (Tzeng, 1973; Bjork \& Whitten, 1974; Baddeley \& Hitch, 1974, 1977; Glenberg \& Kraus, 1981). Darüber hinaus wurde deutlich, daß die Annahme einer rein phonologischen Kodierung im Kurzzeitgedächtnis eine zu starke Vereinfachung der Realität darstellte (Baddeley, 1986). Schließlich ergaben sich neurologische Evidenzen von Beobachtungen hirngeschädigter Patienten, die gegen die angenommene Abhängigkeit der Langzeitspeicherung von einem korrekt arbeitenden Kurzzeitgedächtnis sprachen (Shallice \& Warrington, 1970; Basso, Spinnler, Vallar \& Zanobio, 1982; Vallar \& Baddeley, 1984).

Obwohl einige Kritikpunkte am Mehrspeichermodell in nachfolgenden Analysen entkräftet werden konnten (Raaijmakers, 1993; Shiffrin, 1993), hatte sich die rein strukturelle Konzeptualisierung des Gedächtnisses insgesamt als zu problembehaftet erwiesen; sie wurde daher durch eine mehr prozeßorientierte Sichtweise ersetzt, in der die Trennung der Speichersysteme jedoch in modifizierter Form bestehen blieb. In ihrem "levels of processing"-Ansatz hoben Craik und Lockhart (1972) die Bedeutung der Art und des Ausmaßes der Elaboration des Lernmaterials für die Behaltensleistung hervor: Je tiefer die erreichte Verarbeitungsstufe, desto besser sollten Items erinnert werden können. Nach einer minimalen Basiskodierung, bei der bereits erste semantische Analysen stattfänden, sollte die Reizverarbeitung unter der Kontrolle eines zentralen Prozessors mit begrenzter, jedoch flexibler Kapazität stehen. Der Einsatz dieses Prozessors wurde insofern als entscheidend für die Verarbeitungstiefe angenommen, als hier zwischen reiner Erhaltungswiederholung und einer elaborativen Wiederholung des zu lernenden Materials unterschieden werden konnte.

Zahlreiche Experimente z. B. zum inzidentellen Lernen bestätigten den Effekt der Verarbeitungstiefe (Hyde \& Jenkins, 1969; Johnson \& Jenkins, 1971; Craik \& Tulving, 1975 u. a.), betrafen aber vorwiegend die Rolle der Kodierung im Langzeitgedächtnis. Die Vernachlässigung von Kurzzeitgedächtnisproblemen bei der Weiterentwicklung des von Craik und Lockhart vorgeschlagenen "Rahmens für die Gedächtnisforschung" ist 
einer der wichtigsten zu kritisierenden Aspekte dieses Ansatzes. Weitere betreffen die Frage nach der Meßbarkeit der Verarbeitungstiefe, die stark vereinfachte Unterscheidung zwischen Erhaltungs- und elaborativer Wiederholung sowie die Annahme der Linearität der Verarbeitungsstufen (Baddeley, 1986). Darüber hinaus wurde der postulierte generelle Vorteil semantischer gegenüber nicht-semantischer Elaboration durch die Ergebnisse zur "Transfer-angemessenen Verarbeitung" (Morris, Bransford \& Franks, 1977) in Frage gestellt.

Obwohl der "levels of processing"-Ansatz keine befriedigende Alternative zu einem adäquaten Modell des Arbeitsgedächtnisses lieferte, enthielt auch er ein Konzept der Kontrollprozesse im Kurzzeitgedächtnis; zudem blieb die stärkere Beachtung des Verhältnisses von Wahrnehmung und Gedächtnis und der Möglichkeit flexibler Enkodierungsprozesse richtungsweisend für die nachfolgende gedächtnispsychologische Forschung. Etwa zur gleichen Zeit, als dieser Ansatz entwickelt und überarbeitet wurde, begannen Baddeley und Hitch (1974) der weit verbreiteten, jedoch bis dahin wenig geprüften Annahme nachzugehen, daß das Kurzzeitgedächtnis als temporäres Arbeitsgedächtnis ("working memory") tätig sei. Unter Arbeitsgedächtnis verstanden sie "a system for the temporary holding and manipulation of information during the performance of a range of cognitive tasks such as comprehension, learning and reasoning" (Baddeley, 1986, S. 34). Dieses System sollte eine begrenzte Kapazität haben und bei zahlreichen Aufgaben mit verschiedenen Verarbeitungsarten und Inputmodalitäten agieren. Die Prüfung dieser Hypothesen, insbesondere der Kapazitätsbegrenzung, gelang durch die Einführung des sogenannten "Zweitaufgabenparadigmas": Probanden hatten z. B. eine Verständnisaufgabe zu lösen, während sie gleichzeitig eine zweite, das Kurzzeitgedächtnis belastende und "ausfüllende" Aufgabe - wie z. B. das Behalten von sechs Ziffern - durchführen sollten (sog. "Gedächtnisspannenaufgabe"). Anhand solcher Versuche mit variierenden primären und sekundären Aufgaben fanden Baddeley und Hitch (ebd.), daß die zusätzliche Gedächtnislast die Leistung der Probanden zwar allgemein herabsetzte, jedoch nicht in dem nach dem modalen Modell zu erwartendem Ausmaß. Insbesondere sprachen eine konstant geringe Fehlerquote und ein stabiler Rezenzeffekt dafür, daß das Kurzzeitgedächtnis nicht einfach mit dem für die Gedächtnisspanne verantwortlichen System gleichzusetzen ist, sondern hier lediglich Überlappungen aufweist (Baddeley, 1986). Diese und andere Ergebnisse veranlaßten Baddeley und Hitch, die Idee eines einheitlichen Kurzzeitgedächtnisses aufzugeben und ein aus mehreren Subkomponenten zusammengesetztes Arbeitsgedächtnismodell zu entwerfen (s. Abb. 1). Dabei wurde betont, daß die Modellkomponenten durchaus neuronalen Strukturen entsprechen könnten.

Im Mittelpunkt ihres Modells steht die sogenannte "zentrale Exekutive", ein an das "supervisory activating system (SAS)"-Modell von Norman und Shallice (1986) an- 
gelehntes Aufmerksamkeitssystem. Es kontrolliert und koordiniert mindestens zwei Subsysteme, nämlich die "phonologische Schleife", die speziell sprachliches Material verarbeitet, und den "visuell-räumlichen Skizzenblock", der entsprechend für die Aufnahme und Verarbeitung nonverbaler Informationen zuständig ist. Für Baddeley (1986) sind die beiden Subsysteme dabei keine Gedächtnisse im herkömmlichen Sinn, sondern aktivitätsabhängige strategische Kontrollprozesse.

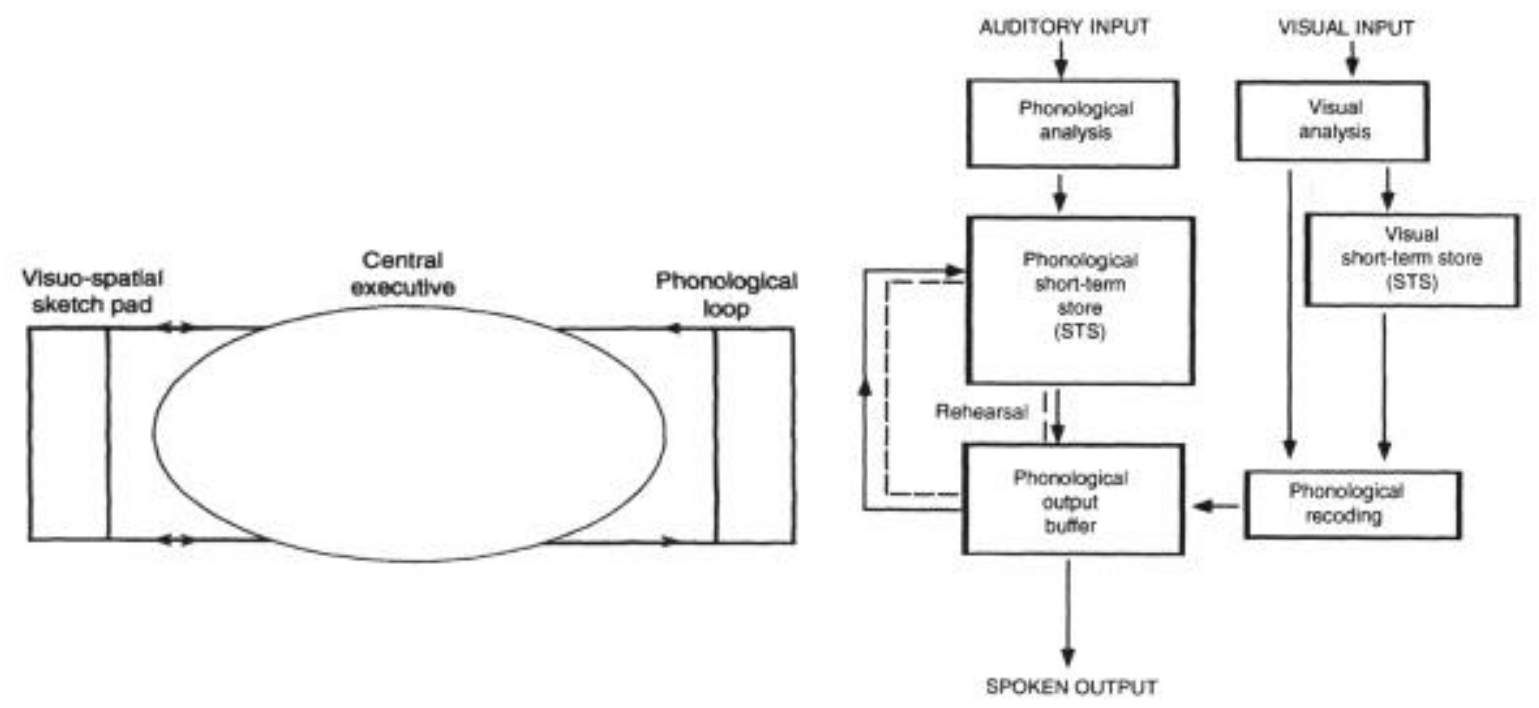

Abbildung 1. Links: Das Arbeitsgedächtnismodell von Baddeley und Hitch (1974; aus Baddeley, 1995). Rechts: Das funktionale Modell des phonologischen und visuellen Kurzzeitgedächtnisses von Vallar und Cappa (1987). Die gestrichelte Linie steht für den artikulatorischen Kontrollprozeß.

Die phonologische Schleife ist die bislang am besten untersuchte Komponente des Arbeitsgedächtnismodells. Zum einen bieten sich hier im Gegensatz zur zentralen Exekutive viele einfachere und leichter zu prüfende Fragestellungen an, zum anderen können die Ergebnisse zahlreicher Untersuchungen, die bereits mit sprachlichem Material durchgeführt wurden, zur Prüfung der theoretischen Annahmen herangezogen werden. Nach Baddeley umfaßt die phonologische Schleife zwei Komponenten, und zwar einen "phonologischen Speicher" und einen "artikulatorischen Kontrollprozeß". Gedächtnisspuren im phonologischen Speicher sollen nach etwa 1,5 bis 2 Sekunden verblassen, wenn sie nicht in den auf subvokalem Wiederholen basierenden artikulatorischen Kontrollprozeß eingelesen und auf diese Weise "aufgefrischt" werden. Versuche, in denen Probanden trotz artikulatorischer Unterdrückung (d. h. trotz Störung der phonologischen Schleife durch Aussprechen eines irrelevanten Items) bestimmte Klangaspekte von Wörtern beurteilen konnten (Besner, Davies \& Daniels, 1975), hatten diese Unterscheidung notwendig gemacht. Zwischen der Artikulationsrate und der Gedächtnisspanne besteht eine systematische Beziehung: Die Gedächtnisspanne entspricht ungefähr derjenigen Anzahl von Items, die innerhalb von 2 Sekunden artikuliert werden können (Baddeley, Thomson und Buchanan, 1975). Während akustisch dargebotene verbale Items nach der phonologischen Analyse einen obligatorischen direkten Zugang zum phonologischen 
Speicher besitzen, sorgt der artikulatorische Kontrollprozeß dafür, daß visuell dargebotene verbale Items nach der visuellen Analyse und nach der phonologischen Rekodierung ebenfalls in den phonologischen Speicher gelangen (vgl. Abb. 1).

Experimentelle Evidenz für das Konzept der phonologischen Schleife lieferten mehrere empirische Phänomene, u. a. der "Ähnlichkeitseffekt", der "Wortlängeneffekt" und die Auswirkungen artikulatorischer Unterdrückung auf Gedächtnisleistungen. In zahlreichen Untersuchungen konnte zudem die Beteiligung der phonologischen Schleife bei der Entwicklung der Lesefähigkeit (Conrad, 1971; Shankweiler \& Liberman, 1976; Mann \& Liberman, 1984; Morais, Allegria \& Content, 1987; Ellis, 1988) und beim Verstehen komplexer sprachlicher Information (Hardyk \& Petrinovich, 1970; Baddeley, 1976; Slowiaczek \& Clifton, 1980) gezeigt werden. Neuropsychologische Evidenzen für die Existenz einer phonologischen Schleife stammen aus klinisch-anatomischen Studien hirngeschädigter Patienten mit selektiven Beeinträchtigungen des Kurzzeitgedächtnisses bzw. aus entsprechenden Untersuchungen mit bildgebenden Verfahren (vgl. Kap. 3.1.1). Zur eigentlichen Beschaffenheit des phonologischen Speichers ist jedoch recht wenig bekannt. Insbesondere sind seine zeitlichen Eigenschaften, die Art und Weise, wie sprachliche Information hineingelangt und wieder abgerufen wird und die Rolle, die er bei der Sprachwahrnehmung und -produktion spielt, noch ungeklärt (Baddeley, 1998). Es kann jedoch davon ausgegangen werden, daß akustisch-verbale Informationen nach der phonologischen Analyse einen direkten Zugang zum phonologischen Speicher besitzen, während visuelle Informationen vor der phonologischen Speicherung zunächst der visuellen Analyse, der phonologischen Rekodierung (Umwandlung von Grapheme in Phoneme) und dem artikulatorischen Kontrollprozeß unterzogen werden (Vallar \& Cappa, 1987; vgl. Abb. 1).

Der phonologischen Schleife wird im Arbeitsgedächtnimodell das sogenannte "visuospatial sketchpad" (visuell-räumlicher Notizblock) gegenübergestellt. Neben klinischanatomischen Studien (vgl. Kap. 3.1.2) legten spezielle Versuche im Zweitaufgabenparadigma, die an frühe Untersuchungen von Brooks (1967) zu bildhaften Vorstellungen angelehnt waren, die Existenz dieses visuell-räumlichen Systems nahe, das - analog der phonologischen Schleife bei sprachlichem Material - Bilder generieren und manipulieren kann (Baddeley, Thomson \& Buchanan, 1975). Es ist allerdings u. a. noch unklar, wie die Aufrechterhaltung der bildhaften Vorstellungen erfolgt bzw. ob es überhaupt ein räumliches Äquivalent zum artikulatorischen Kontrollprozeß gibt und auf welchen Mechanismen dieses beruhen könnte. Baddeley (1998) nimmt an, daß der entsprechende Mechanismus auf demselben System basieren könnte, das auch für die Kontrolle der Augenbewegungen zuständig ist. Mehrere Studien weisen darauf hin, daß der Notizblock ebenfalls zwei Komponenten umfaßt, eine visuelle, die für die Mustererkennung ("was") zuständig ist, und eine räumliche, die Positionsinformationen ("wo") vermittelt 
(Farah, Hammond, Levine \& Calvanio, 1988; Humphreys \& Riddoch, 1987; Levine, Warach \& Farah, 1985). Obwohl die Bedeutung mentaler Bilder als wichtiges Merkmal unseres geistigen Innenlebens seit Paivios dualer Kodierungshypothese (Paivio, 1969) unumstritten ist, wurde erst kürzlich mit einer Zusammenschau psychophysiologischer, neuro- und experimentalpsychologischer Erkenntnisse dazu begonnen. Das Wissen um die Beteiligung des visuell-räumlichen Systems an geographischer Orientierung und der Planung visuell-räumlicher Aufgaben führte jedoch schon früh dazu, daß entsprechende Aufgaben in Intelligenz- und Eignungstests aufgenommen wurden (Baddeley, 1998).

Die beiden dargestellten Subsysteme des Arbeitsgedächtnismodells können nur einen relativ geringen Teil unserer bewußten mentalen Vorgänge erklären. Die zentrale Exekutive soll, so wird angenommen, als komplexeste Komponente dieses Modells den bei weitem größten Teil mentaler Vorgänge bewältigen. Obwohl Untersuchungen mit dem Zweitaufgabenparadigma auch hier die experimentelle Evidenz für die Existenz dieses Kontrollsystems lieferten (Baddeley, 1966), blieb die zentrale Exekutive aus den bereits genannten Gründen die bislang am wenigsten erforschte Komponente des Arbeitsgedächtnismodells. Baddeley ist der Ansicht, daß "the Norman and Shallice model gives a good account of the functioning of the central executive in working memory ..." (1998, S. 102). In ihrem Modell gehen Norman und Shallice (1986) davon aus, daß Handlungsabläufe auf zwei verschiedene Arten kontrolliert werden können: Zum einen können Konflikte zwischen gut gelernten Fertigkeiten, die relativ automatisch vonstatten gehen, durch einen halbautomatischen Konfliktlösungsprozeß bewältigt werden (sog. "contention scheduling"), zum anderen kann ein willentlich einsetzbares, "supervisory activating system" (SAS) in das Geschehen eingreifen und ablaufende Handlungen unterbrechen und modifizieren. Das SAS soll eine begrenzte Kapazität haben und bei Planungs-, Problemlösungs- und Entscheidungsaufgaben sowie bei neuen oder weitgehend unbekannten Handlungsabläufen zum Einsatz kommen. In diesem Sinne belegen Untersuchungen z. B. zur Generierung von Zufallsfolgen (Baddeley, 1966; Robbins et al., 1996), zum Autofahren (Brown, Tickner \& Simmonds, 1969) und zum Schachspiel (Holding, 1985; Robbins et al., 1996), daß das SAS in seiner Funktionsweise mit der zentralen Exekutive gleichgesetzt werden kann.

Das Arbeitsgedächtnismodell von Baddeley und Hitch (1974) ist trotz einer beachtlichen Akzeptanz unter den Gedächtnispsychologen nicht unkritisiert geblieben. Gegenstand der Diskussion ist $u$. a. die zugrundeliegende Annahme eines einheitlichen Arbeitsgedächtnisses. Im Gegensatz zu Baddeley gehen z. B. Allport (1980), Barnard (1985) sowie Schneider und Detweiler (1988) davon aus, daß das Arbeitsgedächtnis kurzlebige neuronale Netzwerke mehrerer interagierender, jedoch relativ unabhängiger Subsysteme beinhaltet, die sich je nach Aufgabenart unterscheiden. Damit dürften verschiedene 
Konstellationen aktivierter Subsysteme den Anforderungen an das Arbeitsgedächtnis nachkommen, je nachdem, welche Arten sensorischer, motorischer und kognitiver Codes für die Aufgabenlösung gerade relevant sind. Die Interaktionen der verschiedenen Komponenten sollen hierbei auch Kontrollfunktionen einschließen (vgl. auch Posners (1990) Annahme separater neuroanatomischer Strukturen für verschiedene Aufmerksamkeitsmechanismen). Just und Carpenter (1992) gehen in einem verwandten, ebenfalls physiologisch begründeten Ansatz noch weiter und beschreiben das Arbeitsgedächtnis lediglich mittels einer begrenzten Aktivitätsmenge, die für mentale Vorgänge zur Verfügung steht und unter den verschiedenen aktuellen kognitiven Anforderungen aufgeteilt werden muß. Es definiert sich so über diejenigen neuronalen Strukturen, die gerade an der Bewältigung einer Aufgabe beteiligt sind. Neuropsychologische Studien mit bildgebenden Verfahren untermauern diese dynamische Sichtweise der Arbeitsgedächtnisstruktur. So konnte gezeigt werden, daß verschiedene Arbeitsgedächtnisaufgaben charakteristische Aktivationskonstellationen in multiplen kortikalen Arealen hervorrufen (Goldman-Rakic, 1988).

Neue experimentelle Befunde unterstützen Forderungen nach weiteren Differenzierungen des Arbeitsgedächtnismodells. So legen Untersuchungsergebnisse nahe, eine zusätzliche, vom phonologischen Speicher getrennte Arbeitsgedächtnis-Komponente anzunehmen, die spezifisch für das Behalten semantischer und syntaktischer Informationen zuständig ist (Martin, 1993). Außerdem häufen sich Belege für die Notwe ndigkeit einer stärkeren Berücksichtigung des Langzeitgedächtnisses bei Arbeitsgedächtnisprozessen (Cowan, 1993; Potter, 1993; Ericsson \& Kintsch, 1995). Schließlich zeigen Untersuchungen von Cowan und Hulmes (z. B. Hulme, Maughan \& Brown, 1991; Cowan, 1992; Hulme et al., 1997) sowie von Lass und Mitarbeitern (Lass et al., 2000; Lass, Lüer, Becker, Fang \& Chen, 2001), daß die kognitiven Prozesse, die die Gedächtnisspanne bedingen, im Modell der phonologischen Schleife zu stark vereinfacht werden. Cowan und Hulme sowie Lass et al. konnten zeigen, daß für Gedächtnisspannenaufgaben nicht nur der artikulatorische Kontrollprozeß von Bedeutung ist, sondern auch andere Verarbeitungsmechanismen eine wichtige Rolle spielen. Dabei soll es sich um schnelle Suchprozesse zur Aktivierung der Gedächtnisspuren handeln sowie um Mechanismen, mit denen während des Abrufprozesses teilweise verblaßte Gedächtnisspuren wiederhergestellt werden (vgl. Cowan et al., 1998; Hulme et al., 1999).

Derzeit gibt es keine einheitliche psychologische Theorie des Arbeitsgedächtnisses, sondern nur Modellansätze, deren Strukturen sich an älteren universellen Theorien einerseits und an Ergebnissen empirisch-experimenteller Analysen andererseits orientieren.

Die Schwierigkeit im Verständnis dieser Gedächtniskomponente besteht darin, daß der Begriff des Arbeitsgedächtnisses heute, mehr oder weniger implizit das Kurzzeitgedächtnis einschließend, auf ein sehr breites Spektrum an Strukturen 
und Prozessen menschlicher Informationsverarbeitung angewendet wird. Er umfaßt im Grunde alles, was menschliche Informationsverarbeitung ausmacht. Ein umfassendes erfolgreiches Modell des Kurzzeit- und/oder Arbeitsgedächtnisses wäre demnach auch ein Modell der menschlichen Kognition ... . Ein Modell dieses Allgemeinheitsgrades gibt es jedoch nicht. (Kluwe, 1997, S. 144)

\subsubsection{Abruf aus dem Kurzzeitgedächtnis}

Bei der Untersuchung des Abrufs von Informationen aus dem Gedächtnis sind schon früh zwei Kategorien unterschieden worden: ein aktiver Suchprozeß, der dem Problemlösen ähnelt, und ein mehr automatisierter Prozeß, der schnell abläuft und der Introspektion nicht zugänglich ist ( $\mathrm{vgl}$. Ebbinghaus, 1885, und James, 1890, beide zit. nach Kintsch, 1982). Dieser automatisierte Prozeß ist charakteristisch für den Abruf von im Kurzzeitspeicher gehaltenen Informationen; angesichts der Bedeutung des Kurzzeitgedächtnisses für die Lösung aktueller Aufgaben (Funktion als Arbeitsgedächtnis) ist er aber auch Teil aktiver Suchprozesse. In diesem Sinne wird der Abruf aus dem Gedächtnis heute als Zusammenspiel zwischen augenblicklich verfügbarer und bereits gespeicherter Information und damit als ein rekonstruktiver Prozeß bzw. als geleiteter Suchprozeß betrachtet (Parkin, 1993).

Ein großer Teil der Forschung zum Gedächtnisabruf hat sich auf das Vergessen und damit auf Situationen und Bedingungen konzentriert, in denen das Gedächtnis versagt. Sternberg betonte schon 1966, daß diese Vorgehensweise es nicht ermöglicht, diejenigen Mechanismen zu verstehen, die einem erfolgreichen Gedächtnisabruf zugrundeliegen. Er plädierte daher für eine Hinwendung zu Versuchen, in denen die erfaßten Fehler nur noch der Selektion interpretierbarer Antworten dienten und die Reaktionszeit die wichtigste abhängige Variable darstellte. Zu diesem Zweck führte er ein neues, grundlegendes experimentelles Paradigma für die Untersuchung des Abrufs aus dem Kurzzeitgedächtnis ein (ebd.). Probanden hatten verschiedene kurze Listen von ein bis sechs Items zu memorieren (sog. "varied set"-Prozedur) und im darauffolgenden Wiedererkennenstest über Tastendruck so schnell und genau wie möglich zu entscheiden, ob ein Testitem zu der gelernten Itemgruppe (sog. "positiver Set") gehörte oder nicht. Bei den Items handelte es sich um Zahlwörter oder Ziffern. Da die Gedächtnisspanne nicht überschritten wurde, war die Aufgabe einfach und Fehler relativ selten; sie wurden zur Kontrolle eines möglichen "speed-accuracy trade off" erfaßt.

Im Zentrum der Aufmerksamkeit stand der Effekt, der sich durch die Variation der Größe des positiven Sets unter Konstanthaltung der Häufigkeit positiver und negativer Antworten ergab. Sternberg (ebd.) fand einen linearen Anstieg der durchschnittlichen Reaktionszeit mit der Größe des positiven Sets; der Steigungsgradient war für positive und negative Antworten gleich und betrug für jedes Item etwa $40 \mathrm{~ms}$. Abweichungen 
von diesem Durchschnittswert konnten auf Variationen des Stimulusmaterials zurückgeführt werden (Sternberg, 1969a; Cavanagh, 1972). Positive und negative Antworten unterschieden sich lediglich bezüglich der durchschnittlichen Reaktionszeit, die für negative Antworten um etwa 30 ms höher lag. Dieses Ergebnis trat auch unter Verwendung der sogenannten "fixed-set"-Prozedur auf, bei der die Probanden durchschnittlich zehn Minuten mit demselben Set arbeiteten, so daß hier von einer Langzeitspeicherung ausgegangen werden konnte:

Nonetheless, the similarity of the data led to the conjecture that the same memory was being searched in the two procedures: even if a set is well learned, when it is needed in the item-recognition task its members are 'activated', or transferred into an 'active memory', perhaps equivalent to the short-term store, where they are more rapidly accessible (Sternberg, 1975, S. 6).

Für Sternberg (1966) legten die Ergebnisse seiner zahlreichen Versuche einen Suchprozeß innerhalb des positiven Sets nahe, in dem das Testitem seriell mit jedem memorierten Item verglichen und der Vergleich auch nach dem Auftreten einer Übereinstimmung noch fortgesetzt würde. Die Vergleichsgeschwindigkeit sollte konstant sein und die Reaktionszeit daher linear mit der Größe des positiven Sets zunehmen. Die Höhe des Intercepts (etwa 400 ms) deutete jedoch darauf hin, daß ein großer Teil der Reaktionszeit neben dem Such- und Vergleichsvorgang auch die Dauer anderer Prozesse widerspiegelte. Selbst unter Berücksichtigung von Input- und Output-Zeiten aus einfachen visuellen Reaktionszeitversuchen blieben noch mehr als 200 ms erklärungsbedürftig (Sternberg, 1975). Daher zerlegte Sternberg in seinem Abrufmodell die Itemwiedererkennensaufgabe in insgesamt vier aufeinander folgende Teilprozesse, nämlich Reizkodierung, Vergleich, Entscheidung und Antwortrealisierung (s.Abb.2). Mit der Methode des additiven Faktors konnte er zeigen, daß die Annahme dieser separaten Verarbeitungsstufen empirisch gerechtfertigt war: Für jede Verarbeitungsstufe fand er eine experimentelle Variable, die nur die entsprechende Stufe beeinflußte und keine statistische Interaktion mit den Effekten der anderen Variablen aufwies (Sternberg, 1969b).

Während dieser Teil seines Modells offensichtlich wenig umstritten ist, gab es bei der Erklärung des Vergleichprozesses verschiedene Standpunkte. Kritisiert wurde sowohl die Annahme eines seriellen Prüfvorgangs als auch das intuitiv wenig einleuchtende, erschöpfende Vorgehen bei der Suche im Falle positiver Antworten, da zahlreiche Ergebnisse zu Itemwiederholungs-, Wahrscheinlichkeits- und seriellen Positionseffekten im Widerspruch zu Sternbergs Annahmen standen (z. B. Corballis, Kirby \& Miller, 1972; Klatzky \& Smith, 1972; Baddeley \& Ecob, 1973; Theios, Smith, Haviland, Traupmann \& Moy, 1973; Briggs, 1974). 


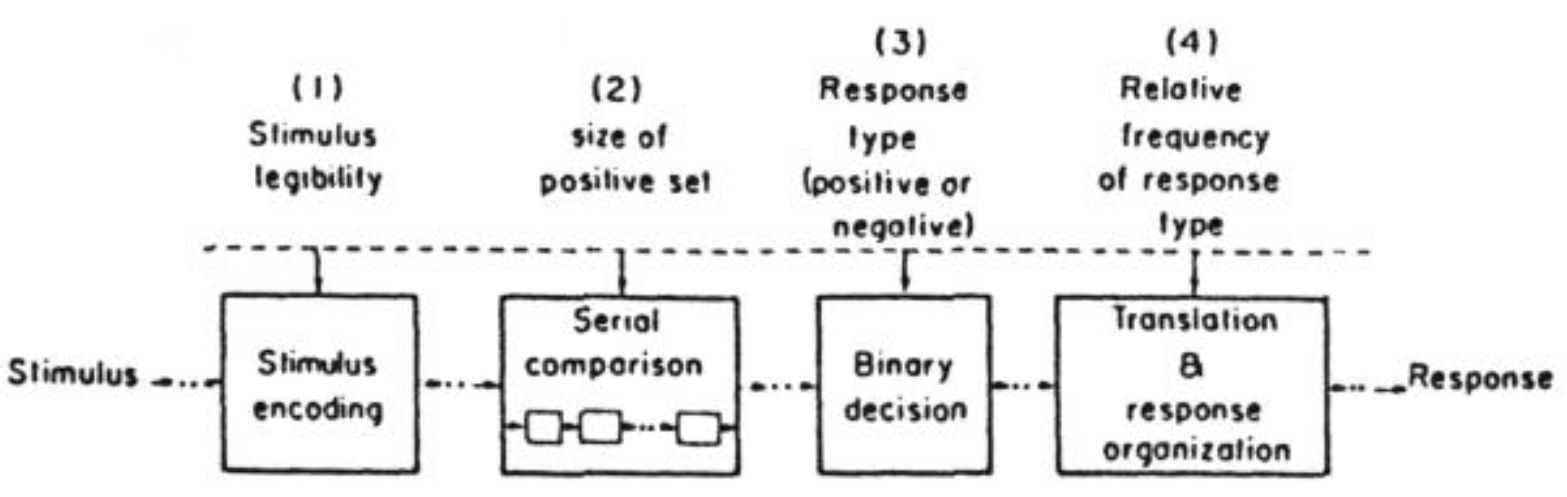

Abbildung 2. Verarbeitungsstufen bei der Itemwiedererkennung nach Sternberg (1975)

Sternberg (1975) selber begegnete solchen empirischen Schwierigkeiten mit dem Hinweis, daß der serielle erschöpfende Prüfvorgang nur einen Teil seines Modells darstelle und daß die abweichenden Ergebnisse auf Vorgänge in den übrigen Teilprozessen zurückzuführen seien. In Nachuntersuchungen bereits vorliegender Daten fand er jedoch einen Zusammenhang zwischen der Vergleichsgeschwindigkeit (sog. Scanning-Zeit) und der Qualität des Such- und Vergleichsprozesses (erschöpfend vs. sich selbst beendend): Je geringer der Steigungsgradient (d. h. je langsamer der Scanning-Prozeß) ausfiel, umso eher ließen sich in den Reaktionszeitkurven der Probanden Hinweise auf einen sich selbst beendenden Such- und Vergleichsprozeß finden. Diese traten nur bei positiven Testitems auf und zeigten sich in einem vergleichsweise flacheren Anstieg der entsprechenden Reaktionszeitkurven (im Durchschnitt $50 \%$ geringere Steigung als bei negativen Testitems).

Während Sternberg (1975) also für positive Testitems immerhin die Möglichkeit eines sich selbst beendenden Vergleichsprozesses einräumte, zogen es andere Forscher vor, seinen Ansatz zu erweitern oder alternative Modelle des Vergleichsprozesses vorzuschlagen (z. B. Corballis, Kirby \& Miller, 1972; Theios, 1973; Murdock, 1974; Ratcliff, 1978). So ist z. B. der lineare Anstieg der Reaktionszeit mit der Setgröße im Prinzip auch durch parallele (statt serielle) Verarbeitungsprozesse erklärbar. In entsprechenden Modellen wird davon ausgegangen, daß die Schnelligkeit, mit der Simultanvergleiche durchgeführt werden können, eine Funktion des Aktivierungsgrades der Items im Gedächtnis ist (Atkinson, Holmgren \& Juola, 1969; Corcoran, 1971; Townsend, 1971; Anderson, 1973). Die begrenzte Verarbeitungskapazität des Gedächtnisses soll so mit jedem zusätzlichen Simultanvergleich weiter verringert werden, wodurch die Vergleichsdauer (und damit die Antwortlatenz) erhöht wird. Ähnlich wird in Spurenstärke-Modellen davon ausgegangen, daß ein feststehender Betrag der Reizrepräsentationsstärke im Gedächtnis zwischen den Elementen des gelernten Sets aufgeteilt wird; die funktionelle Beziehung zwischen der Spurenstärke und der Dauer des Stärke-Unterscheidungsprozesses ist in diesen Ansätzen ursächlich für die Variationen der Antwort- 
latenzen (z. B. Baddeley \& Ecob, 1973; Reed, 1976). Allerdings wird dabei nicht von einem Suchprozeß, sondern von einem direkten Zugang zur inneren Testreizrepräsentation ausgegangen.

Sternberg (1975) selber räumte die Möglichkeit einer probabilistischen Mischung von Abrufprozessen ein. Danach wäre eine frühe Reizanalyse dafür ausschlaggebend, daß in einem Teil der Durchgänge ein serieller und im übrigen Teil ein alternativer (z. B. paralleler) Vergleichsprozeß stattfindet. Atkinson und Juola (1974) entwickelten in einer Erweiterung des Sternbergschen Ansatzes ein solches probabilistisches Mischmodell. Es geht davon aus, daß jedes Item im Gedächtnis durch einen Bekanntheitswert gekennzeichnet ist, das quantitativ variieren kann. Die Höhe dieses Bekanntheitswertes, der kurz nach Darbietung des Testitems im Gedächtnis aufgesucht wird, bestimmt, ob das Testitem vom Probanden wiedererkannt wird oder nicht. Eine Wiedererkennensantwort erfolgt jedoch nur, wenn der Bekanntheitswert des Testitems sehr hoch oder sehr niedrig ausfällt. Im dazwischenliegenden (Unsicherheits-)Bereich wird die Antwort aufgeschoben und das Gedächtnis zunächst wie bei Sternberg seriell und erschöpfend durchsucht. Der Effekt der Setgröße wird auf diese ausgedehnte Gedächtnisprüfung in einem bestimmten Prozentsatz der Durchgänge zurückgeführt. Die unverkennbaren Vorteile dieses Modells liegen darin, daß es viele Daten erklärt, die mit dem SternbergModell unvereinbar sind (z. B. Itemwiederholungs- und serielle Positionseffekte) und daß es leicht auf andere Aspekte des Wiedererkennensgedächtnisses ausgedehnt werden kann. Darüber hinaus ist es auf Entscheidungsprozesse im Langzeitgedächtnis übertragbar (Atkinson \& Juola, 1974). Bei der Erklärung einiger Ergebnisse (z. B. Wahrscheinlichkeitseffekte) versagt jedoch auch dieser Ansatz (Kintsch, 1982).

Insgesamt wurde aus vielen Studien zum Sternberg-Paradigma deutlich, daß anhand der alleinigen Betrachtung der mittleren Reaktionszeit die Frage nach serieller vs. paralleler Verarbeitung nicht beantwortet werden kann (Meyer, 1970; Rips, Shoben \& Smith, 1973; Ratcliff \& Murdock, 1976; Townsend \& Ashby, 1983; Ashby, Jein \& Balakrishnan, 1993). Daher rückten Reaktionszeitverteilungen, Positionseffekte und auch wieder Fehlerraten und -latenzen in den Mittelpunkt des Interesses, wie z. B. in Ratcliffs (1978) Untersuchungen zu der von ihm entwickelten Theorie des Gedächtnisabrufs. Diese Theorie bezieht sich vorwiegend auf Wiedererkennensprozesse im Kurzzeitgedächtnis, obwohl Ratcliff nach der Revision einer Studie von Burrows und Okada (1975) keine Notwendigkeit für die Annahme separater Abrufprozesse im Langzeitgedächtnis sieht (ebd., S. 92). In seinem Modell ruft die Darbietung des Testreizes eine Menge von Items im Gedächtnis hervor, die umfangreicher als der sogenannte positive Set sein kann. Damit erklärt er die längeren Antwortlatenzen und höheren Fehlerraten beim falschen Wiedererkennen kürzlich dargebotener Items in Sternberg-Versuchen (Atkinson, Hermann \& Wescourt, 1974). Der anschließende Vergleichsprozeß erfolgt parallel und ist das Er- 
gebnis einer Interaktion des Testreizes mit allen Repräsentationen der im Gedächtnis hervorgerufenen Items. Ziel ist die Feststellung eines Verwandtschaftswertes zwischen Gedächtnisspur und Testreiz; Ratcliff nennt das Vorgehen hierbei einen "random walk":

A count is kept of the combined sum of the number of feature matches and nonmatches, so that for a feature match, a counter is incremented, and for a feature nonmatch, the counter is decremented. The counter begins at some starting value $Z$, and if a total of $A$ counts are reached, the probe is declared to match the memory-set item ( $A-Z$ more feature matches than nonmatches). But if a total of zero counts are reached, an item nonmatch is declared. (Ratcliff, 1978, S. 63).

Variationen der für den Vergleich benötigten Zeit werden auf die Reihenfolge der Vergleiche und auf die Verteilung von Treffern und Nichttreffern in dieser Reihenfolge zurückgeführt. Außerdem wird angenommen, daß die Übereinstimmungsgrenze umso schneller erreicht wird, je größer der Verwandtschaftswert zwischen gespeicherten Items und Testreiz ausfällt. Der Entscheidungsprozeß soll bei positiven Items selbstbeendend und bei negativen Items erschöpfend verlaufen. Da der kritische Grenzwert für Nichtübereinstimmungen nach Ratcliff näher am Startwert liegt als für Übereinstimmungen, können negative Antworten in seinem Modell ebenso schnell oder sogar schneller als positive ausfallen.

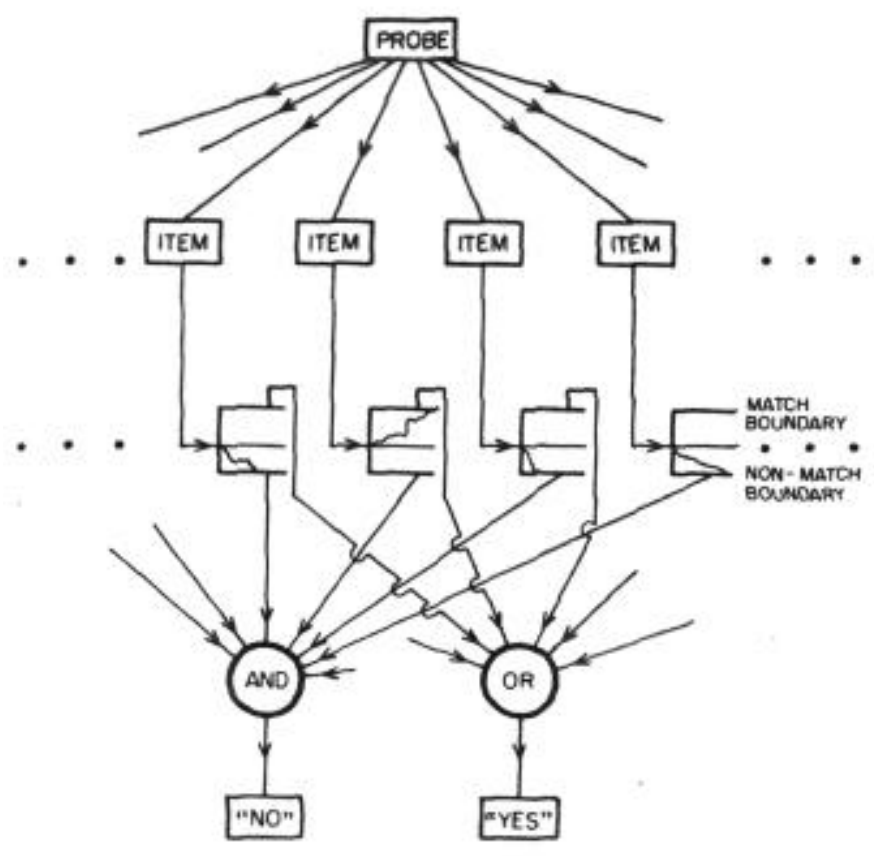

PROBE INPUT

EVOCATION OF THE

SEARCH SET

EVOKED SET

(POTENTIALLY VERY LARGE)

PARALLEL COMPARISON

(DIFFUSION PROCESSES)

UPPER BOUNDARY MATCH

LOWER BOUNDARY NON-MATCH

DECISION PROCESS

SELF TERMINATING ON MATCH

(OR gate)

EXHAUSTIVE ON NON - MATCH

(ANO gote)

RESPONSE OUTPUT

Abbildung 3. Überblick über das Itemwiedererkennensmodell von Ratcliff (1978)

Ratcliffs Theorie des Gedächtnisabrufs enthält eine mathematische Komponente, die Vorhersagen bezüglich mittlerer Antwortlatenzen, Reaktionszeitverteilungen, Fehlerraten und -latenzen ermöglicht. Sie hat sich für verschiedene Paradigmen des Itemwie- 
dererkennens (darunter auch die Sternberg-Aufgabe) bewährt und weist enge Beziehungen zu Theorien neuronaler Netzwerke auf (Ratcliff, 1978).

Heute scheint klar zu sein, daß die Art des Verarbeitungsprozesses von der gestellten Abrufaufgabe abhängt. So konnten z. B. McElree und Dosher (1993) anhand der Analyse des gesamten Zeitverlaufs beim Abruf mittels der sogenannten "Antwort-Signal-Prozedur" zeigen, daß die Verarbeitung parallel (oder direkt - vgl. Reed, 1976) erfolgt, wenn - wie im Sternberg-Paradigma - nur eine Wiedererkennensleistung gefordert ist, und seriell, wenn die Reihenfolgeinformation für die Aufgabenlösung benötigt wird. Die Deutung solcher Ergebnisse im Rahmen neuerer mathematischer Gedächtnismodelle wie SAM (Search of Associative Memory; Gillund \& Shiffrin, 1984) oder TODAM (Theory of Distributed Associative Memory; Murdock, 1982) gestaltet sich jedoch schwierig. Zwar wird in diesen Modellen wie in Ratcliffs Ansatz davon ausgegangen, daß die Itemwiedererkennung über die innere Erstellung eines globalen Stärke- oder Verwandtschaftswertes und dessen Vergleich mit einem Kriteriumswert erfolgt. Für eine wirklich adäquate Erklärung von Reaktionszeit-Verlaufsdaten sind die globalen Modelle jedoch zu statisch, da sie hauptsächlich für die Deutung von Gedächtnisleistungen aus Versuchen ohne Zeitdruck entwickelt wurden und daher keine Aussagen zu den Zeiteigenschaften zugrundeliegender Abrufprozesse machen (Gronlund \& Ratcliff, 1989; McElree \& Dosher, 1993). "In comparison with models of long-term retrieval, however, such models for short-term retrieval are still at an early stage of development" (Shiffrin, 1993, S. 196).

\subsection{Das Kurzzeitgedächtnis als Gegenstand neurowissenschaftlicher Forschung}

Die wissenschaftliche Erforschung des Neokortex und dessen Bedeutung für die höheren intellektuellen Leistungen des Menschen begann in der zweiten Hälfte des 19. Jahrhunderts mit verschiedenen deskriptiven Studien, histologischen Nachweisen struktureller Unterschiede einzelner Hirnrindenteile und Analysen kortikaler Funktionen aufgrund neurologischer Ausfallserscheinungen (Creutzfeldt, 1983). Von Bedeutung war auch die Aufnahme neurophysiologischer und neuroanatomischer empirischer Ergebnisse in die Psychologie durch Wilhelm Wundt (1874, zit. nach Creutzfeldt, 1983). Entscheidende Fortschritte wurden jedoch erst im 20. Jahrhundert erzielt: Die Einführung der Reiztechnik in die Neurochirurgie ermöglichte die Erstellung von Funktionskarten der menschlichen Hirnrinde (Foerster, 1937; Penfield, 1937; beide zit. nach Creutzfeldt, 1983), und die Entdeckung des EEGs beim Menschen durch Berger (1929) ermöglichte die zeitgetreue Abbildung hirnelektrischer Korrelate psychischer Prozesse. Die Weiterentwicklung der elektrophysiologischen Technik, die intrazelluläre Ableitungen von einzelnen kortikalen Nervenzellen möglich machte (Phillips, 1956), und die gleichzeitige Einführung der Golgi-Färbetechnik (Scholl, 1956; Szentágothai, 1973) ließen die quan- 
titative und funktionell ausgerichtete Hirnrindenanalyse wieder aufleben und führten unter Ausnutzung des Wissens um den Stofftransport in Nervenzellen zu einem wirklichen Durchbruch in der Neurobiologie (Creutzfeldt, 1983).

Heute lassen sich verschiedene neurowissenschaftliche Forschungsstrategien nach der räumlichen und zeitlichen Auflösung der verwendeten Untersuchungsverfahren unterscheiden (Rösler, 1997). Während sich die Neurophysiologie über molekularbiologische und biochemische Analysen einzelner Nervenzellen mit der Frage befaßt, wie Informationen gelernt und gespeichert werden, konzentriert sich die Neuropsychologie auf größere Struktureinheiten; sie versucht über die Herstellung eines unmittelbaren Bezugs zwischen biologischer und psychologischer Analyseebene herauszufinden, wie und wo gespeicherte Informationen auf Gehirnebene abgelegt werden. Neben der traditionellen Untersuchung umschriebener krankhafter Läsionen der Hirnrinde und deren Auswirkung auf das Verhalten rücken dabei zusehends Biosignalanalysen unter Einsatz bildgebender Verfahren in den Vordergrund. Die Betrachtung des Kurzzeitgedächtnisses als Gegenstand neurowissenschaftlicher Forschung erfordert daher neben einer Zusammenfassung wichtiger neuropsychologischer Untersuchungsergebnisse (vgl. Kap. 3) auch eine Darstellung neurophysiologischer und anatomischer Grundlagen des Gedächtnisses sowie der darauf basierenden Modelle der Informationsverarbeitung. Darüber hinaus darf auch die Beschreibung der aktuell eingesetzten Verfahren zur Untersuchung des Gedächtnisses nicht fehlen.

\subsubsection{Neurophysiologische und anatomische Grundlagen des Gedächtnisses}

D. O. Hebb gilt als Vater aller neurophysiologischen Gedächtnistheorien; seine 1949 aufgestellte synaptische Theorie spezifischer Gedächtnisinhalte bildet bis heute den Ausgangspunkt aller Überlegungen zu neurobiologischen Grundlagen des Gedächtnisses (Birbaumer \& Schmidt, 1996). Seit langem war bekannt, daß der Übergang von Informationen vom Kurz- in das Langzeitgedächtnis in der Regel eine Wiederholung (rehearsal) des Lernmaterials erforderte; das Wiederholen bzw. der Prozeß des zyklischen "Kreisens" einer Information im selben Kurzzeitgedächtnis-Abschnitt bis zur Überschreitung einer kritischen Schwelle zum Langzeitgedächtnis wurde Konsolidierung genannt, und Interferenz bei der Konsolidierung galt seit Müllers und Pilzeckers Arbeit (1900) als eine der Hauptursachen von Vergessen.

Hebb übertrug den psychologischen Gedanken kreisender Information auf die physiologische Ebene. Physiologisch gesehen werden unter Gedächtnis strukturelle Veränderungen durch elektrochemische Vorgänge innerhalb des Organismus verstanden; für Hebb (1949) schien es daher notwendig, einen Mechanismus für die Aufrechterhaltung des Gedächtnisinhaltes bis zum Eintritt dieser strukturellen Änderungen zu postulieren. Nach der Entdeckung von Fasern, die in geschlossenen, potentiell sich 
selbst erregenden Kreisen angeordnet waren (de Nó, 1938, zit. nach Creutzfeldt, 1990), war die Annahme eines darauf beruhenden Kurzzeitgedächtnis-Mechanismus für Hebb die notwendige Folge. Er unterschied zwischen Aktivitätsspuren, die dem autonomen Zerfall unterliegen (Zellen werden z. B. refraktär) und das Kurzzeitgedächtnis ausmachen, und strukturellen Langzeitspuren, die stabil sind und dann entstehen, wenn Aktivitätsspuren lange genug ungestört andauern. Dabei sollten kreisförmig geschlossene Erregungsverläufe von Nervenzellen (reverberatory circuits) nach dem Ende der aktuellen Reizung die neurophysiologische Basis dieser Konsolidierung darstellen. Hebb (ebd.) nahm an, daß sich in dieser Phase die zunächst schwachen synaptischen Verbindungen zwischen den Neuronen bis zur Stabilisierung bzw. bis zur Bildung des sogenannten strukturellen Engramms (Gedächtnisspur) durch eine mehrmalige gemeinsame assoziative Erregung verstärken; danach würde auch eine Teilaktivität im entsprechenden "cell-assembly" genügen, um das gesamte Ensemble zu aktivieren. Die Spezifität eines Gedächtnisinhaltes sollte durch den Ort des aktivierten Zellensembles im Zentralnervensystem und durch die Frequenz und Rhythmuseigenschaften der kreisenden Erregung bestimmt werden.

Obwohl sich die Grundgedanken der Theorie von Hebb erstaunlich gut bestätigen ließen, weiß man inzwischen, daß es verschiedene Realisationsmöglichkeiten von dauerhaften synaptischen Verknüpfungen im Zentralnervensystem gibt und daß damit neben reverberatorischen Erregungskreisen noch andere Grundlagen für die Konsolidierung existieren. Oft genügt schon eine Neuronenerregung in wiederholter zeitlicher Nachbarschaft für die Änderung der Stärke synaptischer Verbindungen (Birbaumer \& Schmidt, 1990). Es kann zudem davon ausgegangen werden, daß erst das gleichförmige, kohärente Entladungsverhalten eines ganzen Zellensembles speicherbare Information für das Gedächtnis darstellt, während die Aktivität einzelner Zellen im elektrochemischen Rauschen des Zentralnervensystems untergeht (John, 1967).

Viele Forschungsarbeiten haben sich mit der Frage beschäftigt, wie aus dem dynamischen ein strukturelles Engramm wird bzw. wie die kreisende oder räumlich-zeitlich wiederholte neuronale Erregung zu einer strukturellen Veränderung an den beteiligten Synapsen führt. Nach Birbaumer und Schmidt (1990) ist die wichtigste Voraussetzung für die Übertragung von Informationen in das Langzeitgedächtnis die kontrollierte Verarbeitung und die Zuweisung von Aufmerksamkeitsressourcen, damit der Lerninhalt länger im Kurzzeitgedächtnis aktiv bleiben und eine tiefere bzw. elaboriertere Kodierung desselben stattinden kann. Zuständig ist hier ein hypothetisches System limitierter Aufmerksamkeitskontrolle (Limited Capacity Control System - LCCS; vgl. auch das SASKonzept von Norman \& Shallice, 1986), das in neuen oder komplexen Situationen aktiviert wird und die beteiligten informationsverarbeitenden und reaktionsplanenden Systeme des Gehirns in Abhängigkeit motivationaler Einflüsse zusätzlich erregt, während 
nicht beteiligte Systeme gehemmt werden (Shiffrin \& Schneider, 1977). Diese willentliche Anstrengung koordiniert dann die sensorische und motorische Aktivierung und geht mit Bewußtsein und verstärktem Energieverbrauch im Zentralnervensystem einher. Sie erfordert aktive Bewegungsplanung (Wille) oder kontrollierte Suche (ständiger Vergleich von Kurzzeit- und Langzeitgedächtnisinhalten); letztere ist von der Ressourcen-Zuteilung des LCCS abhängig, die vor allem bei der Überlappung verschiedener Verarbeitungsdimensionen (z. B. durch simultane Aufgabendarbietung) eine wichtige Rolle spielt (Gopher \& Donchin, 1985). Neurophysiologisch gesehen liegt dem LCCS im wesentlichen die Aktivierung der Verbindungen zwischen Thalamus und Kortex zugrunde (s. Abschnitt zu anatomischen Grundlagen); sie äußert sich u. a. in der erhöhten Feuerungsrate von Neuronen mit langen Axonen, in der erhöhten Erregbarkeit thalamo-kortikaler Zellen und in der Einengung und Focussierung inhibitorischer Prozesse am Kortex (Steriade, 1981). Hinzu kommt als Entscheidungsinstanz der präfrontale Kortex, der aus allen Teilen des Neokortex Meldungen über eingelaufene Informationen sowie über die Ergebnisse nicht bewußter Vergleichsprozesse und deren motivationaler Bedeutung erhält. Außerdem spielen rückwirkende Systeme, die über die Topographie der Erregungsverteilung am Neokortex informieren, eine Rolle: Sie verhindern, daß bereits erregte Areale weiter erregt werden (für eine detailliertere Darstellung siehe Edelman, 1989, und Birbaumer \& Schmidt, 1996).

Erkenntnisse zu zellulären Korrelaten des eigentlichen Konsolidierungsprozesses stammen in der Regel aus Tierversuchen, da gemeinhin davon ausgegangen wird, daß es sich auch bei komplexeren Lernvorgängen in höheren Organismen nur um Variationen einiger weniger fundamentaler neurophysiologischer Vorgänge handelt. Für das Lernen einfacher konditionierter Reflexe konnte so gezeigt werden, daß prä- und postsynaptische molekulare Prozesse (Änderung der Entladungsrate, vermehrter Transmitterausstoß) in den am Verhalten beteiligten sensomotorischen Systemen den langanhaltenden Änderungen zugrundeliegen (Kandel \& Schwartz, 1982; Hawkins \& Kandel, 1984; Black et al., 1987; Squire, 1987; Teyler, 1991). Wiederholte Benutzung einer Synapse kann z. B. zu einer beträchtlichen Erhöhung des synaptischen Potentials und damit zu einer synaptischen Bahnung führen, die funktionell gesehen als ein durch Üben erleichterter Abruf eines zentralnervösen Vorgangs und damit als Lernprozeß betrachtet wird (Schmidt, 1987). Die Dauer der Bahnung hängt dabei vor allem von der Dauer und Frequenz der wiederholten Reizung ab; sie kann als sogenannte "posttetanische Potenzierung" an bestimmten Synapsen für viele Stunden und länger anhalten und zu vermehrten dendritischen Verzweigungen bzw. zum Wachstum dendritischer Fortsätze (sog. "spines") führen (Chang \& Greenough, 1982; Birbaumer \& Schmidt, 1990; Rosenzweig \& Bennett, 1996). Die Herstellung und Aufrechterhaltung neuer synaptischer Verbindungen setzt eine Steigerung der Proteinbiosynthese an den entsprechenden Zellen voraus. Über eine Hemmung der cerebralen Proteinbiosynthese 
durch bestimmte Antibiotika konnte ein kausaler Einfluß dieses biochemischen Prozesses auf das Gedächtnis nachgewiesen werden (Bennett, Orne \& Hebert, 1972; Davis \& Roitblat, 1984; Davis \& Squire, 1982). Damit entspricht die minimale Dauer von Kurzzeitgedächtnis-Prozessen jener Zeit, die nötig ist, um Proteinmoleküle aufzubauen und über axonalen Transport zu den relevanten interneuronalen Kommunikationsstellen zu befördern; diese Zeitverhältnisse (minimal einige Minuten) sollen gut mit der Dauer derjenigen biochemischen Prozesse übereinstimmen, die bei posttetanischer Potenzierung gefunden wurden (Birbaumer \& Schmidt, 1996). Neben der Herstellung neuer soll im übrigen auch der Abbruch alter synaptischer Verbindungen und die Aktivierung stiller Zellen an Lernvorgängen beteiligt sein (Rosenzweig \& Leiman, 1982).
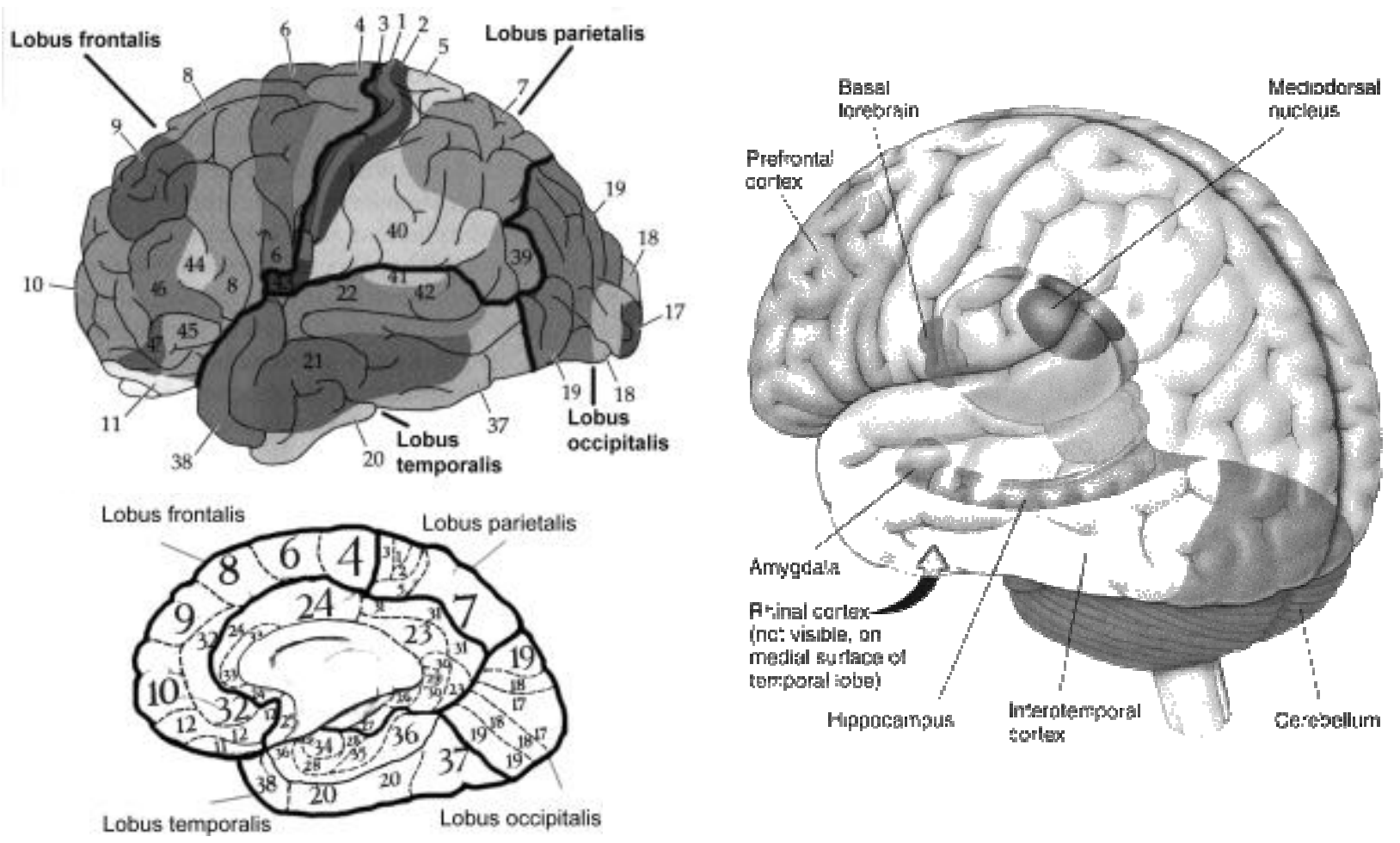

Abbildung 4. Links: Laterale (oben) und mediale (unten) Ansicht der Brodmannschen zytoarchitektonischen Einteilung der Hirnrinde (nach Rosenzweig, Leiman \& Breedlove, 1996, und Rösler, 1997; die Bezeichnung der verschiedenen Hirnlappen wurde ergänzt). Rechts: Für Gedächtnisfunktionen wesentliche Hirnstrukturen (aus Pinel, 1997).

Besonders lange posttetanische Potenzierungen wurden im Hippocampus entdeckt, so daß dieser zentralnervösen Struktur (vgl. Abb. 4 rechts) und ihren Afferenzen aus den Assoziationscortices zusätzlich zu einigen anderen neuronalen Strukturen der medialen Temporallappenregion (Amygdala, G. hippocampus, entorhinaler Kortex) eine wichtige Beteiligung an der Konsolidierung von Gedächtnisinhalten beim Menschen zugeschrieben wird (z. B. Bloom, Lazerson \& Hofstadter, 1985; Markowitsch, 1992; Birbaumer \& Schmidt, 1996). Hierfür sprechen auch zahlreiche Läsionsbefunde; Läsionen des Hippocampus und umliegender neokortikaler Strukturen des Temporallappens führen zu 
schweren anterograden Amnesien (vgl. den klassischen Fall H. M., Scoville \& Milner, 1957; Milner, 1970), gehen aber nicht mit Beeinträchtigungen der über Sternberg- und Gedächtnisspannenaufgaben erfaßten Kurzzeitgedächtnis-Leistung einher (Corkin et al., 1985; Cave \& Squire, 1992). Der mediale Temporallappen stellt somit nur eine der sogenannten "Flaschenhalsstrukturen" für die Langzeiteinspeicherung dar, nicht jedoch für das kurzzeitige Behalten von Informationen. Patienten, bei denen eine selektive Beeinträchtigung des auditiv-verbalen Kurzzeitspeichers bzw. eine stark verminderte Gedächtnisspanne vorliegt, weisen hingegen Schädigungen im linken unteren Parietalund linken oberen Temporallappen auf (Areale 7, 37 und 40 der linken Hemisphäre nach Brodmann, s. Abb. 4 links; vgl. Warrington, Logue \& Pratt, 1971; Ojemann, 1978; Shallice, 1988; Vallar \& Papagno, 1995; Smith \& Jonides, 1997). Ergebnisse weiterer Einzelfallstudien zeigen jedoch, daß je nach Aufgabenanforderung bzw. Verarbeitungsdimension ein breites Spektrum von neuronalen Strukturen und Prozessen an der Bewältigung von Kurzzeitgedächtnis-Aufgaben beteiligt ist (vgl. Kap. 3): So können z. B. für jeden Verarbeitungsschritt (Enkodierung, zentrale Verarbeitung, Reaktionsvorbereitung/-ausführung) mindestens je zwei Verarbeitungsarten (verbal-nonverbal), je zwei Sinnesmodalitäten (visuell-akustisch) und je zwei Reaktionsmodalitäten (manuell-vokal) angenommen werden (Gopher \& Donchin, 1985), die zu unterschiedlichen Aktivationskonstellationen in kortikalen Arealen führen. Das Kurzzeitgedächtnis wird daher heute zu recht als vielfältige Kollektion temporärer Verarbeitungskapazitäten (Squire, 1992) betrachtet und verstärkt unter Berücksichtigung seiner Arbeitsgedächtnisfunktionen untersucht.

Für das Verständnis und die Einordnung entsprechender neurowissenschaftlicher Ergebnisse sind Grundkenntnisse insbesondere der Funktionen neokortikaler Assoziationsareale und cerebraler Funktionsasymmetrien sehr wichtig, die im folgenden kurz skizziert werden sollen. Die Assoziationsareale der Großhirnrinde (Temporal-, Parietalund Frontallappen) spielen für die höheren, integrativen Leistungen des menschlichen Zentralnervensystems eine bedeutende Rolle (Schmidt, 1987), wobei es fast keine Funktion gibt, für die nicht eine - wenn auch geringe - Hemisphärendominanz nachweisbar wäre (Kolb \& Whishaw, 1995).

Der Temporallappen ${ }^{1}$ kann "... wegen der vielfältigen Afferenzen und Efferenzen seiner verschiedenen Unterfelder funktionell weniger als die anderen großen Hirnlappen in einem einheitlichen Zusammenhang gesehen werden" (Creutzfeldt, 1983, S. 296). Mit seinen Verbindungen zum limbischen System wird inm aber neben der bereits beschriebenen Gedächtnisfunktion (Konsolidierung von Lerninhalten) noch die Einordnung auditorischer, visueller und viszeraler Reize in den emotionalen Erlebnisraum sowie die

\footnotetext{
${ }^{1}$ Siehe Anmerkungsapparat ab Seite 145
} 
Repräsentation dieser Einordnungen im temporo-parietalen, sogenannten "interpretativen" Kortex zugeschrieben (Creutzfeldt, 1983). Effekte von Läsionen und elektrischer Reizung weisen ihn $u$. a. als wichtig für akustische und visuelle Wahrnehmung, Reizselektion sowie -organisation und -kategorisierung aus (vgl. Tab. 1).

Tabelle 1

Überblick über Funktionsausfälle nach Läsion des Temporallappens (links) und Parietallappens (rechts) (nach Kolb \& Whishaw, 1995; zit. nach Birbaumer \& Schmidt, 1996)

\begin{tabular}{|c|c|c|c|}
\hline Symptome & Möglicher Läsionsort & Symptome & $\begin{array}{c}\text { Wahrscheinlicher } \\
\text { Ort der Läsion }\end{array}$ \\
\hline $\begin{array}{l}\text { Störung der akustischen Wahr- } \\
\text { nehmung .................................. } \\
\text { Störung der Selektion visueller und } \\
\text { akustischer Reize .................... } \\
\text { Störungen der visuellen Wahrneh- } \\
\text { mung ........................................ } \\
\text { Gestörte Organisation und Katego- } \\
\text { risierung ............................... } \\
\text { Störung der Sprachwahrnehmung } \\
\text { Schlechtes Langzeitgedächtnis ....... } \\
\text { Änderungen der Persönlichkeit } \\
\text { und des Affektes ........................ } \\
\text { Änderungen sexuellen Verhaltens }\end{array}$ & $\begin{array}{l}\text { Areal 22, 41, } 42 \\
\text { Areal 20, 21, 22, 37, } 38 \\
\text { Areal 20, } 21 \\
\text { Areal 21, } 38 \text { links } \\
\text { Areal } 22 \text { links } \\
\text { Areal } 21 \text { (Hippocampus und } \\
\text { möglicherweise Amygdala }\end{array}$ & 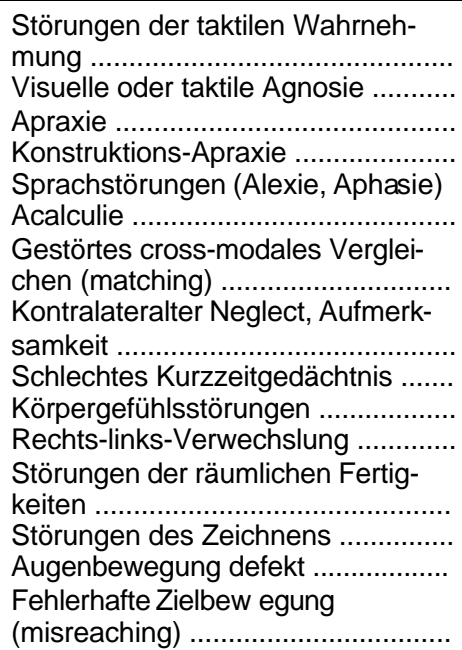 & $\begin{array}{l}\text { Areale } 1,2,3 \\
\text { Areale } 5,7,37 ? \\
\text { Areale } 7,40 \text { links } \\
\text { Areale } 7,40 \\
\text { Areale } 39,40 \text { links } \\
\text { Areale } 39,40 \text { links } \\
\text { Areale } 37,40 \\
\text { Areale } 7,40 \text { rechts } \\
\text { Areale } 37,40 \\
\text { Areal } 7 ? \\
\text { Areale } 7,40 \text { links } \\
\text { Areale } 7,40 \text { rechts } \\
\text { Areal } 40 \\
\text { Areal } 7,40 \\
\text { Areale } 5,7\end{array}$ \\
\hline
\end{tabular}

Vom Parietallappen ${ }^{2}$ wird angenommen, daß er an einem weitgestreuten corticosubkortikalen Aufmerksamkeitssystem beteiligt ist (LCCS, s. o.). Der bereits beschriebene enge Zusammenhang zwischen kontrollierter Aufmerksamkeit und Kurzzeitgedächtnis sowie die multisensorische Informationsintegration im parietalen Neokortex legen somit nahe, daß dieser Bereich eine Basis für wichtige Verarbeitungsschritte im Rahmen der Prozesse des deklarativen Kurzzeitgedächtnisses bildet. Entsprechend treten nach Läsionen des Parietallappens vielfältige Funktionsausfälle auf, die von Störungen der taktilen Wahrnehmung über Beeinträchtigungen des Kurzzeitgedächtnisses bis zu defekten Augenbewegungen reichen (vgl. Tab. 1).

Der Frontallappen ${ }^{3}$ kann nach funktionellen Gesichtspunkten grob in drei Subsysteme unterteilt werden, die motorische Aufgaben, Aufmerksamkeitsfunktionen und motivationale Funktionen erfüllen. "Da der präfrontale Kortex hinsichtlich seiner afferenten Organisation, zumindest in seinem vorderen Anteil eine relativ homogene Organisation aufweist, sind differenzierte Ausfallserscheinungen nach umschriebenen Läsionen nur schwer voneinander abzugrenzen. ..." (Creutzfeldt, 1983, S. 314). Die Hauptsymptome nach Läsionen des Frontallappens können als Störungen der Kontrolle zweckgerichteten motorischen und adäquaten emotionalen Verhaltens zusammengefaßt werden, denn die dorsolateralen und orbitalen Regionen des Frontallappens (sogenannter prä- 
frontaler Kortex, "PFC") sind im wesentlichen für die zeitliche Steuerung von Informationen und motorischen Akten bzw. für die zeitliche Abschätzung zukünftiger Ereignisse zuständig (vgl. Tab.2) ${ }^{4}$. Folgeerscheinungen sind Störungen des kontingenten Abrufens von Kurzzeitgedächtnis-Informationen bzw. auch Störungen der exekutiven Kontrolle des Gedächtnisses (Baddeley, 1990). Vorgänge im Frontallappen werden daher häufig mit Funktionen der sogenannten "zentralen Exekutive" in Baddeleys Arbeitsgedächtnismodell in Verbindung gebracht (Parkin, 1993; s. auch Kap. 3.1.3).

\section{Tabelle 2}

Überblick über Funktionsausfälle nach Läsion des Frontallappens (links) und entsprechende funktionelle Zonen (rechts) (nach Birbaumer \& Schmidt, 1996)

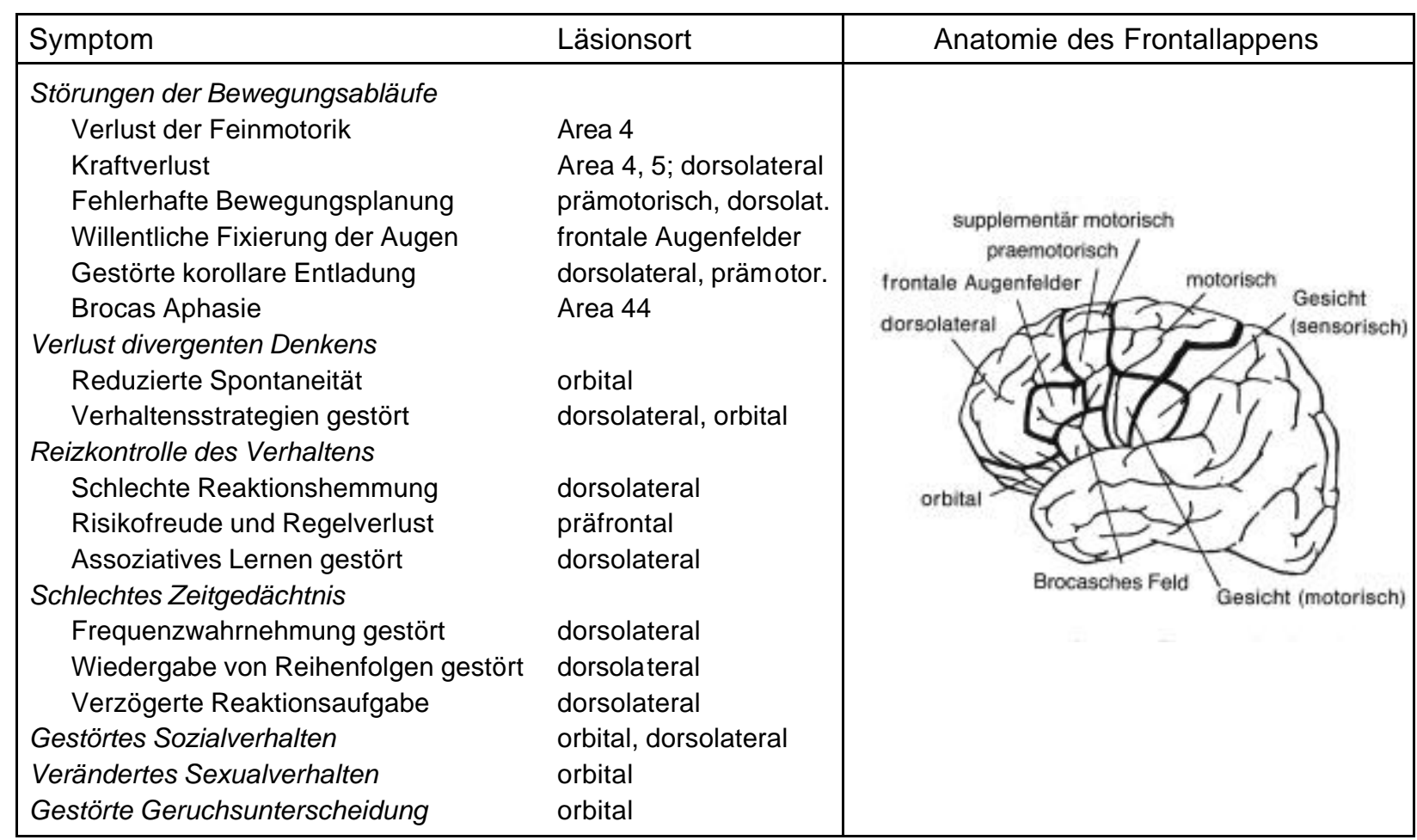

Obwohl der Zusammenhang zwischen Händigkeit und Lateralität nicht perfekt ist, kann die bevorzugte Benutzung einer Hand beim Menschen als das auffälligste Zeichen für die funktionelle Asymmetrie der Hemisphären betrachtet werden (Creutzfeldt, 1983). Daneben gibt es sehr eindeutige anatomische Korrelate einzelner lateralisierter Funktionen $^{5}$. Die Untersuchung sogenannter "Split-Brain-Patienten" hat neben Versuchen mit elektrischer Hirnreizung und reversibler einseitiger Ausschaltung einer Hemisphäre zahlreiche gesicherte Erkenntnisse zu Funktionslateralisierungen erbracht (vgl. Tab. 3): Während die linke Hemisphäre bei ungefähr 95 \% der Bevölkerung für Sprache und darüber hinaus auch für komplexe gelernte Bewegungen dominant ist, fallen visuelle und taktile Formerkennung, Abstraktions- und räumliches Vorstellungsvermögen sowie musikalische Leistungen in den Zuständigkeitsbereich der rechten Hemisphäre (Schmidt, 1987). 
Tabelle 3

Funktionslateralisierungen der Hemisphären bei Rechtshändern (links) und entsprechende anatomische Unterschiede zwischen rechter und linker Hemisphäre (nach Birbaumer \& Schmidt, 1996)

\begin{tabular}{|c|c|c|c|}
\hline Funktion & Linke Hemisphäre & Rechte Hemisphäre & Anatomische Unterschiede (siehe Text) \\
\hline Vis. System & Buchstaben, Wörter & $\begin{array}{l}\text { Komplexe geometr. } \\
\text { Muster }\end{array}$ & \\
\hline Auditor. System & Sprachbezog. Laute & $\begin{array}{l}\text { Nichtsprachbezog. } \\
\text { externe Geräusche, } \\
\text { Musik }\end{array}$ & \\
\hline $\begin{array}{l}\text { Somatosensor. } \\
\text { System }\end{array}$ & $?$ & $\begin{array}{l}\text { Taktiles Wiederer- } \\
\text { kennen von komple- } \\
\text { xen Mustern }\end{array}$ & \\
\hline Bewegung & $\begin{array}{l}\text { Komplexe Willkür- } \\
\text { bewequng }\end{array}$ & $\begin{array}{l}\text { Bewegungen in } \\
\text { räumlichen Mustern }\end{array}$ & \\
\hline Gedächtnis & $\begin{array}{l}\text { Verbales } \\
\text { Gedächtnis }\end{array}$ & $\begin{array}{l}\text { Nonverbales } \\
\text { Gedächtnis }\end{array}$ & \\
\hline Sprache & $\begin{array}{l}\text { Sprechen, Lesen, } \\
\text { Schreiben, Rechnen }\end{array}$ & Prosodie & \\
\hline $\begin{array}{l}\text { Räumliche } \\
\text { Prozesse }\end{array}$ & & $\begin{array}{l}\text { Geometrie, Rich- } \\
\text { tungssinn, mentale }\end{array}$ & \\
\hline Emotionen & neutral-positiv & $\begin{array}{l}\text { Rotation von Formen } \\
\text { negativ-depressiv }\end{array}$ & \\
\hline
\end{tabular}

Kognitionspsychologisch interessant ist nicht nur die erwiesene Funktionslateralisierung, sondern auch der Unterschied in der Art der Informationsverarbeitung: Untersuchungen weisen darauf hin, daß die linke Hemisphäre eher sequentiell, analytisch und kausal verarbeitet, während die rechte eher ganzheitlich-holistisch, parallel und intuitiv vorgeht (Levy \& Trevarthen, 1976; Gazzaniga \& LeDoux, 1978). Wie stark sich beide Hemisphären dabei ergänzen, geht ebenfalls aus Split-Brain-Studien hervor: So spielt die rechte Hemisphäre zwar die wesentliche Rolle bei der Erfassung visueller Information, isoliert ist sie jedoch im Gegensatz zur linken Hemisphäre nicht in der Lage, komplexe visuelle Vorstellungsbilder zu erzeugen (Posner, Petersen, Fox \& Raichle, 1988).

Die neurophysiologischen Erkenntnisse zur Informationsenkodierung auf zellulärer Ebene und die aus der Anatomie- bzw. Läsionsforschung abgeleiteten Hypothesen zur Organisation des menschlichen Gedächtnisses finden sich heute in neueren Modellbildungen zur Informationsspeicherung wieder. Diese Modelle bilden zwar das neurowissenschaftliche Pendant zu psychologischen Gedächtnistheorien, befassen sich aber im wesentlichen mit der Übertragung von Informationen vom Kurzzeit- in das Langzeitgedächtnis (z. B. Kornhuber, 1988; Markowitsch, 1985; Mesulam, 1990). Dabei ist "allen neueren Modellannahmen ... eigen, daß sie die Verwobenheit zwischen Hirnregionen betonen und das "Level"-Denken aufgeben ... . Information wird nicht mehr als von bestimmten Einzelorten abrufbar betrachtet, sondern als durch das Zusammenwirken von eng verquickten Systemen repräsentiert betrachtet." (Markowitsch, 1992, S. 237). Diese Sichtweise wird besonders in Damasios (1990) Abrufmodell deutlich: Damasio lehnt die verbreitete Annahme ab, daß integrierte Gedächtnisinhalte in multimodalen kortikalen As- 
soziationsregionen gespeichert und von dort auch wieder abgerufen werden. Er nimmt im Gegenteil dazu an, daß die Komponenten eines Perzepts in unterschiedliche kortikale Regionen aufgenommen, für den Abruf jedoch synchron reaktiviert werden, so daß sie dann wieder den einheitlichen Wahrnehmungsinhalt repräsentieren. Es sollen mit anderen Worten beim Gedächtnisabruf Aktivationsmuster in genau jenen kortikalen Gebieten zeitgleich erzeugt werden, in denen die Merkmale des Gedächtnisinhaltes auch bei einer rein perzeptuellen Analyse verarbeitet werden. (Die Synchronisation der Erregungsmuster zur Prüfung dieser Annahme läßt sich heute besonders gut über elektrophysiologische Verfahren erfassen, siehe Kapitel 2.2.2.) Damit wird in den neuesten Modellen nicht mehr zwischen kortikalen Arealen unterschieden, die für Verarbeitung vs. Speicherung von Informationen spezialisiert sind, sondern es werden Strukturen postuliert, die gleichermaßen für die Verarbeitung und Repräsentation bestimmter Merkmale wie Farbe, Form, Ort usw. zuständig sind (Rösler, 1997). Die neurophysiologische Unterscheidung zwischen Kurz- und Langzeitgedächtnis entfällt somit bzw. betrifft in diesem Sinne nur noch den Aktivationszustand der Gedächtnisrepräsentation (vgl. z. B. Shiffrin \& Schneider, 1977).

Vor dem Hintergrund der beschriebenen Hemisphärenasymmetrie und aus der Kenntnis von Funktionsausfällen nach umschriebenen Läsionen neorkortikaler Bereiche lassen sich Hypothesen zu möglichen neuroanatomischen Korrelaten des Arbeitsgedächtnisses bzw. zur Topographie der aktivierten Gedächtnisrepräsentationen ableiten (vgl. Kap. 3 u. 4). Die Untersuchung von kognitiven Aktivationen und deren Änderungen in Abhängigkeit verschiedener Anforderungen an das Arbeitsgedächtnis setzt jedoch den Einsatz angemessener Methoden voraus: Im folgenden sollen daher die wichtigsten Verfahren zur Untersuchung des Gedächtnisses beschrieben und kurz bewertet werden.

\subsubsection{Verfahren zur Untersuchung des Gedächtnisses}

In der biologischen und physiologischen Psychologie werden Hirn-Verhaltensbeziehungen am intakten System meist mittels invasiver Methoden im Tierversuch untersucht (z. B. Analyse von Verhaltensstörungen nach zugefügten Hirnschäden, intrakranielle Ableitungen hirnelektrischer Aktivität). Im Humanbereich konzentrierte sich die Psychophysiologie bis Ende der 70er Jahre vorwiegend auf die Registrierung hirnelektrischer oder magnetischer Prozesse, darüber hinaus aber auch auf Reizmethoden und Läsionsstudien (Untersuchung der Auswirkung von elektrischen, magnetischen oder chemischen Reizen und von erlittenen Hirnschäden auf Verhalten). Läsionsstudien ermöglichen jedoch nur indirekte Schlüsse über die Struktur-Funktionsbeziehungen einzelner Hirngebiete, da sich die Ergebnisinterpretation wegen der verschiedenen möglichen Bedeutung einzelner Hirnstrukturen für die beobachtete Verhaltensänderung schwierig gestaltet (Birbaumer \& Schmidt, 1996; Rösler, 1997): 
Keine dieser Vorgehensweisen führt jedoch zu befriedigenden Antworten, was primär daran liegt, daß Hirnregionen nie für sich alleine existieren und arbeiten, sondern, daß der Netzwerkcharakter mit einem Mehrfachen an synaptischen Verbindungen gegenüber der Zahl vorhandener Neurone bedeutet, daß man immer beachten muß, welche direkten (und nachfolgend welche indirekten) Aufschaltungen und Efferenzen ihren Beitrag zur Funktionsweise einer Hirnregion liefern. Daneben ist festzuhalten, daß Regionen ontogenetisch, krankheitsbedingt, durch Umwelteinflüsse ausgelöst, etc. ihre primäre Funktion oder Funktionszuständigkeit einbüßen oder ändern können. (Markowitsch, 1992, S. 227)

Erst die Kombination von angemessenen experimentalpsychologischen Untersuchungsstrategien mit (möglichst mehreren) hochauflösenden bildgebenden Verfahren erlaubt methodisch einwandfreie Zuordnungen von Funktion und Struktur im Gehirn (Markowitsch, 1992; Birbaumer \& Schmidt, 1996).

Mit bildgebenden Verfahren lassen sich strukturell-anatomische und metabolische Veränderungen im Gehirn sichtbar machen (mittels Röntgencomputertomographie / CAT, Positron-Emissions-Tomographie / PET und funktioneller Magnetresonanztomographie / fMRT). Elektrische und magnetische Prozesse der Großhirnrinde können mittels Elektroenzephalographie (EEG) und Magnetoenzephalographie (MEG) erfaßt werden. Wegen ihrer höheren zeitlichen Auflösung und damit größeren Bedeutung für die Untersuchung schnell ablaufender kognitiver Prozesse soll hier nach einer kurzen Darstellung der erstgenannten Verfahren vor allem auf MEG und EEG eingegangen werden.

\section{Bildgebende Verfahren: CAT, rCBF, PET und fMRT}

Die computerisierte Axial-Tomographie bzw. Röntgencomputertomographie (CAT) hat die klassiche neuroradiologische Untersuchung ersetzt, die für den Patienten recht gefährlich und belastend gewesen war. Bei der CAT wird der Patient in einem sich langsam drehenden Ring zwischen einer Röntgenröhre und einem Röntgendetektor plaziert; die sukzessiv an den Kopf des Patienten abgegebenen und vom Detektor gemessenen (Rest-)Strahlen ermöglichen die Erstellung zweidimensionaler Bilder des Gehirns in verschiedenen horizontalen und vertikalen Schnittebenen. Die Einführung der CAT machte erstmals das Studium von Verhaltensstörungen nach exakt definierten Läsionen möglich; da die einzelnen Aufnahmen einer Schnittebene jedoch Minuten dauern und sich die Röntgenabsorptionskoeffizienten nur bei deutlichen Gewebeveränderungen ändern, dient die CAT hauptsächlich der Diagnose raumforderner Prozesse und der Ermittlung von Läsionen (Birbaumer \& Schmidt, 1996).

Für die Messung regionaler zerebraler Durchblutungsänderungen ( $r C B F)$ während verschiedener Hirnaktivitäten wird ein schwach und kurz radioaktives Gas (z. B. Xenon) in die Blutbahn injiziert. Das Auftauchen dieses Gases in verschiedenen Hirnregionen wird mit Geigerzählern gemessen; aus der Strahlungsverteilung und dem Gesamtsau- 
erstoffverbrauch des Gehirns läßt sich die lokale Hirndurchblutung errechnen (ebd.). Eine analoge Methode ist die Positronen-Emissions-Tomographie (PET). Durch die Injektion biologisch wichtiger, radioaktiv markierter Substanzen (Wasser, Glucose, Aminosäuren) werden verschiedene Aspekte des Hirnstoffwechsels sichtbar gemacht, die eng mit lokalen Durchblutungsänderungen korreliert sind. Die injizierten Moleküle setzen Positrone frei, die nach einer Distanz von 2 bis $8 \mathrm{~mm}$ mit Elektronen kollidieren. Der sich daraus ergebende Teilchenuntergang erfolgt unter Aussendung zweier $\gamma$-Strahlen (unter einem Winkel von genau $180^{\circ}$ ), die von Photodetektoren aufgefangen werden und die Berechnung der Abstrahlungsdichte bzw. der Verteilung der jeweiligen Substanzen im Gehirn ermöglicht (ebd.). Der unbestreitbare Vorteil der PET liegt bei dem vergleichsweise hohen räumlichen Auflösungsvermögen (ca. 4-8 mm) dieser Methode, dem die für die Untersuchung kognitiver Prozesse viel zu geringe Zeitauflösung von etwa einer Sekunde entgegensteht. Anlaß zur Kritik gibt aber auch die Tatsache, daß die neuronale Aktivität nur indirekt erfaßt wird und daß man bei der Auswertung wegen der meist schwachen Durchblutungsänderungen auf die problematische Differenzbildung zwischen den Messungen aus zwei Bedingungen angewiesen ist (vgl. Rösler, 1997). Darüber hinaus handelt es sich bei der PET um ein recht kostspieliges Verfahren, da das Zyklotron zur Isotopen-Herstellung wegen der kurzen Halbwertszeit der benötigten Isotope in unmittelbarer Nähe liegen muß.

Eine weitere Methode zur indirekten Messung des lokalen zerebralen Blutflusses ist die funktionelle Magnetresonanztomographie (fMRT), die sich in den letzten Jahren zum wichtigsten nichtinvasiven Verfahren der diagnostischen Bildgebung entwickelt hat (Frahm, 2000). Mittels starker Elektromagneten wird die Auslenkung der Richtung von Wasserstoffatomen erzielt, die besonders zahlreich in durchblutetem Gewebe vorhanden sind. Gemessen werden die schwachen hochfrequenten Radiowellen, die von den Protonen der Wasserstoffatome bei der Rückkehr in ihre Ausgangslage (sog. Relaxation) abgegeben werden. Durch die Kombination vieler Einzelmessungen mit unterschiedlichen Ortskodierungen lassen sich so Aktivierungskarten des Gehirns erstellen. Trotz der mit diesem Verfahren möglichen sehr präzisen anatomischen Lokalisation metabolischer Aktivitäten und einer gegenüber der PET verbesserten zeitlichen Auflösung ist der Einsatz der fMRT für die Untersuchung kognitiver Tätigkeiten problematisch. Zum einen bedingt die notwendige vollständige Abschirmung von störenden externen elektromagentischen Quellen und die hohe Bewegungsempfindlichkeit des Meßverfahrens eine psychisch recht belastende Untersuchungssituation und schränkt gleichzeitig die verschiedenen Möglichkeiten experimenteller Versuchsanordnungen erheblich ein. Zum anderen wird bei diesem Verfahren (wie bei der PET) das zeitliche Auflösungsvermögen durch die hämodynamische Antwortfunktion begrenzt, was eine Untersuchung schnell ablaufender kognitiver Prozesse unmöglich macht. 


\section{MEG und EEG}

Eine zeitgetreue Abbildung informationsverarbeitender Prozesse im Gehirn ist nur mittels Elektroenzephalographie (EEG) und Magnetoenzephalographie (MEG) möglich (Birbaumer \& Schmidt, 1996; Rösler, 1997). Mit diesen Verfahren werden hirnelektrische Ströme bzw. deren schwache Magnetfelder mit angemessen hoher zeitlicher Auflösung (im Bereich von Millisekunden) erfaßt, so daß genaue Zuordnungen zwischen elektrischen Änderungen und Verarbeitungsprozessen beim Menschen möglich sind. Die Ableitung erfolgt extrakranial, beim MEG über hochempfindliche Detektoren, beim EEG mittels sensibler Elektroden, die nach bestimmten Vorschriften (sog. 10-20-System, vgl. Kap. 5.6) auf der Schädeloberfläche angebracht werden. Daneben existieren auch Verfahren zur intrakranialen Ableitung von hirnelektrischen Strömen (vgl. Rösler, 1997), die ihren Einsatz vorwiegend bei der Klärung neurophysiologischer und molekularbiologischer Fragestellungen finden, auf die hier aber nicht weiter eingegangen werden soll.

Die Magnetoenzephalographie wird in der Neuropsychologie hauptsächlich zur präzisen Lokalisation der Stromquellen im Gehirn eingesetzt. In Kombination mit dem EEG ermöglicht es eine sehr genaue Lokalisierung von Aktivitätsquellen im Gehirn (bis zu 2 mm genau): $M E G$ und EEG liefern komplementäre Informationen über die Stromquellenverteilung, da über MEG die orthogonal und über EEG die vertikal zur Schädeldecke gelegenen elektrischen Ströme aufgezeichnet werden (Birbaumer \& Schmidt, 1996). Die Erfassung magnetischer Felder auch in tieferen Hirnregionen stellt mittels MEG kein Problem dar, weil es - im Gegensatz zum EEG - durch umliegende Schichten (Hirnhaut, Schädelknochen usw.) nicht beeinflußt wird (ebd.). Da die Stärke der magnetischen Felder, die von Nervenzellaktivitäten im Körper ausgehen, jedoch extrem niedrig ist, sind zum einen wie bei der FMRT wirksame Abschirmmaßnahmen erforderlich (Aufzeichnung in magnetisch abgeschirmten Kabinen), zum anderen müssen für die exakte Erfassung hochempfindliche, heliumgekühlte Detektoren eingesetzt werden. Aus diesem Grunde handelt es sich auch beim MEG um ein recht kostspieliges und aufwendiges Verfahren (Gevins, Leong, Smith, Le \& Du, 1995), das zudem erst in den letzten Jahren technisch soweit fortentwickelt werden konnte, "... daß die spontane und ereigniskorrelierte Hirnaktivität für den klinischen Einsatz mit hinreichend gutem Signalrauschverhältnis, befriedigender räumlicher Auflösung und vertretbarem zeitlichen Aufwand untersucht werden kann." (Scherg, 1999, S. 1). Obwohl die MEG selber noch Gegenstand der Forschung ist (hier wird insbesondere nach Muster und Wellen gesucht, die den bekannten Erscheinungen beim EEG entsprechen), gilt sie heute im Prinzip als dasjenige nichtinvasive Verfahren, das die beste örtliche und zeitliche Auflösung erreicht (Birbaumer \& Schmidt, 1996). 
Die Ableitung des Elektroenzephalogramms erlaubt relativ genaue Zuordnungen zwischen elektrischen Spannungsschwankungen der Großhirnrinde und einzelnen kognitiven Prozessen. "Die Aufzeichnung der elektrischen Aktivität des Gehirns ist zusammen mit der Aufzeichnung der Magnetfelder ... der wichtigste methodische Zugang zur Erforschung der Zusammenhänge zwischen Hirn und Verhalten beim Menschen" (Birbaumer \& Schmidt, 1996, S. 488). Durch das EEG "... ist eine Aufklärung der funktionalen Topographie der Großhirnrinde in vivo bei Gesunden in engem Bezug zu Konstrukten der Kognitiven Psychologie möglich, dies zudem bei Normalpersonen und ohne gesundheitliche Risiken" (Rösler, 1997, S. 99). Die Kompaktheit und Einfachheit der EEGTechnologie, die in wissenschaftlichen Darstellungen oft unberücksichtigt bleibt, ermöglicht zudem Hirnaktivitätsuntersuchungen während der Bewältigung alltäglicher Aufgaben außerhalb des Labors (Gevins et al., 1995). Diesen Vorteilen stand bislang der Nachteil einer relativen örtlichen Ungenauigkeit der herkömmlichen EEG-Technik gegenüber: Da die erfaßten Signale primär im Neokortex generiert und von recht umfänglichen Netzwerken abgeleitet werden, konnten subkortikale Potentialverschiebungen nur indirekt erschlossen werden. Diesem Nachteil kann heute durch die Nutzung der fMRT und PET für die Elektrodenpositionierung, durch die Erhöhung der Elektrodenanzahl und durch die Korrektur der durch Kopfhaut und Schädel verursachten Signalverzerrung begegnet werden (ebd.).

Die abgeleitete kortikale elektrische Aktivität entspricht einer summierten Tätigkeit von Tausenden von Zellen und ist das Ergebnis exzitatorischer und inhibitorischer postsynaptischer Potentiale der Zellkörper und der großen Dendriten der senkrecht ausgerichteten Pyramidenzellen (Cooper, Osselton \& Shaw, 1984). Die an den oberen apikaIen Dendriten (Schicht I und II) endenden exzitatorischen Fasern aus den "unspezifischen" thalamischen Kernen spielen dabei, verglichen mit den übrigen Zellanteilen, eine dominierende Rolle (Creutzfeldt, 1983). Voraussetzung für die Ableitbarkeit des EEGs ist eine zeitliche Synchronisation der afferenten Impulssalven bzw. die Bildung starker Feldpotentiale durch gleichzeitige synaptische Aktivierung mehrerer Tausend Pyramidenzellen (Birbaumer \& Schmidt, 1996). Das EEG kann als bedeutsames Äquivalent von Kognition und Verhalten angesehen werden: Trotz der zugrundeliegenden Vielfalt zellulärer Prozesse ergibt sich ein geordnetes Muster elektrischer Potentiale, so daß davon ausgegangen werden kann, daß diejenigen zentralnervösen Eigenheiten und Strukturen, die diese Ordnung bedingen, auch unserem Verhalten und Denken zugrundeliegen (ebd.). Dafür sprechen u.a. statistische Untersuchungen der Hirnrindenstruktur, in denen nachgewiesen werden konnte, daß die wesentliche Aufgabe der Hirninde in der Bildung einer möglichst hohen Konvergenz und Divergenz von Signalen besteht (Petsche, 1995). In verschiedenen tierexperimentellen Studien konnte zudem gezeigt werden, daß Synchronisierungsphänomene auf kortikaler Ebene das Korrelat spezifischer Informationsverarbeitung sind (Espinosa \& Gerstein, 1988; Singer, 1990). Diese Erkenntnis hat 
sich in den letzten Jahren zunehmend auf den Einsatz bestimmter rechnerischer Verarbeitungsmethoden von EEG-Signalen ausgewirkt, so daß der EEG-Auswertung und der damit einhergehenden Problematik an dieser Stelle ein Exkurs gewidmet werden soll.

\section{Exkurs: EEG-Analyse}

Bei der hirnelektrischen Aktivität (EEG) handelt es sich um ein kontinuierliches, rhythmisches Signal, das jedoch nicht exakt reproduzierbar und schwierig quantitativ zu beschreiben ist (Cooper, Osselton \& Shaw, 1984). Quantitative EEG-Analysen liefern daher immer nur statistische Abschätzungen des zugrundeliegenden, generierenden Prozesses, ähnlich wie die Größenmessung einer Menschengruppe nur eine statistische Abschätzung der Größe der Gesamtpopulation sein kann (ebd.).

Die wichtigsten Kennwerte des EEGs sind Frequenz und Amplitude sowie deren zeitliche und örtliche Verteilung (Birbaumer \& Schmidt, 1996). Zusätzlich zu verschiedenen Techniken zur Quantifizierung des EEGs (z. B. die Berechnung von Amplitudenmaßen und Wellenindizes) stellt die Spektralanalyse eine der gängigsten Methoden zur rechnerischen Verarbeitung von EEG-Signalen dar. Sie macht sich die Tatsache zunutze, daß rhythmische Muster im EEG durch Additionen verschiedener Sinuswellen simulierbar sind: In der Spektralanalyse wird das Originalsignal im umgekehrten Prozeß mittels bestimmter mathematischer Techniken, sogenannter Fourier-Analysen, in seine (sinusförmigen) Frequenzkomponenten zerlegt. Das Ergebnis ist ein Spektrum, dessen Amplitude eine Funktion der Frequenz (Anzahl der vollständigen Schwingungen pro Sekunde) und nicht mehr wie beim Originalsignal eine Funktion der Zeit ist (sog. "Amplitudenspektrum"; Cooper, Osselton \& Shaw, 1984). Das Quadrat des Amplitudenspektrums gibt die "Stärke" oder Leistung des Signals wieder (Leistungsspektrum), die in der EEGAnalyse von großer Bedeutung ist, da die Summe der Amplitudenwerte des Leistungsspektrums dem quadratischen Mittelwert des ursprünglichen Signals entspricht (ebd.). Die Form komplexer Wellen hängt jedoch nicht nur von den Frequenzen der sinusförmigen Komponenten, sondern auch von deren Zeitbeziehungen bzw. Phasen ab; die Phasenwinkel aller Frequenzkomponenten ergeben das Phasenspektrum, das neben dem Amplitudenspektrum für die vollständige Signalbeschreibung unerläßlich ist (ebd.).

Die Fouriertransformation von Signalen bildet die Grundlage für weitere wichtige EEG-Analyseverfahren, darunter die sogenannte Kohärenzanalyse. Die Kohärenz ist ein Maß für den Grad der Synchronisierung der von verschiedenen kortikalen Regionen abgeleiteten elektrophysiologischen Signale. Da diese Synchronisierung von zugrundeliegenden strukturellen Verbindungen bzw. von der funktionellen Kopplung der entsprechenden Hirnregionen abhängt (s. o.), können über die Berechnung von Kohärenzfunktionen elektrographische Korrelate der funktionellen Organisation des Gehirns gewonnen werden (Busk \& Galbraith, 1975; Cooper, Osselton \& Shaw, 1984; Petsche, 1995). 
Dies hat in den letzten Jahren zu einer vermehrten Anwendung der Kohärenzanalyse bei der elektrophysiologischen Untersuchung kognitiver Informationsverarbeitungsprozesse geführt (z. B. Rappelsberger \& Petsche, 1988; Petsche, Lacroix, Lindner, Rappelsberger \& Schmidt-Heinrich, 1992; Trembley et al., 1994; Weiss \& Rappelsberger, 1994; Petsche, 1995; Krause, Schack, Gibbons \& Kriese, 1997), wobei wiederholt gezeigt werden konnte, daß sich die Kohärenz bei kognitiven Prozessen spezifisch verändert. Über die Frage, ob sich eine kortikale Aktivierung im EEG in einer Synchronisierung oder Desynchronisierung äußert, herrscht allerdings noch Uneinigkeit (von Stein, Rappelsberger, Filz \& Petsche, 1993). Die hohe Dynamik der schnell ablaufenden kognitiven Prozesse begrenzte bislang die Einsatzmöglichkeiten der auf Fourier-Analysen basierenden Kohärenzschätzungen, da eine bestimmte, durch dieses Verfahren bedingte zeitliche Auflösung von etwa 125 ms nicht unterschritten werden kann (Schack, 1997). Andere Verfahren, die eine höhere zeitliche Auflösung erreichen, nutzen die Tatsache, daß sich EEG-Signale durch sogenannte autoregressive Modelle approximieren lassen: Über eine bi- oder multivariate lineare Regression, bei der vereinfachend gesagt die ablaufenden Prozesse zu früheren Zeitpunkten die Prädiktoren und die Prozesse selbst die Kriterien bilden, erfolgt eine kontinuierliche parametrische Schätzung der Kohärenz (Isaksson, Wennberg \& Zetterberg, 1981; Gersch, 1987; Arnold, Miltner, Witte, Bauer \& Braun, 1998). Diese zeitreihenanalytischen Verfahren wiesen bislang jedoch den Nachteil einer (trotz des parametrischen Vorgehens) fehlenden Rekursivität und/oder eines immensen Rechenaufwandes für die Schätzung der spektralen Dichtematrix jedes Abtastpunktes auf (Schack, Grieszbach \& Krause, 1998).

Relativ neu ist die Entwicklung eines in der Zeit kontinuierlichen Schätzverfahrens der Kohärenzfunktion mit gleichzeitiger hoher Frequenzauflösung, das nur einen vergleichsweise geringen Rechenaufwand erfordert (Schack, Grieszbach, Arnold \& Bolten, 1995). Dieses Verfahren wird der fehlenden Stationarität biologischer Signale besonders gut gerecht, indem es eine adaptive Anpassung eines bivariaten autoregressiven "moving-average" Modells (ARMA-Modell) mit zeitvarianten Parametern vornimmt (Schack, 1997). Das Kriterium für die Modellanpassung ist die Minimierung des Vorhersagefehlers; dies geschieht zu jedem Abtastzeitpunkt "... by means of modification and generalization of the least mean square (LMS) algorithm ..." (Schack, Grieszbach \& Krause, 1998, S. 219), wodurch die Schätzgenauigkeit erhöht wird. Einen Überblick über das mathematische Vorgehen geben Schack, Grieszbach und Krause (1998); für eine detailliertere Darstellung siehe Schack (1997). Die auf diese Weise berechnete Kohärenz hat sich für das Studium schnell ablaufender elementarer Denkprozesse als sehr sensitiv herausgestellt (Schack \& Krause, 1995, 1996; Krause, 1997; Krause, Gibbons \& Schack, 1998). Darüber hinaus ermöglichen die Ergebnisse der Kohärenzanalyse verschiedener Elektrodenpaare unter Anwendung sogenannter Mapping-Verfahren die Erstellung topographischer Karten aktivierter neokortikaler Areale (Gevins et al., 
1995; Schack, Grieszbach \& Krause, 1998). Will man die Vorteile der EEG-Ableitung (s. o.) für die Analyse der funktionellen Kopplung verschiedener Hirnregionen im Verlauf von Kurzzeitgedächtnisprozessen in angemessener Weise nutzen, so wird man in Zukunft bei der kohärenzanalytischen Auswertung nicht mehr auf das zeitlich hochauflösende Schätzverfahren verzichten können. In diesem Zusammenhang stehen jedoch noch weitere Untersuchungen aus, insbesondere zur Feststellung derjenigen Kohärenzmaße, die sich besonders für die Beschreibung schnell ablaufender kognitiver Prozesse eignen. 


\section{Neuropsychologische Untersuchungen zum Arbeitsgedächtnis}

Der Frage nach den neuronalen Korrelaten verschiedener Komponenten des Arbeitsgedächtnisses wird traditionell in klinisch-anatomischen Studien nachgegangen, in denen Zusammenhänge zwischen umgrenzten Hirnläsionen einerseits und Lern- und Gedächtnisdefiziten andererseits untersucht werden (Vallar \& Papagno, 1995). Die daraus resultierenden Befunde werden heute durch neuropsychologische Studien mit bildgebenden Verfahren ergänzt, in denen physiologische Parameter gesunder Probanden in kontrollierten Versuchsanordnungen erhoben und für die bildliche Darstellung aktivierter Himstrukturen verwendet werden (Rösler, 1997). Diesem zweigleisigen Vorgehen der Neurowissenschaft folgend sollen hier repräsentative Befunde aus Läsionsstudien und aus Untersuchungen an gesunden Personen getrennt referiert werden (Kap. 3.1 und 3.2). Im Anschluß folgt eine zusammenfassende Bewertung der empirischen Untersuchungen und die Ableitung der dieser Arbeit zugrundeliegenden Fragestellung (Kap. 3.3 und Kap. 4).

\subsection{Klinisch-anatomische Studien}

Obwohl Baddeley und Hitch (1974) in ihrem modularen Modell des Arbeitsgedächtnisses (vgl. Kap. 2.1.1) das Vorhandensein von weiteren, über die phonologische Schleife und das visuell-räumliche Sketchpad hinausgehenden Komponenten nicht ausschlieBen, hat sich die neuropsychologische Forschung bislang im wesentlichen auf diese beiden Subsysteme konzentriert. Die zentrale Exekutive ist als schwierigste Systemkomponente erst in den letzten Jahren etwas mehr in den Mittelpunkt der Aufmerksamkeit gerückt, so daß die Anzahl der Untersuchungen zur Klärung ihrer Struktur und Funktionsweise vergleichsweise gering ausfällt.

Die Existenz der verschiedenen Komponenten des Arbeitsgedächtnismodells läßt sich heute anhand zahlreicher Untersuchungen hirngeschädigter Patienten mit selektiver Beeinträchtigung des Kurzzeitgedächtnisses untermauern. Bedauerlicherweise gilt für die meisten Studien das von Smith und Jonides (1997) Gesagte:

The vast majority of research on working memory has been carried out solely using behavioral experiments. This is clearly true to the many behavioral studies with normal subjects, and it applies as well to behavioral experiments with braininjured patients that are primarily concerned with functional dissociation, with little focus on the specific brain regions involved. (S.6)

Dies mag zum einen methodische Gründe haben, da entscheidende Fortschritte bei der Entwicklung bildgebender Verfahren zur nichtinvasiven Untersuchung des menschlichen Gehirns erst in den letzten 20 Jahren erzielt wurden. Zum anderen gestaltet sich die Interpretation mutmaßlicher Funktionen lädierter Hirnbereiche problematisch, da das 
Ausmaß und die genaue Lage von Hirnschäden von Patient zu Patient in beachtlichem Ausmaß variieren (Raichle, 1997). Unberücksichtigt bleiben zudem mögliche interindividuelle Differenzen hinsichtlich der Organisation des Gehirns (ebd.). Aufgrund der netzwerkartigen Verknüpfung verschiedener Hirnregionen kann darüber hinaus nicht eindeutig geklärt werden, ob die lädierte Region tatsächlich für die beeinträchtigte Funktion zuständig ist oder ob nicht andere, damit verbundene Hirnbereiche als Ursache in Frage kommen (vgl. Kap. 2.2.1). Klinisch-anatomische Studien sollten vor diesem Hintergrund stets mit Vorsicht interpretiert werden.

\subsubsection{Beeinträchtigung des phonologischen Speichers}

Obwohl der Zusammenhang zwischen Läsionen der linken Hemisphäre und der Unfähigkeit, verbale Stimuli zu wiederholen, bereits aus verschiedenen Arbeiten zur sogenannten "Leitungsaphasie"6 bekannt gewesen war (z. B. Wernicke, 1874; Dubois, Hécaen, Angelergues, Manfras du Chatelier \& Marcie, 1964; Geschwind, 1965), hatten sich bis Ende der 60er Jahre nur wenige Forscher mit dem isolierten Auftreten dieser Wiederholungsschwierigkeiten befaßt (z. B. Luria, Sokolov \& Klimkowski, 1967). Warrington gelang als erste die methodisch einwandfreie Beschreibung des sogenannten "verbalen Kurzzeitgedächtnis-Syndroms" (Warrington \& Shallice, 1969; Shallice, 1988): Im Rahmen einer routinemäßigen Anwendung der Wechsler Intelligenstest-Batterie ${ }^{7}$ waren bei einem 28jährigen Patienten, K. F., der seit mehreren Jahren unter epileptischen Anfällen infolge einer linkshemisphärischen parieto-occipitalen Hirnverletzung litt (Fraktur mit subduralem Hämatom, vgl. Abb. 5 a), größere Leistungsdefizite beim Test "Zahlennachsprechen" verglichen mit den anderen Untertests zur verbalen Intelligenz aufgefallen. Eingehendere Gedächtnisprüfungen schlossen sich an: So wurde neben der Wiederholbarkeit von ein bis vier, akustisch oder visuell dargebotenen Zahlen, Buchstaben oder Wörtern ${ }^{8}$ auch der Effekt der Darbietungsrate der Stimuli untersucht, darüber hinaus wurden die Fähigkeit zur akustischen Wahrnehmung, die Artikulation und verschiedene Aspekte des Langzeitgedächtnisses (LZG) geprüft. Die zuverlässige verbale Gedächtnisspanne von K. F. lag im Falle akustischer Präsentation bei einem, im Falle visueller Präsentation bei zwei Items. (Bei gesunden Probanden ist die akustische Gedächtnisspanne größer als die visuelle; Conrad, 1964.) Da die Beeinträchtigung der akustischverbalen Gedächtnisspanne sich weder auf eine Störung der akustischen Wahrnehmungsfähigkeit noch auf eine Störung der Artikulation zurückführen ließ, deuteten Warrington und Shallice das Ergebnis als Folge einer erheblich reduzierten Kapazität des Kurzzeitspeichers für akustisch-verbales Material bzw. einer Störung des phonologischen Speichers. Die Tatsache, daß das verbale Lernen und das verbale Langzeitgedächtnis sich dabei als intakt herausstellten, war insbesondere für die kognitive Gedächtnispsychologie bedeutsam, da die in verschiedenen damaligen Modellansätzen 
angenommene Abhängigkeit der Langzeitspeicherung von einem korrekt arbeitenden Kurzzeitgedächtnis daraufhin revidiert werden mußte (Shallice, 1988).

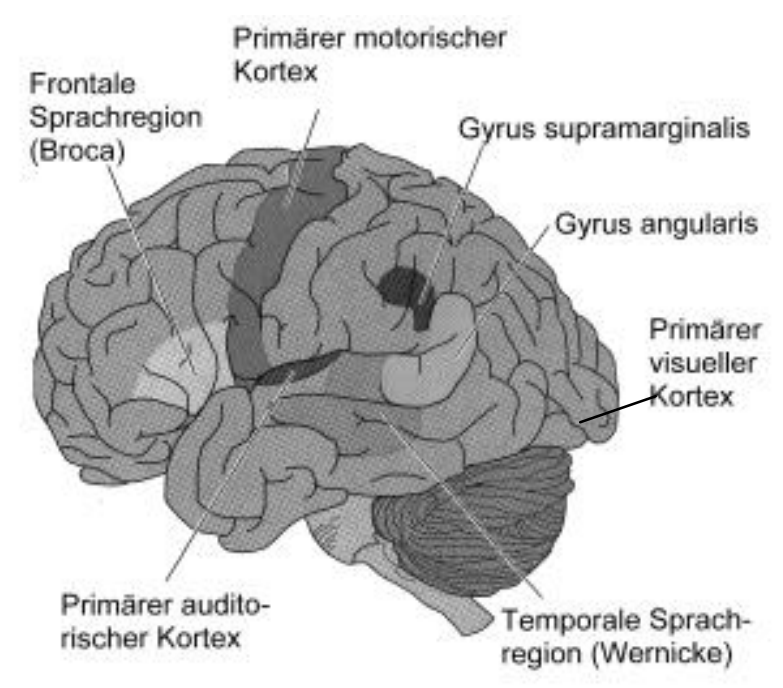

a)

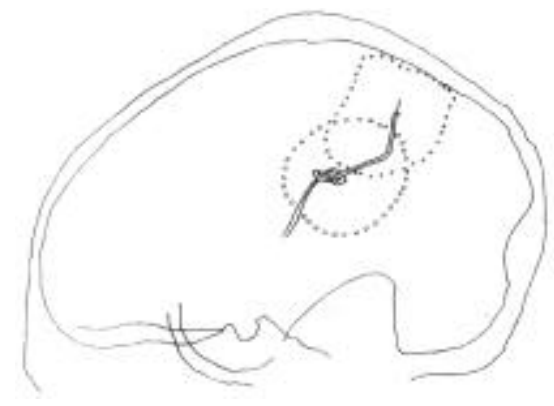

b)

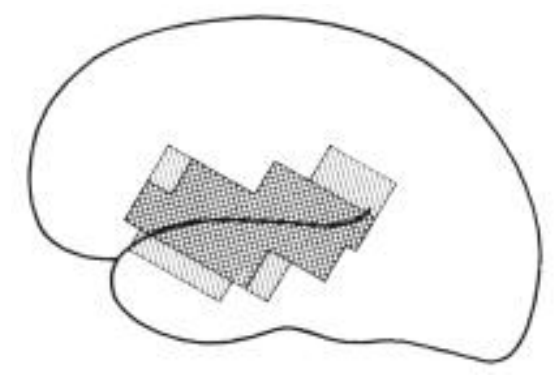

Abbildung 5. Links: Grobanantomie der an Sprache beteiligten Hirnregionen (nach Rosenzweig, Leiman \& Breedlove, 1996). Rechts: a) Hirnläsionsorte der Patienten K. F. und J. B. (aus Warrington, Logue \& Pratt, 1971): XXXX = lädiertes Areal bei K. F.; OOOO = Tumorposition bei J. B.; zusätzlich zu sehen: Verlauf der Angularis-Arterie von K. F. b) CT-Map hypodenser Bereiche bei Patientin P. V. (aus Basso et al., 1982): dunkel schraffiert = tiefe und oberflächliche Läsion; hell schraffiert = nur tiefe Läsion.

Aus K. F.'s vergleichsweise besserer kurzzeitiger Behaltensleistung für visuell dargebotene sprachliche Items schlossen Warrington und Shallice (1969), daß der unmittelbare Abruf visuellen Materials aus einem separaten visuellen Kurzzeitspeicher möglich sein müsse, und zwar ohne eine notwendige Beteiligung des phonologischen Kurzzeitspeichers. Diese Vermutung konnte in späteren Untersuchungen bestätigt werden, in denen phonologische Ähnlichkeits- und Wortlängeneffekte sowie Auswirkungen artikulatorischer Suppression auf den unmittelbaren seriellen Abruf akustisch und visuell dargebotenen sprachlichen Materials bei Patienten mit beeinträchtigter akustisch-verbaler Gedächtnisspanne untersucht wurden (z. B. Vallar \& Baddeley, 1984; Campbell \& Butterworth, 1985; Bisiacchi, Cipolotti \& Denes, 1989; Vallar, Basso \& Bottini, 1990).

An Warrington und Shallices Arbeit schlossen sich weitere Einzelstudien an, in denen der Zusammenhang zwischen linksseitigen parietalen (z. T. zustätzlich noch temporalen und/oder occipitalen) Läsionen und Defiziten der akustisch-verbalen Gedächtnisspanne untersucht wurde. 1971 fanden Warrington, Logue und Pratt bei zwei weiteren Patienten ebenfalls selektive, unterdurchschnittliche Leistungen bei der Zahlengedächtnisspanne. J. B. (weibl., 35 Jahre) war mit 23 Jahren ein Tumor entfernt worden, der etwa zu zwei Dritteln unterhalb und zu einem Drittel oberhalb der Sylvischen Furche gelegen und damit unter anderem den Gyrus supramarginalis und Gyrus ang ularis überlagert hatte (vgl. Abb. 5). Bei W. H. (männl., 39 Jahre) war die genaue Ätio- 
logie seiner neurologischen Störungen unklar; Untersuchungen legten die Vermutung nahe, daß sie die Folge eines Schlaganfalls in der linken temporo-parietalen Region gewesen sein könnten. Beide Patienten zeigten wie K. F. Defizite in allen akustischverbalen Gedächtnisspannenaufgaben; bei visueller Stimulus-Darbietung war die Leistung etwas besser, akustische Wahrnehmung, Artikulation und LZG waren erneut nicht beeinträchtigt. Andere sprachliche Funktionen wie die Benennung abgebildeter Gegenstände, das Lesen und Buchstabieren sowie der sprachliche Ausdruck fielen bei den drei Patienten unterschiedlich aus. Bei J. B. konnten so gut wie keine weiteren sprachlichen Störungen gefunden werden. Warrington und Mitarbeiter (ebd.) kamen daher zu dem Schluß, daß "it seems unlikely then that an expressive speech defect or reading and spelling difficulties are necessary concomitants of a much-reduced verbal span" $(\mathrm{S} .385)^{9}$. Defizite der drei genannten Patienten in den Tests zum Sprachverständnis führten sie auf den beeinträchtigten phonologischen Kurzzeitspeicher zurück.

Die Untersuchung des kurzzeitigen Vergessens anhand der Brown-Peterson-Technik (vgl. Lezak, 1995) mit akustischem und vergleichbarem, visuell präsentierten Material erbrachte bei K. F., J. B. und W. H. eine größere Vergessensrate bei akustischer als bei visueller Darbietung; dieses Ergebnis tritt bei gesunden Probanden in umgekehrter Weise auf (Craik, 1970) und unterstreicht die Modalitätsspezifität des beobachteten KZGDefizits. Zusätzliche Evidenz für diese Modalitätsspezifität erbrachte eine weitere Studie, in der K. F.'s relativ normale Vergessensrate bei visueller Darbietung eingehender untersucht wurde (Warrington und Shallice, 1972). Während gesunde Probanden dazu neigen, ähnlich klingende Buchstaben auch in visuellen Gedächtnisspannen-Aufgaben zu verwechseln, verwechselte K. F. auffallend häufig Buchstaben, die ähnlich aussahen (z. B. O und Q, P und R). Aus dem fehlenden phonologischen Ähnlichkeitseffekt bei visueller Stimulus-Darbietung zogen die Autoren zwei Schlüsse: 1. Bei gesunden Probanden erfolgt auch im Falle visueller Darbietung sprachlichen Materials eine phonologische Rekodierung und eine Speicherung im phonologischen KZG (vgl. Kap. 2.1.1, Abb. 1). 2. Die Kodierung im visuellen KZG richtet sich wahrscheinlich nach der Form der zu merkenden Stimuli. Da K. F. für die Behaltensaufgaben aufgrund des beeinträchtigten phonologischen Speichers lediglich der visuelle Speicher zur Verfügung stand, bezogen sich seine Fehler auf visuelle und nicht auf akustische Charakteristika der Buchstaben.

In vielen weiteren klinischen Studien wurde über ähnliche Patienten mit einer Dissoziation zwischen Kurz- und Langzeitgedächtnis für akustisch-verbales Material und einer besseren Leistung bei visueller Stimulus-Darbietung berichtet (z. B. Kinsbourne, 1972; Strub \& Gardner, 1974; Tzortzis \& Albert, 1974; Saffran \& Marin, 1975; Caramazza, Basili, Koller \& Berndt, 1981). In den meisten Fällen lag die Diagnose einer Leitungsaphasie vor. Einige Symptome dieser Störung (nämlich das paraphrasierende spontane Sprechen und die Benennungs-, Lese- und Schreibstörungen) lassen sich jedoch nicht allein auf ein selektives Defizit des phonologischen Kurzzeitspeichers zt- 
rückführen, sondern implizieren eine zusätzliche Gestörtheit anderer Mechanismen wie z. B. phonologischer Enkodierungsprozesse (Basso, Spinnler, Vallar \& Zanobio, 1982). Angesichts der Diskussion um die theoretischen und neuronalen Grundlagen eines beeinträchtigten phonologischen Speichers sind jedoch besonders diejenigen klinischen Fälle von Interesse, bei denen diese Störung relativ isoliert, $d$. h. unabhängig von weiteren sprachlichen Beeinträchtigungen (und nicht als deren mögliche Folge), auftritt (sog. "primäres KZG-Defizit"; Vallar, Corno \& Basso, 1992). Basso et al. (1982) berichten in diesem Sinne von einem weiteren Fall: P. V., eine damals 28jährigen Patientin, hatte infolge eines Hirnschlags vorübergehend das Bewußtsein verloren. Bei der sprachlichen Untersuchung 14 und 23 Monate später zeigte sie lediglich Schwierigkeiten bei der Wiederholung von Sätzen mit mehr als acht Silben sowie Defizite im sogenannten "Token Test", wenn die Anweisungen mündlich erteilt wurden (DeRenzi \& Faglioni, 1978) ${ }^{10}$. Weitergehende Untersuchungen umfaßten u. a. eine umfangreiche Analyse serieller Positionseffekte für die Wiedergabe von Zahlen, Buchstaben und Wörtern. Während gesunde Probanden einen deutlichen Rezenzeffekt zeigen (die letzten Items einer Liste werden am besten erinnert), fehlte dieser bei P. V. sowohl bei der seriellen als auch bei der freien Wiedergabe akustisch-verbaler Items (Basso et al., 1982), während er bei visueller Präsentation derselben erhalten war (Vallar \& Papagno, 1986). Da Rezenzeffekte typischerweise auf das Vorhandensein eines modalitätsspezifischen Kurzzeitspeichers zurückgeführt werden (Miller, 1956; Baddeley \& Hitch, 1993), konnte dieses Ergebnis als weiterer Beleg für die Existenz des phonologischen Kurzzeitspeichers bzw. für dessen selektive Beeinträchtigung im genannten Fall gewertet werden (s. auch Bisiacchi, Cipolotti \& Denes, 1989). Dafür spricht auch P. V.'s Schwierigkeit, eine neue Sprache zu lernen: Durch die Beeinträchtigung des phonologischen Speichers können neue Wörter nicht lange genug im KZG gehalten werden, um eine Konsolidierung ins LZG zu ermöglichen (Baddeley, Papagno \& Vallar, 1988). Im CT der Patientin fand sich ein hypodenser Bereich, der praktisch die gesamte Sprachregion betraf (vgl. Abb. 5 b). Ungefähre Überschneidungen zu den Läsionsorten von J. B. sind danach für den Bereich der mittleren und oberen Temporalwindung, für den Gyrus supramarginalis und Gyrus angularis anzune hmen.

Die topographischen Daten aus Einzelstudien konnten in Gruppenstudien bestätigt werden. So untersuchten De Renzi und Nichelli (1975) 125 Patienten mit links- oder rechtshemisphärischen Hirnschäden und verglichen sie mit 30 gesunden Kontrollpersonen. Neben einem visuell-räumlichen Gedächtnistest (Corsi's "Block Tapping Test") ${ }^{11}$ und dem Token Test wurden drei verbale Gedächtnistests (Zahlennachsprechen; vorgelesene Zahlen durch Zeigen wiederholen; nach vorgelesenen Wörtern auf die entsprechenden Bilder zeigen) durchgeführt. Linkshemisphärisch geschädigte Patienten zeigten in den verbalen Gedächtnistests signifikant schlechtere Leistungen als rechtshemisphärisch Geschädigte, die sich nicht von den Kontrollpersonen unterschieden, 
dafür aber eine verringerte visuell-räumliche Gedächtnisspanne aufwiesen (vgl. Abschnitt zu Beeinträchtigungen des visuell-räumlichen Speichers). Die verbale Gedächtnisspanne der Patienten mit globaler Aphasie (Schädigung der gesamten perisylvanischen Region) lag unter derjenigen von Patienten mit Wernicke-Aphasie, die ihrerseits schlechtere Leistungen als Patienten mit Broca-Aphasie erbrachten. Die Antwortmodalität (nachsprechen vs. zeigen) hatte keinen Einfluß auf die Leistung; daraus schlossen De Renzi und Nichelli, daß die beobachteten KZG-Defizite der Aphasiker keine Folge der beeinträchtigten Sprache darstellten und bei selektivem Auftreten folglich auch nicht als einfache Manifestation der Aphasie anzusehen seien.

Risse, Rubens und Jordan (1984) verglichen 20 Aphasiker, deren Hirnschäden entweder im unteren Frontallappen und/oder in den Basalganglien ("frontale Gruppe") oder im posterioren temporoparietalen Bereich ("posteriore Gruppe") lokalisiert waren, hinsichtlich ihrer verbalen Lernfähigkeit ${ }^{12}$. Zusätzlich wurde die akustische und visuelle Zifferngedächtnisspanne erfaßt ${ }^{13}$. Während die frontale Gruppe deutliche Beeinträchtigungen beim Lernen und langzeitlichen Behalten der Wörterlisten zeigte, trat bei der posterioren Gruppe der umgekehrte Effekt auf: Trotz einer mittleren Zifferngedächtnisspanne von nur drei Items, waren diese Patienten in der Lage, eine Liste von neun Wörtern zu lernen und auch noch nach einer Stunde korrekt wiederzugeben. Die Autoren werteten dieses Ergebnis als weiteren Beleg für die funktionelle und anatomische Unterscheidbarkeit von Kurz- und Langzeitgedächtnis.
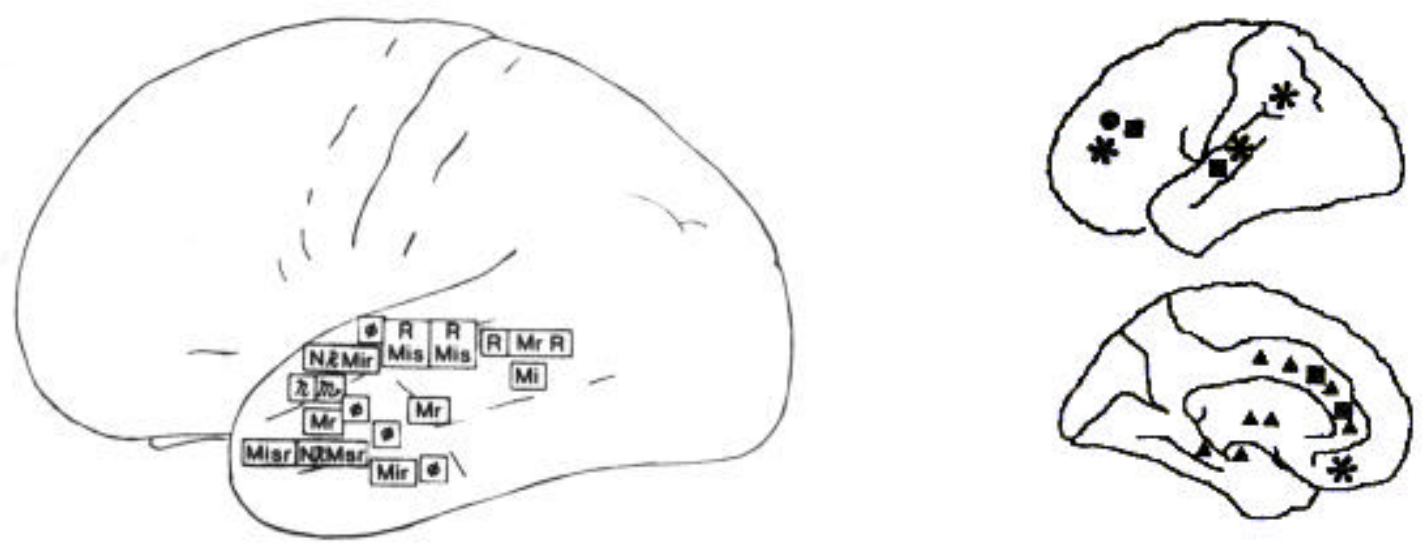

Abbildung 6. Links: Signifikante neuronale Aktivitätszunahmen im vorderen Temporallappen epileptischer Patienten (aus Ojemann et al., 1988). $\mathrm{N}=$ Benennungsaufgabe, $\mathrm{R}=$ Leseaufgabe, $\mathrm{Mi}=\mathrm{KZG}$-Aufgabe (Enkodierungsphase), Ms = KZG-Aufgabe (Memorierphase), Mr = KGZ-Aufgabe (Abrufphase); die kursiven Buchstaben stehen für entsprechende signifikante Aktivitätsabnahmen. Rechts: Ergebnisse einer PET-Untersuchung an Alzheimer Patienten (Perani et al., 1993). Oben ist die laterale, unten die mediale Ansicht der linken Hemispäre zu sehen. $\mathbf{\square}=$ semantisches Gedächtnis, $\boldsymbol{\Delta}=$ episodisches Gedächtnis, - = implizites Gedächtnis, * = verbales Kurzzeitgedächtnis.

Einige neuere klinisch-anatomischen Studien liefern vergleichsweise genauere topographische Informationen zu den neuronalen Grundlagen der phonologischen Schleife. So führten Ojemann, Creutzfeldt, Lettich und Haglund (1988) an dreizehn Patienten, die 
sich infolge von medikamentös nicht mehr zu behandelden epileptischen Anfällen einer Hirnoperation unterziehen mußten (Resektion des linken vorderen Temporallappens unter Lokalanästhesie), extrazelluläre Messungen der neuronalen Aktivitätsänderungen in den betroffenen Hirnregionen während verschiedener visuell dargebotener Arbeitsgedächtnis-Aufgaben durch: Die Patienten sollten u. a. ein einfaches Objekt benennen, Wörter laut oder leise lesen und die Bezeichnung eines Objekts während einer interferierenden Satzergänzungsaufgabe behalten. Die Autoren fanden für jede Aufgabe sgnifikante Änderungen der neuronalen Aktivität in verschiedenen Zellpopulationen (vgl. Abb. 6 links). Daß neuronale Aktivitätsänderungen auch in Hirnregionen gefunden werden konnten, denen normalerweise keine grundlegende Bedeutung für sprachliche und Kurzzeitgedächtnis-Funktionen zugeschrieben wird, unterstreicht nach Meinung der Autoren das Ausmaß der an diesen Funktionen beteiligten Areale des temporalen Kortex. Außerdem geht aus ihrer Untersuchung hervor, daß Zellpopulationen, die für eine bestimmte Funktion (z. B. Kurzzeitgedächtnis) entscheidend sind, durchaus auch an anderen Funktionen beteiligt sein können (z. B. Sprache).
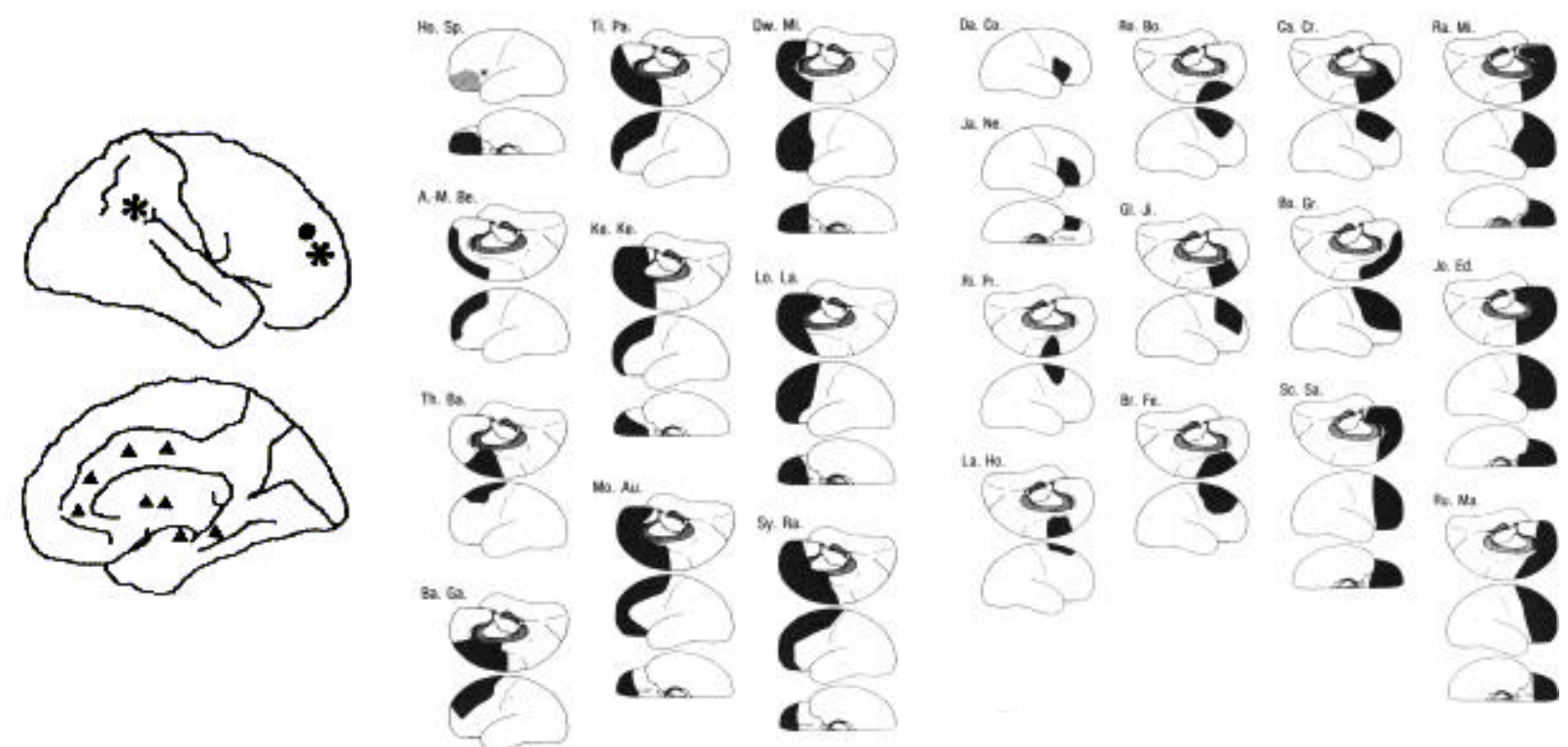

Abbildung 7. Links: Aktivierte Areale während einer PET-Untersuchung an Alzheimer Patienten (Perani et al., 1993). Oben ist die laterale, unten die mediale Ansicht der rechten Hemisphäre zu sehen. $\boldsymbol{\Lambda}=$ episodisches Gedächtnis, $\mathbf{0}=$ implizites Gedächtnis, * = räumliches Kurzzeitgedächtnis. Mitte/rechts: Übersicht über exstirpierte Frontallappenareale bei einzelnen Patienten mit Beeinträchtigung der Mustergedächtnisspanne im Versuch von Pigott und Milner (1994). Neben der lateralen ist pro Patient z. T. auch die mediale (oben) und ventrale (unten) Ansicht zu sehen.

Welche spezifischen Hirnregionen darüber hinaus an verschiedenen KZG-Funktionen beteiligt sind, geht aus einer Studie von Perani und Mitarbeitern (1993) hervor. Sie untersuchten mittels PET regionale zerebrale Durchblutungsänderungen während verschiedener Kurz- und Langzeitgedächtnisaufgaben an 18 Patienten, bei denen erst kürzlich die Diagnose einer möglichen Alzheimer-Erkrankung gestellt worden war. Signifikante zerebrale Durchblutungsänderungen während der akustisch-verbalen Zifferngedächt- 
nisspannenaufgabe taten linkshemisphärisch frontal, temporal und parietal auf (vgl. Abb. 6 rechts); eine entsprechende Aufgabe zur Erfassung der visuell-räumlichen Gedächtnisspanne ("Block Tapping Test") führte zu signifikanten Durchblutungsänderungen im rechten frontalen und rechten parietalen Bereich (s. Abb. 7). Diese Ergebnisse stehen im Einklang mit den zuvor von De Renzi und Nichelli (1975) publizierten Daten (s. o.). Die frontalen Aktivationen weisen darüber hinaus auf eine verstärkte Beteiligung aktiver Kontrollprozesse (zentrale Exekutive) an KZG-Aufgaben bei Alzheimer-Patienten hin. Die Funktionen der zentralen Exekutive gelten als besonders sensitiv für die Auswirkungen der Alzheimerschen Krankheit (Morris, 1994; vgl. auch Abschnitt zu Beeinträchtigungen der zentralen Exekutive).

Insgesamt lassen sich die Erkenntnisse aus klinisch-anatomischen Studien zur selektiven Beeinträchtigung des phonologischen Speichers wie folgt zusammenfassen:

1. Die Patienten weisen linkshemisphärische Schäden, insbesondere im Bereich der mittleren und oberen Temporalwindung, des Gyrus supramarginalis und Gyrus angularis auf (vgl. auch Warrington, James \& Maciejewski, 1986).

2. Sprachverständnis, Langzeitgedächtnis, Artikulation und visuelles Kurzzeitgedächtnis sind nicht gestört.

3. Die selektive Beeinträchtigung des Kurzzeitgedächtnisses für akustisch-verbales Material bei erhaltener verbaler Lernfähigkeit und erhaltenem Langzeitgedächtnis unterstreicht die funktionelle und anatomische Unterscheidbarkeit von phonologischem Kurz- und Langzeitgedächtnis.

4. Die größere Vergessensrate und der fehlende Rezenzeffekt bei akustischer StimulusDarbietung sprechen neben dem fehlenden phonologischen Ähnlichkeitseffekt bei $\dot{\mathrm{u}}$ sueller Darbietung für die Modalitätsspezifität des beobachteten KZG-Defizits.

\subsubsection{Beeinträchtigung des visuell-räumlichen Speichers}

Nachdem Milner 1958 (zit. nach Warrington \& James, 1967) als erste über Patienten berichtet hatte, die nach Schädigungen des rechten Temporallappens Beeinträchtigungen in visuellen Wahrnehmungsaufgaben zeigten, widmeten sich viele Forscher in den folgenden Jahren vermehrt der Untersuchung des visuell-räumlichen Gedächtnisses.

1962 beschrieben Kinsbourne und Warrington vier Patienten mit linkshemisphärischen Läsionen (einschließlich posteriorer Areale), die visuelle, bedeutungsvolle Reize (Buchstaben, Zahlen, geometrische Figuren) nur erkennen konnten, wenn diese einzeln nacheinander dargeboten wurden. Mit zunehmendem zeitlichen Abstand zwischen den Stimuli machten die Patienten weniger Wiedererkennungsfehler. Bei gleichzeitiger Präsentation zweier Reize wurde einer davon jedesmal nicht oder falsch identifiziert. Kontralateraler Neglekt ${ }^{14}$ konnte als Ursache ausgeschlossen werden, so daß die Autoren 
zunächst "Simultanagnosie" (Störung der simultanen Formwahrnehmung) diagnostizierten. Sie räumten jedoch ein, daß es unklar sei, ob die Störung die frühe visuelle Wahrnhemung oder den nachfolgenden Wiedererkennungsprozeß betreffe. In einer neueren Veröffentlichung interpretieren sie die Störung der simultanen Formwahrnehmung als mögliche Auswirkung einer eingeschränkten Kapazität des visuellen Kur zzeitspeichers (McCarthy \& Warrington, 1990).

Warrington und James veröffentlichten 1967 die Ergebnisse einer Gruppenstudie, in der 36 Patienten mit Läsionen der rechten und 29 Patienten mit Läsionen der linken Hemisphäre sowie 10 Kontrollpersonen untersucht worden waren. Neben verschiedenen visuellen Wahrnehmungstests wurde eine visuelle Behaltensaufgabe vorgegeben: Die Patienten hatten entweder 2 oder $10 \mathrm{sec}$ Zeit, um sich einen tachistoskopisch dargebotenen nonverbalen Reiz ${ }^{15}$ zu merken und ihn anschließend aus einer Gruppe von vier Zielreizen (Testreiz plus drei Distraktoren) heraus wiederzuerkennen. Dabei wurde die Ähnlichkeit der Distraktoren mit dem Testreiz manipuliert, so daß leichte und schwierige Aufgabenversionen entstanden. Patienten mit rechtshemisphärischer Läsion des Parietallappens machten mehr Wiedererkennensfehler als Kontrollpersonen und Patienten mit Schädigungen des linken Parietallappens, unabhängig davon, ob sie sich den Testreiz nur 2 oder $10 \mathrm{sec}$ lang gemerkt hatten. Diese beiden Patientengruppen unterschieden sich darüber hinaus signifikant bezüglich ihrer Wiedererkennensleistungen in den schwierigen Durchgängen, in denen die Patienten mit rechtshemisphärischen Parietallappenläsionen schlechter abschnitten. Diese Patienten zeigten außerdem geringere Leistungen bei einer räumlichen Konstruktionsaufgabe ("Mosaiktest" aus der Wechsler-Intelligenztestbatterie; Wechsler, 1987). Für Warrington und James (ebd.) unterstrich dieses Ergebnis die Bedeutung des rechten Parietallappens für die Verarbeitung der räumlichen Wahrnehmungskomponenten visueller Behaltensaufgaben. In ihrer Ergebnisdiskussion stellten sie fest, daß "the right hemisphere is critical for the processing of visual sensory data and the left hemisphere for recording information in terms of linkages with verbal symbols" (S. 265).

In der bereits erwähnten Gruppenstudie fanden De Renzi und Nichelli (1975) ähnliche Zusammenhänge. Sie berichten darüber hinaus von zwei Patienten mit selektiver Beeinträchtigung des visuell-räumlichen Gedächtnisses: Es handelte sich um einen 21jährigen Mann mit Epilepsie, der sich einer rechtsseitigen occipitalen Lobektomie und einer Koagulation der rechten Amygdala und des rechten Fornix unterzogen hatte, sowie um einen 73jährigen Mann, der erst kurz zuvor einen Schlaganfall mit Schädigung der rechten Hemisphäre erlitten hatte. Beide wiesen eine Halbseitenblindheit (links) auf, zeigten jedoch keinerlei Aufmerksamkeits- oder räumliche Wahrnehmungsstörungen. Sie waren nicht desorientiert und hatten auch keine Schwierigkeit, den Weg durch ein Labyrinth in einer angemessenen Anzahl von Durchgängen zu lernen und zu behalten. Während ihre akustische Zifferngedächtnisspanne bei sieben Items lag, umfaßte die vi- 
suell-räumliche Gedächtnisspanne ("Block Tapping Test", s. o.) jedoch nur durchschnittlich 2.5 Items. De Renzi und Nichelli folgerten, daß

... the two right brain-damaged patients who scored so low on the spatial pointing test without showing evidence of space perceptual or of general memory derangement suggest that there may exist an elective impairment of spatial span in all respects similar to that described for verbal span. (ebd., S. 353)

Die selektive Beeinträchtigung des visuell-räumlichen Speichers führten sie auf Schäden im rechten posterioren Neokortex zurück.

1977 untersuchten De Renzi, Faglioni und Previdi 40 Patienten mit rechtshemisphärischen und 40 Patienten mit linkshemisphärischen Schäden mit und ohne Beeinträchtigung des Gesichtsfeldes ${ }^{16}$ sowie 40 Kontrollpersonen, um den Zusammenhang zwischen Läsionsort und räumlichen Gedächtnisleistungen zu analysieren. Anhand verschiedener Versionen des "Block Tapping Tests" wurde die visuell-räumliche Gedächtnisspanne, die verzögerte Behaltensleistung nach einem 8- bzw. 16-sec-Intervall mit und ohne Zählaufgabe und die räumliche Lernfähigkeit ${ }^{17}$ erfaßt. Die varianzanalytische Auswertung verdeutlichte einen signifikanten Einfluß des Vorhandenseins von Gesichtsfeldstörungen auf die visuell-räumliche Gedächtnisspanne, und zwar unabhängig von der Läsionsseite. Die Daten zur verzögerten Behaltensleistung und zur räumlichen Lernfähigkeit unterstrichen jedoch die Bedeutung rechter posteriorer Areale für das Lernen und Behalten visuell-räumlicher Information: Die Leistungen der Patienten mit rechtshemisphärischen Schäden einschließlich Beeinträchtigung des Gesichtsfeldes lagen nicht nur unter denen der Kontrollpersonen, sondern auch unter denen aller übrigen hirngeschädigten Patienten. Darüber hinaus fanden sich aber auch Hinweise auf eine Beteiligung linksseitiger posteriorer Areale: Von den 20 Patienten mit linksseitigen Schäden und Gesichtsfeldstörungen konnten sechs das Lernkriterium nicht erreichen, alle anderen waren nach fünf Minuten nicht mehr in der Lage, die gelernte Block-Sequenz fehlerfrei wiederzugeben, "... as if the traces in long-term storage were weak or rehearsable with difficulty" (ebd., S. 431).

Viele Studien legen einen Zusammenhang zwischen visuell-räumlicher Kurzzeitspeicherung und visuell-räumlichem Vorstellungsvermögen nahe (z. B. Brooks, 1968; Baddeley, Grand, Wight \& Thomson, 1975; Baddeley \& Lieberman, 1980; Johnson, 1982; Mathews, 1983; Logie, 1986). Ergebnisse aus Untersuchungen zu den an mentalen Vorstellungsbildern beteiligten kognitiven Mechanismen unterstreichen die Bedeutung posteriorer Areale für das Erkennen und Generieren bildhaften Materials. Eine Arbeitsgruppe um Farah (Levine, Warach \& Farah, 1985; Farah, 1988; Farah, Hammond, Levine \& Calvanio, 1988; Farah, 1989) weist in diesem Zusammenhang auf die Notwendigkeit einer Unterscheidung zwischen visuellen und räumlichen Komponenten mentaler Vorstellungen hin. So berichteten Levine, Warach und Farah (1985) über zwei 
Patienten mit posterioren Hirnschäden und verschiedenen, selektiven neuropsychologischen Ausfallserscheinungen: Während der eine Patient unter Prosopagnosie (Störung des Gesichterkennens) und Achromatopsie (totale Farbenblindheit) ohne räumliche Desorientierung litt, fand sich bei dem anderen das umgekehrte Bild. Nach CT-Auswertungen und Reanalysen bereits publizierter Fälle kamen Levine und Mitarbeiter (ebd.) zu dem Schluß, daß es sich beim Verlust des visuell-räumlichen Vorstellungsvermögens um eine Folge parieto-occipitaler Hirnschäden handelt, während temporo-occipitale Schäden zu gestörtem Gesichterkennen und Verlust der Farberkennung führen. Entscheidend sei somit nicht die Lateralisierung der Läsionen, sondern deren genaue Lokalisation in rechten und/oder linken posterioren Hirnarealen. Newcombe, Ratcliff und Damasio (1987) sowie Farah et al. (1988) lieferten weitere Evidenzen für die Unterscheidung visueller und räumlicher Komponenten von mentalen Vorstellungen.

Die umfassendste Einzelstudie über einen Fall mit selektiver Beeinträchtigung des visuell-räumlichen Speichers stammt von Hanley, Pearson und Young (1990, 1991). Die untersuchte 54jährige Patientin E. L. D. hatte sich einer Operation zur Entfernung eines Aneurysmas unterziehen müssen, das rechtshemisphärisch zu einem subarachnoiden Hämatom im vorderen Bereich der sylvischen Fissur und damit zu einem Hirnschlag geführt hatte. Die Patientin klagte in der Folge über Gedächtnisprobleme, insbesondere über die Schwierigkeit, sich neue Gesichter zu merken (Prosopagnosie) und ihren Weg nach Hause zu finden. In einer umfangreichen Untersuchung prüften Hanley, Pearson und Young (1990) daher zunächst E.L.D.'s Fähigkeit, Gesichter, Gegenstände und Wörter zu erinnern. Sie fanden eine beeindruckende Dissoziation zwischen der Fähigkeit, früher (d. h. vor der Erkrankung) bekannte visuelle Formen einerseits und Abbildungen unbekannter visueller Formen andererseits wiederzuerkennen: Während E. L. D. ihr bekannte Gegenstände und Gesichter ebenso wie verbales Material (visuell bzw. akustisch präsentierte Wörter und aussprechbare Nichtwörter) gut erinnern konnte, fiel ihr das Behalten neuer visueller Stimuli (Gegenstände, Gesichter) außerordentlich schwer. Sie zeigte jedoch keinerlei nennenswerte visuelle Wahrnehmungsstörungen, auch war die Wiedererkennungsleistung nicht generell für alle unbekannten visuellen Reize beeinträchtigt (E. L.D. hatte keine Schwierigkeiten, aussprechbare Nichtwörter zu erinnern). In einem Test zur Wiedererkennung unbekannter Stimmen schnitt die Patientin jedoch ebenfalls schlecht ab. Hanley et al. (ebd.) interpretierten die Daten als Folge einer selektiven Beeinträchtigung des Kurzzeitspeichers für nonverbales visuelles Material; sie schlossen aber aufgrund der letztgenannten Ergebnisse nicht aus, daß es sich auch um eine Störung eines fundamentaleren Speichersystems handeln könnte, das generell und unabhängig von der Darbietungsmodalität für nonverbale Informationen zuständig sei.

In einer nachfolgenden Studie untersuchten Hanley, Young und Pearson (1991) noch eingehender E.L.D.'s Kurzzeitgedächtnisleistungen sowie ihr räumliches Vor- 
stellungsvermögen. E. L.D. erbrachte in der Matrixaufgabe von Brooks ${ }^{18}$, in Corsis "Block Tapping Test" und in mentalen Rotationsaufgaben ${ }^{19}$ unterdurchschnittliche Leistungen, darüber hinaus fiel ihr die Anwendung mentaler Vorstellungsbilder als Mnemotechnik schwer. In einem Test, in dem sie sich die Darbietungsreihenfolge von vier unbekannten Gesichtern merken sollte, machte E. L.D. mehr Fehler als sieben gesunde Kontrollpersonen, außerdem fehlte bei ihr der visuelle Rezenzeffekt. Somit lag bei dieser rechtshemisphärisch geschädigten Patientin eine Störung sowohl der visuellen als auch der visuell-räumlichen Gedächtnisspanne vor. Beim unmittelbaren seriellen Abruf von gelernten Buchstaben (visuelle und akustische Darbietung) schnitt sie jedoch erneut gut ab und zeigte sowohl phonologische Ähnlichkeitseffekte als auch Effekte artikulatorischer Suppression. Der Abruf visuell-räum lichen Materials aus dem LZG bereitete E. L. D. wie bereits erwähnt keine Schwierigkeiten, solange es sich dabei um hformationen handelte, die ihr schon vor der Erkrankung bekannt gewesen waren. Hanley und Mitarbeiter (ebd.) interpretierten die Ergebnisse als Dissoziation zwischen der phonologischen Schleife und dem visuell-räumlichen Sketchpad und zwischen dem Kurz- und dem Langzeitspeicher für visuell-räumliches Material.

An der Speicherung visuell-räumlicher Information scheinen damit im wesentlichen rechtshemisphärisch posteriore Areale beteiligt zu sein. Darauf weisen auch die Ergebnisse der bereits erwähnten PET-Untersuchung von Perani und Mitarbeitern (1993) an Alzheimer-Patienten hin (vgl. Kap. 3.1.1, Abb. 7). Diese Autoren fanden jedoch neben einer rechtsseitig posterioren Aktivation (Übergang vom Parietal- zum Occipitallappen, etwa Area 39 nach Brodmann, s. Kap. 2.2.1, Abb. 4) während des "Block Tapping Tests" auch einen aktivierten rechtsfrontalen Bereich. Aus zahlreichen Untersuchungen geht hervor, daß Frontallappenregionen einbezogen werden, wenn aktive Kontrollprozesse für die erfolgreiche Bewältigung von Gedächtnisspannenaufgaben erforderlich sind (Alivasatos \& Milner, 1989; Wiegersma, Van der Scheer \& Human, 1990; Owen, Evans \& Petrides, 1996). Pigott und Milner (1994) interessierten sich in diesem Zusammenhang für die Veränderungen der visuellen KZG-Kapazität nach rechts- oder linksseitiger Resektion des Frontal- oder vorderen Temporallappens. Sie erfaßten diesbezüglich die Ziffern- und sogenannte "Muster-Gedächtnisspanne"20 von insgesamt 106 Patienten und 21 gesunden Kontrollpersonen und fanden sowohl bei unmittelbarem (2 sec) als auch bei verzögertem Abruf (10 sec) mit und ohne Interferenzaufgabe signifikante Zusammenhänge zwischen rechtshemisphärischen Frontallappen-Schädigungen (vgl. Kap. 3.1.1, Abb.7) und Leistungseinbußen bei der visuell-räumlichen Gedächtnisaufgabe. Aufmerksamkeitsstörungen und Beeinträchtigungen willentlicher Augenbewegungen bei der Wahrnehmung der komplexeren Muster konnten als Ursache ausgeschlossen werden. Die Autoren führten daher die reduzierte visuelle KZG-Kapazität auf eine Störung der aktiven Kontrollprozesse bei der Verarbeitung der komplexeren Muster zurück. 
Die Erkenntnisse aus klinisch-anatomischen Studien zur selektiven Beeinträchtigung des visuell-räumlichen Speichers lassen sich wie folgt zusammenfassen:

1. Bei Störungen des visuell-räumlichen Systems (Baddeleys "visuospatial sketchpad") weisen die Patienten rechtshemisphärische Läsionen der unteren posterioren parietalen und frontalen Assoziationsareale auf. Während dabei die parietalen Bereiche vermutlich eine größere Rolle für die Speicherung von lokalisatorischer Information spielen, wird den frontalen Arealen eine Beteiligung an zentralen Kontrollprozessen zugesprochen.

2. Patienten mit linkshemisphärischen Schäden im Bereich des Parietallappens und seines Übergangs zum Occipitallappen zeigen Störungen des visuellen Systems bzw. der Fähigkeit, verbale und nonverbale Muster zu speichern.

3. Die selektive Beeinträchtigung des Kurzzeitspeichers für visuell-räumliches Material bei erhaltenem Langzeitgedächtnis spricht für die funktionelle und anatomische Unterscheidbarkeit zwischen visuell-räumlichem Kurz- und Langzeitgedächtnis.

4. Die höhere Fehleranzahl bei der Wiedererkennung visueller Stimuli und bei der Wiedergabe räumlicher Informationen verglichen mit der intakten Behaltensleistung für verbale Reize unterstreicht neben den fehlenden Rezenzeffekten für visuell dargebotenes nonverbales Material die Modalitätsspezifität dieses KZG-Defizits.

\subsubsection{Beeinträchtigung der zentralen Exekutive}

Die Aufgaben, die in Baddeleys Arbeitsgedächtnismodell der zentralen Exekutive zugeschrieben werden, sind sehr vielfältig; sie betreffen die Kontrolle und Koordination des phonologischen und des visuell-räumlichen Speichers sowie des Informationsflusses zwischen den entsprechenden Kurz- und Langzeitspeichern, das Einschätzen und Abwägen von Informationen, die Problemlösung und Entscheidungsbildung sowie die ständige Supervision bei der Durchführung dieser Aufgaben. Die sich daraus ergebende Komplexität dieser Modellkomponente stellt das größte Problem für die Untersuchung ihrer neuroanatomischen Grundlagen dar und macht die Einbeziehung verschiedener Aufgabenstellungen notwendig, die ihre Kontroll-, Verarbeitungs- und Speicherfunktionen betreffen. Im Rahmen dieser Arbeit kann jedoch nur ein kurzer Überblick über die vielen verschiedenen Arbeitsgedächtnisaufgaben vermittelt werden, die in kinisch-anatomischen Studien zur Untersuchung zentralexekutiver Funktionen eingesetzt werden.

Baddeley setzt die zentrale Exekutive in ihrer Funktionsweise dem von Norman \& Shallice (1986) eingeführten "Supervisory Activating System" (SAS) gleich (vgl. Kap. 2.1.1). Für Shallice (1982) selber bietet das SAS-Modell eine angemessene Erklärungsgundlage für die vielfältigen kognitiven Defizite, die bei frontallappengeschädigten Patienten beschrieben werden (sog. "Frontallappen-Syndrom" ${ }^{21}$ ). Funktionen der zentralen Exe- 
kutive werden daher heute meist mit Vorgängen in den Frontallappen in Verbindung gebracht, wenngleich Baddeley (1998) es vorzieht, die Funktions - von der Lokalisationsfrage zu trennen und in diesem Zusammenhang von einem "dysexekutiven Syndrom" zu sprechen.

Eine Aufgabe, deren erfolgreiche Bewältigung ganz besonders vom intakten Funktionieren des frontalen Kortex abhängen soll, ist die Kartensortieraufgabe (z. B. "Wisconsin Card Sorting Test"). Sie erfordert die Fähigkeit, verschiedene Sortierkategorien zu behalten und bei jeder Karte neu über die Kategorienzugehörigkeit zu entscheiden ${ }^{22}$. Nachdem bereits verschiedentlich über Zusammenhänge zwischen Frontal- oder Parietallappenschädigungen und Leistungsbeeinträchtigungen bei Sortieraufgaben berichtet worden war (Weigl, 1927; Halstead, 1940; Goldstein, 1944; Teuber, Battersby \& Bender, 1951; McFie \& Piercy, 1952), widmete sich Milner 1963 der genaueren Analyse dieses kognitiven Defizits. Sie untersuchte 41 Patienten mit Läsionen des dorsolateralen frontalen Kortex und verglich sie mit 53 Kontrollpersonen, die links- oder rechtshemisphärische Schäden im Bereich des Temporal- oder Parietallappens aufwiesen. Im Mittelpunkt standen potentielle Leistungsbeeinträchtigugen beim "Wisconsin Card Sorting Test". Milner (ebd.) fand, daß Patienten sowohl mit rechts- als auch mit linkshemisphärischen dorsolateralen Frontallappenschädigungen signifikant mehr Fehler (insbesondere perseverativer Art) machten; sie erreichten zudem weniger Kategorien als $\mathrm{Pa}$ tienten mit Läsionen in anderen Hirnregionen (einschließlich orbitofrontal-temporaler Areale). Diese Ergebnisse wurden als Beleg für ein spezifisches intellektuelles Defizit nach Schädigung des dorsolateralen Frontallappens gewertet: In der Unfähigkeit, das Sortierprinzip zu wechseln bzw. die Angemessenheit der eigenen Antwort einzuschätzen und daraus entsprechende Konsequenzen zu ziehen, spiegelt sich eine Beeinträchtigung der Aktivitätenkoordination wieder, eine der wichtigsten Funktionen der zentralen Exekutive (Della Sala \& Logie, 1993).

Eine Störung zentraler Koordinationsfunktionen soll auch die defizitären Leistungen verursachen, die Alzheimer-Patienten bei der Bewältigung konkurrierender Aufgaben zeigen. In verschiedenen Studien befaßten sich z. B. Baddeley und Mitarbeiter (1986, 1991; s. auch Morris \& Baddeley, 1988, und Spinnler, Della Sala, Bandera \& Baddeley, 1988) mit den Auswirkungen der Alzheimerschen Krankheit auf das Arbeitsgedächtnis. Sie fanden neben dem klassischen amnestischen Syndrom eine leicht reduzierte verbale und nonverbale Gedächtnisspanne bei erhaltenem auditorischen Pezenzeffekt. Im Doppelaufgabenparadigma ${ }^{23}$ führten besonders Reaktionszeit- und Memorieraufgaben zu starken Leistungsbeeinträchtigungen; diese nahmen in einem langzeitlichen Vergleich (über ein Jahr hinweg) zudem signifikant kontinuierlich zu, während die Leistung in den Einzelaufgaben sich nur geringfügig verschlechterte. Die Ergebnisse konnten nicht auf die größere Schwierigkeit bei der Bewältigung konkurrierender Aufgaben (verglichen mit den Einzelaufgaben) zurückgeführt werden. Damit folgerte Badde- 
ley (1993), daß Alzheimer-Patienten "... show a particularly marked deficit in the operation of the central-executive component of working memory ..." (S. 165). Ähnliche Ergebnisse wurden auch bei Parkinson-Patienten gefunden (Dalrymple-Alford, Kalders, Jones \& Watson, 1994).

Mit verschiedenen anderen Arbeitsgedächtnisaufgaben, deren erfolgreiche Bewältigung ebenfalls verstärkt zentrale Kontrollfunktionen voraussetzt, haben sich Petrides und Mitarbeiter in den achtziger und neunziger Jahren befaßt. 1982 ließen Petrides und Milner zunächst 79 Patienten mit unilateralen Frontal- oder Temporallappenexzisionen und 18 gesunde Kontrollpersonen Reihen von nonverbalen Reizen (abstrakte und konkrete Zeichnungen) sowie von verbalen Reizen (Wörter mit hoher versus niedriger Bildhaftigkeit) bilden (sog. "Self-Ordered Tasks") ${ }^{24}$. Wie erwartet machten Patienten mit Frontallappenläsionen bei allen Reizarten mehr Fehler als die übrigen Patienten bzw. als die Kontrollpersonen (einzige Ausnahme: Patienten mit Schädigung des hippokampalen Systems). Entgegen der Hypothese erbrachten linkshemisphärisch-frontal geschädigte Patienten jedoch nicht nur bei den verbalen, sondern auch bei den nonverbalen Reizarten stark unterdurchschnittliche Leistungen, während Patienten mit rechtshemisphärisch-frontalen Läsionen nur bei den längsten nonverbalen Tests Defizite zeigten. Petrides und Milner (ebd.) kontrastierten diese Ergebnisse mit denen zweier früherer Studien, in denen frontallappengeschädigte Patienten die durch den Versuchsleiter vorgegebene Reihenfolge verschiedener Reize wiederzugeben hatten (sog. "Recency Tests"; Milner, 1971; Milner, 1982). In diesen Studien schnitten Patienten mit linkshemisphärischen Läsionen lediglich bei verbalen Reizen schlecht ab, während rechtshemisphärisch beeinträchtigte Patienten starke Leistungseinbußen bei allen nonverbalen Reizen aufwiesen. Die Autoren sahen darin nicht nur einen bedeutenden materialspezifischen Effekt, sondern vermuteten darüber hinaus, daß

... it may be the case that an active working memory is more critically dependent on the left frontal cortex than the right, perhaps because some degree of verbal mediation may be necessary to this process. By verbal mediation we do not wish to imply that the nonverbal stimuli must be coded verbally, but rather that the strategy to be employed or being employed may be aided by some degree of verbal formulation. (Petrides \& Milner, 1982, S. 260)

In zwei weiteren Untersuchungen ging Petrides (1985, 1990) der Frage nach, inwiefern frontal- oder temporallappengeschädigte Patienten Beeinträchtigungen bei konditionierten Assoziationsaufgaben zeigten. Die Patienten hatten dabei verschiedene Assoziationen zu lernen, und zwar zwischen räumlichen Reizen (gleichfarbige Lämpchen) und räumlichen Antworten (auf eine von sechs weißen Karten zeigen) sowie zwischen visuellen Reizen (verschiedenfarbige Hütchen) und Handbewegungen bzw. verschiedenen abstrakten Zeichnungen. Die Ergebnisse bestätigten die kritische Rolle, die der frontale Kortex für die Bewältigung konditionierter Assoziationsaufgaben spielt: Frontallappen- 
geschädigte Patienten machten mehr Fehler als temporallappengeschädigte Patienten mit intaktem hippokampalen System. Die Leistungsbeeinträchtigungen waren nicht auf gestörte Diskriminationsfähigkeit oder auf Probleme mit der Antwortrealisierung zurückzuführen. Vielmehr fiel es den Patienten schwer, aus einem Satz vorgegebener Antwortmöglichkeiten die zu einem bestimmten Stimulus passende Antwort auszuwählen. In den Studien zeigte sich zudem erneut ein Zusammenhang zwischen Lateralisierung der Läsion und Aufgabenart (rechtsfrontal geschädigte Patienten zeigten etwas größere Defizite in räumlichen, linksfrontal geschädigte Patienten in nichträumlichen Aufgaben). Patienten mit Läsionen des hippokampalen Systems wiesen im wesentlichen dasselbe Ergebnismuster auf, was die Beteiligung eines ausgedehnten neuronalen Netzwerkes an der Bewältigung der beschriebenen Assoziationsaufgaben nahelegt.

Mehrere Untersuchungen heben die Bedeutung der Frontallappen für das Behalten der zeitlichen Abfolge kürzlich stattgefundener Ereignisse hervor (Lavadas, Umilta \& Provinciali, 1979; Milner, 1971; Prisko, 1963, zit. in Milner, 1964; Shimamura, Janowsky \& Squire, 1988; Yntema \& Trask, 1963). In einer neueren Studie fanden Milner, McAndrews und Leonard (1990) in einer Gruppe von 117 frontallappengeschädigten Patienten neben dem bereits beschriebenen materialspezifischen Lateralitätseffekt einen Zusammenhang zwischen links- bzw. rechtsseitig frontaler Schädigung und Defiziten in der Beurteilung der zeitlichen Abfolge verschiedener verbaler und nonverbaler Reize ${ }^{25}$. Dabei stellte sich das Vorhandensein einer Läsion in einem bestimmten frontalen Areal (v. a. linksseitig) als besonders relevant für das beobachtete Defizit heraus (vgl. Abb. 8).

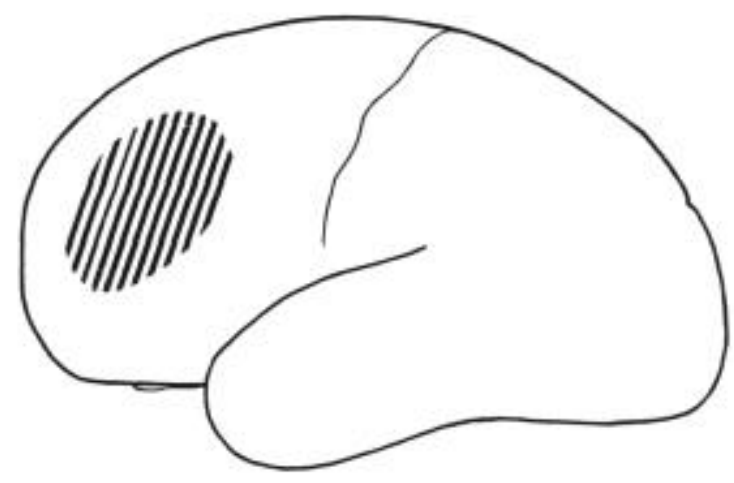

Abbildung 8. Schematische Darstellung desjenigen Areals im linken frontalen Kortex, das für die Beurteilung zeitlicher Abfolgen eine wesentliche Rolle spielen soll (aus Milner, McAndrews \& Leonard, 1990).

Verschiedene Autoren schreiben den Frontallappen eine wichtige Rolle bei der Planung und Ausführung von Antwortstrategien zu (Brody \& Pribram, 1978; Luria, 1969; Pribram, 1973, 1987; Shallice, 1982; 1988). Karnath, Wallesch und Zimmermann (1991) konnten diesen Zusammenhang in einem eleganten Experiment bestätigen. Sie ließen 32 Patienten mit akuten oder chronischen Läsionen des Frontallappens, 16 Patienten mit temporalen oder parietalen Schäden sowie 16 gesunde Kontrollpersonen am Bildschirm 
sieben teilmaskierte Labyrinthe lernen ${ }^{26}$ und erfaßten dabei verschiedene Verhaltensdaten wie die Gesamtreaktionszeit, die Entscheidungszeit an verschiedenen Kreuzungspunkten der Labyrinthe, Art und Anzahl der Fehler usw. Patienten mit akuten und chronischen frontomedialen Läsionen hatten die größten Schwierigkeiten, mentale Pläne (kognitive Landkarten) der Labyrinthe zu erzeugen. Signifikante Unterschiede zeigten sich lediglich im zweiten Durchgang: Akut frontomedial geschädigte Patienten machten mehr Fehler als die übrigen (einschließlich frontolateral geschädigter) Patienten und die Kontrollpersonen und benötigten daher mehr Durchgänge bis zum Erreichen des Aufgabenkriteriums; chronisch frontomedial geschädigte Patienten trafen hingegen häufiger als die übrigen Patienten regelwidrige Entscheidungen. Karnath und Mitarbeiter (ebd.) führten die beobachteten Schwierigkeiten der frontallappengeschädigten Patienten auf Beeinträchtigungen antizipatorischer Prozesse zurück: Zwar sei der Umgang dieser Patienten mit neuen, unbekannten Situationen nicht generell gestört, die beeinträchtigte Erwartungsbildung führe jedoch zu ungewohnten intuitiven Verhaltensweisen und Entscheidungen.

Frontallappengeschädigte Patienten scheinen somit besonders in denjenigen Aufgaben schlecht abzuschneiden, die eine aktive Informationsmanipulation im Gedächtnis oder die Bildung und den Abruf von Assoziationen zwischen räumlichen und visuellen Reizen erfordern. Dies geht auch aus einer neueren Untersuchung von Owen und Mitarbeiter (1995) hervor. Sie verglichen die Leistung von 20 temporal- und 16 frontallappengeschädigten Patienten sowie von 11 Patienten mit Läsionen des amygdalo-hippokampalen Systems mit denen zweier gesunder Kontrollgruppen (44 bzw. 37 Probanden) u. a. hinsichtlich verschiedener Wiedererkennungs- und Paarassoziationsaufgaben sowie ihres räumlichen Arbeitsgedächtnisses. Während Patienten mit Frontallappenläsionen Schwierigkeiten bei räumlichen, nicht jedoch bei Muster-Wiedererkennungsaufgaben zeigten, fand sich bei den übrigen Patienten das umgekehrte Bild. Darüber hinaus korrelierten die Paarassoziationsleistungen der frontallappengeschädigten Patienten stärker mit den entsprechenden Ergebnissen aus Tests zum räumlichen Wiedererkennen, während sie bei den hippokampal/temporallappengeschädigten Patienten stärker mit deren Testergebnissen aus der Musterwiedererkennungsaufgabe zusammenhingen. In einer räumlichen Arbeitsgedächtnisaufgabe ${ }^{27}$ zeigten alle Patienten defizitäre Leistungen, die jedoch unterschiedlichen Ursachen zuzuschreiben waren: Während bei den Frontallappenpatienten die Anwendung einer ineffizienten Suchstrategie zu Fehlern führte, wandten die übrigen Patienten normale, systematische Suchstrategien an, so daß die Autoren deren Defizite in schwierigeren Durchgängen auf eine fundamentale Beeinträchtigung des Speicherprozesses zurückführten. Owen et al. (ebd.) werteten dies als weiteren Beleg dafür, daß dem räumlichen Arbeitsgedächtnis ein Netzwerk funktionell verknüpfter kortikaler und subkortikaler Areale zugrundeliegt, das den prä- 
frontalen Kortex und den Hippokampus einschließt (vgl. auch die Untersuchungen von Petrides, 1985, 1990).

Die Erkenntnisse aus klinisch-anatomischen Studien zu Beeinträchtigungen der zentralen Exekutive lassen sich wie folgt zusammenfassen:

1. Leistungsdefizite in verschiedenen kognitiven Arbeitsgedächtnisaufgaben, die im wesentlichen zentrale Koordinations- und Kontrollprozesse erfordern, liefern Evidenzen für das Vorliegen einer selektiven Beeinträchtigung der zentralen Exekutive.

2. Patienten mit Störungen der exekutiven Kontrolle des Gedächtnisses weisen Schädigungen des rechten und/oder linken frontalen Kortex auf.

3. Differentielle Testergebnisse z.B. von rechts- versus linksfrontal oder frontolateral versus frontomedial geschädigten Patienten deuten auf eine funktionelle Spezialisierung verschiedener Frontallappenareale hin (vgl. auch Milner, 1982).

\subsection{Untersuchungen an gesunden Personen}

Obwohl der Einsatz bildgebender Verfahren erst seit den 70er Jahren systematische Analysen des normal funktionierenden Gehirns ermöglicht (Raichle, 1997), gibt es inzwischen auf diesem Gebiet eine Fülle von Untersuchungen, die sich mit verschiedenen neuropsychologischen Fragestellungen befassen. Im Mittelpunkt des Forschungsinteresses stehen dabei topographische Aspekte der og. Arbeitsgedächtniskomponenten sowie die zeitlichen Eigenschaften der postulierten Verarbeitungsprozesse. Anhand einer Auswahl repräsentativer Befunde zu diesen Schwerpunkten soll nachfolgend ein Überblick über den derzeitigen Forschungsstand gegeben werden.

\subsubsection{Topographische Aspekte}

In den meisten Studien zur Klärung der Frage, welche Hirnregionen an verbalen bzw. nonverbalen Kurzzeitgedächtnisaufgaben beteiligt sind, wurde die Positronen-Emissions-Tomographie (vgl. Kap. 2.2.2) als bildgebendes Verfahren eingesetzt. Die Logik des experimentellen Designs von PET-Studien gründet auf der sog. "Subtraktionsmethode": Dabei wird die Differenz der mittleren Durchblutungsänderungen interpretiert, die während zweier verschiedener Aufgaben (experimentelle und Kontrollaufgabe) gemessenen wurden (s. Buckner \& Tulving, 1995). Um eindeutige Ergebnisse zu erhalten, müssen die Aufgaben dabei so gewählt werden, daß sie sich in nur einer einzigen Prozeßkomponente voneinander unterscheiden.

Paulesu, Frith und Frackowiak führten 1993 eine PET-Studie an sechs Probanden durch, um die neuronalen Korrelate des verbalen Arbeitsgedächtnisses aufzudecken. Dazu verwendeten sie eine Variante der klassischen Sternberg-Aufgabe (vgl. 
Kap. 2.1.2): In der ersten experimentellen Aufgabe ließen sie die Probanden zunächst sechs lateinische Buchstaben still memorieren (phonologische Memorieraufgabe); im Anschluß wurde ein Testreiz dargeboten, und die Probanden hatten darüber zu entscheiden, ob dieser Testreiz im gelernten Set vorhanden gewesen war oder nicht. In der entsprechenden Kontrollaufgabe wurden den Probanden statt lateinischen koreanische Buchstaben präsentiert, um eine phonologische Verarbeitung auszuschließen (nonverbale Memorieraufgabe - die Probanden waren des Koreanischen nicht mächtig). In der zweiten experimentellen Aufgabe sollten die Probanden lediglich beurteilen, ob sich zwei dargebotene Konsonanten reimten; ein Memorieren war somit nicht erforderlich. In der dazugehörigen Kontrollaufgabe hatten die Probanden jeweils zwei koreanische Buchstaben auf ihre Ähnlichkeit hin zu beurteilen. Die Autoren fanden während der phonologischen Memorieraufgabe signifikante Zunahmen regionaler Durchblutung beidseitig in den Brodmann-Arealen 22/42, 40 und 44 (vgl. Kap. 2.2.1, Abb. 4) sowie im Bereich der Insulae und schlugen vor, dieses Areal-Ensemble als anatomische Grundlage der phonologischen Schleife zu betrachten. Da sowohl bei der phonologischen Memorieraufgabe als auch bei der Reimaufgabe besonders linksseitig signifikante Aktivitätszunahmen in Areal 44 (Broca) zu verzeichnen waren, nahmen Paulesu et al. (ebd.) an, daß dieses Areal eine wesentliche Rolle für das subvokale Wiederholen (sog. "Rehearsal"-System) spielen müsse. Damit einhergehende beidseitige Aktivitätsänderungen im supplementären motorischen Areal, im Kleinhirn und in den primären sensomotorischen Gebieten von Mund und Kehlkopf (Areale 1-4 und 6) wurden unter Berücksichtigung der Ergebnisse verschiedener Läsionsstudien an anarthrischen Patienten auf motorische Anteile bei der Sprachplanung und -durchführung zurückgeführt; entsprechend konnten linksseitig occipitale Aktivitätszunahmen (Area 18) der visuellen Verarbeitung von Buchstaben zugeschrieben werden. Aus der Tatsache, daß bei der Reimaufgabe (im Gegensatz zur phonologischen Memorieraufgabe) linkshemisphärisch keine Aktivation im Gyrus Supramarginalis (Areal 40) zu verzeichnen war, schlossen die Autoren schließlich, daß es sich bei diesem Gebiet um den Hauptort des phonologischen Speichers handeln müsse.

Die Ergebnisse von Paulesu und Mitarbeitern haben sich als recht robust erwiesen. Insbesondere konnte wiederholt gezeigt werden, daß der linkshemisphärische Parietalbereich (Area 40) bei verschiedenen Arbeitsgedächtnisaufgaben aktiviert wird, wenn diese die Speicherung verbalen Materials erfordern (Awh et al., 1996; Petrides, Alivisatos, Meyer \& Evans, 1993b; Salmon et al., 1996; Smith \& Jonides, 1997). Aufgaben, die das stille Wiederholen verbaler Informationen beinhalten, führen darüber hinaus zu verstärkten linksseitigen Aktivationen im sogenannten Broca-Areal (Area 44; Braver et al., 1997; Frackowiak, 1994; Petersen et al., 1988; Salmon et al., 1996; Smith \& Jonides, 1997; Smith, Jonides \& Koeppe, 1996). Smith und Jonides (1997) konnten in diesem Zusammenhang zeigen, daß die Zunahme der Gedächtnislast zu Aktivitätszu- 
nahmen in bestimmten, festgelegten Arealen führt: Sie werteten die Daten ihrer zahlreichen PET-Versuche nicht nur mit der Subtraktionsmethode, sondern auch mit der weniger problematischen Methode der "parametrischen Variation" ${ }^{28}$ aus. Nach der Variation eines einzelnen experimentellen Faktors, nämlich der Anzahl zu erinnernder Buchstaben in einer "n-back"-Aufgabe ${ }^{29}$, untersuchten sie, inwieweit regionale Hirnaktivationen von diesem Faktor beeinflußt worden waren. Die Betrachtung prozentualer Aktivitätsänderungen in Abhängigkeit von der Gedächtnislast machte deutlich, daß monotone Aktivationszunahmen nur in denjenigen Arealen aufgetreten waren, denen verbale Arbeitsgedächtnisfunktionen zugeschrieben werden (z. B. linksseitig Area 46/10, 40, 44 und 6/32 sowie rechtsseitig Area 6, 7, 9/46 und 46/10; vgl. Abb. 9). Entsprechend fanden die Autoren im primären visuellen, motorischen und somatosensorischen Kortex keine von der Gedächtnislast abhängigen Aktivationsänderungen. Mit zunehmender Anzahl zu erinnernder Items wurden aber nicht nur bestimmte Areale stärker aktiviert, sondern auch zwei neue Regionen mit einbezogen, nämlich der dorsolaterale PFC und das Broca-Areal. Diese Areale wiesen erst bei den "2-back"- und "3-back"-Aufgaben stärkere Aktivationszunahmen auf, was als Folge veränderter Aufgabenanforderungen (größere Bedeutung von zeitlicher Informationskodierung und stillem Wiederholen) interpretiert wurde. Insgesamt konnten Smith und Jonides (ebd.) mit der Methode der parametrischen Variation die zuvor mit dem Subtraktionsverfahren erhaltenen Befunde bestätigen. Braver et al. (1997) erhielten in einer vergleichbaren fMRT-Studie analoge Ergebnisse.
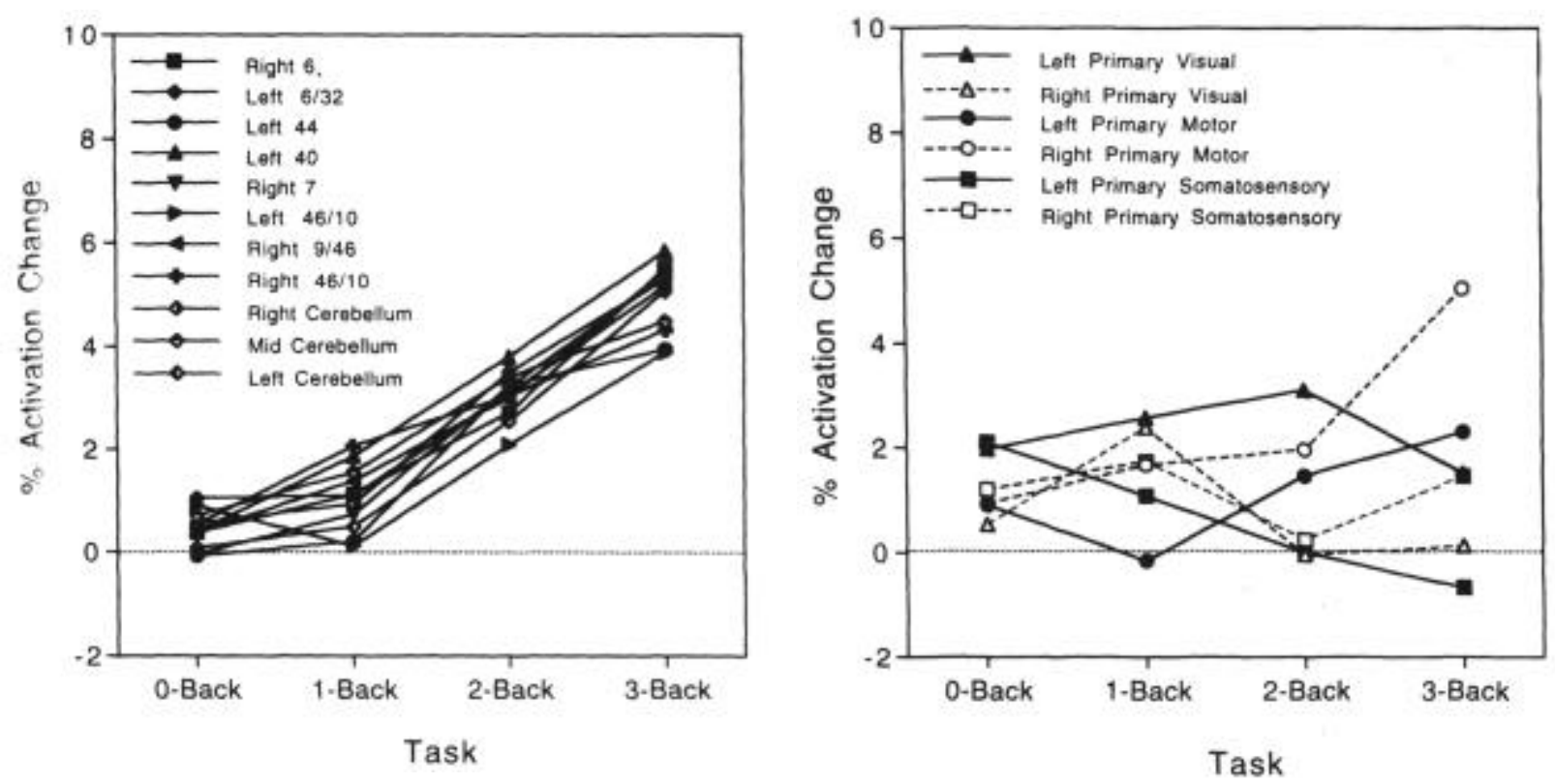

Abbildung 9. Zusammenhang zwischen prozentualer Aktivationsänderung und Gedächtnislast in Arealen, denen verbale Arbeitsgedächtnisfunktionen zugeschrieben (links) bzw. nicht zugeschrieben (rechts) werden (Smith \& Jonides, 1997). 
Der Dissoziation zwischen Speicherung und stillem Wiederholen im phonologischen Arbeitsgedächtnis gingen Awh et al. (1996) in ihrer PET-Studie nach: Sie ließen neun Probanden verschiedene "n-back"-Aufgaben absolvieren; diese erforderten entweder das fortlaufende Memorieren verbalen Materials im Arbeitsgedächtnis oder (in den Kontrollbedingungen) das Vergleichen bzw. stille Wiederholen dargebotener Buchstaben. Mit der Subtraktionsmethode konnten Awh et al. (ebd.) zeigen, daß verschiedene Hirnbereiche an der Speicherung bzw. am stillen Wiederholen verbalen Materials beteiligt sind (vgl. Abb. 10 links): Bei der Differenzbildung zwischen Memorier- und Wiederholungsaufgabe verschwand die signifikante Aktivation im Broca-Areal und im prämotorischen Kortex (Area 44 und 6), während sie im posterioren und superioren Parietallappen (Area 40 und 7) erhalten blieb. Daß darüber hinaus noch Aktivationen im supplementären motorischen Areal (SMA; in der Tiefe der Fissura interhemispherica liegender Teil von Area 6) und im rechten zerebellären Kortex zu verzeichnen waren, weist nach Meinung der Autoren darauf hin, daß diese Areale nicht nur an der motorischen Programmierung, sondern auch an anderen Prozessen beteiligt sind (z. B. Fehlerfindung, übungsbezogenes Lernen; vgl. Fiez, Petersen, Cheney \& Raichle, 1992).
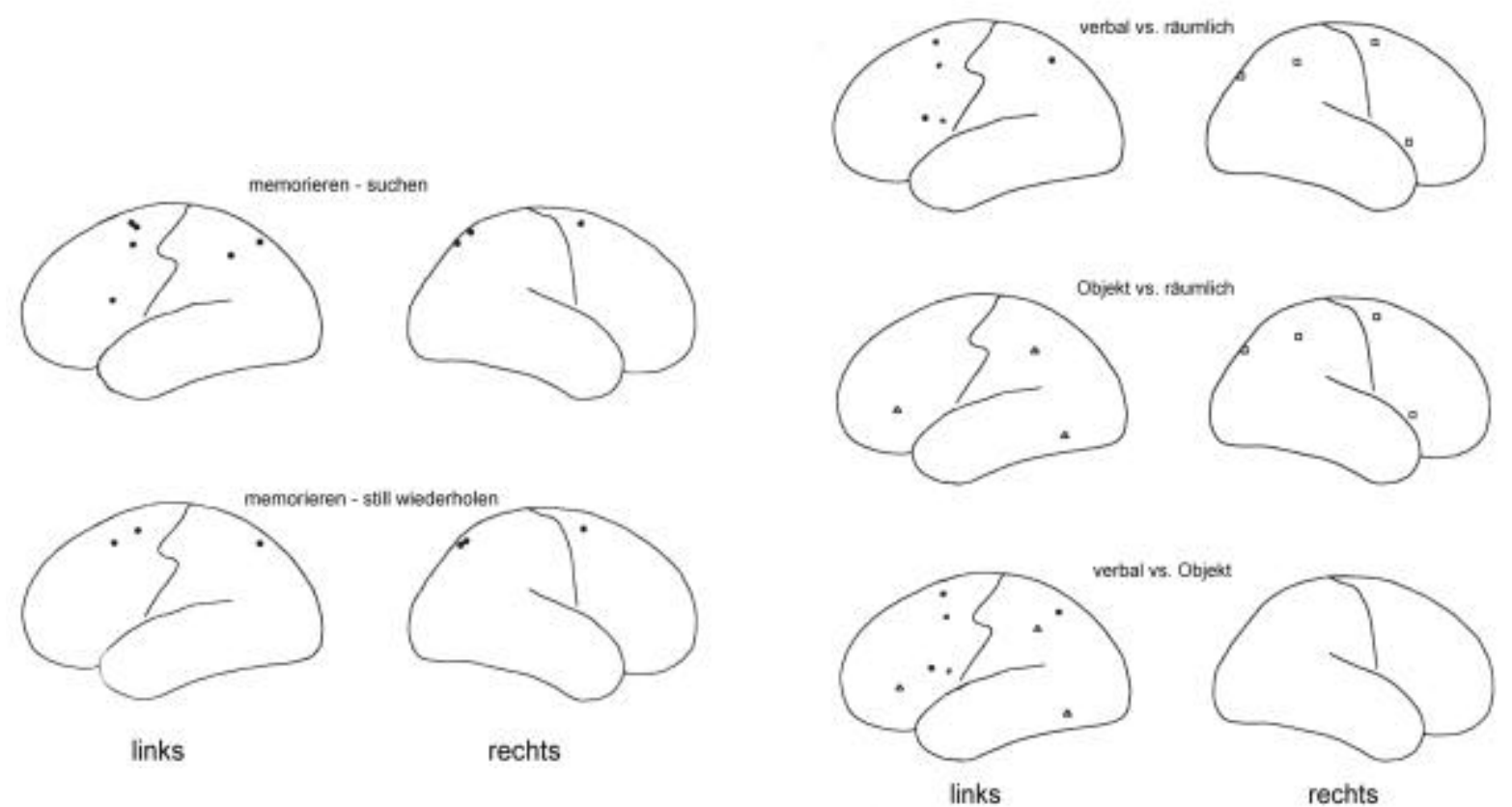

Abbildung 10. Schematische Darstellung kortikaler PET-Aktivationen: Links: Gegenüberstellung von Memorier- und Rehearsal-Prozessen im Versuch von Awh et al. (1996; aus Smith \& Jonides, 1997). Oben: Aktivierte Areale nach Differenzbildung zwischen Memorier- und Suchprozessen; unten: aktivierte Areale nach Differenzbildung zwischen Memorier- und Rehearsalprozessen. Rechts: Überblick über die bei verbalen, räumlichen und Objektgedächtnisaufgaben signifikant aktivierten Regionen (nach Abzug der Kontrollaufgaben) in den Versuchen von Smith \& Jonides (1997).

Wie Paulesu et al. (1993) fanden auch Awh et al. (1996) während der phonologischen Memorieraufgabe Aktivationen in einigen, den linksseitig aktivierten Arealen entsprechenden rechtshemisphärischen Regionen (oberer Parietalkortex, prämotorischer Kor- 
tex und SMA). Dabei lag das Aktivationsausmaß dieser rechtsseitigen Areale jedoch unter dem der entsprechenden linkshemisphärischen Areale. Smith und Mitarbeiter (1996), die dieses Ergebnis in ihrer PET-Untersuchung bestätigen konnten, offerieren zwei mögliche Erklärungen für die Aktivation korrespondierender Areale: "... the additional areas tend to be homologs of areas that typically mediate the task, which suggests that the homologs may be recruited to assist in the processing or they may reflect excessive activation that spills over to the opposite hemisphere" (S. 18). Welche dieser beiden Erklärungen Gültigkeit hat, ist bislang noch ungeklärt.

Eine - wenn auch nicht immer signifikante - Aktivierung homologer Areale fand sich auch in PET-Analysen, die sich mit den am räumlichen Arbeitsgedächtnis beteiligten kortikalen Strukturen befassen. Jonides et al. (1993) ließen ihre Probanden die Position dreier schwarzer Punkte memorieren, die auf einem Bildschirm um ein Fixationskreuz angeordnet erschienen. Nach anschließender Darbietung eines Testreizes (Kreis) sollten die Probanden entscheiden, ob dieser an einer der zuvor gemerkten Positionen aufgetreten war oder nicht (räumliche Memorieraufgabe). In der Referenzaufgabe erschienen die Punkte zusammen mit dem Testreiz, so daß ein Memorieren für die Aufgabenlösung nicht erforderlich war. Die Autoren fanden vier rechtshemisphärische Areale, bei denen die regionale zerebrale Durchblutung während der räumlichen Memorieraufgabe signifikant erhöht war: Dabei handelte es sich um Area 40 (Parietalkortex), Area 19 (Occipitalkortex), Area 6 (prämotorischer Kortex) sowie Area 47 (ventrolateraler präfrontaler Kortex). Gleichzeitig waren die homologen Areale der linken Hemisphäre aktiviert, wenngleich das gesetzte Signifikanzkriterium nicht erreicht wurde. Diese Ergebnisse konnte die Arbeitsgruppe um Jonides in weiteren Studien bestätigen (Koeppe, Minoshima, Jonides, Smith \& Awh, 1993; Smith et al., 1995; Smith et al., 1996), in denen vor allem die rechtshemisphärischen Areale 6 und 40 während verschiedener räumlicher Gedächtnisaufgaben zuverlässig stä rker aktiviert erschienen.

Im Gegensatz zu Jonides et al. (1993) fanden Petrides, Alivisatos, Evans \& Meyer (1993a) in ihrer PET-Studie zum visuell-räumlichen Arbeitsgedächtnis beidseitig signifikant aktivierte Regionen. In einem ihrer Versuche boten sie den Probanden acht abstrakte Zeichungen dar, wobei deren Positionen von Karte zu Karte wechselte. Die Probanden hatten sich jedoch nicht die räumlichen Positionen zu merken, sondern bei jeder Karte auf eine neue, vorher nicht ausgewählte Zeichnung zu zeigen. Gefordert war damit neben der Speicherung bereits ausgewählter Reize auch eine verstärkte Überwachung der eigenen Antworten. In der Kontrollaufgabe zeigten die Probanden in jedem Durchgang auf eine vom Versuchsleiter vorbestimmte Zeichnung. Mit der Subtraktionsmethode fanden die Autoren signifikante Aktivationen im mittleren dorsolateralen frontalen Kortex, und zwar links in Area 9 sowie beidseitig in Area 46. Darüber hinaus erschienen der posteriore Parietallappenbereich (beidseitig Area 7) und der vordere Gy- 
rus cinguli (Area 32) aktiviert. Die Aktivationen in Area 46 waren in der rechten Hemisphäre signifikanter.

Paulesu und Mitarbeiter interessierten sich in einer Komplementärstudie zu ihrer PET-Analyse der neuronalen Korrelate des verbalen Arbeitsgedächtnisses (Paulesu et al., 1993) ebenfalls für die funktionelle Anatomie des visuellen Speichers (sog. "Objektgedächtnis"). Sie ließen ihre Probanden, die des Koreanischen nicht mächtig waren, (nicht verbalisierbare) koreanische Buchstaben memorieren und verglichen das Ergebnis mit dem einer entsprechenden verbalen Gedächtnisaufgabe aus ihrer vorherigen Untersuchung (ebd.). Folgende Regionen erschienen bei der nonverbalen visuellen Arbeitsgedächtnisaufgabe signifikant aktiviert: rechtshemisphärisch die Areale 19/39 und 19/40, beidseitig das inferotemporale Areal 19/37 sowie links das präfrontale Areal 47 und der vordere Gyrus cinguli (Area 32).

Die Ergebnisse der skizzierten PET-Untersuchungen zur visuell-räumlichen Komponente des Arbeitsgedächtnismodells von Baddeley (1986) legen nahe, daß getrennte Speicher für visuell-räumliches und für nonverbales visuelles Material existieren. Während räumliche Arbeitsgedächtnisaufgaben hauptsächlich zu rechtsseitigen Aktivationen in den Arealen 6, 19, 40 und 45/47 führen, werden bei visuellen Arbeitsgedächtnisaufgaben meist linksseitig die kortikalen Areale 6, 32, 37, 40 und 47 (z. T. auch das rechte Areal 19/40) aktiviert. Postle, Stern, Rosen und Corkin (1997) verglichen in ihrer fMRTStudie visuelle bzw. räumliche "n-back"-Aufgaben mit entsprechenden Kontrollaufgaben. In beiden experimentellen Bedingungen waren der präfrontale Kortex (Area 9, 46), beidseitig der prämotorische Kortex (Area 6, 8) sowie links der untere Teil des Parietallappens (Area 40) aktiviert. Erst über den direkten Vergleich der räumlichen mit der Objektgedächtnisaufgabe konnten Unterschiede gefunden werden: Während in der vंsuellen Bedingung links die Areale 44 und 45 sowie beidseitig 21 und 37 stärker aktiviert waren, fanden die Autoren in der räumlichen Bedingung Aktivitätszunahmen vor allem in den oberen Parietallappen (beidseitig Area 7). "These results suggest that the pattern of PFC activation is not a good predictor of VWM (visual working memory; Anm. der Autorin) test condition, and that material-specific VWM computations may be supported by posterior cortex" (S. 1679).

Zu ähnlichen Ergebnissen kamen auch Smith und Jonides (1997), die ebenfalls die während einer räumlichen Gedächtnisaufgabe (siehe Jonides et al., 1993) und einer Objektgedächtnisaufgabe ${ }^{30}$ aktivierten Areale verglichen: An der Bewältigung der räumlichen Gedächtnisaufgabe waren in ihrem PET-Versuch rechtsseitig zwei frontale, eine parietale und eine occipitale Region beteiligt, bei der Objektgedächtnisaufgabe traten Aktivationen linkshemisphärisch u. a. im inferotemporalen, parietalen und frontalen (prämotorischen) Bereich sowie im linken Gyrus cinguli auf (vgl. Abb. 10 rechts). Der aktivierte Teil des inferotemporalen Kortex soll maßgeblich an der Wiedererkennung von Objekten beteiligt sein (Mishkin, Ungerleider \& Macko, 1983), Aktivationen im Gyrus 
cinguli werden selektiven Aufmerksamkeitsprozessen zugeschrieben (Pardo, Pardo, Janer \& Raichle, 1990; Paus, Petrides, Evans \& Meyer, 1993; Posner \& Petersen, 1990). Die übrigen, während der Objektgedächtnisaufgabe linksseitig aktivierten Bereiche stimmten im wesentlichen mit denjenigen Arealen überein, die auch bei verbalen Gedächtnisaufgaben eine wichtige Rolle spielen (links prämotorisch, links posterior parietal). Smith und Jonides (ebd.) führten dieses Ergebnis auf die mögliche verbale Beschreibung der zu lernenden Objekte durch die Probanden und auf die anschließende stille Wiederholung dieser Beschreibungen zurück. In einem weiteren, kontrollierteren Experiment, in dem die Autoren für beide Aufgaben neue nonverbale Reize verwendeten, erhielten sie bei der Objektgedächtnisaufgabe nur noch zwei linksseitig aktivierte Areale (inferotemporal, posterior parietal). In der räumlichen Bedingung erschien zusätzlich zu den im ersten Versuch beteiligten Arealen die linksseitige posteriore Parietalregion aktiviert. In einer Zusammenschau ihrer Ergebnisse heben Smith und Jonides (ebd.) die Existenz unterschiedlicher neuronaler Schaltkreise für das visuell-räumliche und für das Objektgedächtnis hervor, wobei der erstere hauptsächlich rechtshemisphärisch, der letztere hingegen ebenso wie das verbale Gedächtnis im wesentlichen linkshemisphärisch angelegt sein soll.

Während die bei der Ausführung visuell-räumlicher Gedächtnisaufgaben auftretenden Aktivationen im rechten occipitalen Kortex (Area 17/18/19) in der Regel Wahrnehmungs- bzw. visuellen Kodierungsprozessen zugeschrieben (Courtney, Ungerleider, Keil \& Haxby, 1996; Jonides et al., 1993; Kosslyn et al., 1993) oder zusammen mit Aktivationen in Area 40 als Teil eines parietal-occipitalen Schaltkreises zur Aufrechterhaltung der bildlichen Vorstellung betrachtet werden (Smith \& Jonides, 1997), bedürfen die Funktionen der frontal aktivierten Areale noch weiterer Klärung. Ergebnisse aus verschiedenen Untersuchungen an Primaten legten zunächst nahe, daß auch beim Menschen der dorsolaterale Anteil des präfrontalen Kortex an der temporären Speicherung räumlicher, der ventrale Teil hingegen an der temporären Speicherung visueller Information (Form, Farbe) maßgeblich beteiligt sein könnte (Fuster, 1989; Goldman-Rakic, 1987; Wilson, O'Scalaidhe \& Goldman-Rakic, 1993). fMRT- und PET-Studien aus dem humanen Bereich verdeutlichten zwar eine konsistente Aktivierung des präfrontalen Kortex (PFC) bei Aufgaben, die die Manipulation kurzzeitig gespeicherter Information erforderten; bezüglich der Frage nach den spezifischen, präfrontal aktivierten Regionen und ihrer hemisphärischen Lateralisierung erbrachten die Untersuchungen jedoch widersprüchliche Ergebnisse (Cohen et al., 1994; Courtney et al., 1996; Jonides et al., 1993; McCarthy et al., 1994; Paulesu et al., 1993; Petrides et al., 1993a, b; Smith et al., 1995). Neuere fMRT-Untersuchungen (s. o. D'Esposito \& Postle, 1998; Postle et al., 1997) und eine Analyse von 24 Studien, in denen bildgebende Verfahren eingesetzt worden waren (D'Esposito et al., 1998a), konnten keine Evidenz für die Zuordnung ver- 
schiedener präfrontaler Areale zu räumlichen bzw. visuellen Speicherfunktionen des Arbeitsgedächtnisses liefern.

Heute wird angenommen, daß die funktionelle Organisation des PFC beim Menschen davon abhängt, wie die im Arbeitsgedächtnis gehaltenen Informationen verarbeitet werden. Petrides (1994) geht davon aus, daß im ventrolateralen PFC (Area 45/47) die aus den hinteren Assoziationsarealen ankommenden Informationen in einem aktiven Zustand gehalten werden, um so einen Vergleich der memorierten Reize und eine aktive Organisation von Antwortsequenzen zu ermöglichen. Der dorsolaterale PFC (Area 9/46) soll hingegen erst dann eine Rolle spielen, wenn die im Arbeitsgedächtnis gehaltene Information überwacht bzw. manipuliert werden muß. In beiden Fällen handelt es sich um Aufgaben, die in Baddeleys (1986) Arbeitsgedächtnismodell der zentralen Exekutive zugeschrieben werden (vgl. Kap. 2.1.1) und die sowohl bei der Bewältigung phonologischer als auch visuell-räumlicher Aufgaben erforderlich sein können. So fanden z. B. Salmon et al. (1996), die in ihrer PET-Untersuchung die Ergebnisse von Paulesu et al. (1993) replizieren konnten, zusätzliche Aktivitätszunahmen bei der nonverbalen Aufgabe linkshemisphärisch im Bereich des mittleren frontalen Gyrus (Area 10 und 46), bei der verbalen Aufgabe links im Areal 10 und im parahippokampalen Bereich. Sie deuteten dieses Ergebnis als Zeichen für eine Beteiligung der zentralen Exekutive an den Gedächtnisspannenaufgaben: Ihr Einsatz könne z. B. in der verbalen Bedingung der Erhöhung der Effizienz der phonologischen Schleife (durch Chunking oder durch Bereitstellung eigener Speicherkapazität) oder der Koordination zwischen Inhalten aus dem Kurz- und Langzeitgedächtnis dienen, wodurch auch die Aktivationen im parahippokampalen Bereich erklärt würden (vgl. auch Hulme, Maughan \& Brown, 1991). Eine weitere verbale Gedächtnisaufgabe, die neben der phonologischen Schleife explizit auch die zentrale Exekutive beanspruchen sollte ${ }^{31}$, führte ebenfalls $u$. a. zu präfrontalen Aktivationen (rechtes Areal 9, linke Areale 10 und 46). "From that perspective, our data confirm that the prefrontal cortex ... is a key structure for the central executive" (Salmon et al., 1996, S. 1623).

In zahlreichen weiteren Arbeiten wurde die Bedeutsamkeit der Rolle, die der frontale Kortex sowohl bei der Bewältigung visuell-räumlicher als auch phonologischer Arbeitsgedächtnisaufgaben spielt, bestätigt (z. B. D'Esposito et al., 1995a; Petrides et al., 1993a, b; Owen et al., 1996). Braver et al. (1997) interessierten sich in ihrer fMRTStudie u. a. für den Zusammenhang zwischen Gedächtnislast (Anzahl zu memorierender Items) und präfrontaler Aktivität. Sie ließen ihre Probanden verbale Arbeitsgedächtnisaufgaben ("n-back"-Aufgaben) absolvieren und fanden eine mit der Gedächtnislast monoton ansteigende Aktivität beidseitig im dorsolateralen PFC (Area 46/9) und im Broca-Areal (links Area 44). Aktivitätszunahmen in diesen Regionen korrelierten zudem als einzige signifikant mit der mittleren Antwortlatenz, "suggesting that behavioral performance can be used as a psychologically relevant reference function ... by which to iso- 
late activity related to WM (working memory; Anm. der Autorin)" (ebd., S. 54). Über Reanalysen der Daten und über weitere Experimente konnten Alternativerklärungen (z. B. Fehlerverarbeitung, Aufgabenschwierigkeit) für den gefundenen Zusammenhang ausgeschlossen werden.

Die Arbeitsgruppe um Petrides bestätigte ihre Hypothese bezüglich der funktionellen Dissoziation unterschiedlicher präfrontaler Areale in verschiedenen Studien. In ihrer Untersuchung von 1993 hatten Petrides et al. gezeigt, daß die Durchführung einer visuellen Arbeitsgedächtnisaufgabe, die eine verstärkte Überwachung der eigenen Antworten erfordert, zu Aktivationen u. a. im dorsolateralen PFC (Area 46 und 9) führt (s. o.); wenn visuelle Stimuli nur beurteilt, jedoch nicht in irgendeiner Form manipuliert werden müssen, sind lediglich im ventralen PFC signifikante Durchblutungsänderungen zu verzeichnen (Petrides et al., unveröffentlichte Beobachtung, zitiert in Owen et al., 1996). Die PET-Studie von Owen et al. (1996) macht zudem deutlich, daß das Modell der frontolateralen Dissoziation auch auf verschiedene räumliche Arbeitsgedächtnisaufgaben anwendbar ist: Aufgaben, die die Organisation und Ausführung einer Reihe von gelernten räumlichen Bewegungen erforderten, führten zu signifikanten Durchblutungsänderungen beidseitig in Area 47; wenn hingegen eine aktive Überwachung und Manipulation räumlicher Information im Arbeitsgedächtnis für die Aufgabenbewältigung notwendig war, fanden die Autoren zusätzliche signifikante Aktivationen rechtshemisphärisch in Area 46 und 9.

D'Esposito, Postle, Ballard und Lease (1999) untersuchten die Hypothese von Petrides (1994) anhand einer phonologischen Arbeitsgedächtnisaufgabe. Sie ließen ihre Probanden fünf simultan dargebotene Konsonanten memorieren und anschließend prüfen, ob eines dieser Buchstaben mit einem Testreiz übereinstimmte. Gleichzeitig wurde eine ereignisbezogene funktionelle Magnetresonanztomographie (fMRT) durchgeführt ${ }^{32}$. Während in der ersten Bedingung nur das Memorieren und Vergleichen erforderlich war, mußten die Probanden in der zweiten Bedingung die Buchstaben während der Memorierphase nach ihrer alphabetischen Reihenfolge sortieren, um korrekt zu antworten. D'Esposito et al. (ebd.) fanden in beiden Bedingungen gesteigerte Aktivationen sowohl im dorsolateralen als auch im ventrolateralen PFC (Area 9, 46 bzw. 44, 45, 47); die dorsolaterale Aktivation war jedoch in der Manipulationsbedingung signifikant höher als in der reinen Memorierbedingung. Die verschiedene Aufgabenschwierigkeit konnte als mögliche Ursache dieses unterschiedlichen Aktivierungsausmaßes ausgeschlossen werden. Die Autoren werteten das Ergebnis als weiteren Beleg dafür, daß der dorsolaterale und der ventrolaterale Anteil des PFC unterschiedlichen Arbeitsgedächtnisfunktionen zugeordnet werden können. Die gleichzeitige Aktivation beider Gebiete in der Memorieraufgabe führten sie auf eine Überlastung der Kurzzeitgedächtniskapazität zurück, die zum Einsatz zusätzlicher, manipulatorischer (Memorier-)Prozesse geführt haben könnte. In einer weiteren Studie konnten D'Esposito und Postle (1998) zeigen, daß der dorsolate- 
rale PFC nicht nur auf gesteigerte Verarbeitungsanforderungen, sondern auch auf eine zunehmende Gedächtnislast sensitiv reagiert (vgl. auch oben, Braver et al., 1997).

Während also je nach Verarbeitungsanforderung entweder dorsolaterale und/oder ventrolaterale Anteile des PFC aktiviert zu sein scheinen (vgl. auch Berman, AustinLane, Esposito, Van Horn \& Weinberger, 1996; Klingberg, O'Sullivan \& Roland, 1997; Salmon et al., 1996), sprechen viele Studien auch dafür, daß die Art der zu verarbeitenden Information die hemisphärische Lateralisierung dieser Aktivation bestimmt. So kamen D'Esposito et al. (1998a) nach Durchsicht der Literatur zu dem Schluß, daß der ventrolaterale PFC während räumlicher Gedächtnisaufgaben meist stärker rechtshemisphärisch, während nichträumlicher Aufgaben jedoch stärker linkshemisphärisch aktiviert ist.

Wenngleich die dargestellten Untersuchungen die Bedeutung des PFC für die sogenannte "exekutive" Informationsverarbeitung im Arbeitsgedächtnis unterstreichen, kann daraus nicht die Schlußfolgerung gezogen werden, daß die Funktionen des frontalen Kortex sich hauptsächlich und spezifisch auf das Arbeitsgedächtnis beziehen. So fanden D'Esposito, Balard, Aguirre und Zarahn (1998b) in ihrer fMRT-Studie signifikante Aktivationszunahmen im lateralen PFC nicht nur während Aufgaben, die das phonologische oder visuell-räumliche Arbeitsgedächtnis beanspruchten, sondern auch während nicht-mnestischer Aufgaben, wenn diese mit einer Ruhephase verglichen wurden. Andere Autoren berichten ebenfalls über Aktivationen im lateralen PFC während verschiedener nicht-mnestischer Aufgaben, die z. B. die Aufrechterhaltung einer gesteigerten Aufmerksamkeit erforderten (Cohen et al., 1988; Lewin et al., 1996; McCarthy et al., 1994, 1996; Pardo, Fox \& Raichle, 1991; Tzourio et al., 1997; Zarahn, Aguirre \& D'Esposito, 1999). Aktivationen im PFC sind z. T. auch der semantischen (Petersen, Fox, Snyder \& Raichle, 1990; Raichle, 1990) bzw. der grammatikalischen (Grossman et al., 1993) Informationsverarbeitung zugeschrieben oder auf die intrinsische Antworterzeugung zurückgeführt worden (Frith, Friston, Liddle \& Frackowiak, 1991). Insgesamt weisen die Befunde "... auf lokale Spezialisierungen und auf eine funktionale Heterogenität im Frontalkortex für verschiedene Aspekte der exekutiven Steuerung und Aufmerksamkeitskontrolle" hin (Kluwe, 1997, S. 158). Zusammenfassend folgern D'Esposito et al. (1998b), $\mathrm{da} B$ "the function of human lateral PFC is not specific for working memory. An important area for further research will be to define more narrowly the specific component processes that engage PFC" (S. 281).

Die Ergebnisse der Studien zu neuronalen Korrelaten verschiedener Arbeitsgedächtniskomponenten lassen sich wie folgt zusammenfassen:

1. Die Aktivationen, die verbale Arbeitsgedächtnisaufgaben hervorrufen, verteilen sich in der Regel auf die an Sprache beteiligten kortikalen Strukturen. Es sind dies der obere Temporallappen (Area 22, 42), der untere Parietallappen (Area 40), das Broca-Areal (Area 44), der primäre motorische Kortex (Area 6) sowie der occipitale Kor- 
tex (Area 18). Darüber hinaus werden auch Teile des präfrontalen Kortex aktiviert (Area 9/10, 46). Obwohl der Schwerpunkt in der linken Hemisphäre liegt, wurden auch Aktivationen homologer Areale der rechten Hemisphäre beobachtet. Studien, in denen die phonologische Schleife isoliert untersucht wurde, weisen die linksseitigen Bereiche um Area 40 und 44 als neuronale Korrelate dieser Arbeitsgedächtniskomponente aus. Dabei konnte das Vorhandensein einer Dissoziation zwischen Speicherung (Area 40) und stillem Wiederholen (Area 44) aufgezeigt werden.

2. Nonverbale Arbeitsgedächtnisaufgaben führen zu Aktivationen im occipitalen Kortex (Area 19), im Parietal- und unteren Temporallappen (Area 7/40 und 37), im vorderen Gyrus cinguli (Area 32) sowie im prämotorischen und präfrontalen Kortex (Area 6 und 46 oder 45/47). Der Schwerpunkt liegt dabei für visuell-räumliches Material in der rechten, für nichträumliche (Objekt-)Informationen hingegen in der linken Hemisphäre, wenngleich auch hier von der Aktivation homologer Areale berichtet wird. Die anfängliche Vermutung, daß bestimmte Teile des präfrontalen Kortex für die temporäre Speicherung räumlichen Materials, während andere Teile für die temporären Speicherung nichträumlicher Information zuständig seien, konnte in neueren Arbeiten nicht bestätigt werden. Vielmehr scheinen sich materialspezifische Aktivationsunterschiede im posterioren Kortex (insbesondere in den Parietallappen, Area 7/40) widerzuspiegeln.

3. Der präfrontale Kortex spielt sowohl bei phonologischen als auch bei visuell-räumlichen Arbeitsgedächtnisaufgaben eine bedeutsame Rolle und gilt als entscheidendes neuronales Korrelat der zentralen Exekutive. Vieles spricht dafür, daß seine funktionelle Organisation vom Verarbeitungsmodus der im Arbeitsgedächtnis gehaltenen Informationen abhängt: Während dem ventrolateralen PFC (Area 45/47) Erhaltungsfunktionen zugeschrieben werden, soll der dorsolaterale PFC (Area 9/46) für die Überwachung und Manipulation der zu verarbeitenden Informationen zuständig sein. Dabei könnte die auftretende hemisphärische Lateralisierung der Aktivation mit der Art des zu verarbeitenden Materials zusammenhängen. Die Funktionen des präfrontalen Kortex sind jedoch nicht spezifisch für das Arbeitsgedächtnis: Aktivationen im lateralen PFC sind auch während verschiedener nicht-mnestischer Aufgaben beobachtet wo rden, so daß von einer funktionalen Heterogenität des PFC für verschiedene Aspekte der exekutiven Steuerung und Aufmerksamkeitskontrolle ausgegangen werden kann.

4. Mit dem Umfang der zu memorierenden Information nimmt die Aktivität in bestimmten Hirnregionen zu; außerdem werden auch weitere, zuvor nicht oder weniger aktivierte Areale in den Verarbeitungsprozeß einbezogen. Mnestische Prozesse spielen sich somit in einem weit verzweigten neuronalen Netzwerk ab, das je nach Art des Reizmaterials spezifische kortikale Strukturen umfaßt. 


\subsubsection{Dynamik der Verarbeitungsprozesse}

PET-Studien vermitteln eindrucksvolle Einsichten in die funktionelle Anatomie des Gehirns, geben jedoch keinerlei Auskunft über die zeitliche Abfolge der Informationsverarbeitung in den abgebildeten neuronalen Schaltkreisen. Die Betrachtung des Zeitverlaufs ist jedoch unerläßlich, wenn man die unserem Verhalten zugrundeliegende Koordination zentralnervöser Prozesse verstehen will (Raichle, 1997). Darüber hinaus lassen sich über Zeitverlaufsanalysen aktivierter Hirnareale Rückschlüsse auf die Funktionen der entsprechenden Areale ziehen (Smith \& Jonides, 1997). Dabei wird zwischen einem frühen, mittleren und späten Verarbeitungsstadium bzw. zwischen Enkodierungs-, Memorier- und Abrufphase unterschieden. Da solche Analysen den Einsatz zeitlich hochauflösender Meßverfahren erfordern, kommt in der Regel das EEG als Methode der Wahl zum Einsatz. Im Mittelpunkt steht dabei die Untersuchung sogenannter "ereignisbezogener Potentiale" (event-related potentials, ERP), wobei Unterschiede in der Amplitude und Latenz bekannter ERP-Komponenten an verschiedenen Elektroden interessieren ${ }^{33}$.
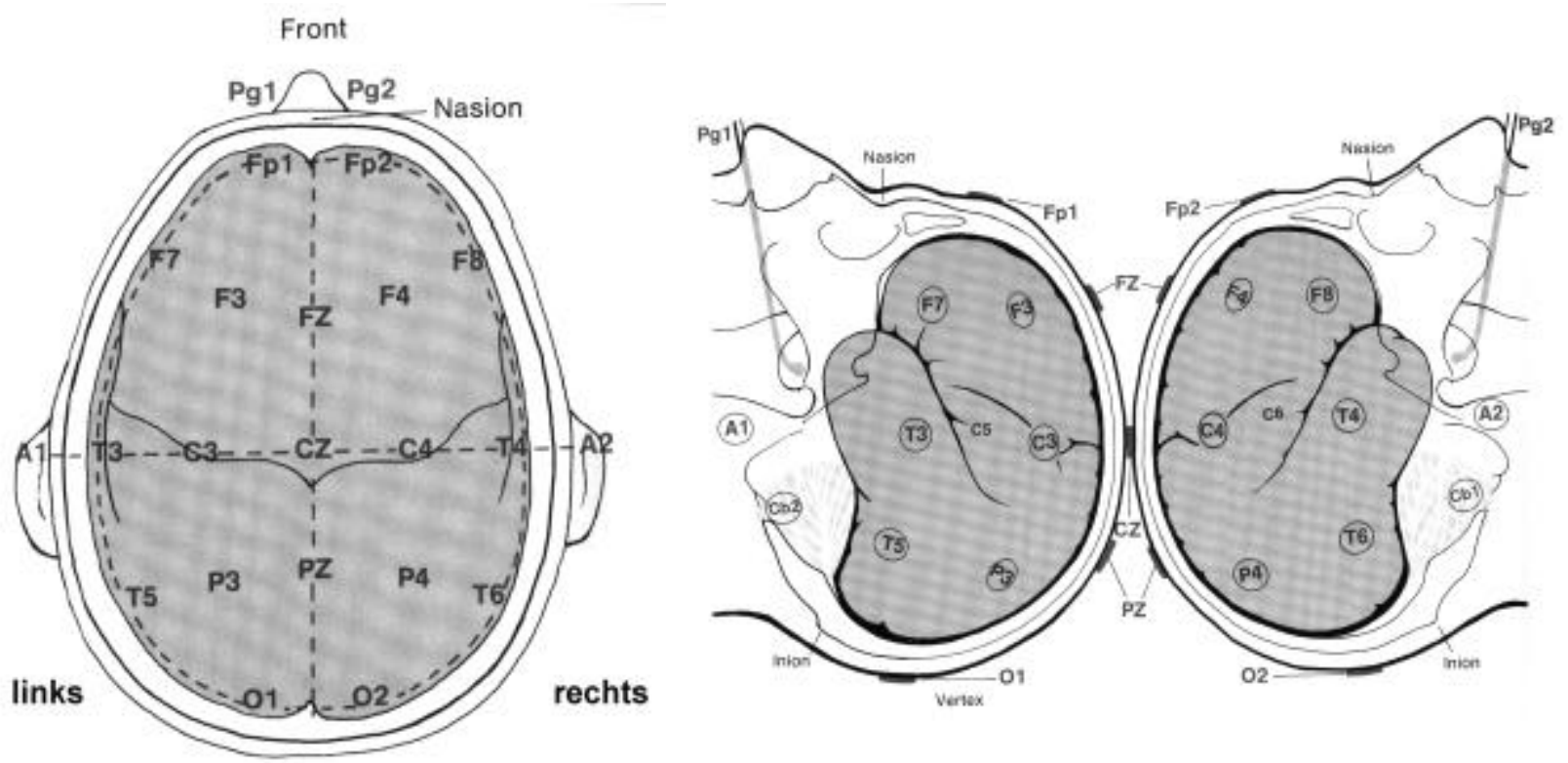

Abbildung 11. Übersicht über die Elektrodenpositionen nach dem 10-20-System (aus Birbaumer \& Schmidt, 1996)

In vielen elektrophysiologischen Studien zum Arbeitsgedächtnis wurde das klassische Sternberg-Paradigma verwendet, von dem bekannt ist, daß der Testreiz eine P300-Komponente hervorruft. Es konnte gezeigt werden, daß die Amplitude dieser Komponente bei der Elektrode $\mathrm{Cz}$ (vgl. Abb. 11) mit zunehmender Anzahl zu memorierender Items abnimmt, während ihre Latenz zunimmt (Adam \& Collins, 1978; Ford, Roth, Mohs, Hopkins \& Kopell, 1979; Gomer, Spicuzza \& O'Donnell, 1976; Karrer, McDonough, Warren \& Cone, 1980; Kramer, Schneider, Fisk \& Donchin, 1986; Marsh, 1975; Pratt, Michlewski, 
Barret \& Starr, 1989a; Starr \& Barrett, 1987). Starr, Barrett, Pratt, Michalewski und Patterson (1990) fanden in der Abrufphase zentro-parietal (Elektrode Pz) eine ausgeprägte P450, die in ihrem Sternberg-Versuch mit ein bis fünf visuell dargebotenen Ziffern oder Noten etwa 250 ms nach dem Testreiz begann. Die Latenz dieser Komponente nahm mit der Gedächtnislast zu, bei den entsprechenden Steigungsgradienten für die verschiedenen Reizarten konnte jedoch kein Unterschied gefunden werden. Starr und Mitarbeiter (ebd.) schlossen daraus, daß die Pz-Latenz der P450 ein frühes Verarbeitungsstadium im Arbeitsgedächtnis widerspiegelt, das hauptsächlich von dem Umfang, nicht jedoch von den Merkmalen der zu memorierenden Reize beeinflußt wird. Wie in dieser Studie bezog sich die Ergebnisinterpretation auch in den übrigen Untersuchungen im wesentlichen auf den Zusammenhang zwischen Gedächtnislast und P300/P450Latenz; Vergleiche mit nicht mnestischen Kontrollaufgaben wurden nicht durchgeführt, und topographische Aspekte blieben meist unberücksichtigt. Anspruchsvollere Untersuchungen zu raumzeitlichen Aspekten der Verarbeitungsprozesse im Arbeitsgedächtnis sind erst in den letzten Jahren durchgeführt worden.

So unterzogen z. B. Ruchkin, Johnson, Grafman, Canoune und Ritter (1992) ihre Probanden einer Variante der klassischen Sternberg-Aufgabe und leiteten dabei das EEG ab. Den Probanden wurde für 1,5 sec entweder phonologisches Material (aussprechbare Nichtwörter, die aus 3, 4 oder 5 Konsonant-Vokal-Abfolgen bestanden) oder visuell-räumliche Informationen (räumliche Anordnung von 3, 4 oder 5 Konsonanten) dargeboten, die sie bis zum Erscheinen des Testreizes (ca. $5 \mathrm{sec}$ ) memorieren sollten. Die Analyse der in der Enkodierungs- und Memorierphase erhaltenen ereignisbezogenen Potentiale ergab sowohl topographische als auch dynamische Unterschiede zwischen den beiden experimentellen Aufgaben (vgl. Abb. 12): Die visuell-räumlichen Stimuli riefen rechtshemisphärisch größere N220-Komponenten hervor, während diese bei der Verarbeitung phonologischer Reize beidseitig symmetrisch erschienen. In beiden Fällen lag das Maximum dieser frühen negativen Welle über dem Occipitallappen und kann daher der visuellen Reizverarbeitung zugeordnet werden. Kürzere Latenzen der P300 (etwa -97 bis -75 ms) und der sich anschließenden langsamen negativen Potentiale an den Elektroden $\mathrm{Cz}$ und $\mathrm{Pz}$ machten deutlich, daß in der visuell-räumlichen Bedingung Enkodierungsprozesse schneller abliefen und Rehearsalprozesse früher einsetzten als in der phonologischen Bedingung. Langsame negative Wellen traten dabei zunächst hauptsächlich über der rechten Hemisphäre auf und verschoben sich erst später (in der mutmaßlichen Memorierphase) weiter zur Mitte, was die Autoren auf anhaltende Enkodierungsprozesse in den früheren Stufen der visuell-räumlichen Verarbeitung zurückführten. Das phonologische Material rief eine vergleichsweise komplexere Aktivität der langsamen Potentiale hervor: Zwischen 600 und 1500 ms nach Stimulusdarbietung fanden sich langsame negative Potentiale im Occipital- und linken posterioren Temporallappen, beides Regionen, die an Prozessen zur Aufnahme phonologi- 
scher Information beteiligt sein sollen. Zwischen 1000 und 3000 ms traten über dem parietalen Kortex beidseitig langsame positive Potentiale auf, deren Amplitude mit der Zahl zu verarbeitender Silben zunahm, so daß diese Aktivität als Zeichen länger andauernder Enkodierungsprozesse gewertet wurde. Ab 3000 ms nach Stimulusdarbietung, also im wahrscheinlichen Rehearsal-Intervall, erschienen links frontal und beidseitig centro-parietal langsame negative Wellen, die mit den verschiedenen Komponenten der phonologischen Schleife (artikulatorischer Kontrollprozeß und phonologischer Speicher) in Zusammenhang gebracht wurden. Neben den genannten Unterschieden fanden sich auch übereinstimmende Potentialänderungen: So konnte z. B. etwa 1000 ms nach Stimulusdarbietung in beiden Bedingungen eine linksseitige frontale Negativität beobachtet werden. Aus der Ähnlichkeit ihrer Latenz und Topographie schlossen die Autoren, daß diese Negativität für einen materialunspezifischen, zentralen Kontrollprozeß stehen könnte. Zusammenfassend unterstrichen sie, daß "the current ERP data from normal subjects adds to converging evidence from behavioral studies with normal subjects and neuropsychological studies with brain-lesion subjects that working memory involves specialized visuo-spatial and phonological subsystems" (Ruchkin et al., 1992, S. 65). Auf die in der Abrufphase zwischen Testreiz und Antwort stattfindenden Prozesse wurde in dieser Untersuchung nicht eingegangen.
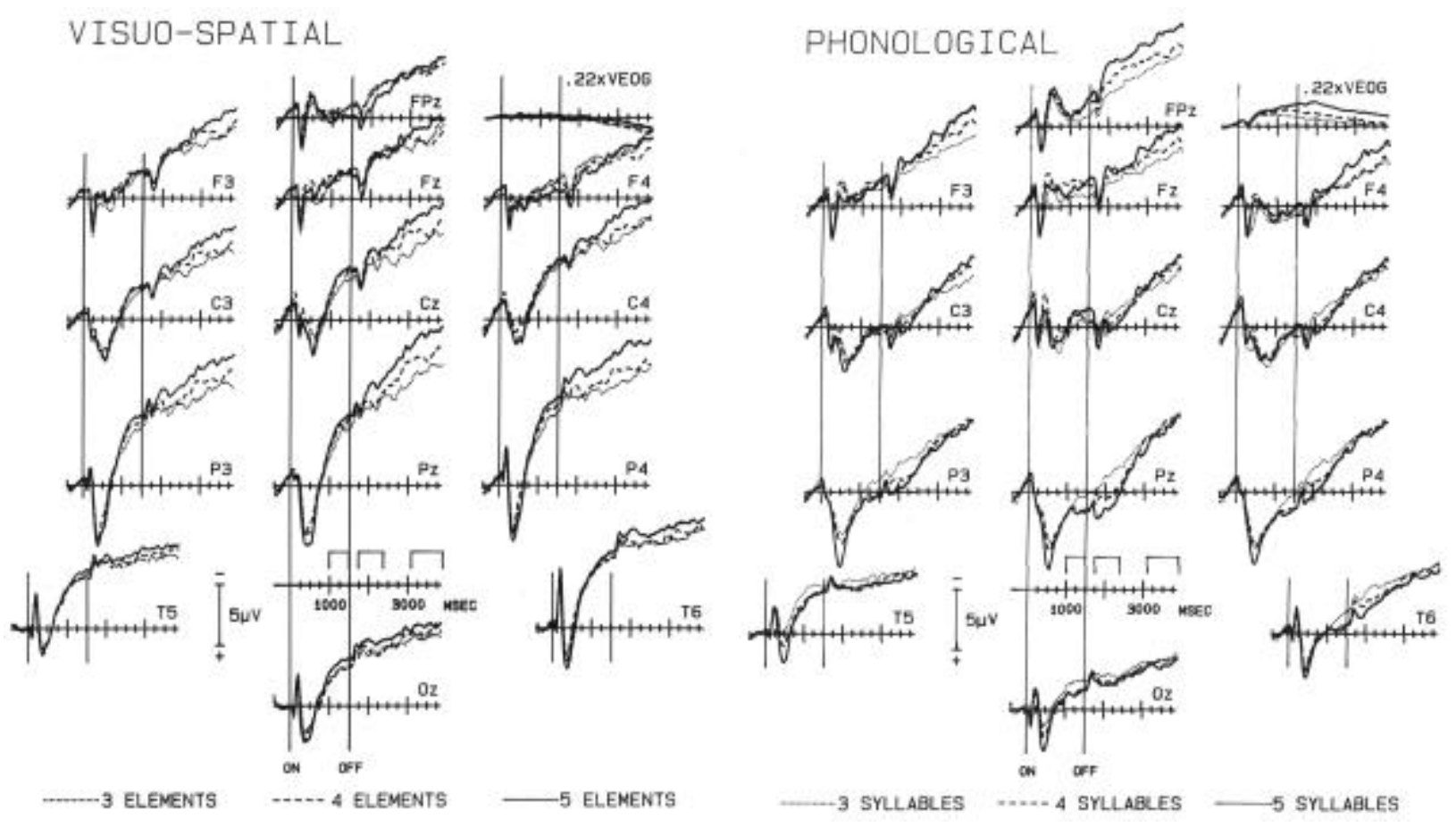

Abbildung 12. Mittlere ereignisbezogene Potentiale der Memorierphase von 12 Probanden während einer visuell-räumlichen (links) bzw. phonologischen (rechts) Arbeitsgedächtnisaufgabe mit zunehmender Gedächtnislast (aus Ruchkin et al., 1992)

Gevins et al. (1996) werteten die während verschiedener "3-back"- und entsprechender Kontrollaufgaben erhaltenen ereignisbezogenen Potentiale aus. Als Reize verwendeten 
sie einzelne Großbuchstaben, deren Name oder Erscheinungsort sich die Probanden merken sollten. Alle Reize riefen zentral eine P200 hervor, die jedoch in der visuell-räumlichen Bedingung größer als in der phonologischen Bedingung ausfiel. Weitere Unterschiede erhielten die Autoren beim Vergleich von Stimuli, die mit dem Zielreiz übereinstimmten (positive Items), und Stimuli, die mit dem Zielreiz nicht übereinstimmten (negative Items): Negative Reize riefen beidseitig frontal einen Peak bei etwa 305 ms hervor, der in der visuell-räumlichen Bedingung am größten war; zusätzlich trat in beiden Bedingungen links frontal eine P450-Komponente auf, die von einem anhaltenden Potential über dem oberen Parietallappen gefolgt wurde. Positive Reize führten hingegen zu einer posterioren P390-Komponente, deren Amplitude sowohl bei der phonologischen als auch bei der visuell-räumlichen Aufgabe kleiner als bei den Kontrollaufgaben ausfiel. Diese Ergebnisse verdeutlichen, daß das dem Arbeitsgedächtnis zugrundeliegende neuronale Netzwerk sowohl aufgabenspezifische als auch aufgabenunspezifische Komponenten umfaßt.

In anderen EEG-Studien wurde die auf anatomischen Untersuchungen basierende Erkenntnis genutzt, daß eine Analyse der elektrischen Beziehungen zwischen verschiedenen kortikalen Regionen mehr Einblicke in die Funktion der Hirnrinde erlaubt als die Feststellung lokaler funktioneller EEG-Änderungen (Braitenberg \& Schüz, 1991; Petsche, 1995). Gevins und Cutillo (1993) führten zum Beispiel eine kovarianzanalytische Auswertung ${ }^{34}$ von ereignisbezogenen Potentialen durch, die es ihnen ermöglichte, gleichzeitig topographische und zeitdynamische Aspekte kortikaler Synchronisierungsphänomene zu berücksichtigen. In ihrem Versuch verwendeten sie eine "2-back"-Aufgabe mit alphanumerischen Ziffern, bei der die Probanden auf die jeweils vorletzte Ziffer mit entsprechendem Druck des rechten Zeigefingers zu antworten hatten, sofern diese nicht mit der aktuellen Ziffer übereinstimmte. In der Kontrollbedingung sollten die Probanden auf jede Ziffer (außer der Null) mit graduellem Zeigefingerdruck reagieren. Ausgewertet wurden vier verschiedene Zeitintervalle, die unterschiedlichen Verarbeitungsstadien zugeordnet wurden: 1. Reizerwartung, 2. Reizverarbeitung, 3. Antwortunterdrückung (bei regativen Testreizen) und 4. Antwortausführung (bei positiven Testreizen). Während keine bedeutsamen Unterschiede zwischen experimenteller und Kontrollbedingung in den frühen Stadien der Reizverarbeitung und bei der Antwortausführung gefunden wurden, erwies sich das Kohärenzmuster der evozierten Potentiale beider Bedingungen im ersten und dritten Intervall (-500 bis -125 ms vor bzw. 203 bis 578 ms nach der Reizdarbietung) als signifikant verschieden: Im ersten Intervall führte die Arbeitsgedächtnisaufgabe zu komplexeren Kovarianzmustern als die Kontrollaufgabe. Es waren besonders linkshemisphärisch höhere Kovarianzen zwischen (antero-)zentralen und occipitalen sowie parietalen Elektroden zu verzeichnen, während in der Kontrollbedingung rechts antero-occipitale und parietale Bereiche beteiligt waren. Dieses Ergebnis konnte als antizipatorische Aktivation der für die Verarbeitung des jeweils erwarteten Stimulus u- 
ständigen Areale gedeutet werden. Im dritten Intervall fiel die Latenz der P300 in der experimentellen Bedingung um etwa 140 ms kürzer aus als in der Kontrollbedingung, und der Schwerpunkt ihres Kovarianzmusters lag mehr linksseitig und frontal. Unter Berücksichtigung ähnlicher Ergebnisse aus anderen Studien wurde dies auf die unterschiedlichen Aufgabenanforderungen und Antwortstrategien in beiden Bedingungen zurückgeführt. Schließlich lagen die Gesamtkovarianzen antero-zentraler Elektroden sowohl im ersten als auch im dritten Intervall in der experimentellen Bedingung höher als in der Kontrollbedingung, so daß auf eine lediglich transiente Beteiligung des präfrontalen Kortex bei der Bewältigung von Arbeitsgedächtnisaufgaben geschlossen wurde. "These results suggest that distinct EPC (evoked potential covariance; Anm. der Autorin) patterns associated with WM (working memory; Anm. der Autorin) only occur during intervals in which the information in an active state is being utilized for task performance" (Gevins \& Cutillo, 1993, S. 1).

Krause, Gibbons und Schack (1998) nutzten einen anderen statistischen Kennwert der linearen Verwandtschaft zweier Signale, und zwar die sogenannte Kohärenz (vgl. Kap. 2.2.2). Sie konnten zeigen, daß die Aktivierung eines Begriffes und die Koordination dieser Aktivierung unterschiedliche neuronale Netzwerke beanspruchen. Ihren Probanden wurden zwei gleiche oder verschiedene Groß- bzw. Kleinbuchstaben dargeboten, die danach beurteilt werden sollten, ob sie identisch aussahen (Mustervergleich) oder einen identischen Namen hatten (Begriffsaktivation). Dabei wurde das Interstimulus-Intervall (ISI) von 100 bis 600 ms variiert. Die Autoren fanden in der Abrufphase signifikante Kohärenzdauerunterschiede ${ }^{35}$ im $\beta$-Band $(13-20 \mathrm{~Hz})$ zwischen den beiden Bedingungen; diese Unterschiede traten bei einem ISI von 100 ms zentro-frontal, bei einem ISI von $300 \mathrm{~ms}$ jedoch links parieto-temporal auf (vgl. Abb. 13). Die Entscheidungszeitdifferenz zwischen Begriffsaktivation und Mustervergleich betrug ISI-unabhängig etwa 128 ms; somit konnte die zentro-frontale Aktivation bei dem darunterliegenden ISI von 100 ms dem gesteigerten Koordinationsaufwand zugeschrieben werden, der vermutlich infolge einer Überschneidung der zwei Begriffsaktivationen (Lern- und Testitem) geleistet werden mußte.

Untersuchungen dieser Art unterstreichen besonders, wie ergiebig der Einsatz zeitlich hochauflösender Meß- und Auswertungsverfahren für die Analyse der neuronalen Korrelate von kognitiven Prozessen im Arbeitsgedächtnis ausfallen kann. Dessen ungeachtet kommt heute vermehrt die fMRT zur Untersuchung der Zeitdynamik regionaler Hirnaktivitätsänderungen zum Einsatz. Aufgrund des gröberen zeitlichen Auflösungsvermögens (etwa 2,5 sec) dieses Verfahrens müssen die Interstimulus-Intervalle vergrößert werden, um mehrere Scans hintereinander durchführen und so Aussagen über den Zeitverlauf der Verarbeitungsprozesse machen zu können (Smith \& Jonides, 1997). Dadurch wird jedoch die methodisch einwandfreie Zuordnung kortikaler Aktivitäts- 
änderungen zu einzelnen kognitiven Verarbeitungsschritten erschwert, so daß die Ergebnisse dieser Studien mit Vorsicht zu interpretieren sind.

ISI: $100 \mathrm{~ms}$

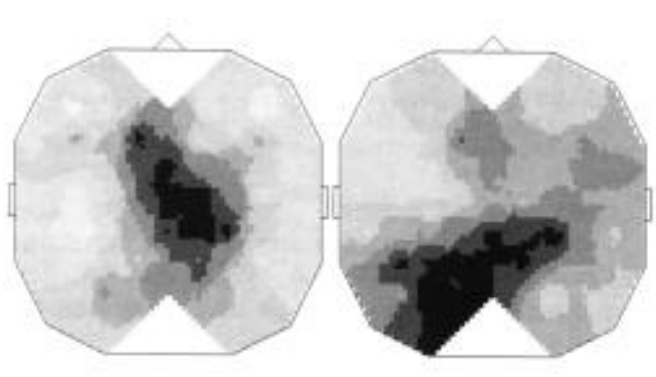

Table 3. Selected foci of activation coherence duration difference $\mathrm{Na}_{-} \mathrm{Aa}$ minus Au_AA (ms) at selected electrode pairs for interstimulus intervals from 100 to $600 \mathrm{~ms}$ $(n=120)$

\begin{tabular}{llllll}
\hline ISI & 100 & 200 & 300 & 500 & 600 \\
\hline F3F7 & 37 & -18 & 8 & 28 & 15 \\
FZF4 & 33 & -7 & 38 & 50 & 14 \\
F4F8 & 27 & -7 & 27 & 15 & -9 \\
F3FZ & $72^{*}$ & 0 & 40 & 42 & $57^{*}$ \\
FZCZ & $77^{* *}$ & 31 & $47^{*}$ & $60^{*}$ & $49^{*}$ \\
C3CZ & $53^{*}$ & -8 & 13 & 2 & 35 \\
CZC4 & $66^{*}$ & 6 & 20 & 10 & -2 \\
CZPZ & $59^{*}$ & -21 & 33 & 11 & 21 \\
T5P3 & 13 & 2 & $63^{* *}$ & 13 & 29 \\
P3PZ & 31 & $42^{*}$ & $64^{*}$ & 23 & $82^{* *}$ \\
P3O1 & 28 & 21 & $57^{* *}$ & $64^{*}$ & 45 \\
T5O1 & 14 & 1 & 29 & 6 & 6 \\
PZP4 & $54^{*}$ & 38 & 48 & 52 & 58 \\
P4T6 & -8 & -13 & 29 & 12 & 38 \\
T6O2 & 9 & -28 & 15 & 30 & 52 \\
P4O2 & 30 & -17 & -1 & 35 & $71^{* *}$ \\
\hline
\end{tabular}

Significant $(*)$ coherence duration differences: Na_Aa minus $A u$ AA at $p=0.05$ confidence level. $(* * ; p=0.01)$ ISI, interstimulus interval, $n=$ number of trials for one condition and one interstimulus interval

Abbildung 13. Übersicht über Verteilung (links) und Signifikanzen (rechts) der Kohärenzdauerunterschiede zwischen Begriffsaktivation und Mustervergleich bei verschiedenen Interstimulus-Intervallen (ISI; Krause, Gibbons \& Schack, 1998).

Cohen et al. (1997) verlängerten das Interstimulus-Intervall ihrer phonologischen "n-back"Aufgaben auf $10 \mathrm{sec}$ und variierten gleichzeitig die Anzahl zu memorierender Items (0 bis 3 Buchstaben). Zeitabhängige Aktivitätsänderungen fanden sich in denjenigen Arealen, in denen sensorische und motorische Prozesse ablaufen (beidseitig Area 17/18, links Area 1 - 4, rechts Area 22/42; vgl. Abb. 14 links). Die von der Gedächtnislast abhängigen Aktivitätsänderungen traten hingegen in den für das verbale Arbeitsgedächtnis relevanten Regionen auf (links Area 39, rechts Area 7, 8, 9/46 und beidseitig Area 6, 40, 44). In einigen Bereichen konnte auch eine (zusätzliche) Interaktion zwischen Zeit und Gedächtnislast verzeichnet werden (Area 32, links Area 44 sowie beidseitig Area 6, 40). Diese Ergebnisse zeigen, wie sehr sich die Spezialisierung verschiedener Hirnregionen in entsprechend unterschiedlichen zeitlichen Aktivitätsmustern widerspiegelt: Während die pro Durchgang (Trial) einmalig ablaufenden kognitiven Prozesse (Stimulusenkodierung, Antwortausführung) zu einer transienten Aktivität im visuellen und primären motorischen Kortex führen, rufen diejenigen Operationen, die während des gesamten Trials benötigt werden (Informationsspeicherung, Aktualisierung und Speicherung zeitlicher Kodes), längeranhaltende und intensivere Aktivitätszunahmen in präfrontalen und parietalen Regionen hervor. 
verbales Material

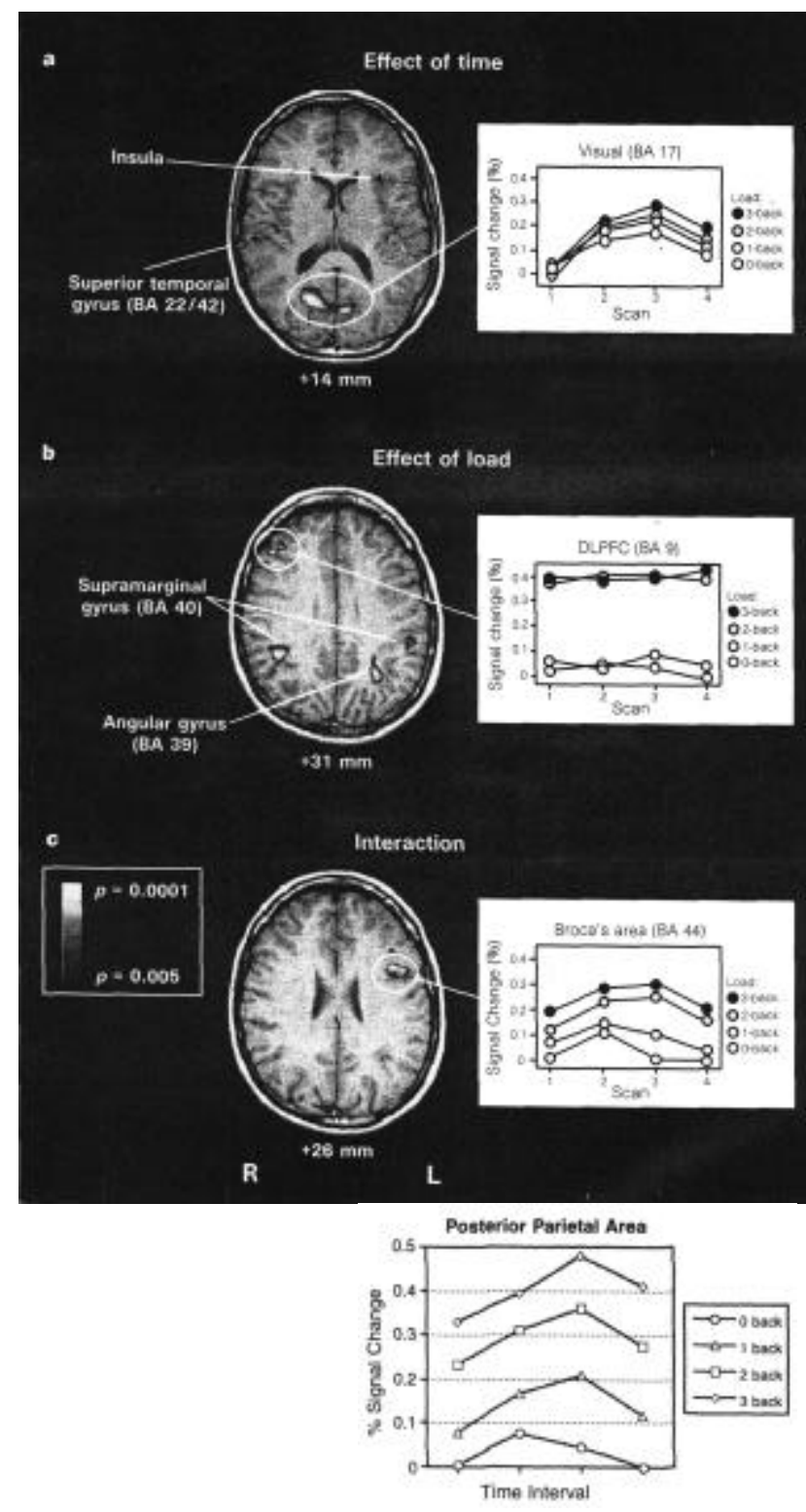

nonverbales Material
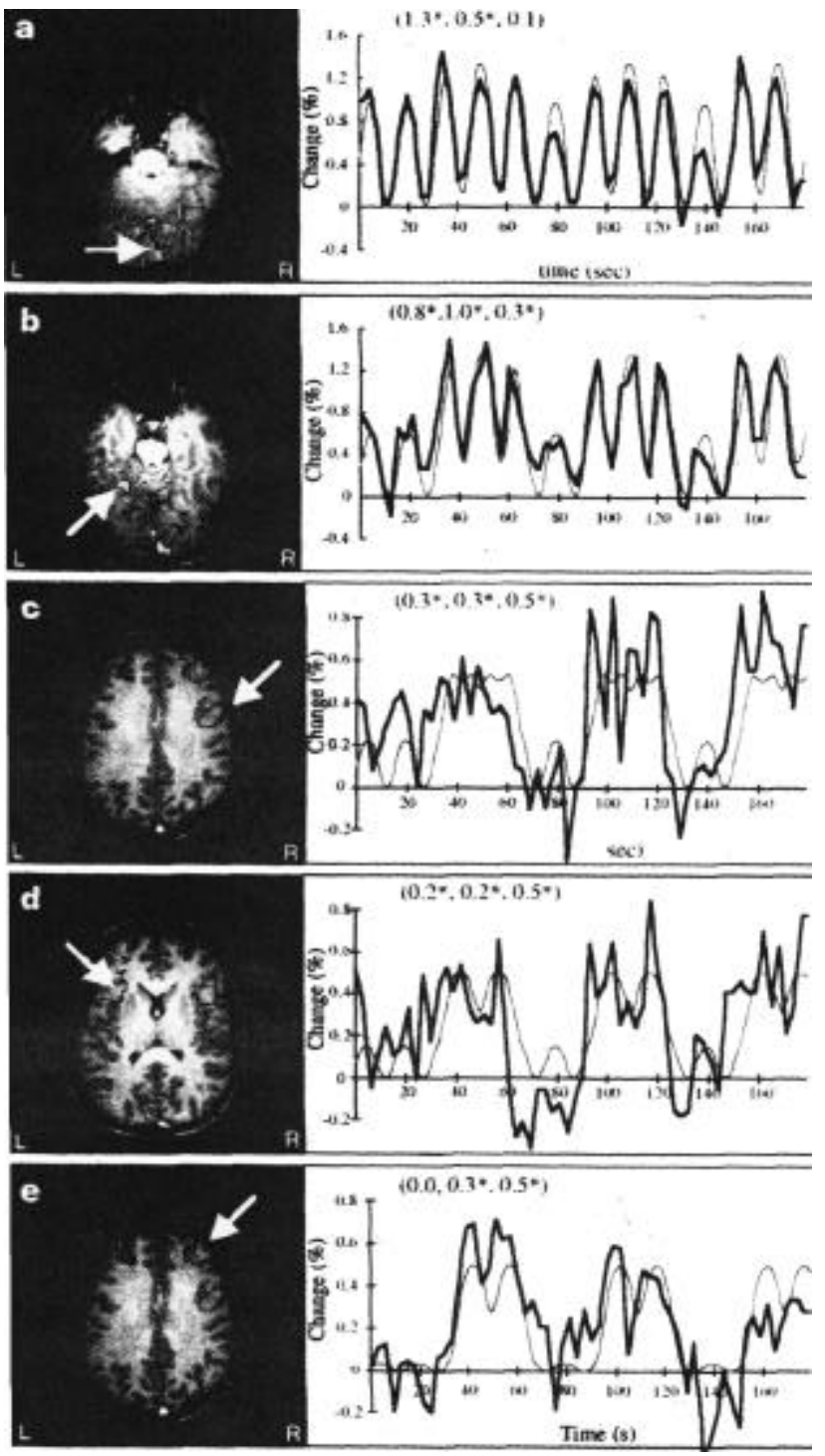

Abbildung 14. Ergebnisse aus fMRT-Untersuchungen zur Dynamik der Verarbeitungsprozesse beim Memorieren von verbalem und nonverbalem Material (linke Darstellung aus Cohen et al., 1997, mit einer Ergänzung aus Smith \& Jonides, 1997; rechte Darstellung aus Courtney et al., 1997)

Ein analoges Ergebnis erhielten auch Courtney, Ungerleider, Keil und Haxby, die sich in ihrer fMRT-Studie von 1997 für die Dynamik visueller Verarbeitungsprozesse interessierten. Sie verwendeten zwei einfache nonverbale Itemwiedererkennungsaufgaben, in denen sich die Probanden in jedem Trial $8 \mathrm{sec}$ lang ein verschlüsselt oder unverschlüsselt dargebotenes Gesicht merken und mit einem Testreiz vergleichen sollten. Dabei kam es in occipito-temporalen Hirnarealen (Area 18, 18/19 sowie 37 links) zu transienten, in präfrontalen Regionen (rechts Area 9/44 und 46, links Area 45/47) hingegen zu anhaltenden Aktivitätsänderungen. Darüber hinaus wiesen verschiedene Bereiche des occipito-temporalen Kortex unterschiedliche Selektivitätsgrade auf (bei unverschlüsselt 
gezeigten Gesichtern nahm die Aktivität in Area 18/19 sowie links in Area 37 stärker zu als bei verschlüsselt gezeigten Gesichtern), während das Ausmaß der anhaltenden Aktivität in verschiedenen präfrontalen Arealen schwankte (am größten fiel es rechtshemisphärisch in Area 46 aus). "There was a systematic progression in the relative strengths of perception- and memory-related acitivity from posterior extrastriate through prefrontal areas, indicating that this distributed neural system for working memory is hierarchically organized" (Courtney et al., 1997, S. 608; vgl. Abb. 14 rechts).

Obwohl Unterschiede in der Methodik und im experimentellen Design die Vergleichbarkeit der wenigen vorhandenen Zeitverlaufsstudien erschweren, wird aus diesen Studien insgesamt sehr deutlich, wie wichtig es ist, bei der Untersuchung des Arbeitsgedächtnisses neben topographischen Informationen auch zeitdynamische Aspekte zu berücksichtigen.

Die Befunde zum Zeitverlauf der im Arbeitsgedächtnis stattfindenden Prozesse lassen sich wie folgt zusammenfassen:

1. In elektrophysiologischen Studien wurden bei der Verarbeitung von Reizen verschiedener Art und verschiedenen Umfangs nicht nur topographische, sondern auch zeitdynamische Unterschiede gefunden. Je nach Untersuchungsdesign konnten diese Unterschiede für alle oder nur einige der Verarbeitungsphasen aufgezeigt werden.

2. Als besonders sensitiv hat sich in herkömmlichen EEG-Untersuchungen die P300-Komponente erwiesen, deren zentro-parietale Amplitude und Latenz in der Memorierphase von der Reizart, in der Abrufphase hingegen von der Anzahl der memorierten Items abzuhängen scheint. Insgesamt liefern Analysen ereignisbezogener Potentiale weitere Evidenz für das Vorhandensein verschiedener Arbeitsgedächtniskomponenten, die sowohl aufgabenspezifische als auch aufgabenunspezifische Funktionen überne hmen.

3. Vergleichsweise ergiebiger, jedoch seltener ist der Einsatz von zeitlich hochauflösenden EEG-Kovarianz- und Kohärenzanalysen, die millisekundengenau Auskunft über Ausmaß und Änderung kortikaler Synchronisierungsphänomene in Abhängigkeit verschiedener kognitiver Anforderungen geben. Das Ergebnis einer solchen Studie legt nahe, daß sich verschiedene, mit dem Arbeitsgedächtnis assoziierte Aktivitätsmuster nur in denjenigen Zeitintervallen ergeben, in denen die aktivierte Information zur Aufgabenbewältigung genutzt wird. In einer anderen Untersuchung konnte gezeigt werden, daß eine erschwerte Reizverarbeitung zu einem vermehrten Koordinationsaufwand führt, der sich in einer Zunahme zentro-frontaler Aktivation widerspiegelt.

4. fMRT-Studien lassen den Schluß zu, daß sich die einmalig ablaufenden kognitiven Prozesse (wie Stimulusenkodierung und Antwortausführung) in einer transienten Aktivität sensorischer und primär motorischer Areale (Area 17/18, 22/42, 37 und 1-4) niederschlagen, während andauernde (Speicherungs- und Kontroll-)Operationen zu einer längeranhaltenden Aktivität vor allem im präfrontalen, z. T. aber auch im parieta- 
len Kortex führen (Area 9/46, 45/47, 44 sowie 40). Insgesamt sprechen die Ergebnisse für eine hierarchische Organisation der neuronalen Korrelate des Arbeitsgedächtnisses.

Die meisten neuropsychologischen Studien liefern übereinstimmende Befunde bezüglich des materialspezifischen Einflusses der gelernten Items auf die Prozesse im Arbeitsgedächtnis. Damit täuschen die Ergebnisse oft über interindividuelle Unterschiede in der Topographie und/oder Dynamik aktivierter Hirnareale hinweg. Abschließend sollen kurz einige Befunde zu diesem Thema dargestellt werden, bevor eine Kritik und zusammenfassende Bewertung der empirischen Untersuchungen erfolgt.

\subsubsection{Interindividuelle Unterschiede}

Die interindividuellen Unterschiede, die im Rahmen verschiedener Arbeiten beobachtet wurden, sind nicht auf Geschlechts-, Alters- oder Ausbildungsdifferenzen bei den Probanden zurückzuführen: Diese Variablen werden in der Regel konstant gehalten, nachdem wiederholt der Nachweis ihres Einflusses z. B. auf das EEG erbracht werden konnte (z. B. Matsuura et al., 1985; Petsche, 1990; Petsche, Pockberger \& Rappelsberger, 1985; Pratt, Michlewski, Barret \& Starr, 1989b; Rappelsberger, Krieglsteiner, Mayerweg, Petsche \& Pockberger, 1987; Vögel \& Götze, 1962). Auch der Einfluß der Übung bzw. der Vertrautheit mit dem zu lernenden Material wird in den meisten Studien berücksichtigt. Beide Variablen beeinflussen nicht nur die Abrufgeschwindigkeit, sondern auch den Zeitverlauf der Verarbeitungsprozesse und die Topographie aktivierter Hirnareale. So konnte zum einen in elektrophysiologischen Studien nachgewiesen werden, daß der Einfluß der phonologischen Gedächtnislast auf die Latenz der P300-Komponente an zentralen Elektroden mit zunehmender Übung zurückgeht (z. B. Kramer et al., 1986). Zum anderen fand Raichle (1990) in einer PET-Studie mit phonologischem Material, daß ein Gebiet im linken unteren präfrontalen Kortex zusammen mit dem Gyrus cinguli (vgl. Abb. 15) eine entscheidende Rolle beim Erwerb neuer Fähigkeiten spielt: Die genannten Gebiete waren nur bei der erstmaligen Bewältigung einer Worterzeugungsaufgabe ${ }^{36}$ aktiviert, d. h. nur dann, als die Aufgabe noch neu war und ein vergleichsweise größeres Ausmaß an Aufmerksamkeit erforderte.

Ruchkin et al. fanden in ihrer Studie von 1992 (s. o.; vgl. auch Ruchkin, Johnson, Canoune \& Ritter, 1990) trotz der herkömmlichen Kontrollen größere interindividuelle Varianzen im ERP-Muster der phonologischen verglichen mit der visuell-räumlichen Memorierphase. Diese Varianzen schwächten den Effekt der Gedächtnislast auf die Aktivität der ereignisbezogenen Potentiale. Die Ergebnisse legten nahe, die Varianzen als Ausdruck des individuell verschiedenen Timings bzw. der unterschiedlichen Strategien beim stillen Wiederholen des phonologischen Materials zu interpretieren. So könn- 
ten einige Probanden die Rehearsalrate mit dem Umfang der zu lernenden Items variiert haben, andere hingegen durchweg dieselbe schnelle Rate benutzt und damit den Zusammenhang zwischen der Latenz später negativer Potentiale und der Gedächtnislast beeinflußt haben. Im Gegensatz dazu erschienen in der visuell-räumlichen Bedingung die Effekte der Gedächtnislast in der Memorierphase insgesamt robuster und wurden auf den durchweg früheren (und damit weniger variablen) Beginn der Rehearsalprozesse zurückgeführt.

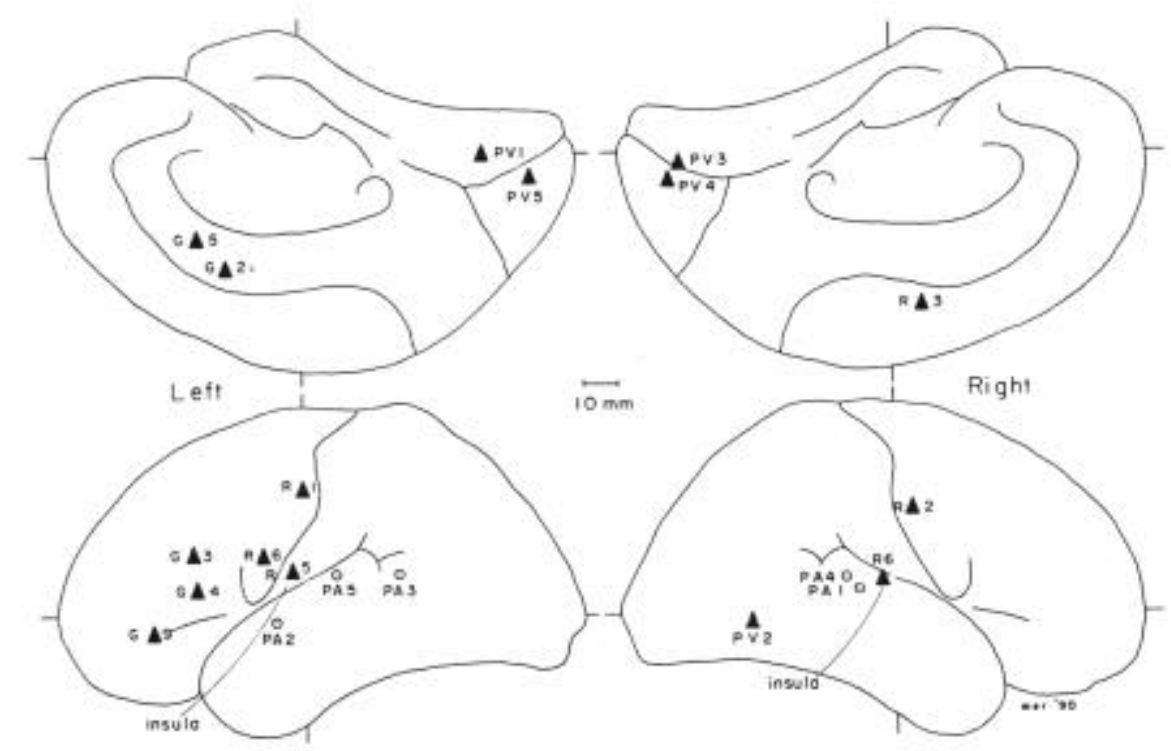

Abbildung 15. Laterale (unten) und mediale (oben) schematische Darstellung kortikaler PET-Aktivationen während verschiedener Arbeitsgedächtnisaufgaben (aus Raichle, 1990): PV/PA = Rezeption visuell bzw. akustisch dargebotener Wörter; $R=$ Wiederholung visuell dargebotener Wörter; $G=$ Erzeugung von Verben auf visuell dargebotene Substantive. In der Kontrollaufgabe hatten die Probanden einen Punkt auf dem Monitor zu fixieren.

Die Verwendung unterschiedlicher Strategien wird auch als Erklärung für die in PETStudien gefundenen Aktivationsunterschiede bei Probanden mit guter und schlechter Leistung (definiert über die Fehleranzahl) angeführt. Raife, Fiez, Raichle, Balota und Petersen (1992) ließen ihre Probanden phonologisches Material (fünf Wörter bzw. Nichtwörter) memorieren und anschließend frei wiedergeben. Bei allen Probanden waren in der Memorierphase der rechte präfrontale Kortex und das supplementäre motorische Areal (SMA) aktiviert. Probanden mit guter Leistung wiesen darüber hinaus Aktivationszunahmen im linken prämotorischen Kortex und im Gyrus cinguli auf, während bei Probanden mit schlechter Leistung Bereiche des linken visuellen Kortex und des Kleinhirns stärker aktiviert waren. Dieses Ergebnis führte zu der Annahme, daß leistungsstarke Probanden beim Memorieren eine phonologische, leistungsschwache Probanden hingegen eine visuelle Strategie benutzen (s. auch Raichle, 1993).

Andere Autoren fanden größere interindividuelle Unterschiede bezüglich der präfrontal aktivierten Areale (z. B. Cohen et al., 1994; D'Esposito et al., 1995b; Postle, 
Stern, Rosen \& Corkin, 1997). Die Mehrzahl der Probanden von D'Esposito et al. (1998b) wiesen beispielsweise sowohl bei der phonologischen als auch bei der visuell-räumlichen "n-back"-Aufgabe Aktivationen innerhalb des dorsolateralen PFC (Area 9 oder 46) auf, bei einigen erstreckte sich die Aktivation jedoch mehr nach dorsal (Area 8) oder ventral (bis Area 44/45). Über die Ursache waren zunächst nur Spekulationen möglich: So nahmen D'Esposito et al. (ebd.) an, daß diese interindividuellen Differenzen auf verschiedene Verhaltensstrategien bei der Bewältigung der komplexen Memorieraufgabe oder aber auf verschiedene Beziehungen zwischen den anatomischen Strukturen und entsprechenden kognitiven Funktionen zurückzuführen seien. Inzwischen sind Rypma und D'Esposito (1999) in einer neuen fMRT-Studie gezielt den Einflüssen interindividueller Unterschiede auf die Aktivation präfrontaler Areale nachgegangen. Sie fanden einen Zusammenhang zwischen der Geschwindigkeit, mit der die Probanden ihre Gedächtnisinhalte abriefen, und den Mustern aktivierter Hirnareale: Bei besonders langsam reagierenden Probanden spielte der dorsale PFC in der Abrufphase eine größere Rolle als der ventrale PFC. Als mögliche Ursachen wurden ineffizientere Abrufprozesse oder die geringere Qualität mnestischer Repräsentationen angeführt.

Insgesamt zeigen diese Studien, daß interindividuelle Unterschiede trotz der Konstanthaltung bestimmter Variablen (wie Geschlecht, Alter, Ausbildung und Übung) die Ergebnisse neuropsychologischer Arbeitsgedächtnisuntersuchungen beeinflussen können. Als mögliche Ursachen werden heute folgende Faktoren angenommen: Varianz der Rehearsalraten, Art der Strategie beim Memorieren, Effizienz der Abrufprozesse, Qualität der mnestischen Repräsentationen sowie Unterschiede in den Beziehungen zwischen anatomischen Strukturen und entsprechenden kognitiven Funktionen. Der Einfluß der genannten Variablen spiegelt sich physiologisch in der Topographie und Dynamik aktivierter Hirnareale und psychologisch in der Qualität der erbrachten Leistung sowie in der Geschwindigkeit der Abrufprozesse wider.

\subsection{Kritik der neuropsychologischen Untersuchungen zum Arbeitsgedächtnis}

Die Vielfalt der neuropsychologischen Untersuchungen zum Arbeitsgedächtnis unterstreicht die große Bedeutung, die diesem Themenbereich nicht nur in der psychologischen, sondern auch in der physiologisch-anatomischen Forschung beigemessen wird. Sie erschwert aber auch gleichzeitig die Formulierung zusammenfassender Aussagen zum Stand der empirischen Arbeitsgedächtnisforschung: Zum einen schränken verschiedene Kombinationen unterschiedlicher Arbeitsgedächtisaufgaben und bildgebender Verfahren die Vergleichbarkeit der vorliegenden Untersuchungen ein, zum anderen weisen viele Studien, insbesondere jene aus dem medizinischen Bereich, methodische Mängel auf, die ihre Aussagekraft aus kognitionspsychologischer Sicht erheblich min- 
dern. Vor der zusammenfassenden Bewertung sollen daher die wichtigsten Kritikpunkte der vorliegenden empirischen Untersuchungen erläutert werden.

Die traditionellen klinisch-anatomischen Studien lieferten zwar erste Evidenzen für die Existenz unterschiedlicher Arbeitsgedächtniskomponenten, die Interpretation ihrer Ergebnisse ist aber aus heutiger Sicht am stärksten problembehaftet. Die untersuchten Patienten weisen in der Regel ausgedehnte Läsionen auf, so daß eine genaue Zuordnung zwischen bestimmten Verarbeitungsprozessen und den zuständigen, meist hochspezialisierten und eng umschriebenen Hirnarealen nur bedingt möglich ist. Vorhandene Zusammenhänge zwischen lädierten Bereichen und erhaltenen kognitiven Funktionen können außerdem durch die Nutzung verschiedener Strategien zur Bewältigung kognitiver Aufgaben verschleiert werden. Darüber hinaus gestaltet es sich schwierig, die Rolle, die ein geschädigtes Hirnareal bei der Bewältigung verschiedener kognitiver Aufgaben spielt, genau zu spezifizieren: So lassen sich z. B. Enkodierungs- und Abrufprozesse bei einem hirngeschädigten Patienten nur schwer isolieren.

Aufgrund dieser und ähnlicher Kritikpunkte werden klinisch-anatomische Studien heute durch Untersuchungen an gesunden Probanden ergänzt. Bildgebende Verfahren ermöglichen dabei die nichtinvasive Erfassung der Aktivität einzelner Hirnstrukturen, die direkt mit kognitiven Verarbeitungsprozessen in Verbindung gebracht werden kann. Dies setzt allerdings kontrollierte experimentelle Anordnungen voraus. Dazu gehört die Abforderung einer genau spezifizierten Hirnleistung, deren Durchführung nach Möglichkeit mittels neuropsychologischer Testverfahren überprüft werden sollte. Die genaue Präsentation der Aufgabenstellung spielt auch bei scheinbar einfachen Hirnleistungen eine wichtige Rolle, wie Frahm (2000) an zwei Beispielen aus der visuellen Sprachwahrnehmung und der Repräsentation von Fingerbewegungen eindrucksvoll für die fMRT zeigen konnte.

Obwohl die meisten der Untersuchungen an gesunden Probanden übereinstimmende Ergebnisse liefern, die auch mit Daten klinisch-anatomischer Studien im Einklang stehen, bleibt ihr Nutzen für die Prüfung psychologischer Modelle des Arbeitsgedächtnisses eher gering. Dies liegt einerseits an der Methodik der Studien und andererseits daran, daß topographische Aspekte infolge des gröberen zeitlichen Auflösungsvermögens der verwendeten Verfahren im Mittelpunkt des Interesses stehen.

In den allermeisten Fällen kommt als bildgebendes Verfahren die PET zum Einsatz, deren Auswertung auf der Subtraktionslogik mentaler Prozesse beruht (Donders, 1868). Bei der Anwendung dieser Subtraktionslogik wird von der Prämisse ausgegangen, daß die Einführung einer weiteren Verarbeitungsstufe (z. B. einer Memorieranforderung) innerhalb einer kognitiven Aufgabe keine Auswirkung auf andere Verarbeitungsstufen (z. B. auf Wahrnehmungs- und Antwortprozesse) oder auf die vom Probanden verwendete Bearbeitungsstrategie hat. Über eine Differenzbildung zwischen zwei entsprechend 
ausgewählten Aufgaben sollen sich demzufolge diejenigen aktivierten Hirnareale abbilden lassen, die funktional mit der eingeführten Verarbeitungsstufe verknüpft sind. $\mathrm{Ob}$ wohl die Problematik ihrer zugrundeliegenden Annahmen hinlänglich bekannt ist, stellt die Subtraktionsmethode nach wie vor die klassische Auswertungsstrategie nicht nur für PET-, sondern auch für viele fMRT-Studien dar. Nur wenige Forscher umgehen die damit verknüpften Interpretationsschwierigkeiten durch den Einsatz eines hierarchischen Subtraktionskonzepts (z. B. Raichle, 1997) oder indem sie die Methode der parametrischen Variation verwenden (z. B. Braver, 1997; Smith \& Jonides, 1997), die zu den klassischen Verfahren der experimentellen Psychologie gehört. Die Ergebnisse solcher Studien zeigen jedoch, wie fruchtbar die Kombination ausgereifter psychologischer Versuchsplanung und moderner bildgebender Verfahren sein kann.

Daß vergleichsweise wenige Befunde zum Zeitverlauf der Aktivationen in neuronalen Netzwerken vorliegen, ist aus kognitionspsychologischer Sicht besonders bedauerlich, da zeitdynamische Aspekte insbesondere bei der Erstellung und Prüfung von Informationsverarbeitungsmodellen eine wichtige Rolle spielen. Darüber hinaus kann die Berücksichtigung der Zeitdimension ergänzende Hinweise auf die mutmaßliche Funktionalität verschiedener Hirnareale liefern (z. B. Cohen et al., 1997; Courtney et al., 1997). Der Einsatz moderner bildgebender Verfahren wie PET oder fMRT zur Untersuchung dynamischer Aspekte von Hirnaktivitäten während verschiedener Arbeitsgedächtnisaufgaben gestaltet sich jedoch einigermaßen problematisch. Wegen des geringen zeitlichen Auflösungsvermögens dieser Verfahren müssen künstliche Verlängerungen der Arbeitsgedächtnisaufgaben vorgenommen werden (z. B. über die Verlängerung der InterstimulusIntervalle), um mehrere PET- bzw. fMRT-Scans hintereinander durchführen und Aussagen über den Zeitverlauf treffen zu können. In solchen Fällen kann nicht ausgeschlossen werden, daß sich der ursprüngliche Aufgabencharakter ändert bzw. daß neben den interessierenden noch andere kognitive Prozesse ablaufen, die die Ergebnisinterpretation erschweren. Aber selbst bei einer Verbesserung der zeitlichen Auflösung lassen sich schnell ablaufende kognitive Prozesse mit der PET oder fMRT streng genommen nicht angemessen untersuchen, da Änderungen des Blutflusses bzw. des Blutsauerstoffgehaltes erst mehrere Sekunden nach Änderungen der neuronalen Aktivität auftreten (Raichle, 1997). Die wichtige differentielle Analyse von Enkodierungs-, Memorierund Abrufprozessen im Arbeitsgedächtnis ist mit solchen Verfahren nicht möglich.

Eine ausreichend hohe Sensitivität weisen lediglich die elektrophysiologischen bzw. elektromagnetischen Verfahren auf, die in der medizinischen Forschung aber nur vereinzelt zum Einsatz kommen, da sie synchrone elektrische bzw. magnetische Änderungen von relativ umfänglichen neuronalen Netzwerken abbilden und sich weniger zur Erfassung interessierender subkortikaler Aktivitäten eignen. EEG und EMG stellen édoch die Mittel der Wahl dar, wenn die Gültigkeit psychologischer Arbeitsgedächtnismodelle auf der physiologischen Ebene geprüft werden soll: Sie liefern eine zeitgetreue 
Abbildung neuronaler Prozesse des Neokortex, der als großer assoziativer Speicher betrachtet werden kann bzw. als Ort des Lernens und Denkens gilt (Birbaumer \& Schmidt, 1996). Damit können sehr genaue Zuordnungen zwischen neuronalen Aktivitätsänderungen und aktuellen Verarbeitungszuständen der Personen vorgenommen werden, so daß auch eine gezielte Untersuchung bestimmter kognitiver Prozesse möglich wird. Als besonders ergiebig hat sich in diesem Zusammenhang der Einsatz des EEGs erwiesen, wenn statt lokaler funktioneller Potentialänderungen die elektrischen Beziehungen zwischen verschiedenen kortikalen Regionen untersucht wurden (z.B. Gevins \& Cutillo, 1993; Krause, Gibbons \& Schack, 1998).

Zusammenfassend kann festgehalten werden, daß sich unter den vielen neuropsychologischen Untersuchungen nur wenige methodisch adäquate Studien befinden, die es erlauben, die Gültigkeit psychologischer Arbeitsgedächtnismodelle auf der physiologischen Ebene in angemessener Weise zu prüfen. 


\section{Empirischer Teil}

\section{$4 \quad$ Ableitung und Konkretisierung der eigenen Fragestellungen}

Die Konvergenz der meisten Ergebnisse aus klinisch-anatomischen Studien und aus Untersuchungen an gesunden Probanden mit bildgebenden Verfahren untermauert die Existenz verschiedener Arbeitsgedächtniskomponenten, die vor dem Hintergrund des Arbeitsgedächtnismodells von Baddeley (1998) als phonologische Schleife, visuell-räumlicher Notizblock und zentrale Exekutive definiert werden können. Während klinischanatomische Studien eine nur grobe Zuordnung dieser Komponenten zu bestimmten Hirngebieten ermöglichen, liefern Untersuchungen gesunder Probanden mit bildgebenden Verfahren genauere und differenziertere Informationen zu topographischen und zeitdynamischen Charakteristika des Arbeitsgedächtnisses. Bei der Durchsicht der neuropsychologischen Untersuchungen zum Arbeitsgedächtnis fällt jedoch auf, daß der Forschungsschwerpunkt bei der Mehrzahl der Studien in der Enkodierungs- und Memorierphase liegt und nur sehr wenige Arbeiten zu Abrufprozessen aus dem Arbeitsgedächtnis vorliegen. Dies ist zum Teil, wie bereits in Kapitel 3.3 kritisch angemerkt, auf die derzeit bevorzugte Verwendung räumlich hochauflösender bildgebender Verfahren zurückzuführen, deren zu geringes zeitliches Auflösungsvermögen die Analyse schnell ablaufender kognitiver Prozesse unmöglich macht.

Ziel der vorliegenden Arbeit soll es daher sein, das Augenmerk stärker auf die Abrufphase zu lenken und einen methodisch ausgefeilten Beitrag zur Aufklärung der zugrundeliegenden neuronalen Prozesse zu liefern. Es soll geprüft werden, ob die vorliegenden Erkenntnisse zu topographischen und zeitdynamischen Charakteristika der verschiedenen Arbeitsgedächtniskomponenten auch für diese Phase Gültigkeit besitzen. Zur Untersuchung dieser Fragestellung wurde als Arbeitsgedächtnisaufgabe die klassische Sternberg-Aufgabe (vgl. Kap. 2.1.2) und als psychophysiologisches Verfahren das EEG ausgewählt.

Das Sternberg-Paradigma ist eines der bestuntersuchten Paradigmen der Gedächtnispsychologie. In zahlreichen Experimenten konnte für die verschiedensten Stimuluskategorien (z. B. Ziffern, Farben, Wörter, Zufallsformen) die von Sternberg gefundene lineare Beziehung zwischen der Anzahl memorierter Items (Größe des positiven Sets) und der Antwortlatenz bestätigt werden (vgl. Cavanagh, 1972; Lass, 1997; Lass et al., 2001). Die Verwendung der Sternberg-Aufgabe bringt zwei wichtige Vorteile mit sich:

1. Die Merkmale der linearen Funktion (Steigungsgradient und Intercept) liefern Informationen hinsichtlich der Zeit, die die Probanden im Durchschnitt für das Durchsuchen des gelernten Sets einerseits und für weitere, von der Setgröße unabhängige Prozesse (z. B. Enkodierung, Antworterzeugung) andererseits benötigen. 
2. Der Aufbau der Sternberg-Aufgabe ermöglicht - im Gegensatz z. B. zu den weit verbreiteten "n-back"-Aufgaben - die gezielte neuropsychologische Untersuchung von Abrufprozessen aus dem Arbeitsgedächtnis.

Das EEG stellt dank seines hohen zeitlichen Auflösungsvermögens heute mehr denn je das geeignete Instrument für die Untersuchung neuronaler Korrelate des Arbeitsgedächtnisses dar. Verbesserte Analyseverfahren und die Möglichkeit, topographische Karten aktivierter Hirnregionen zu erstellen (vgl. Kap. 2.2.2), rechtfertigen darüber hinaus dessen Einsatz in der vorliegenden Untersuchung. Statt der ereignisbezogenen Potentiale sollen dabei jedoch die aussagekräftigeren elektrischen Beziehungen zwischen verschiedenen kortikalen Regionen im Mittelpunkt des Interesses stehen, so daß eine kohärenzanalytische Auswertung der elektrophysiologischen Daten angezeigt ist. Hierfür kommt ein neu entwickeltes kontinuierliches Schätzverfahren der Kohärenzfunktion zum Einsatz (vgl. Schack et al., 1995), das sich für das Studium schnell ablaufender elementarer Denkprozesse bereits bewährt hat und dessen Nutzen für die Untersuchung höherer kognitiver Prozesse in diesem Zusammenhang ebenfalls geprüft werden soll.

Die Verwendung der Sternberg-Aufgabe legt zunächst nahe, den Zusammenhang zwischen Setgröße und Antwortlatenz für das gewählte verbale und nonverbale Reizmaterial zu prüfen. Es wird vermutet, daß sich die von Sternberg beschriebene lineare Beziehung zwischen der Anzahl memorierter Items und der Antwortlatenz sowohl für positive Testitems (Testreiz in gelerntem Set vorhanden) als auch für negative Testitems (Testreiz in gelerntem Set nicht vorhanden) finden läßt. Ein solches Ergebnis würde bestätigen, daß die Probanden der Memorieraufgabe tatsächlich nachgekommen sind und daß der Versuch korrekt durchgeführt wurde.

Die geplante kohärenzanalytische Auswertung eines während der Durchführung einer Sternberg-Aufgabe aufgezeichneten EEGs ist bislang einmalig und erfordert eine eingehende Tauglichkeitsprüfung, bevor sie zur Prüfung topographischer und zeitdynamischer Hypothesen herangezogen werden kann. Über eine Analyse verschiedener kohärenzanalytischer Kennwerte soll daher festgestellt werden, ob es sich bei der unter Einsatz eines neuen Schätzverfahrens der Kohärenzfunktion durchgeführten Kohärenzanalyse um ein angemessenes Verfahren zur Untersuchung neuronaler Korrelate schnell ablaufender kognitiver Prozesse handelt.

Einen zentralen Schwerpunkt dieser Arbeit bildet die Frage nach den während der $A b-$ rufphase aktivierten Hirnarealen. In neuropsychologischen Untersuchungen besteht Einigkeit darüber, daß beim Memorieren verbalen Materials die linkshemisphärischen Areale 22, 42, 40 und 44 eine wesentliche Rolle spielen, wobei insbesondere Area 40 für die phonologische Speicherung und Area 44 für das stille Wiederholen des zu behaltenden Materials zuständig sein soll. Es wird daher erwartet, daß der Abruf verbaler 
Information zu Aktivationen in den an Sprache beteiligten kortikalen Strukturen der linken Hemisphäre führt. Hier sollten die Areale 22, 42 und 40, nicht aber das Areal 44 aktiviert sein, da in der Abrufphase kein stilles Wiederholen der gelernten Items erforderlich ist.

Beim Memorieren nonverbaler Information werden regelmäßig Aktivationen in Area 7, 40 und 37 beobachtet, wobei diese beim Lernen visuell-räumlichen Materials hauptsächlich rechtshemisphärisch, beim Lernen visuellen nichträumlichen Materials hauptsächlich linkshemisphärisch lokalisiert sind. Bezüglich des für diese Arbeit ausgewählten nonverbalen Reizmaterials wird daher angenommen, daß rechtshemisphärisch kortikale Aktivationen in den Arealen 7, 40 und 37 auftreten, nicht jedoch in den Sprachstrukturen der linken Hemisphäre.

Unabhängig von der Art des zu lernenden Materials wird in neuropsychologischen Studien immer wieder von Aktivationen im frontalen, occipitalen sowie prämotorischen Kortex berichtet. Der frontale Kortex rückt dabei mehr und mehr in den Mittelpunkt der Aufmerksamkeit, da insbesondere der präfrontale Bereich im Sinne einer "zentralen Exekutive" für die Bewältigung verschiedener kognitiver Aufgaben von größerer Bedeutung zu sein scheint. Dabei werden dem ventrolateralen präfrontalen Kortex (Area 45/47) Erhaltungsfunktionen, dem dorsolateralen präfrontalen Kortex (Area 9/46) hingegen Überwachungs- und Manipulationsfunktionen zugeschrieben. Die Art des Reizmaterials soll insofern von Bedeutung sein, als sie zu hemisphärischen Lateralisierungen der Aktivation führen kann, wobei nonverbales Reizmaterial eher rechtsseitige und verbales Reizmaterial eher linksseitige Aktivationen hervorrufen soll. In der Abrufphase dürfte sowohl das Behalten (des Testreizes) als auch das Überwachen des Vergleichsprozesses eine Rolle spielen. Es müßten also sowohl ventrolaterale als auch dorsolaterale Bereiche des präfrontalen Kortex in dieser Phase aktiviert sein. Das vergleichsweise geringe räumliche Auflösungsvermögen des EEGs reicht jedoch für die Prüfung einer solchen differenzierten Hypothese nicht aus. Es wird daher lediglich angenommen, daß während des Abrufs verbaler bzw. nonverbaler Information Aktivationen im präfrontalen Kortex auftreten, wobei sich diese Aktivationen beim Abruf verbaler Information auf den linken, beim Abruf nonverbaler Information hingegen auf den rechten präfrontalen Kortex konzentrieren sollten.

Fragen zur Dynamik kognitiver Verarbeitungsprozesse bleiben in vielen neuropsychologischen Studien vor allem aus methodischen Gründen unberücksichtigt. Dabei könnten sich heute über den Einsatz angemessener Auswertungsverfahren (z. B. EEG-Kohärenzanalysen) bzw. über gut durchdachte experimentelle Designs (z. B. parametrische Variationen) relevante Befunde zum Zeitverlauf der im Arbeitsgedächtnis stattfindenden Prozesse erzielen lassen. Vor diesem Hintergrund soll auch in dieser Untersuchung versucht werden, zur weiteren Aufklärung zeitdynamischer Aspekte des Arbeitsgedächt- 
nisses beizutragen. Da bislang keine Erkenntnisse zum Zeitverlauf elektrophysiologischer Synchronisationsprozesse speziell in der Abrufphase vorliegen, werden auch keine Hypothesen dazu aufgestellt. Stattdessen soll nach einer umfassenden Beschreibung dieser Prozesse exploratorisch nach Unterschieden in der Verarbeitung der gewählten Materialarten gesucht werden.

Die wesentlichen Ziele dieser Arbeit lassen sich wie folgt zusammenfassen:

1. Replikation der von Sternberg gefundenen linearen Beziehung zwischen Antwortlatenz und Anzahl zu memorierender Items für das gewählte verbale und nonverbale Reizmaterial

2. Prüfung der Tauglichkeit eines kontinuierlichen Schätzverfahrens der Kohärenzfunktion zur Untersuchung schnell ablaufender kognitiver Prozesse

3. Prüfung der Gültigkeit bisheriger Erkenntnisse zu topographischen Aspekten des Arbeitsgedächtnisses unter Einsatz kohärenzanalytischer Auswertungsverfa hren

4. Exploratorische Untersuchung des Zeitablaufes von Synchronisationsprozessen in der Abrufphase 


\section{$5 \quad$ Methode}

\subsection{Untersuchungsansatz}

EEG-Kohärenzanalysen erfordern die Mittelung über eine große Anzahl von Wiederholungen des Einzelversuches (Krause et al., 1997). Angesichts des potentiellen Einflusses der Übung (vgl. Kap. 3.2.3) sind zudem mehrfache Untersuchungen der Probanden zu unterschiedlichen Meßzeitpunkten angezeigt. Für die geplante Studie erschien daher eine Verlängerung der herkömmlichen Sternberg-Aufgabe erforderlich. Dabei mußte aus Motivationsgründen und wegen der begrenzten kognitiven Belastbarkeit des Menschen ein Kompromiß zwischen der Mindestanzahl benötigter Durchgänge und Meßzeitpunkte und der maximal zumutbaren Versuchslänge gefunden werden. Dieser Kompromiß ergab sich aus mehreren Vorversuchen mit freiwilligen Probanden und aus der Diskussion mit einschlägig ausgewiesenen Experten (Uta Lass und Dietrich Becker, GeorgElias-Müller-Institut für Psychologie, Göttingen; Werner Krause, Institut für Psychologie der Friedrich-Schiller-Universität, Jena; Bärbel Schack, Institut für Medizinische Statistik, Informatik und Dokumentation, Jena): Es wurde ein Untersuchungsverlaufsplan mit zwei Meßzeitpunkten bzw. Versuchssitzungen festgelegt, in denen die Probanden jeweils 768 Durchgänge absolvierten, die nicht länger als drei Stunden in Anspruch nahmen. Die Beschreibung der einzelnen Durchgänge und der Versuchsplan kann Kapitel 5.4 bzw. 5.5 entnommen we rden.

\subsection{Probanden}

Insgesamt nahmen zehn Personen an der Untersuchung teil. Bei den Probanden handelte es sich um acht Studentinnen der Fachrichtung Psychologie an der FriedrichSchiller-Universität in Jena sowie eine wissenschaftliche Mitarbeiterin und eine DiplomIngenieurin. Die Probandinnen waren zwischen 18 und 31 Jahre alt, das Durchschnittsalter lag bei 22,8 Jahre. Alle gaben an, daß ihre rechte Hand die dominante und ihre Muttersprache Deutsch sei. Fünf Probandinnen benötigten eine Sehhilfe (Brille/Kontaktlinsen), die sie während des Versuchs auch benutzten. Sieben der zehn Probandinnen hatten schon Erfahrung mit dem EEG gemacht bzw. an Versuchen teilgenommen, bei denen das EEG abgeleitet worden war. Die Teilnahme der Probandinnen am Versuch war freiwillig. Aufgrund der Versuchslänge wurden die Probandinnen mit einer Pauschale von DM 200,00 entlohnt. Einzelheiten zu den erhobenen Probandenmerkmalen sind der Tabelle A-1 (Anhang A) zu entnehmen. 


\subsection{Apparate}

Der Versuch wurde mittels eines Computers der Marke "Apple" (Typ Macintosh II FX) durchgeführt, mit dem gewährleistet war, daß die Reizdarbietungen und Antwortaufzeichnungen auf $1 \mathrm{~ms}$ genau erfolgten. Als Bildschirm wurde ein Standardfarbmonitor von Apple (14" RGB Trinitron, $67 \mathrm{~Hz}$ ) verwendet. Statt der regulären Tastatur kam eine spezielle Versuchstastatur mit nur drei Tasten zum Einsatz: Die Taste "L" diente dabei der "Ja"-Antwort, während die Taste "D" für die Antwort "Nein" verwendet wurde. Zur Orientierung waren entsprechende Kennzeichnungen über den Tasten angebracht. Als "neutrale" bzw. "weiter"-Taste fungierte die Leertaste. Alle Probandinnen bedienten die "Ja"-Taste mit dem Zeigefinger der rechten, die "Nein"-Taste mit dem Zeigefinger der linken Hand. Eine Ausbalancierung der Zuordnung der "Ja"/"Nein"-Tasten zur dominanten Hand wurde absichtlich nicht vorgenommen, um die Anzahl der möglichen Fehler im Versuch zusätzlich gering zu halten.

Für die dem ersten Versuchsdurchgang vorangestellte Übungsphase wurde statt der beschriebenen Tastatur ein sogenannter "Touch Screen" mit passendem Stift eingesetzt. Der Touch Screen ermöglicht bei nonverbalem Reizmaterial eine ebenfalls nonverbale Fragenbeantwortung, indem entsprechende Felder auf dem Bildschirm mit dem Spezialstift berührt werden (vgl. Kap. 5.5).

Zur EEG-Ableitung wurden 19 nicht polarisierbare AgCl-Elektroden (Silberplättchen-Elektroden) verwendet. Zusätzlich kamen zwei Referenzelektroden, eine Elektrode zur Erdung und zwei Augenelektroden zur Artefaktkontrolle zum Einsatz. Für das Ausmessen des Kopfes wurde ein herkömmliches Maßband und für das Markieren der Elektrodenpositionen auf der Kopfhaut ein blauer Filzstift verwendet.

Die Aufzeichnung des EEGs erfolgte über die bewährte Hard- und Software der Marke "Brainstar". Die Anlage bestand aus einem Rechner mit umfangreicher Speicherkapazität und einem Standardbildschirm zur unmittelbaren Kontrolle des abgeleiteten EEGs. Bedauerlicherweise wurde nach der Untersuchung der ersten beiden Probandinnen eine neuere Programmversion installiert, so daß die Aufzeichnungsbedingungen nicht für alle Versuchsteilnehmerinnen konstant gehalten werden konnten. Tabelle 4 gibt einen Überblick über die entsprechenden Einstellungen.

Tabelle 4

EEG-Aufzeichnungsbedingungen bei den verschiedenen Probandinnen

\begin{tabular}{|l|c|c|}
\cline { 2 - 3 } \multicolumn{1}{c|}{} & Pb 1 und 2 & Pb 3 bis 10 \\
\hline Empfindlichkeit: & $119 \mu \mathrm{V} / \mathrm{cm}$ & $303 \mu \mathrm{V} / \mathrm{cm}$ \\
\hline Filter: & $70 \mathrm{~Hz}$ & $70 \mathrm{~Hz}$ \\
\hline Zeitkonstante: & $1.0 \mathrm{sec}$ & $0.3 \mathrm{sec}$ \\
\hline Abtastrate: & $250 \mathrm{~Hz}$ & $256 \mathrm{~Hz}$ \\
\hline Marker: & Port & Port \\
\hline
\end{tabular}




\subsection{Versuchsmaterial}

Um die Untersuchung verbaler und nonverbaler Informationsverarbeitung beim Abruf kurzzeitig gespeicherten Reizmaterials zu ermöglichen, mußten passende Items aus verschiedenen Reizkategorien ausgewählt werden. Dabei fiel die Wahl zum einen auf deutsche Zahlwörter und zum anderen auf sogenannte "Zufallsfiguren". Für den Versuch wurden die Zahlwörter "Null" bis "Neun" und zehn verschiedene, von Vanderplas und Garvin (1959) entworfene Zufallsfiguren verwendet. Der Einsatz der sehr geläufigen Zahlwörter sollte sicherstellen, daß eine verbale Informationsverarbeitung seitens der Probanden erfolgt. Im Gegenzug sollte durch die als nicht oder nur schwer verbalisierbar angesehenen Zufallsfiguren eine nonverbale Informationsverarbeitung nahegelegt und ein verbales Vorgehen beim Memorieren und Abrufen des Reizmaterials weitgehend ausgeschlossen werden. Abbildung 16 gibt einen Überblick über das verwendete Reizmaterial.

\begin{tabular}{|c|c|}
\hline \multicolumn{2}{|c|}{ Verwendete Reizkategorien und Lernitems } \\
\hline Zahlwörter & Zufallsfiguren \\
\hline Null & \\
\hline Eins & \\
\hline Zwei & \\
\hline Drei & \\
\hline Vier & \\
\hline Fünf & \\
\hline Sechs & \\
\hline Sieben & \\
\hline Acht & \\
\hline Neun & \\
\hline
\end{tabular}

Abbildung 16. Verwendetes Reizmaterial 
Die Zahlwörter und Zufallsfiguren erschienen den Probanden in schwarz auf weißem Hintergrund. Die Buchstabenhöhe der im Schrifttyp "Helvetica" dargebotenen Zahlwörter betrug 6,5 mm, die Wortlänge variierte je nach Anzahl der Buchstaben zwischen 14 und $24 \mathrm{~mm}$. Die im Durchschnitt von den Zufallsfiguren bedeckte Bildschirmfläche belief sich auf 12,5 x 21,9 mm (Breite x Höhe). Da der durchschnittliche Abstand der Augen vom Bildschirm etwa $60 \mathrm{~cm}$ betrug, kann somit von einem Sehwinkel von maximal $2.5^{\circ}$ ausgegangen werden.

\section{5 Übungsphase und Versuchsaufgabe}

Während Zahlwörter sehr geläufige verbale Reize darstellen, die bereits im Langzeitgedächtnis repräsentiert sind, handelt es sich bei den Zufallsfiguren um wenig vertrautes (bzw. im Langzeitgedächtnis nicht repräsentiertes) Reizmaterial. Daher diente die dem eigentlichen Versuch vorangestellte Übungsphase dazu, die Probandinnen mit den Zufallsfiguren soweit vertraut zu machen, daß sie im Versuch selbst möglichst wenige Falschantworten gaben. In jedem Durchgang wurde ihnen eine einzelne, zufällig ausgewählte Figur gezeigt, die gemerkt werden sollte. Anschließend erschienen alle zehn Figuren in einer Zufallsverteilung gleichzeitig auf dem Bildschirm, und die Probandinnen hatten die zuvor dargebotene Figur mit dem Spezialstift auf dem Touch Screen zu berühren. Dabei kam es nicht darauf an, möglichst schnell zu antworten, sondern sich intensiv mit den wenig geläufigen Zufallsfiguren zu befassen und keine Fehler zu machen.

Nachfolgend wird ein Überblick über die genaue Ereignisabfolge in einem Durchgang der Übungsaufgabe wiedergegeben:

- Auf dem Bildschirm erscheint folgender Text: "Bitte zum Starten eine Taste drücken."

- Die Probandin drückt eine der Tasten auf der Versuchstastatur.

- Pausenintervall von 500 ms.

- Eine der zehn Zufallsfiguren erscheint für 1000 ms auf dem Bildschirm.

- Alle zehn Zufallsfiguren werden dargeboten und bleiben so lange sichtbar, bis die Probandin eine von ihnen mit dem Spezialstift auf dem Touch Screen berührt.

- Die Probandin tippt eine der zehn Figuren mit dem Spezialstift auf dem Touch Screen an.

- Bei einer richtigen Antwort erscheint auf dem Bildschirm keine explizite Rückmeldung. Bei einer falschen Antwort erscheint für 1500 ms die Meldung "Leider nicht richtig".

- Es folgt die Meldung: "Bitte zum Starten eine Taste drücken."

- Die Probandin drückt eine der Tasten auf der Versuchstastatur.

- Auf dem Bildschirm erscheint wieder eine der zehn Zufallsfiguren usw.

Die im Versuch verwendete Aufgabe bestand darin, sich eine kurze Abfolge der oben beschriebenen Stimuli (Zahlwörter oder Zufallsfiguren) zu merken und nach anschlie- 
Bender Darbietung eines Test- bzw. Prüfreizes so schnell wie möglich zu entscheiden, ob dieser Teil der zuvor gesehenen Itemabfolge war oder nicht. Dieses Vorgehen entspricht der von Sternberg eingeführten Itemwiedererkennungsaufgabe und wird daher im folgenden als "Sternberg-Aufgabe" bezeichnet.

Die Sternberg-Aufgabe kann, wie bereits in Kapitel 2.1.2 erläutert, auf zwei verschiedene Arten durchgeführt werden. Im vorliegenden Fall wurde die sogenannte "variedset procedure" verwendet, bei der sich der Proband in jedem Durchgang eine neue Itemliste (positiver Set) merken muß. Die zu lernenden Items wurden seriell dargeboten; ihre Anzahl (im folgenden "Setgröße" genannt) variierte von eins bis vier, so daß die Gedächtnisspanne nicht überschritten wurde. Nach Darbietung des positiven Sets erschien auf dem Bildschirm ein Vorwarnreiz, der den Testreiz bzw. das Testitem ankündigte. Die Probandinnen drückten die ihrer Entscheidung entsprechende Taste auf der Versuchstastatur, und der Rechner speicherte die Antwortlatenz, die Antwortart (Ja/ Nein) sowie die Falschantworten in ein Datenfile.

Jede Probandin absolvierte 768 Durchgänge pro Sitzung, die sich auf 12 Blöcke verteilten. Mit jedem Block wechselte die zu memorierende Reizart, wobei der Versuch aber immer mit den Zufallsfiguren begonnen wurde. Innerhalb eines Blocks wurden die vier Setgrößen jeweils insgesamt 16 mal dargeboten; dabei war in acht Durchgängen eine positive und in weiteren acht Durchgängen eine negative Antwort gefordert. Die Abfolge der Setgrößen und der beiden Antwortarten innerhalb eines Blocks wurde durch das Versuchssteuerungsprogramm randomisiert. Auch die Position, die positive Testitems innerhalb des positiven Sets einnahmen, wurde nach dem Zufallsprinzip variiert. Jedes Lernitem kam nur einmal pro Set vor.

Die exakte Ereignisabfolge in einem Durchgang der Versuchsaufgabe gestaltete sich wie folgt:

- Auf dem Bildschirm erscheint folgender Text: "Bitte zum Starten eine Taste drücken."

- Die Probandin drückt eine der Tasten auf der Versuchstastatur.

- Pausenintervall von 500 ms.

- Die Items des positiven Sets erscheinen einzeln nacheinander für jeweils $1000 \mathrm{~ms}$ auf dem Bildschirm. Das Interitemintervall beträgt $200 \mathrm{~ms}$.

- Es folgt eine Pause von 2000 ms. 200 ms vor dem Ende dieser Pause erscheint ein Vorwarnreiz $(\otimes)$, der das Testitem ankündigt.

- Das Testitem wird dargeboten. Es bleibt so lange sichtbar, bis die Probandin eine der Antworttasten gedrückt hat.

- Die Probandin drückt entweder die Ja- oder die Nein-Taste auf der Versuchstastatur.

- Bei einer richtigen Antwort erscheint auf dem Bildschirm für 1000 ms die gemessene Antwortlatenz. Bei einer falschen Antwort wird die Rückmeldung "Falsch!" erteilt.

- Es folgt eine Pause von 2000 ms.

- Auf dem Bildschirm erscheint wieder der Text: "Bitte zum Starten eine Taste drücken." 


\subsection{Versuchsdurchführung}

Die Untersuchung der zehn Probandinnen fand in der Zeit von Ende September bis Anfang Dezember 1997 im Institut für Psychologie der Friedrich-Schiller-Universität in Jena statt. Jede Probandin wurde einzeln in zwei Sitzungen untersucht, zwischen denen in allen Fällen ein Tag Pause lag. Der genaue Versuchsplan kann Tabelle 5 entnommen werden.

\section{Tabelle 5}

Versuchsplan

\begin{tabular}{|c|c|c|c|c|c|c|c|c|c|c|c|c|c|c|c|}
\hline \multirow[b]{2}{*}{$\mathrm{Pb}$} & \multirow[b]{2}{*}{$\begin{array}{l}\text { Sit- } \\
\text { zung }\end{array}$} & \multirow[b]{2}{*}{ Tag } & \multirow[b]{2}{*}{$\begin{array}{l}\text { Übungs- } \\
\text { aufgabe }\end{array}$} & \multicolumn{12}{|c|}{ Block } \\
\hline & & & & 1 & 2 & 3 & 4 & 5 & 6 & 7 & 8 & 9 & 10 & 11 & 12 \\
\hline 1 & 1 & 30.09 .97 & ja & $\mathrm{F}$ & W & $\mathrm{F}$ & W & $\mathrm{F}$ & W & $\mathrm{F}$ & W & $\mathrm{F}$ & W & $\mathrm{F}$ & W \\
\hline 2 & 1 & 30.09 .97 & ja & $\mathrm{F}$ & W & $\mathrm{F}$ & W & $\mathrm{F}$ & W & $\mathrm{F}$ & W & $\mathrm{F}$ & W & $\mathrm{F}$ & W \\
\hline 1 & 2 & 02.10 .97 & nein & $\mathrm{F}$ & W & $F$ & W & $F$ & W & $F$ & W & $F$ & W & $F$ & W \\
\hline 2 & 2 & 02.10 .97 & nein & $\mathrm{F}$ & W & $\mathrm{F}$ & W & $\mathrm{F}$ & W & $\mathrm{F}$ & W & $\mathrm{F}$ & W & $\mathrm{F}$ & W \\
\hline 3 & 1 & 03.11 .97 & ja & $\mathrm{F}$ & W & $\mathrm{F}$ & W & $F$ & W & $\mathrm{F}$ & W & $F$ & W & $F$ & W \\
\hline 4 & 1 & 03.11 .97 & ja & $\mathrm{F}$ & W & $\mathrm{F}$ & W & $\mathrm{F}$ & W & $\mathrm{F}$ & W & $F$ & W & $\mathrm{F}$ & W \\
\hline 5 & 1 & 04.11 .97 & ja & $\mathrm{F}$ & W & $F$ & W & $F$ & W & $F$ & W & $F$ & W & $F$ & W \\
\hline 6 & 1 & 04.11 .97 & ja & $\mathrm{F}$ & W & $F$ & W & $F$ & W & $\mathrm{F}$ & W & $\mathrm{F}$ & W & $F$ & W \\
\hline 3 & 2 & 05.11 .97 & nein & $\mathrm{F}$ & W & $\mathrm{F}$ & W & $\mathrm{F}$ & W & $\mathrm{F}$ & W & $\mathrm{F}$ & W & $\mathrm{F}$ & W \\
\hline 4 & 2 & 05.11 .97 & nein & $\mathrm{F}$ & W & $F$ & W & $F$ & W & $\mathrm{F}$ & W & $\mathrm{F}$ & W & $F$ & W \\
\hline 5 & 2 & 06.11 .97 & nein & $\mathrm{F}$ & W & $\mathrm{F}$ & W & $\mathrm{F}$ & W & $\mathrm{F}$ & W & $\mathrm{F}$ & W & $\mathrm{F}$ & W \\
\hline 6 & 2 & 06.11 .97 & nein & $\mathrm{F}$ & W & $F$ & W & $F$ & W & $F$ & W & $F$ & W & $F$ & W \\
\hline 7 & 1 & 02.12 .97 & ja & $\mathrm{F}$ & W & $F$ & W & $\mathrm{F}$ & W & $\mathrm{F}$ & W & $\mathrm{F}$ & W & $\mathrm{F}$ & W \\
\hline 8 & 1 & 02.12 .97 & ja & $F$ & W & $F$ & W & $F$ & W & $F$ & W & $F$ & W & $F$ & W \\
\hline 9 & 1 & 03.12 .97 & ja & $\mathrm{F}$ & W & $F$ & W & $F$ & W & $\mathrm{F}$ & W & $F$ & W & $F$ & W \\
\hline 10 & 1 & 03.12 .97 & ja & $F$ & W & $F$ & W & $F$ & W & $F$ & W & $F$ & W & $F$ & W \\
\hline 7 & 2 & 04.12 .97 & nein & $F$ & W & $F$ & W & $F$ & W & $\mathrm{F}$ & W & $\mathrm{F}$ & W & $F$ & W \\
\hline 8 & 2 & 04.12 .97 & nein & $\mathrm{F}$ & W & $\mathrm{F}$ & W & $\mathrm{F}$ & W & $\mathrm{F}$ & W & $\mathrm{F}$ & W & $\mathrm{F}$ & W \\
\hline 9 & 2 & 05.12 .97 & nein & $\mathrm{F}$ & W & $F$ & W & $F$ & W & $\mathrm{F}$ & W & $\mathrm{F}$ & W & $F$ & W \\
\hline 10 & 2 & 05.12 .97 & nein & $\mathrm{F}$ & W & $\mathrm{F}$ & W & $\mathrm{F}$ & W & $\mathrm{F}$ & W & $\mathrm{F}$ & W & $\mathrm{F}$ & W \\
\hline
\end{tabular}

Anmerkungen: F = Reizart Zufallsfiguren, W = Reizart Zahlwörter

Das Setzen der Elektroden erfolgte nach dem internationalen 10-20-System (vgl. Kap. 3.2.2, Abb. 11). Dieses System geht von vier Standardpunkten des Kopfes aus: dem Nasion, dem Inion und dem linken und rechten präaurikulären Punkt. Die Positionen aller Elektroden sind durch prozentuale Abstände (10\% oder $20 \%$ ) zu diesen Standardpunkten festgelegt. Durch die gleichen Elektrodenabstände entlang jeder antero-posterioren oder transversalen Reihe gilt das 10-20-System als exakt und gewährleistet eine umfassende Überdeckung der Hemisphären. Das Ausmessen des Kopfes und Markieren der Elektrodenpositionen sowie das Kleben der Elektroden nahm die 
Versuchsleiterin vor, wobei ihr eine erfahrene EEG-Assistentin zur Seite stand. Diese Prozedur nahm etwa 45 Minuten in Anspruch. Nach Anbringen und Anschließen der Elektroden verließ die EEG-Assistentin den Raum, so daß während des Versuches neben der Probandin nur noch die Versuchsleiterin anwesend war.

Die Dauer der Übungsphase, die lediglich in der ersten Sitzung stattfand, betrug etwa 15 Minuten. Die Dauer des Sternberg-Versuchs belief sich in den Erstsitzungen auf etwa drei Stunden. Aufgrund der bereits bestehenden Vertrautheit mit den Aufgabenanforderungen bewältigten die Probandinnen den Versuch in den Zweitsitzungen innerhalb von 2 1/2bis 2 3/4Stunden.

Alle Versuche fanden in demselben Raum statt, der über zwei Fenster verfügte. Um eventuelle Spiegelungen auf dem Versuchsbildschirm durch eintretendes Tageslicht zu vermeiden, wurde der Raum durch Vorhänge mäßig verdunkelt. In den Nachmittagssitzungen wurde rechtzeitig vor Einbruch der Dunkelheit für eine indirekte Beleuchtung durch drei kleinere Lampen gesorgt.

\subsection{Versuchsablauf}

Jede Probandin wurde zunächst durch die Versuchsleiterin und durch die EEG-Assistentin begrüßt und gebeten, auf einem Stuhl Platz zu nehmen. Alle Teilnehmerinnen waren schon vorweg darüber informiert worden, daß es sich um eine EEG-Untersuchung handelte. Daher konnte nach einer kurzen Erläuterung der Vorgehensweise direkt mit dem Anbringen der Elektroden begonnen werden. Der Kopf wurde zunächst mit einem Maßband ausgemessen, und die einzelnen Elektrodenpositionen wurden mit einem blauen Filzstift gekennzeichnet. Anschließend wurde die Kopfhaut an den markierten Stellen mit einer Spezialemulsion leicht aufgerauht, bevor die einzelnen Elektroden mit einer Spezialpaste aufgeklebt wurden. Das schmerzfreie Aufrauhen der Kopfhaut verbessert die Haftung und vermindert die Übergangswiderstände der Elektroden. Zur Sicherheit wurden alle Elektroden zusätzlich mit einem Pflaster auf der Kopfhaut befestigt.

Das Anbringen der Elektroden erfolgte immer nach demselben Schema: Als erstes wurden, mit den frontalen Elektroden Fp1 und Fp2 beginnend, die Skalpelektroden gesetzt; anschließend wurden die Referenzelektroden hinter den Ohren, die Elektroden zur Artefaktkontrolle am äußeren Ende der Augenbrauen und schließlich eine Elektrode zur Erdung am Hals der Probandin angebracht. Nach dem Setzen der Elektroden wurde die Probandin gebeten, sich in einen bequemen Stuhl vor den Versuchscomputer zu setzen. Die Tastatur wurde auf ein speziell angefertigtes Brett gelegt, das quer über die Armlehnen des Stuhls positioniert wurde und ein müheloses Erreichen der benötigten 
Tasten ermöglichte. Der etwa in Augenhöhe aufgestellte Bildschirm stand ungefähr $60 \mathrm{~cm}$ von der Probandin entfernt (vgl. Anhang A, Abb. A-1).

Während die Probandin ihre optimale Sitzposition suchte, erfolgte das AnschlieBen der Elektroden an den EEG-Verstärker, wobei selbstverständlich auf die richtige Verknüpfung zwischen den Elektroden und den einzelnen Kanälen am Verstärker geachtet wurde. Die Zeit, die für die Vorbereitungen zur EEG-Ableitung erforderlich war, wurde von der Versuchsleiterin und von der EEG-Assistentin dazu genutzt, die Probandin in ein zwangloses Gespräch zu verwickeln, um so eventuell vorhandene Ängste und Spannungen abzubauen.

Vor dem eigentlichen Versuchsbeginn verabschiedete sich die EEG-Assistentin und verließ den Raum. Die Probandin erhielt ein erstes Instruktionsblatt, mit dem sie über das Untersuchungsziel und die voraussichtliche Untersuchungsdauer aufgeklärt und in dem inr die Anonymität der Datenspeicherung zugesichert wurde (s. Anhang A, Seite A2). Darüber hinaus bekam sie in der ersten Sitzung einen einseitigen Fragebogen, mit dem Informationen über Alter, Beruf, Geschlecht, Sehtüchtigkeit, Händigkeit sowie über Erfahrungen mit EEG-Ableitungen erhoben wurden (s. Anhang A, Seite A3). In dieser Zeit prüfte die Versuchsleiterin den Übergangswiderstand der Elektroden; dieser lag stets unter $10 \mathrm{k} \Omega$ und damit im zulässigen Bereich.

Nach dem Ausfüllen des Fragebogens wurde der Probandin mitgeteilt, daß nun als erstes etwa zwei Minuten lang ein Ruhe-EEG aufgenommen werden würde. Sie wurde gebeten, nicht zu sprechen, sich zurückzulehnen und sich mit geschlossenen Augen solange entspannt und ruhig zu verhalten, bis sie die Aufforderung zum Öffnen der Augen bekäme.

Im Anschluß an die Aufzeichnung des Ruhe-EEGs bekam die Probandin in der ersten Sitzung weitere schriftliche Instruktionen. Mit dem zweiten Instruktionsblatt wurde ihr die Sternberg-Aufgabe vorgestellt und der genaue Ablauf dessen, was im folgenden auf dem Computerbildschirm zu sehen sein würde, skizziert (s. Anhang A, Seite A4). Die Instruktion enthielt auch einen Hinweis auf die vor dem eigentlichen Versuchsbeginn stattfindende Übungsphase.

Nachdem die Probandin die zweite Instruktion gelesen und verstanden hatte, wurden ihr die im Versuch verwendeten Reize - Zufallsfiguren und Zahlwörter - einzeln auf dem Bildschirm vorgeführt. Auf Wunsch wurde die Vorführung auch wiederholt. Danach erhielt die Probandin die Instruktion für die Übungsphase (vgl. Anhang A, Seite A5), in der sie sich mit den Zufallsfiguren vertraut machen konnte. In der zweiten Sitzung wurden die einzelnen Reize nur bei Bedarf noch einmal vorgeführt. Da hier die Übungsphase aufgrund der bereits bestehenden Vertrautheit mit den Zufallsfiguren entfallen konnte, wurde gleich im Anschluß mit der Sternberg-Aufgabe begonnen. 
In der ersten Sitzung wurde der eigentliche Versuch nach der Übungsphase gestartet. Der Probandin wurde hierfür ein letztes Instruktionsblatt ausgehändigt, das wichtige Hinweise zur geforderten Lernleistung und zum Verhalten während der Versuchsdurchgänge enthielt (s. Anhang A, Seite A6). Dadurch sollte zweierlei sichergestellt werden: Einerseits sollte sich die Probandin die Reize auf eine bestimmte Art und Weise merken (nämlich bei Zufallsfiguren eine "bildliche", bei Zahlwörtern hingegen eine "verbale" Speicherung vornehmen), andererseits sollten Artefakte, die durch Augenzwinkern, Sprechen, unregelmäßige Atmung usw. während der EEG-Aufzeichnung auftreten können, weitgehend vermieden werden.

Nach der Beantwortung etwaiger anstehender Fragen begann die Versuchsleiterin mit der EEG-Aufzeichnung, und die Probandin startete durch Tastendruck die einzelnen Durchgänge. Nach jedem Block fand eine kurze Pause (etwa zwei Minuten) statt, da vor jedem Reizartwechsel eine Datensicherung vorgenommen werden mußte. Weitere kurze Pausen wurden zwangsläufig immer dann eingelegt, wenn Elektroden nachgeklebt oder ausgetauscht werden mußten.

Nach dem fünften und nach dem neunten Block (d. h. nach dem 320. bzw. 576. Durchgang) erfolgte eine längere Pause von etwa 10 bis 15 Minuten, in der der Probandin Kaffee oder Saft und Kekse gereicht wurden. Der Bildschirm wurde zu ihrer Entlastung verdunkelt und der Raum bei Bedarf gelüftet. Die Probandin konnte wegen der angeschlossenen Elektroden nicht aufstehen und wurde daher gebeten, sich in der Pause so weit wie möglich auf ihrem Stuhl zu entspannen. Sie erhielt Gelegenheit, Fragen zu stellen sowie über sich und den Versuch zu sprechen. Kurz vor Pausenende wurde die Probandin noch einmal daran erinnert, auf welche Art und Weise sie sich die Reize merken und wie sie sich während der einzelnen Versuchsdurchgänge verhalten sollte.

Nach dem zwölften und letzten Block stoppte die Versuchsleiterin zunächst die EEGAufzeichnung und sicherte die Daten. Dann nahm sie der Probandin die Elektroden ab und befragte sie dabei noch einmal zum Versuch. Von den Elektroden befreit, erhielt die Probandin Haarwaschmittel, Handtuch und Fön, um an einem Waschbecken Rückstände der Klebepaste von der Kopfhaut entfernen zu können. Anschließend wurde die Probandin (in der ersten Sitzung) an den zweiten Versuchstermin erinnert. Vor der Verabschiedung bedankte sich die Versuchsleiterin für die Teilnahme. Das versprochene Entgelt von DM 200,00 wurde der Probandin am Ende der zweiten Sitzung ausgehändigt.

\subsection{Statistische Auswertung}

Zur Klärung der dieser Arbeit zugrundeliegenden Fragestellungen wurden verschiedene statistische Tests verwendet. Dabei handelte es sich - neben Regressions- und Korre- 
lationsanalysen - in der Regel um t-Tests für abhängige Stichproben. Vereinzelt wurden auch Wilcoxon-Tests durchgeführt, wenn Unsicherheit darüber bestand, ob die auszuwertenden Daten die Voraussetzungen verteilungsgebundener Verfahren erfüllten. Den Ergebnistabellen können unter anderem die genauen Irrtumswahrscheinlichkeiten ( $p$ Werte) der einzelnen Tests und die Effektstärken $(\boldsymbol{\varepsilon})$ entnommen werden. Als signifikant (bzw. sehr signifikant) wurden diejenigen Tests erachtet, die einen $p$-Wert von maximal 0.05 (bzw. 0.01) aufwiesen. Die Größe des experimentellen Effekts wurde in Anlehnung an Cohen (1988) bestimmt: Effekte ab $\varepsilon=0.2$ gelten dabei als klein, ab $\varepsilon=$ 0.5 als mittelgroß und $a b \varepsilon=0.8$ als groß .

Während der einzelnen Durchgänge im Sternberg-Versuch erfolgte eine fortlaufende Aufzeichnung der elektrophysiologischen Signale der Probandinnen (vgl. Kap. 5.7). Dabei wurde automatisch für jedes Ereignis im Versuchsablauf ein sogenannter "Marker" gesetzt: Während mit den Anfangsmarkern 1 bis 4 der Beginn der Darbietung der Lernitems pro Setgröße kodiert wurde, stand der Marker 7 für die Präsentation des Testitems; die Marker 8 bis 10 kodierten die Antwort der Probandinnen ( $8=$ richtige positive Antwort; $9=$ richtige negative Antwort; $10=$ falsche Antwort), und der Marker 14 kennzeichnete das Ende des jeweiligen Durchgangs (vgl. Abb. 17). Auf diese Weise konnten ausgewählte EEG-Abschnitte kohärenzanalytisch untersucht und sehr genaue Zuordnungen des Verlaufs dieser Abschnitte zu aktuellen Verarbeitungszuständen der Probandinnen vorgenommen werden.

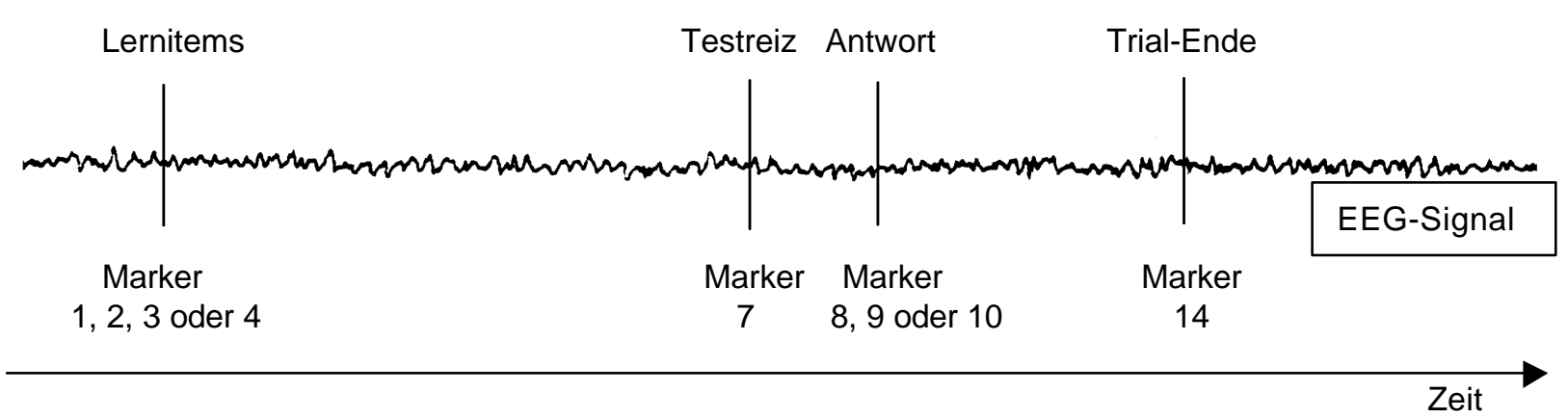

Abbildung 17. Zum EEG-Signal hinzugefügte Marker und deren Bedeutung

Die kohärenzanalytische Auswertung erfolgte unter Einsatz eines in der Zeit kontinuierlichen Schätzverfahrens der Kohärenzfunktion. Dieser von Schack entwickelte Ansatz (vgl. Schack et al., 1995) erlaubt eine hohe Zeit- und Frequenzauflösung. Die Ausgangssignale zweier EEG-Kanäle werden dabei als instationärer, zweidimensionaler Prozeß verstanden, der über die Anpassung eines bivariaten linearen Modells (sogenanntes ARMA-Modell) mit zeitabhängigen Parametern abgebildet wird. Die zeitabhängigen Modellparameter erlauben die parametrische Berechnung der Spektraldichtematrix des angepaßten ARMA-Modells zu jedem Abtastzeitpunkt. Aus dieser momentanen Spektraldichtematrix, die eine Annäherung an die Spektraldichtematrix der zugrundeliegen- 
den EEG-Kanäle darstellt, erfolgt eine kontinuierliche Schätzung der Kohärenz (für eine detaillierte Darstellung des Verfahrens vgl. Schack, 1999, bzw. Schack, Grieszbach \& Krause, 1998).

Momentane Kohärenzwerte sind unabhängig von den Amplituden der zu vergleichenden Signale (Cooper, Osselton \& Shaw, 1984). Sie geben einen Hinweis auf den Grad der Kopplung der elektrischen Tätigkeit zweier Hirnregionen, beziehen sich auf bestimmte Frequenzbänder und liegen wie Korrelationskoeffizienten zwischen 0 und 1. Die Kohärenzwerte wurden für den Frequenzbereich $13-20 \mathrm{~Hz}$ berechnet, da höhere Kognitionen bekanntermaßen vor allem im $\beta$-Band zu charakteristischen Veränderungen führen (v. Stein et al., 1993; Petsche, Rappelsberger \& Pockberger, 1987) und sich dieser Frequenzbereich bereits für kognitive Elementarprozesse als besonders sensibel erwiesen hat (Schack \& Krause, 1995). Untersucht wurden die von 30 lokalen Elektrodenpaaren abgeleiteten elektrophysiologischen Signale (vgl. Abb. 18).

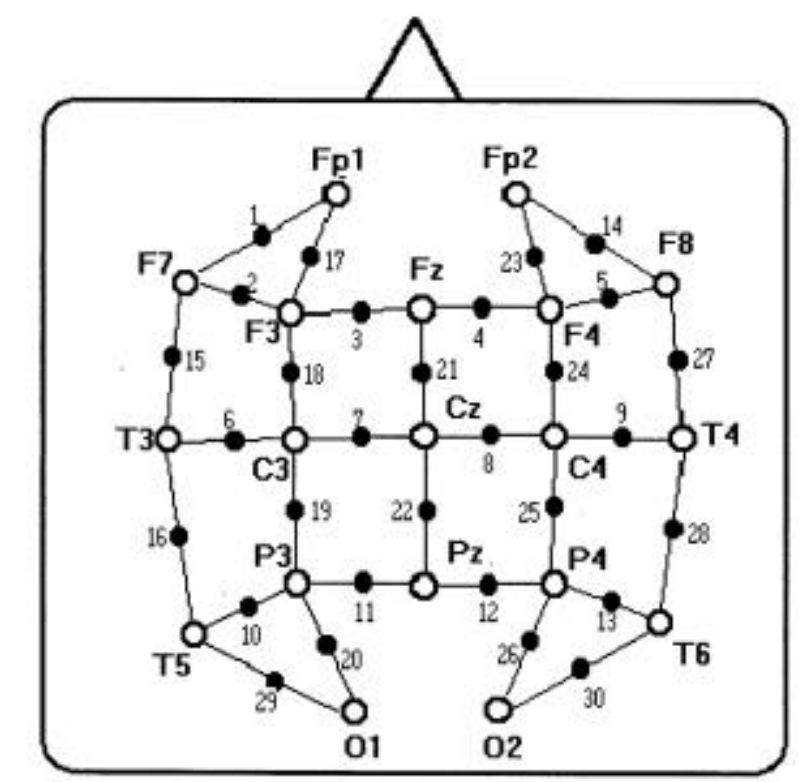

Bezeichnungen und numerische Kodierung der Elektrodenpaare:

Fp1/F7 (1), F7/F3 (2), F3/Fz (3), Fz/F4 (4),

F4/F8 (5), T3/C3 (6), C3/Cz (7), Cz/C4 (8),

C4/T4 (9), T5/P3 (10), P3/Pz (11), Pz/P4 (12),

P4/T6 (13), Fp2/F8 (14), F7/T3 (15), T3/T5 (16),

Fp1/F3 (17), F3/C3 (18), C3/P3 (19), P3/O1 (20),

$\mathrm{Fz} / \mathrm{Cz}$ (21), Cz/Pz (22), Fp2/F4 (23), F4/C4 (24),

C4/P4 (25), P4/O2 (26), F8/T4 (27), T4/T6 (28),

T5/O1 (29), T6/O2 (30)

Abbildung 18. Übersicht über die 30 lokalen Elektrodenpaare, von denen elektrophysiologische Signale abgeleitet und kohärenzanalytisch ausgewertet wurden. Die weißen Kreise stehen für die gesetzten Elektroden; die schwarzen Kreise verbinden jeweils zwei Elektroden, für die mittlere Bandkohärenzen gerechnet wurden.

Die Bandkohärenzen bilden die Grundlage für die Berechnung weiterer kohärenzanalytischer Kennwerte: Es sind dies der mittlere Kohärenzwert, die Kohärenzdauer, der maximale Kohärenzwert und der Zeitpunkt der maximalen Kohärenz. Abbildung 19 gibt einen Überblick über diese Kennwerte, die für jedes der 30 Elektrodenpaare berechnet wurden. 
Der mittlere Kohärenzwert errechnet sich aus der Summe aller Kohärenzwerte dividiert durch die Anzahl von Abtastzeitpunkten. Damit gibt er Auskunft über das in einem definierten Zeitintervall im Mittel aufgetretene Synchronisationsausmaß.

Die Kohärenzdauer ist die kumulierte Zeitdauer, für die die Bandkohärenz eine bestimmte Schwelle im definierten Zeitintervall überschreitet (vgl. Krause, Schack, Gibbons \& Kriese, 1997). Für die vorliegende Untersuchung wurde eine Schwelle von 0.65 gewählt, nachdem sich die übliche Schwelle von 0.8 aufgrund eigener Voruntersuchungen am erhobenen Datenmaterial als wenig ergiebig erwiesen hatte.

Der maximale Kohärenzwert steht für das höchste erreichte Synchronisationsausmaß, der Zeitpunkt maximaler Kohärenz (bzw. die Maximumzeit) für dessen Auftretenszeitpunkt im interessierenen Intervall. Beide Kennwerte wurden für das Zeitintervall ab 120 ms nach Darbietung des Testitems bis zum Drücken der Antworttaste berechnet, um frühe Maximumzeiten, die vermutlich Ausdruck der kognitiven Verarbeitung des Testitems sind, aus der Analyse auszuschließen.

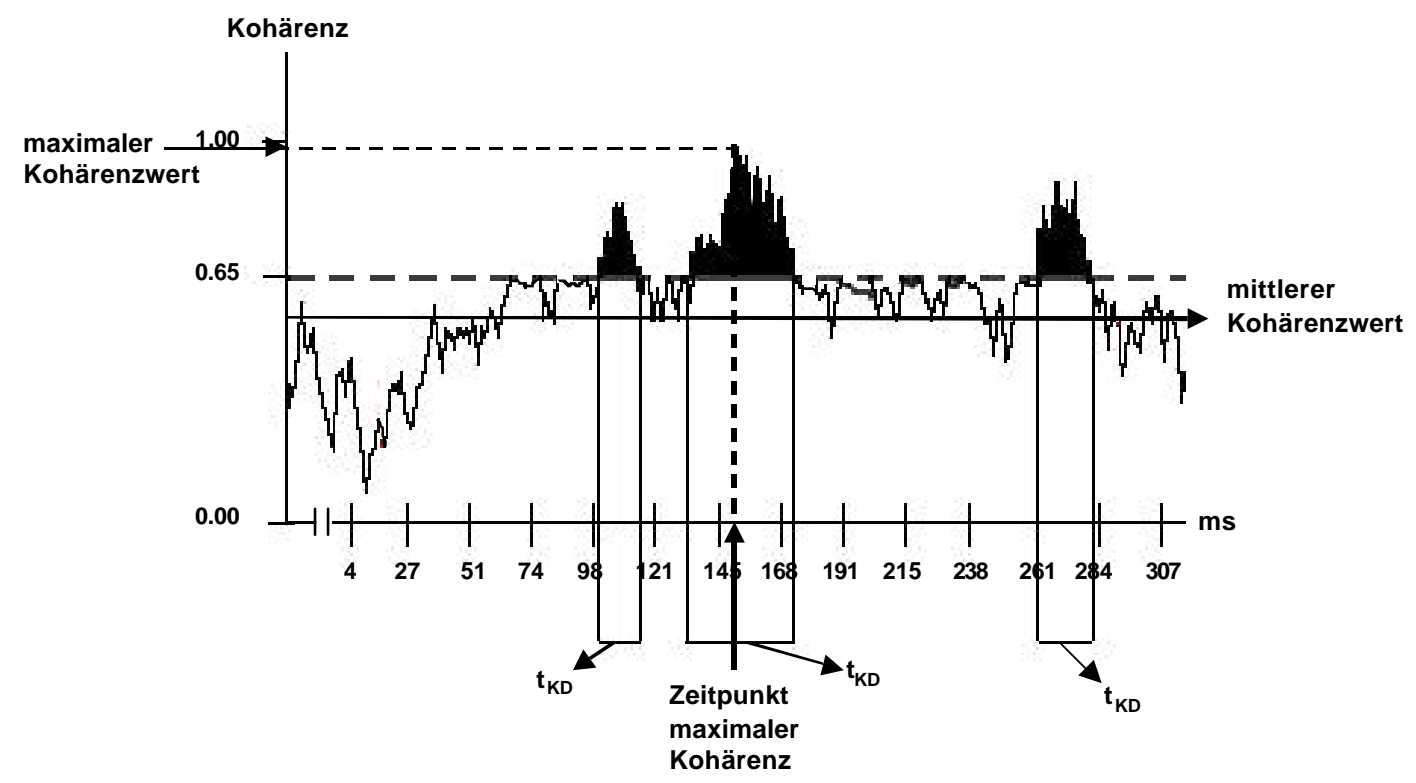

Abbildung 19. Die kohärenzanalytischen Kennwerte: mittlerer Kohärenzwert, Kohärenzdauer $\left(\mathrm{t}_{\mathrm{KD}}\right)$, maximaler Kohärenzwert und Zeitpunkt maximaler Kohärenz 


\section{$6 \quad$ Ergebnisse}

\subsection{Antwortlatenzen}

Die bei der Sternberg-Aufgabe erfaßte "Antwortlatenz" entspricht derjenigen Zeit, die nach dem Memorieren der Lernliste bzw. des positiven Sets zwischen der Darbietung des Testreizes und der Antwort des Probanden (Tastendruck) vergeht (vgl. Kap. 5.5). Die geforderte Antwortart (ja bzw. nein) hängt von dem jeweils vorgegebenen Testreiz bzw. von dessen Vorhandensein in der Lernliste ab. Entsprechend können sogenannte positive und negative Testitems (Testreiz in Lernliste vorhanden bzw. nicht vorhanden) unterschieden werden. Neben den Antwortlatenzen wurden auch die fehlerhaften Antworten erhoben.

Im folgenden werden zunächst die wichtigsten Ergebnisse der Analyse dieser fehlerhaften Antworten und der über- bzw. unterdurchschnittlich langen Antwortlatenzen (sogenannte "Ausreißer") kurz dargestellt. Anschließend wird der Frage nachgegangen, welchen Einfluß die Variablen "Reizart", "Testitem" und "Sitzung" auf die Antwortlatenz hatten und wie sich der Zusammenhang zwischen Setgröße und Antwortlatenz darstellt.

\subsubsection{Falschantworten und Ausreißer}

Tabelle 6 gibt einen Überblick über die Gesamtanzahl der Falschantworten über alle Probandinnen bei den beiden Reizarten "Figuren" und "Wörter" (im folgenden kurz "Figuren" und "Wörter" genannt) für jede Setgröße (Umfang des positiven Sets) und Sitzung sowie für jede Testitemart (vgl. auch Anhang B, Abb. B-1 und B-2).

Die Anzahlen der Falschantworten schwanken beträchtlich vor allem mit der Reizart, der Setgröße und mit dem Testitem. Bei den Figuren wurden in beiden Sitzungen und bei beiden Testitemarten mehr falsche Antworten gegeben als bei den Wörtern. Durchgänge mit Figuren und negativen Testitems riefen darüber hinaus in jeder Sitzung besonders bei den Setgrößen 3 und 4 mehr Falschantworten hervor als Durchgänge mit Figuren und positiven Testitems. Bei den Wörtern ist hier ein gegenläufiger Trend zu verzeichnen: In beiden Sitzungen gaben die Probandinnen bei den Setgrößen 3 und 4 dann mehr falsche Antworten, wenn das Testitem positiv statt negativ war.

In der ersten Sitzung wurden beim Merken der Figuren bei fast allen Setgrößen mehr Falschantworten gegeben als in der zweiten Sitzung. Dies gilt sowohl für positive als auch für negative Testitems. Bei den Wörtern sind bei positiven Testitems keine bedeutenden Unterschiede zwischen der ersten und der zweiten Sitzung zu verzeichnen. Reduktionen der Anzahlen der Falschantworten ergaben sich lediglich im Falle negaiver Testitems bei den Setgrößen 1, 3 und 4. 
Tabelle 6

Gesamtanzahl der Falschantworten über alle Probandinnen pro Versuchsbedingung

\begin{tabular}{|c|c|c|c|c|c|c|c|c|}
\hline \multirow[b]{3}{*}{ Setgröße } & \multicolumn{8}{|c|}{ Positives Testitem (Testreiz in Lernliste vorhanden) } \\
\hline & \multicolumn{4}{|c|}{ Figuren } & \multicolumn{4}{|c|}{ Wörter } \\
\hline & 1 & 2 & 3 & 4 & 1 & 2 & 3 & 4 \\
\hline $\begin{array}{l}\text { Gesamtfehler } \\
\text { 1. Sitzung }\end{array}$ & 12 & 65 & 64 & 90 & 1 & 15 & 16 & 22 \\
\hline Prozentsatz & $2.50 \%$ & $13.54 \%$ & $13.30 \%$ & $18.90 \%$ & $0.21 \%$ & $3.13 \%$ & $3.33 \%$ & $4.58 \%$ \\
\hline $\begin{array}{l}\text { Gesamtfehler } \\
\text { 2. Sitzung }\end{array}$ & 4 & 40 & 66 & 71 & 7 & 16 & 13 & 23 \\
\hline Prozentsatz & $0.83 \%$ & $8.30 \%$ & $13.75 \%$ & $14.80 \%$ & $1.46 \%$ & $3.33 \%$ & $2.71 \%$ & $4.79 \%$ \\
\hline
\end{tabular}

\begin{tabular}{|c|c|c|c|c|c|c|c|c|}
\hline \multirow[b]{3}{*}{ Setgröße } & \multicolumn{8}{|c|}{ Negatives Testitem (Testreiz in Lernliste nicht vorhanden) } \\
\hline & \multicolumn{4}{|c|}{ Figuren } & \multicolumn{4}{|c|}{ Wörter } \\
\hline & 1 & 2 & 3 & 4 & 1 & 2 & 3 & 4 \\
\hline $\begin{array}{l}\text { Gesamtfehler } \\
\text { 1. Sitzung }\end{array}$ & 21 & 57 & 127 & 158 & 13 & 9 & 14 & 18 \\
\hline Prozentsatz & $4.38 \%$ & $11.88 \%$ & $26.46 \%$ & $32.92 \%$ & $2.71 \%$ & $1.88 \%$ & $2.92 \%$ & $3.75 \%$ \\
\hline $\begin{array}{l}\text { Gesamtfehler } \\
\text { 2. Sitzung }\end{array}$ & 26 & 45 & 101 & 152 & 8 & 10 & 6 & 7 \\
\hline Prozentsatz & $5.42 \%$ & $9.38 \%$ & $21.04 \%$ & $31.70 \%$ & $1.70 \%$ & $2.08 \%$ & $1.25 \%$ & $1.46 \%$ \\
\hline
\end{tabular}

Anmerkung: Pro Reizart, Sitzung, Testitem und Setgröße waren maximal 48 Fehler pro Probandin bzw. 480 Fehler über alle Probandinnen möglich.

Insgesamt übersteigen die prozentualen Anzahlen falscher Antworten vor allem bei den Figuren die von Sternberg (1975) und anderen Autoren berichteten Größenordnungen. Daraus lassen sich Schlüsse bezüglich der Lernqualität der verwendeten Reizarten ziehen (vgl. Kap. 7). Für die nachfolgenden statistischen Analysen wurden die Werte von Falschantworten gelöscht; damit ergab sich für jede Versuchsbedingung eine unterschiedliche Anzahl gültiger Durchgänge (vgl. Anhang B, Tab. B-1).

Die Gesamtanzahl der Ausreißer über alle Probandinnen bei den beiden Reizarten für jede Setgröße und Sitzung sowie für jedes Testitem kann der Tabelle B-2 im Anhang B entnommen werden (siehe auch Abb. B-3 und B-4). Als Ausreißer wurden nach Tukey (1977; vgl. auch Bredenkamp, Klein, Hayn \& Vaterrodt, 1988) diejenigen Antwortlatenzen definiert, die Werte größer als $Q_{3}+1.5\left(Q_{3}-Q_{1}\right)$ bzw. kleiner als $Q_{1}-1.5\left(Q_{3}-Q_{1}\right)$ aufwiesen $\left(Q_{3}\right.$ und $Q_{1}$ stehen für das dritte bzw. erste Quartil einer Werteverteilung). Ihre Berechnung erfolgte individuell für jede Probandin und jede Versuchsbedingung.

Die Prozentsätze machen deutlich, daß es keine systematischen Unterschiede zwischen den Ausreißeranzahlen in den verschiedenen Versuchsbedingungen gibt. Die Aus- 
reißerwerte wurden ebenso wie die der Falschantworten gelöscht. Der entsprechend bereinigte Datensatz bildete die Grundlage für die nachfolgenden statistischen Analysen.

\subsubsection{Einfluß von Reizart, Testitem und Sitzung}

Ein Vergleich der über die zehn Probandinnen gemittelten Antwortlatenzen unter den verschiedenen Versuchsbedingungen zeigt, daß alle Werte mit ansteigender Setgröße zunehmen (vgl. Tab. 7; siehe auch Anhang B, Abb. B-5). Die mittleren Antwortlatenzen (M) der Figuren liegen darüber hinaus in allen Versuchsbedingungen ausnahmslos über den mittleren Antwortlatenzen der Wörter. Dasselbe gilt für die erste Sitzung und für das negative Testitem, deren Werte bei der Betrachtung der einzelnen Reizarten in jeder Setgröße über denen der zweiten Sitzung bzw. des positiven Testitems liegen.

Tabelle 7

Mittlere Antwortlatenzen pro Versuchsbedingung (in ms; $\mathrm{N}=10$ )

\begin{tabular}{l|c|c|c|c|c|c|c|c|}
\multicolumn{1}{c}{} & \multicolumn{5}{c|}{ Positives Testitem (Testreiz in Lernliste vorhanden) } \\
\cline { 2 - 9 } Setgröße & 1 & 2 & 3 & 4 & 1 & 2 & 3 & 4 \\
\hline$M$ (1. Sitzung) & 470.16 & 617.71 & 657.19 & 686.66 & 412.11 & 510.74 & 547.11 & 579.58 \\
\hline$S D$ & 92.54 & 125.64 & 120.57 & 140.82 & 58.25 & 73.43 & 76.74 & 91.73 \\
\hline$M$ (2. Sitzung) & 418.78 & 532.02 & 586.10 & 587.87 & 382.59 & 475.08 & 513.61 & 523.10 \\
\hline$S D$ & 66.90 & 75.97 & 99.77 & 118.29 & 59.58 & 57.37 & 60.57 & 62.50 \\
\hline
\end{tabular}

Negatives Testitem (Testreiz in Lernliste nicht vorhanden)

\begin{tabular}{|l|c|c|c|c|c|c|c|c|}
\cline { 2 - 9 } \multicolumn{1}{c|}{} & \multicolumn{4}{c}{ Figuren } & \multicolumn{4}{c|}{ Wörter } \\
\hline Setgröße & 1 & 2 & 3 & 4 & 1 & 2 & 3 & 4 \\
\hline M (1. Sitzung) & 553.30 & 664.09 & 755.99 & 806.94 & 491.26 & 540.09 & 579.71 & 615.75 \\
\hline$S D$ & 97.05 & 108.33 & 139.91 & 150.19 & 66.36 & 73.23 & 79.48 & 76.82 \\
\hline$M$ (2. Sitzung) & 496.62 & 592.94 & 646.10 & 688.96 & 462.36 & 508.34 & 539.86 & 565.69 \\
\hline$S D$ & 52.71 & 51.54 & 68.67 & 100.10 & 45.06 & 57.93 & 31.65 & 32.69 \\
\hline
\end{tabular}

Die Standardabweichungen der Mittelwerte (SD) nehmen in fast allen Versuchsbedingungen - wie die Mittelwerte selbst - mit ansteigender Setgröße zu. Systematische Abnahmen lassen sich bei der Gegenüberstellung der Sitzungen pro Versuchsbedingung beobachten, wobei diese für das negative Testitem größer ausfallen als für das positive Testitem. Die Mittelwertsstreuungen der Wörter liegen in allen Fällen unter den entsprechenden Mittelwertsstreuungen der Figuren. Insgesamt sprechen die Werte für einen recht homogenen Datensatz. 
Die beobachteten Mittelwertsunterschiede wurden inferenzstatistisch mittels t-Tests für abhängige Stichproben auf ihre Signifikanz hin überprüft. Tabelle 8 gibt einen Überblick über den Vergleich der Reizarten pro Versuchsbedingung:

Tabelle 8

Mittlere Antwortlatenzen: Vergleich von Figuren $(F)$ und Wörtern $(W)$ pro Versuchsbedingung (t-Test für abhängige Stichproben, zweiseitig; $N=10$ )

\begin{tabular}{|ccccccccccc|}
\hline Set & Testitem & Sitzung & $\begin{array}{c}\text { Figuren } \\
M_{F}\end{array}$ & $\begin{array}{c}\text { Wörter } \\
M_{W}\end{array}$ & $\begin{array}{c}\text { Differenz } \\
M_{F}-M_{W}\end{array}$ & $\begin{array}{c}\text { SD (max) } \\
\text { t-Wert }\end{array}$ & $p$ (sign.) & $\begin{array}{c}\text { Korrel. } \\
r\end{array}$ & $\begin{array}{c}\text { Effekt- } \\
\text { größe } \varepsilon\end{array}$ \\
\hline 1 & positiv & 1 & 470.16 & 412.11 & 58.05 & 92.54 & 4.65 & 0.001 & 0.97 & 3.35 \\
2 & positiv & 1 & 617.71 & 510.74 & 106.96 & 125.64 & 3.80 & 0.004 & 0.72 & 1.61 \\
3 & positiv & 1 & 657.19 & 547.11 & 110.08 & 120.57 & 4.52 & 0.001 & 0.78 & 1.96 \\
4 & positiv & 1 & 686.66 & 579.58 & 107.08 & 140.82 & 3.59 & 0.006 & 0.75 & 1.52 \\
\hline 1 & negativ & 1 & 553.30 & 491.26 & 62.04 & 97.05 & 3.76 & 0.004 & 0.86 & 1.72 \\
2 & negativ & 1 & 664.09 & 540.09 & 123.99 & 108.33 & 5.38 & 0.000 & 0.74 & 2.25 \\
3 & negativ & 1 & 755.99 & 579.71 & 176.28 & 139.91 & 5.27 & 0.001 & 0.66 & 2.16 \\
4 & negativ & 1 & 806.94 & 615.75 & 191.19 & 150.19 & 6.40 & 0.000 & 0.85 & 3.25 \\
\hline 1 & positiv & 2 & 418.78 & 382.59 & 36.20 & 66.90 & 3.29 & 0.009 & 0.86 & 1.42 \\
2 & positiv & 2 & 532.02 & 475.08 & 56.94 & 75.97 & 4.32 & 0.002 & 0.84 & 1.87 \\
3 & positiv & 2 & 586.10 & 513.61 & 72.50 & 99.77 & 2.91 & 0.017 & 0.61 & 1.17 \\
4 & positiv & 2 & 587.87 & 523.10 & 64.77 & 118.29 & 2.19 & 0.056 & 0.62 & 0.89 \\
\hline 1 & negativ & 2 & 496.62 & 462.36 & 34.26 & 52.71 & 6.88 & 0.000 & 0.96 & 3.25 \\
2 & negativ & 2 & 592.94 & 508.34 & 84.60 & 57.93 & 13.68 & 0.000 & 0.94 & 6.12 \\
3 & negativ & 2 & 646.10 & 539.86 & 106.24 & 68.67 & 5.65 & 0.000 & 0.50 & 2.19 \\
4 & negativ & 2 & 688.96 & 565.69 & 123.28 & 100.10 & 4.63 & 0.001 & 0.61 & 1.98 \\
\hline
\end{tabular}

In fast allen Fällen, d. h. bei fast jeder Kombination von Sitzung, Setgröße und Testitem, unterscheiden sich die mittleren Antwortlatenzen beider Reizarten signifikant bzw. sehr signifikant voneinander $(0.05 \leq p \leq 0.01)$. Eine Ausnahme bildet in der zweiten Sitzung die Setgröße 4, positives Testitem. Hier kann lediglich von einem tendenziellen Unterschied gesprochen werden $(p=0.056)$. Die Probandinnen benötigten also in fast allen Bedingungskombinationen bei nonverbalen Reizen bedeutend mehr Zeit, um richtig zu entscheiden, ob das Testitem in der memorierten Liste vorhanden war oder nicht. Mit $\varepsilon=0.89$ bis 3.35 ergeben sich für den Reizartvergleich in allen Versuchsbedingungen große experimentelle Effekte. Beim Vergleich der Testitems und der Sitzungen (vgl. Tab. 9 und 10) ergeben sich für die negativen Testitems bzw. für die erste Sitzung fast ausnahmslos signifikant bzw. sehr signifikant längere Antwortlatenzen als für die positiven Testitems bzw. für die zweite Sitzung. 
Bei der Betrachtung der Mittelwertsdifferenzen der Antwortlatenzen von Figuren und Wörtern (Tab. 8) fällt auf, daß diese in beiden Sitzungen beim negativen Testitem über alle, beim positiven Testitem hingegen nur über die ersten drei Setgrößen hinweg zunehmen. Dies liegt daran, daß beim positiven Testitem die Differenz zwischen den mittleren Antwortlatenzen der Setgrößen 4 und 3 bei den Wörtern die entsprechende Differenz bei den Figuren übersteigt, während es sich bei den Differenzen der mittleren Antwortlatenzen der übrigen Setgrößen umgekehrt verhält. Der maximale Unterschied zwischen den mittleren Antwortlatenzen beider Reizarten tritt somit beim positiven Testitem bei Setgröße 3, beim negativen Testitem bei Setgröße 4 auf. Auch beim Vergleich der Testitems pro Versuchsbedingung (Tab. 9) gibt es Auffälligkeiten bei den Mittelwertsdifferenzen. Die größte Mittelwertsdifferenz der Antwortlatenzen negativer und positiver Testitems tritt unabhängig von der Sitzung bei Figuren bei Setgröße 4, bei Wörtern hingegen bei Setgröße 1 auf.

Tabelle 9

Mittlere Antwortlatenzen: Vergleich von positiven $(P)$ und negativen $(N)$ Testitems pro Versuchsbedingung (t-Test für abhängige Stichproben, zweiseitig; $N=10$ )

\begin{tabular}{|ccccccccccc|}
\hline Set & Reizart & Sitzung & $\begin{array}{c}\text { negativ } \\
M_{N}\end{array}$ & $\begin{array}{c}\text { positiv } \\
M_{P}\end{array}$ & $\begin{array}{c}\text { Differenz } \\
M_{N}-M_{P}\end{array}$ & $S D$ (max) & $t$-Wert & $p$ (sign.) & $\begin{array}{c}\text { Korrel. } \\
r\end{array}$ & $\begin{array}{c}\text { Effekt- } \\
\text { größe } \varepsilon\end{array}$ \\
\hline 1 & Figuren & 1 & 553.30 & 470.16 & 83.14 & 97.05 & 5.46 & 0.000 & 0.87 & 2.39 \\
2 & Figuren & 1 & 664.09 & 617.71 & 46.38 & 125.64 & 2.93 & 0.017 & 0.92 & 1.30 \\
3 & Figuren & 1 & 755.99 & 657.19 & 98.81 & 139.91 & 4.93 & 0.001 & 0.89 & 2.15 \\
4 & Figuren & 1 & 806.94 & 686.66 & 120.28 & 150.19 & 5.35 & 0.000 & 0.88 & 2.33 \\
\hline 1 & Wörter & 1 & 491.26 & 412.11 & 79.15 & 66.37 & 8.58 & 0.000 & 0.90 & 3.75 \\
2 & Wörter & 1 & 540.09 & 510.74 & 29.35 & 73.43 & 3.13 & 0.012 & 0.92 & 1.40 \\
3 & Wörter & 1 & 579.71 & 547.11 & 32.60 & 79.49 & 3.94 & 0.003 & 0.94 & 1.73 \\
4 & Wörter & 1 & 615.75 & 579.58 & 36.17 & 91.73 & 3.17 & 0.011 & 0.92 & 1.42 \\
\hline 1 & Figuren & 2 & 496.62 & 418.78 & 77.84 & 66.90 & 10.85 & 0.000 & 0.96 & 5.55 \\
2 & Figuren & 2 & 592.94 & 532.02 & 60.92 & 75.97 & 4.30 & 0.002 & 0.82 & 1.89 \\
3 & Figuren & 2 & 646.10 & 586.10 & 60.00 & 99.77 & 4.24 & 0.002 & 0.93 & 2.20 \\
4 & Figuren & 2 & 688.96 & 587.87 & 101.10 & 118.29 & 4.49 & 0.002 & 0.80 & 1.91 \\
\hline 1 & Wörter & 2 & 462.36 & 382.59 & 79.77 & 59.58 & 7.22 & 0.000 & 0.81 & 3.09 \\
2 & Wörter & 2 & 508.34 & 475.08 & 33.27 & 57.93 & 2.89 & 0.018 & 0.80 & 1.28 \\
3 & Wörter & 2 & 539.86 & 513.61 & 26.25 & 60.57 & 2.00 & 0.077 & 0.77 & 0.90 \\
4 & Wörter & 2 & 565.69 & 523.10 & 42.59 & 62.50 & 3.45 & 0.007 & 0.85 & 1.73 \\
\hline
\end{tabular}


Tabelle 10

Mittlere Antwortlatenzen: Vergleich von erster (1) und zweiter (2) Sitzung pro Versuchsbedingung (t-Test für abhängige Stichproben, zweiseitig; $N=10$ )

\begin{tabular}{|ccccccccccc|}
\hline Set & Reizart & Testitem & $\begin{array}{c}\text { Sitzg.1 } \\
M_{1}\end{array}$ & $\begin{array}{c}\text { Sitzg.2 } \\
M_{2}\end{array}$ & $\begin{array}{c}\text { Differenz } \\
M_{1}-M_{2}\end{array}$ & $S D$ (max) & $t$-Wert & $p$ (sign.) & $\begin{array}{c}\text { Korrel. } \\
r\end{array}$ & $\begin{array}{c}\text { Effekt- } \\
\text { größe } \varepsilon\end{array}$ \\
\hline 1 & Figuren & positiv & 470.16 & 418.78 & 51.38 & 92.54 & 3.62 & 0.006 & 0.89 & 1.67 \\
2 & Figuren & positiv & 617.71 & 532.02 & 85.69 & 125.64 & 3.77 & 0.004 & 0.86 & 1.82 \\
3 & Figuren & positiv & 657.19 & 586.10 & 71.09 & 120.57 & 4.67 & 0.001 & 0.92 & 2.11 \\
4 & Figuren & positiv & 686.66 & 587.87 & 98.79 & 140.82 & 5.67 & 0.000 & 0.92 & 2.54 \\
\hline 1 & Wörter & positiv & 412.11 & 382.59 & 29.52 & 59.58 & 2.73 & 0.023 & 0.83 & 1.21 \\
2 & Wörter & positiv & 510.74 & 475.08 & 35.67 & 73.43 & 3.20 & 0.011 & 0.88 & 1.43 \\
3 & Wörter & positiv & 547.11 & 513.61 & 33.51 & 76.74 & 2.62 & 0.028 & 0.85 & 1.14 \\
4 & Wörter & positiv & 579.58 & 523.10 & 56.48 & 91.73 & 4.39 & 0.002 & 0.93 & 2.33 \\
\hline 1 & Figuren & negativ & 553.30 & 496.62 & 56.68 & 97.05 & 3.41 & 0.008 & 0.92 & 2.09 \\
2 & Figuren & negativ & 664.09 & 592.94 & 71.15 & 108.33 & 2.97 & 0.016 & 0.78 & 1.39 \\
3 & Figuren & negativ & 755.99 & 646.10 & 109.89 & 139.91 & 3.87 & 0.004 & 0.84 & 1.99 \\
4 & Figuren & negativ & 806.94 & 688.96 & 117.97 & 150.19 & 4.54 & 0.001 & 0.86 & 2.09 \\
\hline 1 & Wörter & negativ & 491.26 & 462.36 & 28.90 & 66.37 & 2.80 & 0.021 & 0.90 & 1.36 \\
2 & Wörter & negativ & 540.09 & 508.34 & 31.75 & 73.23 & 3.56 & 0.006 & 0.93 & 1.69 \\
3 & Wörter & negativ & 579.71 & 539.86 & 39.86 & 79.49 & 2.33 & 0.045 & 0.87 & 1.41 \\
4 & Wörter & negativ & 615.75 & 565.69 & 50.06 & 76.82 & 3.01 & 0.015 & 0.84 & 1.61 \\
\hline
\end{tabular}

Die unterschiedlichen mittleren Antwortlatenzen beider Sitzungen (vgl. Tab. 10) weisen auf einen Übungs- bzw. Lerneffekt hin, der nicht nur für die Prüfung psychologischer Hypothesen, sondern auch für die Untersuchung physiologisch-anatomischer Fragestellungen eine potentielle Störvariable darstellt (vgl. Kap.3.1.2). Um festzustellen, ob dieser Effekt auch innerhalb der einzelnen Sitzungen auftrat, sind die mittleren Antwortlatenzen des ersten und des dritten Drittels der Durchgänge jeder Sitzung verglichen worden (Block 1/3 vs. 9/11 bei Figuren bzw. Block 2/4 vs. 10/12 bei Wörtern; vgl. Tab. 5 in Kap. 5.6). Während in der ersten Sitzung die mittleren Antwortlatenzen des ersten Drittels der Durchgänge unter jeder Bedingung signifikant bzw. sehr signifikant länger als diejenigen des dritten Drittels der Durchgänge ausfallen, kann ein solcher Unterschied für die zweite Sitzung nicht nachgewiesen werden (vgl. Tab. 11 und 12).

Tabelle 12 verdeutlicht, daß sich die - über die mittleren Antwortlatenzen definierte Leistung der Probandinnen innerhalb der zweiten Sitzung nicht signifikant verbesserte. Ein bedeutender Lerneffekt kann - sofern er über eine Erhöhung der Antwortgeschwindigkeit definiert wird - für diese Sitzung somit ausgeschlossen werden. Aus diesem Grund beziehen sich alle weiteren statistischen Analysen sowohl der Verhaltens- als auch der EEG-Daten auf die in der zweiten Sitzung erhobenen Werte. 
Tabelle 11

Mittlere Antwortlatenzen: Vergleich von erstem (1) und drittem (3) Drittel der Durchgänge in der ersten Sitzung pro Versuchsbedingung (t-Test für abhängige Stichproben, zweiseitig; $\mathrm{N}=10$ )

\begin{tabular}{|ccccccccccc|}
\hline Set & Reizart & Testitem & $\begin{array}{c}\text { 1. Drittel } \\
M_{1}\end{array}$ & $\begin{array}{c}\text { 3. Drittel } \\
M_{3}\end{array}$ & $\begin{array}{c}\text { Differenz } \\
M_{1}-M_{3}\end{array}$ & $\begin{array}{c}S D \\
(\max )\end{array}$ & $\begin{array}{l}\text { t-Wert } \\
\text { p (sign.) }\end{array}$ & $\begin{array}{c}\text { Korrel. } \\
r\end{array}$ & $\begin{array}{c}\text { Effekt- } \\
\text { größe } \varepsilon\end{array}$ \\
\hline 1 & Figuren & positiv & 514.71 & 438.81 & 75.90 & 108.77 & 5.06 & 0.001 & 0.90 & 2.21 \\
2 & Figuren & positiv & 678.35 & 569.88 & 108.47 & 161.11 & 3.48 & 0.007 & 0.79 & 1.48 \\
3 & Figuren & positiv & 714.55 & 625.10 & 89.45 & 133.72 & 3.49 & 0.007 & 0.80 & 1.49 \\
4 & Figuren & positiv & 737.21 & 634.29 & 102.92 & 162.66 & 2.53 & 0.032 & 0.63 & 1.03 \\
\hline 1 & Wörter & positiv & 421.03 & 387.20 & 33.83 & 57.90 & 2.34 & 0.044 & 0.68 & 1.04 \\
2 & Wörter & positiv & 545.67 & 476.61 & 69.06 & 95.36 & 4.08 & 0.003 & 0.84 & 1.81 \\
3 & Wörter & positiv & 577.76 & 518.08 & 59.68 & 91.33 & 3.20 & 0.011 & 0.76 & 1.34 \\
4 & Wörter & positiv & 610.23 & 536.59 & 73.64 & 117.35 & 2.59 & 0.029 & 0.65 & 1.06 \\
\hline 1 & Figuren & negativ & 592.57 & 522.36 & 70.21 & 122.15 & 3.59 & 0.006 & 0.90 & 1.80 \\
2 & Figuren & negativ & 715.07 & 639.36 & 75.71 & 133.35 & 2.42 & 0.039 & 0.69 & 1.02 \\
3 & Figuren & negativ & 815.10 & 730.75 & 84.35 & 176.73 & 2.56 & 0.031 & 0.81 & 1.10 \\
4 & Figuren & negativ & 845.16 & 746.95 & 98.20 & 167.59 & 3.54 & 0.006 & 0.88 & 1.66 \\
\hline 1 & Wörter & negativ & 507.24 & 457.95 & 49.28 & 71.38 & 5.21 & 0.001 & 0.94 & 2.77 \\
2 & Wörter & negativ & 571.73 & 502.30 & 69.43 & 98.24 & 3.76 & 0.005 & 0.88 & 2.01 \\
3 & Wörter & negativ & 619.30 & 551.67 & 67.63 & 99.09 & 3.49 & 0.007 & 0.79 & 1.48 \\
4 & Wörter & negativ & 645.28 & 574.03 & 71.26 & 97.04 & 3.93 & 0.003 & 0.88 & 2.08 \\
\hline
\end{tabular}

Tabelle 12

Mittlere Antwortlatenzen: Vergleich von erstem (1) und drittem (3) Drittel der Durchgänge in der zweiten Sitzung pro Versuchsbedingung ( $\mathrm{t}$-Test für abhängige Stichproben, zweiseitig; $\mathrm{N}=10$ )

\begin{tabular}{|ccccccccccc|}
\hline Set & Reizart & Testitem & $\begin{array}{c}\text { 1. Drittel } \\
M_{1}\end{array}$ & $\begin{array}{c}\text { 3. Drittel } \\
M_{3}\end{array}$ & $\begin{array}{c}\text { Differenz } \\
M_{1}-M_{3}\end{array}$ & $\begin{array}{c}S D \\
(\max )\end{array}$ & $\begin{array}{c}\text { t-Wert } \\
\text { (sign.) }\end{array}$ & $\begin{array}{c}\text { Korrel. } \\
r\end{array}$ & $\begin{array}{c}\text { Effekt- } \\
\text { größe } \varepsilon\end{array}$ \\
\hline 1 & Figuren & positiv & 409.79 & 417.39 & -7.60 & 82.07 & -0.83 & 0.425 & 0.96 & -0.45 \\
2 & Figuren & positiv & 530.13 & 525.89 & 4.24 & 96.36 & 0.34 & 0.741 & 0.92 & 0.16 \\
3 & Figuren & positiv & 592.44 & 585.20 & 7.25 & 109.14 & 0.74 & 0.478 & 0.96 & 0.33 \\
4 & Figuren & positiv & 575.41 & 614.30 & -38.89 & 151.22 & -2.23 & 0.053 & 0.95 & -1.10 \\
\hline 1 & Wörter & positiv & 377.53 & 376.20 & 1.34 & 65.00 & 0.20 & 0.849 & 0.94 & 0.09 \\
2 & Wörter & positiv & 472.15 & 460.57 & 11.58 & 59.09 & 1.14 & 0.283 & 0.84 & 0.49 \\
3 & Wörter & positiv & 517.35 & 509.92 & 7.43 & 71.23 & 0.59 & 0.572 & 0.84 & 0.26 \\
4 & Wörter & positiv & 510.20 & 519.20 & -9.00 & 63.87 & -0.70 & 0.501 & 0.79 & -0.31 \\
\hline 1 & Figuren & negativ & 494.45 & 491.93 & 2.52 & 66.69 & 0.33 & 0.746 & 0.97 & 0.22 \\
2 & Figuren & negativ & 584.59 & 581.52 & 3.07 & 69.39 & 0.46 & 0.656 & 0.96 & 0.21 \\
3 & Figuren & negativ & 645.83 & 645.22 & 0.61 & 87.73 & 0.04 & 0.967 & 0.86 & 0.02 \\
4 & Figuren & negativ & 702.55 & 710.20 & -7.65 & 120.65 & -0.41 & 0.691 & 0.87 & -0.18 \\
\hline 1 & Wörter & negativ & 465.82 & 454.00 & 11.82 & 55.28 & 1.02 & 0.334 & 0.76 & 0.43 \\
2 & Wörter & negativ & 505.40 & 495.12 & 10.27 & 54.88 & 1.05 & 0.323 & 0.83 & 0.45 \\
3 & Wörter & negativ & 543.04 & 536.48 & 6.56 & 39.65 & 1.40 & 0.195 & 0.93 & 0.62 \\
4 & Wörter & negativ & 564.62 & 549.64 & 14.98 & 36.83 & 2.19 & 0.056 & 0.81 & 0.93 \\
\hline
\end{tabular}




\subsubsection{Setgröße und Antwortlatenz}

Eine für diese Arbeit wichtige Frage lautet, ob die von Sternberg (1966) gefundene lineare Beziehung zwischen der Antwortlatenz und der Größe des positiven Sets mit dem gewählten verbalen und nonverbalen Reizmaterial repliziert werden konnte. Um diese Frage zu klären, sind lineare Regressionsanalysen durchgeführt worden. Für jede Versuchsbedingung wurde die Beziehung zwischen den über alle Probandinnen gemittelten Antwortlatenzen und der Setgröße untersucht.

Wie aus Tabelle 13 ersichtlich, besteht im vorliegenden Datensatz zwischen der Antwortlatenz und der Setgröße eine eindeutige lineare Beziehung. Dies gilt für beide Reizarten und beide Testitems gleichermaßen (siehe auch Anhang B, Abb. B-6).

Auf die Variable "Setgröße" können je nach Versuchsbedingung zwischen 31.7 und $51.2 \%$ der gesamten Streuung zurückgeführt werden. Die Regressionskoeffizienten (Slope), die als Schätzer für die pro Itemvergleich benötigte Zeit (sog. "ScanningZeit") angesehen werden, schwanken mit 34.15 bis $63.12 \mathrm{~ms}$ um den von Sternberg (1975) angegebenen Schätzwert von 40 ms. Gleiches gilt für die Regressionskonstanten, die die Dauer anderer kognitiver Prozesse widerspiegeln sollen. Sie liegen mit 390.86 bis $448.61 \mathrm{~ms}$ nahe an dem von Sternberg beschriebenen Durchschnittswert von $400 \mathrm{~ms}$. Beide Kennwerte variieren offensichtlich nicht nur - wie in der Literatur verschiedentlich beschrieben (Cavanagh, 1972; Sternberg, 1969) - mit der Reizart, sondern auch mit der Testitemart.

Tabelle 13

Ergebnisse der linearen Regressionsanalysen der gemittelten Antwortlatenzen ( $\left.\mathrm{M}_{\mathrm{AL}}\right)$ und der Größe des positiven Sets (2. Sitzung, $N=10$ )

\begin{tabular}{|cccccccccc|}
\hline UV & AV & Testitem & Multiple $R$ & $R^{2}$ & Sign. F & Slope & $p$ (sign.) & Intercept & $p$ (sign.) \\
\hline Set & $M_{A L}$ Figuren & positiv & 0.563 & 0.317 & 0.000 & 56.133 & 0.000 & 390.861 & 0.000 \\
Set & $M_{A L}$ Wörter & positiv & 0.647 & 0.418 & 0.000 & 46.006 & 0.000 & 358.578 & 0.000 \\
Set & $M_{A L}$ Figuren & negativ & 0.716 & 0.512 & 0.000 & 63.019 & 0.000 & 448.609 & 0.000 \\
Set & $M_{A L}$ Wörter & negativ & 0.679 & 0.461 & 0.000 & 34.151 & 0.000 & 433.685 & 0.000 \\
\hline
\end{tabular}

Unterschiede in den Regressionskoeffizienten positiver und negativer Testitems sind nach Sternberg (1975) darauf zurückzuführen, daß positive und negative Testitems unterschiedliche Such- und Vergleichsprozesse hervorrufen können. Der sich selbst beendende Suchprozeß, der bei der Beantwortung positiver Testitems auftreten kann, führt dazu, daß die entsprechenden Regressionsgeraden flacher verlaufen als diejenigen negativer Testitems, die stets einen erschöpfenden Suchprozeß hervorrufen sollen (vgl. Kap. 2.1.2). Im vorliegenden Fall kann nur für die Figuren eine Differenz in der von Stern- 
berg beschriebenen Richtung festgestellt werden. Bei den Wörtern fällt der Regressionskoeffizient positiver Testitems dagegen (anders als von Sternberg beschrieben) größer als derjenige negativer Testitems aus.

Um diese Unterschiede auf ihre Signifikanz hin prüfen zu können, sind lineare Regressionsanalysen der individuellen mittleren Antwortlatenzen und der Größe des positiven Sets gerechnet worden. Aus ihnen geht hervor, daß die für die gesamte Stichprobe gefundene lineare Abhängigkeit zwischen diesen Variablen nicht für jeden Einzelfall gleichermaßen gültig ist (vgl. Anhang B, Tab. B-3). Bei den Probandinnen 1 und 3 finden sich in einigen Versuchsbedingungen Regressionskoeffizienten, die mit 11.19 bis 18.57 ms deutlich unter den Werten der übrigen Probandinnen liegen und nicht signifikant sind. In die t-Tests zum Vergleich der mittleren Scanning-Zeiten und Regressionskonstanten der beiden Reizarten und Testitems sind die Werte dieser beiden Probandinnen daher nicht einbezogen worden.

Aus den Tabellen 14 und 15 gehen die Ergebnisse des inferenzstatistischen Vergleichs der mittleren Scanning-Zeiten und Regressionskonstanten beider Reizarten für jedes Testitem hervor. Die mittleren Scanning-Zeiten beider Reizarten unterscheiden sich signifikant bzw. sehr signifikant, wobei sie für Wörter beim positiven Testitem um 18.24 ms, beim negativen Testitem sogar um $38.60 \mathrm{~ms}$ schneller ausfallen als für Figuren. Die mittleren Regressionskonstanten unterscheiden sich nur im Falle positiver Testitems sehr signifikant voneinander. Die entsprechenden experimentellen Effekte sind als groß zu beschreiben.

Tabelle 14

Vergleich der mittleren Regressionskoeffizienten ("Scanning-Zeiten"; $\mathrm{M}_{\mathrm{SL}}$, in ms) von Figuren und Wörtern (t-Test für abhängige Stichproben, zweiseitig; $n=8 ; 2$. Sitzung)

\begin{tabular}{|ccccccccc|}
\hline Testitem & $\begin{array}{c}S L \text { Figuren } \\
M_{\text {SLF }}\end{array}$ & $\begin{array}{c}S L \text { Wörter } \\
M_{\text {SLW }}\end{array}$ & $\begin{array}{c}\text { Differenz } \\
M_{\text {SLF }}-M_{\text {SLW }}\end{array}$ & $\begin{array}{c}S D \\
(\max )\end{array}$ & $t$-Wert & $p$ (sign.) & $\begin{array}{c}\text { Korrel. } \\
r\end{array}$ & $\begin{array}{c}\text { Effekt- } \\
\text { größe } \varepsilon\end{array}$ \\
\hline positiv & 65.63 & 47.40 & 18.24 & 22.36 & 2.58 & 0.036 & 0.47 & 1.12 \\
negativ & 74.44 & 35.84 & 38.60 & 19.34 & 5.45 & 0.001 & -0.05 & 1.95 \\
\hline
\end{tabular}

Tabelle 15

Vergleich der mittleren Regressionskonstanten ( $\mathrm{M}_{\mathrm{IC}}$, in $\mathrm{ms}$ ) von Figuren und Wörtern (t-Test für abhängige Stichproben, zweiseitig; $n=8 ; 2$. Sitzung)

\begin{tabular}{|c|c|c|c|c|c|c|c|c|}
\hline Testitem & $\begin{array}{c}\text { IC Figuren } \\
M_{\text {ICF }}\end{array}$ & $\begin{array}{c}\text { IC Wörter } \\
M_{\text {ICW }}\end{array}$ & $\begin{array}{c}\text { Differenz } \\
M_{I C F}-M_{I C W}\end{array}$ & $\begin{array}{c}S D \\
(\max )\end{array}$ & $t$-Wert & $p$ (sign.) & $\begin{array}{c}\text { Korrel. } \\
r\end{array}$ & $\begin{array}{l}\text { Effekt- } \\
\text { größe } \varepsilon\end{array}$ \\
\hline positiv & 374.69 & 347.57 & 27.12 & 56.51 & 5.67 & 0.001 & 0.97 & 2.92 \\
\hline negativ & 426.74 & 426.96 & -0.22 & 42.05 & -0.02 & 0.982 & 0.79 & -0.01 \\
\hline
\end{tabular}


Bei der Gegenüberstellung der Testitems wird deutlich, daß sich die mittleren Regressionskonstanten positiver und negativer Items bei jeder Reizart sehr signifikant voneinander unterscheiden (Tab. 16). Im Falle der Scanning-Zeiten trifft dieses Ergebnis hingegen nur auf eine Reizart - nämlich auf die Wörter - zu (Tab. 17). Darüber hinaus ist ein umgekehrtes Verhältnis der Scanning-Zeiten positiver und negativer Testitems bei den beiden Reizarten zu verzeichnen: Figuren riefen im Schnitt etwas längere Scanning-Zeiten dann hervor, wenn es sich um negative Testitems handelte, Wörter hingegen dann, wenn die Testitems positiv waren. Die Differenz zwischen den mittleren Regressionskoeffizienten positiver und negativer Testitems ist bei Figuren allerdings nicht signifikant.

Tabelle 16

Vergleich der mittleren Regressionskoeffizienten ("Scanning-Zeiten"; $\mathrm{MSL}_{\mathrm{SL}}$ in $\mathrm{ms}$ ) von positiven und negativen Testitems ( $t$-Test für abhängige Stichproben, zweiseitig; $n=8$; 2 . Sitzung)

\begin{tabular}{|ccccccccc|}
\hline Reizart & $\begin{array}{c}S L \text { negativ } \\
M_{S L N}\end{array}$ & $\begin{array}{c}S L \text { positiv } \\
M_{S L P}\end{array}$ & $\begin{array}{c}\text { Differenz } \\
M_{\text {SLN }}-M_{S L P}\end{array}$ & $\begin{array}{c}S D \\
(\max )\end{array}$ & $t$-Wert & $p$ (sign.) & $\begin{array}{c}\text { Korrel. } \\
r\end{array}$ & $\begin{array}{c}\text { Effekt- } \\
\text { größe } \varepsilon\end{array}$ \\
\hline Figuren & 74.44 & 65.63 & 8.81 & 22.36 & 1.11 & 0.305 & 0.43 & 0.52 \\
Wörter & 35.84 & 47.40 & -11.56 & 7.32 & -3.77 & 0.007 & -0.05 & -1.55 \\
\hline
\end{tabular}

Tabelle 17

Vergleich der mittleren Regressionskonstanten $\left(\mathrm{M}_{\mathrm{IC}}\right.$, in $\mathrm{ms}$ ) von positiven und negativen Testitems ( $t$-Test für abhängige Stichproben, zweiseitig; $n=8 ; 2$. Sitzung)

\begin{tabular}{|ccccccccc|}
\hline Reizart & $\begin{array}{c}\text { IC negativ } \\
M_{I C N}\end{array}$ & $\begin{array}{c}\text { IC positiv } \\
M_{I C P}\end{array}$ & $\begin{array}{c}\text { Differenz } \\
M_{I C N}-M_{I C P}\end{array}$ & $\begin{array}{c}S D \\
(\max )\end{array}$ & $t$-Wert & $p$ (sign.) & $\begin{array}{c}\text { Korrel. } \\
r\end{array}$ & $\begin{array}{c}\text { Effekt- } \\
\text { größe } \varepsilon\end{array}$ \\
\hline Figuren & 426.74 & 374.69 & 52.04 & 51.75 & 3.74 & 0.007 & 0.67 & 1.74 \\
Wörter & 426.96 & 347.57 & 79.38 & 56.51 & 5.48 & 0.007 & 0.69 & 2.52 \\
\hline
\end{tabular}

Zusammenfassend kann festgehalten werden, daß eine lineare Beziehung zwischen der Antwortlatenz und der Anzahl zu memorierender Items sowohl für Figuren als auch für Wörter nachgewiesen werden konnte. Die Ergebnisse des Vergleichs der mittleren Regressionskoeffizienten und -konstanten zeigen darüber hinaus, daß eine Wechselwirkung zwischen Testitem und Reizart vorliegt: Die Art des Testitems hat einen bedeutenden Einfluß auf die Scanning-Zeiten bei Wörtern, nicht jedoch bei Figuren. Was die Regressionskonstanten betrifft, so tritt ein signifikanter Unterschied zwischen den Reizarten nur bei positivem, nicht jedoch bei negativem Testitem auf. Diese Ergebnisse legen nahe, auch die weiteren Datenauswertungen für beide Testitems getrennt durchzuführen. 


\subsection{Synchronisationsprozesse elektrophysiologischer Signale}

\subsubsection{Aufbereitung der EEG-Daten}

Entsprechend dem Vorgehen bei den Verhaltensdaten (vgl. Kap. 6.1.1) wurden auch die EEG-Daten vor der statistischen Auswertung aufbereitet. Durchgänge mit falschen Antworten wurden nicht ausgewertet. Die Anfangsmarker derjenigen Durchgänge, die über- bzw. unterdurchschnittlich lange Antwortlatenzen beinhalteten und/oder Artefakte wie z. B. Augenbewegungen innerhalb des interessierenden EEG-Abschnittes (d. h. zwischen Marker 7 und 8 bzw. 9, vgl. Kap. 5.8) aufwiesen, wurden umkodiert (Marker 5) und so ebenfalls von der Auswertung ausgeschlossen. Tabelle 18 gibt einen Überblick über die Anzahl der Durchgänge der zweiten Sitzung, die nach der Datenaufbereitung für die weitere Auswertung genutzt werden konnten:

Tabelle 18

Anzahl auswertbarer Durchgänge nach Datenaufbereitung (maximal mögliche Anzahl: 480)

\begin{tabular}{|c|c|c|c|c|c|c|c|c|}
\hline \multirow[b]{3}{*}{ Setgröße } & \multicolumn{8}{|c|}{ Positives Testitem (Testreiz in Lernliste vorhanden) } \\
\hline & \multicolumn{4}{|c|}{ Figuren } & \multicolumn{4}{|c|}{ Wörter } \\
\hline & 1 & 2 & 3 & 4 & 1 & 2 & 3 & 4 \\
\hline $\begin{array}{l}\text { gültige } \\
\text { Durchgänge }\end{array}$ & 407 & 381 & 360 & 345 & 404 & 396 & 403 & 394 \\
\hline Prozentsatz & $84.79 \%$ & $79.38 \%$ & $75.00 \%$ & $71.86 \%$ & $84.17 \%$ & $82.50 \%$ & $83.96 \%$ & $82.08 \%$ \\
\hline
\end{tabular}

\begin{tabular}{|c|c|c|c|c|c|c|c|c|}
\hline \multirow[b]{3}{*}{ Setgröße } & \multicolumn{8}{|c|}{ Negatives Testitem (Testreiz in Lernliste nicht vorhanden) } \\
\hline & \multicolumn{4}{|c|}{ Figuren } & \multicolumn{4}{|c|}{ Wörter } \\
\hline & 1 & 2 & 3 & 4 & 1 & 2 & 3 & 4 \\
\hline $\begin{array}{l}\text { gültige } \\
\text { Durc hgänge }\end{array}$ & 381 & 373 & 320 & 278 & 393 & 403 & 396 & 401 \\
\hline Prozentsatz & $79.38 \%$ & $77.71 \%$ & $66.67 \%$ & $57.92 \%$ & $81.88 \%$ & $83.96 \%$ & $83.50 \%$ & $83.54 \%$ \\
\hline
\end{tabular}

Die Anzahl der pro Versuchsbedingung gültigen Durchgänge kann insgesamt als gut betrachtet werden und ist für eine aussagekräftige kohärenzanalytische Datenauswertung ausreichend (Beurteilung durch Bärbel Schack und Eva Möller, Institut für Medizinische Statistik, Informatik und Dokumentation, Jena).

\subsubsection{Sensitivität kohärenzanalytischer Kennwerte}

Bislang konnte nicht zuverlässig geklärt werden, welcher kohärenzanalytische Kennwert sich besonders für die Beschreibung schnell ablaufender kognitiver Prozesse eignet. In 
vorangegangenen Studien stand meist die mit einer Schwelle von 0,8 berechnete Kohärenzdauer im Mittelpunkt des Interesses (Krause, 1997; Krause, Gibbons \& Schack, 1998; Krause, Schack, Gibbons \& Kriese, 1997). In einer Untersuchung von Schack, Chen, Mescha und Witte (1999) stellten sich hingegen die Kohärenz, die maximale Kohärenz und die Zeitpunkte maximaler Kohärenz als besonders aussagekräftig heraus.

Im vorliegenden Fall sollte daher zunächst die Sensitivität der genannten kohärenzanalytischen Kennwerte zur Beschreibung möglicher reizartspezifischer topographischer Unterschiede geprüft werden. Hierfür sind die folgenden vier für die zweite Sitzung berechneten Kennwerte exemplarisch für das positive Testitem verglichen worden (vgl. auch Kap. 5.8):

- Mittlere Kohärenz

- Mittlere maximale Kohärenz (gerechnet ab 120 ms nach Marker 7)

- Mittlere Kohärenzdauern (Schwelle 0,65)

- Mittlere Zeitpunkte maximaler Kohärenz (gerechnet ab 120 ms nach Marker 7)

Für jede Setgröße und Reizart wurde ein sogenanntes "Map" erstellt, aus dem hervorgeht, welche Ausprägung der entsprechende Kennwert bei den verschiedenen Elektrodenpaaren im Mittel angenommen hat (vgl. Abb. 20 bis 23).

Der optische Vergleich zeigt, daß bei der unmittelbaren Gegenüberstellung nur diejenigen Kennwerte für jede Setgröße deutliche Unterschiede zwischen den Reizarten liefern, die rechnerisch gesehen von der Antwortlatenz abhängig sind (nämlich die mittlere Kohärenzdauer und der mittlere Zeitpunkt maximaler Kohärenz, vgl. Abb.22 und 23). Entsprechend lassen sich bei den zugehörigen graphischen Veranschaulichungen auch Zunahmen über die Setgrößen hinweg finden. Für eine eindeutige Interpretation müssen die gefundenen Unterschiede jedoch von der Antwortlatenz unabhängig sein. In den Maps der mittleren Kohärenz und mittleren maximalen Kohärenz (vgl. Abb.20 und 21), die - rechnerisch gesehen - von der Antwortgeschwindigkeit der Probanden unabhängige Synchronisationsinformationen liefern, sind nur fronto-zentral bis zentroparietal vereinzelte Zunahmen über die Setgrößen auszumachen. Diese treten zudem sowohl bei Figuren als auch bei Wörtern auf, so daß sich bei der direkten Gegenüberstellung der Reizarten pro Setgröße keine systematischen topographischen Unterschiede ergeben. 

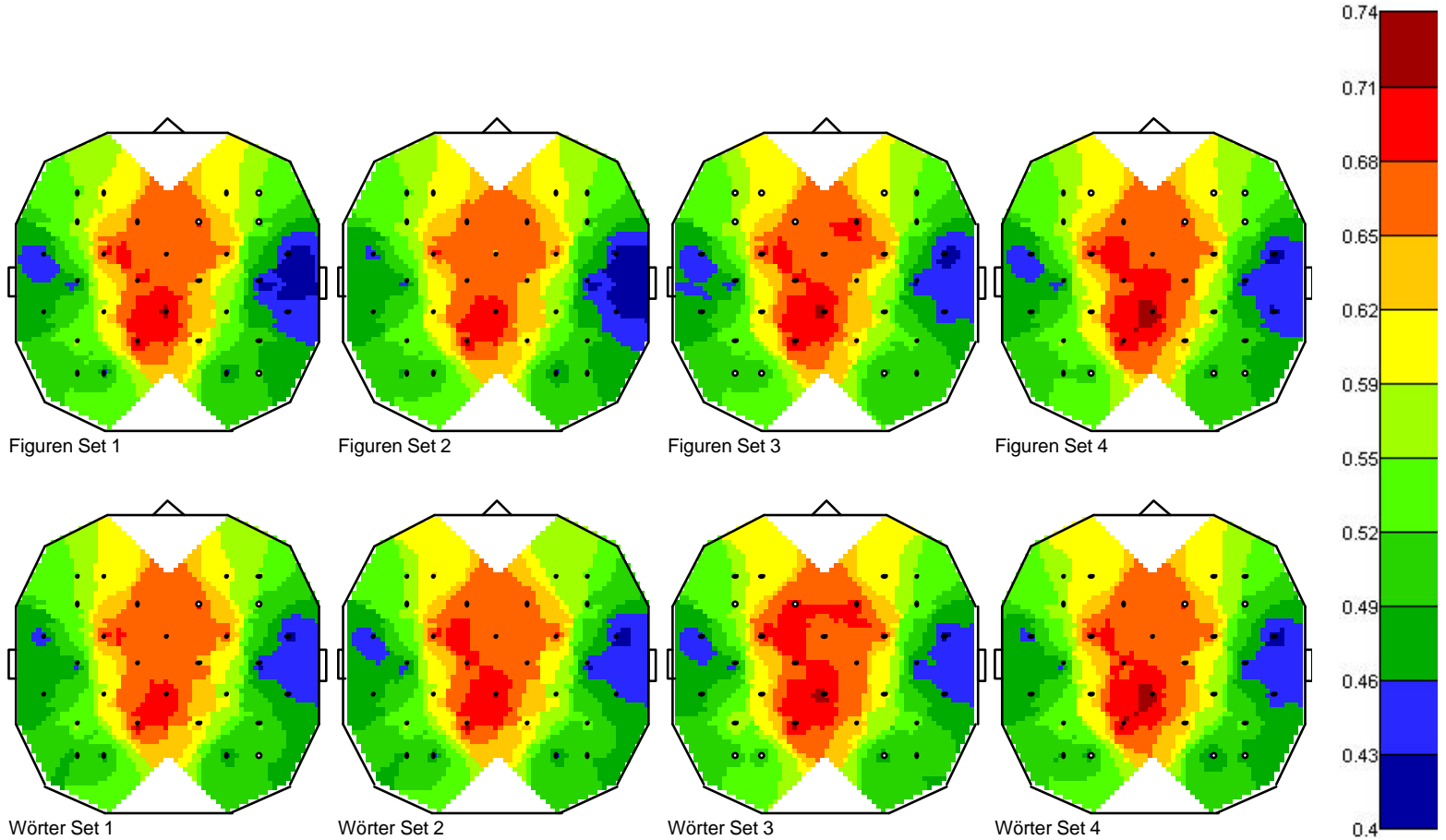

Abbildung 20. Maps der mittleren Kohärenz pro Reizart und Setgröße ( $N=10$; positives Testitem; 2. Sitzung)
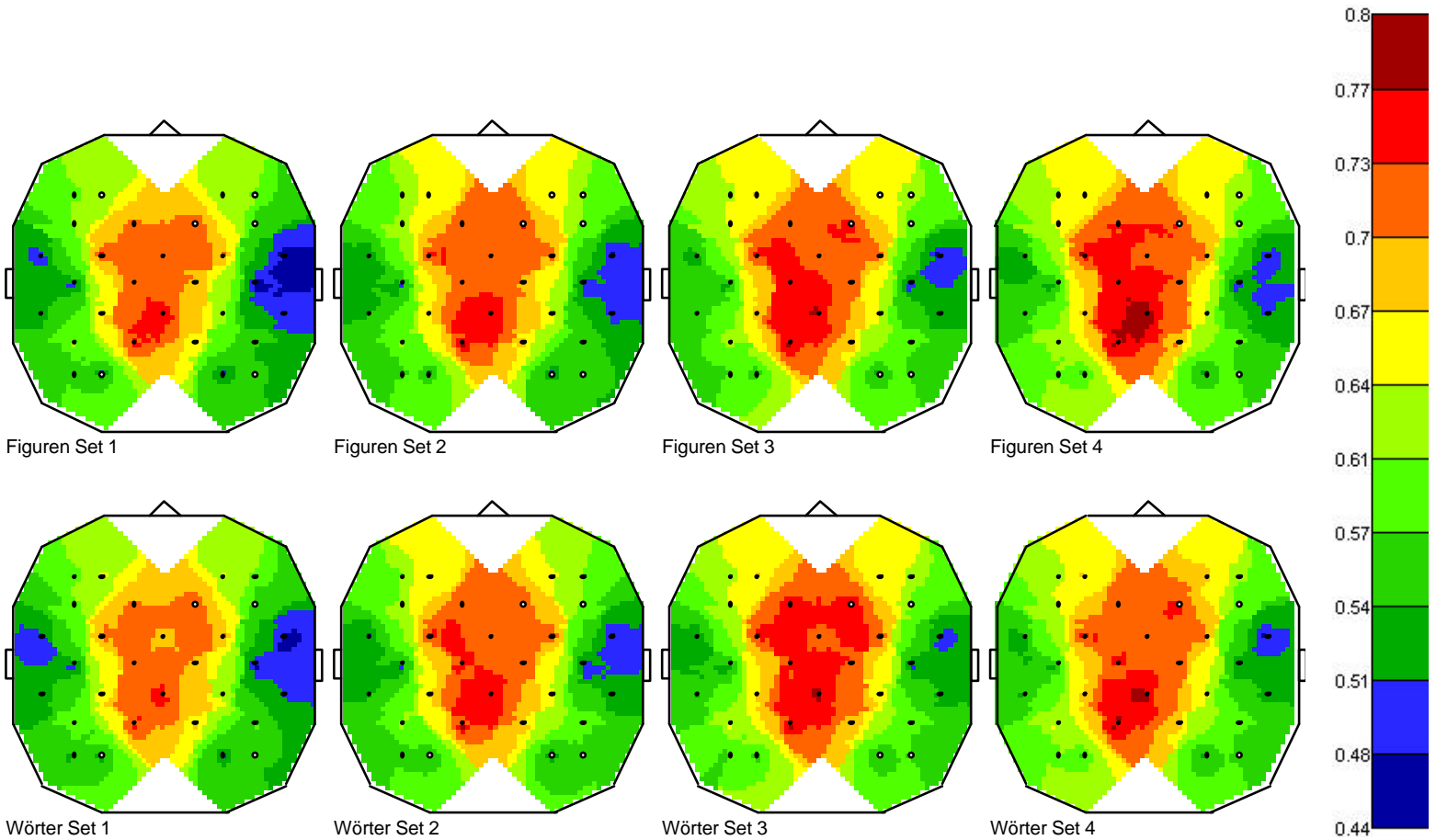

Abbildung 21. Maps der mittleren maximalen Kohärenz pro Reizart und Setgröße $(\mathrm{N}=10$; positives Testitem; 2. Sitzung; die maximale Kohärenz wurde ab 120 ms nach Marker 7 gerechnet) 


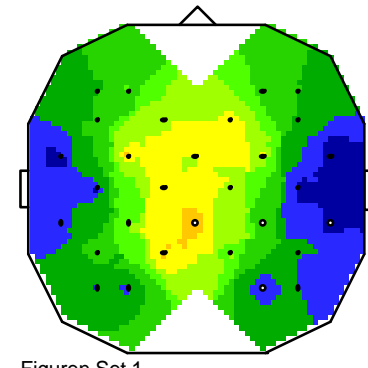

Figuren Set 1

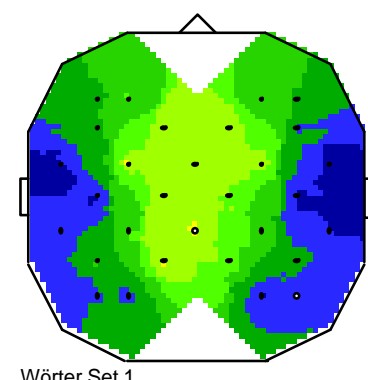

Wörter Set 1

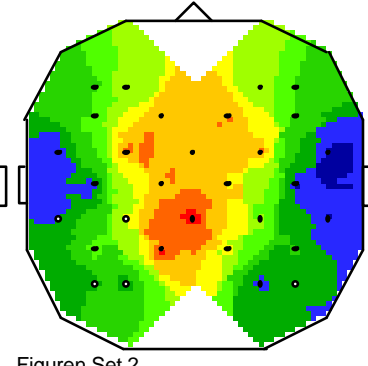

Figuren Set 2

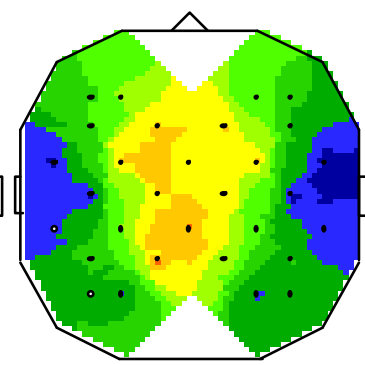

Wörter Set 2
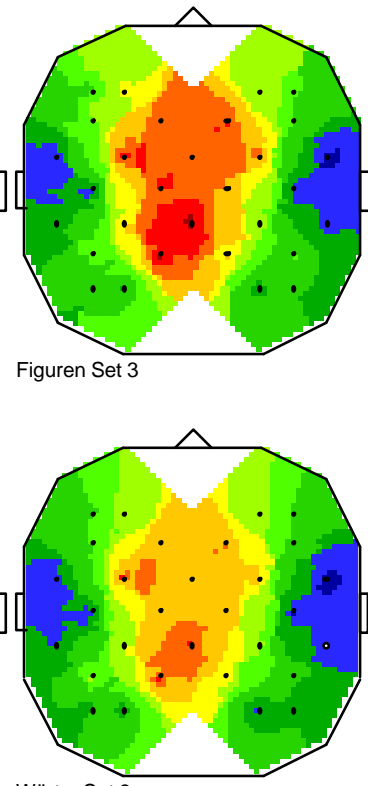

Wörter Set 3
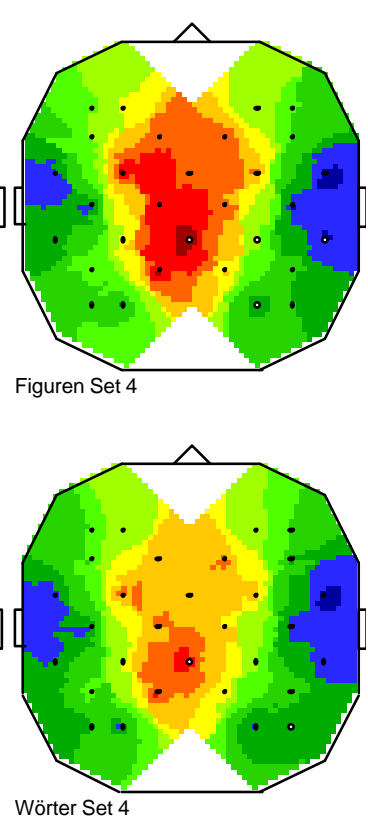

Wörter Set 4

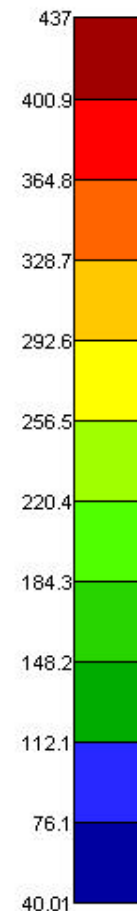

Abbildung 22. Maps der mittleren Kohärenzdauern (in $\mathrm{ms}$ ) pro Reizart und Setgröße $(\mathrm{N}=10$; positives Testitem; 2. Sitzung; Schwelle: 0.65)
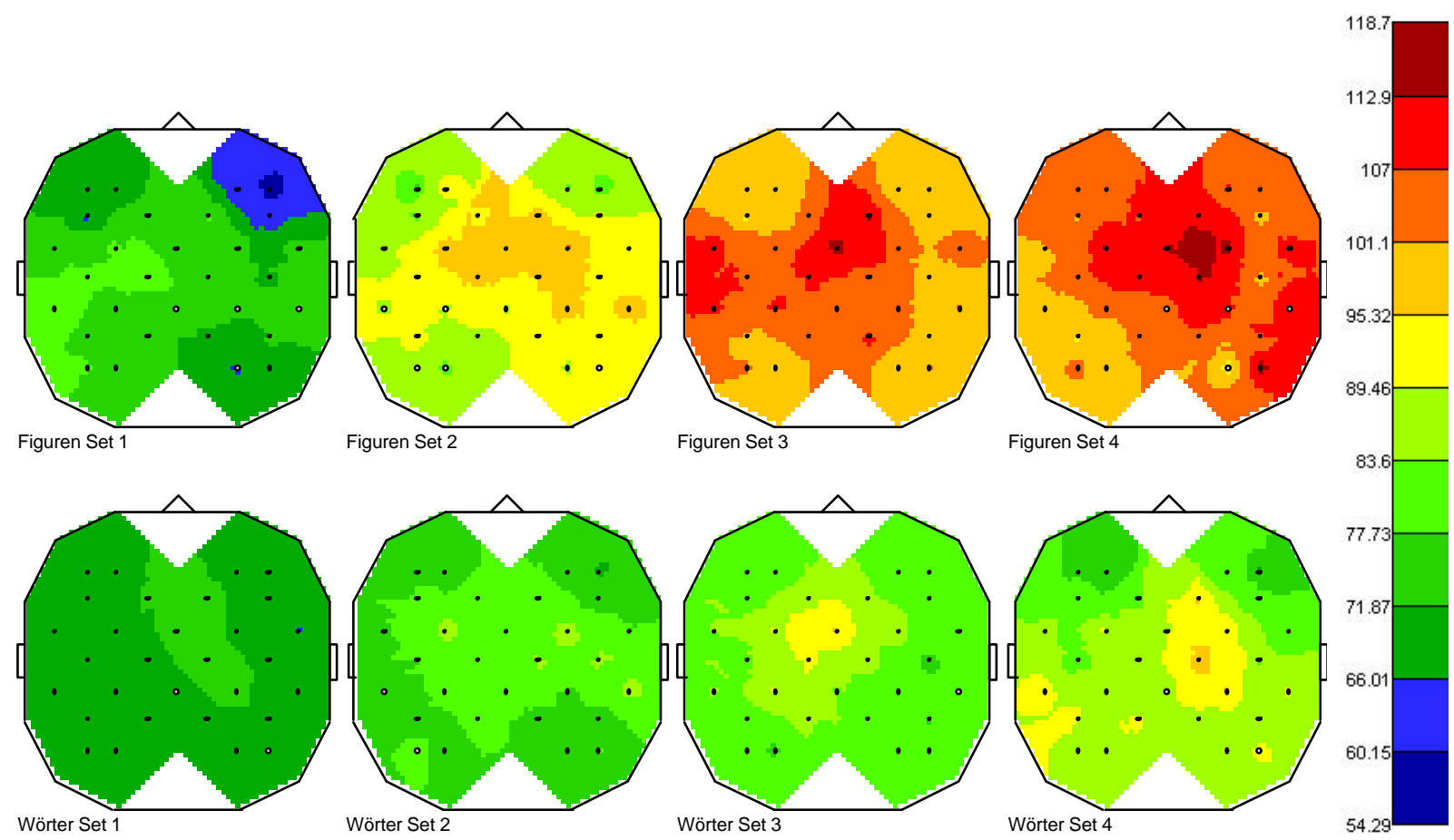

Abbildung 23. Maps der mittleren Zeitpunkte maximaler Kohärenz (in ms) pro Reizart und Setgröße ( $N=10$; positives Testitem; 2. Sitzung; die Zeitpunkte maximaler Kohärenz wurden ab $120 \mathrm{~ms}$ nach Marker 7 gerechnet) 
Der erste, aus dem optischen Vergleich gewonnene Eindruck wurde in statistischen Tests zum Vergleich der kohärenzanalytischen Kennwerte der Reizarten pro Setgröße bestätigt. Hierfür sind zunächst der Übersichtlichkeit halber bestimmte Elektrodenpaare nach Rücksprache mit einer EEG-Expertin (BärbelSchack, Institut für Medizinische Statistik, Informatik und Dokumentation, Jena) zu globalen "Arealen" zusammengefaßt worden (siehe Tab. 19):

Tabelle 19

Übersicht über die Elektrodenpaare, die zu globalen Arealen zusammengefaßt wurden

\begin{tabular}{|c|l|l|l|}
\hline $\begin{array}{c}\text { Areal- } \\
\text { Nr. }\end{array}$ & Bezeichnung & Elektrodenpaare & \\
\hline 1 & Mitte & 21,22 & Graphische Veranschaulichung \\
2 & links frontal & $1,2,3,17$ \\
3 & rechts frontal & $4,5,14,23$ \\
4 & links frontoparietal & $7,11,18,19$ \\
6 & rechts frontoparietal & $8,12,24,25$ \\
7 & parietal & $10,11,12,13,22$
\end{tabular}

Für jedes Areal wurden die Kennwerte der betreffenden Elektroden pro Probandin und Versuchsbedingung gemittelt. Anschließend wurden die Werte der Figuren und der Wörter für jede Setgröße mittels Wilcoxon-Tests verglichen. Tabelle 20 gibt die signifikanten Ergebnisse dieser Tests wieder (für die vollständigen Ergebnisse siehe Anhang B, Tab. B-4 bis B-7). Signifikante bis sehr signifikante Unterschiede zwischen den Reizarten sind wie erwartet nur bei den mittleren Kohärenzdauern und bei den mittleren Zeitpunkten maximaler Kohärenz zu verzeichnen. Obwohl diese Unterschiede in fast jedem Areal auftreten, fällt auf, daß immer nur vereinzelte Setgrößen betroffen sind. Dieses uneinheitliche Ergebnis und die mögliche Abhängigkeit der genannten Kennwerte von der Antwortlatenz erlauben keine schlüssige Interpretation der gefundenen Unterschiede und legen nahe, zur Aufdeckung möglicher topographischer Synchronisationsunterschiede andere kohärenzanalytischen Kennwerte sowie eine andere Strategie der Datenauswertung zu wählen.

Bei der Betrachtung der Abbildungen 20 bis 22 sticht ein Gebiet ins Auge, in dem die kohärenzanalytischen Kennwerte sehr hohe Ausprägungen aufweisen. Dieses Gebiet umfaßt im wesentlichen fronto-zentrale Areale. Die hier zugrundeliegenden ausgeprägten Synchronisationsprozesse könnten die eventuell in anderen Arealen bestehenden reizartspezifischen Unterschiede überlagern. Da sie bei beiden Reizarten und allen Setgrößen auftreten, erscheint eine unmittelbare Gegenüberstellung der verschiedenen Versuchsbedingungen insgesamt wenig ergiebig. Stattdessen bietet sich ein mittelbarer 
Vergleich an. Dieser ist für diejenigen Kennwerte durchgeführt worden, die rechnerisch gesehen von der Antwortlatenz unabhängig sind (mittlere Kohärenz und mittlere maximale Kohärenz). Der methodische Ansatz, das Vorgehen und die Ergebnisse werden im folgenden Abschnitt referiert.

Tabelle 20

Ränge der mittleren Kohärenzdauern und der mittleren Zeitpunkte maximaler Kohärenz: Signifikante Ergebnisse der Wilcoxon-Tests

(Vergleich der Reizarten pro Setgröße; $N=10$; positives Testitem; 2. Sitzung)

\begin{tabular}{|ccccccccccc|}
\multicolumn{9}{|c}{ Mittlere Kohärenzdauern } & \multicolumn{1}{c|}{ Anzahl Rangunterschiede } & \multicolumn{7}{c|}{ Mittelwerte } \\
Areal & Set & 1. Var. & 2. Var. & W $<F$ & $W>F$ & verbundene & $W<F$ & $W>F$ & $z$-Wert & $p$ (zweis.) \\
\hline 1 & 1 & Figuren & Wörter & 9 & 1 & 0 & 6.00 & 1.00 & -2.701 & 0.007 \\
1 & 2 & Figuren & Wörter & 8 & 2 & 0 & 6.13 & 3.00 & -2.192 & 0.028 \\
1 & 3 & Figuren & Wörter & 8 & 2 & 0 & 6.00 & 3.50 & -2.090 & 0.037 \\
2 & 2 & Figuren & Wörter & 8 & 2 & 0 & 6.50 & 1.50 & -2.497 & 0.013 \\
4 & 1 & Figuren & Wörter & 8 & 2 & 0 & 6.50 & 1.50 & -2.497 & 0.013 \\
4 & 2 & Figuren & Wörter & 8 & 2 & 0 & 6.00 & 3.50 & -2.090 & 0.037 \\
4 & 3 & Figuren & Wörter & 7 & 3 & 0 & 6.71 & 2.67 & -1.988 & 0.047 \\
5 & 2 & Figuren & Wörter & 9 & 1 & 0 & 5.56 & 5.00 & -2.293 & 0.022 \\
6 & 1 & Figuren & Wörter & 9 & 1 & 0 & 5.89 & 2.00 & -2.599 & 0.009 \\
6 & 2 & Figuren & Wörter & 8 & 2 & 0 & 6.25 & 2.50 & -2.293 & 0.022 \\
6 & 3 & Figuren & Wörter & 9 & 1 & 0 & 5.33 & 7.00 & -2.090 & 0.037 \\
7 & 1 & Figuren & Wörter & 9 & 1 & 0 & 5.67 & 4.00 & -2.395 & 0.017 \\
7 & 2 & Figuren & Wörter & 7 & 3 & 0 & 6.71 & 2.67 & -1.988 & 0.047 \\
\hline
\end{tabular}

\begin{tabular}{|c|c|c|c|c|c|c|c|c|c|c|}
\hline \multicolumn{11}{|c|}{ Mittlerer Zeitpunkt maximaler Kohärenz } \\
\hline \multirow[b]{2}{*}{ Areal } & \multirow[b]{2}{*}{ Set } & \multirow[b]{2}{*}{ 1. Var. } & \multirow[b]{2}{*}{ 2. Var. } & \multicolumn{3}{|c|}{ Anzahl Rangunterschiede } & \multicolumn{2}{|c|}{ Mittelwerte } & \multirow[b]{2}{*}{$z$-Wert } & \multirow[b]{2}{*}{$p$ (zweis.) } \\
\hline & & & & $W<F$ & $W>F$ & verbundene & $W<F$ & $W>F$ & & \\
\hline 1 & 2 & Figuren & Wörter & 8 & 2 & 0 & 6.00 & 3.50 & -2.090 & 0.037 \\
\hline 1 & 3 & Figuren & Wörter & 7 & 3 & 0 & 6.86 & 2.33 & -2.090 & 0.037 \\
\hline 2 & 1 & Figuren & Wörter & 7 & 3 & 0 & 7.00 & 2.00 & -2.192 & 0.028 \\
\hline 2 & 3 & Figuren & Wörter & 9 & 1 & 0 & 5.89 & 2.00 & -2.599 & 0.009 \\
\hline 3 & 2 & Figuren & Wörter & 8 & 2 & 0 & 6.38 & 2.00 & -2.395 & 0.017 \\
\hline 3 & 3 & Figuren & Wörter & 9 & 1 & 0 & 6.00 & 1.00 & -2.701 & 0.007 \\
\hline 4 & 3 & Figuren & Wörter & 9 & 1 & 0 & 5.44 & 6.00 & -2.192 & 0.028 \\
\hline 4 & 4 & Figuren & Wörter & 8 & 2 & 0 & 5.88 & 4.00 & -1.988 & 0.047 \\
\hline 5 & 2 & Figuren & Wörter & 8 & 2 & 0 & 6.50 & 1.50 & -2.497 & 0.013 \\
\hline 5 & 3 & Figuren & Wörter & 8 & 2 & 0 & 6.13 & 3.00 & -2.192 & 0.028 \\
\hline 6 & 2 & Figuren & Wörter & 7 & 3 & 0 & 6.86 & 2.33 & -2.090 & 0.037 \\
\hline 6 & 3 & Figuren & Wörter & 8 & 2 & 0 & 5.88 & 4.00 & -1.988 & 0.047 \\
\hline 6 & 4 & Figuren & Wörter & 7 & 3 & 0 & 6.71 & 2.67 & -1.988 & 0.047 \\
\hline 7 & 2 & Figuren & Wörter & 8 & 2 & 0 & 6.25 & 2.50 & -2.293 & 0.022 \\
\hline 7 & 3 & Figuren & Wörter & 10 & 0 & 0 & 5.50 & 0.00 & -2.803 & 0.005 \\
\hline
\end{tabular}




\subsubsection{Setgröße, mittlere Kohärenz und mittlere maximale Kohärenz}

Eine Möglichkeit des mittelbaren Vergleichs der Reizarten läßt sich aus der Methode der "parametrischen Variation" ableiten, auf der auch der vorliegende Versuch basiert. Bei der parametrischen Variation wird ein einzelner experimenteller Faktor variiert und anschließend untersucht, inwieweit diese Variation eine oder mehrere der beobachteten Variablen systematisch beeinflußt. Smith und Jonides (1997; vgl. auch Kap. 3.2.1) haben dieses Vorgehen erfolgreich zur topographischen Auswertung ihrer PET-Daten angewendet, indem sie für verschiedene Hirnareale prozentuale Aktivationsänderungen in Abhängigkeit von der variierten Gedächtnislast berechneten und graphisch darstellten. Im vorliegenden Fall bot sich - analog dem Vorgehen bei den Antworlatenzen - die regressionsanalytische Untersuchung des Einflusses der Setgröße auf die kohärenzanalytischen Kennwerte an. Für jede Versuchsbedingung sind daher für alle 30 definierten Elektrodenpaare (vgl. Kap. 5.8) Regressionsanalysen der mittleren Kohärenz sowie der mittleren maximalen Kohärenz der Größe des positiven Sets gerechnet worden. Die Tabellen 21 und 22 geben einen Überblick über die signifikanten Ergebnisse (vgl. auch Anhang B, Tab. B-8 bis B-15).

Tabelle 21

Signifikante Ergebnisse der linearen Regressionsanalysen der gemittelten Kohärenz $\left(M_{K}\right)$ und der Größe des positiven Sets ( $N=10 ; 2$. Sitzung)

\begin{tabular}{|c|c|c|c|c|c|c|c|c|c|}
\hline $\begin{array}{c}\text { Elektr.- } \\
\text { Paar }\end{array}$ & $A V$ & Testitem & Multiple $R$ & $R^{2}$ & Sign. $F$ & Slope & $p$ (sign.) & Intercept & $p$ (sign.) \\
\hline $9(C 4 / T 4)$ & $M_{K}$ Figuren & positiv & 0.971 & 0.943 & 0.029 & 0.008 & 0.029 & 0.396 & 0.000 \\
\hline $13(P 4 / T 6)$ & $M_{k}$ Figuren & positiv & 0.965 & 0.931 & 0.035 & 0.007 & 0.035 & 0.511 & 0.000 \\
\hline 17 (Fp1/F3) & Mk Figuren & positiv & 0.971 & 0.943 & 0.029 & 0.006 & 0.029 & 0.451 & 0.000 \\
\hline $29(T 5 / 01)$ & $M_{K}$ Figuren & positiv & 0.999 & 0.999 & 0.001 & 0.005 & 0.001 & 0.524 & 0.000 \\
\hline $9(C 4 / T 4)$ & $M_{k}$ Wörter & positiv & 0.960 & 0.922 & 0.040 & 0.006 & 0.040 & 0.413 & 0.000 \\
\hline $21(F z / C z)$ & Mk Wörter & positiv & 1.000 & 1.000 & 0.000 & 0.010 & 0.000 & 0.694 & 0.000 \\
\hline 29 (T5/O1) & $M_{K}$ Wörter & positiv & 0.967 & 0.935 & 0.033 & 0.005 & 0.033 & 0.536 & 0.000 \\
\hline $7(C 3 / C z)$ & Mk Figuren & negativ & 0.965 & 0.932 & 0.035 & 0.008 & 0.035 & 0.655 & 0.000 \\
\hline $11(P 3 / P z)$ & Mk Figuren & negativ & 0.954 & 0.910 & 0.046 & 0.006 & 0.046 & 0.651 & 0.000 \\
\hline $21(F z / C z)$ & Mk Wörter & negativ & 0.992 & 0.983 & 0.008 & 0.007 & 0.008 & 0.705 & 0.000 \\
\hline 29 (T5/O1) & $M_{K}$ Wörter & negativ & 0.962 & 0.925 & 0.038 & 0.005 & 0.038 & 0.538 & 0.000 \\
\hline
\end{tabular}

Zwischen der Setgröße und der mittleren Kohärenz sind nur vergleichsweise wenige, signifikant lineare Beziehungen zu verzeichnen (vgl. Tab.21). Die Differenzierungsmöglichkeit zwischen den beiden untersuchten Reizarten ist besonders im Falle positiver Testitems gering; hier finden sich von insgesamt neun Elektrodenpaaren, deren mittlere Kohärenzen eine signifikant lineare Beziehung zur Setgröße aufweisen, nur drei Elektrodenpaare, die sich bei Figuren und Wörtern unterscheiden. Es sind dies die 
Elektrodenpaare 13 (P4/T6) und 17 (Fp1/F3) bei den Figuren und das Elektrodenpaar 21 (Fz/Cz) bei den Wörtern. Bei der Betrachtung der Tabelle 22 fällt auf, daß alle diese Elektrodenpaare auch bei der Untersuchung des Zusammenhangs zwischen der Setgröße und den maximalen Kohärenzen zwischen den Reizarten unterscheiden. Darüber hinaus treten hier weit mehr signifikant lineare Beziehungen auf als bei den mittleren Kohärenzen. Der Überschneidungsgrad der Elektrodenpaare mit signifikanten Ergebnissen bei den verschiedenen Reizarten ist für jedes Testitem sehr gering: Beim positiven Testitem betrifft dies nur das Elektrodenpaar 3 (F3/Fz), beim negativen Testitem das Elektrodenpaar 26 (P4/O2). Insgesamt scheint die maximale Kohärenz am besten dazu geeignet zu sein, topographische Synchronisationsunterschiede zwischen den untersuchten Reizarten aufzudecken.

Tabelle 22

Signifikante Ergebnisse der linearen Regressionsanalysen der gemittelten maximalen Kohärenz $\left(\mathrm{M}_{\mathrm{MK}}\right)$ und der Größe des positiven Sets ( $=10 ; 2$. Sitzung)

\begin{tabular}{|c|c|c|c|c|c|c|c|c|c|}
\hline $\begin{array}{c}\text { Elektr.- } \\
\text { Paar }\end{array}$ & $A V$ & Testitem & Multiple $R$ & $R^{2}$ & Sign. $F$ & Slope & $p$ (sign.) & Intercept & $p$ (sign.) \\
\hline $3(F 3 / F z)$ & MМк Figuren & positiv & 0.996 & 0.993 & 0.004 & 0.010 & 0.004 & 0.753 & 0.000 \\
\hline $4(F z / F 4)$ & $M_{M K}$ Figuren & positiv & 0.986 & 0.972 & 0.014 & 0.013 & 0.014 & 0.654 & 0.000 \\
\hline $7(C 3 / C z)$ & MMK Figuren & positiv & 0.963 & 0.927 & 0.037 & 0.009 & 0.037 & 0.710 & 0.000 \\
\hline $8(C z / C 4)$ & $M_{M K}$ Figuren & positiv & 0.996 & 0.992 & 0.004 & 0.013 & 0.004 & 0.674 & 0.000 \\
\hline 13 (P4/T6) & $M_{M K}$ Figuren & positiv & 0.960 & 0.921 & 0.040 & 0.018 & 0.040 & 0.541 & 0.000 \\
\hline 14 (Fp2/F8) & $M_{M K}$ Figuren & positiv & 0.966 & 0.933 & 0.034 & 0.007 & 0.034 & 0.553 & 0.000 \\
\hline 17 (Fp1/F3) & $M_{M K}$ Figuren & positiv & 0.965 & 0.932 & 0.035 & 0.014 & 0.035 & 0.494 & 0.000 \\
\hline 19 (С3/Р3) & MMK Figuren & positiv & 0.995 & 0.989 & 0.005 & 0.008 & 0.005 & 0.737 & 0.000 \\
\hline $22(C z / P z)$ & $M_{M K}$ Figuren & positiv & 0.988 & 0.977 & 0.012 & 0.007 & 0.012 & 0.700 & 0.000 \\
\hline $28(T 4 / T 6)$ & $M_{M K}$ Figuren & positiv & 0.987 & 0.973 & 0.014 & 0.012 & 0.014 & 0.437 & 0.000 \\
\hline $29(T 5 / 01)$ & $M_{M K}$ Figuren & positiv & 0.990 & 0.981 & 0.010 & 0.014 & 0.010 & 0.566 & 0.000 \\
\hline 1 (Fp1/F7) & $M_{M K}$ Wörter & positiv & 0.984 & 0.968 & 0.016 & 0.016 & 0.016 & 0.519 & 0.000 \\
\hline $3(F 3 / F z)$ & MMK Wörter & positiv & 0.999 & 0.999 & 0.001 & 0.012 & 0.001 & 0.744 & 0.000 \\
\hline 6 (Т3/C3) & $M_{M K}$ Wörter & positiv & 0.997 & 0.995 & 0.003 & 0.010 & 0.003 & 0.482 & 0.000 \\
\hline $9(C 4 / T 4)$ & $M_{M K}$ Wörter & positiv & 0.999 & 0.998 & 0.001 & 0.016 & 0.001 & 0.447 & 0.000 \\
\hline 16 (T3/T5) & $M_{M K}$ Wörter & positiv & 0.988 & 0.976 & 0.012 & 0.013 & 0.012 & 0.475 & 0.000 \\
\hline $21(F z / C z)$ & $M_{M K}$ Wörter & positiv & 0.992 & 0.984 & 0.008 & 0.013 & 0.008 & 0.737 & 0.000 \\
\hline 26 (P4/O2) & MMK Wörter & positiv & 0.952 & 0.906 & 0.048 & 0.016 & 0.048 & 0.619 & 0.000 \\
\hline 2 (F7/F3) & MMK Figuren & negativ & 0.954 & 0.909 & 0.046 & 0.012 & 0.046 & 0.597 & 0.000 \\
\hline $4(F z / F 4)$ & $M_{M K}$ Figuren & negativ & 0.993 & 0.986 & 0.007 & 0.011 & 0.007 & 0.674 & 0.000 \\
\hline 5 (F4/F8) & MMK Figuren & negativ & 0.963 & 0.928 & 0.037 & 0.006 & 0.037 & 0.570 & 0.000 \\
\hline $11(P 3 / P z)$ & $M_{M K}$ Figuren & negativ & 0.972 & 0.945 & 0.028 & 0.013 & 0.028 & 0.703 & 0.000 \\
\hline 14 (Fp2/F8) & MMK Figuren & negativ & 0.992 & 0.984 & 0.008 & 0.012 & 0.008 & 0.554 & 0.000 \\
\hline 16 (T3/T5) & $M_{M K}$ Figuren & negativ & 0.966 & 0.934 & 0.034 & 0.010 & 0.034 & 0.500 & 0.000 \\
\hline 17 (Fp1/F3) & MMK Figuren & negativ & 0.975 & 0.951 & 0.025 & 0.010 & 0.025 & 0.507 & 0.000 \\
\hline $26(P 4 / O 2)$ & $M_{M K}$ Figuren & negativ & 0.958 & 0.919 & 0.042 & 0.011 & 0.042 & 0.636 & 0.000 \\
\hline 30 (T6/O2) & $M_{M K}$ Figuren & negativ & 0.977 & 0.955 & 0.023 & 0.014 & 0.023 & 0.590 & 0.000 \\
\hline
\end{tabular}


Fortsetzung Tabelle 22

Signifikante Ergebnisse der linearen Regressionsanalysen der gemittelten maximalen Kohärenz $\left(\mathrm{M}_{\mathrm{MK}}\right)$ und der Größe des positiven Sets ( $N=10 ; 2$. Sitzung)

\begin{tabular}{|llllllllll|}
\hline $\begin{array}{c}\text { Elektr.- } \\
\text { Paar }\end{array}$ & AV & Testitem Multiple $R$ & $R^{2}$ & Sign. F & Slope & $p$ (sign.) & Intercept & $p$ (sign.) \\
\hline $3(F 3 / F z)$ & $M_{\text {MK }}$ Wörter & negativ & 0.970 & 0.941 & 0.030 & 0.005 & 0.030 & 0.769 & 0.000 \\
$10(T 5 / P 3)$ & $M_{\text {MK }}$ Wörter & negativ & 0.954 & 0.910 & 0.046 & 0.009 & 0.046 & 0.600 & 0.000 \\
$13(P 4 / T 6)$ & $M_{\text {MK }}$ Wörter & negativ & 0.992 & 0.984 & 0.008 & 0.011 & 0.008 & 0.556 & 0.000 \\
$20(P 3 / O 1)$ & $M_{\text {MK Wörter }}$ & negativ & 0.990 & 0.980 & 0.010 & 0.008 & 0.010 & 0.655 & 0.000 \\
$21(F z / C z)$ & $M_{\text {MK }}$ Wörter & negativ & 0.981 & 0.962 & 0.019 & 0.012 & 0.019 & 0.745 & 0.000 \\
$26(P 4 / O 2)$ & $M_{\text {MK }}$ Wörter & negativ & 0.997 & 0.995 & 0.003 & 0.008 & 0.003 & 0.634 & 0.000 \\
\hline
\end{tabular}

Für den mittelbaren Vergleich der Reizarten wurden diejenigen Elektrodenpaare gemappt, deren Regressionskoeffizienten aus der Regression der maximalen Kohärenzen und der Größe des positiven Sets signifikante Ergebnisse aufwiesen (vgl. Abb. 24 und Tab. 22). Anhand dieser Maps können die Hypothesen zu den topographischen Aspekten des Arbeitsgedächtnisses geprüft werden.

In der ersten Hypothese wurde angenommen, daß am Abruf kurzzeitig gespeicherter verbaler Information die linksseitigen Areale 22, 40 und 42 (oberer Temporallappen und unterer Parietallappen) beteiligt sind. Diese kortikalen Strukturen sollen eine entscheidende Rolle bei der Speicherung verbaler Information spielen, so daß damit gerechnet werden kann, daß die Synchronisierung hier mit der Größe des positiven Sets zunimmt. Für Area 44 (Broca-Areal) wurde hingegen keine erhöhte Aktivität (bzw. keine Zunahme der Synchronisierung) erwartet, da diesem Areal eine wesentliche Rolle beim stillen Wiederholen verbalen Materials zugeschrieben wird und in der Abrufphase der SternbergAufgabe kein stilles Wiederholen der gelernten Items erforderlich ist.

Aus Abbildung 24 geht hervor, daß der Abruf verbaler Information (Wörter) beim positiven Testitem in den linksseitigen Arealen 1 bis 3, 40 und 22 (Elektrodenpaar T3/C3) sowie 22/42 bis 37 (Elektrodenpaar T3/T5) zu signifikanten Zunahmen der maximalen Kohärenz mit der Größe des positiven Sets führt (vgl. Abb. 25 und Kap. 3.2.2, Abb. 11). In Area 44 (Elektrodenpaar F3/F7) kann keine solche Zunahme festgestellt werden. Neben den genannten Arealen lassen sich signifikante Synchronisationszunahmen linkshemisphärisch in Area 10/46 (Elektrodenpaar Fp1/F7) sowie rechtshemisphärisch in Area 1/2/3 bis 22 (Elektrodenpaar C4/T4) und 19 bis 17 (Elektrodenpaar $\mathrm{P} 4 / \mathrm{O} 2)$ finden. Schließlich können auch in einem ausgedehnten zentro-frontalen Bereich signifikante Zunahmen der maximalen Kohärenz mit der Größe des positiven Sets festgestellt werden. Dieser Bereich schließt linksseitig Area 8/9 mit ein (Elektrodenpaare $\mathrm{F} 3 / \mathrm{Fz}$ und $\mathrm{Fz} / \mathrm{Cz}$ ) und weist auch beim negativen Testitem signifikante Ergebnisse auf. Im übrigen weichen die Ergebnisse des negativen Testitems jedoch von denen des 
positiven Testitems ab. Linkshemisphärisch sind beidseitig in den Arealen 7/39/37 (Elektrodenpaare P3/T5 und P4/T6) und 7 bis 17 (Elektrodenpaare P3/O1 und P4/O2) signifikante Synchronisationszunahmen zu verzeichnen, nicht jedoch in den linkshemisphärischen Arealen 22, 40 und 42. Damit haben die in der ersten Hypothese enthaltenen Annahmen nur für die Bedingung "Wörter, positives Testitem" Gültigkeit.

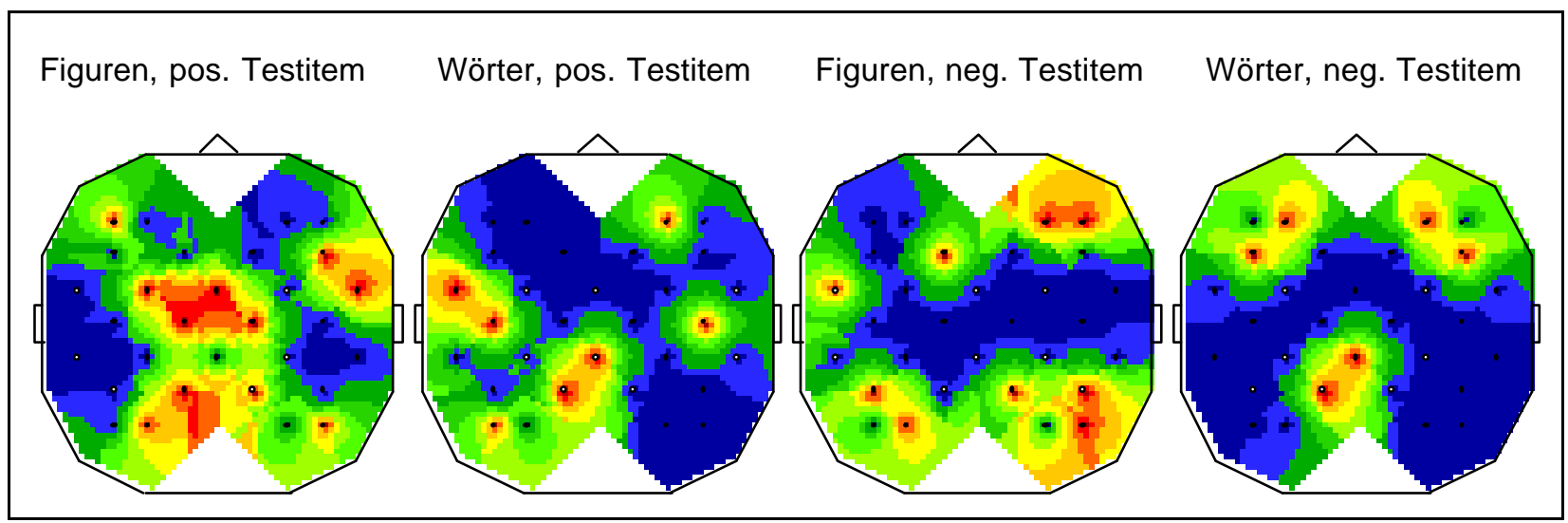

Abbildung 24. Elektrodenpaare, für die in der linearen Regressionsanalyse der maximalen Kohärenz und der Größe des positiven Sets signifikante Regressionskoeffizienten gefunden wurden (in der Abbildung rot hervorgehoben; $N=10 ; 2$. Sitzung)

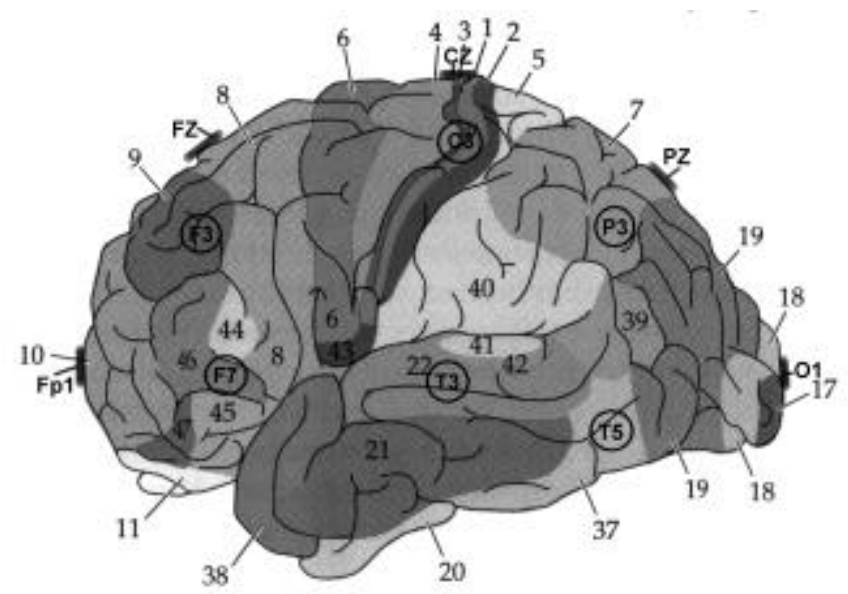

Abbildung 25. Überblick über die zytoarchitektonischen Areale und ungefähre Elektrodenpositionen (am Beispiel der linken Hemisphäre)

In der zweiten Hypothese wurde postuliert, daß der Abruf nonverbaler Information aus dem Arbeitsgedächtnis rechtshemisphärisch zu kortikalen Aktivationen in den Arealen 7, 40 und 37 führt. Diese Areale sollen beim Lernen visuell-räumlichen Materials von zentraler Bedeutung sein. Weiterhin wurde vermutet, daß sich für die Sprachstrukturen der linken Hemisphäre (Area 22, 40, 42, 44) keine Beteiligung am Abruf nonverbaler Information nachweisen ließe.

Wie aus Abbildung 24 ersichtlich, treten während des Abrufs nonverbaler Information (Figuren) beim positiven Testitem in den rechtshemisphärischen Arealen 22/42/37 (Elektrodenpaar T4/T6) und 7/39/37 (Elektrodenpaar P4/T6) signifikante Synchronisati- 
onszunahmen mit der Größe des positiven Sets auf. Darüber hinaus sind linksseitig die Areale 8/9/10 (Elektrodenpaare Fp1/F3 und F3/Fz), 1/2/3 bis 7 (Elektrodenpaar C3/P3) und 37 bis 17 (Elektrodenpaar T5/O1) sowie rechtsseitig die Areale 8/9 (Elektrodenpaar F4/Fz) und 10/46 (Elektrodenpaar Fp2/F8) beteiligt. Schließlich lassen sich beidseitig zentro-parietal ausgedehnte Synchronisationsprozesse finden (Areale 1/2/3, 5 und 7).

Der Vergleich positiver und negativer Testitems offenbart auch bei den Figuren Unterschiede in der Verteilung der Synchronisationsprozesse. Bei negativen Testitems treten signifikante Synchronisationszunahmen beidseitig in frontalen Arealen auf (Area 9, 10, 46 und 44; Elektrodenpaare Fp1/F7, Fp2/F8 und F4/F8), während die zentro-parietalen und rechtsseitig temporo-parietalen Synchronisationen fehlen. Dafür lassen sich rechtsseitig in den Arealen 7 bis 17 und 37 bis 17 (Elektrodenpaare P4/O2 und T6/O2) sowie linksseitig in den Arealen 7 und 22/42/37 (Elektrodenpaare Pz/P3 und T3/T5) Synchronisationsprozesse finden, die beim positiven Testitem nicht auftreten.

Insgesamt kann somit ein Teil der zweiten Hypothese als bestätigt angesehen werden, allerdings wiederum nur für das positive Testitem. In den rechtshemisphärischen Arealen 7 und 37 finden sich erwartungsgemäß verstärkte Synchronisationsprozesse, jedoch nicht in Area 40. Wie vermutet, zeigten sich beim Abruf nonverbaler Information (positives Testitem) in den Sprachstrukturen der linken Hemisphäre keine signifikanten Synchronisationszunahmen.

In der dritten Hypothese wurde angenommen, daß beim Abruf sowohl verbaler als auch nonverbaler Information aus dem Arbeitsgedächtnis Areale im präfrontalen Kortex aktiviert sind (etwa Area 45/47 und 9/46). Dabei handelt es sich um Strukturen, denen Erhaltungs- bzw. Überwachungsfunktionen zugeschrieben werden. Die Aktivationen sollten sich beim Abruf verbaler Information auf den linken, beim Abruf nonverbaler Information auf den rechten präfrontalen Kortex konzentrieren.

Ein Vergleich der einzelnen Maps aus Abbildung 24 macht deutlich, daß in allen vier Bedingungen in präfrontalen Arealen signifikante Synchronisationszunahmen mit der Größe des positiven Sets zu finden sind. Allerdings treten diese Synchronisationszunahmen in unterschiedlichen Bereichen des präfrontalen Kortex auf und sind nicht immer auf eine Hemisphäre beschränkt. Ein durchweg hypothesenkonformes Ergebnis läßt sich für die Wörter aufzeigen: Bei beiden Testitems nehmen die Synchronisationen linksseitig etwa in Area 8/9 (Elektrodenpaar F3/Fz) signifikant mit der Größe des positiven Sets zu, beim positiven Testitem zusätzlich auch in Area 10/46 (Elektrodenpaar Fp1/F7). Für die Figuren kann die dritte Hypothese nur zum Teil als bestätigt angesehen werden: Zwar lassen sich hier Synchronisationszunahmen in präfrontalen Arealen finden (Area 8/9, 9/10 und 10/46), diese sind jedoch nicht auf die rechte Hemisphäre beschränkt, sondern treten bei beiden Testitemarten auch linksseitig auf (Elektrodenpaare Fp2/F8, Fp1/F3, F4/Fz und F3/Fz). 
Neben der Lokalisation der signifikanten Regressionskoeffizienten aus der Regressionsanalyse der maximalen Kohärenzen und der Größe des positiven Sets ist auch die Größenordnung dieser Regressionskoeffizienten von Interesse. Diese kann den Maps in Abbildung 26 entnommen werden (vgl. auch Tab. 22). Aus den Maps geht hervor, daß bei positiven Testitems der größte Regressionskoeffizient bei den Figuren in Area 7/39/37 auftritt (Elektrodenpaar P4/T6). Bei den Wörtern finden sich die größten Regressionskoeffizienten linksseitig in Area 10/46 (Elektrodenpaar Fp1/F7) sowie rechtsseitig in Area 1/2/3, 40 und 22 und 7 bis 17 (Elektrodenpaare C4/T4 und P4/O2).

Beim negativen Testitem sind die stärksten Zunahmen der maximalen Kohärenz bei den Figuren linkshemisphärisch in Area 46 bis 22 und 7, rechtshemisphärisch in Area 10/46 und 37 bis 17 zu verzeichnen. Bei den Wörtern treten die höchsten Regressionskoeffizienten an einem fronto-zentralen Elektrodenpaar $(\mathrm{Fz} / \mathrm{Cz})$ und rechtshemisphärisch in Area 7/39/37 auf (Elektrodenpaar P4/T6).

Insgesamt führt die Zunahme der Größe des positiven Sets in den verschiedenen Versuchsbedingungen zu unterschiedlich starken Zunahmen der maximalen Kohärenz. Die Unterschiede zwischen den Regressionskoeffizienten sind allerdings sehr klein: Es handelt sich dabei um Kohärenzunterschiede von 0.001 bis maximal 0.011.

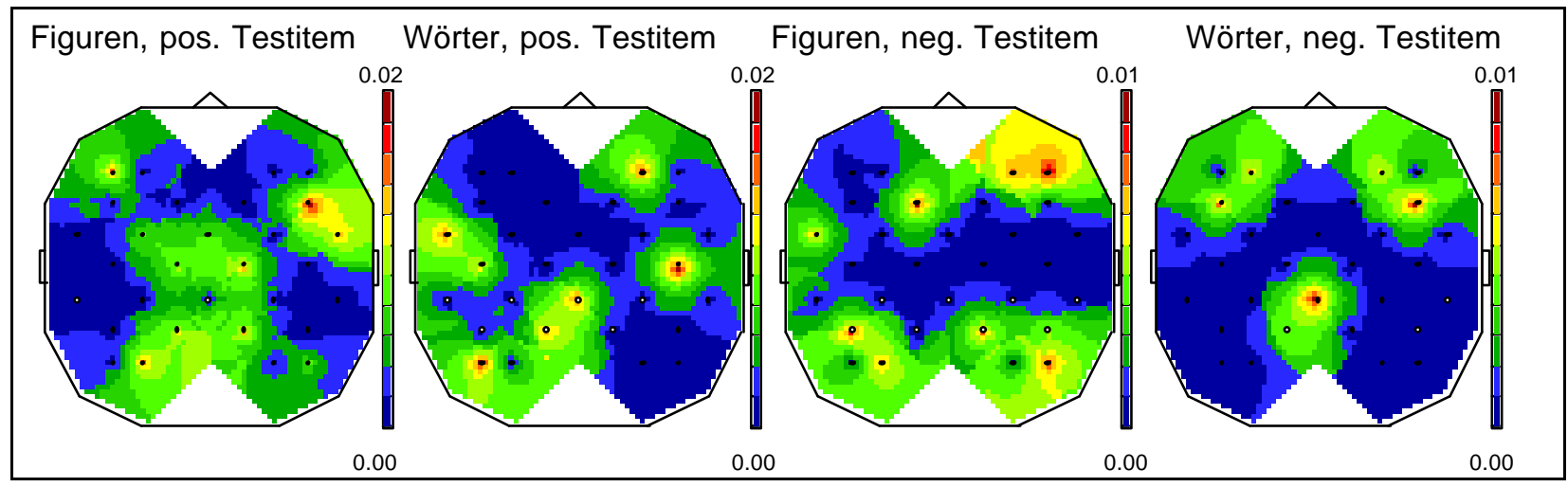

Abbildung 26. Lineare Regressionsanalyse der maximalen Kohärenz und der Größe des positiven Sets: signifikante Regressionskoeffizienten ( $N=10 ; 2$. Sitzung)

Zusammenfassend kann festgehalten werden, daß sich topographische Synchronisationsunterschiede während des Abrufs kurzzeitig behaltenen verbalen bzw. nonverbalen Reizmaterials am besten durch eine Analyse der mittels eines kontinuierlichen Schätzverfahrens der Kohärenzfunktion erhaltenen maximalen Kohärenzen aufdecken lassen. Von entscheidender Bedeutung ist dabei der mittelbare Vergleich der entsprechenden Werte: Während eine direkte inferenzstatistische Gegenüberstellung der qualitativ sehr verschiedenen Reizarten pro Setgröße zu keinen aussagekräftigen Ergebnissen geführt hat, konnten im mittelbaren Vergleich der Regressionskoeffizienten bzw. der Elektrodenpaare, die in der Regressionsanalyse der maximalen Kohärenzen und der Größe des positiven Sets signifikante Ergebnisse aufweisen, reizartspezifische topographische 
Synchronisationsunterschiede aufgezeigt werden. So sind im Falle des positiven Testitems am Abruf nonverbaler Information erwartungsgemäß unter anderem rechts frontopolar-frontale und parieto-temporale Areale, am Abruf verbaler Information links frontopolar-frontale und parieto-temporale Areale beteiligt gewesen. Damit konnten die drei in Kapitel 4 formulierten Hypothesen zu topographischen Aspekten des Arbeitsgedächtnisses zumindest teilweise für das positive Testitem bestätigt werden.

\section{3 Zeitverlauf der Synchronisationsprozesse}

\subsubsection{Verlauf der gemittelten Bandkohärenzen}

Ein bedeutendes Ziel dieser Arbeit besteht unter anderem darin, Erkenntnisse zum Zeitverlauf der Synchronisationsprozesse elektrophysiologischer Signale in der Abrufphase der Sternberg-Aufgabe zu liefern. Erste Anhaltspunkte für Unterschiede im zeitlichen Verlauf der Verarbeitungsprozesse bei Figuren und Wörtern kann eine Verlaufsbetrachtung der mittleren Bandkohärenzen $(13-20 \mathrm{~Hz})$ liefern. Diese wurde für ein bestimmtes Zeitintervall zwischen dem Testitem und der Antwort vorgenommen, das 120 ms nach der Darbietung des Testitems - und damit mutmaßlich nach dessen kognitiver Verarbeitung beginnt (vgl. Kap. 5.8; für die vollständigen Verläufe vgl. Anhang $\mathrm{C}$ ). Da sich die mittleren Kohärenzen aufgrund unterschiedlicher Antwortlatenzen nicht zu jedem Abtastzeitpunkt aus den Werten aller sechs Probandinnen zusammensetzen, wurden hintere Verlaufsabschnitte, denen lediglich Werte von fünf oder weniger Probandinnen ugrundelagen, gekappt. Das in den Abbildungen 27 und 28 dargestellte Zeitintervall endet daher je nach Reizart und Setgröße zwischen 265.68 ms und $332.10 \mathrm{~ms}$.

Um den Rahmen dieser Arbeit nicht zu sprengen, wurde nur eine Testitemart einbezogen. Dabei fiel die Entscheidung für das positive Testitem, da sich bei der Analyse der maximalen Kohärenz nur für dieses Testitem hypothesenkonforme Ergebnisse aufzeigen ließen (vgl. Kap. 6.2.3). Die Kohärenzen wurden über die Probandinnen 4, 5, 7, 8, 9 und 10 gemittelt. Die Probandinnen 1 und 2 wurden aufgrund abweichender EEGAufzeichnungsbedingungen, die Probandinnen 3 und 6 aufgrund überdurchschnittlich kurzer Antwortlatenzen ausgeschlossen.

Verschiedene kognitive Funktionen neokortikaler Areale sollen sich unter anderem in unterschiedlichen zeitlichen Aktivationsverläufen dieser Areale niederschlagen (vgl. z. B. Smith \& Jonides, 1997). In diesem Sinne müßten im untersuchten Zeitintervall der Sternberg-Aufgabe in den Kohärenzverläufen derjenigen Areale, die für den Vergleichs- bzw. Abrufprozeß von Bedeutung sind, andauernde hohe Synchronisationen und SetgrößenEffekte zu finden sein. Folgt man dem Forschungsstand, können dabei je nach Reizart unterschiedliche Areale betroffen sein. Für die in jedem Durchgang einmalig stattfinden- 
de (motorische) Antworterzeugung sollten hingegen bei Figuren und bei Wörtern dieselben Areale zuständig sein und neben einem mit der Zeit zunehmenden Synchronisationsausmaß einen fehlenden Setgrößen-Effekt aufweisen. Damit ist für die Analyse der Kohärenzverläufe in der Abrufphase der Sternberg-Aufgabe der Einfluß der Setgröße und der Reizart von zentraler Bedeutung.

Bei den Figuren (vgl. Abb. 27) läßt sich der deutlichste Anstieg der mittleren Kohärenzen mit zunehmender Setgröße rechtsseitig zentro-temporal beim Elektrodenpaar C4/T4 verzeichnen. Bei genauerer Betrachtung finden sich allerdings weitere Elektrodenpaare mit einer zumindest zeitweisen und wenigstens über drei aufeinanderfolgenden SetgröBen zunehmenden Synchronisation: Es sind dies die fronto-zentral liegenden Elektrodenpaare $\mathrm{Fz} / \mathrm{Cz}$ (Set 2-4) und F4/C4 (Set 1-3) sowie ein Elektrodenpaar im linken parieto-occipitalen Bereich (P3/O1, Set $1-3)$. Berücksichtigt man jedoch die generelle Verlaufstendenz der mittleren Bandkohärenzen an diesen Elektrodenpaaren, kann nur bei $\mathrm{C} 4 / \mathrm{T} 4, \mathrm{Fz} / \mathrm{Cz}$ und F4/C4 von einem andauernden hohen Synchronsationsausmaß bei gleichzeitigem Setgrößen-Effekt gesprochen werden.

Eine deutliche und bei allen Setgrößen gleichermaßen ausgeprägte, stetige ఒnahme der mittleren Kohärenzen findet sich zentral bei $\mathrm{C} 3 / \mathrm{Cz} \mathrm{Cz} / \mathrm{C} 4$. Darüber hinaus fallen verschiedene Elektrodepaare auf, bei denen die Kohärenzwertverläufe im interessierenden Intervall mit der Zeit stark abfallen, so zum Beispiel frontopolar bei Fp1/F3 und Fp2/F4, rechts parieto-temporal bei P4/T6, rechts temporal bei T4/T6 und im gesamten parieto-occipitalen Bereich (T5/O1, P3/O1, P4/O2 und T6/O2). Schließlich finden sich auch Elektrodenpaare, bei denen die Synchronisation mit zunehmender Setgröße (Set 1 - 3) zeitweise abnimmt (C3/P3 und T6/O2).

Bei den Wörtern (vgl. Abb. 28) lassen sich Setgrößen-Effekte lediglich dann ausmachen, wenn nur die ersten ca. 90 bis 115 ms der Verläufe betrachtet werden. In diesem Fall kann eine anhaltende und wenigstens über drei Setgrößen hinweg zunehmende Synchronisation frontopolar bis fronto-zentral bei den Elektrodenpaaren Fp1/F7 (Set 2 4), Fz/F4 (Set $2-4$ ), F3/C3 (Set 1-3) und Fz/Cz (Set 1-3), rechts zentro-temporal bei C4/T4 (Set 2 - 4) sowie links zentro-parietal bis temporo-occipital bei C3/P3 (Set 1 4), T5/P3 (Set 1 - 4), P3/Pz (Set 1 - 3) und T5/O1 (Set 2 - 4) festgestellt werden.

Eine bei allen Setgrößen gleichermaßen ausgeprägte, stetige Zunahme der mittleren Kohärenzen findet sich auch hier nur im zentralen Bereich bei $\mathrm{Cz} / \mathrm{C} 4$ und bei $\mathrm{C} 3 / \mathrm{Cz}$. Mit der Zeit mehr oder weniger deutlich abfallende Verläufe weisen die temporal und temporo-parietal bis temporo-occipital liegenden Elektrodenpaare T4/T6, P4/T6, P3/O1 und T6/O2 auf. Schließlich zeigt sich auch bei dieser Reizart eine über drei Setgrößen abnehmende Synchronisation, wenn man bei $\mathrm{Cz} / \mathrm{Pz}$ die Setgröße 1 unberücksichtigt läßt. 


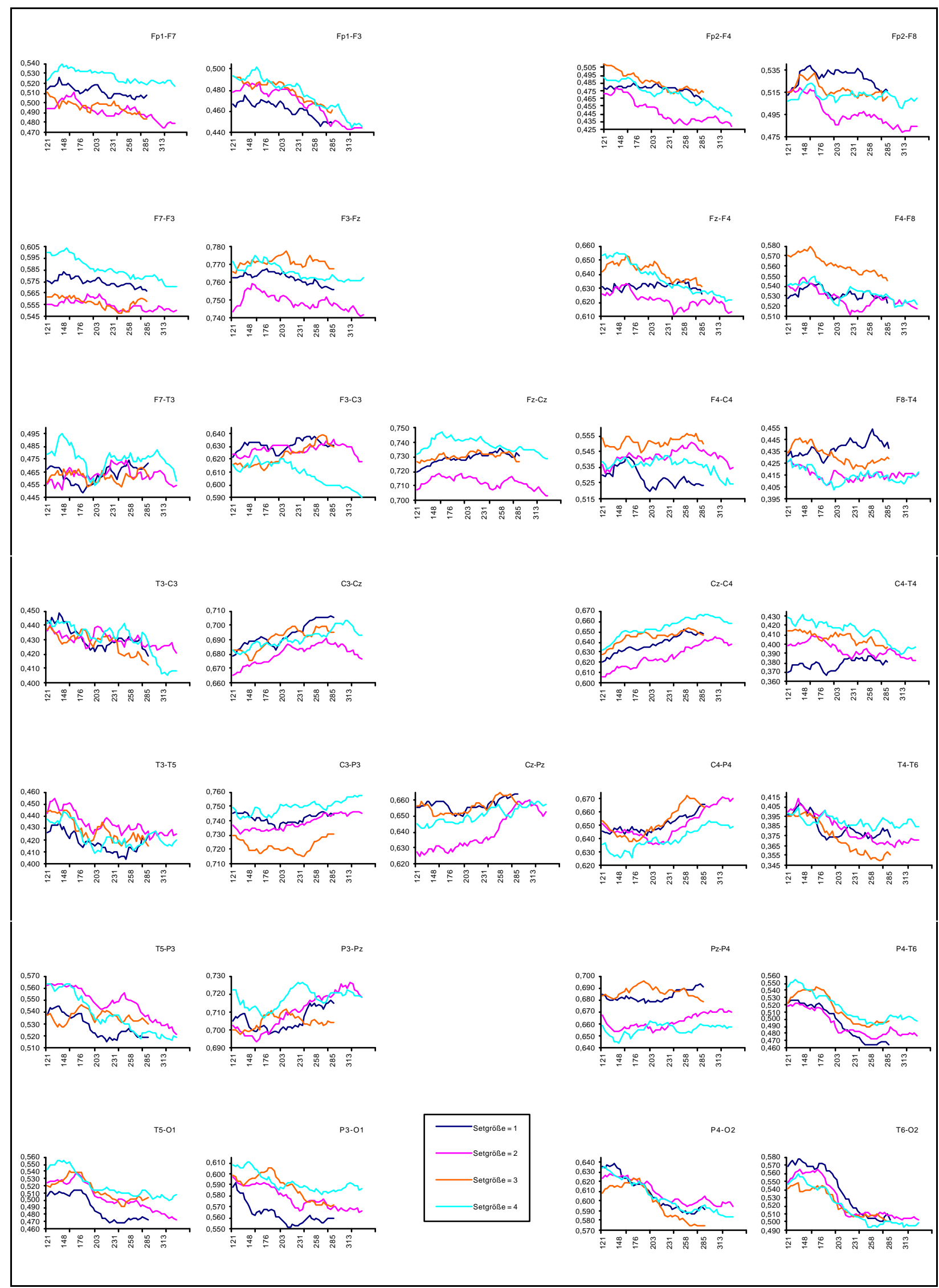

Abbildung 27. Verlauf der über die Probandinnen 4, 5, 7, 8, 9 und 10 gemittelten Kohärenzwerte im Intervall zwischen 121.12 und maximal 332.10 ms nach dem positiven Testitem: Reizart Figuren (2. Sitzung) 


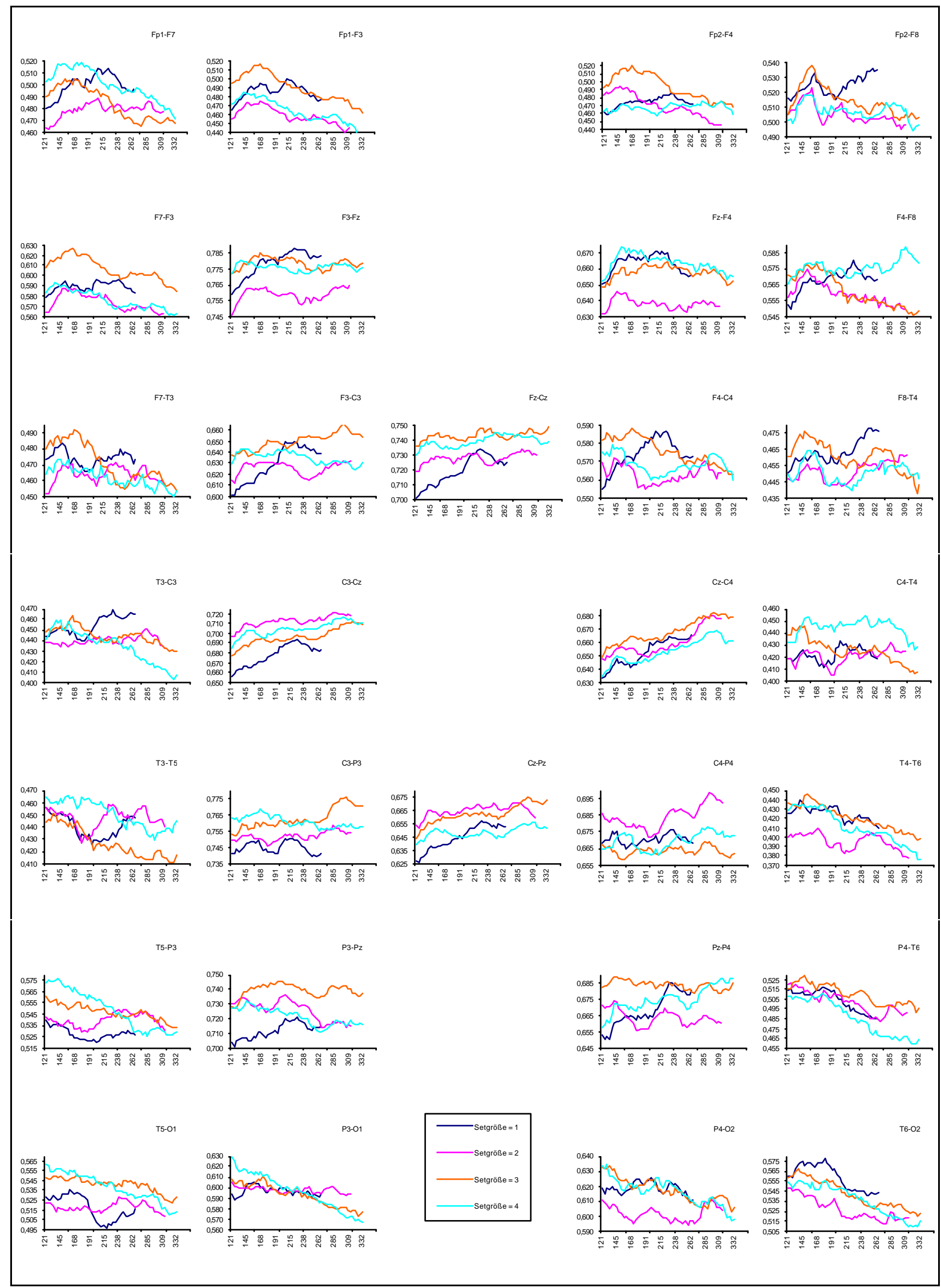

Abbildung 28. Verlauf der über die Probandinnen 4, 5, 7, 8, 9 und 10 gemittelten Kohärenzwerte im Intervall zwischen 121.12 und maximal 332.10 ms nach dem positiven Testitem: Reizart Wörter (2. Sitzung) 
Interessante Ergebnisse liefert der mittelbare Vergleich der Verläufe der mittleren Bandkohärenzen beider Reizarten. Während die Zahlworte deutliche Setgrößen-Effekte u. a. in linken zentro-parietalen bis temporo-occipitalen Arealen aufweisen (Elektrodenpaare C3/P3, T5/P3, P3/Pz und T5/O1), treten diese bei den Figuren schwerpunktmäßig im fronto-zentralen bis rechts zentro-temporalen Bereich auf (Elektrodenpaare $\mathrm{Fz} / \mathrm{Cz}$, F4/C4 und C4/T4). Am deutlichsten wird dieses Ergebnis, wenn diejenigen Elektrodenpaare beider Reizarten gegenübergestellt werden, bei denen sich der Setgrößen-Effekt über alle vier Setgrößen erstreckt: Dies trifft bei den Figuren nur auf C4/T4, bei den Wörtern hingegen auf C3/P3 und T5/P3 zu.

Übereinstimmungen lassen sich hinsichtlich derjenigen Elektrodenpaare finden, die bei allen Setgrößen im Verlauf eine vergleichbar ausgeprägte, stetige Zunahme der mittleren Kohärenzen zeigen. Es sind dies $\mathrm{C} 3 / \mathrm{Cz}$ und $\mathrm{Cz} / \mathrm{C} 4$, beides Elektrodenpaare, die über dem motorischen Areal liegen. Bei allen Setgrößen steil abfallende Kohärenzwertverläufe finden sich sowohl bei Figuren als auch bei Wörtern vor allem rechts parieto-temporal bis temporo-occipital (P4/T6, P4/O2 und T6/O2), zum Teil auch links temporo-occipital (T5/O1 und P3/O1). Dabei treten charakteristische Unterschiede hinsichtlich der Kurvenformen auf: Während die Synchronisationsabnahmen bei den Wörtern durch einen eher linearen Verlauf charakterisiert sind, zeigen sie bei den Figuren einen eher kurvilinearen Verlauf.

Hervorzuheben sind schließlich die bei beiden Reizarten andauernden hohen Synchronisationen im linken fronto-zentralen und linken zentro-parietalen Bereich ( $\mathrm{Fz} / \mathrm{Cz}$ und F3/Fz bzw. C3/P3 und P3/Pz). Hier liegen die Kohärenzen im gesamten betrachteten Intervall bei allen Setgrößen über 0.69, während sie an anderen Elektrodenpaaren zum Teil bis unter 0.41 abfallen.

Zusammenfassend kann gesagt werden, daß beim Abruf verbaler und nonverbaler $\mathrm{h}$ formation sowohl Übereinstimmungen als auch Unterschiede hinsichtlich der Verläufe der mittleren Bandkohärenzen zu verzeichnen sind. Bei beiden Reizarten steigen die mittleren Kohärenzen über das betrachtete Zeitintervall hinweg in zentralen Bereichen an (Area 1 - 4, vgl. Kap, 6.2.3, Abb. 25), während die Kohärenzen rechtshemisphärisch parieto-temporal bis temporo-occipital mit der Zeit abnehmen (Area 7, 39 und 37 bis 17). Topographische Unterschiede zwischen den Kohärenzwertverläufen von Figuren und Wörtern werden erst durch die Analyse des Einflusses der Setgröße deutlich: Bei den Wörtern treten die auffälligsten Setgrößen-Effekte links zentro-parietal bis temporoparietal (Area 1 bis 3, 5 und 7 sowie 37 und 39), bei den Figuren hingegen rechts zentro-temporal auf (Area 1 bis 3, 40 und 22). 


\subsubsection{Setgröße und Zeitpunkt maximaler Kohärenz}

Zur exploratorischen Untersuchung des Zeitablaufs der Synchronisationsprozesse bietet sich neben der Betrachtung der Kohärenzwertverläufe eine Analyse der Zeitpunkte maximaler Kohärenz an. Dieser Parameter beschreibt den Zeitpunkt, an dem die maximale Kohärenz im interessierenden Zeitintervall aufgetreten ist, und zwar unabhängig von dessen absoluten Wert (vgl. Kap. 5.8).

$\mathrm{Daß}$ sich der mittlere Zeitpunkt maximaler Kohärenz (gerechnet ab 120 ms nach Marker 7) an vielen Elektrodenpaaren mit zunehmender Setgröße verschiebt, konnte bereits in einer ersten optischen Analyse festgestellt werden (vgl. Kap.6.2.2). Inwiefern dieses Ergebnis statistische Signifikanz erreicht, wurde über lineare Regressionsanalysen der mittleren Zeitpunkte maximaler Kohärenz und der Größe des positiven Sets geprüft. Die Ergebnisse dieser Regressionsanalysen können den Tabellen D-1 bis D-4 im Anhang $D$ entnommen werden. Für eine übersichtlichere Darstellung wurden Absolutmaps der signifikanten Regressionskoeffizienten sowie Maps der Elektrodenpaare mit signifikanten Ergebnissen in der Regressionsanalyse erstellt (vgl. Abb. 29 bis 30).

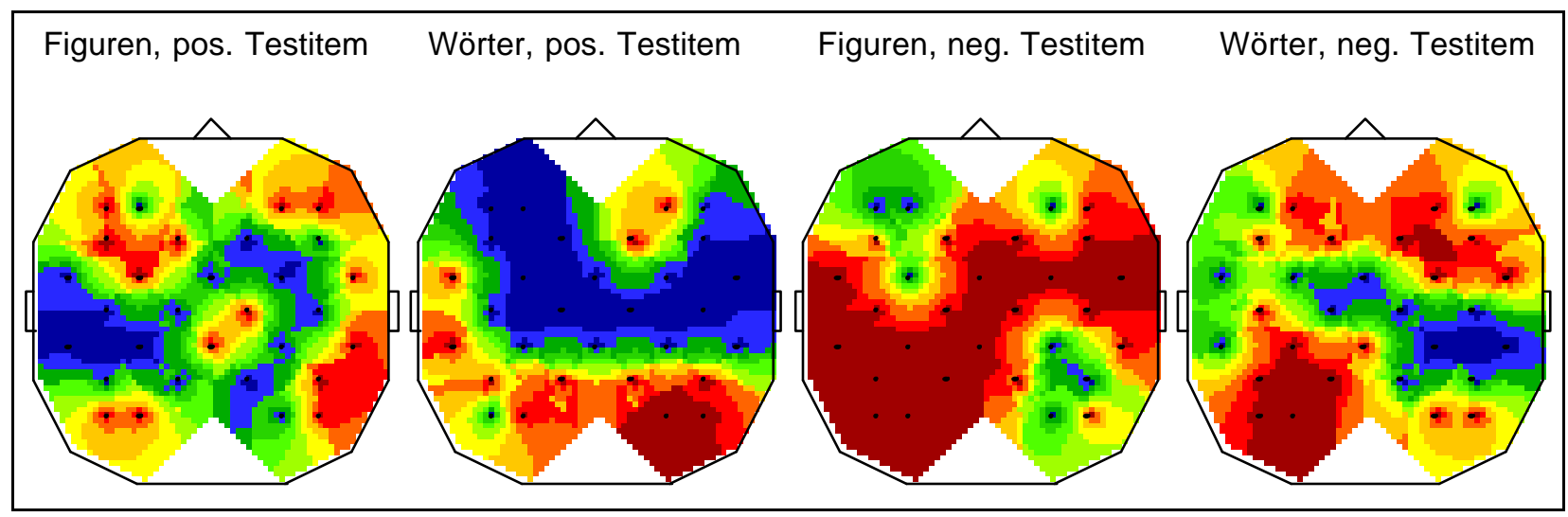

Abbildung 29. Elektrodenpaare, für die in der linearen Regressionsanalyse der Zeitpunkte maximaler Kohärenz und der Größe des positiven Sets signifikante Regressionskoeffizienten gefunden wurden (in der Abbildung rot hervorgehoben; $N=10 ; 2$. Sitzung)

Aus Abbildung 29 geht hervor, daß im Falle positiver Testitems bei den Figuren links frontopolar und links zentro-parietal bis temporo-occipital lineare Zusammenhänge zwischen der Setgröße und den Zeitpunkten maximaler Kohärenz auftreten. Rechtshemisphärisch sind bei fast allen Elektrodenpaaren in frontalen bis parieto- und temporooccipitalen Bereichen derartige Zusammenhänge auszumachen. Schließlich sind auch fronto-zentral zwei signifikante Ergebnisse $(\mathrm{Fz} / \mathrm{Cz}, \mathrm{Cz} / \mathrm{C} 4)$ zu verzeichnen, die bei den Wörtern (positive Testitems) nicht auftreten. Hier finden sich im wesentlichen beidseitig frontal und links fronto-temporal bedeutsame Zusammenhänge zwischen der Setgröße 
und den Zeitpunkten maximaler Kohärenz. Hinzu kommen rechtsseitig die zentro-parietal und parieto-occipital liegenden Elektrodenpaare Pz/P4 und P4/O2.

Im Falle negativer Testitems treten bei beiden Reizarten, vor allem aber bei den Figuren vermehrt signifikante lineare Beziehungen zwischen der Setgröße und den Zeitpunkten maximaler Kohärenz auf. Bei den Figuren weisen alle links frontalen bis links temporalen sowie alle zentralen bis rechts zentro-parietalen und rechts temporalen Areale signifikante Ergebnisse auf. Bei den Wörtern sind in links frontopolaren bis frontozentralen sowie beidseitig parietalen bis parieto-occipitalen Arealen signifikante Ergebnisse zu verzeichnen.

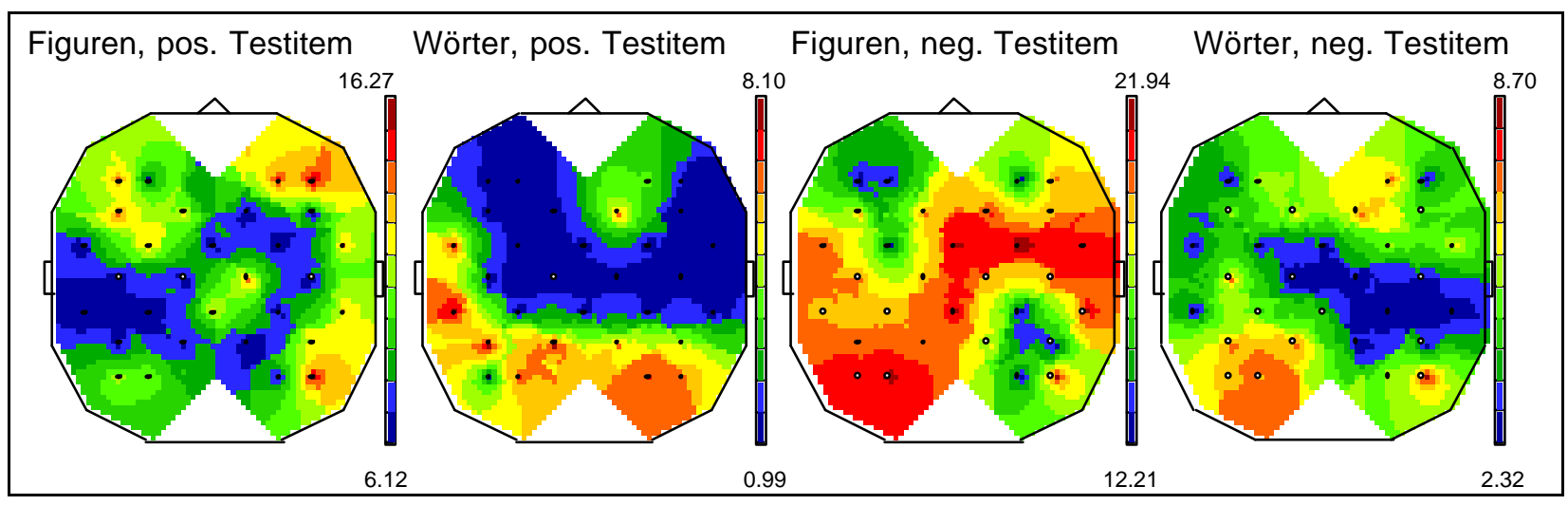

Abbildung 30. Linearen Regressionsanalyse der Zeitpunkte maximaler Kohärenz und der Größe des positiven Sets: signifikante Regressionskoeffizienten ( $N=10 ; 2$. Sitzung)

Die Maps der signifikanten Regressionskoeffizienten machen deutlich, daß sich - ungeachtet der vielen signifikanten Ergebnisse - eine Zunahme der Größe des positiven Sets nicht überall gleich stark auf die Zeitpunkte maximaler Kohärenz auswirkt (s. Abb. 30). Im Falle des positiven Testitems hat die Setgröße bei den Figuren rechts frontopolar sowie rechts parieto- und temporo-occipital den größten Einfluß auf die Zeitpunkte maximaler Kohärenz. Dies entspricht in etwa den Brodmannschen Arealen 10/46, 17 bis 37 und 17 bis 7 (vgl. Kap. 6.2.3, Abb. 25). Anders sieht es bei den Wörtern aus, bei denen sich an links frontopolaren, frontalen, fronto-temporalen und temporalen sowie an rechts frontopolaren Elektrodenpaaren die höchsten Regressionskoeffizienten finden lassen (Area 8/9/10, 46 bis 22, 22/42/37 und 10/46).

Beim negativen Testitem fallen die Regressionskoeffizienten vergleichsweise hoch aus. Der stärkste Einfluß der Zunahme der Setgröße auf die Zeitpunkte maximaler Kohärenz ist bei den Figuren für die Elektrodenpaare Fp1/F7, Fp1/F3, Fz/Cz, Cz/Pz, C4/P4 T4/T6, F8/F4 und Fp2/F8 zu verzeichnen. Linkshemisphärisch sind somit vor allem die Areale 10/46 und 9/10, rechtshemisphärisch die Areale 10/46, 46 bis 22, 22 bis $37,1 / 2 / 3$ bis 7 sowie zentral die Areale $8 / 6 / 4,1 / 2 / 3$ bis 7 betroffen. Bei den Wörtern treten die höchsten Regressionskoeffizienten bei Fp1/F3 und Fp2/F8 und damit in Area 9/10 bzw. 10/46 auf. 
Aus den Skalen der Maps in Abbildung 30 geht hervor, daß die Größenordnung der Regressionskoeffizienten mit der Reizart und mit dem Testitem stark schwankt. Dies legt nahe, einen inferenzstatistischen Vergleich der Regressionskoeffizienten für diejenigen lokalen Elektrodenpaare vorzunehmen, die pro Testitem bei beiden Reizarten bzw. pro Reizart bei beiden Testitems signifikante Ergebnisse in der Regressionsanalyse aufwiesen. Die signifikanten Ergebnisse der t-Tests können Tabelle 23 und 24 bzw. Abbildung 31 entnommen werden.

Tabelle 23

Signifikante Ergebnisse des Vergleichs der mittleren Regressionskoeffizienten ( $\mathrm{M}_{\mathrm{SL}}$, in $\mathrm{ms}$ ) der Zeitpunkte maximaler Kohärenz ( $\mathrm{M}_{\mathrm{SL}}$, in $\mathrm{ms}$ ) von Figuren und Wörtern (t-Test für abhängige Stichproben, zweiseitig; $N=10 ; 2$. Sitzung)

\begin{tabular}{|c|c|c|c|c|c|c|c|c|}
\hline \multicolumn{2}{|c|}{ Positives Testitem } & \multirow[b]{2}{*}{$\begin{array}{c}\text { SL Wörter } \\
\text { MSLW }\end{array}$} & \multirow[b]{2}{*}{$\begin{array}{c}\text { Differenz } \\
\text { MSLF - MSLW }\end{array}$} & \multirow[b]{2}{*}{$\begin{array}{c}S D \\
(\max )\end{array}$} & \multirow[b]{2}{*}{$t$-Wert } & \multirow[b]{2}{*}{$p$ (sign.) } & \multirow[b]{2}{*}{$\begin{array}{c}\text { Korrel. } \\
r\end{array}$} & \multirow[b]{2}{*}{$\begin{array}{l}\text { Effekt- } \\
\text { größe E }\end{array}$} \\
\hline $\begin{array}{l}\text { Elektroden- } \\
\text { paar }\end{array}$ & $\begin{array}{c}\text { SL Figuren } \\
\text { MSLF }\end{array}$ & & & & & & & \\
\hline P4/O2 & 14.26 & 3.40 & 10.87 & 10.48 & 2.69 & 0.025 & -0.69 & 0.80 \\
\hline \multicolumn{2}{|c|}{ Negatives Testitem } & & & & & & & \\
\hline $\begin{array}{l}\text { Elektroden- } \\
\text { paar }\end{array}$ & $\begin{array}{c}\text { SL Figuren } \\
M_{S L F}\end{array}$ & $\begin{array}{c}S L \text { Wörter } \\
\text { MSLW }\end{array}$ & $\begin{array}{c}\text { Differenz } \\
M_{S L F}-M_{S L W}\end{array}$ & $\begin{array}{c}S D \\
(\max )\end{array}$ & $t$-Wert & $p$ (sign.) & $\begin{array}{c}\text { Korrel. } \\
r\end{array}$ & $\begin{array}{l}\text { Effekt- } \\
\text { größe } \varepsilon\end{array}$ \\
\hline Fp1/F7 & 19.71 & 6.12 & 13.60 & 14.75 & 2.54 & 0.032 & -0.18 & 0.85 \\
\hline$F 3 / F z$ & 17.06 & 6.99 & 10.07 & 15.36 & 2.63 & 0.027 & 0.69 & 1.19 \\
\hline T3/C3 & 16.90 & 6.56 & 10.34 & 12.59 & 2.39 & 0.041 & -0.11 & 0.78 \\
\hline T5/P3 & 14.94 & 4.32 & 10.61 & 14.45 & 2.41 & 0.039 & 0.33 & 0.90 \\
\hline$P 3 / P z$ & 16.78 & 5.43 & 11.34 & 13.42 & 2.66 & 0.026 & 0.33 & 1.03 \\
\hline$P z / P 4$ & 18.32 & 6.62 & 11.70 & 8.48 & 3.86 & 0.004 & -0.02 & 1.36 \\
\hline P4/T6 & 16.53 & 3.06 & 13.47 & 10.23 & 3.10 & 0.013 & -0.18 & 1.21 \\
\hline$F p 2 / F 8$ & 21.35 & 8.70 & 12.65 & 9.69 & 3.43 & 0.007 & -0.24 & 1.17 \\
\hline Fp1/F3 & 20.78 & 6.28 & 14.50 & 13.24 & 2.89 & 0.018 & -0.46 & 0.91 \\
\hline F3/C3 & 14.21 & 2.83 & 11.38 & 13.26 & 2.57 & 0.030 & 0.00 & 0.86 \\
\hline$F z / C z$ & 20.03 & 2.32 & 17.71 & 9.94 & 6.58 & 0.000 & 0.53 & 2.60 \\
\hline$C 4 / P 4$ & 21.94 & 3.97 & 17.97 & 10.98 & 5.52 & 0.000 & 0.37 & 2.06 \\
\hline T4/T6 & 19.04 & 5.34 & 13.70 & 8.52 & 5.70 & 0.000 & 0.50 & 2.28 \\
\hline
\end{tabular}

Die meisten Unterschiede zwischen den Regressionskoeffizienten von Figuren und Wörtern treten beim negativen Testitem auf. Sie sind hauptsächlich in den linken frontopolaren, fronto-zentralen und zentro-temporalen sowie in den rechten parieto-zentralen bis parieto-temporalen Arealen zu finden. Hier übersteigen die mittleren Regressionskoeffizienten der Figuren diejenigen der Wörter im Mittel um 13 ms. Beim positiven Testitem kann nur ein signifikanter Unterschied festgestellt werden: Rechts parieto-occipital (Elek- 
trodenpaar P4/O2) nimmt der Zeitpunkt, an dem die maximale Kohärenz im Verlauf auftritt, bei den Figuren im Mittel um 14.26 ms, bei den Wörtern im Mittel nur um 3.40 ms pro Item zu.

Tabelle 24

Signifikante Ergebnisse des Vergleichs der mittleren Regressionskoeffizienten ( $M_{S L}$, in ms) der Zeitpunkte maximaler Kohärenz von positiven und negativen Testitems (t-Test für abhängige Stichproben, zweiseitig; $N=10 ; 2$. Sitzung)

\begin{tabular}{|c|c|c|c|c|c|c|c|c|}
\hline \multicolumn{2}{|c|}{ Figuren } & & & & & & & \\
\hline $\begin{array}{l}\text { Elektroden- } \\
\quad \text { paar }\end{array}$ & $\begin{array}{c}S L \text { negativ } \\
\text { MSLN }\end{array}$ & $\begin{array}{c}S L \text { positiv } \\
M S L P\end{array}$ & $\begin{array}{c}\text { Differenz } \\
\text { MSLN - MSLP }\end{array}$ & $\begin{array}{l}S D \\
(\max )\end{array}$ & $t$-Wert & $p$ (sign.) & $\begin{array}{c}\text { Korrel. } \\
r\end{array}$ & $\begin{array}{l}\text { Effekt- } \\
\text { größe } \varepsilon\end{array}$ \\
\hline$F p 1 / F 7$ & 19.71 & 9.15 & 10.56 & 14.75 & 4.47 & 0.002 & 0.89 & 2.15 \\
\hline Fp1/F3 & 20.78 & 8.20 & 12.58 & 13.24 & 5.85 & 0.000 & 0.87 & 2.59 \\
\hline$F z / C z$ & 20.03 & 9.91 & 10.13 & 11.03 & 3.60 & 0.006 & 0.64 & 1.54 \\
\hline F8/T4 & 21.48 & 10.67 & 10.81 & 12.00 & 3.57 & 0.006 & 0.67 & 1.57 \\
\hline \multicolumn{9}{|c|}{ Wörter } \\
\hline $\begin{array}{l}\text { Elektroden- } \\
\text { paar }\end{array}$ & $\begin{array}{c}S L \text { negativ } \\
M_{S L N}\end{array}$ & $\begin{array}{c}S L \text { positiv } \\
M_{S L P}\end{array}$ & $\begin{array}{c}\text { Differenz } \\
M_{S L N}-M_{S L P}\end{array}$ & $\begin{array}{c}S D \\
(\max )\end{array}$ & $t$-Wert & $p$ (sign.) & $\begin{array}{c}\text { Korrel. } \\
r\end{array}$ & $\begin{array}{l}\text { Effekt- } \\
\text { größe } \varepsilon\end{array}$ \\
\hline P4/O2 & 6.82 & 3.40 & 3.42 & 3.08 & 2.27 & 0.049 & -0.39 & 0.94 \\
\hline
\end{tabular}

Figuren vs. Wörter, positives Testitem

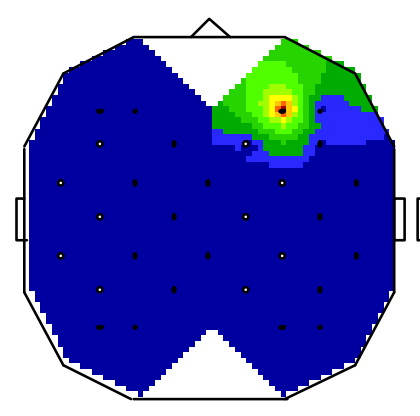

Figuren vs. Wörter, negatives Testitem

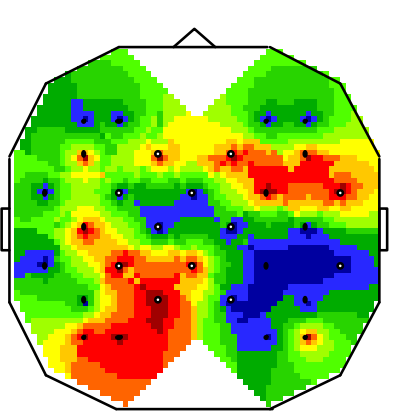

Figuren: Negatives vs. positives Testitem

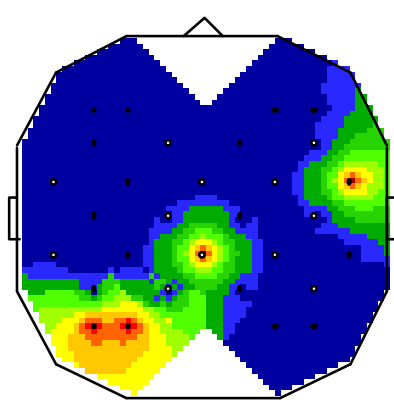

Wörter: Negatives vs. positives Testitem

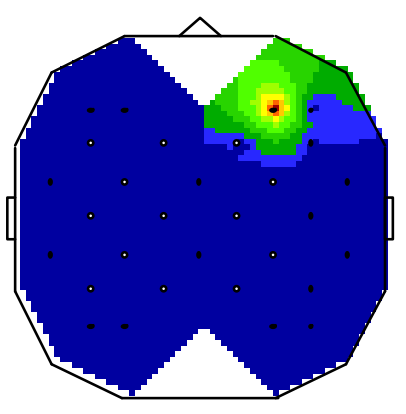

Abbildung 31. Elektrodenpaare, die im inferenzstatistischen Vergleich der Betakoeffizienten (aus der Inearen Regression der Zeitpunkte maximaler Kohärenz und der Größe des positiven Sets) signifikante Ergebnisse aufwiesen (in der Abbildung rot hervorgehoben; $N=10 ; 2$. Sitzung)

Bei der Gegenüberstellung der beiden Testitems sind im Falle der Figuren bei negativen Testitems links frontopolar, zentral und rechts temporal größere Verschiebungen der Zeitpunkte maximaler Kohärenz mit der Setgröße zu verzeichnen. Bei den Wörtern fällt der mittlere Regressionskoeffizient negativer Testitems rechts parieto-occipital (Elektrodenpaar $\mathrm{P} 4 / \mathrm{O} 2$ ) etwa doppelt so groß aus wie derjenige positiver Testitems. Mit $\varepsilon=$ 
0.80 bis 2.60 ergeben sich für alle dargestellten Vergleiche der Reizarten bzw. Testitems große Effekte.

Insgesamt können die Ergebnisse des mittelbaren und unmittelbaren Vergleichs der signifikanten Regressionskoeffizienten beider Reizarten bzw. Testitems (vgl. Abb. 29 und 31) wie folgt zusammengefaßt werden:

1. Bei Figuren und Wörtern bzw. bei positiven und negativen Testitems sind über einen mittelbaren Vergleich lineare Zusammenhänge zwischen der Setgröße und dem Zeitpunkt maximaler Kohärenz an zum Teil unterschiedlichen Elektrodenpaaren auszumachen. Beim positiven Testitem (Ja-Antworten) wirkt sich eine Zunahme der Größe des positiven Sets bei den Figuren rechts frontopolar sowie rechts parieto- und temporo-occipital am stärksten auf die Zeitpunkte maximaler Kohärenz aus, während bei den Wörtern links frontopolare, frontale, fronto-temporale und temporale sowie rechts zentro-parietale Bereiche betroffen sind. Beim negativen Testitem (Nein-Antworten) sind bei Figuren fronto-zentral, zentro-parietal, links und rechts frontopolar sowie rechts fronto-temporal, temporal und zentro-parietal die größten Regressionskoeffizienten zu verzeichnen; bei den Wörtern hingegen nimmt der Zeitpunkt maximaler Kohärenz am stärksten rechts und links frontopolar mit der Größe des positiven Sets zu.

2. Anhand eines unmittelbaren Vergleichs können signifikante Unterschiede zwischen den Regressionskoeffizienten festgestellt werden. Beim positiven Testitem verschiebt sich der Zeitpunkt maximaler Kohärenz der Figuren rechts parieto-occipital signifikant stärker als derjenige der Wörter. Analoge Unterschiede treten beim negativen Testitem links frontopolar, fronto-zentral und zentro-temporal sowie rechts zentro-parietal und parieto-temporal auf. Negative Testitems weisen zudem sowohl bei Figuren als auch bei Wörtern an verschiedenen Elektrodenpaaren signifikant größere Regressionskoeffizienten als positive Testitems auf: Die betroffenen Areale liegen im Falle der Figuren links frontopolar, zentral und rechts temporal, im Falle der Wörter rechts parieto-occipital.

\subsubsection{Antwortlatenz und Zeitpunkt maximaler Kohärenz}

Angesichts der an vielen Elektrodenpaaren gefundenen linearen Beziehung zwischen Setgröße und Zeitpunkt maximaler Kohärenz und des signifikanten Einflusses der Setgröße auf die Antwortlatenz (vgl. Kap.6.1.3) stellt sich abschließend die Frage nach dem Zusammenhang zwischen dem Zeitpunkt maximaler Kohärenz und der Antwortlatenz. Obwohl die Interpretation eines solchen Zusammenhangs sicherlich nicht unproblematisch ist, könnte die Beantwortung dieser Frage der Erforschung weiterer möglicher Unterschiede im Zeitablauf der Verarbeitungsprozesse von verbalem und nonver- 
balem Reizmaterial dienen. Sie schlägt zudem einen Bogen zwischen den bio-physiologischen Kennwerten der Medizin und den Verhaltensparametern der Psychologie.

Zunächst wurde über Korrelationsrechnungen die Enge des Zusammenhangs zwischen den Zeitpunkten maximaler Kohärenz und den Antwortlatenzen für jedes Elektrodenpaar und jede Versuchsbedingung bestimmt. Die gemappten Korrelationskoeffizienten (vgl. Anhang E, Abb. E-1 und Tab. E-1 bzw. E-2) und Signifikanzen (vgl. Anhang E, Abb. E-2 und Tab. E-3 bzw. E-4) zeigten, daß in vielen Arealen signifikante Zusammenhänge zwischen den genannten Kennwerten bestanden. Sie ließen jedoch keine systematischen Unterschiede zwischen den Reizarten bzw. Testitems erkennen. Daher wurde auf eine topographische Analyse verzichtet und stattdessen ein Vergleich der Regressionskoeffizienten durchgeführt. Hierzu sind die einzelnen signifikanten Regressionskoeffizienten aus der Regressionsanalyse der Zeitpunkte maximaler Kohärenz und der Größe des positiven Sets den Regressionskoeffizienten der Antwortlatenzen gegenübergestellt worden (s. Anhang E, Tab. E-5; vgl. auch Kap. 6.1.3 und 6.3.2). Zusätzlich wurden Mittelwerte und Mittelwertsdifferenzen zwischen den Regressionskoeffizienten der Maximumwerte bzw. Antwortlatenzen beider Reizarten und Testitems gebildet. Diese sind in Tabelle 25 wiedergegeben und in Abbildung 32 veranschaulicht.

Tabelle 25

Mittelwerte der signifikanten Regressionskoeffizienten aus der linearen Regressionsanalyse der gemittelten Zeitpunkte maximaler Kohärenz (SLZMK) bzw. der gemittelten Antwortlatenzen (SLLAT) und der Größe des positiven Sets sowie entsprechende Mittelwertsdifferenzen dieser Regressionskoeffizienten in verschiedenen Versuchsbedingungen ( $N=10 ; 2$. Sitzung)

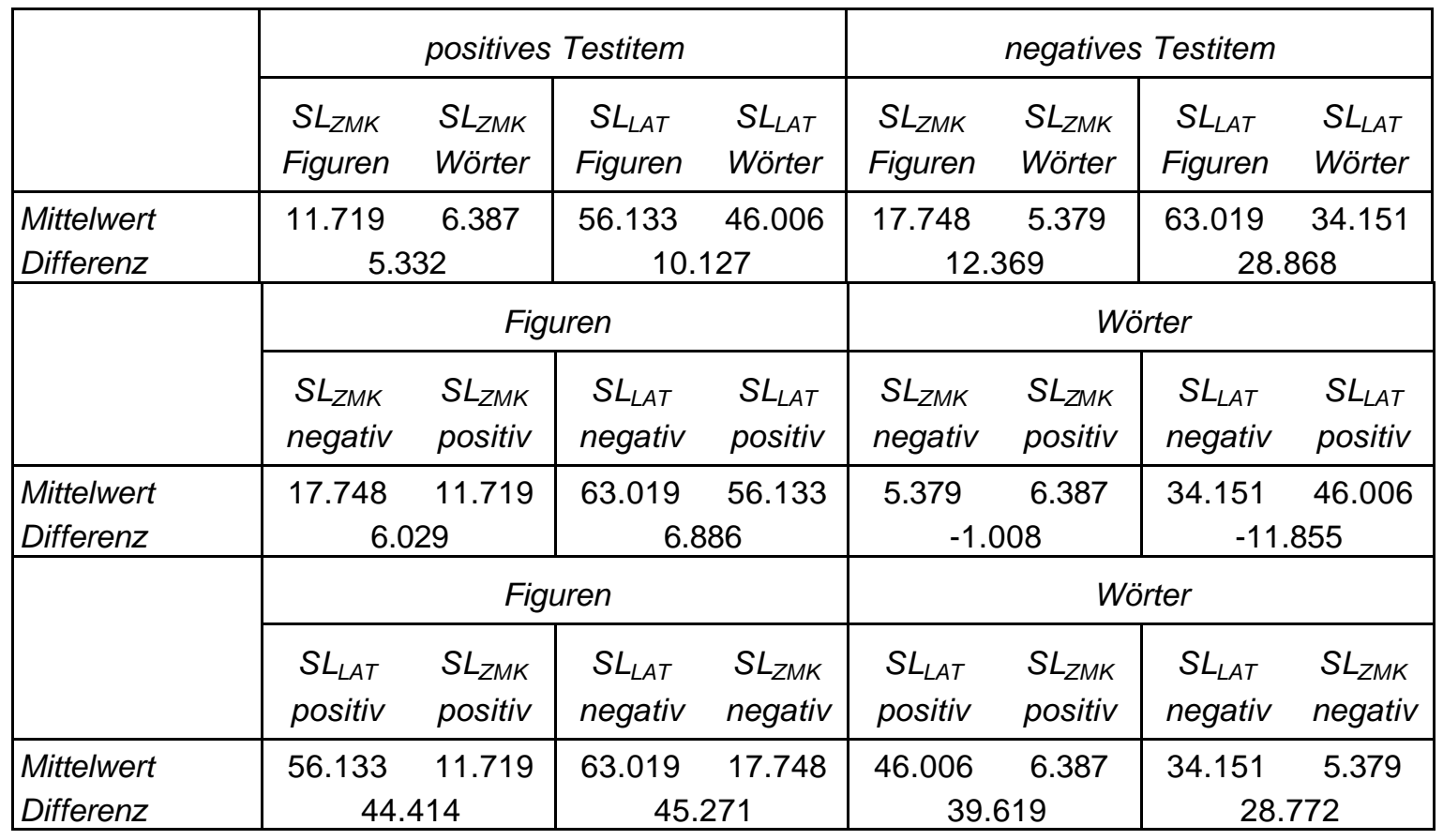


positives Testitem

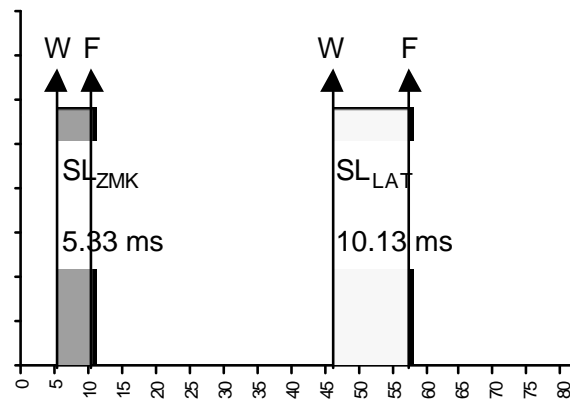

Figuren

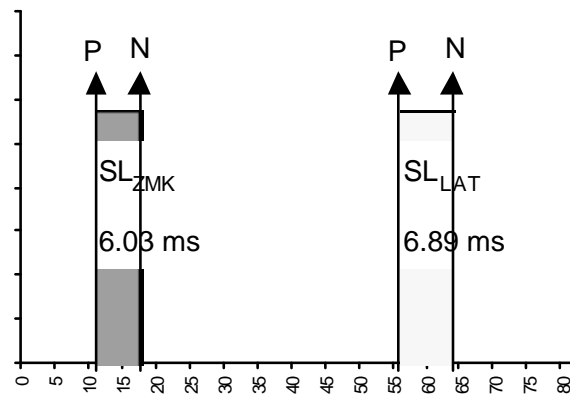

Figuren

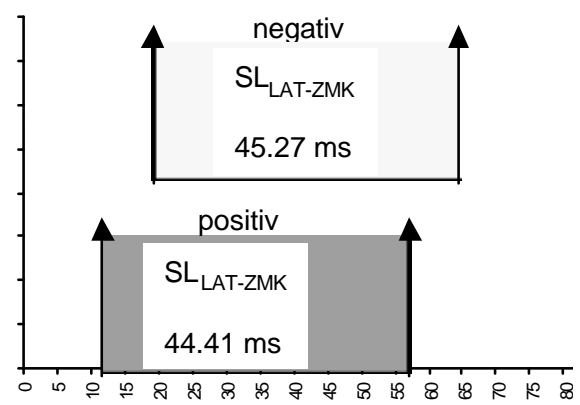

negatives Testitem

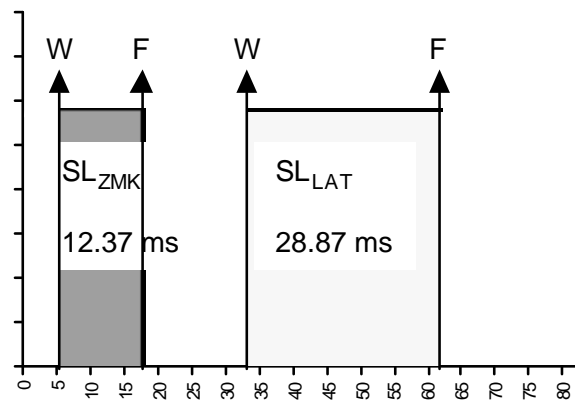

Wörter

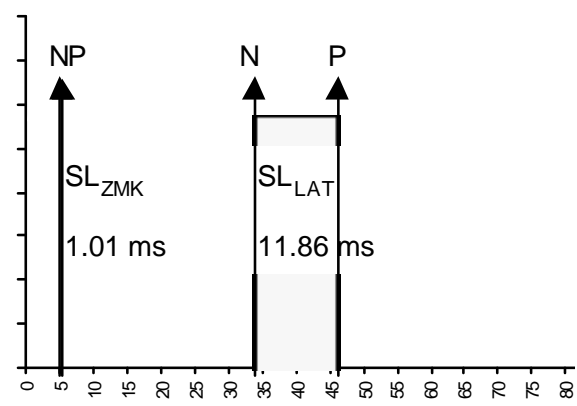

Wörter

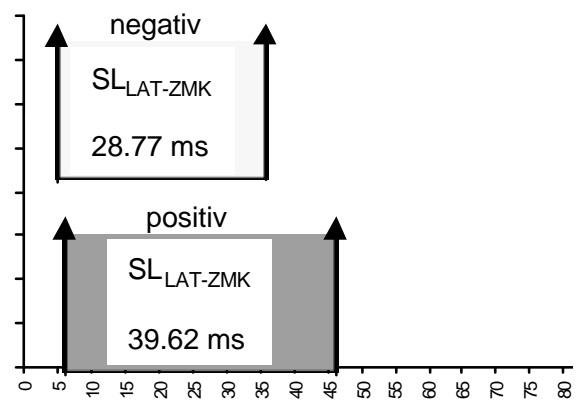

Abbildung 32. Absolute Differenzen zwischen den mittleren signifikanten Regressionskoeffizienten aus der linearen Regressionsanalyse der gemittelten Zeitpunkte maximaler Kohärenz (SLzMk) bzw. der gemittelten Antwortlatenzen (SLLAT) und der Größe des positiven Sets ( $F=$ Figuren; W = Wörter; $P=$ positives Testitem; $N$ = negatives Testitem; SLLAT-ZMK = Differenz zwischen den Regressionskoeffizienten der Antwortlatenzen und der Zeitpunkte maximaler Kohärenz)

Aus dem Vergleich der Regressionskoeffizienten (Tab. E-5) wird deutlich, daß das Ausmaß, mit dem sich die Zeitpunkte maximaler Kohärenz an verschiedenen Elektrodenpaaren mit jedem hinzugekommenen Lernitem verschieben, in allen Versuchsbedingungen erheblich unter der sogenannten "Scanning-Zeit" liegt. Aus den für jede Versuchsbedingung errechneten Mittelwerten (Tab. 25) ist darüber hinaus ersichtlich, daß die Rangordnung, die bei den Regressionskoeffizienten der Antwortlatenzen zwischen den einzelnen Versuchsbedingungen besteht, sich auch bei den Regressionskoeffizienten der Zeitpunkte maximaler Kohärenz wiederfindet (Figuren, neg. Testitem > Figuren, pos. Testitem > Wörter, pos. Testitem > Wörter, neg. Testitem). Allerdings gibt es keine direkte Entsprechung zwischen den absoluten Differenzen beider Reizarten. Während 
die Scanning-Zeiten der Figuren um 10.13 ms (positives Testitem) bzw. 28.87 ms (negatives Testitem) über denjenigen der Wörter liegen, fällt die Differenz zwischen den Regressionskoeffizienten der Zeitpunkte maximaler Kohärenz mit 5.33 ms (positives Testitem) bzw. 12.37 ms (negatives Testitem) nur etwa halb so groß aus.

Die Gegenüberstellung von positivem und negativem Testitem ergibt für die Figuren eine weitgehende Übereinstimmung der Differenzen der Regressionskoeffizienten der Zeitpunkte maximaler Kohärenz und der Antwortlatenzen (6.03 bzw. 6.89). Diese Übereinstimmung fehlt bei den Wörtern, bei denen sich darüber hinaus die Richtung der Mittelwertsdifferenz beider Kennwerte umkehrt. Damit verschieben bzw. verlängern sich die Zeitpunkte maximaler Kohärenz und Antwortlatenzen der Figuren bei Nein-Antworten gegenüber den Ja-Antworten um etwa denselben Betrag (ca. $6 \mathrm{~ms} / \mathrm{ltem}$ ), während bei den Wörtern die Regressionskoeffizienten der Zeitpunkte maximaler Kohärenz beider Testitems fast gleich ausfallen und sich lediglich die Antwortlatenzen positiver Testitems gegenüber denjenigen negativer Testitems um etwa $11.86 \mathrm{~ms} / \mathrm{ltem}$ verlängern. Hier zeigt sich, daß die bereits in Kapitel 6.1.3 für die Antwortlatenz festgestellte Wechselwirkung zwischen Reizart und Testitem auch bei den Maximumwerten vorliegt. Sie wird noch deutlicher, wenn die Differenzen zwischen den Scanning-Zeiten und den Regressionskoeffizienten der Zeitpunkte maximaler Kohärenz beider Reizarten und Testitems einander gegenübergestellt werden (Tab. 25 bzw. Abb. 32 unten).

Die wesentlichen Ergebnisse zur Beziehung zwischen den Zeitpunkten maximaler Kohärenz und den Antwortlatenzen können abschließend wie folgt zusammengefaßt werden: 1. Die Prüfung der Enge des Zusammenhangs zwischen den Zeitpunkten maximaler Kohärenz und den Antwortlatenzen auf direktem Weg, nämlich über entsprechende Korrelationsrechnungen für jede einzelne Setgröße, offenbart keine systematischen Unterschiede zwischen den Reizarten bzw. Testitems.

2. Aus der Analyse der mittleren signifikanten Regressionskoeffizienten der Zeitpunkte maximaler Kohärenz und der Antwortlatenzen sowie der absoluten Differenzen zwischen den Regressionskoeffizienten beider Reizarten bzw. Testitems geht folgendes hervor:

- Nicht nur die Antwortlatenzen, sondern auch die Zeitpunkte maximaler Kohärenz der Figuren verlängern bzw. verschieben sich mit zunehmendem Umfang des Reizmaterials stärker als diejenigen der Wörter, und zwar beim positiven Testitem etwa um den doppelten, beim negativen Testitem etwa um den dreieinhalbfachen Betrag.

- Die Differenz zwischen den Scanning-Zeiten von Figuren und Wörtern fällt sowohl bei positiven als auch bei negativen Testitems doppelt so groß wie die entsprechende Differenz zwischen den Regressionskoeffizienten der Zeitpunkte maximaler Kohärenz aus. 
- Es existiert eine Wechselwirkung zwischen Reizart und Testitemart. Bei den Figuren liegen sowohl die Scanning-Zeiten als auch die Regressionskoeffizienten der Zeitpunkte maximaler Kohärenz von negativen Testitems über denjenigen der positiven Testitems, bei den Wörtern ergibt sich das umgekehrte Bild.

- Im Falle der Figuren entspricht die Differenz zwischen den Scanning-Zeiten negativer und positiver Testitems weitgehend der Differenz zwischen den Regressionskoeffizienten der Zeitpunkte maximaler Kohärenz, bei den Wörtern fehlt diese Entsprechung.

Insgesamt weisen diese Ergebnisse auf einen unterschiedlichen zeitlichen Ablauf der Verarbeitungsprozesse beim Abruf von verbalem und nonverbalem Reizmaterial hin. 


\section{Diskussion}

Mit dieser interdisziplinär angelegten Arbeit über kognitive Prozesse im Arbeitsgedächtnis wurden vier verschiedene Ziele verfolgt, die vor der Diskussion der eigenen Untersuchungsergebnisse noch einmal in Erinnerung gerufen werden sollen: Neben der Replikation der von Sternberg gefundenen linearen Beziehung zwischen Setgröße und Antwortlatenz für das gewählte verbale und nonverbale Lernmaterial bestand ein wichtiges Anliegen darin, über eine Analyse kohärenzanalytischer Kennwerte die Tauglichkeit eines neuen Schätzverfahrens der Kohärenzfunktion für die Untersuchung schnell ablaufender kognitiver Prozesse zu prüfen. Außerdem sollte untersucht werden, ob sich bisherige Erkenntnisse zu topographischen Aspekten des Arbeitsgedächtnisses anhand sensitiver kohärenzanalytischer Kennwerte bestätigen lassen und ob bezüglich des Zeitablaufes der elektrophysiologischen Synchronisationsprozesse Unterschiede in der Verarbeitung verbalen und nonverbalen Reizmaterials gefunden werden können.

Die nachfolgende Ergebnisdiskussion gliedert sich nach den genannten Zielstellungen. Es werden zunächst die Ergebnisse der Verhaltensdaten diskutiert, bevor auf die Sensitivität kohärenzanalytischer Kennwerte und auf topographische sowie zeitdynamische Aspekte der elektrophysiologischen Synchronisationsprozesse eingegangen wird. Im Anschluß daran erfolgt eine zusammenfassende Bewertung der durchgeführten Untersuchung.

Die deskriptive und inferenzstatistische Analyse der Antwortlatenzen hat zu Ergebnissen geführt, die fast ausnahmslos den Erwartungen entsprachen. Schon die tabellarische Gegenüberstellung der mittleren Antwortlatenzen zeigte deutlich, daß die Zeit, die die Probandinnen im Mittel zur Beantwortung des kritischen Reizes benötigten, in allen Versuchsbedingungen mit steigendem Umfang des Lernmaterials zunahm. Dabei lagen die Antwortlatenzen der Wörter stets unter denjenigen der Figuren, die Antwortlatenzen positiver Testitems (Testitems, die eine Ja-Antwort erforderten) durchweg unter denjenigen negativer Testitems (Testitems, die eine Nein-Antwort erforderten) und die Antwortlatenzen der zweiten Versuchssitzung immer unter denjenigen der ersten Versuchssitzung. Die entsprechenden Mittelwertsunterschiede waren in der überwiegenden Mehrzahl aller Fälle signifikant bis sehr signifikant.

Der in allen Versuchsbedingungen auftretende, bedeutende Unterschied zwischen den Antwortlatenzen von Figuren und Wörtern spiegelt die unterschiedliche Qualität des gewählten Reizmaterials wider: Bei den in Druckschrift dargebotenen Zahlwörtern handelte es sich um sehr geläufige verbale Reize, während der Bekanntheitsgrad der schwer verbalisierbaren Zufallsfiguren auch nach der Übungsaufgabe noch vergleichsweise gering sein dürtte. Bekanntheitsgrad und Verbalisierbarkeit sind Reizeigenschaften, die die Dauer von Antwortlatenzen entscheidend beeinflussen: Wenig vertraute, nonverbale 
Reize sind schwieriger zu memorieren und schwieriger wiederzuerkennen als vertraute, verbale Reize. Daß zwischen den gewählten Reizarten ein qualitativer Unterschied besteht, belegen auch die Gesamtanzahlen der Falschantworten. In beiden Sitzungen wurden unabhängig von der Testitemart bei Zufallsfiguren bedeutend mehr Falschantworten gegeben als bei Zahlwörtern. Dies gilt insbesondere für die Setgrößen 2 bis 4 .

Schnellere Antworten auf positive verglichen mit negativen Testitems sind wiederholt auch in anderen Untersuchungen beobachtet worden. Im vorliegenden Fall lag in der zweiten Sitzung die durchschnittliche Antwortlatenz für negative Antworten bei den Zahlwörtern um etwa $45 \mathrm{~ms}$, bei den Zufallsfiguren um etwa $75 \mathrm{~ms}$ höher als für positive Antworten. Beide Werte übersteigen den von Sternberg (1975) angegebenen mittleren Abweichungswert von $30 \mathrm{~ms}$, wobei die Diskrepanz bei den Zufallsfiguren besonders auffallend ist. Hier könnte zusätzlich zur Antwortart die bereits beschriebene besondere Qualität dieser Reize (geringer Bekanntheitsgrad, fehlende Verbalisierbarkeit) eine Rolle gespielt haben. Möglicherweise hat eine Wechselwirkung zwischen Reizart und Testitemart zu einer Erhöhung der Aufgabenschwierigkeit und damit zu einer überproportionalen Verlängerung der Antwortlatenzen geführt.

Die kurzen Antwortlatenzen der zweiten verglichen mit der ersten Versuchssitzung wurden bereits in Kapitel 6.1.2 als Ausdruck eines möglichen Lerneffekts betrachtet. Um einen über die Erhöhung der Antwortlatenz definierten Lern- bzw. Übungseffekt als potentielle Störvariable auszuschalten, sind nur die Daten der zweiten Versuchssitzung in die weiteren statistischen Analysen einbezogen worden. Diese Entscheidung konnte dadurch gerechtfertigt werden, daß sich innerhalb der zweiten Sitzung (im Gegensatz zur ersten Sitzung) keine signifikante Abnahme der mittleren Antwortlatenzen vom ersten zum dritten Drittel der Durchgänge und somit kein bedeutender Lerneffekt nachweisen ließ.

Von besonderem Interesse ist die Art der Beziehung zwischen der Größe des positiven Sets und der Antwortlatenz. Es wurde erwartet, daß sich die von Sternberg (1966) gefundene Linearität am erhobenen Datenmaterial replizieren ließe. Tatsächlich verdeutlichen Regressionsanalysen, daß zwischen der Größe des positiven Sets und der Antwortlatenz in allen Versuchsbedingungen eine eindeutige lineare Beziehung besteht. Dabei schwanken sowohl die Regressionskoeffizienten (Slope) als auch die Regressionskonstanten (Intercept) um die von Sternberg (1975; vgl. auch Cavanagh, 1972) angegebenen globalen Schätzwerte von 40 bzw. 400 ms.

Die Regressionskoeffizienten gelten als Schätzer für die pro Itemvergleich benötigte Zeit (sog. "Scanning-Zeit"). Im vorliegenden Fall hat die Reizart einen Einfluß auf die Scanning-Zeit, die für Zahlwörter bei beiden Testitemarten unter derjenigen für Zufallsfiguren liegt. Das bedeutet, daß der Such- und Vergleichsprozeß (zweite Verarbeitungsstufe in Sternbergs (1975) Modell der Itemwiedererkennung) unabhängig von der 
Art des Testitems bei nonverbalem Reizmaterial deutlich langsamer abläuft als bei verbalem Reizmaterial. Dieses Ergebnis stimmt mit den Befunden früherer Untersuchungen überein (vgl. z. B. Sternberg, 1969a; Cavanagh, 1972). Anders als bei Sternberg hat aber im vorliegenden Datenmaterial auch die Art des Testitems einen Einfluß auf die Scanning-Zeit: Diese fällt bei Zahlwörtern dann länger aus, wenn das zu beantwortende Testitem positiv ist. Nach Sternberg (1975) verursachen Unterschiede in den Such- und Vergleichsprozessen Diskrepanzen in den Regressionskoeffizienten positiver und negativer Testitems. Während der für die Beantwortung negativer Testitems notwendige erschöpfende Suchprozeß sich in vergleichsweise steilen Regressionsgeraden niederschlägt, soll ein sich selbst beendigender Such- und Vergleichsprozeß, der nur bei der Beantwortung positiver Testitems auftreten kann, zu flacher verlaufenden Regressionsgeraden führen können. Für den bei Zahlwörtern vorliegenden Fall (flachere Regressionsgerade bei negativen statt bei positiven Testitems) ist aus Sternbergs Modell allerdings keine Erklärung ableitbar.

Die Regressionskonstanten spiegeln die Dauer anderer kognitiver Prozesse wider, die in der Abrufphase zusätzlich zum Such- und Vergleichsprozeß stattfinden. Nach Sternberg sind dies Prozesse, die die Reizkodierung, die Entscheidung und die Antwortrealisierung betreffen. In dieser Untersuchung zeigt der Vergleich der mittleren Regressionskonstanten, daß die Dauer dieser Prozesse unabhängig von der Reizart bei negativen Testitems um 52 bis 79 ms länger ausfällt als bei positiven. Wenn positive Testitems beantwortet werden, nehmen diese Prozesse darüber hinaus bei Zufallsfiguren mehr Zeit in Anspruch als bei Zahlwörtern. Damit kann auch für die Dauer der übrigen Verarbeitungsstufen bei der Itemwiedererkennung festgehalten werden, daß diese im vorliegenden Fall sowohl von der Art der memorierten Items als auch von der geforderten Antwortart abhängig ist.

Insgesamt weisen die Ergebnisse der inferenzstatistischen Vergleiche der mittleren Regressionskoeffizienten und Regressionskonstanten auf eine Wechselwirkung zwischen Testitemart und Reizart hin. Sie legen somit nahe, daß - abhängig von der Art des Testitems - unterschiedliche Prozesse bei der Verarbeitung von verbalem und nonverbalem Reizmaterial stattfinden, und rechtfertigen somit auch die getrennte Datenauswertung für positive und negative Testitems. Das für diese Arbeit wichtigere Ergebnis ist jedoch, daß die von Sternberg (1966) gefundene lineare Beziehung zwischen Antwortlatenz und Größe des positiven Sets für das gewählte verbale und nonverbale Reizmaterial repliziert werden konnte. Dies bestätigt, daß die Probandinnen der Memorieraufgabe nachgekommen sind, und daß der Sternberg-Versuch korrekt durchgeführt wurde - beides Voraussetzungen, die erfüllt sein müssen, wenn vor dem Hintergrund des Sternberg-Paradigmas Aussagen zu topographischen und zeitdynamischen Aspekten des Arbeitsgedächtnisses gemacht werden sollen. 
Im Rahmen der vorliegenden Untersuchung ist zum ersten Mal eine kohärenzanalytische Auswertung eines während der Durchführung einer Sternberg-Aufgabe aufgezeichneten EEGs vorgenommen worden. Die Kohärenzanalyse wurde mit Hilfe eines neu entwickelten, zeitlich hochauflösenden Schätzverfahrens der Kohärenzfunktion gerechnet, das bisher nur in sehr wenigen Studien zum Einsatz gekommen ist. In diesen Studien haben sich zudem unterschiedliche kohärenzanalytische Kennwert für die Beschreibung schnell ablaufender kognitiver Prozesse als aussagekräftig herausgestellt. Daher wurde die Sensitivität dieser Kennwerte noch einmal am erhobenen Datenmaterial geprüft, wobei ihr Beitrag zur Aufdeckung reizartspezifischer topographischer Synchronisationsunterschiede im Mittelpunkt des Interesses stand. Ein hohes Ausmaß an Synchronisation bzw. eine hohe Kohärenz kann als Ausdruck der Involvierung des betreffenden kortikalen Areals bei der untersuchten Aufgabe angesehen (v. Stein, Rappelsberger, Filz \& Petsche, 1993) und in diesem Sinne als Zeichen verstärkter kortikaler (Funktions-)Aktivierung interpretiert werden.

In einem ersten Schritt sind die vier kohärenzanalytischen Kennwerte "mittlere Kohärenz", "mittlere maximale Kohärenz", "mittlere Kohärenzdauer" und "mittlerer Zeitpunkt maximaler Kohärenz" miteinander verglichen worden: Für jeden Kennwert sind für das positive Testitem pro Reizart und Setgröße Maps erstellt worden, mittels derer in einer unmittelbaren Gegenüberstellung von Zufallsfiguren und Zahlwörtern nach systematischen topographischen Synchronisationsunterschieden gesucht wurde. Zusätzlich sind inferenzstatistische Vergleiche in sieben verschiedenen Arealen durchgeführt worden. Dieses Vorgehen hat erste aufschlußreiche Ergebnisse erbracht. Während sich zum Beispiel in Untersuchungen zu elementaren, hochautomatisierten Denkprozessen die mittlere Kohärenzdauer als ergiebiger kohärenzanalytischer Kennwert erwiesen hat (z. B. Krause, 1997), konnte dies in der vorliegenden Arbeit nicht bestätigt werden. Zwar hat die optische Analyse der Maps der verschiedenen kohärenzanalytischen Kennwerte gezeigt, daß in fronto-zentralen bis zentro-parietalen Arealen eine bei beiden Reizarten unterschiedlich starke Zunahme der mittleren Kohärenzdauer über die vier Setgrößen vorliegt; da die Kohärenzdauer jedoch als kumulierte Zeitdauer, für die die Bandkohärenz eine bestimmte Schwelle im definierten Zeitintervall überschreitet, keinen von der Antwortlatenz unabhängigen Kennwert darstellt, ist eine schlüssige Interpretation dieser unterschiedlich starken Zunahmen über die Setgrößen nicht möglich. Dasselbe gilt für den Kennwert "Zeitpunkt maximaler Kohärenz", der angibt, wann das höchste erreichte Synchronsationsausmaß im interessierenden Intervall aufgetreten ist.

In den Maps der beiden übrigen kohärenzanalytischen Kennwerte "mittlere Kohärenz" und "mittlere maximale Kohärenz", die rechnerisch gesehen von der Antwortlatenz unabhängig sind, ließen sich fronto-zentral bis zentro-parietal nur leichte Zunahmen über die Setgrößen ausmachen. Diese traten jedoch bei Zufallsfiguren und Zahl- 
wörtern in vergleichbarem Ausmaß auf, so daß auch sie wiederum keine Aussagen zu systematischen topographischen Synchronisationsunterschieden ermöglichen.

Drei der vier Kennwerte (mittlere Kohärenz, mittlere maximale Kohärenz und mittlere Kohärenzdauer) wiesen bei beiden Reizarten in fast allen Setgrößen in einem fronto-zentralen Areal sehr hohe Ausprägungen auf. Dieses Areal spielt offenbar eine bedeutende Rolle beim Abruf sowohl verbaler als auch nonverbaler Information aus dem Arbeitsgedächtnis. Die Annahme liegt nahe, daß die starken Synchronisationsprozesse in diesem Areal eventuell die in anderen Arealen bestehenden reizartspezifischen Synchronisationsunterschiede überlagern könnten.

Der erste Versuch, einen für die Untersuchung schnell ablaufender kognitiver (Abruf-) Prozesse tauglichen kohärenzanalytischen Kennwert zu finden, hat zu zwei wichtigen Erkenntnissen geführt: Erstens sollte zur Aufdeckung systematischer topographischer Synchronisationsunterschiede beim Abruf verbaler bzw. nonverbaler Information im Rahmen des Sternberg-Paradigmas ein kohärenzanalytischer Kennwert verwendet werden, der rechnerisch gesehen von der Antwortlatenz unabhängig ist, und der es erlaubt, eventuell vorhandene topographische Synchronisationsunterschiede eindeutig auf die Reizart zurückzuführen. Zweitens sollte mit einem solchen kohärenzanalytischen Kennwert ein mittelbarer Vergleich der Reizarten vorgenommen werden, da die starken Synchronisationsprozesse in einem fronto-zentralen Areal, das sowohl am Abruf verbaler als auch nonverbaler Information beteiligt zu sein scheint, bei einer unmittelbaren Gegenüberstellung der Reizarten das Aufdecken spezifischer Synchronisationsunterschiede in anderen Arealen unmöglich machen.

In einem zweiten Schritt sind daher mit den beiden von der Antwortlatenz unabhängigen Kennwerte "mittlere Kohärenz" und "mittlere maximale Kohärenz" mittelbare Vergleiche zwischen Zufallsfiguren und Zahlwörtern durchgeführt worden. Analog zu dem Vorgehen bei den Antwortlatenzen wurde für jedes Elektrodenpaar regressionsanalytisch die Beziehung zwischen der in diesem Versuch systematisch variierten Setgröße und den genannten Kennwerten untersucht und für beide Reizarten verglichen. Hinter einem solchen Vorgehen stehen zwei Annahmen: Erstens wird davon ausgegangen, daß sich eine Aktivierung der am Abruf verbaler bzw. nonverbaler Information beteiligten Areale im EEG durch eine verstärkte Synchronisierung auszeichnet. Zweitens wird davon ausgegangen, daß die Beziehung zwischen der Setgröße und dem physiologischen Maß (Kohärenz) in den betreffenden Arealen ebenso wie die Beziehung zwischen der Setgröße und dem Verhaltensmaß (Antwortlatenz) linear ausfällt (vgl. hierzu die Ergebnisse von Braver et al., 1997, sowie von Smith \& Jonides, 1997). Da die Ergebnisse zahlreicher Untersuchungen nahelegen, daß je nach Art des Reizmaterials unterschiedliche Areale an der Informationsverarbeitung beteiligt sind, muß vor diesem Hintergrund derjenige kohärenzanalytische Kennwert als sensitiv angesehen werden, 
der nicht nur die meisten signifikant linearen Beziehungen zur Setgröße aufweist, sondern darüber hinaus diesbezüglich auch die wenigsten Überschneidungen zwischen den Reizarten hervorbringt.

Mit der maximalen Kohärenz ließ sich ein solcher Kennwert finden. Über alle Versuchsbedingungen gesehen wies die mittlere maximale Kohärenz an fast dreimal so vielen Elektrodenpaaren wie die mittlere Kohärenz signifikant lineare Beziehungen zur Setgröße auf. Der Überschneidungsgrad zwischen den Reizarten fiel bei beiden Testitemarten mit jeweils zwei Elektrodenpaaren äußerst gering aus. Aufgrund dieser Ergebnisse ist die mittlere maximale Kohärenz als der sensitivste kohärenzanalytische Kennwert betrachtet und für die Prüfung der Hypothesen zu topographischen Aspekten des Arbeitsgedächtnisses verwendet worden.

Die Frage nach den während der Abrufphase im Sternberg-Versuch aktivierten neokortikalen Arealen stellt einen zentralen Schwerpunkt der vorliegenden Arbeit dar. Hierzu sind nach Durchsicht zahlreicher neuropsychologischer Studien drei Hypothesen aufgestellt worden. Sie betreffen diejenigen Areale, die mutmaßlich am Abruf verbaler bzw. nonverbaler Information aus dem Arbeitsgedächtnis beteiligt sind, sowie diejenigen Areale, denen während einer solchen Aufgabe Erhaltungs- bzw. Überwachungsfunktionen zugeschrieben werden.

Die erste Hypothese, in der postuliert worden war, daß der Abruf verbaler Information aus dem Arbeitsgedächtnis zu kortikalen Aktivationen im linken oberen Temporallappen und linken unteren Parietallappen (Area 22/42 und 40), nicht jedoch im Broca-Areal (Area 44) führen sollte, konnte für das positive Testitem bestätigt werden. Die maximalen Kohärenzen nahmen in den Sprachstrukturen der linken Hemisphäre in denjenigen Versuchsdurchgängen, in denen bei der Reizart "Zahlwörter" eine Ja-Antwort gefordert war, linear mit der Größe des positiven Sets zu. Im Broca-Areal, dem eine zentrale Rolle bei der stillen Wiederholung verbaler Information zugeschrieben wird, trat erwartungsgemäß keine solche Synchronisationszunahme auf. Für das negative Testitem ließ sich im Gegensatz dazu die erste Hypothese nicht bestätigten. In den Sprachstrukturen der linken Hemisphäre waren in dieser Bedingung keine verstärkten Synchronisationsprozesse zu verzeichnen.

Was das positive Testitem betrifft, so stehen die Ergebnisse im Einklang mit Befunden anderer neuropsychologischer Untersuchungen zum phonologischen Arbeitsgedächtnis, in denen die Areale 22/42, 40 und 44 als anatomische Korrelate der phonobgischen Schleife ausgewiesen werden konnten (z. B. Paulesu, Frith \& Frackowiak, 1993; Smith \& Jonides, 1997). Die zu Recht fehlende kortikale Aktivation im Broca-Areal spricht darüber hinaus für die Existenz einer Dissoziation zwischen Speicherung und stillem Wiederholen im phonologischen Arbeitsgedächtnis (vgl. Awh et al., 1996). Außerdem 
läßt sich die in der Literatur wiederholt beschriebene Aktivation korrespondierender ("homologer") Areale finden: Die maximale Kohärenz nimmt nicht nur in den Sprachstrukturen der linken Hemisphäre mit der Größe des positiven Sets zu, sondern auch in einigen, diesen Strukturen entsprechenden rechtshemisphärischen Arealen. Diese werden möglicherweise aktiviert, um den Verarbeitungsprozeß zu unterstützen, oder aber um im Falle eines exzessiven Aktivationsanstiegs als "Ventil" zu fungieren (vgl. Smith, Jonides \& Koeppe, 1996).

Die Unterschiede zwischen den beim positiven bzw. negativen Testitem aktivierten kortikalen Arealen lassen sich vor dem Hintergrund des gegenwärtigen Forschungsstandes nur schwer erklären. In neuropsychologischen Studien zum Arbeitsgedächtnis werden meist sogenannte "n-back"-Aufgaben verwendet, die lediglich eine Ja-Antwort erfordern, sobald einer der fortlaufend präsentierten Reize ein vorher festgelegtes Kriterium erfüllt (z. B. Übereinstimmung mit dem drittletzten Reiz). Aber auch aus den wenigen Studien, in denen die klassische Sternberg-Aufgabe oder ähnlich geartete Wiedererkennungsaufgaben zum Einsatz kamen (z. B. Paulesu, Frith \& Frackowiak, 1993; Awh et al., 1996), können keine Erkenntnisse zu topographischen Aktivationsunterschieden bei der Beantwortung positiver und negativer Testitems abgeleitet werden. Das ist auf die eingesetzten bildgebenden Verfahren (z. B. PET) zurückzuführen, deren geringes zeitliches Auflösungsvermögen es nicht erlaubt, Versuchsdurchgänge mit Jabzw. Nein-Antworten getrennt auszuwerten. Stattdessen wird über alle Durchgänge gemittelt; anhand einer passenden Kontrollaufgabe werden anschließend lediglich diejenigen Aktivationen "abgezogen", die von perzeptuellen und motorischen Verarbeitungsprozessen herrühren (sog. "Subtraktionsmethode").

Über die Ursachen der gefundenen topographischen Aktivationsunterschiede bei positiven und negativen Testitems können also nur Vermutungen angestellt werden. Es liegt nahe, sie auf Unterschiede in den Abrufprozessen zurückzuführen. Möglicherweise ändert sich in den Sprachstrukturen der linken Hemisphäre mit der Testitemart auch die Art der Beziehung zwischen der Größe des positiven Sets und der maximalen Kohärenz (z. B. von linear zu exponentiell), so daß sie sich mittels linearer Regressionsanalysen nicht mehr aufzeigen läßt. Eventuell spiegeln sich Unterschiede in den Abrufprozessen auch in der Rekrutierung anderer Hirnareale; dies würde z. B. die beim negativen Testitem auftretende Aktivation homologer Areale im occipitalen Kortex erklären. Auch eine "Verschiebung" der Aktivation innerhalb der beteiligten Areale ist denkbar. So könnten die von den Elektroden T5 und P3 abgeleiteten Signale neben den Arealen 7/39/37 auch Randgebiete von Area 40 einschließen; Area 40 ist beim positiven Testitem eindeutig aktiviert (s. o.) und wird derzeit als Hauptort des phonologischen Speichers gehandelt (Paulesu, Frith \& Frackowiak, 1993; Petrides, Alivisatos, Meyer \& Evans, 1993b; Awh et al., 1996; Salmon et al., 1996; Smith \& Jonides, 1997). Für eine bessere Interpretation der beim positiven bzw. negativen Testitem gefundenen Aktivationsunterschie- 
de müßten gezieltere Untersuchungen unter zusätzlicher Verwendung räumlich hochauflösender Verfahren durchgeführt werden.

In der Literatur wird die Existenz unterschiedlicher neuronaler Schaltkreise für das visuell-räumliche Gedächtnis und das Objektgedächtnis postuliert, wobei das visuell-räumliche Gedächtnis rechtshemisphärisch, das Objektgedächtnis linkshemisphärisch angelegt sein soll (Postle, Stern, Rosen \& Corkin, 1997; Smith \& Jonides, 1997). Obwohl es sich beim Memorieren und Wiedererkennen von Zufallsfiguren streng genommen um eine nicht-räumliche (Objektgedächtnis-)Aufgabe handelt, wurde in der zweiten Hypothese angenommen, daß am Abruf der nonverbalen Information die rechtshemisphärischen Areale 7/40 und 37 beteiligt sein würden. Der Grund für diese Annahme liegt in der Qualität des gewählten nonverbalen Reizmaterials: Die besondere Beschaffenheit der Zufallsfiguren legt es den Probanden nahe, sich die Figuren für eine bessere Behaltensleistung anhand ihrer räumlichen Charakteristika zu merken (z. B. anhand der Ausrichtung der Spitzen; vgl. Abb. 4, Kap. 5.4).

Tatsächlich ließen sich - wiederum nur beim positiven Testitem - in den rechtshemisphärischen Arealen 7/39/37 Aktivationen aufzeigen, nicht jedoch in Area 40. (Die von den Elektroden P4 und T6 abgeleiteten Signale könnten aber Randgebiete von Area 40 mit einschließen, vgl. oben.) Außerdem waren in dieser Bedingung zentral, rechtsseitig temporal sowie linksseitig zentro-parietal und temporo-occipital Zunahmen der maximalen Kohärenz mit der Größe des positiven Sets zu verzeichnen. Während die Aktivationen im oberen Teil des linken Parietallappens und in linken inferotemporalen bis occipitalen Arealen wahrscheinlich die Beteiligung des sogenannten "Objektgedächtnisses" widerspiegeln (Mishkin, Ungerleider \& Macko, 1983; Paulesu, Frith \& Frackowiak, 1993; Postle, Stern, Rosen \& Corkin, 1997; Smith \& Jonides, 1997), ist die zentral auftretende Aktivation nicht einfach zu erklären. Sie erstreckt sich beidseitig vom oberen Teil des primären motorischen bzw. somatosensorischen Projektionsfeldes bis zu den am Sulcus centralis gelegenen Anteilen des posterioren Parietallappens (Area $1 / 2 / 3 / 4$ bis 7). Im primären motorischen bzw. somatosensorischen Kortex sollten sich eigentlich keine von der Gedächtnislast abhängigen Aktivationsänderungen finden lassen, da diesen Arealen in der Regel keine Arbeitsgedächtnisfunktionen zugeschrieben werden. Sie sind dem derzeitigen Kenntnisstand zufolge hauptsächlich für die Ausführung feinmotorischer Bewegungen der Zielmotorik bzw. für die Sinnesempfindungen der zugehörigen Körperteile zuständig (Birbaumer \& Schmidt, 1996). Die zentrale Aktivation könnte aber darauf hinweisen, daß die genannten Areale auch noch an anderen Prozessen beteiligt sind. Awh et al. (1996) interpretieren zum Beispiel die während einer verbalen Memorieraufgabe wider Erwarten gefundene beidseitige Aktivation im supplementären motorischen Areal (SMA; in der Tiefe der Fissura interhemispherica liegender Teil von Area 6) als Zeichen dafür, daß dieses Areal nicht nur für die motorische Pro- 
grammierung, sondern auch für die Fehlerfindung oder für übungsbezogenes Lernen zuständig sein könnte. Möglicherweise trifft eine analoge Erklärung für die während des Abrufs nonve rbaler Information gefundene zentrale Aktivation zu.

Wie bei den Zahlwörtern, so weichen auch bei den Zufallsfiguren die Ergebnisse des negativen von denen des positiven Testitems ab. Beim negativen Testitem traten die rechtsseitigen Aktivationen verglichen mit dem positiven Testitem weiter dorsal in parieto- und temporo-occipitalen Arealen auf (Area 7/19 - 17 und 37/19 - 17). Offenbar spielt das visuelle System in dieser Bedingung eine wichtige Rolle. Linkshemisphärisch konnte zwar im oberen Parietallappen (Area 7) eine Zunahme der maximalen Kohärenz mit der Größe des positiven Sets verzeichnet werden, allerdings trat diese Zunahme parietal bis parieto-zentral und nicht wie beim positiven Testitem zentral bis parietal auf. Immerhin könnte damit auch bei der Beantwortung negativer Testitems auf eine Beteiligung des Objektgedächtnisses geschlossen werden. Die Aktivation in der linkshemisphärischen Area 37, die zusätzlich zum oberen Parietallappen maßgeblich an der Wiedererkennung von Objekten beteiligt sein soll (Mishkin, Ungerleider \& Macko, 1983), reicht jedoch in dieser Bedingung wider Erwarten bis in die Sprachstrukturen hinein (Area 22/42). Außerdem ist auch im Broca-Areal eine Aktivation zu verzeichnen. Beim positiven Testitem ließ sich eine Beteiligung der Sprachstrukturen hingegen nicht nachweisen. Auffällig ist schließlich, daß die beim positiven Testitem gefundene zentrale Aktivation gänzlich fehlt und daß beim negativen Testitem fronto-temporal homologe Areale (Area 46/22) aktiviert sind.

Im Prinzip sind die bei den Zufallsfiguren aufgetretenen topographischen Aktivationsunterschiede zwischen positivem und negativem Testitem auf dieselben möglichen Mechanismen zurückführbar, die bei der Diskussion der Ergebnisse der Zahlwörter erwähnt wurden. Insgesamt sprechen diese Aktivationsunterschiede dafür, daß sich auch im Falle der Zufallsfiguren die Abrufprozesse bei der Beantwortung positiver bzw. negativer Testitems unterscheiden.

Dem präfrontalen Kortex werden unabhängig von der gestellten kognitiven Aufgabe Informationserhaltungs- und -manipulationsfunktionen zugeschrieben (Owen, Evans \& Petrides, 1996; Braver et al., 1997; D'Esposito \& Postle, 1998). Die Art der zu verarbeitenden Information soll dabei die hemisphärische Lateralisierung präfrontaler Aktivationen bestimmen (D'Esposito et al., 1998a). In der dritten Hypothese wurde daher zum einen angenommen, daß der präfrontale Kortex während des Abrufs sowohl verbaler als auch nonverbaler Information aktiviert sein würde. Zum anderen wurde postuliert, daß sich die Aktivation beim Abruf verbaler Information auf den linken, beim Abruf nonverbaler Information auf den rechten präfrontalen Kortex konzentrieren sollte. Der erste Teil dieser Hypothese konnte für beide Reizarten bestätigt werden: Unabhängig von der Testitemart fanden sich sowohl bei Zufallsfiguren als auch bei Zahlwörter in mehreren 
präfrontalen Arealen Zunahmen der maximalen Kohärenz mit der Größe des positiven Sets. Der zweite Teil der Hypothese, der sich auf die Lateralisierung der präfrontalen Aktivation bezieht, konnte jedoch nur für die Zahlwörter verifiziert werden. Bei ihnen lieBen sich unabhängig von der Testitemart nur linksseitig präfrontale Aktivationen verzeichnen. Bei den Zufallsfiguren traten präfrontale Aktivationen hingegen sowohl rechtshemisphärisch als auch linkshemisphärisch auf. Dieses Ergebnis könnte darauf zurückzuführen sein, daß das in dieser Untersuchung verwendete nonverbale Reizmaterial sowohl räumliche als auch nichträumliche Merkmale aufwies (s. o.). Möglicherweise spiegelt es aber auch den größeren Schwierigkeitsgrad der nonverbalen Wiedererkennungsaufgabe wider.

Ein Vergleich der beiden Reizarten zeigte, daß einige präfrontale Areale nur bei den Zufallsfiguren, nicht jedoch bei den Zahlwörtern aktiviert waren. Dabei handelte es sich rechtsseitig um Area 8/9 und linksseitig um Area 9/10. Umgekehrt waren die frontozentralen Areale 8/6/4 nur bei den Zahlwörtern, nicht jedoch bei den Zufallsfiguren aktiviert. Vom Reizmaterial abhängige hemisphärische Aktivationslateralisierungen konnten nur in den Arealen 10 und 46 festgestellt werden. Diese Gebiete waren beim Abruf nonverbaler Information (positives und negatives Testitem) nur rechtshemisphärisch, beim Abruf verbaler Information (positives Testitem) nur linkshemisphärisch aktiviert. Die Areale 10 und 46 gehören zwar zum dorsolateralen präfrontalen Kortex, jedoch könnten Aktivationen in diesen frontopolar-frontalen Gebieten angesichts der gewählten Elektrodenpositionen (vgl. Kap. 6.2.3, Abb. 13, und Kap. 3.2.2, Abb. 11) auch ventrolaterale Anteile des präfrontalen Kortex einschließen (Area 45/47). Differenzierte Schlußfolgerungen bezüglich der in der Literatur wiederholt postulierten Dissoziation unterschiedlicher präfrontaler Areale lassen sich aus den vorliegenden Ergebnissen somit nicht ableiten. Es kann lediglich festgehalten werden, daß die Lateralisierung der Aktivation in Area 10/46 in der vorliegenden Untersuchung reizartspezifisch aufgetreten ist. Im übrigen spiegelten sich die präfrontalen Aktivationsunterschiede zwischen beiden Reizarten eher in der Aktivation unterschiedlicher Areale als in der Lateralisierung dieser Aktivationen wider.

Sowohl bei Zufallsfiguren als auch bei Zahlwörtern ließen sich deutliche Übereinstimmungen hinsichtlich der jeweils beim positiven bzw. negativen Testitem aktivierten präfrontalen Areale finden. Solche Übereinstimmungen konnten für die übrigen kortikalen Areale nicht aufgezeigt werden (vgl. oben). Dieses Ergebnis kann dahingehend interpretiert werden, daß sich der beim positiven Testitem ablaufende Informationsabruf nicht gänzlich von dem beim negativen Testitem ablaufenden Informationsabruf unterscheidet. Beiden Vorgängen sind zumindest diejenigen (Teil-)Prozesse gemein, die von präfrontalen Arealen geleistet werden. Es sind dies Steuerungs- und Aufmerksamkeitskontrollprozesse, die während der Lösung von Itemwiedererkennungsaufgaben, aber auch während der Bearbeitung anderer, nicht-mnestischer Aufgaben erforderlich sind 
(Kluwe, 1997; D'Esposito et al., 1998b). Der Unterschied zwischen den Abrufprozessen bei der Beantwortung positiver bzw. negativer Testitems manifestiert sich also bei beiden Reizarten weniger in frontalen als in den übrigen kortikalen Arealen. Insgesamt stützen diese Ergebnisse den derzeitigen Kenntnisstand bezüglich der Rolle, die dem präfrontalen Kortex bei der Bewältigung kognitiver Aufgaben zugeschrieben wird.

Neben der Analyse reizartspezifischer topographischer Synchronisationsunterschiede in der Abrufphase des Sternberg-Versuchs bestand ein weiteres wichtiges Anliegen dieser Arbeit darin, einen Beitrag zur Aufklärung zeitdynamischer Aspekte des Arbeitsgedächtnisses zu liefern. Da bislang keine Erkenntnisse zum Zeitverlauf elektrophysiologischer Synchronisationsprozesse in der Abrufphase des Sternberg-Versuchs vorliegen, ist statt einer hypothesengeleiteten Untersuchung eine exploratorische Untersuchung durchgeführt worden. Diese exploratorische Untersuchung erfolgte anhand einer Verlaufsbetrachtung der mittleren Bandkohärenzen und über eine Analyse der Zeitpunkte maximaler Kohärenz im interessierenden Intervall (ab 120 ms nach dem Testreiz). Wie bei der Untersuchung der reizartspezifischen topographischen Synchronisationsunterschiede stand dabei der Einfluß des parametrisch variierten Faktors "Setgröße" im Vordergrund.

Da die Analyse der maximalen Kohärenzen nur für das positive Testitem hypothesenkonforme Ergebnisse erbracht hat, wurde die Verlaufsbetrachtung der mittleren Bandkohärenzen nur für diese eine Testitemart durchgeführt. Sie hat gezeigt, daß der Abruf verbaler bzw. nonverbaler Information in unterschiedlichen Arealen zu längeranhaltenden und mit der Größe des positiven Sets zunehmenden Synchronisationsprozessen führt. Der deutlichste Unterschied zwischen den Reizarten konnte beim Vergleich derjenigen Areale festgestellt werden, in denen die mittleren Bandkohärenzen über alle vier Setgrößen zunahmen. Dieser vollständige Setgrößen-Effekt trat bei den Zufallsfiguren rechtshemisphärisch in den Arealen 1/2/3, 40 und 22, bei den Zahlwörtern linkshemisphärisch in den Arealen 1/2/3, 5 und 7 sowie 7/39/37 auf. Er erstreckte sich bei den Zufallsfiguren auf die ersten 170 ms des untersuchten Zeitintervalls, bei den Zahlwörtern trat er hingegen in den ersten 90 bis 115 ms dieses Intervalls auf. Je nach Art des memorierten Reizmaterials lassen sich also nicht nur in unterschiedlichen Arealen längeranhaltende Aktivationen ausmachen, sondern auch Unterschiede in der Dauer dieser Aktivationen aufzeigen. Die betroffenen Areale grenzen dabei an kortikale Strukturen, in denen wiederholt während verbaler bzw. nonverbaler Memorieraufgaben erhöhte Aktivationen nachgewiesen werden konnten. Sie stimmen jedoch - mit Ausnahme von Area 40 (Zufallsfiguren) - nicht direkt mit diesen Strukturen überein; das könnte bedeuten, daß sie zwar am Informationsabruf beteiligt sind, in diesem Zusammenhang aber andere Aufgaben erfüllen als diejenigen Areale, in denen die maximale Kohärenz mit der Größe des positiven Sets zunimmt (s. o.). Neben Arealen, in denen die Bandkohä- 
renzen über die vier Setgrößen hinweg zunahmen, ließen sich auch solche finden, die unabhängig von der Setgröße und unabhängig von der Reizart im Verlauf eine vergleichbar ausgeprägte, stetige Abnahme oder Zunahme der mittleren Bandkohärenzen aufwiesen. Kohärenzabnahmen wurden unter anderem in parieto- bis temporo-occipitalen Arealen beobachtet (Area 7 bis 17 bzw. 37 bis 17). Da diese Areale den visuellen Kortex mit einschließen, wird vermutet, daß die Kohärenzabnahmen noch zum Prozeß der visuellen Enkodierung des Testreizes gehören. Kohärenzzunahmen traten beidseitig in den primären motorischen bzw. somatosensorischen Arealen auf (Area 1 bis 4). Sie könnten möglicherweise Ausdruck einer antizipatorischen Aktivation in Bezug auf die anstehende Beantwortung des Testreizes (Tastendruck) sein. Zusätzlich zu Arealen mit Kohärenzzunahmen bzw. -abnahmen ließen sich auch Areale finden, in denen bei beiden Reizarten und allen vier Setgrößen außerordentlich hohe Bandkohärenzen auftraten (links Area 8/9, 1/2/3/4, 6, 8, 5 und 7). Diese kortikalen Strukturen scheinen Funktionen zu erfüllen, die in der Abrufphase unabhängig von der Reizart und von der Setgröße besonders gefordert sind. Insgesamt zeigen diese Ergebnisse, daß das dem Arbeitsgedächtnis zugrundeliegende neuronale Netzwerk sowohl aufgabenspezifische als auch aufgabenunspezifische Komponenten umfaßt. Darüber hinaus kann die Annahme bestätigt werden, daß sich verschiedene kognitive Funktionen neokortikaler Areale auch in unterschiedlichen zeitlichen Aktivationsverläufen dieser Areale niederschlagen (Cohen et al., 1997; Smith \& Jonides, 1997).

Ein weiterer Beitrag zur Aufklärung zeitdynamischer Aspekte des Arbeitsgedächtnisses konnte durch die Analyse des Einflusses der Setgröße auf die Zeitpunkte maximaler Kohärenz geleistet werden. Sie hat deutlich gemacht, daß die stärksten Verschiebungen des Aktivitätsmaximums im Falle des positiven Testitems bei den Zufallsfiguren rechtshemisphärisch (Area 10/46, 7 bis 17 und 37 bis 17), bei den Zahlwörtern jedoch linkshemisphärisch auftreten (Area 8/9, 9/44/46, 46 bis 22 und 22/42/37). Wenn eine Nein-Antwort gefordert war (negatives Testitem), verschoben sich die Aktivitätsmaxima unabhängig von der Reizart sehr stark in links und rechts frontalen Arealen (Area 10/46 und 9/10); daneben traten bei den Zufallsfiguren rechtshemisphärisch ausgeprägte Zunahmen der Zeitpunkte maximaler Kohärenz auf (Area 46 bis 22, 1/2/3, 5 und 7). Beim positiven Testitem schließen die genannten Gebiete einige derjenigen kortikalen Strukturen ein, die am Abruf verbaler bzw. nonverbaler Information beteiligt sein sollen (z. B. Area 22/42 und 44 bei den Zahlwörtern, Area 7 und 37 bei den Zufallsfiguren). In diesen Strukturen nahm also sowohl die maximale Kohärenz als auch der Zeitpunkt ihres Auftretens im untersuchten Intervall mit der Größe des positiven Sets zu. Die Beteiligung kortikaler Areale am Abruf verbaler bzw. nonverbaler Information äußert sich demnach nicht nur in einem Aktivationsanstieg, sondern auch in dessen späterem Auftreten in der Abrufphase. 
Unterschiede zwischen den Reizarten bzw. Testitemarten manifestierten sich jedoch nicht nur topographisch, sondern auch hinsichtlich des Ausmaßes, mit dem sich die Zeitpunkte maximaler Kohärenz in den betroffenen Arealen verschoben: Je nach Testitemart nahmen sie bei den Zufallsfiguren mit jedem hinzugekommenen Item um 9 bis $22 \mathrm{~ms}$, bei den Zahlwörtern nur um 3 bis $9 \mathrm{~ms}$ zu. Der statistische Vergleich zwischen diesen Beträgen führte in einigen derjenigen Areale, in denen der Zeitpunkt maximaler Kohärenz pro Testitemart bei beiden Reizarten bzw. pro Reizart bei beiden Testitemarten mit der Größe des positiven Sets zunahm, zu signifikanten Ergebnissen. Dabei traten interessante Überschneidungen zwischen den einzelnen Versuchsbedigungen auf: So verschob sich das Aktivitätsmaximum in der rechtshemisphärischen Area 7 bis 17 bei den Zufallsfiguren dann am stärksten, wenn es sich um das positive Testitem handelte, bei den Zahlwörtern hingegen dann, wenn es sich um das negative Testitem handelte. Die meisten Unterschiede offenbarten sich jedoch in der Gegenüberstellung der Reizarten beim negativen Testitem. Während eine solche Gegenüberstellung bei der Analyse der maximalen Kohärenzen lediglich topographische Aktivationsunterschiede ergeben hatte, verdeutlichte die Analyse der Zeitpunkte maximaler Kohärenz unter anderem, daß der Abruf nonverbaler Information links und rechts frontal zu erheblich stärkeren Verschiebungen der Aktivitätsmaxima führt als der Abruf verbaler Information (21 ms vs. 6 ms links in Area 9/10, 21 ms vs. 9 ms rechts in Area 10/46). Beim positiven Testitem waren in diesen Arealen hingegen keine signifikanten Unterschiede zwischen den Reizarten auszumachen. Dieses Ergebnis könnte darauf hinweisen, daß sich der Abruf nonverbaler Information bei geforderten Nein-Antworten schwieriger gestaltet als der Abruf verbaler Information und daher eine verstärkte bzw. längeranhaltende Beteiligung des präfrontalen Kortex erforderlich macht, der für Steuerungs- und Aufmerksamkeitskontrollprozesse zuständig ist (vgl. oben). In diesem Sinne könnte von einer Wechselwirkung zwischen Reizart und Testitemart gesprochen werden, wie sie bereits bei der Diskussion der Verhaltensdaten erwähnt wurde.

Die exploratorische Untersuchung des Zeitablaufes der Verarbeitungsprozesse von verbalem und nonverbalem Reizmaterial wurde mit dem Versuch abgeschlossen, die Frage nach einer möglichen Beziehung zwischen dem Zeitpunkt maximaler Kohärenz und der Antwortlatenz zu beantworten. Die zunächst durchgeführten Korrelationsanalysen ergaben lediglich, daß diese Beziehung in vielen Arealen signifikant ausfällt; topographisch ließen sie jedoch keine systematischen Unterschiede zwischen den verschiedenen Versuchsbedingungen erkennen. Ergiebiger war der globale Vergleich der mittleren Regressionskoeffizienten der Zeitpunkte maximaler Kohärenz mit den mittleren Regressionskoeffizienten der Antwortlatenzen ("Scanning-Zeiten"). Hierüber konnte unter anderem die obengenannte Wechselwirkung zwischen Reizart und Testitemart noch einmal aufgezeigt werden: Beim Abruf nonverbaler Information fielen die Regres- 
sionskoeffizienten beider Kennwerte bei negativen Testitems größer aus als bei positiven Testitems, beim Abruf verbaler Information kehrte sich dieses Verhältnis um.

Insgesamt betrachtet hat die exploratorische Untersuchung des zeitlichen Ablaufes der Synchronisationsprozesse wertvolle zusätzliche Erkenntnisse bezüglich derjenigen elektrophysiologischen Prozesse geliefert, die während des Abrufs verbaler bzw. nonverbaler Information aus dem Arbeitsgedächtnis stattfinden. Zum einen wurde deutlich, daß sich die zeitlichen Aktivationsverläufe in verschiedenen neokortikalen Arealen unterscheiden und daß sich eine Beteiligung derjenigen Areale, die für die Verarbeitung verbaler bzw. nonverbaler Information zuständig sein sollen, in einer Zunahme und gleichzeitigen zeitlichen Verschiebung der maximalen Aktivation niederschlägt. Zum anderen waren mit der Analyse der Zeitpunkte maximaler Kohärenz vergleichsweise differenziertere Aussagen z. B. bezüglich der präfrontal aktivierten Areale möglich. Schließlich konnte gezeigt werden, daß zwischen den Antwortlatenzen und den Zeitpunkten maximaler Kohärenz enge Beziehungen bestehen, die die Sensitivität dieses kohärenzanalytischen Kennwertes für die Analyse kognitiver Prozesse unterstreichen.

Die Güte einer interdisziplinär angelegten wissenschaftlichen Arbeit bemißt sich unter anderem an dem Beitrag, der durch diese Arbeit den betreffenden Fachdisziplinen zuteil wird. In diesem Sinne soll abschließend darauf eingegangen werden, welchen Beitrag die vorliegende Arbeit für die kognitive Psychologie und die Psychophysiologie leisten konnte.

Der für die kognitive Psychologie wichtige Aspekt betrifft die Frage, inwiefern die erhaltenen Ergebnisse mit psychologischen Konzepten des Kurzzeitgedächtnisses in Übereinstimmung gebracht werden können. Klinisch-anatomische Studien und Studien mit bildgebenden Verfahren lieferten Evidenzen dafür, daß den von Baddeley (1998) postulierten Komponenten des Arbeitsgedächtnisses (phonologische Schleife, visuellräumlicher Notizblock und zentrale Exekutive) verschiedene neuronale Strukturen entsprechen könnten. Die in dieser Arbeit beim positiven Testitem gefundenen topographischen Aktivationsunterschiede decken sich weitgehend mit den Ergebnissen dieser Studien. Dasselbe gilt auch für die bei beiden Testitemarten beobachteten präfrontalen Aktivationen, die als neuronales Korrelat der Beteiligung der sogenannten "zentralen Exekutive" gedeutet werden können. Problematisch gestaltet sich allerdings die Interpretation der abweichenden Ergebnisse beim negativen Testitem. Da sich die Memorierphasen von Durchgängen mit positiven bzw. negativen Testitems nicht voneinander unterscheiden, stellt sich die Frage, weshalb bei der Beantwortung negativer Testitems nicht auch (wie beim positiven Testitem) diejenigen kortikalen Areale aktiviert sind, die als neuronale Korrelate des phonologischen Speichers bzw. des visuell-räumlichen Notizblocks gehandelt werden. Eine mögliche Antwort wäre, daß die Aktivationen in der Ab- 
rufphase weniger Informationen bezüglich der "Speicherorte" als bezüglich derjenigen kortikalen Strukturen liefern, die für das "Zurückholen" der gespeicherten Informationen zuständig sind. In diesem Fall stünden die topographischen Aktivationsunterschiede zwischen beiden Testitemarten für entsprechend unterschiedliche Abrufprozesse. Da in dem Arbeitsgedächtnismodell von Baddeley (1998) zur eigentlichen Beschaffenheit der verschiedenen Speicher und insbesondere zur Art und Weise, wie die Informationen aus innen abgerufen werden, keine Angaben gemacht werden, lassen sich die topographischen Aktivationsunterschiede (bzw. die entsprechenden unterschiedlichen Abrufprozesse) zwischen positiven und negativen Testitems mit diesem Modell nicht erklären. Hier offenbart sich die Notwendigkeit zusätzlicher Differenzierungen des Arbeitsgedächtnismodells, wie sie auch im Zusammenhang mit anderen Untersuchungsergebnissen gefordert werden (Cowan, 1993; Martin, 1993; Ericsson \& Kintsch, 1995; Cowan et al., 1998; Hulme et al., 1999; Lass et al., 2001). Dazu bedarf es jedoch noch weiterer Untersuchungen; so bietet sich z. B. eine Analyse der während des Memorierens ablaufenden Synchronisationsprozesse an, um den hypostasierten Unterschied zwischen den in der Memorier- bzw. Abrufphase auftretenden kortikalen Aktivationen zu prüfen. Insgesamt hat sich in dieser Arbeit gezeigt, daß die Inbeziehungsetzung psychologischer Gedächtnismodelle und psychophysiologischer Untersuchungsergebnisse einerseits sinnvoll, andererseits nicht unproblematisch ist und stets mit großer Vorsicht vorgenommen werden sollte.

Was die Psychophysiologie betrifft, so konnte mit dieser Arbeit der Wert des von Schack (1997) entwickelten, hochauflösenden kontinuierlichen Schätzverfahrens der Kohärenzfunktion für die Untersuchung schnell ablaufender kognitiver Prozesse aufgezeigt werden. Durch die Analyse des Einflusses der Gedächtnislast auf die verschiedenen kohärenzanalytischen Kennwerte (Methode der parametrischen Variation) wurde die mit dem Verfahren von Schack berechnete "maximale Kohärenz" als der für die Aufdekkung topographischer Aktivationsunterschiede sensitivste Kennwert ausgewiesen. Bezüglich des Zeitverlaufes der während des Abrufs verbaler bzw. nonverbaler Information stattfindenden Prozesse haben sich die Verläufe der "mittleren Bandkohärenzen" und die "Zeitpunkte maximaler Kohärenz" als aussagekräftig herausgestellt. Das neue Schätzverfahren von Schack ermöglicht somit eine angemessene Untersuchung der funktionellen Kopplung verschiedener Kortexregionen im Verlauf von Arbeitsgedächtnisprozessen. Mit Mapping-Verfahren lassen sich die kohärenzanalytischen Ergebnisse zudem sehr gut veranschaulichen. Bei der Interpretation der topographisch dargestellten Ergebnisse rückt jedoch die begrenzte räumliche Aussagefähigkeit des EEGs ins Bewußtsein: Der Versuch, von Synchronisationsprozessen unter einzelnen Elektrodenpaaren ausgehend Schlüsse bezüglich der Aktivation bestimmter neokortikaler Areale zu ziehen, ist mit Unsicherheiten behaftet. Ein nicht unwesentliches Problem ergibt sich zum Beispiel aus den in der Literatur besonders im präfrontalen Bereich auftretenden 
Unterschieden bei der Aufteilung bzw. Bezeichnung der zytoarchitektonischen Felder (vgl. Birbaumer \& Schmidt, 1996, mit Rosenzweig, Leiman \& Breedlove, 1996). Um vergleichsweise genauere Aussagen bezüglich der am Abruf verbaler bzw. nonverbaler Information beteiligten kortikalen Areale mit dem EEG zu erhalten, müßte ein räumlich hochauflösendes bildgebendes Verfahren (fMRT oder PET) für die Elektrodenpositionierung genutzt werden. Dies würde auch die Vergleichbarkeit der Befunde mit den Ergebnissen anderer neuropsychologischer Untersuchungen verbessern. Das EEG durch eines dieser Verfahren vollständig zu ersetzen, erscheint vor dem Hintergrund ihres schon physiologisch begründeten niedrigen zeitlichen Auflösungsvermögens nach wie vor unrealistisch. Es ist aber angesichts der Geschwindigkeit, mit der viele noch nicht oder nicht vollständig untersuchte kognitive Prozesse ablaufen, auch wenig wünschenswert. In dieser Arbeit dürfte deutlich geworden sein, daß das EEG heute mehr denn je ein unverzichtbares Verfahren für die Erforschung der Zusammenhänge zwischen "Hirn" und "Verhalten" darstellt. 


\section{$8 \quad$ Zusammenfassung}

Die vorliegende neuropsychologische Arbeit befaßt sich mit schnell ablaufenden kognitiven Prozessen im Arbeitsgedächtnis. Im Gegensatz zu der Mehrzahl bisheriger Untersuchungen steht jedoch nicht die Enkodierungs- und Memorierphase, sondern die hformationsabrufphase im Mittelpunkt des Interesses. Dabei geht es insbesondere um die Frage, ob die vorliegenden Erkenntnisse zu topographischen und zeitdynamischen Charakteristika verschiedener Arbeitsgedächtniskomponenten auch für diese Phase Gültigkeit besitzen. Im ersten Teil der Arbeit wird der theoretische und empirische Hintergrund zu dieser Fragestellung erläutert. Im zweiten Teil wird die eigene experimentelle Untersuchung referiert und die erhaltenen Ergebnisse werden diskutiert.

Den Ausgangspunkt der theoretischen Überlegungen bildet das psychologische Konzept des Kurzzeitgedächtnisses. In einem historischen Abriß werden zunächst die wichtigsten psychologischen Gedächtnismodelle erläutert. Dabei wird die Bedeutung der Konzipierung des Kurzzeitgedächtnisses als ein aus mehreren Komponenten bestehendes Arbeitsgedächtnis herausgestellt, wie sie im Arbeitsgedächtnismodell von Baddeley (1998) vorgenommen wird. Anschließend wird auf die Problematik des Abrufs von Informationen aus dem Kurzzeit- bzw. Arbeitsgedächtnis eingegangen. Im Mittelpunkt steht hierbei das sogenannte Sternberg-Paradigma, das ein klassisches psychologisches Paradigma zur Untersuchung von Itemwiedererkennungsprozessen darstellt.

Als Pendant zum psychologischen Konzept des Kurzzeitgedächtnisses wird eine Betrachtung des Kurzzeitgedächtnisses als Gegenstand neurowissenschaftlicher Forschung vorgenommen. Sie umfaßt die Darstellung neurophysiologischer und anatomischer Grundlagen und die Beschreibung und Bewertung der wichtigsten Verfahren zur Untersuchung des Gedächtnisses. Als geeignetes Instrument für die Untersuchung der sehr schnell ablaufenden Arbeitsgedächtnisprozesse erweist sich das EEG aufgrund seines vergleichsweise hohen zeitlichen Auflösungsvermögens.

Den empirischen Hintergrund dieser Arbeit bilden neuropsychologische Untersuchungen zu den verschiedenen Arbeitsgedächtniskomponenten, wobei sowohl repräsentative Befunde aus klinisch-anatomischen Studien als auch Ergebnisse aus Untersuchungen an gesunden Probanden einbezogen werden. Insgesamt liefert die Mehrzahl der neuropsychologischen Untersuchungen übereinstimmende Befunde bezüglich der Existenz unterschiedlicher Arbeitsgedächtniskomponenten. Diese zeigt sich unter anderem darin, daß je nach Art der zu verarbeitenden Information unterschiedliche kortikale Areale aktiviert sind. Darüber hinaus treten bei der Verarbeitung von Reizen verschiedener Art und verschiedenen Umfangs auch Unterschiede im Zeitverlauf der Verarbeitungsprozesse auf. 
Auf der Grundlage der dargestellten Forschungsergebnisse werden die eigenen Fragestellungen abgeleitet und konkretisiert. Im Vordergrund steht die Frage nach den topographischen und zeitdynamischen Charakteristika verschiedener Arbeitsgedächtniskomponenten beim Abruf kurzzeitig memorierter verbaler bzw. nonverbaler Information. Um diese Frage angemessen zu untersuchen, wird als Arbeitsgedächtnisaufgabe die klassische Itemwiedererkennungsaufgabe von Sternberg (1966) und als Verfahren zur Untersuchung des Gedächtnisses das EEG ausgewählt, das kohärenzanalytisch ausgewertet werden soll. Die Kohärenzanalyse erlaubt Aussagen über den Grad der Synchronisierung der von verschiedenen kortikalen Regionen abgeleiteten elektrophysiologischen Signale. Eine hohe Kohärenz kann als Zeichen verstärkter kortikaler Aktivation interpretiert werden.

Aus der Kombination von Sternberg-Aufgabe und kohärenzanalytisch auszuwertendem EEG ergeben sich die weiteren Zielsetzungen der eigenen Arbeit: Dabei handelt es sich zum einen um die Replikation der von Sternberg (1966) gefundenen Inearen Beziehung zwischen Antwortlatenz und Anzahl zu memorierender Items für das gewählte verbale und nonverbale Reizmaterial. Zum anderen soll die Tauglichkeit eines neuen, von Schack (1997) entwickelten kontinuierlichen Schätzverfahrens der Kohärenzfunktion zur Untersuchung schnell ablaufender kognitiver Prozesse geprüft werden.

An der eigenen experimentellen Untersuchung nahmen acht Studentinnen und zwei wissenschaftliche Mitarbeiterinnen im Alter zwischen 18 und 31 Jahren teil, die im Institut für Psychologie der Friedrich-Schiller-Universität in Jena untersucht wurden. Sie absolvierten unter EEG-Ableitung eine verlängerte Sternberg-Aufgabe mit visuell dargebotenen Zahlwörtern und nicht verbalisierbaren Zufallsfiguren. Als Leistungsmaß wurde die Art der Antwort (Ja/Nein) und die Antwortlatenz erhoben. Die Registrierung des EEG erfolgte nach dem 10-20-System über verbundene Ohren als Referenzelektrode (Abtastfrequenz 250 bzw. $256 \mathrm{~Hz}$, Empfindlichkeit 119 bzw. $303 \mu \mathrm{V} / \mathrm{cm}$, Filter $70 \mathrm{~Hz}$, Zeitkonstante $1.0 \mathrm{bzw}$. $0.3 \mathrm{sec}$ ). Bei der EEG-Auswertung wurde der Analyse topographischer und zeitdynamischer Aspekte eine Sensitivitätsprüfung möglicher kohärenzanalytischer Kennwerte vorangestellt.

Die Analyse der Antwortlatenzen hat erwartungsgemäß deutlich gemacht, daß nicht nur der Umfang der zu behaltenden Information (Setgröße) und die Art der geforderten Antwort (Testitem), sondern auch die Art des Lernmaterials einen Einfluß auf die Zeit hatte, die im Mittel zur Beantwortung des kritischen Reizes benötigt wurde. In diesem Ergebnis spiegelt sich die unterschiedliche Qualität der Zufallsfiguren und Zahlwörter wider, die sich im wesentlichen hinsichtlich ihres Bekanntheitsgrades und ihrer Verbalisierbarkeit unterscheiden. 
Die Beziehung zwischen der Anzahl memorierter Items und der erhobenen Antwortlatenz erwies sich in allen Versuchsbedingungen als linear. Damit konnte das entsprechende Ergebnis von Sternberg (1966) für das gewählte Reizmaterial repliziert werden. Bei der Analyse der Regressionskoeffizienten ("Scanning-Zeiten") traten jedoch Abweichungen auf: Anders als bei Sternberg hatte nicht nur die Art des Reizmaterials, sondern auch die Art des Testitems einen Einfluß auf die Scanning-Zeit.

Bei der Sensitivitätsprüfung der kohärenzanalytischen Kennwerte zeigte sich, daß die "maximale Kohärenz" die vergleichsweise engsten Beziehungen zum Umfang der Gedächtnislast aufwies und den größten Beitrag zur Aufdeckung topographischer Aktivationsunterschiede zwischen den untersuchten Reizarten leistete. Die übrigen kohärenzanalytischen Kennwerte erwiesen sich als weniger trennscharf bzw. waren angesichts ihrer Abhängigkeit von der Antwortlatenz topographisch nicht eindeutig interpretierbar.

Die Analyse der maximalen Kohärenz offenbarte deutliche topographische Aktivationsunterschiede beim Abruf verbaler bzw. nonverbaler Information aus dem Arbeitsgedächtnis. Dabei stehen jedoch nur die für das positive Testitem erhaltenen Ergebnisse im Einklang mit Befunden anderer neuropsychologischer Unterschungen: Bei dieser Testitemart traten temporo-parietale Aktivationen während des Abrufs verbaler Information linkshemisphärisch, während des Abrufs nonverbaler Information hingegen rechtshemisphärisch auf. Frontale Aktivationen konnten unabhängig von der Art des Testitems bei beiden Reizarten beobachtet werden, wobei sich eine Aktivationslateralisierung jedoch nur für die Zahlwörter aufzeigen ließ.

Die Untersuchung des Zeitablaufes der elektrophysiologischen Synchronisationsprozesse ergab, daß eine Zunahme der Gedächtnislast nicht nur zu einem Anstieg der maximalen Kohärenz in temporo-parietalen Arealen führt, sondern sich auch in einer zeitlichen Verschiebung dieses Kennwertes niederschlägt. Darüber hinaus konnte gezeigt werden, daß der Abruf nonverbaler Information beim negativen Testitem links und rechts frontal zu stärkeren Verschiebungen der Aktivitätsmaxima führt als der Abruf verbaler Information.

Insgesamt sprechen die Ergebnisse der vorliegenden Untersuchung für die Existenz unterschiedlicher Arbeitsgedächtniskomponenten, denen verschiedene neuronale Strukturen zugrundeliegen. Sie belegen außerdem den hohen Nutzen eines neu entwickelten kontinuierlichen Schätzverfahrens der Kohärenzfunktion für die Analyse schnell ablaufender kognitiver Prozesse und zeigen deutlich, wie fruchtbar eine Kombination psychologischer und physiologischer Methoden für die Untersuchung aktueller Fragestellungen zum Thema "Arbeitsgedächtnis" sein kann. 


\section{Anmerkungen}

1 Der Temporallappen enthält zum einen das Projektionsgebiet der auditorischen Afferenz und die auditorischen Felder (Area 41 und 42), zum anderen steht Area 21 mit visuellen Funktionen in Verbindung und Area 37 kann als tertiäres visuelles Feld angesehen werden. Ventral finden sich die primären afferenten Endigungen des olfaktorischen Systems, wobei das entsprechende Gebiet eigentlich nicht mehr zum Neokortex, sondern zum phylogenetisch älteren Paläokortex gehört (ebd.). Enge Verbindungen zum Hippocampus, zu den Mandelkernen und damit zum Hypothalamus stehen für motivationale Funktionen und affektive Färbung (Birbaumer \& Schmidt, 1990).

2 Der Parietallappen erhält über Afferenzen aus den drei primären und sekundären Projektionsarealen visuelle, auditorische und somatosensorische Informationen, die er an frontale und temporale Assoziationsareale, an Thalamus, Striatum, Mittelhirn und Rückenmark weitergibt (Birbaumer \& Schmidt, 1996).

3 Der Frontallappen umfaßt die Areale 4, 6, 8 bis 12 und 44 bis 47 nach Brodmann, die in folgende Subsysteme unterteilt werden:

a) motorische Aufgaben: motorische und prämotorische Regionen, frontale Augenfelder und BrocaAreal (Areale 4, 6, 8, 44)

b) Aufmerksamkeitsfunktionen: dorsolaterale Regionen (Areale 8 -10, $44-46$ )

c) motivationale Funktionen: orbitale Regionen (Areale 11, 12 und 47)

Durch die Zusammenfassung der dorsolateralen und orbitalen Regionen zum sogenannten "granulären" oder präfrontalen Kortex (PFC) wird das entsprechende Gebiet aufgrund seiner besonders gut entwickelten Schicht aus Sternzellen von den agranulären motorischen und prämotorischen Regionen abgegrenzt (Creutzfeldt, 1983). Es bestehen reziproke Verbindungen des PFC zum Thalamus, Hypothalamus, limbischen System (Amygdalae, Gyrus cinguli) und mesencephalen Retikulärsystem sowie olfaktorische Verbindungen (orbitaler Frontalkortex). Efferenzen gehen insbesonders zu den Arealen 7 (somatisch), 22 (auditorisch) und 21 (visuell) sowie in die Basalganglien zur Steuerung des Aufmerksamkeitsverhaltens (Birbaumer \& Schmidt, 1996).

4 Limbische und subkortikale Strukturen liefern hierfür die nötige Energie, deren Ausfall oder falsche (zeitlich von gelernten Hinweisreizen unabhängige) Verteilung zu einer irregulären zeitlichen Abfolge von Gedanken und Verhaltensabläufen führt.

5 Das eindeutigste anatomische Korrelat der Lateralisierung einer Funktion besteht dabei für sprachliche Leistungen: Durch einen weniger steilen und ausgedehnten Verlauf der linken Sylvischen Furche (Sulcus lateralis), die den Temporallappen vom Frontal- und Parietallappen trennt, ergibt sich bereits bei Neugeborenen ein größeres Planum temporale in der linken verglichen mit der rechten Hemisphäre (Birbaumer \& Schmidt, 1996; s. Abb. in Tab. 3, Kap. 2.2.1). Die Bedeutung der rechten Hemisphäre für die räumliche Informationsverarbeitung und die Steuerung von Zielbewegungen spiegelt sich in einem beim Menschen überproportional vergrößerten posterioren Parietallappen (G. angularis, G. supramarginalis und Lobus parietalis superior) wider. Neben solchen makroskopischen existieren auch mikroskopische Unterschiede in der Anatomie einzelner Neuronen (z. B. verschiedene Somagröße von Pyramidenzellen, unterschiedliche Verzweigungsstruktur ihrer Dendritenbäume), die die Hemisphärenasymmetrie zu bedingen scheinen (Birbaumer \& Schmidt, 1996).

6 Die Definition der Leitungsaphasie geht auf Geschwinds (1972) Weiterentwicklung des von Wernicke und Lichtheim Ende des 19. Jahrhunderts postulierten konnektionistischen Modells der an Sprache beteiligten Hirnregionen zurück (vgl. Abb. 5). Danach lassen sich die verschiedenen Aphasien auf verschiedene Diskonnektionen zwischen Komponenten eines aus mehreren Hirnregionen bestehenden neuronalen Netzwerks zurückführen, das für Sprachverständnis und -produktion zuständig ist (Rosenzweig, Leiman \& Breedlove, 1996). Das Hauptsymptom der Leitungsaphasie ist die beeinträchtigte Wiederholung sprachlichen Materials; weitere diagnostische Kriterien sind das flüssige, aber paraphrasierende spontane Sprechen (Verwendung inkorrekter Phoneme) sowie Benennungs-, Lese- und Schreibstörungen bei meist gut erhaltenem Sprachverständnis. Als Ursache der Leitungsaphasie wird in der Regel die Läsion des Fasciculus arcuatus angenommen, der Broca- und Wernikke-Region verbindet, plus einer Läsion des oberen Temporallappens (primärer auditiver Kortex), des Gyrus supramarginalis und/oder der Insula (vgl. Abb. 5). Die genaue Beschreibung des dieser Aphasie zugrundeliegenden Hirnschadens ist jedoch immer noch Gegenstand kontroverser Diskussionen (ebd.). 
7 Gedächtnisleistungen werden von zahlreichen Faktoren beeinflußt, von denen das Alter und insbesondere die Intelligenz der untersuchten Personen die offensichtlichsten sind (Mayes, 1995). Bei Patienten mit Hirnläsionen werden Beeinträchtigungen des Kurzzeitgedächtnisses (KZG) daher oft schon im Rahmen von Intelligenztest-Untersuchungen sichtbar (z. B. bei den Untertests "Zahlennachsprechen" und "Mosaiktest" aus dem revidierten Wechsler Intelligenztest; Wechsler, 1987).

8 Die gezielte Untersuchung des phonologischen und visuell-räumlichen KZG erfolgt in klinisch-anatomischen Studien in der Regel über die Erfassung der KZG-Kapazität bzw. der sogenannten "Gedächtnisspanne" für verschiedene verbale und nonverbale Stimuli sowie für deren räumliche Anordnung (Vallar \& Papagno, 1995). Dabei werden Patienten z. B. aufgefordert, akustisch und/oder visuell präsentierte Zahlen-, Buchstaben- oder Wörter-Listen unterschiedlichen Umfangs unverzüglich wiederzugeben bzw. verschiedene Anzahlen von Gegenständen in derselben Reihenfolge anzutippen oder hinzulegen wie es zuvor der Versuchsleiter getan hat (für einen Überblick über entsprechende Tests siehe Lezak, 1995). Das Erreichen eines bestimmten Kriteriums wie z. B. der größten Itemanzahl, die noch in $50 \%$ der Fälle korrekt wiedergegeben werden konnte, gilt als Maß für die Gedächtnisspanne. Dieses Maß kann für verschiedene Stimuli- und Darbietungsarten (visuell vs. akustisch) unterschiedlich ausfallen (Cavanagh, 1972; Vallar \& Papagno, 1995).

9 In diesem Sinne belegen auch neuere Studien an anarthrischen Patienten, daß der artikulatorische Kontrollprozeß, der für die "Auffrischung" der Informationen im phonologischen Speicher genutzt wird, nicht an die Fähigkeit zur ungestörten Lautbildung gebunden ist. Er scheint vielmehr auf einer zentraleren Ebene prämotorischer Sprachplanung stattzufinden (Nebes, 1975; Baddeley \& Wilson, 1985; Vallar, \& Cappa, 1987; Bishop \& Robson, 1989; Cubelli \& Nichelli, 1992).

10 Im "Token Test" werden Patienten gebeten, unterschiedlich komplexe Anweisungen hinsichtlich éner Reihe verschiedener geometrischer Formen zu befolgen, z. B. "Berühren Sie das schwarze Quadrat" oder "Berühren sie den großen weißen Kreis und das kleine grüne Quadrat". Bei der visuellen Präsentation werden auf einzelne Karten gedruckte Anweisungen zum Lesen gegeben.

11 In diesem Test wird die visuell-räumliche Gedächtnisspanne erfaßt: Patienten sollen zunehmende Anzahlen von Quadern in derselben Reihenfolge antippen wie zuvor der Versuchsleiter (nach Corsi, zit. in Milner, 1971).

12 Die Patienten lernten wiederholt eine Liste von neun Wörtern, bis diese korrekt wiedergegeben werden konnte. Dabei wurden in jedem Durchgang nur die nicht erinnerten Items vom Versuchsleiter nochmal wiederholt (Methode des selektiven Erinnerns).

13 Aufgrund der Sprachstörungen erfolgte die Wiedergabe durch Deuten: Die Patienten erhielten eine Karte mit den Ziffern 1 bis 9 und sollten unmittelbar nach Darbietung der Lernliste auf die entsprechenden Ziffern in der korrekten Reihenfolge zeigen.

14 Fehlende Reaktion auf visuelle, taktile und akustische Reize kontralateral zur Läsion

15 Es handelte sich dabei um fünf schwarze Quadrate, die in einer Matrix von $4 \times 4$ weißen Quadraten unterschiedlich positioniert waren.

16 Nur die Patienten mit Gesichtsfeldstörungen wiesen (zusätzlich) posteriore Hirnschäden auf.

17 Sie entsprach der Anzahl der Lerndurchgänge, die ein Patient brauchte, bis eine bestimmte, über der individuellen visuell-räumlichen Gedächtnisspanne liegende Itemfolge gelernt worden war.

18 Die Probanden werden gebeten, acht Sätze in der Reihenfolge ihrer Darbietung wiederzugeben. In der einen Bedingung beschreiben die Sätze die Positionen, die verschiedene Zahlen in einer 4x 4Matrix relativ zueinander einnehmen, und die Probanden werden aufgefordert, sich die Sätze über ein mentales Vorstellungsbild der Zahlen zu merken; in der anderen Bedinigung werden die Zahlen durch irrelevante Wörter ersetzt, und die Probanden sollen die Sätze verbal statt räumlich kodieren (vgl. Brooks, 1968).

19 Es handelte sich dabei u. a. um dreidimensionale Formrotationsaufgaben, bei denen die Patientin jedesmal eine Form mit vier weiteren, um verschiedene Grade und in verschiedenen Ebenen rotierten Formen vergleichen mußte, um zwei übereinstimmende herauszufinden (vgl. Shepard \& Metzler, 1971). 
20 Die Patienten sollten sich in jedem Durchgang ein Muster von gleich vielen geschwärzten und ungeschwärzten Kästchen merken und nach Darbietung eines Testreizes auf dasjenige schwarze Kästchen zeigen, das zusätzlich hinzugekommen war. Die Komplexität der Muster wurde über die Anzahl der Kästchen manipuliert.

21 Charakteristisch sind neben Auffälligkeiten im sozialen Bereich (vgl. Birbaumer \& Schmidt, 1996) die hohe Ablenkbarkeit und die gleichzeitige Unfähigkeit, einmal eingeschlagene Reaktionsstrategien aufzugeben. Hinzu kommen Gedächtnisstörungen, die allerdings weniger ausgeprägt sind als bei amnestischen Patienten und wahrscheinlich als eine Folge der Unfähigkeit, Abrufprozesse zu planen und auszuführen, betrachtet werden können (Parkin, 1996). Detailliertere Einzelfallbeschreibungen finden sich z. B. bei Baddeley und Wilson (1988) sowie bei Parkin, Pitchford und Binschaedler (zit. nach Parkin, 1996).

22 Den Patienten werden jeweils vier Reizkarten vorgelegt, die sich bezüglich ihrer Farb-, Form- und numerischen Information unterscheiden. Im Anschluß sollen 128 Antwortkarten, die entlang derselben Dimensionen variieren, den Reizkarten zugeordnet werden (10 Karten nach Farbe, 10 Karten nach Form usw.). Die jeweils relevante Dimension hat der Patient aus der Rückmeldung des Versuchsleiters ("richtig" oder "falsch") zu erschließen.

23 Die Alzheimer-Patienten hatten mit einem Leuchtstift einen Lichtpunkt auf dem Bildschirm zu verfolgen und dabei entweder ein bestimmtes Wort zu wiederholen (artikulatorische Suppression), bei einem akustischen Ton auf ein Pedal zu treten (Reaktionszeitaufgabe) oder sich Zahlenfolgen zu merken (Zifferngedächtnisspanne).

24 Den Patienten wurden auf Papier gedruckt verschiedene Anzahlen $(6,8,10,12)$ der pro Versuchsdurchgang nach Kategorien sortierten Stimuli dargeboten; auf jedem Blatt sollten sie einen einzelnen Reiz berühren, Wiederholungen dabei aber unbedingt vermeiden.

25 Bei den verbalen Reizen handelte es sich um Karten, auf denen jeweils zwei hochbildhafte Wörter gedruckt waren. Als nonverbale Reize wurden entsprechend konkrete und abstrakte Zeichnungen verwendet.

26 In dieser Aufgabe bewegte sich mit dem Cursor ein kleines Fenster über die Maske, das einen Teil des Labyrinths sichtbar machte. Die Patienten hatten in mehreren Durchgängen den Labyrinthverlauf zu lernen, bis sie inm mit dem Cursor fehlerfrei folgen konnten.

27 Bei dieser räumlichen Gedächtnisaufgabe ging es darum, drei auf einem Bildschirm an unterschiedlichen Positionen erscheinende Kästen nach versteckten "blauen Zeichen" zu durchsuchen. Die Zeichen erschienen in den verschiedenen Durchgängen nur einmal in jedem Kasten, so daß die Patienten nur dann fehlerfreie Leistungen erbringen konnten, wenn sie sich den Erscheinungsort der Zeichen gemerkt hatten (für eine detailliertere Beschreibung siehe Owen et al., 1990).

28 Dieses Verfahren entspricht im wesentlichen der Methode des additiven Faktors von Sternberg (1969).

29 Den Probanden werden bei "n-back"-Aufgaben fortlaufend einzelne Reize (z. B. Buchstaben) dargeboten; je nach Bedingung haben sie entweder die Reize still zu wiederholen, mit einem bestimmten, zu Beginn des Versuches präsentierten Reiz zu vergleichen oder aber fortlaufend zu memorieren und bei jedem neuen Reiz die Übereinstimmung mit dem drittletzten, vorletzten oder letzten Reiz zu prüfen.

30 In der Objektgedächtnisaufgabe wurden den Probanden simultan zwei schwer verbalisierbare Reize präsentiert, die gemerkt und mit einem Testreiz verglichen werden sollten.

31 In einer zweiten experimentellen Aufgabe boten Salmon et al. (ebd.) ihren Probanden Listen von 8, 9 oder 10 phonologisch unähnlichen Konsonanten dar, von denen lediglich die letzten sechs seriell memoriert werden sollten. In einer früheren Studie war gezeigt worden, daß diese Aufgabe sowohl die phonologische Schleife als auch die zentrale Exekutive beansprucht (Morris \& Jones, 1990).

32 Dieses Verfahren ermöglicht es, die neuronale Aktivität einzelner kognitiver Prozesse zu erfassen und damit die mit der Subtraktionslogik verknüpften Probleme zu umgehen (vgl. Rösler, 1997; Zarahn et al., 1997). 
33 Umweltereignisse rufen charakteristische transiente EEG-Veränderungen hervor, sogenannte evozierte oder ereignisbezogene Potentiale, die aus zahlreichen Komponenten bestehen. Die Amplituden und Latenzen dieser Komponenten sind von den Bedingungen, unter denen sie ausgelöst wurden, abhängig. Die bekanntesten Komponenten sind die N100, P300 und die CNV (Contingent Negative Variation). N (negativ) und $\mathrm{P}$ (positiv) stehen dabei für die Richtung des Potentialausschlags; die Zahl steht für die Latenz, mit der die jeweilige Komponente in Standardsituationen auftritt: So ist die N100 eine nichtspezifische Antwort auf einen akustischen, visuellen oder taktilen Reiz, die etwa 100 ms nach Stimulusdarbietung auftritt. Die P300 tritt bei einer Reihe von Entscheidungsprozessen (z. B. Auffinden von Zielreizen) mit einer Latenz von etwa $300 \mathrm{~ms}$ auf. CNV steht für das charakteristische leichte Ansteigen der Negativität im Interstimulus-Intervall, wenn Probanden nach Darbietung eines ersten Reizes auf einen Testreiz reagieren müssen.

34 Die Kovarianz ist ein Maß für die Ähnlichkeit zweier Signale. Man erhält sie durch die Multiplikation der betreffenden Signale und durch die Berechnung des Mittelwertes über die Signaldauer (Cooper, Osselton \& Shaw, 1984). Unnormiert ist die Kovarianz von den Signalamplituden abhängig. Gevins und Cutillo (1993) rechtfertigten ihren Einsatz mit dem Hinweis auf die Bedeutung der Amplitudenund Wellenähnlichkeit für die angemessene spatiotemporale Beschreibung aktivierter kortikaler Areale.

35 Krause, Gibbons und Schack (1998) definieren die Kohärenzdauer als kumulierte Zeitdauer, in der die Bandkohärenz eine bestimmte Schwelle im Intervall zwischen Testreiz und Antwort überschreitet. Die bei 0,8 liegende Schwelle hat sich in Voruntersuchungen als besonders trennscharf für die Unterscheidung der begrifflichen von der bildhaft-anschaulichen Modalität erwiesen (Krause et al., 1997).

36 Die Probanden hatten auf visuell oder akustisch dargebotene Substantive mit einem jeweils passenden Verb zu antworten. 
Adam, N. \& Collins, G. I. (1978). Late components of the visual evoked potential to search in short-term memory. Electroencephalography and Clinical Neurophysiology, 44, $147-156$.

Alivisatos, B. \& Milner, B. (1989). Effects of frontal or temporal lobectomy on the use of advance information in a choice reaction time task. Neuropsychologia, 27 (4), 495 -503 .

Allport, D. (1980). Patterns and actions: Cognitive mechanisms are context specific. In G. Claxton (Ed.), Cognitive Psychology: New Directions (pp. 26 - 64). London: Rutledge and Kegan Paul.

Anderson, J. A. (1973). A theory for the recognition of items from short-term memorised lists. Psychological Review, 80, $417-438$.

Anderson, J. R. (1988). Kognitive Psychologie. Eine Einführung. Heidelberg: Spektrum der Wissenschaft.

Arnold, M., Miltner, W., Witte, H., Bauer, R., \& Braun, Ch. (1998). Adaptive AR modeling of non-stationary time series by means of Kalman filtering. IEEE Trans. Biomed. Eng., 45, 553-562.

Ashby, F. G., Jein, I.-Y., \& Balakrishnan, J.D. (1993). Response time distributions in memory scanning. Journal of Mathematical Psychology, 37, 526 - 555.

Atkinson, R. C., Herrmann, D. J., \& Wescourt, K. T. (1974). Search processes in recognition memory. In R. L. Solso (Ed.), Theories in cognitive psychology: The Loyola Symposium. Hillsdale, NJ: Erlbaum.

Atkinson, R. C., Holmgren, J. E., \& Juola, J. F. (1969). Processing time as influenced by the number of elements in a visual display. Perception and Psychophysics, 6, 321 $-327$.

Atkinson, R. C. \& Juola, J. F. (1974). Search and decision processes in recognition memory. In D. H. Krantz, R. C. Atkinson, R. D. Luce, \& P. Suppes (Eds.), Contemporary developments in mathematical psychology (Vol. 1, pp. $242-293$ ). San Francisco: Freeman.

Atkinson, R. C. \& Shiffrin, R. M. (1968). Human memory: A proposed system and its control processes. In K. W. Spence (Ed.), The psychology of learning and motivation: Advances in research and theory (Vol. 2, pp. 89 - 195). New York: Academic Press.

Awh, E., Jonides, J., Smith, E. E., Schumacher, E. H., Koeppe, R. A., \& Katz, S. (1996). Dissociation of storage and rehearsal in verbal working memory: Evidence from positron emission tomography. Psychological Science, 7 (1), 25 - 31.

Baddeley, A.D. (1966). The capacity for generating information by randomization. Quarterly Journal of Experimental Psychology, 18,119-129. 
Baddeley, A. D. (1976). The psychology of memory. New York: Basic Books.

Baddeley, A. D. (1986). Working memory. Oxford, England: Clarendon Press.

Baddeley, A. D. (1993). Working memory or working attention? In A. D. Baddeley \& L. Weiskrantz (Eds.), Attention: Selection, awareness, and control (pp. $152-170)$. Oxfort: Clarendon Press.

Baddeley, A. D. (1995). The psychology of memory. In A. D. Baddeley, B. A. Wilson, \& F. N. Watts (Eds.), Handbook of memory disorders (pp. 3 - 25). Chichester: John Wiley \& Sons.

Baddeley, A. D. (1998). Human memory: Theory and practice. Boston: Allyn and Bacon. Baddeley, A. D., Bressi, S., Della Sala, S., Logie, R. H., \& Spinnler, H. (1991). The decline of working memory in Alzheimer's disease: A longitudinal study. Brain, 114, $2521-2542$.

Baddeley, A. D. \& Ecob, J. R. (1973). Reaction time and short-term memory: A trace strength alternative to the high-speed exhaustive scanning hypothesis. Quarterly Journal of Experimental Psychology, 25, 229 - 240.

Baddeley, A. D., Grand, S., Wight, E., \& Thomson, N. (1975). Imagery and visual working memory. In P. M. A. Rabbitt \& S. Dornic (Eds.), Attention and performance $V$ (pp. 205 - 217). London: Academic Press.

Baddeley, A. D. \& Hitch, G. J. (1974). Working memory. In S. Dornic (Ed.), Attention and performance VI (pp. 647 - 667). Hillsdale, N. J.: Erlbaum.

Baddeley, A. D. \& Hitch, G. J. (1977). Recency re-examined. In S. Dornic (Ed.), Attention and performance VI (pp. 647 - 667). Hillsdale, N. J.: Erlbaum.

Baddeley, A. D. \& Hitch, G. J. (1993). The recency effect: Implicit learning with explicit retrieval? Memory and Cognition, 21, 146 - 155.

Baddeley, A. D. \& Lieberman, K. (1980). Spatial working memory. In R. S. Nickerson (Ed.), Attention and performance, VIII (pp. 521 - 539). Hillsdale, N. J.: Erlbaum.

Baddeley, A.D., Logie, R. H., Bressi, S., Della Sala, S., \& Spinnler, H. Q. (1986). Dementia and working memory. Quarterly Journal of Experimental Psychology, 38 A, $603-618$.

Baddeley, A. D., Papagno, C., \& Vallar, G. (1988). When long-term learning depends on short-term storage. Journal of Memory and Language, 27, 586 - 595.

Baddeley, A. D., Thomson, N., \& Buchanan, M. (1975). Word length and the structure of short-term memory. Journal of Verbal Learning and Verbal Behavior, 14, 575 589.

Barnard, P. (1985). Interacting cognitive subsystems: A psycholinguistic approach to short-term memory. In A. Ellis (Ed.), Progress in the psychology of language (Vol. 2, pp. 197 -258). London: Lawrence Erlbaum. 
Basso, A., Spinnler, J., Vallar, G., \& Zanobio, E. (1982). Left hemisphere damage and selective impairment of auditory verbal short-term memory: A case study. Neuropsychologia, 20, $263-274$.

Bekerian, D. A. \& Baddeley, A. D. (1980). Saturation advertising in the repetition effect. Journal of Verbal Learning and Verbal Behavior, 19, 17 - 25.

Bennett, E. L., Orne, A. E., \& Hebert, M. (1972). Cerebral protein synthesis inhibition and amnesia produced by scopolamine, cycloheximide, streptovitacin A, anisomycin, and emetine in rat. Federation Proceedings, 31, 838.

Berger, H. (1929 - 1938). Über das Elektroenzephalogramm des Menschen. 1. - 9. Mitteilung. Zusammenfassung: Nova Acta Leopoldina, N. F. (1938), 6, $173-309$.

Berman, K. F., Austin-Lane, J. L., Esposito, G., Van Horn, J. D., \& Weinberger, D. R. (1996). Dissecting the "working" and the "memory" in a PET study of working memory using grades tasks and isomorphic stimuli. Neuroimage, 3, S529.

Besner, D., Davies, J., \& Daniels, S. (1981). Reading for meaning: The effects of concurrent articulation. Quarterly Journal of Experimental Psychology, 33 A, 415 - 437.

Birbaumer, N. \& Schmidt, R. F. (1990). Biologische Psychologie. Berlin: Springer.

Birbaumer, N. \& Schmidt, R. F. (1996). Biologische Psychologie (3. Aufl.). Berlin: Springer.

Bisiacchi, P. S., Cipolotti, L., \& Denes, G. (1989). Impairment in processing meaningless verbal material in several modalities: The relationship between short-term memory and phonological skills. Quarterly Journal of Experimental Psychology, 41 A, $293-319$.

Bjork, R. A. \& Whitten, W. B. (1974). Recency-sensitive retrieval processes. Cognitive Psychology, 6, $173-189$.

Black, I. B., Adler, J. E., Deyfus, C. F., Friedman, W. F., Lagamma, E. F., \& Roach, A. M. (1987). Biochemistry of information storage in the nervous system. Science, 236, $1263-1268$.

Bloom, F. E., Lazerson, A., \& Hofstadter, L. (1985). Brain, mind, and behavior. New York: W. H. Freeman and Company.

Braitenberg, V. \& Schüz, A. (1991). Anatomy of the cortex. Statistics and geometry. Berlin: Springer.

Braver, T. S., Cohen, J. D., Nystrom, L. E., Jonides, J., Smith, E. E., \& Noll, D. C. (1997). A parametric study of prefrontal cortex involvement in human working memory. Neuroimage, 5, $49-62$.

Bredenkamp, J., Klein, K.-M., Hayn, S. v. \& Vaterrodt, B. (1988). Gedächtnispsychologische Untersuchungen eines Rechenkünstlers. Sprache \& Kognition, 7, 69- 83.

Briggs, G. E. (1974). On the predictor variable for choice reaction time. Memory \& Cognition, 2, $575-580$.

Broadbent, D. E. (1958). Perception and communication. London: Pergamon Press. 
Brody, B. A. \& Pribram, K. H. (1978). The role of the frontal and parietal cortex in cognitive processing. Brain, 101, $607-633$.

Brooks, L. R. (1967). The suppression of visualization by reading. Quarterly Journal of Experimental Psychology, 19, 289 - 299.

Brooks, L. R. (1968). Spatial and verbal components in the act of recall. Canadian Journal of Psychology, 22, 349 - 368.

Brown, I. D., Tickner, A. H., \& Simmonds, D. C. V. (1969). Interference between concurrent tasks of driving and telephoning. Journal of Applied Psychology, 53, $419-$ 424.

Buckner, R. L. \& Tulving, E. (1995). Neuroimaging studies of memory: Theory and recent PET results. In F. Boller \& J. Grafman (Eds.), Handbook of neuropsychology (Vol. 10, pp. 439 - 465). Amsterdam: Elsevier Science B. V.

Burrows, D. \& Okada, R. (1975). Memory retrieval from long and short lists. Science, 188, 1031 - 1033.

Busk, J. \& Galbraith, G. C. (1975). EEG correlates of visual motor practice in man. Electroencephalography and Clinical Neurophysiology, 38, 415.

Campbell, R. \& Butterworth, B. (1985). Phonological dyslecia and dysgraphia in a highly literate subject: A developmental case with associated deficits of phonemic processing and awareness. Quarterly Journal of Experimental Psychology, 37 A, $435-475$.

Caramazza, A., Basili, A. G., Koller, J. J., \& Berndt, R. S. (1981). An investigation of repetition and language processing in a case of conduction aphasia. Brain and Language, 14, $235-271$.

Cavanagh, J. P. (1972). Relation between the immediate memory span and the memory search rate. Psychological Review, 79, 525 - 530.

Cave, C. \& Squire, L. R. (1992). Intact verbal and nonverbal short-term memory following damage to the human hippocampus. Hippocampus, 2 (2), 151 - 164.

Chang, F. L. \& Grennough, W. T. (1982). Lateralized effects of monocular training on dendritic branching in adult split-brain rats. Brain Research, 232, $283-292$.

Cohen, J. D., Forman, S. D., Braver, T. S., Casey, B. J., Servan-Schreiber, D., \& Noll, D. C. (1994). Activation of prefrontal cortex in a non-spatial working memory task with functinal MRI. Human Brain Mapping, 1, 293 - 304.

Cohen, J. D., Perlstein, W. M., Braver, T. S., Nystrom, L. E., Noll, D. C., Jonides, J., \& Smith, E. E. (1997). Temporal dynamics of brain activation during a working memory task. Nature, 386, $604-607$.

Cohen, R. M., Semple, W. E., Gross, M., Holcomb, H. H., Dowling, M. S., \& Nordahl, T. E. (1988). Funcitonal localization of sustained attention: Comparison to sensory stimulation in the absence of instruction. Neuropsychiatry, Neuropsychology, and Behavioral Neurology, 1, 3-20. 
Conrad, R. (1964). Acoustic confusion in immediate memory. British Journal of Psychology, 55, $75-84$.

Conrad, R. (1971). The chronology of the development of covert speech in children. Developmental Psychology, 5, 398 - 405.

Cooper, R., Osselton, J. W., \& Shaw, J.C. (1984). Elektroenzephalographie. Technik und Methoden (3. Aufl.). Stuttgart: Gustav Fischer Verlag.

Corballis, M. C., Kirby, J., \& Miller, A. (1972). Access to elements of a memorized list. Journal of Experimental Psychology, 94, 185 - 190.

Corcoran, D. W. J. (1971). Pattern recognition. Harmondsworth: Penguin Books.

Corkin, S., Cohen, N. J., Sullivan, E. V., Clegg, R. A., Rosen, T. J., \& Ackerman, R. H. (1985). Analyses of global memory impairments of different aetiologies. Anals of the New York Academy of Sciences, 444, 10 - 40.

Courtney, S. M., Ungerleider, L. G., Keil, K., \& Haxby, J. V. (1996). Object and spatial visual working memory activate separate neural systems in human cortex. Cerebral Cortex, 6, $39-49$.

Courtney, S. M., Ungerleider, L. G., Keil, K., \& Haxby, J. V. (1997). Transient and sustained activity in a distributed neural system for human working memory. Nature, $386,608-611$.

Cowan, N. (1992). Verbal memory span and the timing of spoken recall. Journal of Memory and Language, 31, $668-684$.

Cowan, N. (1993). Activation, attention, and short-term memory. Memory \& Cognition, 21 (2), $162-167$.

Cowan, N., Wood, N. L., Wood, P. K., Keller, T. A., Nugent, L. D., \& Keller, C. V. (1998). Two separate verbal processing rates contributing to short-term memory span. Journal of Experimental Psychology: General, 127, 141 - 160.

Craik, F. I. M. (1970). Modality effects in short-term storage. Journal of Verbal Learning and Verbal Behavior, 8, 658-664.

Craik, F. I. M. \& Lockhart, R. S. (1972). Levels of processing: A framework for memory research. Journal of Verbal Learning and Verbal Behavior, 11, 671 - 684.

Craik, F. I. M. \& Tulving, E. (1975). Depth of processing and the retention of words in episodic memory. Journal of Experimental Psychology: General, 104, 268 - 294.

Craik, F. I. M. \& Watkins, M. J. (1973). The role of rehearsal in short-term memory. Journal of Verbal Learning and Verbal Behavior, 12, 599 - 607.

Creutzfeldt, O. D. (1983). Cortex Cerebri. Berlin: Springer.

Dalrymple-Alford, J. C., Kalders, A. S., Jones, R. D., \& Watson, R. W. (1994). A central executive deficit in patients with Parkinson's disease. Journal of Neurology, Neurosurgery, and Psychiatry, 57, 360 - 367.

Damasio, A. R. (1990). Synchronous activation in multiple cortical regions: A mechanism for recall. Seminars in the Neurosciences, 2, $287-296$. 
Daneman, M. \& Carpenter, P. A. (1980). Individual differences in working memory and reading. Journal of Verbal Learning and Verbal Behavior, 19, $450-466$.

Davis, H. P. \& Roitblat, H. L. (1984). Neuropsychology of memory. New York: Guildford Press.

Davis, H. P. \& Squire, L. R. (1982). Protein synthesis and memory: A review. Psychological Bulletin, 96, $518-559$.

Della Sala, S. \& Logie, R. H. (1993). When working memory does not work: The role of working memory in neuropsychology. In F. Boller \& J. Grafman (Eds.), Handbook of Neuropsychology (Vol. 8, pp. 1-62). Amsterdam: Elsevier.

De Renzi, E., Faglioni, P., \& Previdi, P. (1977). Spatial memory and hemispheric locus of lesion. Cortex, 13, $424-433$.

De Renzi, E. \& Faglioni, P. (1978). Normative data and screening power of a shortened version of the Token Test. Cortex, 14, $41-49$.

De Renzi, E. \& Nichelli, P. (1975). Verbal and non-verbal short-term memory impairment following hemispheric damage. Cortex, 11, 341- 354.

D'Esposito, M., Detre, J.A., Alsop, D.C., Shin, R. K., Atlas, S., \& Grossman, M. (1995a). The neural basis of the central executive system of working memory. Nature, 378, $279-281$.

D'Esposito, M., Detre, J. A., Incledon, S., Annis, D., Aguirre, G. K., Grossman, M., \& Alsop, D. C. (1995b). Object and spatial working memory activated dorsolateral prefrontal cortex: A functional MRI study. Society for Neuroscience Abstracts, 21, 1498.

D'Esposito, M., Aguirre, G. K., Zarahn, E.K., Ballard, D., Shin, R. K., \& Lease, J. (1998a). Functional MRI studies of spatial and non-spatial working memory. Cognitive Brain Research, 7 (1), $1-13$.

D'Esposito, M., Balard, D., Aguirre, G. K., \& Zarahn, E. (1998b). Human prefrontal cortex is not specific for working memory: A functional MRI study. Neuroimage, 8, 274 -282 .

D'Esposito, M., Postle, B. R., Ballard, D., \& Lease, J. (1999). Maintenance versus manipulation of information held in working memory: An event-related fMRI study. Brain \& Cognition, 41 (1), $66-86$.

D'Esposito, M. \& Postle, B. R. (1998). Response of prefrontal cortex to varying load and processing demands during a working memory task: An event-related fMRI study. Society for Neuroscience Abstracts, 24, 1251.

Donders, F. E. (1868). On the speed of mental processes. Reprinted in Acta Psychologica (1969), 30, $412-431$.

Dubois, J., Hécaen, H., Angelergues, A., Manfras du Chatelier, A., \& Marcie, P. (1964). Etude neurolinguistique de l'aphasie de conduction. Neuropsychologia, 2, 9 - 44. 
Edelman, G. M. (1989). The remembered present. A biological theory of consciousness. New York: Basic Books.

Ellis, J. A. (1988). Memory for future intention: Investigating pulses and steps. In M. M. Gruneberg, P. E. Morris, \& R. N. Sykes (Eds.), Practical aspects of memory. Current research and issues, Vol. I: Memory in everyday life (pp. $371-376)$. Chichester: Wiley.

Ericsson, K. A. \& Kintsch, W. (1995). Long-term working memory. Psychological Review, $102(2), 211-245$.

Espinosa, I. E. \& Gerstein, G. L. (1988). Cortical auditory neuron interactions during presentation of 3-tone sequences: Effective connectivity. Brain Research, 450, 39 -50 .

Farah, M. J. (1988). Is visual imagery really visual? Overlooked evidence from neuropsychology. Psychological Review, 95, $307-317$.

Farah, M. J. (1989). The neural basis of mental imagery. Trends in Neurosciences, 12, $395-399$.

Farah, M. J., Hammond, K. M., Levine, D. N., \& Calvanio, R. (1988). Visual and spatial mental imagery: Dissociable systems of representation. Cognitive Psychology, 20, $439-462$.

Fiez, J. A., Petersen, S. E., Cheney, M. K., \& Raichle, M. (1992). Impaired non-motor learning and error detection associated with cerebellar damage. Brain, 115, $155-$ 178.

Ford, J. M., Roth, W. T., Mohs, R. C., Hopkins, W. F., \& Kopell, B. S. (1979). Eventrelated potentials recorded from young and old adults during a memory retrieval task. Electroencephalography and Clinical Neurophysiology, 47, 450 - 459.

Frackowiak, R.S.J. (1994). Functional mapping of verbal memory and language. Trends in Neurosciences, 17, $109-115$.

Frahm, J. (2000). Zur materiellen Organisation menschlichen Denkens: Magnetresonanz-Tomographie des Gehirns. In N. Elsner \& G. Lüer (Hrsg.), Das Gehirn und sein Geist (S. 53 - 70). Göttingen: Wallstein Verlag.

Friedrich, F. J., Glenn, C. G., \& Marin, O. S. M. (1984). Interruption of phonological $\infty-$ ding in conduction aphasia. Brain and Language, 22, $266-291$.

Frith, C. D., Friston, K. J., Liddle, P. F., \& Frackowiak, R. S. J. (1991). A PET study of word finding. Neuropsychologia, 29 (12), $1137-1148$.

Frisk, V. \& Milner, B. (1990). The relationship of working memory to the immediate recall of stories following unilateral temporal or frontal lobectomy. Neuropsychologia, $28(2), 121-135$.

Fuster, J. M. (1989). The prefrontal cortex. Anatomy, physiology and neuropsychology of the frontal lobe. New York: Raven Press.

Gazzaniga, M. \& LeDoux, J. (1978). The integrated mind. New York: Plenum Press. 
Gersch, W. (1987). Non-stationary multichannel time series analysis. In A. S. Gevins \& A. Remond (Eds.), Methods of analysis of brain electrical and magnetic signals. EEG Handbook (pp. 261 - 296). Amsterdam: Elsevier.

Geschwind, N. (1965). Disconnection syndromes in animals and man, Part II. Brain, 88, $585-644$.

Geschwind, N. (1972). Language and the brain. Scientific American, 226 (4), 76 - 83.

Gevins, A. \& Cutillo, B. (1993). Spatiotemporal dynamics of component processes in human working memory. Electroencephalography and Clinical Neurophysiology, $87,128-143$.

Gevins, A., Leong, H., Smith, M. E., Le, J., \& Du, R. (1995). Mapping cognitive brain function with modern high-resolution electroencephalography. TINS, 18 (10), 429 -436 .

Gevins, A., Smith, M. E., Le, J., Leong, H., Bennett, J., Martin, N., McEvoy, L, Du, R., \& Whitfield, S. (1996). High resolution evoked potential imaging of the cortical dynamics of human working memory. Electroencephalography and Clinical Neurophysiology, 98, $327-348$.

Gillund, G. \& Shiffrin, R. M. (1984). A retrieval model for both recognition and recall. Psychological Review, 91,1-67.

Glanzer, M. \& Cunitz, A. R. (1966). Two storage mechanisms in free recall. Journal of Verbal Learning and Verbal Behavior, 5, $351-360$.

Glenberg, A. M. \& Kraus, T. A. (1981). Long-term recency is not found on a recognition test. Journal of Experimental Psychology: Human Learning and Memory, 7, 475 479.

Goldman-Rakic, P. (1987). Circuitry of primate prefrontal cortex and the regulation of behavior by representational memory. In F. Plum \& V. Mountcastle (Eds.), Handbook of physiology (Vol. 1, pp. 373 - 417). Bethesda, MD: American Psychological Society.

Goldman-Rakic, P. (1988). Topography of cognition: Parallel distributed networks in primate association cortex. Annual Review of Neurosciences, 11, 137-156.

Goldstein, K. (1944). The mental changes due to frontal lobe damage. Journal of Psychology, 17, $187-208$.

Gomer, F. E., Spicuzza, R. J., \& O'Donnell, R. D. (1976). Evoked potential correlates of visual item recognition during memory-scanning tasks. Physiological Psychology, $4,61-65$.

Gopher, D. \& Donchin, E. (1985). Workload - an examination of the concept. In K. R. Boff, L. Kaufmann \& J. R. Thomas (Eds.), Handbook of perception and human performance (Vol. 2, pp. 411 -418). New York: Wiley. 
Gronlund, S. D. \& Ratcliff, R. (1989). Time course of item and associative information: Implications for global memory models. Journal of Experimental Psychology: Learning, Memory, and Cognition, 15 (5), 846 - 858.

Grossman, M. Reivich, M., Ding, X. S., Morrison, D., Jaggi, J., Alavi, A., \& Greenberg, J. H. (1993). A cerebral network for sentence comprehension examined with a PET activation paradigm. Journal of Cerebral Blood Flow and Metabolism, 13 (1), S525.

Halstead, W. C. (1940). Preliminary analysis of grouping behavior in patients with cerebral injury by the method of equivalent and non-equivalent stimuli. American Journal of Physiology, 96, $1263-1294$.

Hanley, J. R., Pearson, N. A., \& Young, A. W. (1990). Impaired memory for new visual forms. Brain, 113, $1131-1148$.

Hanley, J. R., Young, A. W., \& Pearson, N. A. (1991). Impairment of the visuo-spatial sketch pad. Quarterly Journal of Experimental Psychology, 43 A (1), 101-125.

Hardyk, C. D. \& Petrinovitch, L. R. (1970). Subvocal speech and comprehension level as a function of the difficulty level of reading material. Journal of Verbal Learning and Verbal Behavior, 9, $647-652$.

Hawkins, R. D. \& Kandel, E. R. (1984). Is there a cell-biological alphabet for simple forms of learning? Psychological Review, 91,375 - 391.

Hebb, D. O. (1949). The organization of behavior. New York: Wiley.

Holding, D. H. (1985). The psychology of chess skill. Hillsdale, NJ: Lawrence Erlbaum Associates Inc.

Hulme, C., Maughan, S., \& Brown, G. D. A. (1991) Memory for familiar and unfamiliar words: Evidence for a long-term contribution to short-term memory span. Journal of Memory and Language, 30, $685-701$.

Hulme, C., Newton, P., Cowan, N., Stuart, G., \& Brown, G. (1999). Think before you speak: Pauses, memory search, and trace redintegration processes in verbal memory span. Journal of Experimental Psychology: Learning, Memory, and Cognition, $25,447-463$.

Hulme, C., Roodenrys, S., Schweickert, R., Brown, G. D. A., Martin, S., \& Stuart, G. (1997). Word frequency effects on short-term memory tasks: Evidence for a redintegration process in immediate recall. Journal of Experimental Psychology: Learning, Memory, and Cognition, 23, 1217 - 1232.

Humphreys, G. W. \& Riddoch, M. J. (1987). Visual object processing: A cognitive neuropsychological approach. Hove, UK: Lawrence Erlbaum Associates Ltd.

Hyde, T. S. \& Jenkins, J. J. (1969). Differential effects of incidental tasks on the organization or recall of a list of highly associated words. Journal of Experimental Psychology, 83, $472-481$. 
Isaksson, A., Wennberg, A., \& Zetterberg, L. H. (1981). Computer analysis of EEG signals with parametric models. Proc. IEEE, 69, 451 - 461.

John, E. R. (1967). Mechanisms of memory. New York: Academic Press.

Johnson, P. (1982). The functional equivalence of imagery and movement. Quarterly Journal of Experimental Psychology, 34 A, 349 - 365.

Johnson, C. D. \& Jenkins, J. J. (1971). Two more incidental tasks that differentially affect associative clustering in recall. Journal of Experimental Psychology, 89, 92 - 95.

Jonides, J., Smith, E. E., Koeppe, R. A., Awh, E. S., Minoshima, S., \& Mintun, M. A. (1993). Spatial working memory in humans as revealed by PET. Nature, 363, 623 -625 .

Just, M. A. \& Carpenter, P. A. (1992). A capacitiy theory of comprehension: Individual differences in working memory. Psychological Review, 99, 122 - 149.

Kandel, E. R. \& Schwartz, J. H. (1982). Molecular biology of learning: Modification of transmitter release. Science, 218, 433 - 442.

Karneth, H. O., Wallesch, C. W., \& Zimmermann, P. (1991). Mental planning and anticipatory processes with acute and chronic frontal lobe lesions: A comparison of maze performance in routine and non-routine situations. Neuropsychologia, 29 (4), 271 - 290.

Karrer, R., McDonough, B., Warren, C., \& Cone, R. (1980). CNV during memory retrieval by normal and retarded adults. In H. H. Kornhuber \& L. Deecke (Eds.), Motivation, motor and sensory processes of the brain: Electrical potentials, behavior and clinical use. Progress in brain research (Vol. 54, pp. 668 - 672). Amsterdam: Elsevier.

Kinsbourne, M. (1972). Behavioural analysis of the repetition deficit in conduction aphasia. Neurology, 22, $1126-1132$.

Kinsbourne, M. \& Warrington, E. K. (1962). A disorder of simultaneous form perception. Brain, 85, 461 - 486.

Kintsch, W. (1982). Gedächtnis und Kognition. Berlin: Springer. (Original erschienen 1977: Memory and cognition)

Klatzky, R. L. \& Smith, E. E. (1972). Stimulus expectancy and retrieval from short-term memory. Journal of Experimental Psychology, 94, $101-107$.

Klingberg, T., O'Sullivan, B. T., \& Roland, P. E. (1997). Bilateral activation of frontoparietal networks by incremental demand in a working memory task. Cerebral Cortex, 7, $465-471$.

Kluwe, R. H. (1997). Komponenten des Arbeitsgedächtnisses: Zum Stand kognitionswissenschaftlicher Forschung. In G. Lüer \& U. Lass (Hrsg.), Erinnern und Behalten. Wege zur Erforschung des menschlichen Gedächtnisses (S. 140 - 171). Göttingen: Vandenhoeck \& Ruprecht. 
Koeppe, R. A., Minoshima, S., Jonides, J., Smith, E. E., Awh, E. S. (1993). Working memory in humans as studied by positron emission tomography: Distinguishing spatial and object buffers. Journal of Cerebral Blood Flow and Metabolism, 13 (1), 500.

Kolb, B. \& Whishaw, I. Q. (1995). Fundamentals of human neuropsychology $\left(4^{\text {th }} \mathrm{ed}\right.$.). New York: Freeman.

Kornhuber, H. H. (1988). The human brain: From dream and cognition to fantasy, will, conscience, and freedom. In H. J. Markowitsch (Ed.), Information processing by the brain (pp. 241 - 258). Toronto: Huber.

Kosslyn, S. M., Alpert, N. M., Thompson, W. L., Maljkovic, V., Weise, S.B., Chabris, C. F., Hamilton, S. E., Rauch, S. L., \& Buonanno, F. S. (1993). Visual mental imagery activates topographically organized visual cortex: PET investigations. Journal of Cognitive Neuroscience, 5, $263-287$.

Kramer, A., Schneider, W., Fisk, A., \& Donchin, E. (1986). The effects of practice and task structure on components of the event-related brain potential. Psychophysiology, $33-47$.

Krause, W. (1997). Elementaranalyse von Gedächtnis- und Denkprozessen mittels EEG - eine experimentelle Studie. In G. Lüer \& U. Lass (Hrsg.), Erinnern und Behalten. Wege zur Erforschung des menschlichen Gedächtnisses (S. 117 - 139). Göttingen: Vandenhoeck \& Ruprecht.

Krause, W., Gibbons, H., \& Schack, B. (1998). Concept activation and coordination of activation procedure require two different networks. NeuroReport, 9, 1649-1653.

Krause, W., Schack., B., Gibbons, H., \& Kriese, B. (1997). Über die Unterscheidbarkeit begrifflicher und bildhaft-anschaulicher Repräsentationen bei elementaren Denkanforderungen. Zeitschrift für klinische Psychologie, 205, 169 - 203.

Lass, U. (1997). Einfluß von Sprache auf Verarbeitungsprozesse im Kurzzeitgedächtnis - eine experimentelle Untersuchung mit deutschen und chinesischen Probanden. In G. Lüer \& U. Lass (Eds.), Erinnern und Behalten. Wege zur Erforschung des menschlichen Gedächtnisses (pp. 244 - 268). Göttingen: Vandenhoeck \& Ruprecht.

Lass, U., Lüer, G., Becker, D., Fang, Y., Chen, G., \& Wang, Z. (2000). Kurzzeitgedächtnisleistungen deutscher und chinesischer Probanden mit verbalen und figuralen Items: zur Funktion von Phonologischer Schleife und visuell-räumlichem Notizblock. Zeitschrift für Experimentelle Psychologie, 47 (2), 77 - 88.

Lass, U., Lüer, G., Becker, D., Fang, Y. \& Chen, G. (2001). Encoding and retrieval components affecting memory span: articulation rate, memory search and trace redintegration. In C. Kaernbach, E. Schröger \& H. Müller (Eds.), Psychophysics beyond sensation: Laws and invariants of human cognition. Scientific Psychology Series. Erlbaum. Manuscript submitted for publication.

Lavadas, E., Umilta, C., \& Provinciali, L. (1979). Hemisphere-dependent cognitive performances in epileptic patients. Epilepsia, 20, 493. 
Levine, D. N., Warach, J., \& Farah, M. J. (1985). Two visual systems in mental imagery: Dissociation of "what" and "where" in imagery disorders due to bilateral posterior cerebral lesions. Neurology, 35, 1010-1018.

Levy, J. \& Trevarthen, C. (1976). Metacontrol of hemispheric functions in human split brain partients. Journal of Experimental Psychology, 2, 299 - 312.

Lewin, J. S., Friedman, L., Wu, D., Miller, D. A., Thompson, L. A., Klein, S. K., Wise, A. L., Hedera, P., Buckley, P., Meltzer, H., Friedlang, R. P., \& Duerk, J. L. (1996). Cortical localization of human sustained attention: Detection with functional MR using a visual vigilance paradigm. Journal of Computer Assisted Tomography, 20, $695-701$.

Lezak, M. D. (1995). Neuropsychological assessment. New York: Oxford University Press. Logie, R. H. (1986). Visuo-spatial processing in working memory. Quarterly Journal of Experimental Psychology, 38 A, $229-247$.

Luria, A. R. (1969). Frontal lobe syndromes. In P. J. Vinken \& G. W. Bruyn (Eds.), Handbook of clinical neurology (Vol. 2, pp. 725 - 757). Amsterdam: North Holland.

Luria, A. R., Sokolov, E. N., \& Klimkowski, M. (1967). Towards a neurodynamic analysis of memory disturbances with lesions of the left temporal lobe. Neuropsychologia, $5,1-10$.

Mann, V. A. \& Liberman, I. Y. (1984). Phonological awareness and verbal short-term memory. Journal of Learning Disabilities, 17, $592-598$.

Markowitsch, H.J. (1985). Hypotheses on mnemonic information processing by the brain. International Journal of Neuroscience, 27, $191-227$.

Markowitsch, H. J. (1992). Neuropsychologie des Gedächtnisses. Göttingen: Hogrefe Verlag für Psychologie.

Marsh, G. R. (1975). Age differences in evoked potential correlates of a memory scanning process. Experimental Aging Research, 1, 3- 16.

Martin, R. C. (1993). Short-term memory and sentence processing: Evidence from neuropsychology. Memory \& Cognition, 21 (2), 176 - 183.

Matsuura, M., Yamamoto, K., Fukuzawa, H., Okubo, Y., Uesugi, H., Moriiwa, M., Kojima, T., \& Shimazono, Y. (1985). Age development and sex differences of various EEG elements in healthy children and adults - Quantification by a computerized wave form recognition method. Electroencephalography and Clinical Neurophysiology, 60, $394-406$.

Mathews, W. A. (1983). The effects of concurrent secondary tasks on the use of imagery in a free recall task. Acta Psychologica, 53, 231-241.

Mayes, A. R. (1995). The assessment of memory disorders. In A. D. Baddeley, B.A. Wilson \& F. N. Watts, Handbook of memory disorders (pp. 367 - 391). Chichester: John Wiley \& Sons. 
McCarthy, G., Blamire, A. M., Puce, A., Nobre, A. C., Bloch, G., Hyder, F., GoldmanRakic, P., \& Shulman, R. (1994). Functional MRI studies of frontal cortex activation during a spatial working memory task in humans. Proceedings of the National Academy of Sciences USA, 91, 8690 - 8694.

McCarthy, G., Puce, A., Constable, R. T., Krystal., J. H., Gore, J. C., \& Goldman-Rakic, P. (1996). Activation of human prefrontal cortex during spatial and nonspatial workings tasks measured by functional MRI. Cerebral Cortex, 6, 600-611.

McCarthy, R. A. \& Warrington, E. K. (1987). The double dissociation of short-term memory for lists and sentences. Brain, 110,1545- 1563.

McCarthy, R. A. \& Warrington, E. K. (1990). Cognitive neuropsychology: A clinical introduction. San Diego: Academic Press.

McElree, B. \& Dosher, B. A. (1993). Serial retrieval processes in the recovery of order information. Journal of Experimental Psychology: General, 122 (3), $291-315$.

McFie, J. \& Piercy, M. F. (1952). The relation of laterality of lesion to performance on Weigl's Sorting Test. Journal of Mental Sciences, 98, $299-308$.

Mesulam, M. M. (1990). Large scale neurocognitive networks and distributed processing for attention, language and memory. Annuals of Neurology, 28, 597-613.

Metlon, A. W. (1963). Implications of short-term memory for a general theory of memory. Journal of Verbal Learning and Verbal Behavior, 2, 1-21.

Meyer, D. E. (1970). On the representation and retrieval of stored semantic information. Cognitive Psychology, 1, $242-300$.

Miller, G. A. (1956). The magical number seven, plus or minus two: Some limits on our capacity for processing information. The Psychological Review, 63, $81-97$.

Milner, B. (1963). Effects of different brain lesions on card sorting. Archives of Neurology, $9,90-100$.

Milner, B. (1964). Some effects of frontal lobectomy in man. In J. M. Warner \& K. Akert (Eds.), The frontal granular cortex and behavior (p. 313). New York: McGraw-Hill.

Milner, B. (1970). Memory and the medial temporal regions of the brain. In K. H. Pribram \& D. E. Braodbent (Eds.), Biology of memory (pp. 29 - 50). New York: Academic Press.

Milner, B. (1971). Interhemispheric differences in the localization of psychological processes in man. British Medical Bulletin, 27, 272 - 277.

Milner, B. (1982). Some cognitive effects of frontal-lobe lesions in man. Philosophical Transactions of the Royal Society London, 298 B, 211 - 226.

Milner, B., McAndrews, M. P., \& Leonard, G. (1990). Frontal lobes and memory for the temporal order of recent events. Cold Spring Harbor Symposia on Quantitative Biology, 55, 987 - 994.

Mishkin, M., Ungerleider, L. G., \& Macko, K. A. (1983). Object vision and spatial vision: Two cortical pathways. Trends in Neuroscience, 6, 414-417. 
Morais, J., Allegria, J., \& Content, A. (1987). The relationship between segmental analysis and alphabetic literacy: An interactive view. Cahiers de Psychologie Cognitive, 7, $415-438$.

Morris, R. G. (1994). Working memory in Alzheimer-type dementia. Neuropsychology, $8,544-554$.

Morris, R. G. \& Baddeley, A. D. (1988). Primary and working memory functioning in Alzheimer-type dementia. Journal of Clinical and Experimental Neuropsychology, 10, $279-296$.

Morris, C.D., Bransford, J. D., \& Franks, J. J. (1977). Levels of processing versus transfer appropriate processing. Journal of Verbal Learning and Verbal Behavior, $16,519-533$.

Morris, N. \& Jones, D. M. (1990). Memory updating in working memory: The role of the central executive. British Journal of Psychology, 81, 111 - 121.

Morton, J. (1967). A singular lack of incidental learning. Nature, 215, $203-204$.

Müller, G. E. \& Pilzecker, A. (1900). Experimentelle Beiträge zur Lehre vom Gedächtnis. Zeitschrift für Psychologie, Ergänzungsband 1.

Murdock, B. B. Jr. (1974). Human memory: Theory and data. Potomac, MD: Erlbaum.

Murdock, B. B. Jr. (1982). A theory for the storage and retrieval of item and associative information. Psychological Review, 89,609-626.

Newcombe, F., Ratcliff, G., \& Damasio, H. (1987). Dissociable visual and spatial impairments following right posterior cerebral lesions: Clinical, neuropsychological and anatomical evidence. Neuropsychologia, 25, 149 - 161.

Nickerson, R. S. \& Adams, M. J. (1979). Long-term memory for a common object. Cognitive Psychology, 11, 287 - 307.

Norman, D. A. \& Shallice, T. (1986). Attention to action: Willed and automatic control of behavior. In. R. J. Davidson, G. E. Schwartz, \& D. Shapiro (Eds.), Consciousness and Self-Regulation: Advances in research and theory (Vol. 4, pp. 1 - 18). New York: Plenum.

Ojemann, G. A. (1978). Organization of short-term verbal memory in language areas of human cortex: Evidence from electrical stimulation. Brain and Language, 5, 331 340.

Ojemann, G. A., Creutzfeldt, O., Lettich, E., \& Haglund, M. M. (1988). Neuronal activity in human lateral temporal cortex related to short-term verbal memory, naming and reading. Brain, 111, $1383-1403$.

Owen, A. M., Downes, J. D., Sahakian, B. J., Polkey, C. E., \& Robbins, T. W. (1992). Planning and spatial working memory following frontal lobe lesions in man. Neuropsychologia, 28, $1021-1034$. 
Owen, A. M., Evans, A. C., \& Petrides, M. (1996). Evidence for a two-stage model of spatial working memory processing within the lateral frontal cortex: A positron emission tomography study. Cerebral Cortex, 6,31-38.

Owen, A. M., Sahakian, B. J., Semple, J., Polkey, C. E., \& Robbins, T. W. (1995). Visuo-spatial short-term recognition memory and learning after temporal lobe excisions, frontal lobe excisions or amygdalo-hippocampectomy in man. Neuropsychologia, 33(1), $1-24$.

Paivio, A. (1969). Mental imagery in associative learning and memory. Psychological Review, 76, $241-263$.

Pardo, J. V., Pardo, P. J., Janer, K. W., \& Raichle, M. E. (1990). The anterior cingulate cortex mediates processing selection in the Stroop attentional conflict paradigm. Proceedings of the National Academy of Sciences, USA, 87, 256 - 259.

Pardo, J. V., Fox, P. T., \& Raichle, M. E. (1991). Localization of a human system for sustained attention by positron emission tomography. Nature, 249, 61-64.

Parkin, A. J. (1993). Gedächtnis. Ein einführendes Lehrbuch. Weinheim: Psychologie Verlags Union.

Paulesu, E., Frith, C. D., \& Frackowiak, R.S. J. (1993). The neuronal correlates of the verbal component of working memory. Nature, 362, $342-345$.

Paus, T., Petrides, M. Evans, A. C., \& Meyer, E. (1993). Role of the human anterior cingulate cortex in the control of oculomotor, manual, and speech responses: A positron emission tomography study. Journal of Neurophysiology, 70, 453-469.

Perani, D., Bressi, S., Cappa, S. F., Vallar, G., Alberoni, M., Grassi, F., Caltagirone, C., Cipolotti, L., Franceschi, M., Lenzi, G. L., \& Fazio, F. (1993). Evidence of multiple memory systems in the human brain. Brain, 116, $903-919$.

Petersen, S. E., Fox, P. T., Posner, M. I., Mintun, M., \& Raichle, M. E. (1988). Positron emission tomographic studies of the cortical anatomy of single-word processing. Nature, 331, $585-589$.

Petersen, S. E., Fox, P. T., Snyder, A. Z., \& Raichle, M. E. (1990). Activation of extrastriate and frontal cortical areas by visual words and word-like stimuli. Science, 249, $1041-1044$.

Peterson, L. R. \& Peterson, M. J. (1959). Short-term retention of individual verbal items. Journal of Experimental Psychology, 58, 193 - 198.

Petrides, M. (1985). Deficits on conditional associative-learning tasks after frontal- and temporal-lobe lesions in man. Neuropsychologia, 23 (5), $601-614$.

Petrides, M. (1990). Nonspatial conditional learning impaired in patients with unilateral frontal but not unilateral temporal lobe excisions. Neuropsychologia, 28 (2), 137 149. 
Petrides, M. (1994). Frontal lobes and working memory: Evidence from investigations of the effects of cortical excisions in nonhuman primates. In F. Boller \& J. Grafman (Eds.), Handbook of neuropsychology (Vol. 9, pp. 59 - 82). Amsterdam: Elsevier.

Petrides, M., Alivisatos, B., Evans, A. C. \& Meyer, E. (1993a). Dissociation of human mid-dorsolateral from posterior dorsolateral frontal cortex in memory processing. Proceedings of the National Academy of Sciences, USA, 90, 873-877.

Petrides, M., Alivisatos, B., Meyer, E., \& Evans, A. C. (1993b). Functional activation of the human frontal cortex during the performance of verbal working memory tasks. Proceedings of the National Academy of Sciences, USA, 90, 878-882.

Petrides, M. \& Milner, B. (1982). Deficits on subject-ordered tasks after frontal- and temporal-lobe lesions in man. Neuropsychologia, 20 (3), $249-262$.

Petsche, H. (1990). EEG und Denken. Zeitschrift für EEG-EMG, 21, 219 - 225.

Petsche, H. (1995). Die flirrende Welt der Aufmerksamkeit: Zur Neurophysiologie kognitiver Prozesse. Zeitschrift für EEG-EMG, 26, $1-18$.

Petsche, H., Lacroix, D., Lindner, K., Rappelsberger, P. \& Schmidt-Heinrich, E. (1992). Thinking with images or thinking with language: A pilot EEG probability mapping study. International Journal of Psychophysiology, 12, $31-39$.

Petsche, H., Pockberger, H. \& Rappelsberger, P. (1985). Musikrezeption, EEG und musikalische Vorbildung. Zeitschrift für EEG-EMG, 16, 183 - 190.

Petsche, H., Rappelsberger, P. \& Pockberger, H. (1987). EEG-Veränderungen beim Lesen. In H. M. Weinmann (Hrsg.), Zugang zum Verständnis höherer Hirnfunktionen durch das $E E G$ (S. 59 - 74). München: Zuckschwert.

Phillips, C. G. (1956). Intracellular records from Betz-cells. Quarterly Journal of Experimental Psychology, 41, 58 - 69.

Pigott, S. \& Milner, B. (1994). Capacitiy of visual short-term memory after unilateral frontal or anterior temporal-lobe resection. Neuropsychologia, 32 (8), $969-981$.

Pinel, J. P. J. (1997). Biopsychology. Boston: Allyn and Bacon.

Posner, M. I. \& Petersen, S. E. (1990). The attention system of the human brain. Annual Review of Neuroscience, 13, $25-42$.

Posner, M. I., Petersen, S. E., Fox, P. T., \& Raichle, M. E. (1988). Localization of cognitive operations in the human brain. Science, 240, $1627-1631$.

Postle, B. R., Stern, C. E., Rosen, B. R., \& Corkin, S. (1997). FMRI of spatial and nonspatial visual working memory reveals differences in posterior, but not prefrontal, cortex. Society for Neuroscience Abstracts, 23, 1679.

Potter, M. C. (1993). Very short-term conceptual memory. Memory \& Cognition, 21 (2), $156-161$.

Pratt, H., Michlewski, H., Barret, G., \& Starr. A. (1989a). Brain potentials in a memoryscanning task. I. Modality and task effects on potentials to the probes. Electroencephalography and clinical Neurophysiology, 72, $407-428$. 
Pratt, H., Michlewski, H., Barret, G., \& Starr. A. (1989b). Brain potentials in a memoryscanning task. II. Effects of aging on potentials to the probes. Electroencephalography and clinical Neurophysiology, 72, $507-517$.

Pratt, H., Michlewski, H., Barret, G., \& Starr. A. (1989c). Brain potentials in a memoryscanning task. III. Potentials to the items being memorized. Electroencephalography and clinical Neurophysiology, 73, $41-51$.

Pribram, K. H. (1973). The primate frontal cortex - executive of the brain. In K. H. Pribram \& A. R. Luria (Eds.), Psychophysiology of the frontal lobes (pp. 293 - 314). New York: Academic Press.

Pribram, K. H. (1987). The subdivisions of the frontal cortex revisited. In E. Perecman (Ed.), The frontal lobes revisited (pp. $11-40$ ). New York: IRBN Press.

Raaijmakers, J. G. W. (1993). The story of the two-store model of memory: Past criticisms, current status, and future directions. In D. E. Meyer \& S. Kornblum (Eds.), Attention and performance XIV. Synergies in experimental psychology, artificial intelligence, and cognitive neuroscience (pp. 467 - 488). London: MIT Press.

Raichle, M. E. (1990). Anatomical explorations of mind: Studies with modern imaging techniques. Cold Spring Harbor Symposia on Quantitative Biology, 55, 983 - 986.

Raichle, M. E. (1993). The scratchpad of the mind. Nature, 363, $583-584$.

Raichle, M.E. (1997). Functional imaging in behavioral neurology and neuropsychology. In T. E. Feinberg \& M. J. Farah (Eds.), Behavioral neurology and neuropsychology (pp. 83 - 100). New York: McGraw-Hill.

Raife, E. A., Fiez, J. A., Raichle, M. E., Balota, D. A., \& Petersen, S. E. (1992). A PET study of verbal working memory. Society for Neuroscience Abstracts, 18, 932.

Rappelsberger, P., Krieglsteiner, S., Mayerweg, M., Petsche, H., \& Pockberger, H. (1987). Probability mapping of EEG changes: Application to spatial imaging studies. Journal of Clinical Monitoring, 3, 320 - 322.

Rappelsberger, P. \& Petsche, H. (1988). Probability mapping: Power and coherence analysis of cognitive processes. Brain Topography, 1, 46-54.

Ratcliff, R. (1978). A theory of memory retrieval. Psychological Review, 85 (2), $59-108$.

Ratcliff, R. \& Murdock, B.B. Jr. (1976). Retrieval processes in recognition memory. Psychological Review, 83, $190-214$.

Reed, A. V. (1976). The time course of recognition in human memory. Memory \& Cognition, 4, $16-30$.

Rips, L. J., Shoben, E. J., \& Smith, E. E. (1973). Semantic distance and the verification of semantic relations. Journal of Verbal Learning and Verbal Behavior, 12, 1-20.

Risse, G. L., Rubens, A. B., \& Jordan, L. S. (1984). Disturbances of long-term memory in aphasic patients. Brain, 107,605-617. 
Robbins, T. W., Anderson, E. J., Barker, D. R., Bradley, A. C., Fearnyhough, C., Henson, R., Hudson, S. R., \& Baddeley, A. D. (1996). Working memory in chess. Memory \& Cognition, 24 (1), 83 - 93.

Rösler, F. (1997). Neuropsychologische Gedächtnisforschung. In G. Lüer \& U. Lass (Hrsg.), Erinnern und Behalten. Wege zur Erforschung des menschlichen Gedächtnisses (S. 79 - 116). Göttingen: Vandenhoeck \& Ruprecht.

Rosenzweig, M. R. \& Bennett, E. L. (1996). Psychobiology of plasticity: Effects of training and experience on brain and behavior. Behavioral Brain Research, 78, 57 - 65.

Rosenzweig, M. R. \& Leiman, A. L. (1982). Physiological psychology. Lexington, Mass.: D. C. Heath.

Rosenzweig, M. R., Leiman, A. L., \& Breedlove, S. M. (1996). Biological Psychology. Sunderland, Mass.: Sinauer Associates.

Ruchkin, D. S., Johnson Jr., R., Canoune, H., \& Ritter, W. (1990). Short-term memory storage and retention: An event-related brain potential study. Electroencephalography and Clinical Neurophysiology, 76, 419-439.

Ruchkin, D. S., Johnson Jr., R., Grafman, J., Canoune, H., \& Ritter, W. (1992). Distinctions and similarities among working memory processes: An event-related potential study. Cognitive Brain Research, 1,53-66.

Rypma, B. \& D'Esposito, M. (1999). The roles of prefrontal brain regions in components of working memory: Effects of memory load and individual differences. Proceedings of the National Academy of Sciences, USA, 96 (11), $6558-6563$.

Saffran, E. M. \& Marin, O.S. M. (1975). Immediate memory for word lists and sentences in a patient with deficient auditory short-term memory. Brain and Language, 2, $420-433$.

Salmon, E., Van der Linden, M., Collette, F., Delfiore, G., Maquet, P., Degueldre, C., Luxen, A., \& Franck, G. (1996). Regional brain activity during working memory tasks. Brain, 119, $1617-1625$.

Schack, B. (1997). Adaptive Verfahren zur Spektralanalyse instationärer mehrdimensionaler biologischer Signale. Unveröff. Habil.-Arbeit, Technische Universität IImenau.

Schack, B. (1999). Dynamic topographic spectral analysis of cognitive processes. In Ch. Uhl (Ed.), Analysis of neurophysiological brain functioning (pp. $230-251$ ). Berlin: Springer.

Schack, B., Chen, A. C. N., Mescha, S., \& Witte, H. (1999). Instantaneous EEG coherence analysis during the Stroop task. Clinical Neurophysiology, 110, 1410-1426.

Schack, B., Grieszbach, G., Arnold, M., \& Bolten, J. (1995). Dynamic cross spectral analysis of biological signals by means of bivariate ARMA processes with tmedependent coefficients. Medical \& Biological Engineering \& Computation, 33, 605 -610 . 
Schack, B., Grieszbach, G. \& Krause, W. (1998). The sensitivity of instantaneous coherence for considering elementary comparison processing. Part I: The relationship between mental activities and instantaneous EEG coherence. International Journal of Psychophysiology, 31, $219-240$.

Schack, B. \& Krause, W. (1995). Dynamic power and coherence analysis of ultra shortterm cognitive processes - A methodical study. Brain Topography, 8 (2), 127 - 136.

Schack, B. \& Krause, W. (1996). Instantaneous coherence as a sensible parameter for considering human information processing. In: Proceedings of the International Conference on Pattern Recognition (Vol. II, pp. 45 - 49). Los Alamitos, Calif.: IEEE Computer Society Press.

Scherg, M. (1999). MEG-Technik Grundlagen. Informationsdokument aus dem World Wide Web (http://www.ukl.uni-heidelberg.de/neuro/meg/german/megtech2.htm). Klinikum der Universität Heidelberg, Sektion Biomagnetismus.

Schmidt, R. F. (1987). Grundriß der Neurophysiologie (6. Aufl.). Berlin: Springer.

Schneider, W. \& Detweiler, M. A. (1988). A connectionist/control architecture for working memory. In G. H. Bower (Ed.), The psychology of learning and motivation (Vol. 21, pp. 53 - 119). San Diego, CA: Academic Press.

Scholl, D.A. (1956). The organization of the cerebral cortex. London: Hafner Publ. Comp. (Nachdruck 1967)

Scoville, W. B. \& Milner, B. (1957). Loss of recent memory after bilateral hippocampal lesions. Journal of Neurology, Neurosurgery, and Psychiatry, 20,11-21.

Shallice, T. (1982). Specific impairments of planning. Philosophical Transactions of the Royal Society London, 298 B, $199-209$.

Shallice, T. (1988). From neuropsychology to mental structure. Cambridge: Cambridge University Press.

Shallice, T. \& Warrington, E. K. (1970). Independent functioning of verbal memory stores: A neuropsychological study. Quarterly Journal of Experimental Psychology, 22, $261-273$.

Shankweiler, D. \& Liberman, I. Y. (1976). Exploring the relations between reading and speech. In R. M. Knights \& D. K. Bakker (Eds.), The neuropsychology of learning disorders: Theoretical approaches. Baltimore: University Park Press.

Shepart, R. N. \& Metzler, J. (1971). Mental rotation of three-dimensional objects. Science, $171,701-703$.

Shiffrin, R. M. (1993). Short-term memory: A brief commentary. Memory \& Cognition, 21 (2) $193-197$.

Shiffrin, R. M. \& Schneider, W. (1977). Controlled and automatic human information processing: II. Perceptual learning, automatic attending, and a general theory. Psychological Review, 84 (29), 127 - 190. 
Shimamura, A. P., Janoswky, J. S., \& Squire, L. R. (1988). Memory for temporal order in patients with frontal-lobe lesions and patients with amnesia. Society of Neurosciences Abstracts, 14, 1043.

Simon, H. A. (1974). How big is a chunk? Science, 183, $482-488$.

Singer, W. (1990). Search for coherence: A basic principle of cortical self-organisation. Concepts in Neuroscience, 1,1-26.

Slowiaczek, M. L. \& Clifton, C. (1980). Subvocalization and reading for meaning. Journal of Verbal Learning and Verbal Behavior, 19, 573 - 582.

Smith, E. E. \& Jonides, J. (1997). Working memory: A view from neuroimaging. Cognitive Psychology, 33, 5 - 42.

Smith, E. E., Jonides, J., Koeppe, R. A., Awh, E. S., Schumacher, E., \& Minoshima, S. (1995). Spatial vs. object working memory: PET investigations. Journal of Cognitive Neurosciences, 7, $337-358$.

Smith, E. E., Jonides, J., \& Koeppe, R. A. (1996). Dissociating verbal and spatial working memory using PET. Cerebral Cortex, 6, 11-20.

Spinnler, H., Della Sala, S., Bandera, R., \& Baddeley, A. D. (1988). Dementia, ageing, and the structure of human memory. Cognitive Neuropsychology, 5, $193-211$.

Squire, L. R. (1987). Memory and brain. Oxford: Oxford University Press.

Squire, L. R. (1992). Memory and hippocampus: A synthesis from findings with rats, monkeys, and humans. Psychological Review, 99, 195-231.

Starr, A. \& Barrett, G. (1987). Disordered auditory short-term memory in man and eventrelated potentials. Brain, 110, $935-959$.

Starr, A., Barrett, G., Pratt, H., Michalewski, H. J., \& Patterson, J. V. (1990). Electrophysiological measures of short-term memory. In G. Vallar \& T. Shallice (Eds.), Neuropsychological impairments of short-term memory (pp. $94-110)$. Cambridge: Cambridge University Press.

Steriade, M. (1981). Mechanism underlying cortical activation: Neuronal organization and properties of the midbrain reticular core and intralaminar thalamic nuclei. In O. Pompeiano \& C. A. Ajmone Marsan (Eds.), Brain mechanisms of perceptual awareness and purposeful behavior (pp. 327 -377). New York: Raven Press.

Sternberg, S. (1966). High speed scanning in human memory. Science, 153, $652-654$.

Sternberg, S. (1969a). Memory-scanning: Mental processes revealed by reaction-time experiments. American Scientist, 57, $421-457$.

Sternberg, S. (1969b). The discovery of processing stages: Extensions of Donders' method. Acta Psychologica, 30, $276-315$.

Sternberg, S. (1975). Memory scanning: New findings and current controversies. Quarterly Journal of Experimental Psychology, 27, 1- 32.

Strub, R. L. \& Gardner, H. (1974). The repetition defect in conduction aphasia: Mnestic or linguistic? Brain and Language, 1, 241-255. 
Szentágothai, J. (1973). Synaptology of the visual cortex. In R. Jung (Ed.), Handbook of sensory physiology (Vol. VII, pp. 267 - 324). Berlin: Springer.

Teuber, H. L., Battersby, W. S., \& Bender, M. B. (1951). Performance of complex visual tasks after cerebral lesions. Journal of Nervous and Mental Disorder, 114, 413 - 429.

Teyler, T.J. (1991). A model of associative interactions mediated by N-Methyl-DAspartale activation. In W. C. Abraham, M. Corballis \& K. G. White (Eds.), Memory mechanisms. A tribute to G. V. Goddard (pp. $27-46$ ). Hillsdale, NJ: LEA.

Theios, J. (1973). Reaction time measurements in the study of memory processes: Theory and data. In G. H. Bower (Ed.), The psychology of learning and motivation (Vol. 7, pp. 43 - 85). New York: Academic Press.

Theios, J., Smith, P. G., Haviland, S. E., Traupmann, J., \& Moy, M. C. (1973). Memory scanning as a serial self-terminating process. Journal of Experimental Psychology, $97,323-336$.

Townsend, J. T. (1971). A note on the identifiability of parallel and serial processes. Perception and Psychophysics, 10, $161-163$.

Townsend, J. T. \& Ashby, F. G. (1983). The stochastic modeling of elementary psychological processes. Cambridge, England: Cambridge University Press.

Trembley, M., Lacroix, D., Chaput, Y., Fraile, V., Lamer, R., \& Albert, J.-M. (1994). Brain activation with a maze test: An EEG coherence analysis study in healthy subjects. NeuroReport, 5, $2449-2453$.

Trojano, L., Santione, M., \& Grossi, D. (1992). Short-term memory and verbal learning with auditory phonological coding defect: A neuropsychological case study. Brain and Cognition, 18, $12-33$.

Tukey, J. W. (1977). Exploratory data analysis ( $2^{\text {nd }}$ ed.). Reading, Mass.: Addison-Wesley.

Tulving, E. (1966). Subjective organization and effects of repetition in multi-trial freerecall learning. Journal of Verbal Learning and Verbal Behavior, 5, $193-197$.

Tzeng, O. J. L. (1973). Positive recency effect in delayed free recall. Journal of Verbal Learning and Verbal Behavior, 12, 436 - 439.

Tzortzis, C. \& Albert, M. L. (1974). Impairment of memory for sequences in conduction aphasia. Neuropsychologia, 12, $355-366$.

Tzourio, N., El Massiouli, F., Crivello, F., Joliot, M., Rnault, B., \& Mazoyer, B. (1997). Functional anatomy of human auditory sustained attention studied with PET. Neuroimage, 31, $1379-1396$.

Vallar, G. \& Baddeley, A. D. (1984). Fractionation of working memory. Neuropsychological evidence for a phonological short-term store. Journal of Verbal Learning and Verbal Behavior, 23, $151-161$.

Vallar, G., Basso, A., \& Bottini, G. (1990). Phonological processing and sentence comprehension: A neuropsychological case study. In G. Vallar \& T. Shallice (Eds.), 
Neuropsychological impairments of short-term memory (pp. 448 -476). Cambridge: Cambridge University Press.

Vallar, G. \& Cappa, S. F. (1987). Articulation and verbal short-term memory: Evidence from anarthria. Cognitive Neuropsychology, 4, 417 - 438.

Vallar, G., Corno, M., \& Basso, A. (1992). Auditory and visual verbal short-term memory in aphasia. Cortex, 28, $383-389$.

Vallar, G. \& Papagno, C. (1986). Phonological short-term store and the nature of the recency effect: Evidence from neuropsychology. Brain and Cognition, 5, 428 - 442.

Vallar, G. \& Papagno, C. (1995). The psychology of memory. In A. D. Baddeley, B. A. Wilson \& F. N. Watts (Eds.), Handbook of memory disorders (pp. 3 - 25). Chichester: John Wiley \& Sons.

Vanderplas, J. M. \& Garvin, E. A. (1959). The association value of random shapes. Journal of Experimental Psychology, 57, 147 - 154.

Vögel, F. \& Götze, W. (1962). Statistische Betrachtungen über die Betawellen im EEG des Menschen. Deutsche Zeitschrift für Nervenheilkunde, 184, 112 - 136.

von Stein, A., Rappelsberger, P., Filz, O. \& Petsche, H. (1993). EEG-Korrelate bildlicher Vorstellung: Eine Amplituden- und Kohärenzuntersuchung. Zeitschrift für EEGEMG, 24, $217-224$.

Warrington, E. K. \& James, M. (1967). Disorders of visual perception in patients with bcalised cerebral lesions. Neuropsychologia, 5, 253-266.

Warrington, E. K., James, M., \& Maciejewski, C. (1986). The WAIS as a lateralising and localising diagnostic instrument: A study of 656 patients with unilateral cerebral lesions. Neuropsychologia, 24, $223-239$.

Warrington, E. K., Logue, V., \& Pratt, R. T. C. (1971). The anatomical localisation of selective impairment of auditory-verbal short-term memory. Neuropsychologia, 9, $377-387$.

Warrington, E. K. \& Shallice, T. (1969). The selective impairment of auditory verbal short-term memory. Brain, 92, 885 - 896.

Warrington, E. K. \& Shallice, T. (1972). Neuropsychological evidence of visual storage in short-term memory tasks. Quarterly Journal of Experimental Psychology, 24, $30-40$.

Waugh, N. C. \& Norman, D. A. (1965). Primary memory. Psychological Review, 72, 89 -104 .

Wechsler, D. (1987). Wechsler Memory Scale-Revised. San Antonio: Psychological Corporation.

Weigl, E. (1927). On the psychology of so-called processes of abstraction. Journal of Abnormal and Social Psychology, 36, 3 - 33.

Weiss, S. \& Rappelsberger, P. (1994). Single word processing: EEG coherence differences in alpha and beta band. Brain Topography, 7 (3), $331-355$.

Wernicke, C. (1874). Der aphasische Symptomkomplex. Breslau: Cohn \& Weigert. 
Wiegersma, S., van der Scheer, E., \& Hijman, R. (1990). Subjective ordering, short-term memory, and the frontal lobes. Neuropsychologia, 28, 95 - 98.

Wilson, F. A. W., O'Scalaidhe, S. P., \& Goldman-Rakic, P. S. (1993). Dissociation of object and spatial processing domains in primate prefrontal cortex. Science, 260, 1955 - 1958.

Wundt, W. (1874). Grundzüge der physiologischen Psychologie. Leipzig: W. Engelmann.

Yntema, D. B. \& Trask, F. P. (1963). Recall as a search process. Journal of Verbal Learning and Verbal Behavior, 2, 65.

Zarahn, E., Aguirre, G. K., \& D'Esposito, M. (1999). A trial-based experimental design for functional MRI. Neuroimage, 6, $122-138$. 


\section{Anhang}

Seite

A • Übersicht über Probandenmerkmale

A1

- Versuchsinstruktionen und Fragebogen

$\mathrm{A} 2-\mathrm{A} 6$

B • Übersicht über Gesamtanzahl und Prozentsatz der Falschantworten

$\mathrm{B} 1-\mathrm{B} 2$

- Übersicht über Anzahl gültiger Durchgänge

B3

- Übersicht über Gesamtanzahl und Prozentsatz der Ausreißer

$\mathrm{B} 4-\mathrm{B} 6$

- Übersicht über mittlere Antwortlatenzen

$\mathrm{B} 7-\mathrm{B} 8$

- Antwortlatenzen: Ergebnisse individueller Regressionsanalysen

B9

- Kohärenzen: Ergebnisse der Wilcoxon-Tests

B10

- Kohärenzdauern: Ergebnisse der Wilcoxon-Tests

B11

- Maximale Kohärenzen: Ergebnisse der Wilcoxon-Tests

B12

- Zeitpunkte maximaler Kohärenz: Ergebnisse der Wilcoxon-Tests

B13

- Kohärenzen: Ergebnisse der Regressionsanalysen

$\mathrm{B} 14-\mathrm{B} 17$

- Maximale Kohärenzen: Ergebnisse der Regressionsanalysen

$\mathrm{B} 18-\mathrm{B} 21$

C • Verläufe gemittelter Kohärenzwerte

C1 - C2

D Zeitpunkte maximaler Kohärenz: Ergebnisse der Regressionsanalysen

D1 - D4

E Korrelationen der Zeitpunkte maximaler Kohärenz mit den Antwortlatenzen

$\mathrm{E} 1-\mathrm{E} 7$ 


\section{Anhang A}

Tabelle A-1

Übersicht über die erhobenen Probandenmerkmale

\begin{tabular}{|c|c|c|c|c|c|}
\hline Proband-Nr. & Alter & Geschlecht & Sehhilfe & $\begin{array}{c}\text { Dominante } \\
\text { Hand }\end{array}$ & $\begin{array}{c}\text { EEG- } \\
\text { Erfahrung }\end{array}$ \\
\hline 1 & 31 & 1 & 1 & 1 & 1 \\
\hline 2 & 29 & 1 & 0 & 1 & 1 \\
\hline 3 & 21 & 1 & 0 & 1 & 0 \\
\hline 4 & 19 & 1 & 1 & 1 & 1 \\
\hline 5 & 19 & 1 & 1 & 1 & 0 \\
\hline 6 & 18 & 1 & 0 & 1 & 1 \\
\hline 7 & 18 & 1 & 0 & 1 & 0 \\
\hline 8 & 23 & 1 & 1 & 1 & 1 \\
\hline 9 & 31 & 1 & 1 & 1 & 1 \\
\hline 10 & 19 & 1 & 0 & 1 & 1 \\
\hline
\end{tabular}

Erläuterungen: Geschlecht:

Sehhilfe:

$1=$ weiblich

Dominante Hand: $1=$ rechts

EEG-Erfahrung: $1=$ ja, $0=$ nein

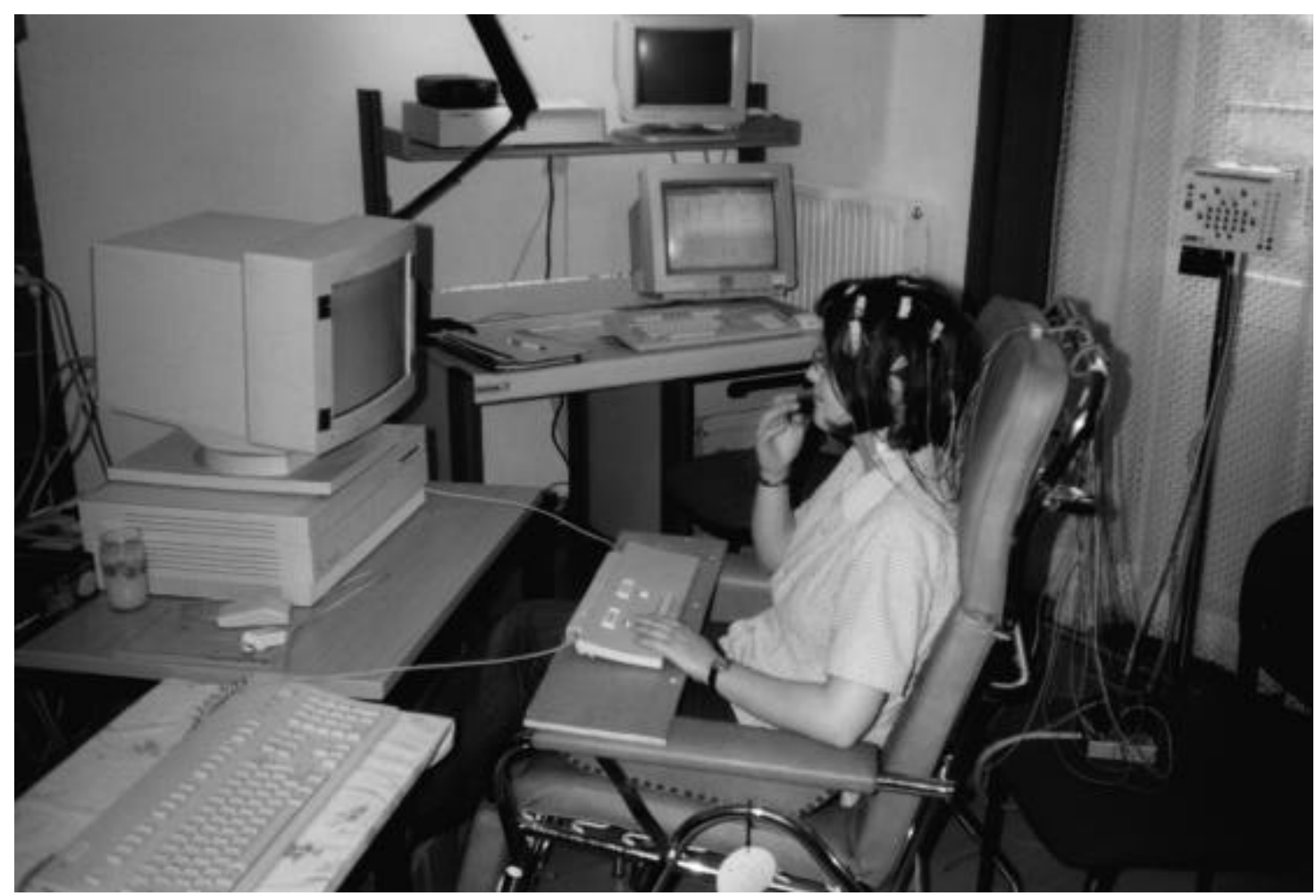

Abbildung A-1. Probandin im Versuchsraum 


\section{Instruktionsblatt}

Liebe Versuchsteilnehmerinnen und Versuchsteilnehmer!

Sie nehmen an einer Untersuchung teil, in der es um das kurzfristige Behalten von unterschiedlichem Reizmaterial geht. Die Untersuchung besteht aus insgesamt zwei Sitzungen, die jeweils mit Pausen circa 3 1/2 bis max. 4 Stunden dauern werden. In jeder Sitzung wird der vor Ihnen stehende Computer benutzt, und es erfolgt gleichzeitig eine EEG-Aufzeichnung, d. h. daß Ihre elektrische Hirnaktivität während der Untersuchung aufgezeichnet wird.

Die Informationen und Anweisungen zu den Versuchen erhalten Sie in diesem Instruktionsheft. Lesen Sie sich den jeweiligen Text bitte genau durch. Falls Sie Fragen haben sollten, stellen Sie diese bitte unmittelbar nach dem Lesen.

Die in der Untersuchung erhobenen Daten werden anonym gespeichert und ausschließlich zu wissenschaftlichen Zwecken verwendet.

Wenn Sie dies gelesen haben, blättern Sie bitte um und füllen zunächst den Fragebogen aus. Anschließend erfolgt die Positionierung der Elektroden. 
Fragebogen

\begin{tabular}{|c|c|c|c|}
\hline Datum _________ & & \multicolumn{2}{|c|}{ Code-Nr. } \\
\hline Alter: & \multicolumn{2}{|c|}{ Jahre } & \\
\hline \multicolumn{4}{|l|}{ Beruf: } \\
\hline Geschlecht: & weiblich & männlich & \\
\hline Sehhilfe benötigt? & nein & ja, wegen ....... & $\begin{array}{l}\text { Weitsichtigkeit } \\
\text { Kurzsichtigkeit }\end{array}$ \\
\hline Dominante Hand: & Rechtshänder & Linkshänder & $\begin{array}{l}\text { beide Hände } \\
\text { gleich }\end{array}$ \\
\hline \multicolumn{4}{|c|}{ Ist bei Ihnen schon einmal eine EEG-Aufzeichnung vorgenommen worden? } \\
\hline & nein & ja & \\
\hline
\end{tabular}




\section{Instruktionsblatt}

In dem folgenden Versuch kommt es auf möglichst rasche und richtige Antworten an. Der Versuch besteht aus 20 einzelnen Abschnitten; nach jedem Abschnitt kann je nach Bedarf eine längere Pause erfolgen. In den Versuchsabschnitten werden Ihnen auf dem Bildschirm in mehreren Durchgängen kurze Reihen bis zu max. 4 (aus insgesamt 10 möglichen) Zufallsfiguren bzw. Zahlwörtern präsentiert, die Sie sich merken sollen. Eine Reihe kann auch aus nur einem Element bestehen. Nach der Darbietung des jeweils letzten Elementes einer Reihe erscheint auf dem Bildschirm folgendes Zeichen:

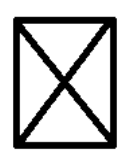

Dieses Zeichen kündigt die Darbietung eines sogenannten "Prüfreizes" an. Sie haben zu entscheiden, ob dieser Prüfreiz in der vorher dargebotenen Reihe enthalten war. War dies der Fall, dann drücken Sie auf der vor Ihnen liegenden Tastatur die Ja-Taste. War dies nicht der Fall, drücken Sie die Nein-Taste.

Drücken Sie bitte die entsprechende Taste so schnell wie möglich, und achten Sie vor allem darauf, so wenig Fehler wie möglich zu machen!

Nach Ihrer Antwort wird die Zeit, die Sie für eine richtige Antwort benötigt haben, kurz auf dem Bildschirm angezeigt (z. B. 0,673 s). Bei einer falschen Reaktion erscheint die Meldung "falsch" auf dem Bildschirm.

Bevor der Versuch beginnt, werden Ihnen die darin verwendeten Reize auf dem Bildschirm vorgeführt. Weil es sich bei Zufallsfiguren um etwas ungewöhnliche "Reize" handelt, erfolgt danach eine kurze Lernphase, damit Sie sich besser mit ihnen vertraut machen können. Die anschließenden Probedurchgänge geben Gelegenheit, die beste Sitzposition für den Versuch zu finden und den Versuchsablauf kennenzulernen.

Sie starten die einzelnen Durchgänge, indem Sie auf der vor Ihnen befindlichen Tastatur eine Taste drücken. 


\section{Instruktionsblatt}

Instruktion für die Lernphase

Wie Sie wissen, werden in dieser Untersuchung neben den bereits bekannten Zahlwörtern noch sogenannte "Zufallsfiguren" verwendet. In jedem der Durchgänge der Lernphase wird jeweils nur eine dieser Figuren kurz gezeigt. Diese Figur soll gemerkt werden. Unmittelbar danach werden gleichzeitig alle 10 Figuren gezeigt und die zuvor dargebotene Figur soll aus diesen identifiziert werden. Hierzu ist die entsprechende Figur mit einem Stift auf dem Bildschirm ("touch screen") anzutippen.

In der Lernphase kommt es nicht wie in den späteren Versuchsabschnitten auf eine möglichst schnelle Bearbeitung an, sondern eher darauf, sich gut mit dem Material vertraut zu machen. 


\section{Instruktionsblatt}

Bevor wir mit dem eigentlichen Versuch beginnen, noch zwei wichtige Informationen:

Sie sollten versuchen, sich die Zahlwörter durch innere Wiederholung der Reihen zu merken. Bei den Zufallsfiguren hingegen sollten Sie sich um eine "bildliche" Speicherung bemühen. Es ist wichtig, daß sie andere mögliche Vorgehensweisen beim Merken der Reihen auf jeden Fall unterdrücken. Sollten dabei Schwierigkeiten auftreten, so können wir darüber während der Pausen zwischen den einzelnen Versuchsabschnitten sprechen.

Für die störungsfreie EEG-Aufzeichnung ist es von großer Bedeutung, daß Sie vor allem während der einzelnen Durchgänge ruhig und gleichmäßig atmen, entspannt sitzen, den Kopf nicht bewegen. möglichst nicht mit den Augen "zwinkern" und nicht laut sprechen. Sollten Sie außerhalb der "größeren" Pausen das Bedürfnis verspüren, Ihre Sitzposition zu korrigieren, sich zu strecken o. ä., so nutzen Sie dafür bitte die Pausen zwischen den einzelnen Durchgängen. Achten Sie darauf, anschließend vor dem Start des nächsten Durchgangs wieder eine bequeme Haltung einzunehmen und sich voll auf den Versuch zu konzentrieren. Nehmen Sie sich ruhig die Zeit, die Sie dafür benötigen, achten Sie jedoch während der einzelnen Durchgänge darauf, daß Ihre Tastenantwort auf den Prüfreiz möglichst rasch und richtig erfolgt!

Wenn Sie Fragen haben, so stellen Sie diese bitte jetzt. Sie starten die einzelnen Durchgänge, indem Sie auf der vor Ihnen befindlichen Tastatur eine Taste drücken. 


\section{Anhang B}
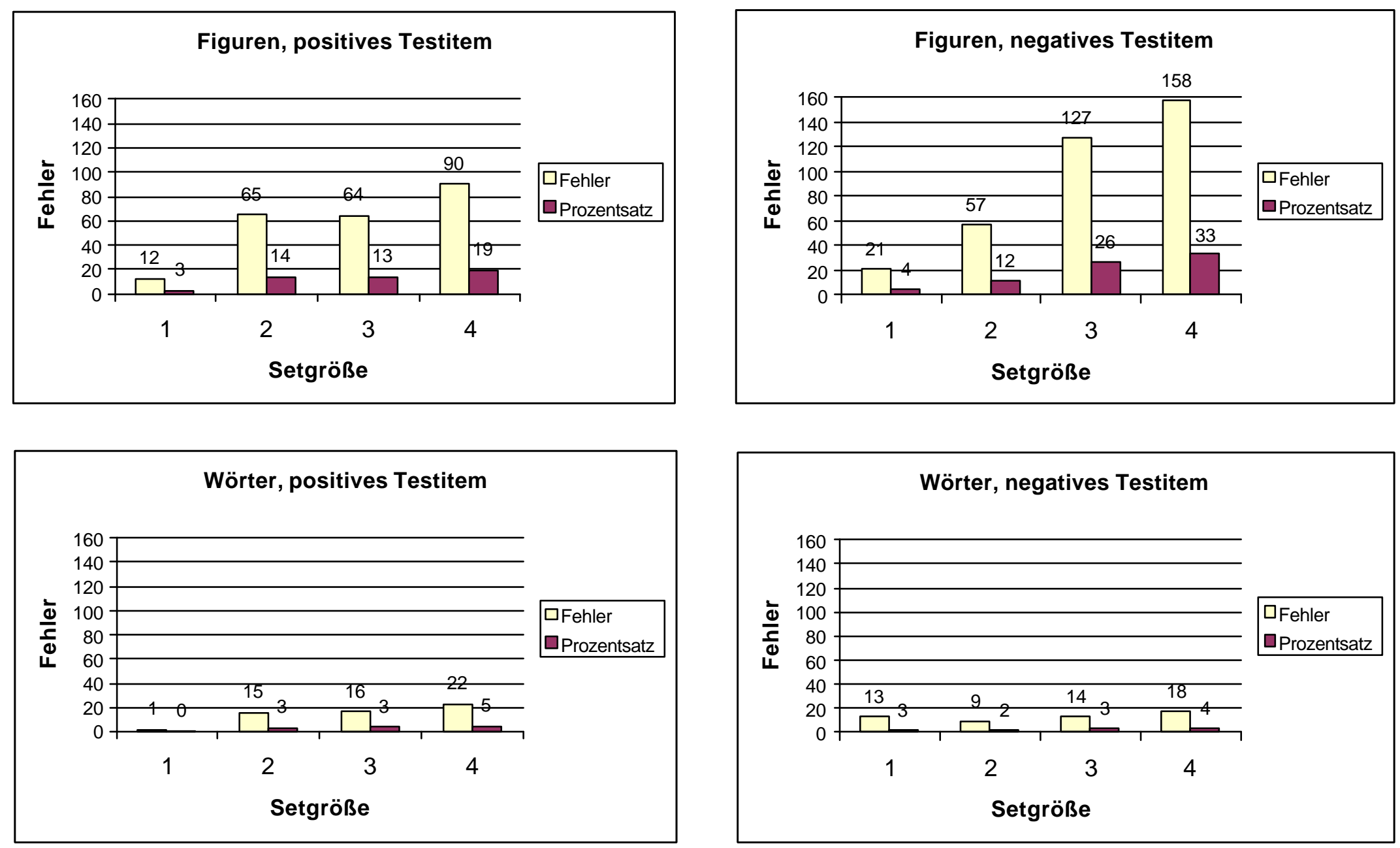

Abbildung B-1. Gesamtanzahl und Prozentsatz der Falschantworten über alle Probandinnen pro Versuchsbedingung (1. Sitzung) 


\section{Anhang B}
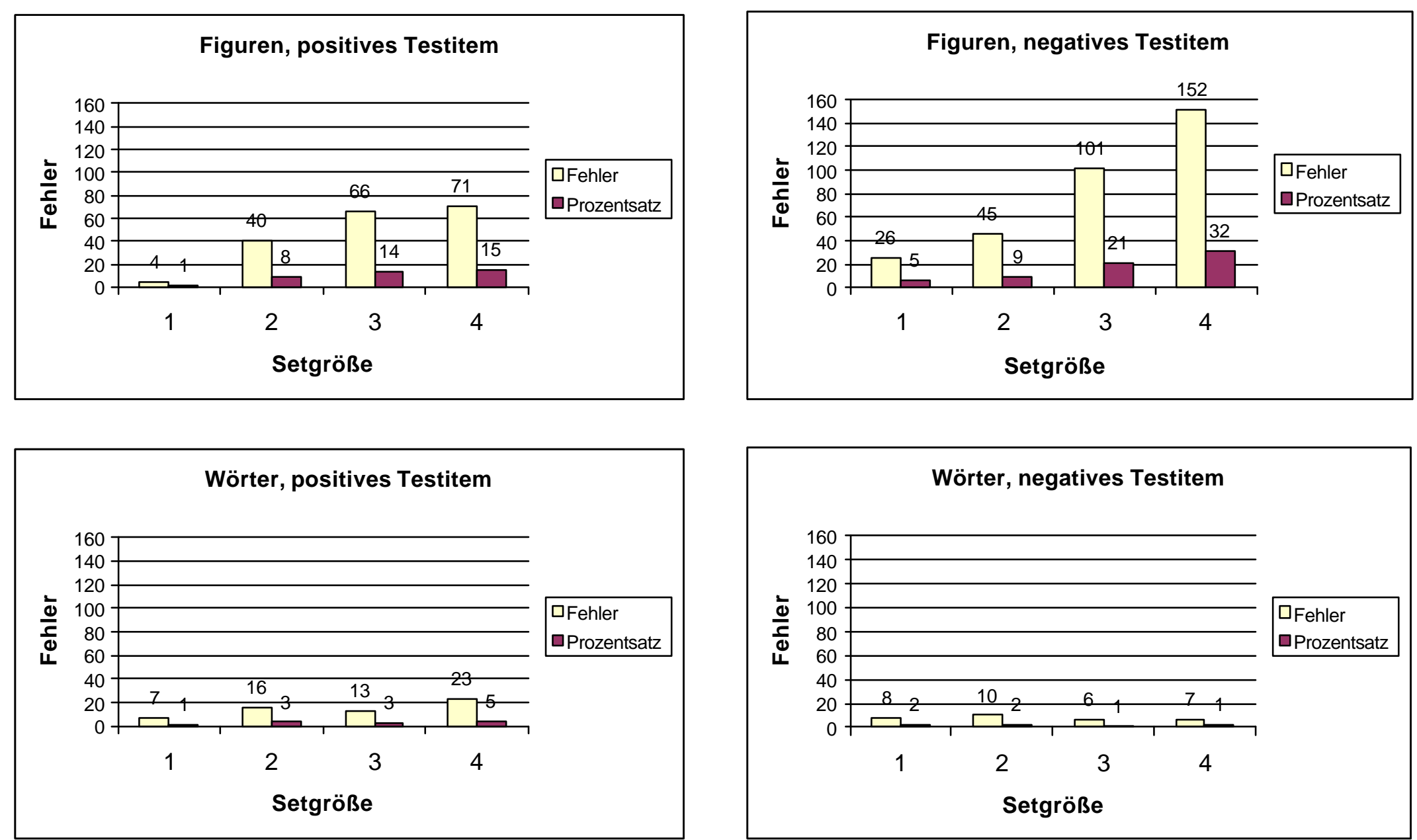

Abbildung B-2. Gesamtanzahl und Prozentsatz der Falschantworten über alle Probandinnen pro Versuchsbedingung (2. Sitzung) 


\section{Anhang B}

\section{Tabelle B-1}

Übersicht über Anzahl gültiger Durchgänge pro Versuchsbedingung

\begin{tabular}{|l|c|c|c|c|c|c|c|c|}
\multicolumn{1}{c}{} & \multicolumn{8}{c|}{ Positives Testitem (Testreiz in Lernliste vorhanden) } \\
\cline { 2 - 9 } \multicolumn{1}{c|}{ Setgröße } & \multicolumn{4}{c|}{} & \multicolumn{4}{c|}{ Wörter } \\
\hline 1. Sitzung & 4 & 2 & 3 & 4 & 1 & 2 & 3 & 4 \\
\hline 2. Sitzung & 468 & 415 & 416 & 390 & 479 & 465 & 464 & 458 \\
\hline
\end{tabular}

Negatives Testitem (Testreiz in Lernliste nicht vorhanden)

\begin{tabular}{|l|c|c|c|c|c|c|c|c|}
\cline { 2 - 9 } \multicolumn{1}{c|}{} & \multicolumn{4}{c|}{ Figuren } & \multicolumn{4}{c|}{ Wörter } \\
Setgröße & 1 & 2 & 3 & 4 & 1 & 2 & 3 & 4 \\
\hline 1. Sitzung & 459 & 423 & 353 & 322 & 467 & 471 & 466 & 462 \\
\hline 2. Sitzung & 454 & 435 & 379 & 328 & 472 & 470 & 474 & 473 \\
\hline
\end{tabular}




\section{Anhang B}

\section{Tabelle $B-2$}

Gesamtanzahl der Ausreißer über alle Probandinnen pro Versuchsbedingung

\begin{tabular}{|l|c|c|c|c|c|c|c|c|}
\multicolumn{1}{c}{} & \multicolumn{7}{c|}{ Positives Testitem (Testreiz in Lernliste vorhanden) } \\
\cline { 2 - 10 } & \multicolumn{7}{c}{ Figuren } & \multicolumn{5}{c|}{ Wörter } \\
Setgröße & 1 & 2 & 3 & 4 & 1 & 2 & 3 & 4 \\
\hline $\begin{array}{l}\text { Ausreißer } \\
\text { 1. Sitzung }\end{array}$ & 22 & 12 & 7 & 8 & 25 & 17 & 22 & 22 \\
\hline $\begin{array}{l}\text { gültige } \\
\text { Durchgänge }\end{array}$ & 468 & 415 & 416 & 390 & 479 & 465 & 464 & 458 \\
\hline Prozentsatz & $4.70 \%$ & $2.89 \%$ & $1.68 \%$ & $2.05 \%$ & $5.22 \%$ & $3.66 \%$ & $4.74 \%$ & $4.80 \%$ \\
\hline $\begin{array}{l}\text { Ausreißer } \\
\text { 2. Sitzung }\end{array}$ & 15 & 7 & 13 & 16 & 19 & 14 & 16 & 17 \\
\hline $\begin{array}{l}\text { gültige } \\
\text { Durchgänge }\end{array}$ & 476 & 440 & 414 & 409 & 473 & 464 & 467 & 457 \\
\hline Prozentsatz & $3.15 \%$ & $1.59 \%$ & $3.14 \%$ & $3.91 \%$ & $4.02 \%$ & $3.02 \%$ & $3.43 \%$ & $3.72 \%$ \\
\hline
\end{tabular}

Negatives Testitem (Testreiz in Lernliste nicht vorhanden)

\begin{tabular}{|c|c|c|c|c|c|c|c|c|}
\hline \multirow[b]{2}{*}{ Setgröße } & \multicolumn{4}{|c|}{ Figuren } & \multicolumn{4}{|c|}{ Wörter } \\
\hline & 1 & 2 & 3 & 4 & 1 & 2 & 3 & 4 \\
\hline $\begin{array}{l}\text { Ausreißer } \\
\text { 1. Sitzung }\end{array}$ & 29 & 19 & 7 & 3 & 20 & 17 & 20 & 19 \\
\hline $\begin{array}{l}\text { gültige } \\
\text { Durchgänge }\end{array}$ & 459 & 423 & 353 & 322 & 467 & 471 & 466 & 462 \\
\hline Prozentsatz & $6.32 \%$ & $4.49 \%$ & $1.98 \%$ & $0.93 \%$ & $4.28 \%$ & $3.61 \%$ & $4.29 \%$ & $4.11 \%$ \\
\hline $\begin{array}{l}\text { Ausreißer } \\
\text { 2. Sitzung }\end{array}$ & 32 & 12 & 11 & 14 & 30 & 19 & 29 & 17 \\
\hline $\begin{array}{l}\text { gültige } \\
\text { Durchgänge }\end{array}$ & 454 & 435 & 379 & 328 & 472 & 470 & 474 & 473 \\
\hline Prozentsatz & $7.05 \%$ & $2.76 \%$ & $2.90 \%$ & $4.27 \%$ & $6.36 \%$ & $4.04 \%$ & $6.12 \%$ & $3.59 \%$ \\
\hline
\end{tabular}

Anmerkung: Die pro Reizart, Sitzung, Testitem und Setgröße maximal möglichen Ausreißer über alle Probandinnen entspricht der Anzahl gültiger Durchgänge. 


\section{Anhang B}
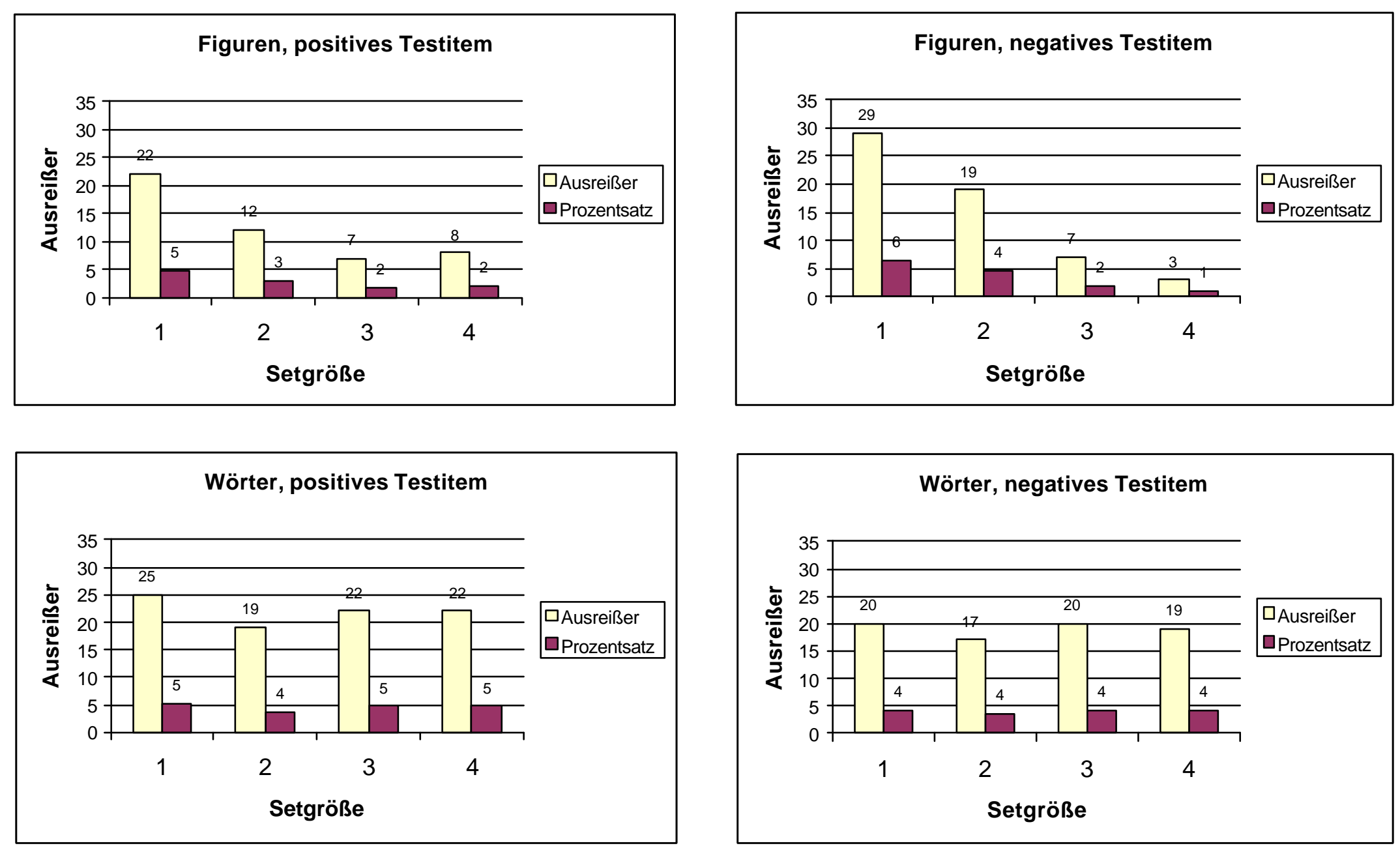

Abbildung B-3. Gesamtanzahl und Prozentsatz der Ausreißer über alle Probandinnen pro Versuchsbedingung (1. Sitzung) 


\section{Anhang B}
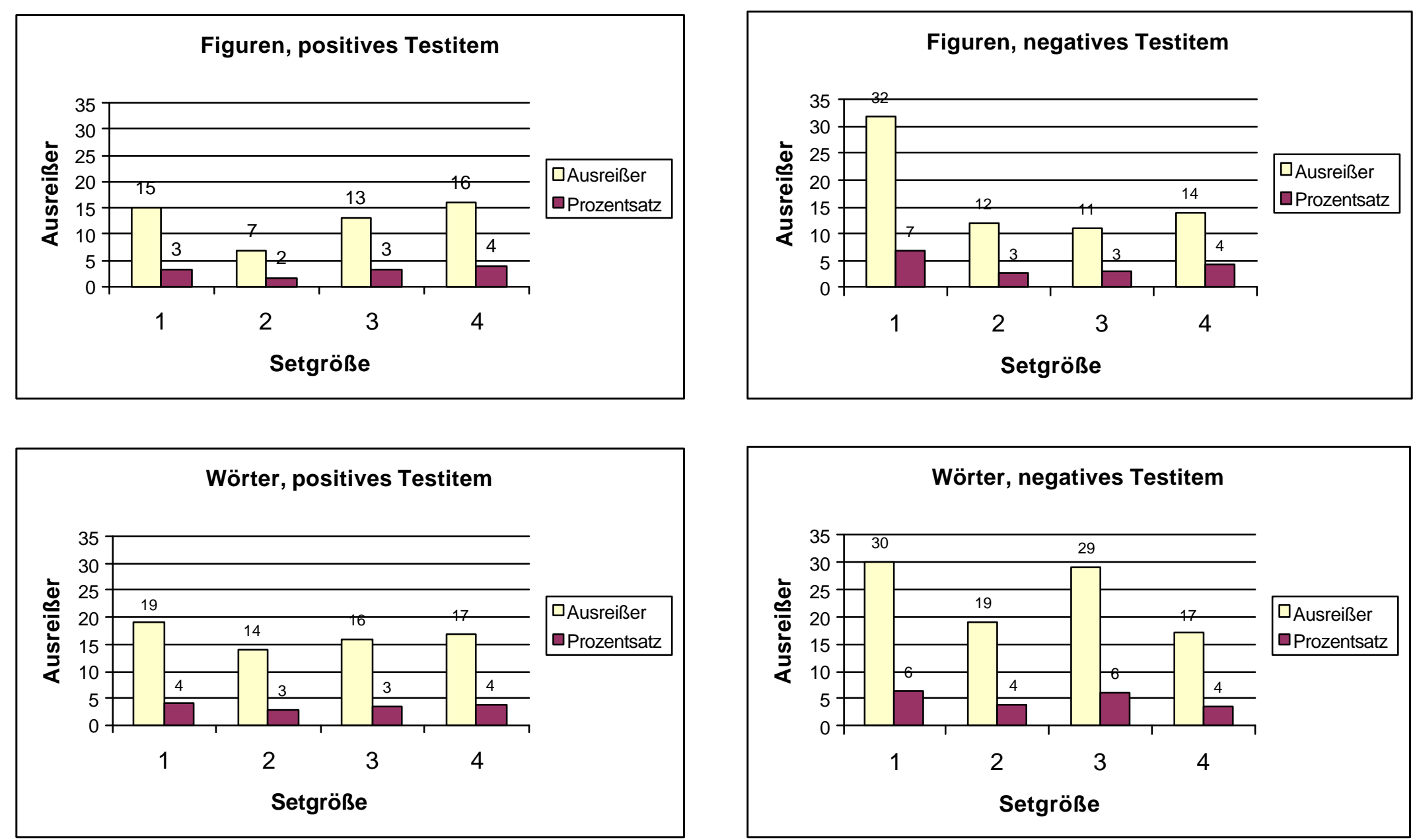

Abbildung B-4. Gesamtanzahl und Prozentsatz der Ausreißer über alle Probandinnen pro Versuchsbedingung (2. Sitzung) 


\section{Anhang B}
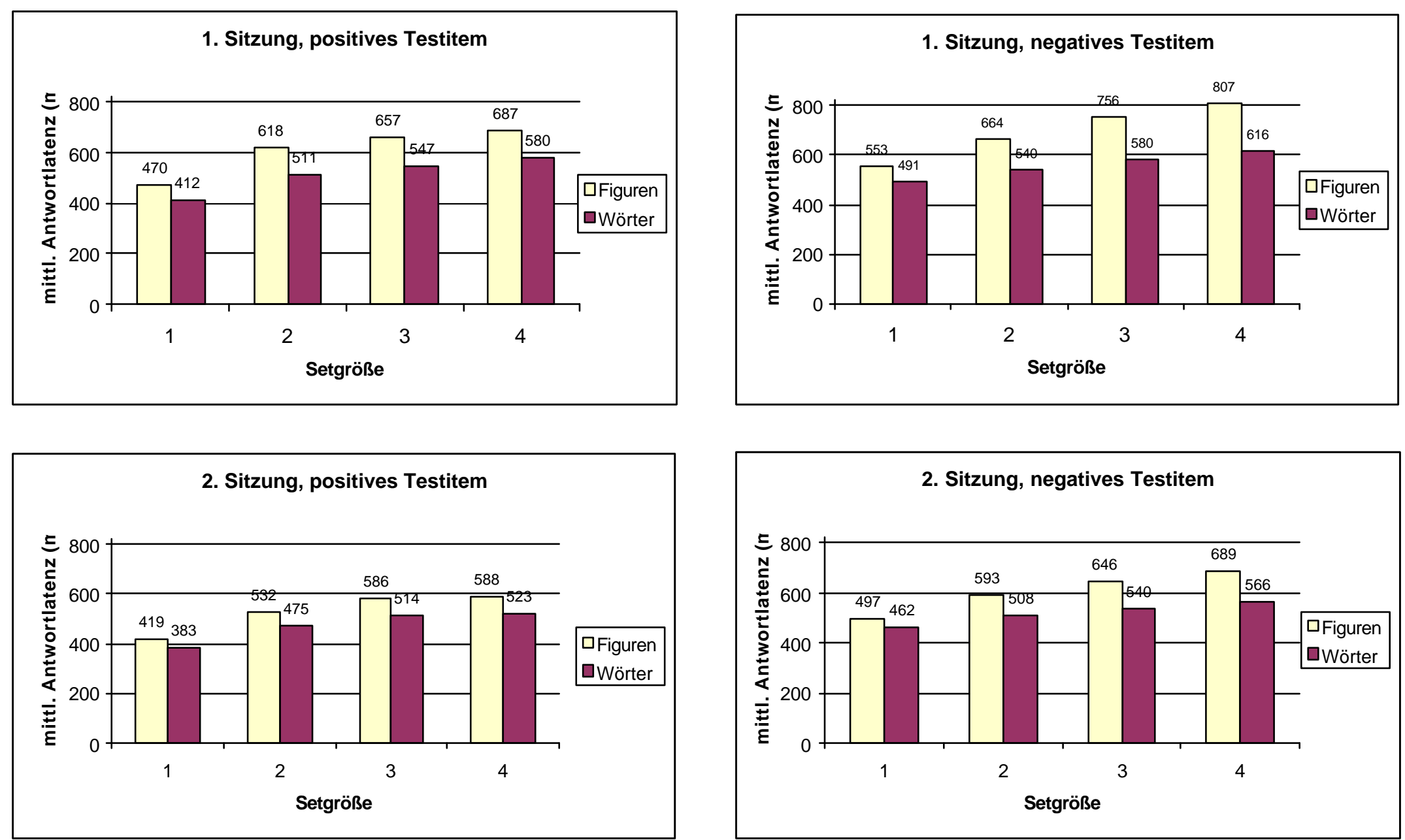

Abbildung B-5. Mittlere Antwortlatenzen über alle Probandinnen pro Versuchsbedingung (ms) 


\section{Anhang B}
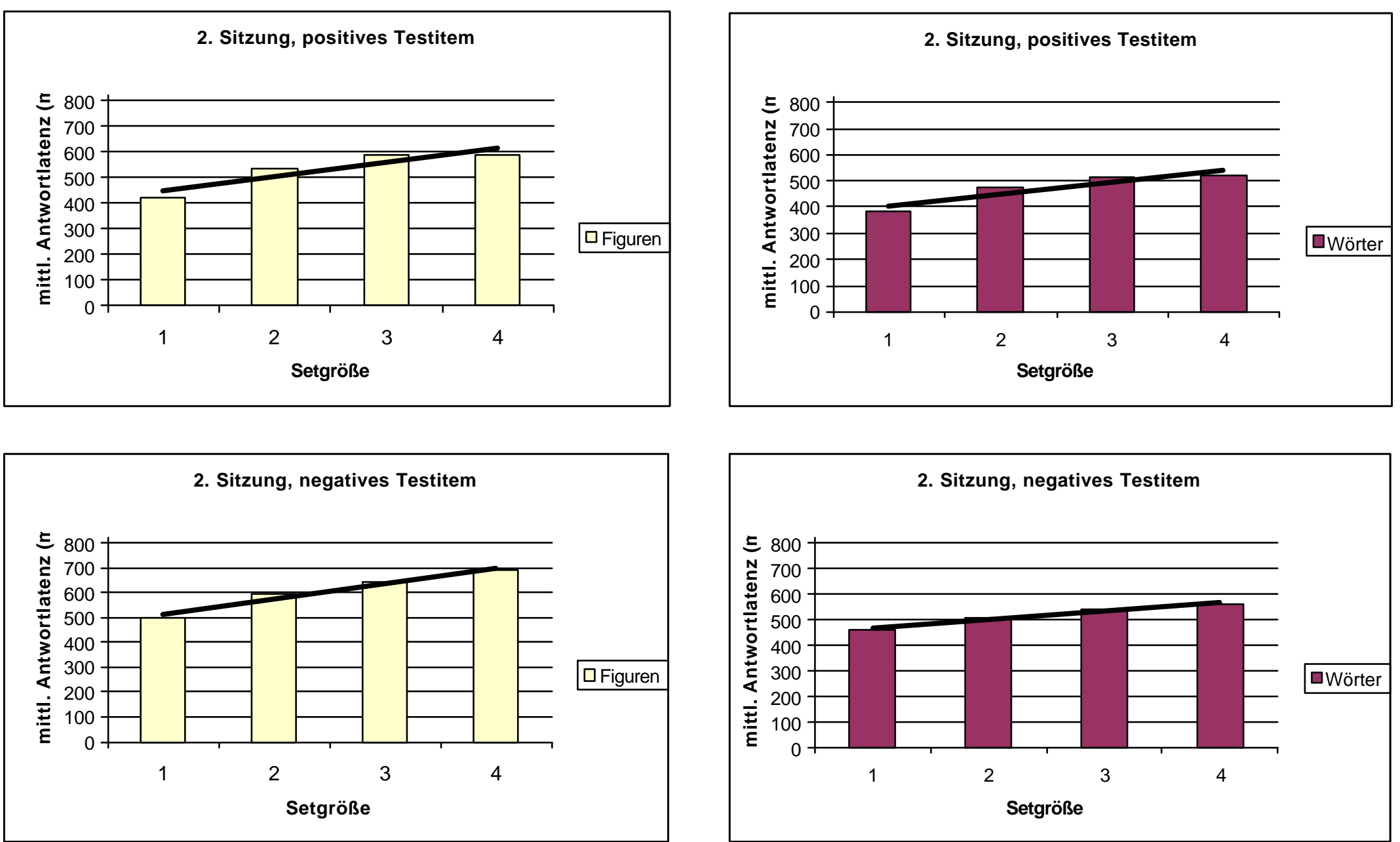

Abbildung B-6. Über alle Probandinnen gemittelte Antwortlatenzen (ms) pro Setgröße 


\section{Anhang B}

Tabelle $B-3$

Ergebnisse individueller Regressionsanalysen der pro Probandin gemittelten Antwortlatenzen und der Größe des positiven Sets

\begin{tabular}{|c|c|c|c|c|c|c|c|c|c|}
\hline$P b$ & Reizart & Testitem & Multiple $R$ & $R^{2}$ & sign. $F$ & Slope & $p$ (sign.) & Intercept & $p$ (sign.) \\
\hline 1 & Figuren & positiv & 0.304 & 0.092 & 0.000 & 36.034 & 0.000 & 522.196 & 0.000 \\
\hline 2 & Figuren & positiv & 0.442 & 0.196 & 0.000 & 43.091 & 0.000 & 350.045 & 0.000 \\
\hline 3 & Figuren & positiv & 0.165 & 0.027 & 0.175 & 12.471 & 0.175 & 357.852 & 0.000 \\
\hline 4 & Figuren & positiv & 0.439 & 0.193 & 0.000 & 70.059 & 0.000 & 466.752 & 0.000 \\
\hline 5 & Figuren & positiv & 0.571 & 0.325 & 0.000 & 81.277 & 0.000 & 359.094 & 0.000 \\
\hline 6 & Figuren & positiv & 0.438 & 0.192 & 0.000 & 47.923 & 0.000 & 306.862 & 0.000 \\
\hline 7 & Figuren & positiv & 0.586 & 0.343 & 0.000 & 109.671 & 0.000 & 324.341 & 0.000 \\
\hline 8 & Figuren & positiv & 0.418 & 0.175 & 0.000 & 61.252 & 0.000 & 406.773 & 0.000 \\
\hline 9 & Figuren & positiv & 0.441 & 0.194 & 0.000 & 44.384 & 0.000 & 373.601 & 0.000 \\
\hline 10 & Figuren & positiv & 0.537 & 0.288 & 0.000 & 67.397 & 0.000 & 410.087 & 0.000 \\
\hline 1 & Wörter & positiv & 0.444 & 0.197 & 0.000 & 50.250 & 0.000 & 447.062 & 0.000 \\
\hline 2 & Wörter & positiv & 0.608 & 0.370 & 0.000 & 38.058 & 0.000 & 333.585 & 0.000 \\
\hline 3 & Wörter & positiv & 0.483 & 0.234 & 0.000 & 33.683 & 0.000 & 352.246 & 0.000 \\
\hline 4 & Wörter & positiv & 0.384 & 0.148 & 0.000 & 45.464 & 0.000 & 462.037 & 0.000 \\
\hline 5 & Wörter & positiv & 0.665 & 0.443 & 0.000 & 50.287 & 0.000 & 321.053 & 0.000 \\
\hline 6 & Wörter & positiv & 0.647 & 0.419 & 0.000 & 56.221 & 0.000 & 271.964 & 0.000 \\
\hline 7 & Wörter & positiv & 0.629 & 0.395 & 0.000 & 56.014 & 0.000 & 306.214 & 0.000 \\
\hline 8 & Wörter & positiv & 0.495 & 0.245 & 0.000 & 52.091 & 0.000 & 373.095 & 0.000 \\
\hline 9 & Wörter & positiv & 0.554 & 0.307 & 0.000 & 42.321 & 0.000 & 348.543 & 0.000 \\
\hline 10 & Wörter & positiv & 0.562 & 0.315 & 0.000 & 38.713 & 0.000 & 364.079 & 0.000 \\
\hline 1 & Figuren & negativ & 0.333 & 0.111 & 0.000 & 31.002 & 0.000 & 590.988 & 0.000 \\
\hline 2 & Figuren & negativ & 0.635 & 0.404 & 0.000 & 62.631 & 0.000 & 393.407 & 0.000 \\
\hline 3 & Figuren & negativ & 0.233 & 0.054 & 0.097 & 18.572 & 0.097 & 455.040 & 0.000 \\
\hline 4 & Figuren & negativ & 0.361 & 0.131 & 0.000 & 57.965 & 0.000 & 521.154 & 0.000 \\
\hline 5 & Figuren & negativ & 0.542 & 0.294 & 0.000 & 62.891 & 0.000 & 429.303 & 0.000 \\
\hline 6 & Figuren & negativ & 0.492 & 0.242 & 0.000 & 49.308 & 0.000 & 422.695 & 0.000 \\
\hline 7 & Figuren & negativ & 0.647 & 0.418 & 0.000 & 104.098 & 0.000 & 417.676 & 0.000 \\
\hline 8 & Figuren & negativ & 0.574 & 0.330 & 0.000 & 81.128 & 0.000 & 427.014 & 0.000 \\
\hline 9 & Figuren & negativ & 0.729 & 0.531 & 0.000 & 96.106 & 0.000 & 379.451 & 0.000 \\
\hline 10 & Figuren & negativ & 0.726 & 0.526 & 0.000 & 81.387 & 0.000 & 423.184 & 0.000 \\
\hline 1 & Wörter & negativ & 0.130 & 0.017 & 0.105 & 11.193 & 0.105 & 559.155 & 0.000 \\
\hline 2 & Wörter & negativ & 0.628 & 0.395 & 0.000 & 39.692 & 0.000 & 364.146 & 0.000 \\
\hline 3 & Wörter & negativ & 0.546 & 0.299 & 0.000 & 41.954 & 0.000 & 367.951 & 0.000 \\
\hline 4 & Wörter & negativ & 0.275 & 0.076 & 0.001 & 30.389 & 0.001 & 501.883 & 0.000 \\
\hline 5 & Wörter & negativ & 0.463 & 0.215 & 0.000 & 32.042 & 0.000 & 406.166 & 0.000 \\
\hline 6 & Wörter & negativ & 0.542 & 0.293 & 0.000 & 39.190 & 0.000 & 406.476 & 0.000 \\
\hline 7 & Wörter & negativ & 0.422 & 0.178 & 0.000 & 31.844 & 0.000 & 447.613 & 0.000 \\
\hline 8 & Wörter & negativ & 0.463 & 0.214 & 0.000 & 41.258 & 0.000 & 423.930 & 0.000 \\
\hline 9 & Wörter & negativ & 0.648 & 0.419 & 0.000 & 38.910 & 0.000 & 420.347 & 0.000 \\
\hline 10 & Wörter & negativ & 0.455 & 0.207 & 0.000 & 33.371 & 0.000 & 445.086 & 0.000 \\
\hline
\end{tabular}




\section{Anhang B}

Tabelle $B-4$

Mittlere Kohärenzwerte: Ergebnisse der Wilcoxon-Tests zum Vergleich der Reizarten pro Setgröße in den Arealen 1 bis 7 ( $N=10$; zweiseitig)

\begin{tabular}{|c|c|c|c|c|c|c|c|c|c|c|}
\hline \multicolumn{11}{|c|}{ Mittlere Kohärenzwerte } \\
\hline \multirow[b]{2}{*}{ Areal } & \multirow[b]{2}{*}{ Set } & \multirow[b]{2}{*}{ 1. Var. } & \multirow[b]{2}{*}{ 2. Var. } & \multicolumn{3}{|c|}{ Anzahl Rangunterschiede } & \multicolumn{2}{|c|}{ Mittelwerte } & \multirow[b]{2}{*}{$z$-Wert } & \multirow[b]{2}{*}{$p$ (zweis.) } \\
\hline & & & & $W<F$ & $W>F$ & verbundene & $W<F$ & $W>F$ & & \\
\hline 1 & 1 & Figuren & Wörter & 7 & 3 & 0 & 5.57 & 5.33 & -1.172 & 0.241 \\
\hline 2 & 1 & Figuren & Wörter & 5 & 5 & 0 & 6.60 & 4.40 & -0.561 & 0.575 \\
\hline 3 & 1 & Figuren & Wörter & 4 & 6 & 0 & 5.00 & 5.83 & -0.765 & 0.445 \\
\hline 4 & 1 & Figuren & Wörter & 6 & 4 & 0 & 5.83 & 5.00 & -0.765 & 0.445 \\
\hline 5 & 1 & Figuren & Wörter & 6 & 4 & 0 & 3.83 & 8.00 & -0.459 & 0.647 \\
\hline 6 & 1 & Figuren & Wörter & 5 & 5 & 0 & 6.80 & 4.20 & -0.663 & 0.508 \\
\hline 7 & 1 & Figuren & Wörter & 5 & 5 & 0 & 5.40 & 5.60 & -0.051 & 0.959 \\
\hline 1 & 2 & Figuren & Wörter & 6 & 4 & 0 & 3.67 & 8.25 & -0.561 & 0.575 \\
\hline 2 & 2 & Figuren & Wörter & 5 & 5 & 0 & 6.60 & 4.40 & -0.561 & 0.575 \\
\hline 3 & 2 & Figuren & Wörter & 3 & 6 & 1 & 4.00 & 5.50 & -1.244 & 0.214 \\
\hline 4 & 2 & Figuren & Wörter & 3 & 7 & 0 & 5.33 & 5.57 & -1.172 & 0.241 \\
\hline 5 & 2 & Figuren & Wörter & 3 & 7 & 0 & 4.67 & 5.86 & -1.376 & 0.169 \\
\hline 6 & 2 & Figuren & Wörter & 5 & 5 & 0 & 4.20 & 6.80 & -0.663 & 0.508 \\
\hline 7 & 2 & Figuren & Wörter & 5 & 5 & 0 & 3.60 & 7.40 & -0.968 & 0.333 \\
\hline 1 & 3 & Figuren & Wörter & 5 & 5 & 0 & 6.20 & 4.80 & -0.357 & 0.721 \\
\hline 2 & 3 & Figuren & Wörter & 3 & 7 & 0 & 5.33 & 5.57 & -1.172 & 0.241 \\
\hline 3 & 3 & Figuren & Wörter & 6 & 4 & 0 & 4.75 & 6.63 & -0.102 & 0.919 \\
\hline 4 & 3 & Figuren & Wörter & 5 & 5 & 0 & 3.80 & 7.20 & -0.866 & 0.386 \\
\hline 5 & 3 & Figuren & Wörter & 3 & 7 & 0 & 5.00 & 5.71 & -1.274 & 0.203 \\
\hline 6 & 3 & Figuren & Wörter & 4 & 5 & 1 & 5.25 & 4.80 & -0.178 & 0.859 \\
\hline 7 & 3 & Figuren & Wörter & 4 & 6 & 0 & 4.00 & 6.50 & -1.172 & 0.241 \\
\hline 1 & 4 & Figuren & Wörter & 6 & 4 & 0 & 6.17 & 4.50 & -0.968 & 0.333 \\
\hline 2 & 4 & Figuren & Wörter & 6 & 4 & 0 & 5.67 & 5.25 & -0.663 & 0.508 \\
\hline 3 & 4 & Figuren & Wörter & 6 & 4 & 0 & 5.00 & 6.25 & -0.255 & 0.799 \\
\hline 4 & 4 & Figuren & Wörter & 6 & 4 & 0 & 4.50 & 7.00 & -0.051 & 0.959 \\
\hline 5 & 4 & Figuren & Wörter & 3 & 7 & 0 & 7.00 & 4.86 & -0.663 & 0.508 \\
\hline 6 & 4 & Figuren & Wörter & 6 & 4 & 0 & 6.67 & 3.75 & -1.274 & 0.203 \\
\hline 7 & 4 & Figuren & Wörter & 4 & 6 & 0 & 4.75 & 6.00 & -0.866 & 0.386 \\
\hline
\end{tabular}




\section{Anhang B}

Tabelle $B-5$

Mittlere Kohärenzdauern: Ergebnisse der Wilcoxon-Tests zum Vergleich der Reizarten pro Setgröße in den Arealen 1 bis $7(\mathrm{~N}=10$; zweiseitig)

\begin{tabular}{|c|c|c|c|c|c|c|c|c|c|c|}
\hline \multicolumn{11}{|c|}{ Mittlere Kohärenzdauern } \\
\hline \multirow[b]{2}{*}{ Areal } & \multirow[b]{2}{*}{ Set } & \multirow[b]{2}{*}{ 1. Var. } & \multirow[b]{2}{*}{ 2. Var. } & \multicolumn{3}{|c|}{ Anzahl Rangunterschiede } & \multicolumn{2}{|c|}{ Mittelwerte } & \multirow[b]{2}{*}{$z$-Wert } & \multirow[b]{2}{*}{$\mathrm{p}$ (zweis.) } \\
\hline & & & & $W<F$ & $W>F$ & verbundene & $W<F$ & $W>F$ & & \\
\hline 1 & 1 & Figuren & Wörter & 9 & 1 & 0 & 6.00 & 1.00 & -2.701 & 0.007 \\
\hline 2 & 1 & Figuren & Wörter & 7 & 3 & 0 & 6.29 & 3.67 & -1.682 & 0.093 \\
\hline 3 & 1 & Figuren & Wörter & 5 & 5 & 0 & 7.20 & 3.80 & -0.866 & 0.386 \\
\hline 4 & 1 & Figuren & Wörter & 8 & 2 & 0 & 6.50 & 1.50 & -2.497 & 0.013 \\
\hline 5 & 1 & Figuren & Wörter & 8 & 2 & 0 & 5.63 & 5.00 & -1.784 & 0.075 \\
\hline 6 & 1 & Figuren & Wörter & 9 & 1 & 0 & 5.89 & 2.00 & -2.599 & 0.009 \\
\hline 7 & 1 & Figuren & Wörter & 9 & 1 & 0 & 5.67 & 4.00 & -2.395 & 0.017 \\
\hline 1 & 2 & Figuren & Wörter & 8 & 2 & 0 & 6.13 & 3.00 & -2.192 & 0.028 \\
\hline 2 & 2 & Figuren & Wörter & 8 & 2 & 0 & 6.50 & 1.50 & -2.497 & 0.013 \\
\hline 3 & 2 & Figuren & Wörter & 6 & 4 & 0 & 5.50 & 5.50 & -0.561 & 0.575 \\
\hline 4 & 2 & Figuren & Wörter & 8 & 2 & 0 & 6.00 & 3.50 & -2.090 & 0.037 \\
\hline 5 & 2 & Figuren & Wörter & 9 & 1 & 0 & 5.56 & 5.00 & -2.293 & 0.022 \\
\hline 6 & 2 & Figuren & Wörter & 8 & 2 & 0 & 6.25 & 2.50 & -2.293 & 0.022 \\
\hline 7 & 2 & Figuren & Wörter & 7 & 3 & 0 & 6.71 & 2.67 & -1.988 & 0.047 \\
\hline 1 & 3 & Figuren & Wörter & 8 & 2 & 0 & 6.00 & 3.50 & -2.090 & 0.037 \\
\hline 2 & 3 & Figuren & Wörter & 8 & 2 & 0 & 5.25 & 6.50 & -1.478 & 0.139 \\
\hline 3 & 3 & Figuren & Wörter & 6 & 4 & 0 & 7.00 & 3.25 & -1.478 & 0.139 \\
\hline 4 & 3 & Figuren & Wörter & 7 & 3 & 0 & 6.71 & 2.67 & -1.988 & 0.047 \\
\hline 5 & 3 & Figuren & Wörter & 7 & 3 & 0 & 5.57 & 5.33 & -1.172 & 0.241 \\
\hline 6 & 3 & Figuren & Wörter & 9 & 1 & 0 & 5.33 & 7.00 & -2.090 & 0.037 \\
\hline 7 & 3 & Figuren & Wörter & 8 & 2 & 0 & 5.63 & 5.00 & -1.784 & 0.075 \\
\hline 1 & 4 & Figuren & Wörter & 8 & 2 & 0 & 5.75 & 4.50 & -1.886 & 0.059 \\
\hline 2 & 4 & Figuren & Wörter & 7 & 3 & 0 & 5.57 & 5.33 & -1.172 & 0.241 \\
\hline 3 & 4 & Figuren & Wörter & 7 & 3 & 0 & 6.29 & 3.67 & -1.682 & 0.093 \\
\hline 4 & 4 & Figuren & Wörter & 7 & 3 & 0 & 6.00 & 4.33 & -1.478 & 0.139 \\
\hline 5 & 4 & Figuren & Wörter & 7 & 3 & 0 & 5.86 & 4.67 & -1.376 & 0.169 \\
\hline 6 & 4 & Figuren & Wörter & 7 & 3 & 0 & 5.86 & 4.67 & -1.376 & 0.169 \\
\hline 7 & 4 & Figuren & Wörter & 7 & 3 & 0 & 5.57 & 5.33 & -1.172 & 0.241 \\
\hline
\end{tabular}




\section{Anhang B}

Tabelle $B-6$

Mittlere maximale Kohärenzwerte: Ergebnisse der Wilcoxon-Tests zum Vergleich der Reizarten pro Setgröße in den Arealen 1 bis $7(\mathrm{~N}=10$; zweiseitig)

\begin{tabular}{|c|c|c|c|c|c|c|c|c|c|c|}
\hline \multicolumn{11}{|c|}{ Mittlere maximale Kohärenzwerte } \\
\hline \multirow[b]{2}{*}{ Areal } & \multirow[b]{2}{*}{ Set } & \multirow[b]{2}{*}{ 1. Var. } & \multirow[b]{2}{*}{ 2. Var. } & \multicolumn{3}{|c|}{ Anzahl Rangunterschiede } & \multicolumn{2}{|c|}{ Mittelwerte } & \multirow[b]{2}{*}{$z$-Wert } & \multirow[b]{2}{*}{ p (zweis.) } \\
\hline & & & & $W<F$ & $W>F$ & verbundene & $W<F$ & $W>F$ & & \\
\hline 1 & 1 & Figuren & Wörter & 7 & 3 & 0 & 5.86 & 4.67 & -1.376 & 0.169 \\
\hline 2 & 1 & Figuren & Wörter & 6 & 4 & 0 & 6.17 & 4.50 & -0.968 & 0.333 \\
\hline 3 & 1 & Figuren & Wörter & 4 & 6 & 0 & 4.75 & 6.00 & -0.866 & 0.386 \\
\hline 4 & 1 & Figuren & Wörter & 7 & 3 & 0 & 5.71 & 5.00 & -1.274 & 0.203 \\
\hline 5 & 1 & Figuren & Wörter & 5 & 5 & 0 & 5.20 & 5.80 & -0.153 & 0.879 \\
\hline 6 & 1 & Figuren & Wörter & 5 & 5 & 0 & 6.40 & 4.60 & -0.459 & 0.647 \\
\hline 7 & 1 & Figuren & Wörter & 5 & 5 & 0 & 4.80 & 6.20 & -0.357 & 0.721 \\
\hline 1 & 2 & Figuren & Wörter & 5 & 5 & 0 & 6.20 & 4.80 & -0.357 & 0.721 \\
\hline 2 & 2 & Figuren & Wörter & 7 & 3 & 0 & 5.57 & 5.33 & -1.172 & 0.241 \\
\hline 3 & 2 & Figuren & Wörter & 5 & 5 & 0 & 3.40 & 7.60 & -1.070 & 0.285 \\
\hline 4 & 2 & Figuren & Wörter & 5 & 5 & 0 & 4.20 & 6.80 & -0.663 & 0.508 \\
\hline 5 & 2 & Figuren & Wörter & 4 & 6 & 0 & 6.25 & 5.00 & -0.255 & 0.799 \\
\hline 6 & 2 & Figuren & Wörter & 4 & 6 & 0 & 7.50 & 4.17 & -0.255 & 0.799 \\
\hline 7 & 2 & Figuren & Wörter & 5 & 5 & 0 & 4.00 & 7.00 & -0.765 & 0.445 \\
\hline 1 & 3 & Figuren & Wörter & 5 & 5 & 0 & 5.00 & 6.00 & -0.255 & 0.799 \\
\hline 2 & 3 & Figuren & Wörter & 4 & 6 & 0 & 6.25 & 5.00 & -0.255 & 0.799 \\
\hline 3 & 3 & Figuren & Wörter & 5 & 5 & 0 & 5.80 & 5.20 & -0.153 & 0.879 \\
\hline 4 & 3 & Figuren & Wörter & 5 & 5 & 0 & 4.60 & 6.40 & -0.459 & 0.647 \\
\hline 5 & 3 & Figuren & Wörter & 3 & 7 & 0 & 4.67 & 5.86 & -1.376 & 0.169 \\
\hline 6 & 3 & Figuren & Wörter & 3 & 7 & 0 & 7.67 & 4.57 & -0.459 & 0.647 \\
\hline 7 & 3 & Figuren & Wörter & 5 & 5 & 0 & 3.60 & 7.40 & -0.968 & 0.333 \\
\hline 1 & 4 & Figuren & Wörter & 6 & 4 & 0 & 7.17 & 3.00 & -1.580 & 0.114 \\
\hline 2 & 4 & Figuren & Wörter & 5 & 5 & 0 & 6.00 & 5.00 & -0.255 & 0.799 \\
\hline 3 & 4 & Figuren & Wörter & 5 & 5 & 0 & 5.60 & 5.40 & -0.051 & 0.959 \\
\hline 4 & 4 & Figuren & Wörter & 7 & 3 & 0 & 5.29 & 6.00 & -0.968 & 0.333 \\
\hline 5 & 4 & Figuren & Wörter & 6 & 4 & 0 & 5.50 & 5.50 & -0.561 & 0.575 \\
\hline 6 & 4 & Figuren & Wörter & 7 & 3 & 0 & 6.14 & 4.00 & -1.580 & 0.114 \\
\hline 7 & 4 & Figuren & Wörter & 5 & 5 & 0 & 5.40 & 5.60 & -0.051 & 0.959 \\
\hline
\end{tabular}




\section{Anhang B}

Tabelle B-7

Mittlere Zeitpunkte maximaler Kohärenz: Ergebnisse der Wilcoxon-Tests zum Vergleich der Reizarten pro Setgröße in den Arealen 1 bis 7 ( $N=10$; zweiseitig)

\begin{tabular}{|c|c|c|c|c|c|c|c|c|c|c|}
\hline \multicolumn{11}{|c|}{ Mittlere Kohärenzwerte } \\
\hline \multirow[b]{2}{*}{ Areal } & \multirow[b]{2}{*}{ Set } & \multirow[b]{2}{*}{ 1. Var. } & \multirow[b]{2}{*}{ 2. Var. } & \multicolumn{3}{|c|}{ Anzahl Rangunterschiede } & \multicolumn{2}{|c|}{ Mittelwerte } & \multirow[b]{2}{*}{ z-Wert } & \multirow[b]{2}{*}{ p (zweis.) } \\
\hline & & & & $W<F$ & $W>F$ & verbundene & $W<F$ & $W>F$ & & \\
\hline 1 & 1 & Figuren & Wörter & 7 & 3 & 0 & 5.86 & 4.67 & -1.376 & 0.169 \\
\hline 2 & 1 & Figuren & Wörter & 7 & 3 & 0 & 7.00 & 2.00 & -2.192 & 0.028 \\
\hline 3 & 1 & Figuren & Wörter & 6 & 4 & 0 & 3.83 & 8.00 & -0.459 & 0.647 \\
\hline 4 & 1 & Figuren & Wörter & 8 & 2 & 0 & 5.50 & 5.50 & -1.682 & 0.093 \\
\hline 5 & 1 & Figuren & Wörter & 6 & 4 & 0 & 6.00 & 4.75 & -0.866 & 0.386 \\
\hline 6 & 1 & Figuren & Wörter & 5 & 5 & 0 & 6.20 & 4.80 & -0.357 & 0.721 \\
\hline 7 & 1 & Figuren & Wörter & 6 & 4 & 0 & 7.00 & 3.25 & -1.478 & 0.139 \\
\hline 1 & 2 & Figuren & Wörter & 8 & 2 & 0 & 6.00 & 3.50 & -2.090 & 0.037 \\
\hline 2 & 2 & Figuren & Wörter & 6 & 4 & 0 & 7.50 & 2.50 & -1.784 & 0.075 \\
\hline 3 & 2 & Figuren & Wörter & 8 & 2 & 0 & 6.38 & 2.00 & -2.395 & 0.017 \\
\hline 4 & 2 & Figuren & Wörter & 8 & 2 & 0 & 5.75 & 4.50 & -1.886 & 0.059 \\
\hline 5 & 2 & Figuren & Wörter & 8 & 2 & 0 & 6.50 & 1.50 & -2.497 & 0.013 \\
\hline 6 & 2 & Figuren & Wörter & 7 & 3 & 0 & 6.86 & 2.33 & -2.090 & 0.037 \\
\hline 7 & 2 & Figuren & Wörter & 8 & 2 & 0 & 6.25 & 2.50 & -2.293 & 0.022 \\
\hline 1 & 3 & Figuren & Wörter & 7 & 3 & 0 & 6.86 & 2.33 & -2.090 & 0.037 \\
\hline 2 & 3 & Figuren & Wörter & 9 & 1 & 0 & 5.89 & 2.00 & -2.599 & 0.009 \\
\hline 3 & 3 & Figuren & Wörter & 9 & 1 & 0 & 6.00 & 1.00 & -2.701 & 0.007 \\
\hline 4 & 3 & Figuren & Wörter & 9 & 1 & 0 & 5.44 & 6.00 & -2.192 & 0.028 \\
\hline 5 & 3 & Figuren & Wörter & 8 & 2 & 0 & 6.13 & 3.00 & -2.192 & 0.028 \\
\hline 6 & 3 & Figuren & Wörter & 8 & 2 & 0 & 5.88 & 4.00 & -1.988 & 0.047 \\
\hline 7 & 3 & Figuren & Wörter & 10 & 0 & 0 & 5.50 & 0.00 & -2.803 & 0.005 \\
\hline 1 & 4 & Figuren & Wörter & 6 & 4 & 0 & 7.17 & 3.00 & -1.580 & 0.114 \\
\hline 2 & 4 & Figuren & Wörter & 5 & 5 & 0 & 6.60 & 4.40 & -0.561 & 0.575 \\
\hline 3 & 4 & Figuren & Wörter & 7 & 3 & 0 & 6.43 & 3.33 & -1.784 & 0.075 \\
\hline 4 & 4 & Figuren & Wörter & 8 & 2 & 0 & 5.88 & 4.00 & -1.988 & 0.047 \\
\hline 5 & 4 & Figuren & Wörter & 6 & 4 & 0 & 6.83 & 3.50 & -1.376 & 0.169 \\
\hline 6 & 4 & Figuren & Wörter & 7 & 3 & 0 & 6.71 & 2.67 & -1.988 & 0.047 \\
\hline 7 & 4 & Figuren & Wörter & 7 & 3 & 0 & 6.29 & 3.67 & -1.682 & 0.093 \\
\hline
\end{tabular}




\section{Anhang B}

Tabelle $B-8$

Ergebnisse der linearen Regressionsanalysen der gemittelten Kohärenzen $\left(M_{K}\right)$ und der Größe des positiven Sets ( $N=10 ; 2$. Sitzung, Figuren, positives Testitem)

\begin{tabular}{|c|c|c|c|c|c|c|c|c|c|}
\hline $\begin{array}{c}\text { Elektr.- } \\
\text { Paar }\end{array}$ & $A V$ & Testitem & Multiple $R$ & $R^{2}$ & Sign. $F$ & Slope & $p$ (sign.) & Intercept & $p$ (sign.) \\
\hline 1 (Fp1/F7) & $M_{K}$ Figuren & positiv & 0.584 & 0.341 & 0.416 & 0.003 & 0.416 & 0.488 & 0.000 \\
\hline 2 (F7/F3) & $M_{K}$ Figuren & positiv & 0.222 & 0.049 & 0.778 & -0.001 & 0.778 & 0.557 & 0.000 \\
\hline $3(F 3 / F z)$ & $M_{k}$ Figuren & positiv & 0.495 & 0.245 & 0.506 & 0.002 & 0.506 & 0.721 & 0.000 \\
\hline $4(F z / F 4)$ & $M_{k}$ Figuren & positiv & 0.854 & 0.730 & 0.146 & 0.002 & 0.146 & 0.625 & 0.000 \\
\hline 5 (F4/F8) & $M_{K}$ Figuren & positiv & 0.629 & 0.396 & 0.371 & 0.007 & 0.371 & 0.494 & 0.001 \\
\hline $6($ T3/C3) & Mk Figuren & positiv & 0.089 & 0.008 & 0.911 & 0.000 & 0.911 & 0.437 & 0.000 \\
\hline $7(C 3 / C z)$ & Mk Figuren & positiv & 0.557 & 0.310 & 0.443 & 0.003 & 0.443 & 0.666 & 0.000 \\
\hline $8(\mathrm{Cz} / \mathrm{C} 4)$ & $M_{K}$ Figuren & positiv & 0.822 & 0.676 & 0.178 & 0.008 & 0.178 & 0.625 & 0.000 \\
\hline $9(C 4 / T 4)$ & $M_{K}$ Figuren & positiv & 0.971 & 0.943 & 0.029 & 0.008 & 0.029 & 0.396 & 0.000 \\
\hline $10(T 5 / P 3)$ & Mk Figuren & positiv & 0.046 & 0.002 & 0.954 & 0.000 & 0.954 & 0.559 & 0.000 \\
\hline $11(P 3 / P z)$ & $M_{K}$ Figuren & positiv & 0.891 & 0.793 & 0.110 & 0.003 & 0.110 & 0.659 & 0.000 \\
\hline $12(P z / P 4)$ & $M_{K}$ Figuren & positiv & 0.211 & 0.045 & 0.789 & 0.002 & 0.789 & 0.681 & 0.001 \\
\hline $13(P 4 / T 6)$ & Mk Figuren & positiv & 0.965 & 0.931 & 0.035 & 0.007 & 0.035 & 0.511 & 0.000 \\
\hline 14 (Fp2/F8) & $M_{K}$ Figuren & positiv & 0.027 & 0.001 & 0.973 & 0.000 & 0.973 & 0.501 & 0.000 \\
\hline 15 (F7/T3) & $M_{K}$ Figuren & positiv & 0.491 & 0.241 & 0.509 & -0.002 & 0.509 & 0.469 & 0.000 \\
\hline 16 (T3/T5) & Mk Figuren & positiv & 0.030 & 0.001 & 0.970 & 0.000 & 0.970 & 0.439 & 0.000 \\
\hline 17 (Fp1/F3) & Mk Figuren & positiv & 0.971 & 0.943 & 0.029 & 0.006 & 0.029 & 0.451 & 0.000 \\
\hline 18 (F3/C3) & $M_{K}$ Figuren & positiv & 0.455 & 0.207 & 0.545 & -0.002 & 0.545 & 0.615 & 0.000 \\
\hline 19 (C3/P3) & Mk Figuren & positiv & 0.223 & 0.050 & 0.777 & 0.001 & 0.777 & 0.701 & 0.000 \\
\hline 20 (P3/O1) & Mk Figuren & positiv & 0.471 & 0.221 & 0.529 & 0.002 & 0.529 & 0.599 & 0.000 \\
\hline $21(F z / C z)$ & $M_{K}$ Figuren & positiv & 0.737 & 0.543 & 0.264 & 0.006 & 0.264 & 0.710 & 0.000 \\
\hline $22(C z / P z)$ & $M_{K}$ Figuren & positiv & 0.508 & 0.258 & 0.492 & 0.004 & 0.492 & 0.652 & 0.000 \\
\hline 23 (Fp2/F4) & $M_{K}$ Figuren & positiv & 0.608 & 0.369 & 0.393 & 0.004 & 0.393 & 0.448 & 0.001 \\
\hline $24(F 4 / C 4)$ & $M_{K}$ Figuren & positiv & 0.887 & 0.786 & 0.113 & 0.003 & 0.113 & 0.569 & 0.000 \\
\hline 25 (C4/P4) & $M_{k}$ Figuren & positiv & 0.048 & 0.002 & 0.952 & 0.000 & 0.952 & 0.667 & 0.000 \\
\hline $26(P 4 / O 2)$ & Mk Figuren & positiv & 0.713 & 0.508 & 0.287 & -0.002 & 0.287 & 0.604 & 0.000 \\
\hline 27 (F8/T4) & $M_{K}$ Figuren & positiv & 0.023 & 0.001 & 0.977 & 0.000 & 0.977 & 0.427 & 0.002 \\
\hline 28 (T4/T6) & $M_{K}$ Figuren & positiv & 0.076 & 0.006 & 0.924 & 0.000 & 0.924 & 0.403 & 0.000 \\
\hline $29(T 5 / 01)$ & Mk Figuren & positiv & 0.999 & 0.999 & 0.001 & 0.005 & 0.001 & 0.524 & 0.000 \\
\hline 30 (T6/O2) & $M_{K}$ Figuren & positiv & 0.916 & 0.840 & 0.084 & -0.006 & 0.084 & 0.565 & 0.000 \\
\hline
\end{tabular}




\section{Anhang B}

Tabelle $B-9$

Ergebnisse der linearen Regressionsanalysen der gemittelten Kohärenzen $\left(M_{K}\right)$ und der Größe des positiven Sets ( $N=10 ; 2$. Sitzung, Wörter, positives Testitem)

\begin{tabular}{|c|c|c|c|c|c|c|c|c|c|}
\hline $\begin{array}{c}\text { Elektr.- } \\
\text { Paar }\end{array}$ & $A V$ & Testitem & Multiple $R$ & $R^{2}$ & Sign. $F$ & Slope & $p$ (sign.) & Intercept & $p$ (sign.) \\
\hline 1 (Fp1/F7) & $M_{K}$ Wörter & positiv & 0.870 & 0.756 & 0.131 & 0.003 & 0.131 & 0.478 & 0.000 \\
\hline 2 (F7/F3) & $M_{K}$ Wörter & positiv & 0.494 & 0.244 & 0.506 & 0.005 & 0.506 & 0.553 & 0.001 \\
\hline $3(F 3 / F z)$ & Mk Wörter & positiv & 0.915 & 0.837 & 0.085 & 0.006 & 0.085 & 0.707 & 0.000 \\
\hline $4(F z / F 4)$ & $M_{k}$ Wörter & positiv & 0.627 & 0.393 & 0.373 & 0.003 & 0.373 & 0.627 & 0.000 \\
\hline 5 (F4/F8) & $M_{K}$ Wörter & positiv & 0.944 & 0.891 & 0.056 & 0.002 & 0.056 & 0.527 & 0.000 \\
\hline $6($ T3/C3) & $M_{\kappa}$ Wörter & positiv & 0.143 & 0.020 & 0.857 & 0.001 & 0.857 & 0.443 & 0.001 \\
\hline $7(C 3 / C z)$ & $M_{\kappa}$ Wörter & positiv & 0.775 & 0.601 & 0.225 & 0.004 & 0.225 & 0.665 & 0.000 \\
\hline $8(\mathrm{Cz} / \mathrm{C} 4)$ & $M_{K}$ Wörter & positiv & 0.295 & 0.087 & 0.705 & 0.001 & 0.705 & 0.650 & 0.000 \\
\hline $9(C 4 / T 4)$ & $M_{K}$ Wörter & positiv & 0.960 & 0.922 & 0.040 & 0.006 & 0.040 & 0.413 & 0.000 \\
\hline 10 (T5/P3) & $M_{\kappa}$ Wörter & positiv & 0.848 & 0.719 & 0.152 & 0.004 & 0.152 & 0.540 & 0.000 \\
\hline $11(P 3 / P z)$ & $M_{k}$ Wörter & positiv & 0.063 & 0.004 & 0.938 & 0.000 & 0.938 & 0.674 & 0.001 \\
\hline $12(P z / P 4)$ & $M_{K}$ Wörter & positiv & 0.382 & 0.146 & 0.618 & 0.003 & 0.618 & 0.679 & 0.000 \\
\hline $13(P 4 / T 6)$ & Mk Wörter & positiv & 0.425 & 0.180 & 0.575 & 0.004 & 0.575 & 0.510 & 0.001 \\
\hline 14 (Fp2/F8) & $M_{K}$ Wörter & positiv & 0.761 & 0.578 & 0.240 & -0.005 & 0.240 & 0.506 & 0.000 \\
\hline 15 (F7/T3) & $M_{K}$ Wörter & positiv & 0.743 & 0.552 & 0.257 & -0.002 & 0.257 & 0.469 & 0.000 \\
\hline 16 (T3/T5) & Mk Wörter & positiv & 0.042 & 0.002 & 0.958 & 0.000 & 0.958 & 0.442 & 0.000 \\
\hline 17 (Fp1/F3) & $M_{\kappa}$ Wörter & positiv & 0.045 & 0.002 & 0.955 & 0.000 & 0.955 & 0.463 & 0.002 \\
\hline 18 (F3/C3) & $M_{K}$ Wörter & positiv & 0.767 & 0.588 & 0.233 & 0.005 & 0.233 & 0.597 & 0.000 \\
\hline 19 (C3/P3) & Mk Wörter & positiv & 0.555 & 0.308 & 0.445 & 0.003 & 0.445 & 0.699 & 0.000 \\
\hline 20 (P3/O1) & $M_{\kappa}$ Wörter & positiv & 0.215 & 0.046 & 0.785 & 0.001 & 0.785 & 0.613 & 0.000 \\
\hline $21(F z / C z)$ & $M_{K}$ Wörter & positiv & 1.000 & 1.000 & 0.000 & 0.010 & 0.000 & 0.694 & 0.000 \\
\hline $22(C z / P z)$ & $M_{K}$ Wörter & positiv & 0.257 & 0.066 & 0.743 & -0.001 & 0.743 & 0.658 & 0.000 \\
\hline 23 (Fp2/F4) & $M_{K}$ Wörter & positiv & 0.397 & 0.158 & 0.603 & -0.003 & 0.603 & 0.458 & 0.001 \\
\hline $24(F 4 / C 4)$ & $M_{k}$ Wörter & positiv & 0.392 & 0.153 & 0.608 & 0.003 & 0.608 & 0.584 & 0.001 \\
\hline 25 (C4/P4) & MK Wörter & positiv & 0.825 & 0.680 & 0.175 & -0.004 & 0.175 & 0.691 & 0.000 \\
\hline $26(P 4 / O 2)$ & $M_{\kappa}$ Wörter & positiv & 0.609 & 0.371 & 0.391 & 0.004 & 0.391 & 0.586 & 0.000 \\
\hline 27 (F8/T4) & $M_{K}$ Wörter & positiv & 0.768 & 0.590 & 0.232 & -0.004 & 0.232 & 0.462 & 0.000 \\
\hline 28 (T4/T6) & $M_{K}$ Wörter & positiv & 0.221 & 0.049 & 0.779 & -0.001 & 0.779 & 0.413 & 0.001 \\
\hline $29(T 5 / 01)$ & Mk Wörter & positiv & 0.967 & 0.935 & 0.033 & 0.005 & 0.033 & 0.536 & 0.000 \\
\hline 30 (T6/O2) & $M_{k}$ Wörter & positiv & 0.437 & 0.191 & 0.564 & 0.002 & 0.564 & 0.548 & 0.000 \\
\hline
\end{tabular}




\section{Anhang B}

Tabelle $B-10$

Ergebnisse der linearen Regressionsanalysen der gemittelten Kohärenzen $\left(\mathrm{M}_{\mathrm{K}}\right)$ und der Größe des positiven Sets ( $N=10 ; 2$. Sitzung, Figuren, negatives Testitem)

\begin{tabular}{|c|c|c|c|c|c|c|c|c|c|}
\hline $\begin{array}{c}\text { Elektr.- } \\
\text { Paar }\end{array}$ & $A V$ & Testitem & Multiple $R$ & $R^{2}$ & Sign. $F$ & Slope & $p$ (sign.) & Intercept & $p$ (sign.) \\
\hline 1 (Fp1/F7) & $M_{k}$ Figuren & negativ & 0.128 & 0.016 & 0.872 & 0.001 & 0.872 & 0.496 & 0.001 \\
\hline 2 (F7/F3) & $M_{K}$ Figuren & negativ & 0.284 & 0.080 & 0.717 & 0.002 & 0.717 & 0.541 & 0.001 \\
\hline $3(F 3 / F z)$ & Mk Figuren & negativ & 0.833 & 0.694 & 0.167 & 0.004 & 0.167 & 0.712 & 0.000 \\
\hline $4(F z / F 4)$ & Mk Figuren & negativ & 0.751 & 0.563 & 0.249 & 0.003 & 0.249 & 0.623 & 0.000 \\
\hline 5 (F4/F8) & $M_{K}$ Figuren & negativ & 0.228 & 0.052 & 0.772 & -0.001 & 0.772 & 0.513 & 0.000 \\
\hline $6($ T3/C3) & Mk Figuren & negativ & 0.453 & 0.205 & 0.547 & 0.003 & 0.547 & 0.432 & 0.001 \\
\hline $7(C 3 / C z)$ & Mk Figuren & negativ & 0.965 & 0.932 & 0.035 & 0.008 & 0.035 & 0.655 & 0.000 \\
\hline $8(\mathrm{Cz} / \mathrm{C} 4)$ & $M_{K}$ Figuren & negativ & 0.026 & 0.001 & 0.975 & 0.000 & 0.975 & 0.649 & 0.001 \\
\hline $9(C 4 / T 4)$ & $M_{K}$ Figuren & negativ & 0.542 & 0.293 & 0.458 & -0.004 & 0.458 & 0.425 & 0.001 \\
\hline $10(T 5 / P 3)$ & Mk Figuren & negativ & 0.401 & 0.161 & 0.599 & 0.003 & 0.599 & 0.545 & 0.001 \\
\hline $11(P 3 / P z)$ & $M_{K}$ Figuren & negativ & 0.954 & 0.910 & 0.046 & 0.006 & 0.046 & 0.651 & 0.000 \\
\hline $12(P z / P 4)$ & $M_{K}$ Figuren & negativ & 0.033 & 0.001 & 0.967 & 0.000 & 0.967 & 0.686 & 0.001 \\
\hline $13(P 4 / T 6)$ & Mk Figuren & negativ & 0.338 & 0.114 & 0.662 & -0.001 & 0.662 & 0.523 & 0.000 \\
\hline 14 (Fp2/F8) & $M_{K}$ Figuren & negativ & 0.620 & 0.384 & 0.380 & 0.003 & 0.380 & 0.488 & 0.000 \\
\hline 15 (F7/T3) & $M_{K}$ Figuren & negativ & 0.446 & 0.199 & 0.554 & 0.005 & 0.554 & 0.453 & 0.002 \\
\hline 16 (T3/T5) & Mk Figuren & negativ & 0.218 & 0.047 & 0.782 & -0.001 & 0.782 & 0.438 & 0.001 \\
\hline 17 (Fp1/F3) & Mk Figuren & negativ & 0.127 & 0.016 & 0.873 & -0.001 & 0.873 & 0.456 & 0.000 \\
\hline 18 (F3/C3) & $M_{K}$ Figuren & negativ & 0.731 & 0.534 & 0.269 & 0.006 & 0.269 & 0.598 & 0.000 \\
\hline 19 (C3/P3) & Mk Figuren & negativ & 0.000 & 0.000 & 0.000 & 0.000 & 1.000 & 0.712 & 0.000 \\
\hline 20 (P3/O1) & Mk Figuren & negativ & 0.341 & 0.116 & 0.659 & 0.002 & 0.659 & 0.600 & 0.000 \\
\hline $21(F z / C z)$ & $M_{K}$ Figuren & negativ & 0.685 & 0.469 & 0.315 & 0.006 & 0.315 & 0.707 & 0.000 \\
\hline $22(C z / P z)$ & $M_{K}$ Figuren & negativ & 0.588 & 0.345 & 0.412 & 0.003 & 0.412 & 0.642 & 0.000 \\
\hline 23 (Fp2/F4) & $M_{K}$ Figuren & negativ & 0.491 & 0.241 & 0.509 & 0.004 & 0.509 & 0.458 & 0.001 \\
\hline $24(F 4 / C 4)$ & $M_{K}$ Figuren & negativ & 0.655 & 0.430 & 0.345 & -0.006 & 0.345 & 0.595 & 0.001 \\
\hline 25 (C4/P4) & Mk Figuren & negativ & 0.070 & 0.005 & 0.930 & -0.001 & 0.930 & 0.676 & 0.001 \\
\hline $26(P 4 / O 2)$ & Mk Figuren & negativ & 0.019 & 0.000 & 0.981 & 0.000 & 0.981 & 0.589 & 0.001 \\
\hline 27 (F8/T4) & $M_{K}$ Figuren & negativ & 0.903 & 0.815 & 0.098 & -0.004 & 0.098 & 0.441 & 0.000 \\
\hline 28 (T4/T6) & $M_{K}$ Figuren & negativ & 0.170 & 0.029 & 0.830 & 0.001 & 0.830 & 0.399 & 0.000 \\
\hline $29(T 5 / 01)$ & Mk Figuren & negativ & 0.865 & 0.747 & 0.135 & -0.002 & 0.135 & 0.542 & 0.000 \\
\hline 30 (T6/O2) & $M_{k}$ Figuren & negativ & 0.507 & 0.257 & 0.493 & 0.003 & 0.493 & 0.537 & 0.000 \\
\hline
\end{tabular}




\section{Anhang B}

Tabelle $B-11$

Ergebnisse der linearen Regressionsanalysen der gemittelten Kohärenzen $\left(\mathrm{M}_{\mathrm{K}}\right)$ und der Größe des positiven Sets ( $N=10 ; 2$. Sitzung, Wörter, negatives Testitem)

\begin{tabular}{|c|c|c|c|c|c|c|c|c|c|}
\hline $\begin{array}{c}\text { Elektr.- } \\
\text { Paar }\end{array}$ & $A V$ & Testitem & Multiple $R$ & $R^{2}$ & Sign. $F$ & Slope & $p$ (sign.) & Intercept & $p$ (sign.) \\
\hline 1 (Fp1/F7) & $M_{K}$ Wörter & negativ & 0.448 & 0.201 & 0.552 & -0.002 & 0.552 & 0.496 & 0.000 \\
\hline 2 (F7/F3) & $M_{K}$ Wörter & negativ & 0.486 & 0.237 & 0.514 & -0.005 & 0.514 & 0.568 & 0.001 \\
\hline $3(F 3 / F z)$ & Mk Wörter & negativ & 0.183 & 0.033 & 0.817 & 0.000 & 0.817 & 0.726 & 0.000 \\
\hline $4(F z / F 4)$ & $M_{k}$ Wörter & negativ & 0.726 & 0.527 & 0.274 & 0.003 & 0.274 & 0.632 & 0.000 \\
\hline 5 (F4/F8) & $M_{K}$ Wörter & negativ & 0.523 & 0.273 & 0.477 & -0.004 & 0.477 & 0.534 & 0.001 \\
\hline $6($ T3/C3) & $M_{\kappa}$ Wörter & negativ & 0.680 & 0.462 & 0.320 & 0.007 & 0.320 & 0.428 & 0.001 \\
\hline $7(C 3 / C z)$ & $M_{\kappa}$ Wörter & negativ & 0.274 & 0.075 & 0.726 & 0.001 & 0.726 & 0.673 & 0.000 \\
\hline $8(\mathrm{Cz} / \mathrm{C} 4)$ & $M_{K}$ Wörter & negativ & 0.024 & 0.001 & 0.976 & 0.000 & 0.976 & 0.657 & 0.000 \\
\hline $9(C 4 / T 4)$ & $M_{K}$ Wörter & negativ & 0.660 & 0.436 & 0.340 & 0.006 & 0.340 & 0.411 & 0.001 \\
\hline 10 (T5/P3) & $M_{\kappa}$ Wörter & negativ & 0.683 & 0.466 & 0.317 & 0.004 & 0.317 & 0.546 & 0.000 \\
\hline $11(P 3 / P z)$ & $M_{k}$ Wörter & negativ & 0.509 & 0.259 & 0.492 & 0.002 & 0.492 & 0.675 & 0.000 \\
\hline $12(P z / P 4)$ & $M_{K}$ Wörter & negativ & 0.850 & 0.722 & 0.150 & -0.010 & 0.150 & 0.711 & 0.000 \\
\hline $13(P 4 / T 6)$ & Mk Wörter & negativ & 0.768 & 0.590 & 0.232 & 0.007 & 0.232 & 0.502 & 0.001 \\
\hline 14 (Fp2/F8) & $M_{K}$ Wörter & negativ & 0.310 & 0.096 & 0.690 & -0.003 & 0.690 & 0.502 & 0.001 \\
\hline 15 (F7/T3) & $M_{K}$ Wörter & negativ & 0.384 & 0.147 & 0.616 & 0.002 & 0.616 & 0.459 & 0.001 \\
\hline 16 (T3/T5) & Mk Wörter & negativ & 0.459 & 0.210 & 0.541 & 0.003 & 0.541 & 0.434 & 0.001 \\
\hline 17 (Fp1/F3) & $M_{\kappa}$ Wörter & negativ & 0.469 & 0.220 & 0.531 & -0.002 & 0.531 & 0.458 & 0.000 \\
\hline 18 (F3/C3) & $M_{K}$ Wörter & negativ & 0.078 & 0.006 & 0.922 & 0.001 & 0.922 & 0.605 & 0.001 \\
\hline 19 (C3/P3) & Mk Wörter & negativ & 0.737 & 0.543 & 0.263 & 0.004 & 0.263 & 0.702 & 0.000 \\
\hline 20 (P3/O1) & Mk Wörter & negativ & 0.841 & 0.707 & 0.159 & 0.003 & 0.159 & 0.609 & 0.000 \\
\hline $21(F z / C z)$ & $M_{K}$ Wörter & negativ & 0.992 & 0.983 & 0.008 & 0.007 & 0.008 & 0.705 & 0.000 \\
\hline $22(C z / P z)$ & $M_{k}$ Wörter & negativ & 0.280 & 0.079 & 0.720 & 0.002 & 0.720 & 0.648 & 0.001 \\
\hline 23 (Fp2/F4) & $M_{K}$ Wörter & negativ & 0.258 & 0.066 & 0.742 & 0.001 & 0.742 & 0.454 & 0.000 \\
\hline $24(F 4 / C 4)$ & $M_{k}$ Wörter & negativ & 0.218 & 0.048 & 0.782 & -0.001 & 0.782 & 0.586 & 0.000 \\
\hline $25(C 4 / P 4)$ & $M_{k}$ Wörter & negativ & 0.079 & 0.006 & 0.921 & 0.001 & 0.921 & 0.676 & 0.000 \\
\hline $26(P 4 / O 2)$ & $M_{\kappa}$ Wörter & negativ & 0.365 & 0.133 & 0.635 & 0.002 & 0.635 & 0.584 & 0.000 \\
\hline $27(F 8 / T 4)$ & $M_{K}$ Wörter & negativ & 0.272 & 0.074 & 0.728 & 0.000 & 0.728 & 0.439 & 0.000 \\
\hline 28 (T4/T6) & $M_{K}$ Wörter & negativ & 0.163 & 0.027 & 0.837 & 0.001 & 0.837 & 0.402 & 0.001 \\
\hline $29(T 5 / 01)$ & Mk Wörter & negativ & 0.962 & 0.925 & 0.038 & 0.005 & 0.038 & 0.538 & 0.000 \\
\hline 30 (T6/O2) & $M_{k}$ Wörter & negativ & 0.024 & 0.001 & 0.976 & 0.000 & 0.976 & 0.553 & 0.001 \\
\hline
\end{tabular}




\section{Anhang B}

Tabelle $B-12$

Ergebnisse der linearen Regressionsanalysen der gemittelten maximalen Kohärenzen ( $\left.\mathrm{M}_{\mathrm{MK}}\right)$ und der Größe des positiven Sets ( $N=10 ; 2$. Sitzung, Figuren, positives Testitem)

\begin{tabular}{|c|c|c|c|c|c|c|c|c|c|}
\hline $\begin{array}{c}\text { Elektr.- } \\
\text { Paar }\end{array}$ & $A V$ & Testitem & Multiple $R$ & $R^{2}$ & Sign. $F$ & Slope & $p$ (sign.) & Intercept & $p$ (sign.) \\
\hline 1 (Fp1/F7) & $M_{M K}$ Figuren & positiv & 0.927 & 0.859 & 0.073 & 0.013 & 0.073 & 0.538 & 0.000 \\
\hline 2 (F7/F3) & $M_{M K}$ Figuren & positiv & 0.778 & 0.605 & 0.222 & 0.006 & 0.222 & 0.600 & 0.000 \\
\hline $3(F 3 / F z)$ & Ммк Figuren & positiv & 0.996 & 0.993 & 0.004 & 0.010 & 0.004 & 0.753 & 0.000 \\
\hline $4(F z / F 4)$ & Mмк Figuren & positiv & 0.986 & 0.972 & 0.014 & 0.013 & 0.014 & 0.654 & 0.000 \\
\hline 5 (F4/F8) & $M_{M K}$ Figuren & positiv & 0.774 & 0.599 & 0.226 & 0.014 & 0.226 & 0.534 & 0.002 \\
\hline $6($ T3/C3) & MMK Figuren & positiv & 0.866 & 0.749 & 0.135 & 0.009 & 0.135 & 0.482 & 0.001 \\
\hline $7(C 3 / C z)$ & Ммк Figuren & positiv & 0.963 & 0.927 & 0.037 & 0.009 & 0.037 & 0.710 & 0.000 \\
\hline $8(\mathrm{Cz} / \mathrm{C} 4)$ & $M_{M K}$ Figuren & positiv & 0.996 & 0.992 & 0.004 & 0.013 & 0.004 & 0.674 & 0.000 \\
\hline $9(C 4 / T 4)$ & $M_{M K}$ Figuren & positiv & 0.899 & 0.808 & 0.101 & 0.016 & 0.101 & 0.439 & 0.001 \\
\hline $10(T 5 / P 3)$ & Ммк Figuren & positiv & 0.764 & 0.584 & 0.236 & 0.009 & 0.236 & 0.598 & 0.001 \\
\hline $11(P 3 / P z)$ & $M_{M K}$ Figuren & positiv & 0.891 & 0.794 & 0.109 & 0.009 & 0.109 & 0.701 & 0.000 \\
\hline $12(P z / P 4)$ & $M_{M K}$ Figuren & positiv & 0.803 & 0.644 & 0.197 & 0.006 & 0.197 & 0.722 & 0.000 \\
\hline $13(P 4 / T 6)$ & MMK Figuren & positiv & 0.960 & 0.921 & 0.040 & 0.018 & 0.040 & 0.541 & 0.000 \\
\hline 14 (Fp2/F8) & $M_{M K}$ Figuren & positiv & 0.966 & 0.933 & 0.034 & 0.007 & 0.034 & 0.553 & 0.000 \\
\hline 15 (F7/T3) & $M_{M K}$ Figuren & positiv & 0.918 & 0.843 & 0.082 & 0.007 & 0.082 & 0.518 & 0.000 \\
\hline 16 (T3/T5) & Ммк Figuren & positiv & 0.855 & 0.731 & 0.145 & 0.010 & 0.145 & 0.486 & 0.001 \\
\hline 17 (Fp1/F3) & MMK Figuren & positiv & 0.965 & 0.932 & 0.035 & 0.014 & 0.035 & 0.494 & 0.000 \\
\hline 18 (F3/C3) & $M_{M K}$ Figuren & positiv & 0.686 & 0.470 & 0.314 & 0.006 & 0.314 & 0.663 & 0.000 \\
\hline 19 (C3/P3) & Ммк Figuren & positiv & 0.995 & 0.989 & 0.005 & 0.008 & 0.005 & 0.737 & 0.000 \\
\hline 20 (P3/O1) & MMK Figuren & positiv & 0.855 & 0.731 & 0.145 & 0.009 & 0.145 & 0.637 & 0.000 \\
\hline $21(F z / C z)$ & $M_{M K}$ Figuren & positiv & 0.939 & 0.882 & 0.061 & 0.011 & 0.061 & 0.748 & 0.000 \\
\hline $22(C z / P z)$ & $M_{M K}$ Figuren & positiv & 0.988 & 0.977 & 0.012 & 0.007 & 0.012 & 0.700 & 0.000 \\
\hline 23 (Fp2/F4) & MMK Figuren & positiv & 0.855 & 0.732 & 0.145 & 0.010 & 0.145 & 0.491 & 0.001 \\
\hline $24(F 4 / C 4)$ & $M_{M K}$ Figuren & positiv & 0.890 & 0.792 & 0.110 & 0.011 & 0.110 & 0.613 & 0.000 \\
\hline $25(C 4 / P 4)$ & MмK Figuren & positiv & 0.794 & 0.630 & 0.206 & 0.006 & 0.206 & 0.708 & 0.000 \\
\hline $26(P 4 / O 2)$ & Ммк Figuren & positiv & 0.575 & 0.331 & 0.425 & 0.005 & 0.425 & 0.642 & 0.001 \\
\hline 27 (F8/T4) & $M_{M K}$ Figuren & positiv & 0.644 & 0.415 & 0.356 & 0.006 & 0.356 & 0.484 & 0.001 \\
\hline $28(T 4 / T 6)$ & $M_{M K}$ Figuren & positiv & 0.987 & 0.973 & 0.014 & 0.012 & 0.014 & 0.437 & 0.000 \\
\hline $29(T 5 / 01)$ & Ммк Figuren & positiv & 0.990 & 0.981 & 0.010 & 0.014 & 0.010 & 0.566 & 0.000 \\
\hline 30 (T6/O2) & $M_{M K}$ Figuren & positiv & 0.861 & 0.742 & 0.139 & 0.007 & 0.139 & 0.593 & 0.000 \\
\hline
\end{tabular}




\section{Anhang B}

Tabelle $B-13$

Ergebnisse der linearen Regressionsanalysen der gemittelten maximalen Kohärenzen $\left(\mathrm{M}_{\mathrm{MK}}\right)$ und der Größe des positiven Sets ( $N=10 ; 2$. Sitzung, Wörter, positives Testitem)

\begin{tabular}{|c|c|c|c|c|c|c|c|c|c|}
\hline $\begin{array}{c}\text { Elektr.- } \\
\text { Paar }\end{array}$ & $A V$ & Testitem & Multiple $R$ & $R^{2}$ & Sign. $F$ & Slope & $p$ (sign.) & Intercept & $p$ (sign.) \\
\hline 1 (Fp1/F7) & MMK Wörter & positiv & 0.984 & 0.968 & 0.016 & 0.016 & 0.016 & 0.519 & 0.000 \\
\hline 2 (F7/F3) & MMK Wörter & positiv & 0.938 & 0.880 & 0.062 & 0.015 & 0.062 & 0.588 & 0.000 \\
\hline $3(F 3 / F z)$ & Mмк Wörter & positiv & 0.999 & 0.999 & 0.001 & 0.012 & 0.001 & 0.744 & 0.000 \\
\hline $4(F z / F 4)$ & MMK Wörter & positiv & 0.633 & 0.401 & 0.367 & 0.005 & 0.367 & 0.674 & 0.000 \\
\hline 5 (F4/F8) & MMK Wörter & positiv & 0.658 & 0.432 & 0.342 & 0.005 & 0.342 & 0.580 & 0.000 \\
\hline $6($ (T3/C3) & MMK Wörter & positiv & 0.997 & 0.995 & 0.003 & 0.010 & 0.003 & 0.482 & 0.000 \\
\hline $7(C 3 / C z)$ & Mмк Wörter & positiv & 0.572 & 0.327 & 0.428 & 0.006 & 0.428 & 0.714 & 0.001 \\
\hline $8(\mathrm{Cz} / \mathrm{C} 4)$ & $M_{M K}$ Wörter & positiv & 0.577 & 0.333 & 0.423 & 0.006 & 0.423 & 0.695 & 0.001 \\
\hline $9(C 4 / T 4)$ & MMK Wörter & positiv & 0.999 & 0.998 & 0.001 & 0.016 & 0.001 & 0.447 & 0.000 \\
\hline 10 (T5/P3) & MMK Wörter & positiv & 0.850 & 0.723 & 0.150 & 0.013 & 0.150 & 0.575 & 0.001 \\
\hline $11(P 3 / P z)$ & $M_{M K}$ Wörter & positiv & 0.182 & 0.033 & 0.818 & 0.003 & 0.818 & 0.719 & 0.001 \\
\hline $12(P z / P 4)$ & MMK Wörter & positiv & 0.791 & 0.626 & 0.209 & 0.010 & 0.209 & 0.716 & 0.000 \\
\hline 13 (P4/T6) & MMK Wörter & positiv & 0.738 & 0.544 & 0.262 & 0.013 & 0.262 & 0.542 & 0.002 \\
\hline 14 (Fp2/F8) & $M_{M K}$ Wörter & positiv & 0.891 & 0.794 & 0.109 & 0.006 & 0.109 & 0.547 & 0.000 \\
\hline 15 (F7/T3) & MMK Wörter & positiv & 0.763 & 0.582 & 0.237 & 0.008 & 0.237 & 0.511 & 0.001 \\
\hline 16 (T3/T5) & MMK Wörter & positiv & 0.988 & 0.976 & 0.012 & 0.013 & 0.012 & 0.475 & 0.000 \\
\hline 17 (Fp1/F3) & MMK Wörter & positiv & 0.906 & 0.820 & 0.094 & 0.015 & 0.094 & 0.492 & 0.001 \\
\hline 18 (F3/C3) & MMK Wörter & positiv & 0.775 & 0.600 & 0.226 & 0.007 & 0.226 & 0.654 & 0.000 \\
\hline 19 (С3/P3) & Mмк Wörter & positiv & 0.698 & 0.488 & 0.302 & 0.008 & 0.302 & 0.734 & 0.001 \\
\hline 20 (P3/O1) & MMK Wörter & positiv & 0.785 & 0.616 & 0.215 & 0.007 & 0.215 & 0.647 & 0.000 \\
\hline $21(F z / C z)$ & MMK Wörter & positiv & 0.992 & 0.984 & 0.008 & 0.013 & 0.008 & 0.737 & 0.000 \\
\hline $22(C z / P z)$ & MMK Wörter & positiv & 0.378 & 0.143 & 0.622 & 0.004 & 0.622 & 0.699 & 0.001 \\
\hline 23 (Fp2/F4) & $M_{M K}$ Wörter & positiv & 0.944 & 0.891 & 0.056 & 0.009 & 0.056 & 0.492 & 0.000 \\
\hline $24(F 4 / C 4)$ & MMK Wörter & positiv & 0.723 & 0.522 & 0.277 & 0.008 & 0.277 & 0.630 & 0.001 \\
\hline $25(C 4 / P 4)$ & MMK Wörter & positiv & 0.498 & 0.248 & 0.502 & 0.005 & 0.502 & 0.719 & 0.001 \\
\hline $26(P 4 / O 2)$ & MMK Wörter & positiv & 0.952 & 0.906 & 0.048 & 0.016 & 0.048 & 0.619 & 0.000 \\
\hline 27 (F8/T4) & MMK Wörter & positiv & 0.890 & 0.792 & 0.110 & 0.009 & 0.110 & 0.495 & 0.000 \\
\hline 28 (T4/T6) & MMK Wörter & positiv & 0.587 & 0.344 & 0.413 & 0.005 & 0.413 & 0.455 & 0.001 \\
\hline $29(T 5 / 01)$ & Mмк Wörter & positiv & 0.793 & 0.629 & 0.207 & 0.008 & 0.207 & 0.580 & 0.001 \\
\hline 30 (T6/O2) & MMK Wörter & positiv & 0.826 & 0.683 & 0.174 & 0.009 & 0.174 & 0.588 & 0.000 \\
\hline
\end{tabular}




\section{Anhang B}

Tabelle $B-14$

Ergebnisse der linearen Regressionsanalysen der gemittelten maximalen Kohärenzen $\left(\mathrm{M}_{\mathrm{MK}}\right)$ und der Größe des positiven Sets ( $N=10 ; 2$. Sitzung, Figuren, negatives Testitem)

\begin{tabular}{|c|c|c|c|c|c|c|c|c|c|}
\hline $\begin{array}{c}\text { Elektr.- } \\
\text { Paar }\end{array}$ & $A V$ & Testitem & Multiple $R$ & $R^{2}$ & Sign. $F$ & Slope & $p$ (sign.) & Intercept & $p$ (sign.) \\
\hline 1 (Fp1/F7) & $M_{M K}$ Figuren & negativ & 0.878 & 0.771 & 0.122 & 0.009 & 0.122 & 0.561 & 0.000 \\
\hline 2 (F7/F3) & $M_{M K}$ Figuren & negativ & 0.954 & 0.909 & 0.046 & 0.012 & 0.046 & 0.597 & 0.000 \\
\hline $3(F 3 / F z)$ & Ммк Figuren & negativ & 0.942 & 0.888 & 0.058 & 0.011 & 0.058 & 0.760 & 0.000 \\
\hline $4(F z / F 4)$ & Ммк Figuren & negativ & 0.993 & 0.986 & 0.007 & 0.011 & 0.007 & 0.674 & 0.000 \\
\hline 5 (F4/F8) & MMK Figuren & negativ & 0.963 & 0.928 & 0.037 & 0.006 & 0.037 & 0.570 & 0.000 \\
\hline $6($ (T3/C3) & Ммк Figuren & negativ & 0.937 & 0.879 & 0.063 & 0.011 & 0.063 & 0.498 & 0.000 \\
\hline $7(C 3 / C z)$ & Ммк Figuren & negativ & 0.933 & 0.870 & 0.067 & 0.012 & 0.067 & 0.715 & 0.000 \\
\hline $8(\mathrm{Cz} / \mathrm{C} 4)$ & $M_{M K}$ Figuren & negativ & 0.685 & 0.469 & 0.315 & 0.007 & 0.315 & 0.704 & 0.001 \\
\hline $9(C 4 / T 4)$ & MMK Figuren & negativ & 0.766 & 0.586 & 0.234 & 0.008 & 0.234 & 0.480 & 0.001 \\
\hline 10 (T5/P3) & Ммк Figuren & negativ & 0.812 & 0.660 & 0.188 & 0.013 & 0.188 & 0.594 & 0.001 \\
\hline $11(P 3 / P z)$ & $M_{M K}$ Figuren & negativ & 0.972 & 0.945 & 0.028 & 0.013 & 0.028 & 0.703 & 0.000 \\
\hline $12(P z / P 4)$ & $M_{M K}$ Figuren & negativ & 0.742 & 0.551 & 0.258 & 0.011 & 0.258 & 0.721 & 0.001 \\
\hline $13(P 4 / T 6)$ & Ммк Figuren & negativ & 0.880 & 0.774 & 0.120 & 0.009 & 0.120 & 0.574 & 0.000 \\
\hline 14 (Fp2/F8) & $M_{M K}$ Figuren & negativ & 0.992 & 0.984 & 0.008 & 0.012 & 0.008 & 0.554 & 0.000 \\
\hline 15 (F7/T3) & $M_{M K}$ Figuren & negativ & 0.899 & 0.808 & 0.101 & 0.010 & 0.101 & 0.529 & 0.000 \\
\hline 16 (T3/T5) & Ммк Figuren & negativ & 0.966 & 0.934 & 0.034 & 0.010 & 0.034 & 0.500 & 0.000 \\
\hline 17 (Fp1/F3) & MMK Figuren & negativ & 0.975 & 0.951 & 0.025 & 0.010 & 0.025 & 0.507 & 0.000 \\
\hline 18 (F3/C3) & $M_{M K}$ Figuren & negativ & 0.886 & 0.784 & 0.115 & 0.012 & 0.115 & 0.664 & 0.000 \\
\hline 19 (С3/P3) & Ммк Figuren & negativ & 0.840 & 0.706 & 0.160 & 0.007 & 0.160 & 0.756 & 0.000 \\
\hline 20 (P3/O1) & Ммк Figuren & negativ & 0.920 & 0.847 & 0.080 & 0.011 & 0.080 & 0.642 & 0.000 \\
\hline $21(F z / C z)$ & MMK Figuren & negativ & 0.944 & 0.891 & 0.056 & 0.013 & 0.056 & 0.754 & 0.000 \\
\hline $22(C z / P z)$ & $M_{M K}$ Figuren & negativ & 0.949 & 0.901 & 0.051 & 0.011 & 0.051 & 0.695 & 0.000 \\
\hline 23 (Fp2/F4) & $M_{M K}$ Figuren & negativ & 0.801 & 0.641 & 0.199 & 0.009 & 0.199 & 0.519 & 0.001 \\
\hline $24(F 4 / C 4)$ & $M_{M K}$ Figuren & negativ & 0.635 & 0.404 & 0.365 & 0.006 & 0.365 & 0.640 & 0.001 \\
\hline $25(C 4 / P 4)$ & Mмк Figuren & negativ & 0.841 & 0.707 & 0.159 & 0.010 & 0.159 & 0.717 & 0.000 \\
\hline $26(P 4 / O 2)$ & Ммк Figuren & negativ & 0.958 & 0.919 & 0.042 & 0.011 & 0.042 & 0.636 & 0.000 \\
\hline 27 (F8/T4) & $M_{M K}$ Figuren & negativ & 0.504 & 0.254 & 0.496 & 0.002 & 0.496 & 0.508 & 0.000 \\
\hline 28 (T4/T6) & $M_{M K}$ Figuren & negativ & 0.816 & 0.665 & 0.184 & 0.011 & 0.184 & 0.454 & 0.001 \\
\hline $29(T 5 / 01)$ & Ммк Figuren & negativ & 0.870 & 0.756 & 0.130 & 0.010 & 0.130 & 0.593 & 0.000 \\
\hline 30 (T6/O2) & $M_{M K}$ Figuren & negativ & 0.977 & 0.955 & 0.023 & 0.014 & 0.023 & 0.590 & 0.000 \\
\hline
\end{tabular}




\section{Anhang B}

Tabelle $B-15$

Ergebnisse der linearen Regressionsanalysen der gemittelten maximalen Kohärenzen $\left(M_{M K}\right)$ und der Größe des positiven Sets ( $N=10 ; 2$. Sitzung, Wörter, negatives Testitem)

\begin{tabular}{|c|c|c|c|c|c|c|c|c|c|}
\hline $\begin{array}{c}\text { Elektr.- } \\
\text { Paar }\end{array}$ & $A V$ & Testitem & Multiple $R$ & $R^{2}$ & Sign. $F$ & Slope & $p$ (sign.) & Intercept & $p$ (sign.) \\
\hline 1 (Fp1/F7) & MMK Wörter & negativ & 0.927 & 0.859 & 0.073 & 0.006 & 0.073 & 0.549 & 0.000 \\
\hline 2 (F7/F3) & MMK Wörter & negativ & 0.102 & 0.010 & 0.898 & 0.001 & 0.898 & 0.620 & 0.001 \\
\hline $3(F 3 / F z)$ & MMK Wörter & negativ & 0.970 & 0.941 & 0.030 & 0.005 & 0.030 & 0.769 & 0.000 \\
\hline $4(F z / F 4)$ & MMK Wörter & negativ & 0.804 & 0.647 & 0.196 & 0.008 & 0.196 & 0.683 & 0.000 \\
\hline 5 (F4/F8) & MMK Wörter & negativ & 0.661 & 0.436 & 0.340 & 0.003 & 0.340 & 0.585 & 0.000 \\
\hline $6($ (T3/C3) & MMK Wörter & negativ & 0.876 & 0.768 & 0.124 & 0.010 & 0.124 & 0.495 & 0.001 \\
\hline $7(C 3 / C z)$ & Mмк Wörter & negativ & 0.722 & 0.521 & 0.278 & 0.002 & 0.278 & 0.735 & 0.000 \\
\hline $8(\mathrm{Cz} / \mathrm{C} 4)$ & MMK Wörter & negativ & 0.816 & 0.665 & 0.184 & 0.003 & 0.184 & 0.711 & 0.000 \\
\hline $9(C 4 / T 4)$ & MMK Wörter & negativ & 0.874 & 0.764 & 0.126 & 0.012 & 0.126 & 0.469 & 0.001 \\
\hline 10 (T5/P3) & MMK Wörter & negativ & 0.954 & 0.910 & 0.046 & 0.009 & 0.046 & 0.600 & 0.000 \\
\hline $11(P 3 / P z)$ & $M_{M K}$ Wörter & negativ & 0.870 & 0.757 & 0.130 & 0.005 & 0.130 & 0.724 & 0.000 \\
\hline 12(Pz/P4) & MMK Wörter & negativ & 0.649 & 0.422 & 0.351 & -0.005 & 0.351 & 0.757 & 0.000 \\
\hline $13(P 4 / T 6)$ & MMK Wörter & negativ & 0.992 & 0.984 & 0.008 & 0.011 & 0.008 & 0.556 & 0.000 \\
\hline 14 (Fp2/F8) & MMK Wörter & negativ & 0.485 & 0.235 & 0.515 & 0.005 & 0.515 & 0.555 & 0.001 \\
\hline 15 (F7/T3) & MMK Wörter & negativ & 0.734 & 0.539 & 0.266 & 0.007 & 0.266 & 0.524 & 0.001 \\
\hline 16 (T3/T5) & MMK Wörter & negativ & 0.694 & 0.482 & 0.306 & 0.008 & 0.306 & 0.493 & 0.001 \\
\hline 17 (Fp1/F3) & MMK Wörter & negativ & 0.631 & 0.398 & 0.369 & 0.004 & 0.369 & 0.508 & 0.000 \\
\hline $18(F 3 / C 3)$ & $M_{M K}$ Wörter & negativ & 0.510 & 0.260 & 0.490 & 0.005 & 0.490 & 0.667 & 0.001 \\
\hline 19 (C3/P3) & Mмк Wörter & negativ & 0.899 & 0.807 & 0.102 & 0.007 & 0.102 & 0.752 & 0.000 \\
\hline 20 (P3/O1) & MMK Wörter & negativ & 0.990 & 0.980 & 0.010 & 0.008 & 0.010 & 0.655 & 0.000 \\
\hline $21(F z / C z)$ & $M_{M K}$ Wörter & negativ & 0.981 & 0.962 & 0.019 & 0.012 & 0.019 & 0.745 & 0.000 \\
\hline $22(C z / P z)$ & MMK Wörter & negativ & 0.734 & 0.539 & 0.266 & 0.005 & 0.266 & 0.699 & 0.000 \\
\hline 23 (Fp2/F4) & $M_{M K}$ Wörter & negativ & 0.806 & 0.649 & 0.194 & 0.005 & 0.194 & 0.505 & 0.000 \\
\hline $24(F 4 / C 4)$ & MMK Wörter & negativ & 0.879 & 0.772 & 0.121 & 0.005 & 0.121 & 0.636 & 0.000 \\
\hline $25(C 4 / P 4)$ & MMK Wörter & negativ & 0.592 & 0.351 & 0.408 & 0.003 & 0.408 & 0.724 & 0.000 \\
\hline 26 (P4/O2) & MMK Wörter & negativ & 0.997 & 0.995 & 0.003 & 0.008 & 0.003 & 0.634 & 0.000 \\
\hline 27 (F8/T4) & MMK Wörter & negativ & 0.926 & 0.858 & 0.074 & 0.004 & 0.074 & 0.505 & 0.000 \\
\hline 28 (T4/T6) & $M_{M K}$ Wörter & negativ & 0.476 & 0.227 & 0.524 & 0.005 & 0.524 & 0.470 & 0.001 \\
\hline 29 (T5/O1) & Mмк Wörter & negativ & 0.911 & 0.829 & 0.089 & 0.009 & 0.089 & 0.596 & 0.000 \\
\hline 30 (T6/O2) & MMK Wörter & negativ & 0.468 & 0.219 & 0.532 & 0.003 & 0.532 & 0.619 & 0.000 \\
\hline
\end{tabular}




\section{Anhang C}

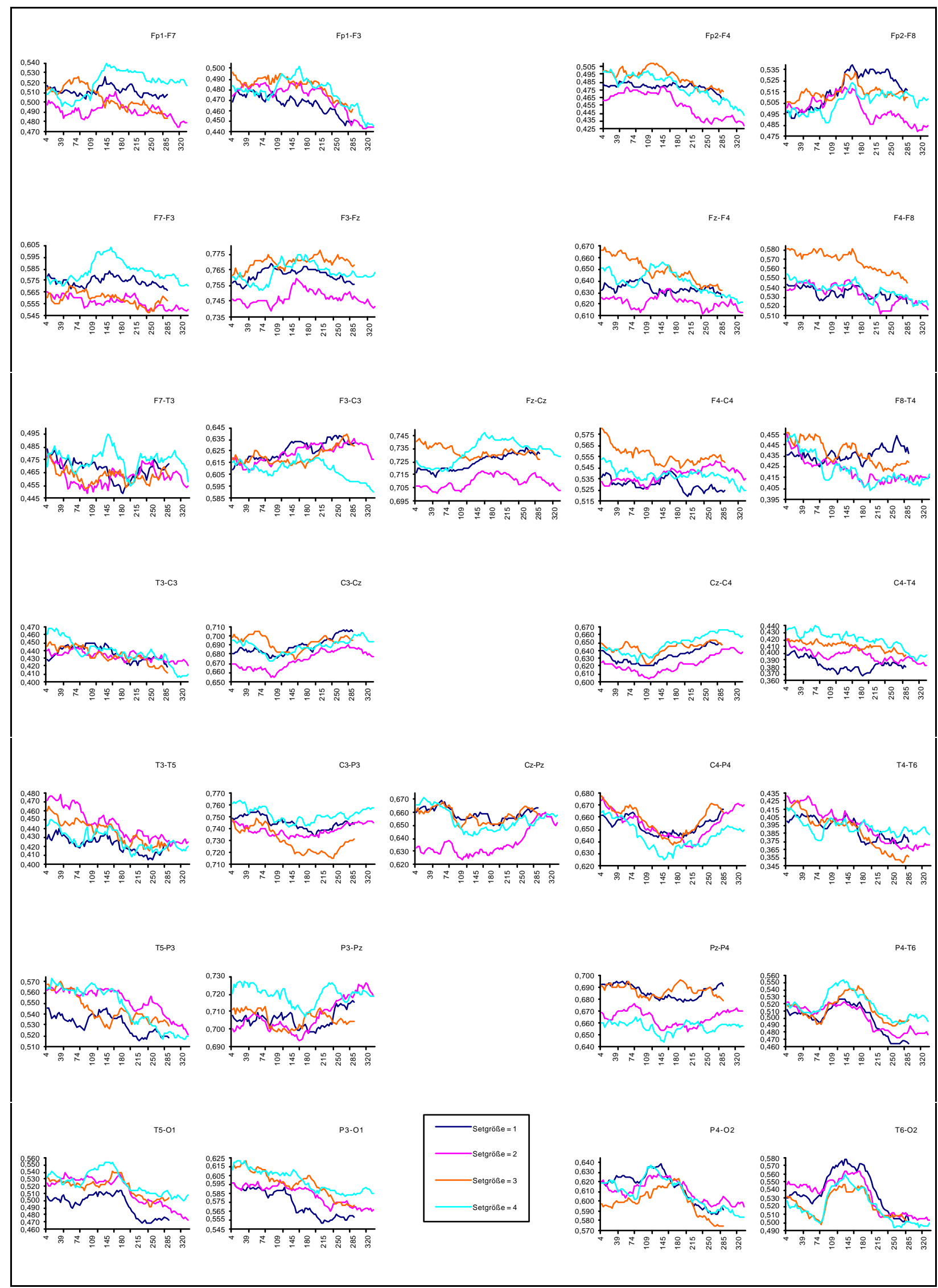

Abbildung C-1. Verlauf der über die Probandinnen 4, 5, 7, 8, 9 und 10 gemittelten Kohärenzwerte im Intervall zwischen 121.12 und maximal $332.10 \mathrm{~ms}$ nach dem positiven Testitem: Reizart Figuren (2. Sitzung) 


\section{Anhang C}

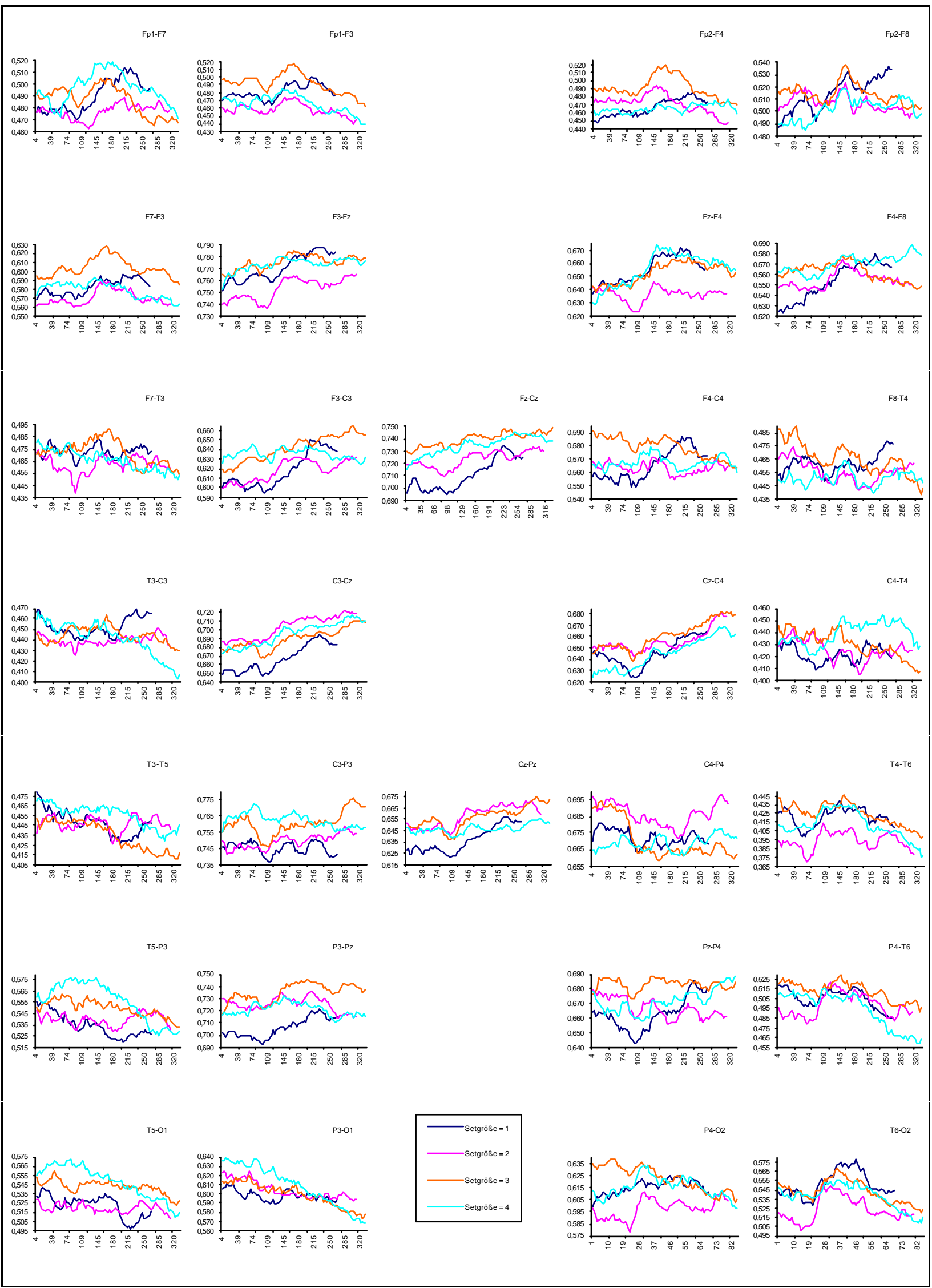

Abbildung C-2. Verlauf der über die Probandinnen 4, 5, 7, 8, 9 und 10 gemittelten Kohärenzwerte im Intervall zwischen 3.91 und maximal 332.10 ms nach dem positiven Testitem: Reizart Wörter (2. Sitzung) 


\section{Anhang D}

Tabelle $D-1$

Ergebnisse der linearen Regressionsanalysen der gemittelten Zeitpunkte maximaler Kohärenz $\left(\mathrm{M}_{\mathrm{ZMK}}\right)$ und der Größe des positiven Sets ( $N=10 ; 2$. Sitzung, Figuren, positives Testitem)

\begin{tabular}{|c|c|c|c|c|c|c|c|c|c|}
\hline $\begin{array}{c}\text { Elektr.- } \\
\text { Paar }\end{array}$ & $A V$ & Testitem & Multiple $R$ & $R^{2}$ & Sign. $F$ & Slope & $p$ (sign.) & Intercept & $p$ (sign.) \\
\hline 1 (Fp1/F7) & MzMK Figuren & positiv & 0.971 & 0.943 & 0.029 & 9.152 & 0.029 & 71.791 & 0.004 \\
\hline 2 (F7/F3) & MZMK Figuren & positiv & 0.765 & 0.586 & 0.235 & 6.118 & 0.235 & 74.843 & 0.017 \\
\hline $3(F 3 / F z)$ & МZмк Figuren & positiv & 0.925 & 0.856 & 0.075 & 10.874 & 0.075 & 64.827 & 0.017 \\
\hline $4(F z / F 4)$ & Mzмк Figuren & positiv & 0.896 & 0.804 & 0.104 & 12.759 & 0.104 & 63.044 & 0.036 \\
\hline 5 (F4/F8) & MZMK Figuren & positiv & 0.963 & 0.927 & 0.037 & 9.835 & 0.037 & 67.048 & 0.006 \\
\hline $6($ T3/C3) & MZMK Figuren & positiv & 0.918 & 0.842 & 0.082 & 8.796 & 0.082 & 73.347 & 0.010 \\
\hline $7(C 3 / C z)$ & MzMK Figuren & positiv & 0.926 & 0.857 & 0.074 & 9.216 & 0.074 & 75.582 & 0.009 \\
\hline $8(\mathrm{Cz} / \mathrm{C} 4)$ & MZMK Figuren & positiv & 0.980 & 0.960 & 0.020 & 11.927 & 0.020 & 67.681 & 0.005 \\
\hline $9(C 4 / T 4)$ & MZMK Figuren & positiv & 0.873 & 0.762 & 0.127 & 8.932 & 0.127 & 65.920 & 0.021 \\
\hline 10 (T5/P3) & Mzмк Figuren & positiv & 0.979 & 0.958 & 0.021 & 11.855 & 0.021 & 56.481 & 0.007 \\
\hline $11(P 3 / P z)$ & MzMK Figuren & positiv & 0.950 & 0.903 & 0.050 & 9.869 & 0.050 & 69.844 & 0.008 \\
\hline $12(P z / P 4)$ & MzMK Figuren & positiv & 0.920 & 0.847 & 0.080 & 11.907 & 0.080 & 71.246 & 0.018 \\
\hline $13(P 4 / T 6)$ & MZMK Figuren & positiv & 0.853 & 0.728 & 0.147 & 10.901 & 0.147 & 60.096 & 0.043 \\
\hline 14 (Fp2/F8) & MzMK Figuren & positiv & 0.965 & 0.930 & 0.036 & 16.269 & 0.036 & 54.877 & 0.024 \\
\hline 15 (F7/T3) & MZMK Figuren & positiv & 0.780 & 0.608 & 0.220 & 7.017 & 0.220 & 77.775 & 0.019 \\
\hline 16 (T3/T5) & Mzмк Figuren & positiv & 0.792 & 0.627 & 0.208 & 9.370 & 0.208 & 71.057 & 0.037 \\
\hline 17 (Fp1/F3) & Mzмк Figuren & positiv & 0.964 & 0.929 & 0.036 & 8.195 & 0.036 & 65.611 & 0.004 \\
\hline 18 (F3/C3) & MzMK Figuren & positiv & 0.913 & 0.834 & 0.087 & 10.983 & 0.087 & 66.467 & 0.020 \\
\hline 19 (C3/P3) & Mzмк Figuren & positiv & 0.967 & 0.935 & 0.033 & 10.919 & 0.033 & 72.041 & 0.006 \\
\hline 20 (P3/O1) & Mzмк Figuren & positiv & 0.912 & 0.832 & 0.088 & 11.394 & 0.088 & 63.234 & 0.024 \\
\hline $21(F z / C z)$ & MZMK Figuren & positiv & 0.980 & 0.960 & 0.020 & 9.906 & 0.020 & 68.282 & 0.003 \\
\hline $22(C z / P z)$ & MZMK Figuren & positiv & 0.914 & 0.835 & 0.086 & 12.966 & 0.086 & 69.035 & 0.025 \\
\hline 23 (Fp2/F4) & MzMK Figuren & positiv & 0.782 & 0.612 & 0.218 & 8.191 & 0.218 & 63.912 & 0.037 \\
\hline 24 (F4/C4) & MzMK Figuren & positiv & 0.903 & 0.816 & 0.097 & 11.284 & 0.097 & 67.931 & 0.023 \\
\hline 25 (C4/P4) & Mzмк Figuren & positiv & 0.934 & 0.872 & 0.066 & 12.574 & 0.066 & 67.967 & 0.018 \\
\hline $26(P 4 / O 2)$ & Mzмк Figuren & positiv & 0.993 & 0.985 & 0.007 & 14.265 & 0.007 & 51.994 & 0.004 \\
\hline 27 (F8/T4) & MZMK Figuren & positiv & 0.960 & 0.922 & 0.040 & 10.674 & 0.040 & 71.694 & 0.007 \\
\hline 28 (T4/T6) & MZMK Figuren & positiv & 0.977 & 0.955 & 0.023 & 12.165 & 0.023 & 67.005 & 0.006 \\
\hline $29(T 5 / 01)$ & Mzмк Figuren & positiv & 0.986 & 0.973 & 0.014 & 12.892 & 0.014 & 55.367 & 0.006 \\
\hline 30 (T6/O2) & MZMK Figuren & positiv & 0.953 & 0.909 & 0.047 & 16.138 & 0.047 & 43.319 & 0.049 \\
\hline
\end{tabular}




\section{Anhang D}

Tabelle $D-2$

Ergebnisse der linearen Regressionsanalysen der gemittelten Zeitpunkte maximaler Kohärenz $\left(\mathrm{M}_{\mathrm{ZMK}}\right)$ und der Größe des positiven Sets ( $N=10 ; 2$. Sitzung, Wörter, positives Testitem)

\begin{tabular}{|c|c|c|c|c|c|c|c|c|c|}
\hline $\begin{array}{c}\text { Elektr.- } \\
\text { Paar }\end{array}$ & $A V$ & Testitem & Multiple $R$ & $R^{2}$ & Sign. $F$ & Slope & $p$ (sign.) & Intercept & $p$ (sign.) \\
\hline $1(F p 1 / F 7)$ & Mzмк Wörter & positiv & 0.926 & 0.857 & 0.074 & 4.313 & 0.074 & 70.519 & 0.002 \\
\hline 2 (F7/F3) & MzMK Wörter & positiv & 0.992 & 0.985 & 0.008 & 7.686 & 0.008 & 58.164 & 0.001 \\
\hline 3 (F3/Fz) & MzмK Wörter & positiv & 0.974 & 0.949 & 0.026 & 7.538 & 0.026 & 62.379 & 0.003 \\
\hline $4(F z / F 4)$ & Mzмк Wörter & positiv & 0.988 & 0.975 & 0.013 & 5.941 & 0.013 & 62.917 & 0.001 \\
\hline 5 (F4/F8) & Mzмк Wörter & positiv & 0.977 & 0.954 & 0.023 & 4.428 & 0.023 & 65.056 & 0.001 \\
\hline $6($ (T3/C3) & MzMK Wörter & positiv & 0.797 & 0.635 & 0.203 & 2.777 & 0.203 & 69.604 & 0.003 \\
\hline $7(C 3 / C z)$ & Mzмк Wörter & positiv & 0.844 & 0.712 & 0.156 & 6.226 & 0.156 & 65.990 & 0.013 \\
\hline $8(C z / C 4)$ & Mzмk Wörter & positiv & 0.916 & 0.840 & 0.084 & 6.965 & 0.084 & 68.671 & 0.007 \\
\hline 9 (C4/T4) & Mzмk Wörter & positiv & 0.598 & 0.357 & 0.402 & 3.478 & 0.402 & 68.908 & 0.017 \\
\hline $10(T 5 / P 3)$ & MzмK Wörter & positiv & 0.710 & 0.504 & 0.290 & 3.608 & 0.290 & 67.386 & 0.010 \\
\hline $11(P 3 / P z)$ & MzMK Wörter & positiv & 0.760 & 0.578 & 0.240 & 3.882 & 0.240 & 71.457 & 0.008 \\
\hline $12(P z / P 4)$ & Mzмk Wörter & positiv & 0.972 & 0.945 & 0.028 & 6.260 & 0.028 & 69.208 & 0.002 \\
\hline 13 (P4/T6) & Mzмк Wörter & positiv & 0.877 & 0.770 & 0.123 & 4.027 & 0.123 & 65.554 & 0.004 \\
\hline 14 (Fp2/F8) & Mzмк Wörter & positiv & 0.986 & 0.972 & 0.014 & 6.441 & 0.014 & 64.807 & 0.001 \\
\hline 15 (F7/T3) & Mzмк Wörter & positiv & 0.992 & 0.984 & 0.008 & 8.095 & 0.008 & 61.311 & 0.001 \\
\hline 16 (T3/T5) & MzмK Wörter & positiv & 0.974 & 0.948 & 0.026 & 7.057 & 0.026 & 60.619 & 0.003 \\
\hline 17 (Fp1/F3) & Mzмк Wörter & positiv & 0.951 & 0.904 & 0.049 & 6.657 & 0.049 & 59.594 & 0.005 \\
\hline 18 (F3/C3) & MzмK Wörter & positiv & 0.864 & 0.747 & 0.136 & 6.935 & 0.136 & 63.927 & 0.015 \\
\hline 19 (C3/P3) & MzмK Wörter & positiv & 0.850 & 0.723 & 0.150 & 7.133 & 0.150 & 65.575 & 0.017 \\
\hline 20 (P3/O1) & Mzмк Wörter & positiv & 0.394 & 0.155 & 0.606 & 0.988 & 0.606 & 72.300 & 0.004 \\
\hline $21(F z / C z)$ & MZMK Wörter & positiv & 0.907 & 0.823 & 0.093 & 5.509 & 0.093 & 66.044 & 0.006 \\
\hline $22(C z / P z)$ & Mzмк Wörter & positiv & 0.755 & 0.570 & 0.245 & 5.610 & 0.245 & 70.392 & 0.018 \\
\hline 23 (Fp2/F4) & Mzмк Wörter & positiv & 0.973 & 0.946 & 0.028 & 6.755 & 0.028 & 61.138 & 0.003 \\
\hline 24 (F4/C4) & MZMK Wörter & positiv & 0.912 & 0.831 & 0.088 & 6.615 & 0.088 & 65.299 & 0.008 \\
\hline 25 (C4/P4) & MzмK Wörter & positiv & 0.940 & 0.884 & 0.060 & 7.636 & 0.060 & 65.135 & 0.007 \\
\hline $26(P 4 / O 2)$ & Mzмk Wörter & positiv & 0.975 & 0.950 & 0.025 & 3.395 & 0.025 & 68.075 & 0.001 \\
\hline 27 (F8/T4) & Mzмк Wörter & positiv & 0.835 & 0.697 & 0.165 & 6.529 & 0.165 & 65.750 & 0.016 \\
\hline $28(T 4 / T 6)$ & MzMK Wörter & positiv & 0.888 & 0.788 & 0.112 & 4.627 & 0.112 & 63.926 & 0.005 \\
\hline $29(T 5 / 01)$ & Mzмk Wörter & positiv & 0.758 & 0.574 & 0.242 & 3.047 & 0.242 & 68.245 & 0.006 \\
\hline 30 (T6/O2) & Mzмк Wörter & positiv & 0.485 & 0.235 & 0.515 & 2.079 & 0.515 & 66.575 & 0.012 \\
\hline
\end{tabular}




\section{Anhang D}

Tabelle $D-3$

Ergebnisse der linearen Regressionsanalysen der gemittelten Zeitpunkte maximaler Kohärenz $\left(\mathrm{M}_{\mathrm{ZMK}}\right)$ und der Größe des positiven Sets ( $N=10 ; 2$. Sitzung, Figuren, negatives Testitem)

\begin{tabular}{|c|c|c|c|c|c|c|c|c|c|}
\hline $\begin{array}{c}\text { Elektr.- } \\
\text { Paar }\end{array}$ & $A V$ & Testitem & Multiple $R$ & $R^{2}$ & Sign. $F$ & Slope & $p$ (sign.) & Intercept & $p$ (sign.) \\
\hline 1 (Fp1/F7) & MzMK Figuren & negativ & 0.971 & 0.943 & 0.029 & 19.715 & 0.029 & 59.036 & 0.024 \\
\hline 2 (F7/F3) & MZMK Figuren & negativ & 0.959 & 0.919 & 0.041 & 16.945 & 0.041 & 61.540 & 0.024 \\
\hline $3(F 3 / F z)$ & Mzмк Figuren & negativ & 0.976 & 0.953 & 0.024 & 17.063 & 0.024 & 64.637 & 0.013 \\
\hline $4(F z / F 4)$ & Mzмк Figuren & negativ & 0.961 & 0.924 & 0.039 & 13.359 & 0.039 & 69.363 & 0.011 \\
\hline 5 (F4/F8) & MZMK Figuren & negativ & 0.945 & 0.893 & 0.055 & 14.801 & 0.055 & 64.546 & 0.023 \\
\hline 6 (T3/C3) & Mzмк Figuren & negativ & 0.959 & 0.920 & 0.041 & 16.898 & 0.041 & 64.985 & 0.021 \\
\hline $7(C 3 / C z)$ & Mzмk Figuren & negativ & 0.981 & 0.962 & 0.019 & 15.534 & 0.019 & 72.282 & 0.007 \\
\hline $8(\mathrm{Cz} / \mathrm{C} 4)$ & MZMK Figuren & negativ & 0.984 & 0.969 & 0.016 & 15.627 & 0.016 & 74.336 & 0.005 \\
\hline $9(C 4 / T 4)$ & MZMK Figuren & negativ & 0.999 & 0.998 & 0.001 & 17.186 & 0.001 & 66.873 & 0.001 \\
\hline 10 (T5/P3) & Mzмк Figuren & negativ & 0.960 & 0.921 & 0.041 & 14.935 & 0.041 & 62.318 & 0.018 \\
\hline $11(P 3 / P z)$ & MzMK Figuren & negativ & 0.999 & 0.998 & 0.001 & 16.776 & 0.001 & 65.989 & 0.001 \\
\hline 12(Pz/P4) & MzMK Figuren & negativ & 0.997 & 0.994 & 0.003 & 18.322 & 0.003 & 71.958 & 0.001 \\
\hline $13(P 4 / T 6)$ & MZMK Figuren & negativ & 0.963 & 0.926 & 0.038 & 16.525 & 0.038 & 56.306 & 0.025 \\
\hline 14 (Fp2/F8) & MzMK Figuren & negativ & 0.996 & 0.991 & 0.004 & 21.354 & 0.004 & 55.689 & 0.005 \\
\hline 15 (F7/T3) & MZMK Figuren & negativ & 0.971 & 0.943 & 0.029 & 15.157 & 0.029 & 73.873 & 0.009 \\
\hline 16 (T3/T5) & Mzмк Figuren & negativ & 0.992 & 0.984 & 0.008 & 17.442 & 0.008 & 62.693 & 0.005 \\
\hline 17 (Fp1/F3) & Mzмк Figuren & negativ & 0.996 & 0.992 & 0.004 & 20.777 & 0.004 & 50.463 & 0.005 \\
\hline 18 (F3/C3) & MZMK Figuren & negativ & 0.954 & 0.910 & 0.046 & 14.210 & 0.046 & 74.717 & 0.013 \\
\hline 19 (C3/P3) & Mzмк Figuren & negativ & 0.909 & 0.826 & 0.091 & 16.273 & 0.091 & 75.318 & 0.035 \\
\hline 20 (P3/O1) & Мzмк Figuren & negativ & 0.933 & 0.870 & 0.067 & 12.777 & 0.067 & 64.874 & 0.021 \\
\hline $21(F z / C z)$ & MZMK Figuren & negativ & 0.983 & 0.967 & 0.017 & 20.033 & 0.017 & 56.762 & 0.016 \\
\hline $22(C z / P z)$ & MZMK Figuren & negativ & 0.980 & 0.961 & 0.020 & 20.971 & 0.020 & 63.905 & 0.016 \\
\hline 23 (Fp2/F4) & $M_{Z M K}$ Figuren & negativ & 0.852 & 0.725 & 0.148 & 12.212 & 0.148 & 66.658 & 0.045 \\
\hline $24(F 4 / C 4)$ & MZMK Figuren & negativ & 0.938 & 0.881 & 0.062 & 17.472 & 0.062 & 64.607 & 0.035 \\
\hline 25 (C4/P4) & Mzмк Figuren & negativ & 0.990 & 0.980 & 0.010 & 21.936 & 0.010 & 66.323 & 0.008 \\
\hline 26 (P4/O2) & Mzмк Figuren & negativ & 0.916 & 0.840 & 0.084 & 16.912 & 0.084 & 60.552 & 0.052 \\
\hline 27 (F8/T4) & MzMK Figuren & negativ & 0.992 & 0.984 & 0.008 & 21.482 & 0.008 & 56.925 & 0.008 \\
\hline 28 (T4/T6) & MZMK Figuren & negativ & 0.995 & 0.990 & 0.005 & 19.042 & 0.005 & 54.416 & 0.005 \\
\hline $29(T 5 / 01)$ & MzmK Figuren & negativ & 0.903 & 0.815 & 0.097 & 14.229 & 0.097 & 62.255 & 0.042 \\
\hline 30 (T6/O2) & MZMK Figuren & negativ & 0.960 & 0.922 & 0.040 & 16.923 & 0.040 & 54.390 & 0.029 \\
\hline
\end{tabular}




\section{Anhang D}

Tabelle $D-4$

Ergebnisse der linearen Regressionsanalysen der gemittelten Zeitpunkte maximaler Kohärenz $\left(\mathrm{M}_{\mathrm{ZMK}}\right)$ und der Größe des positiven Sets $(N=10 ; 2$. Sitzung, Wörter, negatives Testitem)

\begin{tabular}{|c|c|c|c|c|c|c|c|c|c|}
\hline $\begin{array}{c}\text { Elektr.- } \\
\text { Paar }\end{array}$ & $A V$ & Testitem & Multiple $R$ & $R^{2}$ & Sign. $F$ & Slope & $p$ (sign.) & Intercept & $p$ (sign.) \\
\hline 1 (Fp1/F7) & Mzмk Wörter & negativ & 0.965 & 0.930 & 0.035 & 6.118 & 0.035 & 67.857 & 0.002 \\
\hline 2 (F7/F3) & Mzмк Wörter & negativ & 0.952 & 0.906 & 0.048 & 6.818 & 0.048 & 64.950 & 0.004 \\
\hline $3(F 3 / F z)$ & Mzмк Wörter & negativ & 0.969 & 0.938 & 0.031 & 6.991 & 0.031 & 65.654 & 0.003 \\
\hline $4(F z / F 4)$ & Mzмк Wörter & negativ & 0.759 & 0.576 & 0.241 & 3.547 & 0.241 & 71.526 & 0.007 \\
\hline 5 (F4/F8) & MzMK Wörter & negativ & 0.814 & 0.662 & 0.186 & 5.749 & 0.186 & 65.969 & 0.014 \\
\hline $6($ T3/C3) & Mzмk Wörter & negativ & 0.982 & 0.965 & 0.018 & 6.559 & 0.018 & 69.831 & 0.001 \\
\hline $7(C 3 / C z)$ & Mzмк Wörter & negativ & 0.820 & 0.672 & 0.180 & 3.210 & 0.180 & 82.202 & 0.003 \\
\hline $8(\mathrm{Cz} / \mathrm{C} 4)$ & MzMK Wörter & negativ & 0.828 & 0.686 & 0.172 & 3.666 & 0.172 & 78.582 & 0.004 \\
\hline $9(C 4 / T 4)$ & Mzмk Wörter & negativ & 0.861 & 0.740 & 0.140 & 4.374 & 0.140 & 75.756 & 0.004 \\
\hline $10(T 5 / P 3)$ & Mzмk Wörter & negativ & 0.972 & 0.944 & 0.028 & 4.324 & 0.028 & 68.573 & 0.001 \\
\hline $11(P 3 / P z)$ & Mzмk Wörter & negativ & 0.984 & 0.969 & 0.016 & 5.435 & 0.016 & 76.378 & 0.001 \\
\hline $12(P z / P 4)$ & Mzмk Wörter & negativ & 0.999 & 0.997 & 0.001 & 6.624 & 0.001 & 72.817 & 0.000 \\
\hline $13(P 4 / T 6)$ & Mzмk Wörter & negativ & 0.976 & 0.952 & 0.024 & 3.058 & 0.024 & 74.891 & 0.000 \\
\hline 14 (Fp2/F8) & Mzмк Wörter & negativ & 0.983 & 0.966 & 0.017 & 8.701 & 0.017 & 60.405 & 0.003 \\
\hline 15 (F7/T3) & MzMK Wörter & negativ & 0.876 & 0.767 & 0.124 & 4.404 & 0.124 & 71.451 & 0.004 \\
\hline 16 (T3/T5) & Mzмk Wörter & negativ & 0.907 & 0.822 & 0.093 & 3.363 & 0.093 & 73.699 & 0.002 \\
\hline 17 (Fp1/F3) & Mzмк Wörter & negativ & 0.969 & 0.940 & 0.031 & 6.280 & 0.031 & 62.919 & 0.002 \\
\hline 18 (F3/C3) & Mzмk Wörter & negativ & 0.962 & 0.925 & 0.038 & 2.828 & 0.038 & 81.215 & 0.000 \\
\hline 19 (C3/P3) & Mzмk Wörter & negativ & 0.719 & 0.517 & 0.281 & 3.754 & 0.281 & 81.577 & 0.007 \\
\hline 20 (P3/O1) & Mzмk Wörter & negativ & 0.962 & 0.926 & 0.038 & 4.736 & 0.038 & 70.335 & 0.001 \\
\hline $21(F z / C z)$ & Mzмk Wörter & negativ & 0.958 & 0.918 & 0.042 & 2.322 & 0.042 & 77.940 & 0.000 \\
\hline $22(C z / P z)$ & Mzмk Wörter & negativ & 0.922 & 0.850 & 0.078 & 5.465 & 0.078 & 74.213 & 0.004 \\
\hline 23 (Fp2/F4) & MZмк Wörter & negativ & 0.978 & 0.957 & 0.022 & 4.525 & 0.022 & 68.050 & 0.001 \\
\hline $24(F 4 / C 4)$ & Mzмk Wörter & negativ & 0.863 & 0.745 & 0.137 & 4.683 & 0.137 & 69.855 & 0.006 \\
\hline 25 (C4/P4) & Mzмк Wörter & negativ & 0.961 & 0.923 & 0.039 & 3.968 & 0.039 & 82.497 & 0.001 \\
\hline $26(P 4 / O 2)$ & Mzмk Wörter & negativ & 0.978 & 0.957 & 0.022 & 6.818 & 0.022 & 67.741 & 0.002 \\
\hline 27 (F8/T4) & Mzмk Wörter & negativ & 0.797 & 0.635 & 0.203 & 4.458 & 0.203 & 75.324 & 0.008 \\
\hline 28 (T4/T6) & MzMK Wörter & negativ & 0.987 & 0.975 & 0.013 & 5.345 & 0.013 & 65.477 & 0.001 \\
\hline $29(T 5 / 01)$ & Mzмк Wörter & negativ & 0.947 & 0.897 & 0.053 & 5.449 & 0.053 & 64.925 & 0.003 \\
\hline 30 (T6/O2) & MzMK Wörter & negativ & 0.862 & 0.743 & 0.138 & 3.794 & 0.138 & 70.995 & 0.004 \\
\hline
\end{tabular}




\section{Anhang $\mathrm{E}$}

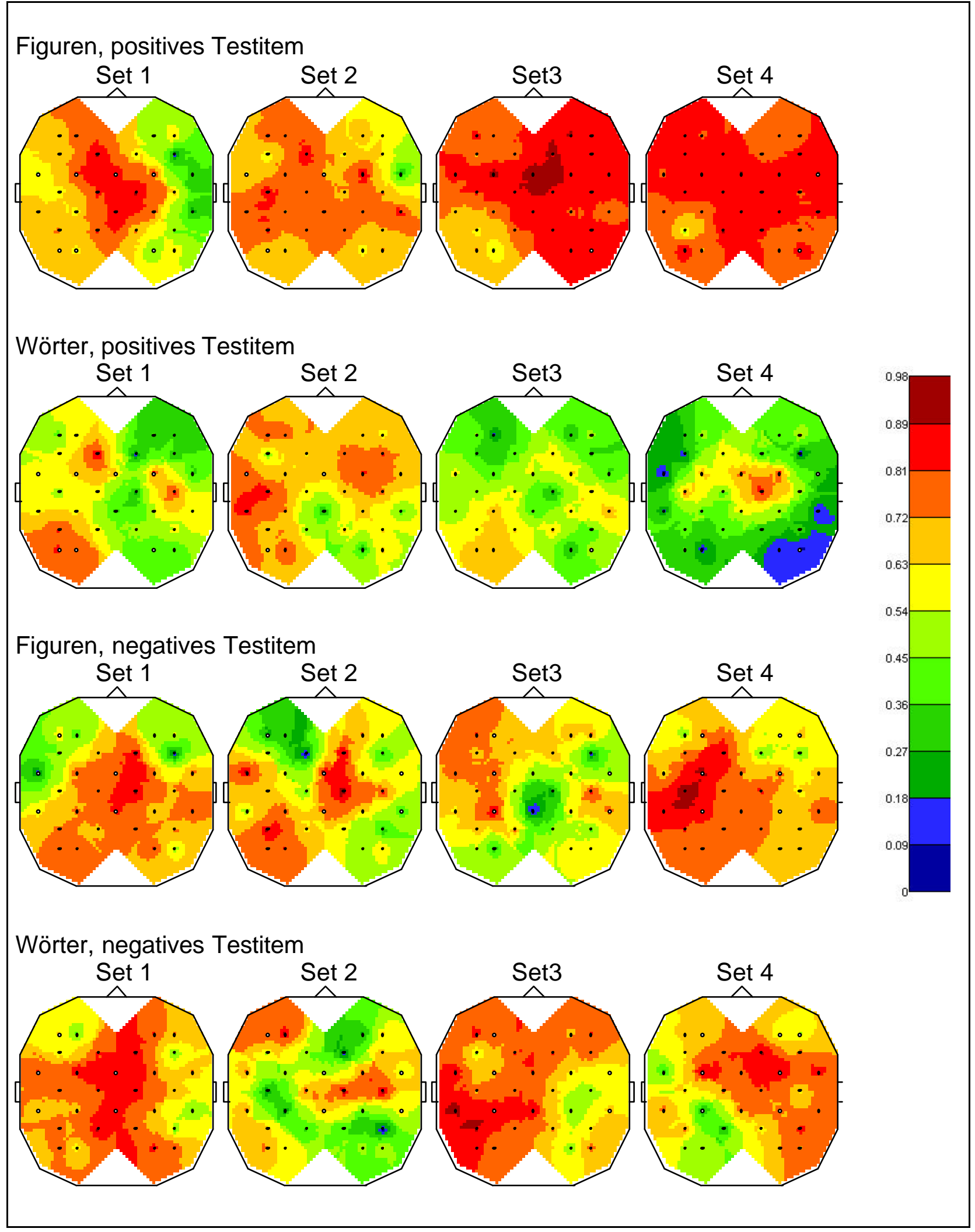

Abbildung E-1. Korrelationen der Zeitpunkte maximaler Kohärenz mit den Antwortlatenzen: Korrelationskoeffizienten ( $\mathrm{N}=10 ; 2$. Sitzung) 


\section{Anhang E}

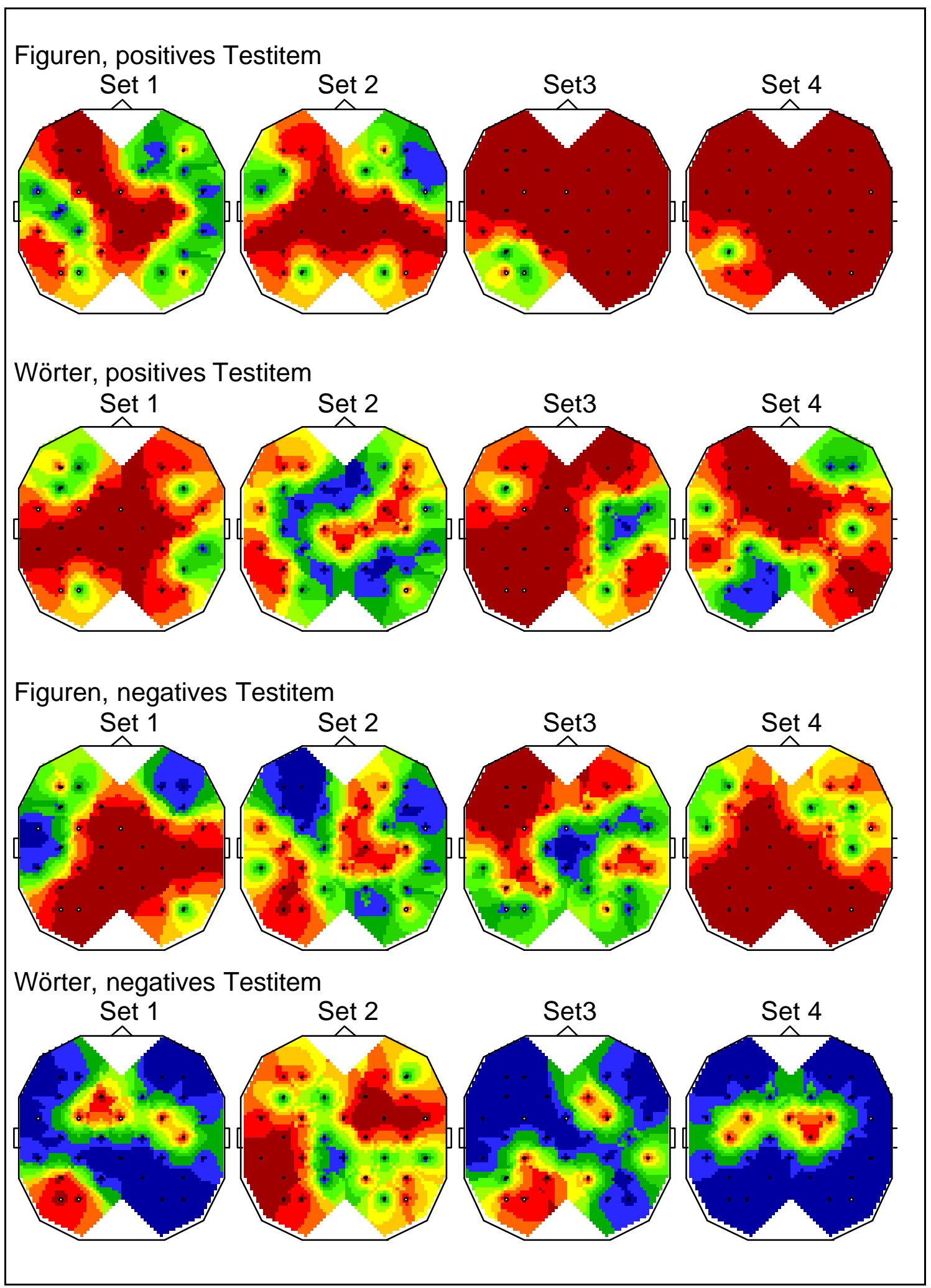

Abbildung E-2. Korrelationen der Zeitpunkte maximaler Kohärenz mit den Antwortlatenzen: Irrtumswahrscheinlichkeiten unter $5 \%$ (in der Abbildung rot hervorgehoben; $N=10 ; 2$. Sitzung) 
Tabelle E-1

Korrelationen der Zeitpunkte maximaler Kohärenz mit den Antwortlatenzen:

Korrelationskoeffizienten positives Testitem ( $N=10 ; 2$. Sitzung)

\begin{tabular}{|c|c|c|c|c|c|c|c|c|c|}
\hline & & Korr & tionskot & zienten: $f$ & uren & Korr & tionsko & zienten: & rter \\
\hline $\begin{array}{l}\text { Elektr.- } \\
\text { Paar }\end{array}$ & Testitem & Set 1 & Set 2 & Set 3 & Set 4 & Set 1 & Set 2 & Set 3 & Set 4 \\
\hline $1(F p 1 / F 7)$ & positiv & 0.684 & 0.656 & 0.752 & 0.964 & 0.771 & 0.769 & 0.838 & 0.518 \\
\hline 2 (F7/F3) & positiv & 0.744 & 0.858 & 0.608 & 0.500 & 0.803 & 0.704 & 0.901 & 0.776 \\
\hline 3 (F3/Fz) & positiv & 0.831 & 0.748 & 0.802 & 0.910 & 0.912 & 0.387 & 0.753 & 0.360 \\
\hline $4(F z / F 4)$ & positiv & 0.727 & 0.791 & 0.871 & 0.807 & 0.766 & 0.422 & 0.761 & 0.620 \\
\hline 5 (F4/F8) & positiv & 0.440 & 0.672 & 0.839 & 0.755 & 0.496 & 0.109 & 0.651 & 0.850 \\
\hline $6(T 3 / C 3)$ & positiv & 0.602 & 0.889 & 0.809 & 0.867 & 0.762 & 0.280 & 0.704 & 0.662 \\
\hline $7(C 3 / C z)$ & positiv & 0.824 & 0.757 & 0.860 & 0.838 & 0.803 & 0.800 & 0.784 & 0.743 \\
\hline $8(C z / C 4)$ & positiv & 0.895 & 0.660 & 0.888 & 0.834 & 0.847 & 0.891 & 0.684 & 0.799 \\
\hline $9(C 4 / T 4)$ & positiv & 0.634 & 0.721 & 0.847 & 0.784 & 0.700 & 0.921 & 0.543 & 0.614 \\
\hline 10 (T5/P3) & positiv & 0.656 & 0.547 & 0.698 & 0.868 & 0.598 & 0.562 & 0.516 & 0.644 \\
\hline $11(P 3 / P z)$ & positiv & 0.906 & 0.889 & 0.735 & 0.818 & 0.831 & 0.518 & 0.822 & 0.781 \\
\hline 12(Pz/P4) & positiv & 0.575 & 0.583 & 0.892 & 0.762 & 0.889 & 0.234 & 0.822 & 0.891 \\
\hline 13 (P4/T6) & positiv & 0.126 & 0.624 & 0.837 & 0.810 & 0.445 & 0.719 & 0.786 & 0.769 \\
\hline 14 (Fp2/F8) & positiv & 0.675 & 0.720 & 0.839 & 0.852 & 0.839 & 0.636 & 0.802 & 0.730 \\
\hline 15 (F7/T3) & positiv & 0.687 & 0.774 & 0.699 & 0.817 & 0.671 & 0.734 & 0.924 & 0.769 \\
\hline 16 (T3/T5) & positiv & 0.517 & 0.607 & 0.868 & 0.787 & 0.836 & 0.757 & 0.926 & 0.390 \\
\hline 17 (Fp1/F3) & positiv & 0.567 & 0.604 & 0.508 & 0.714 & 0.584 & 0.630 & 0.740 & 0.521 \\
\hline 18 (F3/C3) & positiv & 0.575 & 0.700 & 0.714 & 0.721 & 0.687 & 0.284 & 0.894 & 0.286 \\
\hline 19 (C3/P3) & positiv & 0.812 & 0.763 & 0.947 & 0.889 & 0.870 & 0.523 & 0.737 & 0.904 \\
\hline $20(P 3 / 01)$ & positiv & 0.718 & 0.727 & 0.748 & 0.791 & 0.459 & 0.923 & 0.758 & 0.676 \\
\hline $21(F z / C z)$ & positiv & 0.854 & 0.812 & 0.876 & 0.826 & 0.842 & 0.700 & 0.876 & 0.784 \\
\hline $22(C z / P z)$ & positiv & 0.836 & 0.705 & 0.966 & 0.912 & 0.883 & 0.511 & 0.813 & 0.806 \\
\hline 23 (Fp2/F4) & positiv & 0.430 & 0.561 & 0.834 & 0.744 & 0.857 & 0.556 & 0.451 & 0.734 \\
\hline 24 (F4/C4) & positiv & 0.766 & 0.789 & 0.783 & 0.882 & 0.740 & 0.481 & 0.463 & 0.756 \\
\hline 25 (C4/P4) & positiv & 0.813 & 0.916 & 0.885 & 0.884 & 0.785 & 0.761 & 0.629 & 0.908 \\
\hline 26 (P4/O2) & positiv & 0.530 & 0.676 & 0.906 & 0.752 & 0.828 & 0.276 & 0.690 & 0.587 \\
\hline 27 (F8/T4) & positiv & 0.205 & 0.849 & 0.759 & 0.818 & 0.494 & 0.627 & 0.690 & 0.758 \\
\hline 28 (T4/T6) & positiv & 0.288 & 0.232 & 0.833 & 0.908 & 0.741 & 0.550 & 0.588 & 0.873 \\
\hline $29(T 5 / 01)$ & positiv & 0.687 & 0.829 & 0.842 & 0.830 & 0.661 & 0.805 & 0.885 & 0.717 \\
\hline 30 (T6/O2) & positiv & 0.686 & 0.593 & 0.857 & 0.798 & 0.735 & 0.670 & 0.832 & 0.552 \\
\hline
\end{tabular}


Tabelle E-2

Korrelationen der Zeitpunkte maximaler Kohärenz mit den Antwortlatenzen:

Korrelationskoeffizienten negatives Testitem ( $N=10 ; 2$. Sitzung)

\begin{tabular}{|c|c|c|c|c|c|c|c|c|c|}
\hline \multirow{2}{*}{$\begin{array}{c}\text { Elektr.- } \\
\text { Paar }\end{array}$} & \multirow[b]{2}{*}{ Testitem } & \multicolumn{4}{|c|}{ Korrelationskoeffizienten: Figuren } & \multicolumn{4}{|c|}{ Korrelationskoeffizienten: Wörter } \\
\hline & & Set 1 & Set 2 & Set 3 & Set 4 & Set 1 & Set 2 & Set 3 & Set 4 \\
\hline 1 (Fp1/F7) & negativ & 0.784 & 0.771 & 0.571 & 0.757 & 0.828 & 0.677 & 0.716 & 0.385 \\
\hline 2 (F7/F3) & negativ & 0.744 & 0.899 & 0.720 & 0.819 & 0.821 & 0.633 & 0.586 & 0.436 \\
\hline 3 (F3/Fz) & negativ & 0.703 & 0.606 & 0.744 & 0.776 & 0.570 & 0.497 & 0.635 & 0.392 \\
\hline $4(F z / F 4)$ & negativ & 0.737 & 0.608 & 0.493 & 0.816 & 0.469 & 0.662 & 0.672 & 0.295 \\
\hline 5 (F4/F8) & negativ & 0.762 & 0.324 & 0.447 & 0.664 & 0.592 & 0.642 & 0.360 & 0.357 \\
\hline $6(T 3 / C 3)$ & negativ & 0.621 & 0.707 & 0.692 & 0.983 & 0.483 & 0.826 & 0.519 & 0.770 \\
\hline $7(C 3 / C z)$ & negativ & 0.761 & 0.421 & 0.522 & 0.749 & 0.572 & 0.584 & 0.567 & 0.383 \\
\hline $8(C z / C 4)$ & negativ & 0.915 & 0.973 & 0.266 & 0.841 & 0.198 & 0.685 & 0.194 & 0.864 \\
\hline $9(C 4 / T 4)$ & negativ & 0.715 & 0.857 & 0.837 & 0.602 & 0.853 & 0.760 & 0.574 & 0.511 \\
\hline $10(T 5 / P 3)$ & negativ & 0.478 & 0.626 & 0.674 & 0.785 & 0.562 & 0.428 & 0.350 & 0.123 \\
\hline $11(P 3 / P z)$ & negativ & 0.713 & 0.068 & 0.669 & 0.878 & 0.889 & 0.621 & 0.387 & 0.539 \\
\hline 12(Pz/P4) & negativ & 0.905 & 0.875 & 0.716 & 0.455 & 0.113 & 0.730 & 0.661 & 0.270 \\
\hline $13(P 4 / T 6)$ & negativ & 0.152 & 0.345 & 0.183 & 0.438 & 0.289 & 0.821 & 0.318 & 0.036 \\
\hline 14 (Fp2/F8) & negativ & 0.483 & 0.713 & 0.630 & 0.692 & 0.363 & 0.692 & 0.541 & 0.056 \\
\hline 15 (F7/T3) & negativ & 0.616 & 0.498 & 0.627 & 0.896 & 0.622 & 0.851 & 0.467 & 0.172 \\
\hline 16 (T3/T5) & negativ & 0.200 & 0.922 & 0.800 & 0.626 & 0.602 & 0.813 & 0.582 & 0.068 \\
\hline 17 (Fp1/F3) & negativ & 0.741 & 0.828 & 0.299 & 0.746 & 0.766 & 0.874 & 0.636 & 0.075 \\
\hline 18 (F3/C3) & negativ & 0.752 & 0.793 & 0.919 & 0.875 & 0.625 & 0.738 & 0.685 & 0.408 \\
\hline 19 (C3/P3) & negativ & 0.806 & 0.598 & 0.826 & 0.921 & 0.681 & 0.712 & 0.395 & 0.705 \\
\hline 20 (P3/O1) & negativ & 0.402 & 0.338 & 0.724 & 0.769 & 0.628 & 0.782 & 0.142 & 0.529 \\
\hline $21(F z / C z)$ & negativ & 0.835 & 0.786 & 0.087 & 0.804 & 0.345 & 0.235 & 0.362 & 0.566 \\
\hline $22(C z / P z)$ & negativ & 0.784 & 0.927 & 0.532 & 0.752 & 0.670 & 0.664 & 0.575 & 0.803 \\
\hline 23 (Fp2/F4) & negativ & 0.843 & 0.516 & 0.637 & 0.642 & 0.386 & 0.237 & 0.195 & 0.093 \\
\hline 24 (F4/C4) & negativ & 0.666 & 0.654 & 0.658 & 0.640 & 0.551 & 0.622 & 0.630 & 0.436 \\
\hline 25 (C4/P4) & negativ & 0.769 & 0.579 & 0.615 & 0.798 & 0.841 & 0.823 & 0.681 & 0.831 \\
\hline $26(\mathrm{P} 4 / \mathrm{O} 2)$ & negativ & 0.516 & 0.741 & 0.719 & 0.643 & 0.374 & 0.656 & 0.220 & 0.422 \\
\hline 27 (F8/T4) & negativ & 0.821 & 0.405 & 0.776 & 0.854 & 0.537 & 0.377 & 0.749 & 0.005 \\
\hline 28 (T4/T6) & negativ & 0.806 & 0.618 & 0.476 & 0.699 & 0.301 & 0.651 & 0.318 & 0.331 \\
\hline $29(\mathrm{~T} / \mathrm{O} / \mathrm{1})$ & negativ & 0.643 & 0.270 & 0.846 & 0.441 & 0.390 & 0.819 & 0.493 & 0.234 \\
\hline 30 (T6/O2) & negativ & 0.572 & 0.591 & 0.771 & 0.705 & 0.303 & 0.580 & 0.517 & 0.621 \\
\hline
\end{tabular}


Tabelle E-3

Korrelationen der Zeitpunkte maximaler Kohärenz mit den Antwortlatenzen: Irrtumswahrscheinlichkeiten positives Testitem ( $\mathrm{N}=10 ; 2$. Sitzung)

\begin{tabular}{|c|c|c|c|c|c|c|c|c|c|}
\hline \multirow{2}{*}{$\begin{array}{c}\text { Elektr.- } \\
\text { Paar }\end{array}$} & \multirow[b]{2}{*}{ Testitem } & \multicolumn{4}{|c|}{ Signifikanzen: Figuren } & \multicolumn{4}{|c|}{ Signifikanzen: Wörter } \\
\hline & & Set 1 & Set 2 & Set 3 & Set 4 & Set 1 & Set 2 & Set 3 & Set 4 \\
\hline 1 (Fp1/F7) & positiv & 0.029 & 0.039 & 0.012 & 0.000 & 0.009 & 0.009 & 0.002 & 0.125 \\
\hline 2 (F7/F3) & positiv & 0.014 & 0.002 & 0.062 & 0.141 & 0.005 & 0.023 & 0.000 & 0.008 \\
\hline 3 (F3/Fz) & positiv & 0.003 & 0.013 & 0.005 & 0.000 & 0.000 & 0.269 & 0.012 & 0.307 \\
\hline $4(F z / F 4)$ & positiv & 0.017 & 0.006 & 0.001 & 0.005 & 0.010 & 0.225 & 0.011 & 0.056 \\
\hline 5 (F4/F8) & positiv & 0.203 & 0.033 & 0.002 & 0.012 & 0.144 & 0.765 & 0.041 & 0.002 \\
\hline $6(T 3 / C 3)$ & positiv & 0.066 & 0.001 & 0.005 & 0.001 & 0.010 & 0.433 & 0.023 & 0.037 \\
\hline $7(C 3 / C z)$ & positiv & 0.003 & 0.011 & 0.001 & 0.002 & 0.005 & 0.005 & 0.007 & 0.014 \\
\hline $8(C z / C 4)$ & positiv & 0.000 & 0.038 & 0.001 & 0.003 & 0.002 & 0.001 & 0.029 & 0.006 \\
\hline $9(C 4 / T 4)$ & positiv & 0.049 & 0.019 & 0.002 & 0.007 & 0.024 & 0.000 & 0.105 & 0.059 \\
\hline $10($ T5/P3) & positiv & 0.040 & 0.102 & 0.025 & 0.001 & 0.068 & 0.091 & 0.127 & 0.044 \\
\hline $11(P 3 / P z)$ & positiv & 0.000 & 0.001 & 0.016 & 0.004 & 0.003 & 0.125 & 0.004 & 0.008 \\
\hline 12(Pz/P4) & positiv & 0.082 & 0.077 & 0.001 & 0.010 & 0.001 & 0.516 & 0.004 & 0.001 \\
\hline $13(P 4 / T 6)$ & positiv & 0.728 & 0.054 & 0.003 & 0.005 & 0.197 & 0.019 & 0.007 & 0.009 \\
\hline 14 (Fp2/F8) & positiv & 0.032 & 0.019 & 0.002 & 0.002 & 0.002 & 0.048 & 0.005 & 0.016 \\
\hline 15 (F7/T3) & positiv & 0.028 & 0.009 & 0.024 & 0.004 & 0.034 & 0.016 & 0.000 & 0.009 \\
\hline 16 (T3/T5) & positiv & 0.126 & 0.063 & 0.001 & 0.007 & 0.003 & 0.011 & 0.000 & 0.265 \\
\hline 17 (Fp1/F3) & positiv & 0.088 & 0.064 & 0.134 & 0.020 & 0.076 & 0.051 & 0.015 & 0.123 \\
\hline 18 (F3/C3) & positiv & 0.082 & 0.024 & 0.020 & 0.019 & 0.028 & 0.426 & 0.000 & 0.423 \\
\hline 19 (C3/P3) & positiv & 0.004 & 0.010 & 0.000 & 0.001 & 0.001 & 0.121 & 0.015 & 0.000 \\
\hline 20 (P3/O1) & positiv & 0.019 & 0.017 & 0.013 & 0.006 & 0.182 & 0.000 & 0.011 & 0.032 \\
\hline $21(F z / C z)$ & positiv & 0.002 & 0.004 & 0.001 & 0.003 & 0.002 & 0.024 & 0.001 & 0.007 \\
\hline $22(C z / P z)$ & positiv & 0.003 & 0.023 & 0.000 & 0.000 & 0.001 & 0.131 & 0.004 & 0.005 \\
\hline 23 (Fp2/F4) & positiv & 0.215 & 0.092 & 0.003 & 0.014 & 0.002 & 0.095 & 0.191 & 0.016 \\
\hline 24 (F4/C4) & positiv & 0.010 & 0.007 & 0.007 & 0.001 & 0.014 & 0.160 & 0.178 & 0.011 \\
\hline 25 (C4/P4) & positiv & 0.004 & 0.000 & 0.001 & 0.001 & 0.007 & 0.011 & 0.051 & 0.000 \\
\hline 26 (P4/O2) & positiv & 0.115 & 0.032 & 0.000 & 0.012 & 0.003 & 0.441 & 0.027 & 0.075 \\
\hline 27 (F8/T4) & positiv & 0.570 & 0.002 & 0.011 & 0.004 & 0.146 & 0.052 & 0.027 & 0.011 \\
\hline 28 (T4/T6) & positiv & 0.420 & 0.519 & 0.003 & 0.000 & 0.014 & 0.100 & 0.074 & 0.001 \\
\hline $29(T 5 / 01)$ & positiv & 0.028 & 0.003 & 0.002 & 0.003 & 0.037 & 0.005 & 0.001 & 0.020 \\
\hline 30 (T6/O2) & positiv & 0.028 & 0.071 & 0.002 & 0.006 & 0.015 & 0.034 & 0.003 & 0.098 \\
\hline
\end{tabular}


Tabelle E-4

Korrelationen der Zeitpunkte maximaler Kohärenz mit den Antwortlatenzen: Irrtumswahrscheinlichkeiten negatives Testitem ( $N=10 ; 2$. Sitzung)

\begin{tabular}{|c|c|c|c|c|c|c|c|c|c|}
\hline \multirow{2}{*}{\begin{tabular}{|c} 
Elektr.- \\
Paar
\end{tabular}} & \multirow[b]{2}{*}{ Testitem } & \multicolumn{4}{|c|}{ Signifikanzen: Figuren } & \multicolumn{4}{|c|}{ Signifikanzen: Wörter } \\
\hline & & Set 1 & Set 2 & Set 3 & Set 4 & Set 1 & Set 2 & Set 3 & Set 4 \\
\hline 1 (Fp1/F7) & negativ & 0.007 & 0.009 & 0.084 & 0.011 & 0.003 & 0.032 & 0.020 & 0.272 \\
\hline 2 (F7/F3) & negativ & 0.014 & 0.000 & 0.019 & 0.004 & 0.004 & 0.049 & 0.075 & 0.207 \\
\hline $3(F 3 / F z)$ & negativ & 0.023 & 0.063 & 0.014 & 0.008 & 0.086 & 0.144 & 0.048 & 0.263 \\
\hline $4(F z / F 4)$ & negativ & 0.015 & 0.062 & 0.148 & 0.004 & 0.171 & 0.037 & 0.033 & 0.409 \\
\hline 5 (F4/F8) & negativ & 0.010 & 0.361 & 0.195 & 0.036 & 0.071 & 0.046 & 0.307 & 0.312 \\
\hline $6(T 3 / C 3)$ & negativ & 0.056 & 0.022 & 0.027 & 0.000 & 0.157 & 0.003 & 0.125 & 0.009 \\
\hline $7(C 3 / C z)$ & negativ & 0.011 & 0.225 & 0.121 & 0.013 & 0.084 & 0.076 & 0.087 & 0.275 \\
\hline $8(C z / C 4)$ & negativ & 0.000 & 0.000 & 0.457 & 0.002 & 0.584 & 0.029 & 0.592 & 0.001 \\
\hline $9(C 4 / T 4)$ & negativ & 0.020 & 0.002 & 0.003 & 0.066 & 0.002 & 0.011 & 0.083 & 0.131 \\
\hline $10(T 5 / P 3)$ & negativ & 0.163 & 0.053 & 0.033 & 0.007 & 0.091 & 0.217 & 0.321 & 0.735 \\
\hline $11(P 3 / P z)$ & negativ & 0.021 & 0.851 & 0.034 & 0.001 & 0.001 & 0.056 & 0.269 & 0.108 \\
\hline 12(Pz/P4) & negativ & 0.000 & 0.001 & 0.020 & 0.186 & 0.755 & 0.017 & 0.037 & 0.451 \\
\hline $13(P 4 / T 6)$ & negativ & 0.676 & 0.328 & 0.613 & 0.205 & 0.419 & 0.004 & 0.370 & 0.922 \\
\hline 14 (Fp2/F8) & negativ & 0.158 & 0.021 & 0.051 & 0.027 & 0.303 & 0.027 & 0.106 & 0.878 \\
\hline 15 (F7/T3) & negativ & 0.058 & 0.143 & 0.052 & 0.000 & 0.055 & 0.002 & 0.174 & 0.635 \\
\hline 16 (T3/T5) & negativ & 0.580 & 0.000 & 0.005 & 0.053 & 0.066 & 0.004 & 0.078 & 0.851 \\
\hline 17 (Fp1/F3) & negativ & 0.014 & 0.003 & 0.401 & 0.013 & 0.010 & 0.001 & 0.048 & 0.837 \\
\hline 18 (F3/C3) & negativ & 0.012 & 0.006 & 0.000 & 0.001 & 0.054 & 0.015 & 0.029 & 0.241 \\
\hline 19 (C3/P3) & negativ & 0.005 & 0.068 & 0.003 & 0.000 & 0.030 & 0.021 & 0.259 & 0.023 \\
\hline 20 (P3/O1) & negativ & 0.250 & 0.340 & 0.018 & 0.009 & 0.052 & 0.007 & 0.695 & 0.116 \\
\hline $21(F z / C z)$ & negativ & 0.003 & 0.007 & 0.811 & 0.005 & 0.329 & 0.513 & 0.304 & 0.088 \\
\hline $22(C z / P z)$ & negativ & 0.007 & 0.000 & 0.114 & 0.012 & 0.034 & 0.036 & 0.082 & 0.005 \\
\hline 23 (Fp2/F4) & negativ & 0.002 & 0.127 & 0.048 & 0.045 & 0.271 & 0.509 & 0.589 & 0.798 \\
\hline $24(F 4 / C 4)$ & negativ & 0.035 & 0.040 & 0.039 & 0.046 & 0.099 & 0.055 & 0.051 & 0.208 \\
\hline 25 (C4/P4) & negativ & 0.009 & 0.079 & 0.058 & 0.006 & 0.002 & 0.003 & 0.030 & 0.003 \\
\hline $26(P 4 / O 2)$ & negativ & 0.127 & 0.014 & 0.019 & 0.045 & 0.287 & 0.039 & 0.541 & 0.225 \\
\hline 27 (F8/T4) & negativ & 0.004 & 0.245 & 0.008 & 0.002 & 0.109 & 0.284 & 0.013 & 0.990 \\
\hline $28(T 4 / T 6)$ & negativ & 0.005 & 0.057 & 0.164 & 0.025 & 0.398 & 0.042 & 0.371 & 0.350 \\
\hline $29(T 5 / 01)$ & negativ & 0.045 & 0.451 & 0.002 & 0.202 & 0.265 & 0.004 & 0.147 & 0.516 \\
\hline 30 (T6/O2) & negativ & 0.084 & 0.072 & 0.009 & 0.023 & 0.395 & 0.079 & 0.126 & 0.055 \\
\hline
\end{tabular}




\section{Anhang E}

Tabelle E-5

Signifikante Regressionskoeffizienten aus der linearen Regressionsanalyse der gemittelten Zeitpunkte maximaler Kohärenz ( $S L_{Z M K}$ ) bzw. der gemittelten Antwortlatenzen (SL $\left.L_{L A T}\right)$ und der Größe des positiven Sets ( $N=10 ; 2$. Sitzung)

\begin{tabular}{|c|c|c|c|c|c|c|c|c|}
\hline \multirow[b]{2}{*}{$\begin{array}{c}\text { Elektr.- } \\
\text { Paar }\end{array}$} & \multicolumn{4}{|c|}{ positives Testitem } & \multicolumn{4}{|c|}{ negatives Testitem } \\
\hline & $\begin{array}{l}S L_{Z M K} \\
\text { Figuren }\end{array}$ & $\begin{array}{l}S L_{L A T} \\
\text { Figuren }\end{array}$ & $\begin{array}{l}S L_{Z M K} \\
\text { Wörter }\end{array}$ & $\begin{array}{c}S L_{L A T} \\
\text { Wörter }\end{array}$ & $\begin{array}{l}S L_{Z M K} \\
\text { Figuren }\end{array}$ & $\begin{array}{l}S L_{L A T} \\
\text { Figuren }\end{array}$ & $\begin{array}{l}S L_{\text {ZMK }} \\
\text { Wörter }\end{array}$ & $\begin{array}{l}S L_{L A T} \\
\text { Wörter }\end{array}$ \\
\hline 1 (Fp1/F7) & 9.152 & 56.133 & & 46.006 & 19.715 & 63.019 & 6.118 & 34.151 \\
\hline 2 (F7/F3) & & 56.133 & 7.686 & 46.006 & 16.945 & 63.019 & 6.818 & 34.151 \\
\hline $3(F 3 / F z)$ & & 56.133 & 7.538 & 46.006 & 17.063 & 63.019 & 6.991 & 34.151 \\
\hline 4 (Fz/F4) & & 56.133 & 5.941 & 46.006 & 13.359 & 63.019 & & 34.151 \\
\hline 5 (F4/F8) & 9.835 & 56.133 & 4.428 & 46.006 & & 63.019 & & 34.151 \\
\hline $6(T 3 / C 3)$ & & 56.133 & & 46.006 & 16.898 & 63.019 & 6.559 & 34.151 \\
\hline $7(C 3 / C z)$ & & 56.133 & & 46.006 & 15.534 & 63.019 & & 34.151 \\
\hline $8(C z / C 4)$ & 11.927 & 56.133 & & 46.006 & 15.627 & 63.019 & & 34.151 \\
\hline $9(C 4 / T 4)$ & & 56.133 & & 46.006 & 17.186 & 63.019 & & 34.151 \\
\hline 10 (T5/P3) & 11.855 & 56.133 & & 46.006 & 14.935 & 63.019 & 4.324 & 34.151 \\
\hline $11(P 3 / P z)$ & 9.869 & 56.133 & & 46.006 & 16.776 & 63.019 & 5.435 & 34.151 \\
\hline 12(Pz/P4) & & 56.133 & 6.260 & 46.006 & 18.322 & 63.019 & 6.624 & 34.151 \\
\hline $13(P 4 / T 6)$ & & 56.133 & & 46.006 & 16.525 & 63.019 & 3.058 & 34.151 \\
\hline $14(F p 2 / F 8)$ & 16.269 & 56.133 & 6.441 & 46.006 & 21.354 & 63.019 & 8.701 & 34.151 \\
\hline 15 (F7/T3) & & 56.133 & 8.095 & 46.006 & 15.157 & 63.019 & & 34.151 \\
\hline $16($ (T3/T5) & & 56.133 & 7.057 & 46.006 & 17.442 & 63.019 & & 34.151 \\
\hline $17(F p 1 / F 3)$ & 8.195 & 56.133 & 6.657 & 46.006 & 20.777 & 63.019 & 6.280 & 34.151 \\
\hline 18 (F3/C3) & & 56.133 & & 46.006 & 14.210 & 63.019 & 2.828 & 34.151 \\
\hline 19 (C3/P3) & 10.919 & 56.133 & & 46.006 & & 63.019 & & 34.151 \\
\hline 20 (P3/O1) & & 56.133 & & 46.006 & & 63.019 & 4.736 & 34.151 \\
\hline $21(F z / C z)$ & 9.906 & 56.133 & & 46.006 & 20.033 & 63.019 & 2.322 & 34.151 \\
\hline $22(C z / P z)$ & & 56.133 & & 46.006 & 20.971 & 63.019 & & 34.151 \\
\hline $23(F p 2 / F 4)$ & & 56.133 & 6.755 & 46.006 & & 63.019 & 4.525 & 34.151 \\
\hline $24(F 4 / C 4)$ & & 56.133 & & 46.006 & & 63.019 & & 34.151 \\
\hline 25 (C4/P4) & & 56.133 & & 46.006 & 21.936 & 63.019 & 3.968 & 34.151 \\
\hline $26(P 4 / O 2)$ & 14.265 & 56.133 & 3.395 & 46.006 & & 63.019 & 6.818 & 34.151 \\
\hline 27 (F8/T4) & 10.674 & 56.133 & & 46.006 & 21.482 & 63.019 & & 34.151 \\
\hline $28(T 4 / T 6)$ & 12.165 & 56.133 & & 46.006 & 19.042 & 63.019 & 5.345 & 34.151 \\
\hline $29(T 5 / 01)$ & 12.892 & 56.133 & & 46.006 & & 63.019 & & 34.151 \\
\hline 30 (T6/O2) & 16.138 & 56.133 & & 46.006 & 16.923 & 63.019 & & 34.151 \\
\hline Mittelwert & 11.719 & 56.133 & 6.387 & 46.006 & 17.748 & 63.019 & 5.379 & 34.151 \\
\hline
\end{tabular}




\section{Lebenslauf}

Nuria Vath, geb. Patzlaff

geboren 19.09.1965 in Hamburg

verheiratet, 2 Kinder

Schulausbildung:

- 1972 - 1978

Grundschule in Kiel-Holtenau und in São Paulo, Brasilien

- 1978 - 1979

Hebbel-Gymnasium in Kiel

- 1979 - 1985

Otto-Hahn-Gymnasium in Gifhorn; Abitur

Berufsausbildung:

- 10.1985 - 03.1987

Ausbildung zur Fremdsprachensekretärin an der Hamburger Fremd-

- 31.03.1987 sprachenschule $\mathrm{GmbH}$

Abschluß Diplom

Studium:

- 10.1988 - 03.1995

Studium der Psychologie an der Georg-August-Universität Göttingen (wissenschaftlicher Studiengang); Thema der Diplomarbeit: Erfassung und Vorhersage therapieresistenter Depressionen

- 27.03.1995 Abschluß Diplom

Berufstätigkeit:

- 01.08 .1985 - 30.09.1985

Zeitarbeit als Hilfskraft bei der Firma ADIA, Hamburg

- 01.05.1987 - 30.09.1988

Fremdsprachensekretärin bei der Firma Metelmann \& Co. GmbH, Hamburg

- 01.04.1992 - 19.07.1999

Wissenschaftliche Hilfskraft am Georg-Elias-Müller-Institut für Psychologie der Universität Göttingen

- 01.12.1999 - 14.07.2000 Wissenschaftliche Mitarbeiterin am Institut für Wirtschaftsinformatik II der Universität Göttingen

- Seit 15.01.2001

Wissenschaftliche Mitarbeiterin im Rahmen des DFG-Projekts „Referenzsysteme des räumlichen Gedächtnisses “ am Georg-EliasMüller-Institut für Psychologie der Universität Göttingen 